

LIBRARY
UNIVERSTY OF
CALIFORNIA
SANTA CRUZ




$$
\begin{aligned}
& Q L \\
& 45 \\
& \angle 28 \\
& 1900 a \\
& v .1 \\
& p \neq 1
\end{aligned}
$$


Digitized by the Internet Archive in 2007 with funding from Microsoft Corporation 


\section{A TREATISE ON ZOOLOGY}

Demy 8vo, Cloth, price 15s. net each; or in Paper Covers, price 12s. 6d. net each.

\section{VOLUMES READY}

Part I. (First Fascicle) INTRODUCTION AND PROTOZOA. By Sir RAy LANKeSTER, K.C.B., F.R.S. ; Prof. S. J. Hickson, M.A., F.R.S. ; F. W. Gamrle, D.Sc., F.R.S. ; A. WILI.ET, M.A., D.Sc., F.R.S. ; J. J. Lister, F.R.S.; H. M. Woodcock, D.Sc. ; and the late Prof. WELDON.

Part I. (Second Fascicle) INTRODUCTION AND PROTOZOA. By J. B. FARMER, D.Sc., Ml.A., F.R.S. ; J. J. Lister, F.R.S. ; E. A. Minchis, M.A. ; and S. J. HICKSON, F.R.S.

Part II. THE PORIFERA AND COELENTERA. By Sir RaY Lankester, K.C.B., F.R.S. ; E. A. Minchin, M.A.; G. Herbert Fowlek, B.A., Ph.D. ; anl Gilbert C. BOULNE, M.A.

Part III. THE ECHINODERMA. By F. A. Batheir, M.A., assistel by J. W. Gregory, D.Sc., ancl. E. S. Goodrich, M.A.

Part IV. THE PLATYhelmiA, THE MESOZOA, and THE NEMERTINI. By Prof. BenhaM, D.Sc.

Part V. MOllusca. By Dr. Padl Pilsener.

Part ViI. CRUSTACEA. By W. T. Calman.

Part IX. VERTEBRATA CRANIATA. By E. $s$. GOODRICH, F.R.S. 

A TREATISE ON ZOOLOGY 


\section{TREATISE ON ZOOLOGY}

EDITED BY

\section{Sir RAY LANKESTER}

K.C.B., M.A., LL.D., F.R.S.

HONORARY FELLOW OF EXETER COLLEGE, OXFORD; CORRESPONDFNT OF THE INSTITUTE OF FRANCE; LATE DIRECTOR OF THE NATURAL HISTORY DIPARTMFNTS

OF THE BRITISH MUSEUM

\section{Part I \\ INTRODUCTION AND PROTOZOA}

\section{FIRST FASCICLE}

BY

S. J. HICKSON, F.R.S.

PROFESSOR OF ZOOLOQY, VICTORIA UXIVERSITY OF MANCHESTEH

J. J. LISTER, F.R.S.

FELLOW OF ST. JUHN'S COLLEGE, CAMBRIDGE

F. W. GAMBLE, D.Sc., F.R.S.

ASSISTANT DIRECTOR OF THE ZOOLOGICAL LABORATORIFS, AND

LECTURER IN ZOOLOGY, UNIVERSITY OF MANCHFSTFR

A. WILLEY, M.A., D.Sc., F.R.S. DIRECTOR OF COLOMBO MUSETM, CEYLON

H. M. WOODCOCK, D.Sc.

ASSISTANT TO THE PROFESSOR OF PROTOZOOLOGY IN THE UNIVERSITY OF LONDON

The Late W. F. R. WELDON, F.R.S.

LINACRE PROFESSOR OF COMPARATIVE ANATOMY, ONFORD

AND

E. RAY LANKESTER, K.C.B., F.R.S. 


\title{
TREATISE ON ZOOLOGY
}

\author{
Edwin EDITED BY \\ Sir $/$ RAY, LA N K ESTER \\ K.C.B., M.A., LL.D., F.R.S.
}

IONORARY FELLOW OF EXETER COLLEGE, OXFORD; CORRFSFONIFNT OF THE INSTITUTE OY FRANCE; LATE DIRECTÓR OF TIE NATURAI. HISTORY DEPARTMEN:S

OF THE BRITISI MUSELM

\section{Part I}

\section{INTRODUCTION AND PROTOZOA}

FIRST FASCICLE

BY

S. J. HICKSON, F.R.S.

PIROFESSOR OF ZOOLOGY, VICTORIA UNIVERSITY OF MANCHESTER

J. J. LISTER, F.R.S.

FELLOW OF ST, IOIIN'S COLLEGE, CAMBRIDFF

F. W. GAMBLE, D.Sc., F.R.S.

ASSISTANT DIRLCTOR OF TIF ZOOLOFICAI, LAIOORITORIF, AND LECTUREIR IN ZOOLGGS, INIVIRSITY OF MANIIIFTEA

A. WILlEY, M.A., D.Sc., F.R.S. DIRECTOR OF COLOMHO MUSETM, CFITLN

H. M. WOODCOCK, D.Sc.

AsSISTANT TO THE PROFFSSOR OF PROTOZOOLOGY IN THE LNIVEIRSITV OF LONDON

The Late W. F. R. WELDON, F.R.S.

IINACRF, PROFESSOR OF (OMPARATIVE ANATONY, ONFONI)

$A N D$

E. RAY LANKESTER, K.C.B., F.R.S.

LONDON

ADAM AND CHARLES BLACK

1909 
Exclusive Agents for U.S.A.

STECHERT-HAFNER SERVICE AGENCY, INC.

31 East 10th Street

New York, New York 10003

Sole agents for India:

Today \& Tomorrow's Book Agency, 22-B/5, Criginal Road, Karol Bagh, New Delhi-5 


\section{PREFACE}

TuE two fascicles of the first part of this treatise give a more complete account of the Protozoa than is to be found in any similar work hitherto published. Especial attention has been given to the treatment of those groups - the Sporozoa, Flagellata, and Hæmoflagellata-which have recently acquired so much importance in consequence of the discovery that some of their constituent members are the causes of important diseases in man and animals.

\section{E. RAY LANKFSTER.}

J)ecember 1908. 


\section{CONTENTS}

INTRODUCTION . . . . . . . . . . . PAG

CHAPTER I.-PROTOZOA

Section A.-The Proteomyxa . . . . . . 1

" B.-The HeliozoA . . . . . . . 14

„ C.-The MycetozoA . . . . . 37

" D.-ThE Lobosa . . . . . . . 68

" E.-The Radiolaria . . . . . . 94

" F.-The Mastigophora . . . . . 154

" G.-The Haemoflagellates and Allied FormS . . . . . . 193

APPENDIX A.-Chlamydomyxa AND Labyrinthula . 274 " B.-The XeNophYophoridak . . . 284

INDEX $\quad . \quad$. $\quad . \quad . \quad . \quad . \quad . \quad 287$ 



\section{A TREATISE ON ZOOLOGY.}

\section{INTRODUCTION. ${ }^{1}$}

THERE are certain matters which require brief treatment by way of introduction to the present treatise on Zoology.

The first concerns the limitation of the subject-matter indicated by the term "Zoology," requiring a statement of what living things are here considered as animals and what are excluded from that title. The second concerns the grouping of animals in large series corresponding to the indications afforded by their structure as to their genetic affinities. The method adopted in the present work has been to take large divisions of the Animal series such as are often called "sub-kingdoms" or "phyla" (or in some instances less comprehensive divisions) one by one for systematic description and for more detailed enumeration and justification of the classes, orders, and families recognised than is usual in handbooks of Zoology. These large divisions have been assigned for treatment to separate authors, and in each case the author has given a description of the characters which justify the recognition of the group which he treats as an independent series; to this he has added a more extended discussion of the range of variety in the structure of the forms held to be reasonably considered as members of the series.

A special chapter written by me forms the introduction to volume ii. of this work. It may be regarded as a continuation of the present chapter, and treats of the division of the higher grade of animals, which is called the Metazoa (the lower being the Protozoa), into two branches, the "Parazoa " and the "Enterozoa." It is, however, chiefly occupied with a discussion of the division of the Enterozoa into two grades of higher and lower structural complexity, which are designated respectively the "Enterocoela" and "Coelomocoela." The chief phyla or large branches of the animal pedigree are there enumerated, whilst each is subsequently treated by independent authors.

In the present introductory chapter I have therefore to consider, besides the question as to what distinctions separate animals from 
other living things, the facts which render it necessary to recognise two great primary grades of animals-a lower called the Protozoa and a higher called the Metazoa.

\section{A. The Dividing-Linf between Plants and Animal.s.}

Living things-Bionta-are without difficulty, and by the general agreement of both skilled naturalists and the observant layman, divided into two greatly differing groups or series, the animals or Zoa and the plants or Phyta, and into those two great groups only. The study of the one series is called Zoology, and of the other Phytology, or more usually Botany. It is easy to lay down certain general propositions by which nearly all animals are distinguished from nearly all plants. The distinctions which can be thus indicated all arise from one great difference in the chemical activity of the living substance of an animal as compared with that of a plant. Although the living substance of both animals and plants, to which Hugo von Mohl gave the name Protoplasm, appears in both series in the form of nucleated corpuscles called cells, and although the formal appearances and the range of chemical activities exhibited both by the general protoplasm and by the nuclear structures of the cells of animals and plants are practically identical, yet there is a predominant difference in the habitual exhibition of their activities which separates animals from plants, and has determined the difference of form and activity characteristic of the living things assigned to either of the two groups.

Living protoplasm, whether of animal or plant, undergoes (when the processes of life are not, as they may be for a short or for a very extended period, suspended) constant chemical change, requiring the access of free oxygen to the protoplasm and the consequent oxydation of some of its material-which becomes "wasted" or lost and carried away by diffusion from the living protoplasm. This loss has to be replaced, and the process by which it is replaced is "nutrition"; the material taken by a living thing for the purposes of nutrition is its "food."

The result of nutrition is not limited to the repair of loss in the living thing, but is for a part or the whole of its existence in excess of the loss; so that increase of the bulk of the living material or "growth" is a result. The elements carbon, hydrogen, oxygen, and nitrogen, combined to form molecules of the highest degree of complexity, are the essential constituents of living material. It is these that are oxydised and wasted and pass from the living thing during life : it is these which have to be replaced.

Animals are unable to assimilate, that is, to utilise as food, the simpler chemical compounds of carbon or of nitrogen. They can only take their nitrogen from food which is in the elaborate form of 
combination which is called a proteid; they can only take their carbon either from a proteid or from a carbohydrate or a hydrocarbon.

These elaborate compounds only occur in the bodies of other animals or of plants. Hence animals absolutely depend for their food on other living things. Plants, on the contrary, are (with certain exceptions) able to take up as food the compounds of carbon and of nitrogen which may be called the stable or resting condition of those elements-namely, the simple oxide of carbon-carbonic acid gas and the simple compound of nitrogen with hydrogen which is called ammonia, or the oxide of nitrogen which forms nitrates. This "food" of plants is diffused throughout the earth's surface in air and water; hence they need to expose a large absorbing surface to those media; hence their branches and leaves spread in treelike form to the air or to the water, whilst their roots are spread to the water contained in the soil. Their food is ever moving and flowing around them: they have neither to move in search of it nor to seize it. Hence the majority of plants are nixed and find safety and protection in stability. Animals, on the other hand, have to obtain their food from the scattered, solid, separate bodies of plants or of other animals. They have to move in search of it, they have to seize it when found, and they have to act chemically on the solid or viscous body or fragment of their prey so as to dissolve it and to enable the dissolved material containing the precious carbon and nitrogen in a high state of chemical combination to diffuse into their living substance and there be further assimilated and built up into the material of protoplasm. For these purposes animals possess structures enabling them to move more or less rapidly, and others enabling them to seize or grasp. Further, and of even more fundamental a character as determining their whole shape and organisation, they possess (with rare and intelligible exceptions) an aperture, the mouth, leading into a relatively extensive cavity, the gut, into which the solid or viscous mass of food is introduced, and when there is chemically dissolved or "digested."

The obvious and predominant difference in the make and habit of plants as compared with animals is thus connected with the very great and definite difference in the nature of the food of the two groups.

These statements are true in a general way, but require qualification. In the first place, we find it necessary to regard as g^netically part of the great Plant series many organisms which are not able to procure their carbon from carbonic acid nor their nitrogen from ammonia. Only the green plants are able to perform this constructive feat. The protoplasm of the more superficial cells of green plants contains corpuscles impregnated with a transparent green matter known as chlorophyll. In the presence of and in virtue of the physical action of sunlight screened by their chloro- 
phyll, the protoplasm of these cells has the property of decomposing carbonic acid, liberating free oxygen, and combining the carbon with hydrogen and oxygen to form starch. This is the critical step in the interaction of chemical elements on the earth's surface, by which life is at present determined. Were there no assimilation of carbon from carbonic acid to form starch-by the green plantsthe whole fabric of the living world would tumble to the ground-in truth, become mineralised. All living matter breaks down, within a short space of hours or days, to the resting or mineral condition of carbonic acid and ammonia (or nitrates). Were the building-up process, the raising to higher potentiality, not incessantly performed by green plants-a power which chlorophyll and chlorophyll alone confers on them-all carbon must pass from the reach of the organic world and living matter come to an abrupt end.

And this is equally true of nitrogen. The nitrogen present in living protoplasm tends inevitably to the stable inert condition-as a nitrate, as ammonia, or as the pure dissociated atmospheric gas. It is only by a subtle chemical process which occurs in the green plant-as a result of and in connection with the fixation of carbon as starch-that nitrogen taken up in water by the roots of the plant as nitrate and as ammonia is brought into combination as part of an "organic" compound or molecule. Thus in the ultimate history of the chemistry of living things the animal depends for its necessary food-proteids, carbohydrates, and hydrocarbons-on chlorophyll, the "leaf-green" of green plants. Vegetarian animals swallow and digest these substances built up by plants; carnivorous animals swallow and digest animals which have already profited by the work of the green plant. No animal can take up cven a fraction of a grain of carbon or nitrogen from a stomachful of carbonates, nitrates, and ammonia.

There are, however, as exceptions plants which are devoid of chlorophyll and depend upon the results of the constructive activity of other plants and of animals, just as per contra there are exceptional parasitic animals which have no mouths or gut and live in the diffusible nutritive juices elaborated by other animals, which they absorb by the surface of their bodies. The chemical life of those plants which are devoid of chlorophyll-the fungi, the bacteria, and a few others-may be considered as corresponding in character to that of those tissues or cell-groups of green plants which lie within the green plant and are devoid themselves of chlorophyll. Both these tissues and the autonomous fungi and the saprophytes depend for their food on the products supplied to them by the chlorophyll-holding cells of green plants. There are minute filamentous and rod-like plants devoid of chlorophyll (Bacteria and others) which can take their carbon as tartaric acid and their nitrogen as ammonia. It is probable that all such non- 
chlorophylligerous plants must be regarded as derived from chlorophyll-bearing ancestors-by adaptation to a food already somewhat raised by other organisms above the lowest stage of carboncombination.

Again, there are amongst the most highly developed flowering plants examples here and there of the exceptional and special development of stomach-like organs with mouth-like openings into which insects are attracted, and when once entrapped are held either by the actual movement of a grasping organ or by other mechanical apparatus, and are digested by chemical secretions identical in character with those of the animal stomach, the digested product being absorbed and serving to nourish the plant. Such cases, whilst they demonstrate in a most striking way the essential identity of the faculties of the living protoplasm of plant and animal, do not invalidate the fundamental proposition, that plants are a series, of organisms which have developed their distinctive form and structure as feeders on the diffused carbonic acid, ammonia, and nitrates of the circumambient medium; whilst animals are a series which have developed their distinctive form and structure as feeders on scattered-often elusive-live or dead bodies or solid particles of other animals or of plants, that form being essentially a locomotive sac with a mouth. Amongst the larger animals, those visible to the naked eye, there are few exceptions to this rule. Such exceptions are found in the obviously exceptional and therefore aberrant internal parasites which require no mouth nor digestive sac.

But there are a few, very rare cases of small aquatic animals which are provided with chlorophyll-corpuscles and obtain a part (in one case, the worm Convoluta, the whole) of their nutriment in the same way as does the green plant, namely, in virtue of the assimilation of carbon from carbonic acid in the chlorophyllbearing tissue when under the influence of sunlight. The chlorophyll-bearing cells of the worm Convoluta and of many Anthozoa have been shown to be unicellular parasites which have established the closest relationship to their hosts. But it is by no means demonstrated that the chlorophyll-corpuscles of Spongilla and of Hydra are parasitic in origin. ${ }^{1}$ The fact that they are not chlorophyll-bearing cells, but simple non-nucleated corpuscles with a cortex impregnated with chlorophyll precisely comparable to the chlorophyll corpuscles of green plants, does not permit us to consider them as parasites which have effected a lodgment and association with Spongilla and Hydra with any more reason than we can adduce for so regarding the similar corpuscles in green plants. The view has been seriously advanced that the latter are,

1 See on this subject my memoir on "The Chlorophyll-corpuscles and Amyloid Deposits of Spongilla and Hydra" in vol. xxii. (1882) of the Quart. Journal of Microsc. Science. 
in fact, also parasites. This may prove eventually to be susceptible of something like demonstration, but in the meantime we must ask where the limit to this assumption that chlorophyll is of parasitic origin is to be placed.

It cannot be that all chlorophyll-even that observed in all unicellular plants and animals -is to be regarded as "parasitic." And if we are once able to distinguish certain independent unicellular organisms which actually manufacture chlorophyll within themselves by the activity of their own protoplasm, we shall be able to study the steps of that process and to judge as to whether the protoplasm of the green cells of green plants and of the freshwater sponge and of the green Hydra do or do not form chlorophyll plastids in the same way and in virtue of the same protoplasmic capacity as do minute unicellular algae.

There is no reason, a priori, for refusing to ascribe to a tissuecell of a Sponge or a Hydra the same capacity to form a chemical deposit of any kind which a free unicellular organism possesses. Unfortunately this is not a case in which the simple test of observation can be applied, so that the question as to whether the rissuecell does construct a chlorophyll-corpuscle or does not can be settled by inspection. The intricacies of structure and growth are in this matter such as to render direct observation difficult and illusive.

Whilst there are, then, exceptional cases in both plants and animals as to the great nutritional distinction between the two series, it is comparatively easy in all excepting the very lowest forms to satisfy ourselves that the departures from the rule are specialised derivatives from the main series. The colourless or greenless plants are descended from green chlorophylligerous ancestors; mouthless, gutless animals are descended from mouthbearing, gut-hollow animals.

When, however, we come to the very lowest unicellular microscopic forms of life, there is greater difficulty in assigning some of the minuter organisms to one side or the other, and to some extent our decision in the matter must depend on the theory we may provisionally adopt as to the nature of the earliest living material, which was the common ancestral matrix from which both the Plant series and the Animal series have developed. The real question in regard to such a theory is as to whether we find reason to suppose that the combination of carbon and nitrogen to build up proteid, and so protoplasm, required, in the earliest state of the earth's surface, the action of sunlight and the chlorophyll screen. We must remember that, though these are now necessary for the purpose of raising carbon, and indirectly nitrogen, from the mineral resting state to the high elahoration of the organic molecule, yet it is, after all, living protoplasm which effects this marvel with their assistance; and it seems (though possibly there are some 
who would deny this) that it is protoplasm which has, so to speak, invented or produced chlorophyll. Accordingly, I incline to the view that chlorophyll as we now know it is a definitely later evolution-an apparatus to which protoplasm attained, and as a consequence of that attainment we have the arborescent, filamentous, foliaceous, fixed series of living things called plants. But before protoplasm possessed chlorophyll it had a history. It had in the course of that history to develop the nucleus with its complex mechanism of chromosomes, and it had during that period to feed.

The suggestion has been made long ago (see article "Protozoa," Ency. Brit., 6th edition), and appears to me not improbable, that by whatever steps of change that high complex of organic molecules which we call protoplasm-the physical basis of life-came into existence, it very probably fed in the first few æons of its existence on the masses of proteid-like material which, it may be supposed, were formed in no small quantity as antecedents to the final evolution of living matter. If this were the case, the mode of nutrition of the first living things must have been similar to that of animals and unlike that of plants. At a later stage chlorophyll was evolved, the decomposition of carbonic acid became possible, and the Plant series was started.

In accordance with this conception, we must look for the representatives of the most primitive forms of life amongst the minute Protozoa, possessing the simplest methods of nourishing themselves by the digestion of already elaborated proteid. Such are the Mycetozoa, which digest dead organic material by contact, creeping in the form of naked plasmodia of many inches in area over organic debris; such, too, are the minute single cells of naked protoplasm taking in particles of proteid food by extemporised mouths and digesting them in the cell-body, whilst prehensile and motor organs are furnished by the extension of the cell-protoplasm in the form of lobose processes, radiating filaments, or single or double vibratile flagella. The earliest plants, the Protophyta, were, it seems most probable, derived from flagellate colonybuilding Protozoa (similar to the Volvocineæ), which had, at first without discarding their animal-mode of nutrition (Zootrophic), acquired the faculty of manufacturing chlorophyll and supplementing their ingested nutriment by the decomposition of carbonic acid and the fixation of nitrogen (Mixotrophic). The step from this to a purely chlorophyll-given nutrition (Phytotrophic) was not a long one, and indeed occurs in the life-history of some of the Flagellata at the present day. With the establishment of pure Phytotrophic nutrition ensued the formation-by simple cell-division and elementary variation of cell-aggregation - of filamentous green plants consisting of chains of cells in single series; to these followed networks of 
such chains, then growth and division of the still-connected cells in two and finally in three dimensions, producing first sheet-like and finally more solid structures, the constituent cells of which became variously differentiated and specialised.

Those extremely minute, thread-like (Leptuthrix, Spirillum), or rod-like (Bacillus) plants devoid of chlorophyll, which often break up without losing vitality into spherules or into granules of even ultramicroscopic tenuity, known as the Schizomycetes (or colloquially Bacteria), cannot be considered as primitive. Like the Fungi and many of the most highly organised plants, they have descended from chlorophyll-bearing forms, and have become adapted to a parasitic or saprophytic mode of nutrition whilst retaining the general characteristics of growth and form of their ancestors. The intimate connection of the Schizomycetes with the Oscillatoriæ does not seem to admit of any doubt, and forms closely allied to them develop chlorophyll as well as peculiar blue and red pigmentary substances, the function of which is obscure but may be related to their modified nutritional processes. We are thus led to regard all the non-filamentous, non-chlorophylligerous microscopic forms which are not referable to the Schizomycetes or to the simpler Fungi as "Protozoa." The debatable ground is limited to the chlorophyll-forming Flagellata, amongst which are some which, being devoid of mouth and at all periods of their growth incapable of zootrophic activity, are yet so closely allied in life-history and structure with truly zootrophic species that it is not possible to draw a sharp line and assign them definitely either to the Animal or to the Plant series. Such are the Volvocineans, which zoologists will probably for some time to come consider it desirable (as we do in the present treatise) to treat of in the description of the Animal series, whilst botanists will find it equally desirable to discuss them in connection with closely allied minute Plants.

In view of these considerations, we consider the following groups of the simplest organisms as belonging to the Animal series, and as constituting a lowest "grade" of animal organisation, to which the term Protozoa is applicable. The groups in question are given the title of "classes," but it will readily be understood that it is not intended to imply by that term that they have any exact equivalence in the amount of divergence from one another to that which is presented by the "classes" of any one of the phyla of the Metazoa.

ProtozoA.-Class 1, Proteomyxa; Class 2, Heliozoa; Class 3, Mycetozoa; Class 4, Lobosa; Class 5, Radiolaria; Class 6, Mastigophora; Class 7, Sporozoa; Class 8, Ciliata ; Class 9, Acinetaria. 


\section{B. Separation of the Grade Protozoa from the Grade Metazoa.}

Formerly the name Protozoa was used for a sub-kingdom of the Animal Kingdom equivalent in value to other sub-kingdoms which were enumerated as the Coelentera, the Vermes, the Arthropoda, the Echinoderma, the Mollusca, and the Vertebrata. In its earlier use the great division "Protozoa" was made to include the Sponges, which we now assign to a divergent line of descent, the Parazoa, opposed to the main line, the Enterozoa, in the higher grade of animals called the Metazoa. The removal of the Sponges from assciation with the Protozoa is chiefly due to the initiative of Ernst Haeckel. By this step it became possible to give something like a definite characterisation of the Protozoa and to mark them off from all the higher animals. They are definitely characterised by the fact expressed in the English name Cell-animals (Plastidozoa), or less correctly unicellular animals, whilst all the higher animals or Metazoa (inclusive of the Sponges) are Tissue-animals (Histozoa). The fact indicated in these terms is that in Protozoa a single cell or a colony of equi-pollent cells is the organic "individual," whilst in the Metazoa the "individual" is built up by cells which are differentiated into at least two layers or tissues, the cells of each tissue being of like value and origin with its fellow-cells of that tissue, but differing essentially in structure, function, and origin from the cells of the other tissue or tissues. These statements will be found on critical examination to hold good in view of our present knowledge of both Protozoa and Metazoa. Most of the Protozoa are unicellular, and in those which form many-celled colonies, such as the Mycetozoa, some of the Radiolaria, Mastigophora, Ciliata, and Acinetaria, there is no tendency for those cells to differentiate into groups of cells of like structure and function to one another, but differing in structure and function from another group or groups present in the sime colony. The only approach to an exception to this generalisation is found in the specialisation of a cell here and there in the colony as a reproductive cell; but, on the other hand, it is to be noted that any cell in the colony is potentially a reproductive cell, and there is no differentiation of a congeries or tissue of cells for reproductive purposes in the general plan of the colonial structure. ${ }^{1}$ It appears to be the fact that we do not know of any forms at present existing which furnish a transition from Protozoa to the Metazoa. There

1 Thongh the existence of at least two "tissues" in the Metazon suffices to distinguish them from all Protozoa; it may legitimately be contended that the congeries of cells forming the colony of certain Protozoa (e.g. Volvox) is rather of the nature of a "tissue" than of a merely loosely acherent association of cells which, as we see in many Protozoan colonies, can and do separate freely and irregularly from such association. 
have been descriptions of supposed independent organisms suggesting such intermediate character (Trichoplax and others), but the true nature and history of these structures have not been placed on a definite basis, and do not really admit of discussion. The nearest case of a transitional form appears to be the Choanoflagellate "Proterospongia" of Savile Kent, which has been observed on several different occasions from different localities. It combines in one colony "amoebocytes" and "choanocytes," but it appears that the one form of cell develops into the other. It is certainly not unreasonable to regard Proterospongia as a step forward from the Choanoflagellata in the direction of the Parazoa. There is no instance of equally definite character tending to connect Protozoa of any class with the Enterozoa.

Until recently it was possible to add to this distinction between Protozoa and Metazoa the very striking one that all Metazoa reproduce by means of fertilised egg-cells (as well as by other processes), such fertilised cells being the result of the union of specially developed egg-cells and sperm-cells. Conjugation of two cells similar to one another as a preparation to multiplication by fission was known and described in several Protozoa, but the special units, the static female egg-cell and the motile male "spermatozoid," were unknown in Protozoa. The apparent exception to this presented by some of the Volvocinean Flagellata was regarded as a reason for assigning these organisms to the pedigree or great series of Plants, thus removing them from association with the other Flagellata. In the Plant series, though many groups both among the highest and lowest do not present sexual reproductive elements under the typical forms of egg-cell and spermatozoid (antherozoid), yet some of the lowest and simplest, as well as some of the higher, plants do develop motile conjugating "male" cells, which seem to render the relegation of Volvox to the vegetable series a reasonable proceeding. Within the last decade, however, we have not only becoine acquainted among undoubted Protozoa with instances of the development of "microgametes" or small conjugating cells, which are distinguished by their size from the larger egg-cells or "macrogametes" with which they fuse in order to form a fertilised "germ," but we now know undoubted Protozoa which exhibit the breaking up of a parent male unicellular individual into a number of motile microgametes. These have the appearance and characteristics of the spermatozoa of higher animals, are developed from the parent male cell by the same steps as are spermatozoa from sperm-mother-cells, and proceed to fertilise the female macrogametes in the same manner as occurs in the fertilisation of the egg-cell in Metazoa.

The Coccidiidae among the Sporozoa and certain of the Haemoflagellata are the Protozoa in which this phenomenon has been 
carefully observed. It is identical in its essential features with the sexual reproductive phenomena of the colonial Flagellate, Volvox globator. Not only so, but the egg-cells and spermatozoa thus developed and uniting are identical in character with the egg-cells and antherozoids of a vast series of lower and higher plants, and with those of the whole series of Metazoa. A very important link in the genetic relationships of Plants and Animals is thus established. There is no occasion to suppose that they have independently developed the typical form of the male and the female reproductive particles. The plants have inherited this from the Protozoa which gave rise to the earliest chlorophylligerous, phytotrophic organisms.

It is perhaps necessary to remark that further observation is necessary in these lowest forms as to the precise steps in the preparation of the nucleus and its chromatin in each of the conjugating gametes for the definite union of fertilisation. There is abundant evidence that it is of the same nature as that which occurs in the sexual cells of higher organisms, but in special details we may have to recognise some differences.

\section{Separation of the Classes of Protozon into Grades of Lower and Higher Structure.}

The question as to whether the various classes of Protozoa are to be regarded as nine separately divergent lines of descent, starting from a common primitive ancestry not represented at the present time by any one of them, or whether some of them possess closer genetic relationship inter se than do others, is a very difficult one. It has been proposed at various times to seek for evidence of such closer affinity in the development of a cortical firmer layer of the cell-protoplasm (as in most Sporozoa and in the Ciliata), as opposed to the retention of the uniform viscid character of the protoplasm (Lankester, Ency. Brit., article "Protozoa"), and again it has been considered probable that all those forms which produce temporary lobose or filamentar extensions of the protoplasm, as locomotor or grasping organs, may have a genetic community of origin which separates them from those provided with either isolated flagella or with "cilia" of vibratile protoplasm. Some or other, however, of the forms which it is found necessary, on account of the affinities indicated by their life-histories and other details of structure, to class as Flagellata (Mastigophora) exhibit combinations of characters which render both these attempts at grouping unsatisfactory.

We find Flagellata (see the section on this group) which produce extensive amoeboid processes, and yet possess a flagellum, whilst the majority have a distinctly corticate protoplasm. Among the Sporozoa (for which refer to the section on that group in the second fascicle of Part I. of this treatise), which are with these rare exceptions strongly corticate, we find genera which produce lobe-like and 
pointed "pseudopodia" from their superficial protoplasm (Zygocometes and others). It seems that in any attempt at a phylogeny of the Protozoa we should have to treat the assemblage of forms now classed as Mastigophora (Flagellata) as a central group from which the other eight classes have been derived, whilst embracing in itself several specialised lines of descent, including that which has given rise to the primitive green plants.

The indication of a higher and later elaboration of structure, as distinct from a lower and more primitive, by means of the classificatory artifice of "grades," has, however, been introduced in the present work by Professor Hickson in regard to the classes of Protozoa by a consideration of the cell-nucleus. The condition of this important structure justifies, he considers, the separation of the classes of Protozoa into a lower and a higher grade-the Homokaryota and the Heterokaryota - and it is not improbable that further study of the lower grade will lead to the subdivision of that assemblage into sub-grades.

The history of the nucleus of the corpuscle of protoplasm, that corpuscle which it is customary to regard under the name of "the cell " as the unit of living structure, is at present absolutely unknown and altogether a matter of conjecture. It may perhaps be conceded as highly probable that the earliest protoplasm was without nucleus or differentiated nuclear material. It is a legitimate contention that such a substance should not be called "protoplasm" at all, since Hugo von Mohl invented this term to describe the viscid contents of a vegetable cell expressly including the nucleus as part of it. It was proposed some twenty-five years ago by Ed. van Beneden to call the earlier non-nucleated stage of living matter "plasson," and it seems to me by adopting this term we can preserve the word "protoplasm" for its original use. At the same time it is important to avoid using the word "protoplasm," as is not unfrequently done, to signify the critical chemical body which undoubtedly is present in living protoplasm and is the apex of the pyramid or the top of the fountain, to which a variety of chemical bodies are leading and from which another series of chemical bodies are receding at every moment of the chemical activity of living protoplasm. Protoplasm is not a chemical body but a structure, and its nuclear particles, as well as its definitely formed nucleus consisting of chromatin and other constituents, are parts of it. It seems necessary to have a word by which to refer to the highest group of chemical molecules to which one set of chemical processes in the cell are always leading and from which another series are receding. I proposed some years ago (Ency. Brit., article "Zoology") to speak of this hypothetical body as "plasmogen." In the same way it is necessary to avoid the tendency which exists to employ the word "protoplasm" to describe cell-substance both when con- 
sidered as apart from the nucleus and when actually existing in an unmanipulated simplest living thing without any nucleus or nuclear matter. We have seen that "plasson" is the name which has been proposed for the latter; for the former the word "cytoplasm" is frequently used, whilst "nucleoplasm" is applied to that part of the cell-protoplasm which is the nucleus. The use of the word "cytoplasm " in this sense is certainly objectionable, as it signifies "the cell-plasm" and is merely a synonym of "protoplasm." It would be better to term the extra-nuclear substance of the protoplasmic corpuscle "periplasm."

As a hypothesis we may assume that living matter was at one time in the condition of "plasson," though it has yet to be shown that "plasson" is in existence at all at the present day. The next hypothetical stage is the development in distinct granular form of the material which later became aggregated as a nucleus. We may apply the word "protoplasm" to this stage, with a qualifying adjective, "konio-karyote" (powder-nucleated). This condition is known as actually existing in certain phases of the ciliate Protozoa (Trachelocerca), and possibly is to be recognised in some degenerate Protophyta and in some of the Proteomyxa (whether degenerate or archaic) amongst Protozoa. The third stage in the hypothetical development of protoplasm consists in the aggregation of the scattered nuclear granules to form one or more nuclei of definite structure and properties. Usually but one such nucleus is formed, but to cover the case of the existence of two or more similarly organised nuclei the term Homokaryote (proposed by Professor Hickson) may be used for this condition. The nucleus of the Homokaryote cell is in leading features of its structure identical with that of the tissue-cells of higher organisms. It consists of nuclear capsule, nuclear hyaloplasm, and of chromatin elements. The optical, chemical, and physiological analysis of the nuclei of Protozoa and Protophyta has not been extended to a sufficient number of instances, at present, to render it possible to trace the steps (if they are still traceable) by which the complete structure of the nucleus and its activity in cell-division were evolved. It is not yet clear whether there are among Protozoa and Protophyta any surviving simpler phases of the nucleus, or whether apparently primitive phases which are described are so interpreted owing to incomplete observation or, on the other hand, owe their simplicity to a degeneration from a more highly developed condition of the nucleus. It is, however, certain that there are cases amongst the Protozoa in which the structure and activity of the nucleus in cell-division conforms very closely to those of the tissue-cells of higher animals and plants, if not absolutely identical with them.

There are, however, in certain Protozoa special modifications of the nuclear structure which have not yet been shown to occur in 
Metazoa, nor in plants. The most striking of these is the division of the nucleus in Ciliata and Acinetaria into two unequal and dissimilar portions, the mega-nucleus and the micro-nucleus, which appear to be the portions of the primary nucleus which preside over the somatic (the larger) and reproductive activities (the smaller) respectively. Professor Hickson has made use of this differentiation of the nucleus into two parts in order to establish a higher grade of the Protozoa - the Heterokaryota as distinguished from the Homokaryota.

Amongst those forms, however, which are classed by him as Homokaryota, there are (as he recognises) certain forms amongst the Flagellata which also exhibit a differentiation and segregation of the nucleus, but with functions for the separated elements different from that shown in the Ciliata. This case is that of the formation of a separate nuclear body, the kineto-nucleus, in connection with, and apparently controlling the activities of, the large and powerful flagellum of certain flagellate forms (Trypanosoma, Noctiluca). It seems that the word Heterolsaryote would strictly apply to these forms also, although the "heterosis" is not the same as that seen in Ciliata. It would be premature to attempt to introduce a terminology indicating these different specialisations of nuclear structure in the Protozoa until much further study has been given to the subject. It is not at all improbable that researches which are now in progress will in the course of a few years give us, first of all, a better understanding of the chemical nature and activities of the substances which are merely brought into view by colour-staining as form-elements in the nucleus, ${ }^{1}$ and secondly, a far more critical knowledge than we at present possess of the rudimentarily aggregated and diffuse stainable matter which is interpreted as "nucleus" in some of the Protozoa, in some of the Cyanophyceae, in Schizomycetes, and in the yeasts and hyphae of lower fungi.

Whilst therefore recognising the important separation of the Ciliata and Acinetaria effected by having regard to the nuclear structure of those groups and that of the other classes of Protozoa, so far as we at present know them, I am unwilling to emphasise the arrangement of the Protozoa into grades according to their nuclear structure in the present state of knowledge. I should not wish to go farther at present in grouping the classes of Protozoa than to suggest that they should be considered as diverging lines of descent radiating from a central group which possessed the combination of characters presented at the present, day by the simpler Flagellata.

1 The researches of Professor Macallum of Moutreal in this direction will, it may be hoped, be continued and developed. 


\section{CHAPTER I.-PROTOZOA}

\section{SECTION A. - THE PROTEOMYXA ${ }^{1}$}

IN the study of the Protozoa a number of forms are found which are difficult to place in any of the larger orders or families. The difficulty arises in many cases from what is called their simplicity of structure, and partly from our ignorance of their entire life-history. The more we learn of the structure of the Protozoa, the more hazardous does it become to apply the expression "simple" to any living organism, but what is really meant by the term "simple" as applied to these organisms is that they exhibit no definite structure or structures such as skeleton, flagella, or nuclei that are so constant in their form and character that they can be seized upon by the systematist and used for purposes of classification. When characters of this description appear during one phase only of the life-history of an organism they may indicate its affinities if not its true systematic position, but when the life-history is not completely known there may be no characters which can possibly serve for placing the organism with others in any system of classification. In the early history of Protozoology there was a time when it was considered that some of the very small and obscure organisms consisted of a cytode of protoplasm in which there was no structure corresponding with the nucleus of the higher organisms and cells. Such organisms were placed in a class Monera by Haeckel in 1868. Subsequent researches proved that in many of these organisms one or many minute structures occur which give the same reactions as the chromatin of the nucleus, and the conclusion was, in some cases too hastily, drawn that all of them would in time be shown to be nucleated. Modern researches on the nuclear structures of Protozoa have thrown much light on this vexed question. They have shown that the nucleus may discharge into the cytoplasm, or give rise by total fragmentation to, a number of minute granules of chromatin-the chromidia - and that these granules do not degenerate, but retaining their vitality may again aggregate together in the formation of new nuclei. There may thus occur in the life-history of the higher Protozoa a stage which is strictly speaking non-nucleate (akaryote).

1 By Prof. S. J. Hickson, F.R.S. 
This does not imply, however, that the organism is at this stage devoid of nucleoplasm, but that the nucleoplasm is not concentrated in the form of a definite nucleus or kernel but is scattered or diffused. This conception may be expressed by saying that the stage is akaryote but is not moneran. There is no nucleus but there is nucleoplasm. In Amoeba, Pelomyxa, and others in which such a stage occurs the nucleus is present during the greater part of the life-cycle, the akaryote stage being antecedent only to nuclear multiplication or gametogenesis.

In the Proteomyxa, on the other hand, the akaryote condition is, as a rule, of much longer duration, and it is possible that in some cases the diffused nucleoplasm or scattered chromidia do not collect together in any stage to form a defined nucleus.

It seems probable, then, that the protoplasm of the Proteomyxa really represents the protoplasm of the higher Protozoa and Metazoa plus the substance of the nuclei. It is a substance which van Beneden (9) in 1871 proposed to call "the plasson," that is, the formative substance "which is capable of becoming, either in ontogenetic course or in phylogenetic course, monocellular elements after that the chemical elements of the plasson have been separated to constitute a nucleus and a protoplasmic body."

Our knowledge of the nucleus or chromidia of the genera that are here grouped together in the class Proteomyxa is at present very scanty. Vampyrellidium is said to have a nucleus in all stages of its life-history. Zopf states that a definite clear nucleus is present in all species of Vampyrella, but it is often obscured by chlorophyll and other bodies in the cytoplasm. There seems to be little doubt, however, that the nucleus is not present in all stages of the vegetative life of Vampyrella, as several observers who have carefully re-examined its structure have failed to find any definite nucleus. Recently, however, Dangeard (13) has shown that nuclei are present in the cysts, and that they divide by karyokinesis. In Tetramyxa there are said to be minute nuclei, but these are probably chromidia. In Plasmodiophora true nuclei are undoubtedly present at the time of spore-formation, as they have been observed to divide by karyokinesis. It is probable also that a defined nucleus is present during the flagellate and amoebula phases of most of the Proteomyxa (Fig. 8, B, H), but it is clear that for a time during the plasmodium stage the nuclei are disintegrated. In Endyonema nuclei appear to be wanting during the active vegetative phase in the filaments of its host-plant (Lingbya), but definite nuclei are constituted when the body contracts in the formation of the zoocyst.

Many of the genera included in the group have been seen only once, and we are still in ignorance of their nuclear condition, but in Gymnophrys, Biomyxa, Gloidium, Leptoplirys, and.Protamoeba, 
which have been studied by other observers than their original discoverers, no defined nuclei lave been found.

A considerable number of genera are parasitic upon freshwater algae during at least one stage of their life-history, such as Vampyrellidium, Vampyrella, Leptophrys, Endyonema, Enteromyxa, Colpodella, Pseudospora, Gymnococcus, Aphelidium, Tetramyxa, and Ectobiella. Tetramyxa causes the formation of gall-like growths on Ruppia and other freshwater plants. Bursulla occurs in horsedung. Haplococcus occurs in the muscles of the pig, but is apparently harmless. The only species that is of any economical importance is Plasmodiophora brassicae, Woronin, which attacks turnips and causes the disease known as "Fingersand Toes,"or "Hanburies." A considerable number of genera are not parasitic and feed upon minute animal and vegetable

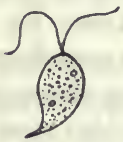

B.

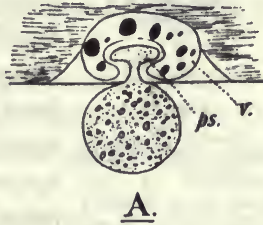

FIG. 1.

Kctobiella plateaui. A, a specimen attacking Licmophora; $p$, the pseudopodiun that is pushed into the substance of the liost; $v$, the vacuole formed by the host containing granules produced by the digestive action of the pseudopodium. B, the bitlagellate zoospore of Ectobiella. (After de Bruyne.) organisms. Such genera are Gymnophrys, Biomyxa, Protomyxa, Gloidium, and others.

In the vegetative condition the body emits pseudopodia. These pseudopodia may be roughly arranged in three categories.

In Protamoeba, Gloidium, Enteromyxa, etc., the pseudopodia are usually lobate like those characteristic of the genus Amocba.

In Vampyrella, Colpodella, Monobia, Myxustrum the pseudopodia are radiate in position, very delicate and rarely anastomosing, like those of an Actinophrys.

In Biomyxa, Gymnophrys, Penardia they are delicate and anastomosing, like the pseudopodia of the Foraminifera.

In Endyonema, Haplococcus, Aphelidium, and other endoparasites the form of the body is adapted to the spaces of the host and true pseudopodia are not formed.

In Protomyxa, Myxastrum, Protomonas, Bursulla, Plasmodiophora a number of amoebulae unite to form a plasmodium, and it is possible that plastogamy also occurs in Vampyrella, Leptophrys, and some others. In Monobia a number of stellate individuals unite to form an open network (Fig. 4).

A contractile vacuole does not usually occur in Proteomyxa, but it appears to be a constant feature in Gloidium and Ciliophrys. Non-contractile vacuoles occur in many of the genera.

Although very litcle is known about the life-history of the Proteomyxa, it seems probable that they all, at some time, form cysts or spores. In Plasnodiophora the protoplasm of the plasmodium breaks up into a large number of simple spores, which are able to 
resist desiccation, and are probably simple hypnocysts. ${ }^{1}$ In other cases the cysts are larger, and the contents give rise to three or four (Vampyrella lateritia, Fig. 2) or a large number (Protomyxa,

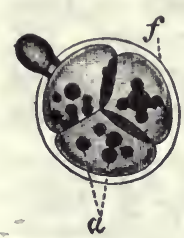

Fra. 2. Fig. 6, and Diplophysalis) of spores, which may be either naked or protected by a membrane. These cysts are protected by one or more cyst-membranes, and the outer of these may be irregular or spiny or gelatinous in texture. Occasionally three or four small areas on the cyst-wall are provided with a thinner membranous coat, and the spores escape by

Cystic phase of Vanipyrella. The contents of the cyst have divider into four equal parts, of which (After Lankester and Cienkowski.) breaking through these areas only (Haplococcus); but usually the cyst-wall breaks down and liberates the spores, or the spore escapes through any part of the membranes. In spore-formation the protoplasm usually discharges all extraneous matters, and one large or a number of smaller granules of these ejecta are found between the wall of the cyst and that of the spores. There is no evidence at present that any process of conjugation occurs between the liberated zoospores, except in Ciliophrys (Cienkowski), and, in the absence of any systematic study of the nuclear substance of the spores, we are not in a position to state that the condition of the nuclei or nucleoplasm of the spores is in any way different to that of the other phases of life. There is therefore no justification whatever for the assumption that any form of cyst-formation indicates or is associated with a sexual process. ${ }^{1}$

A remarkable phenomenon has recently been described by do Bruyne in Leptophrys villosa. After a period of feeding, the animal becomes spherical in shape and enters upon a period of rest. From the surface there are protruded a number of delicate filaments (Fig. 3) which terminate in hyaline globules. These globules are discharged and the filaments after some time are slowly withdrawn.

When conditions are favourable there emerge from the cyst one or more amoebulae (Monadineae azoosporeae, Zopf), or one or more flagellulae (Monadineae zoosporeae, Zopf). The

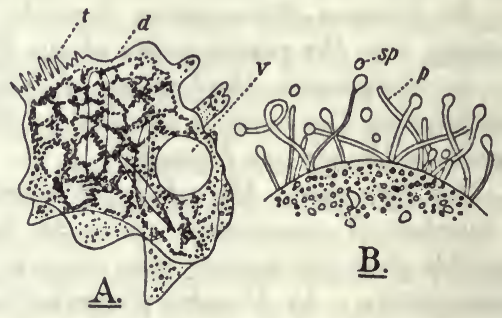

Fic. 3.

Leptophrys villosa. A, a specimen actively feed. ing, showing, $v$, a large non-contractile vacuole; $d$, the diatoms on which it is feeding; and $t$, a tuft of pointed pseudopodia at the posterior end of the body. B, a resting stage of the same animal, provided with fllamentous processes, $p$, which discharge minute globules, s.p, of hyaline protoplasm. (After de Bruyne.)

1 According to von Prowazek the nuclei of the spores of Plasmodiophora are formed by karyogamy (Arb. aus den kaiserl. Gesundheitsamte, xxii., 1905, p. 396). 
amoebulae either grow and become Actinophrys-like in form ( $\mathrm{Vam}$ pyrella) or unite to form plasmodia (Leptophrys, Endyonema, etc.).

The flagellulae are provided usually with one, but sometimes two (Diplophysalis, Gymnococcus) whip-like cilia, and sometimes also with a vacuole. They sometimes swim about actively and attack the organisms on which they feed (Colpodella, Fig. 8, A); but usually they soon withdraw their cilia and become amoeboid in shape, and the amoebulae thus formed either unite to form plasmodia or grow independently into the adult form.

The classification 'of Proteomyxa has always presented innumerable difficultics, and even at the present day our knowledge is so incomplete that nothing better than a tentative arrangement of the genera can be suggested.

A large number of the genera were placed in a division (Monadineae) of the Mycetozoa (Pilzthiere) by Zopf, others are regarded as Foraminifera nuda by Rhumbler, and Bütschli included several of the genera in the Heliozoa.

Zopf further divided his genera into two groups, the Monadineae azoosporeae and the Monadineae zoosporeae. In the former the cysts give rise to amoebulae, and in the latter to flagellulae. It does not appear satisfactory, however, to use the characters of the swarm-spores alone as a basis of classification. Pseudospora, with a flagellate zoospore, is clearly related to Vampyrella and its allies, which have an amoebulate zoospore; and Enteromyxa, Myxastrum, and other genera, with an amoebulate zoospore, appear to have no close relation to Vampyrella.

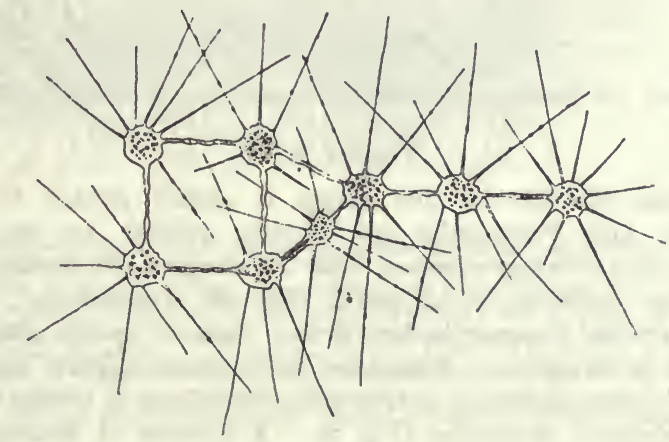

Fro. 4.

Monobia confluens. A number of individuals connected together by protoplasmic strands to form a loose mesliwork colony. (After Schneider.)

In attempting to classify the Proteomyxa, certain genera stand out as clearly related to other groups of Protozoa. Thus Monobia is closely related to the Heliozoa, Protogenes to the Foraminifera, Protamoeba and Gloidium to the Gymnamoebida, and Plasmodiophora 
to the Mycetozoa. Taking into consideration the form assumed by the pseudopodia, the habit of plasmodium-formation, as well as the character of the zoospores, most of the other genera can be arranged around these as central types. But there still remain some forms whose affinities are at present quite obscure, and these must be separated for the present into a group by themselves.

The genera are here arranged in five groups according to their supposed affinities with the other orders of Protozoa.

\section{Groop A.}

The following two genera appear to have affinities with the Gymnainoebida.

Nothing whatever is known concerning their life-history, and it is probable they will prove to be but a stage in the life-history of an Amoeba.

Protamoeba, Haeckel, is like an Amoeba, but without any definite nucleus or contractile vacuole. Freshwater and marine. $110 \mu$ (Penard).

Gloidium, Sorokin (Fig. 5), differs from Protamoeba in possessing a contractile vacuole. Occasionally the surface is denticulated. Freshwater, $71 \mu$. G. inquinatum, Penard, $385 \mu$. The genus Gringa, Frenzel, is probably a species of Gloidium.

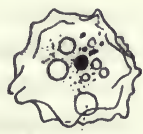

c.V
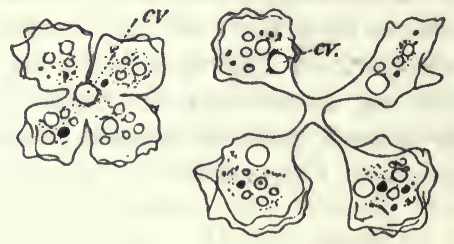

Fia. 5.

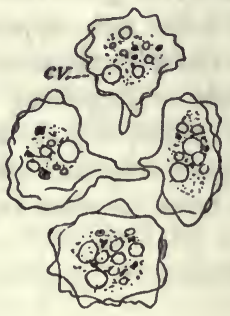

Four stages in the division of Gloidium quculrifidum. c.v, contractile vacuoles. (After Sorokin.)

\section{Group B.}

The genus Monolia in this group is closely related to Heliozoa. Monopodium and Vampyrella are closely related to one another, and agree with Vampyrellidium and Pseudospora in having a stage with delicate radiating pseudopodia like an Actinophrys. Leptophrys has affinities with Vampyrella, but differs from it in the shape of the body, which is irregular. Myxastrum is in some respects intermediate between the genera included in this group and those in Group D.

(IV.) ${ }^{1}$ Monobia, Schneider (Fig. 4). A number of Actinophrys-like individuals, but withont nucleus or contractile vacuoles, and of a bluish

1 As the genera includel in the Proteomyxa in this volume have been shifter about from one class to another by different anthors, the roman figures in brackets liave been introduced to indicate to the reader the position assigned to each genus by the leading writers on Protozoology, when it differs from that given to the same genus in the text. Thus the genera marked (I.) were referrerl to the Monadineae azoosporeae, (II.) to the Monadineae zoosl ioreae of the Mycetozoa by Zopf; (III.) to the Foraminifera nuda by Rhumbler (22); (IV.) to the Heliozoa by Biitschli and Schaudinn. 
colour by transmitted light, are united into a colony by the fusion of the ends of their contiguous pseudopodia. Reproduction by fission has been observed, but no process of spore-formation is known. Freshwater.

(I.), (IV.) Vampyrella, Cienkowski (Fig. 6, 5). Several species of this widely distributed genus are kuown. There is an Actinophrys stage in which, accorling to some authors, there is a nucleus. Vampyrella lateritia attacks Spirogyra by pushing a lobate pseudopodium into the cell and gradually absorbing its contents. $V$. gomphonematis attacks the stalked diatom Gomphonema, completely surrounding the frustules and absorbing their contents. Cysts are formed surrounded by a single smooth membrane, the animal discharges particles of undigested food

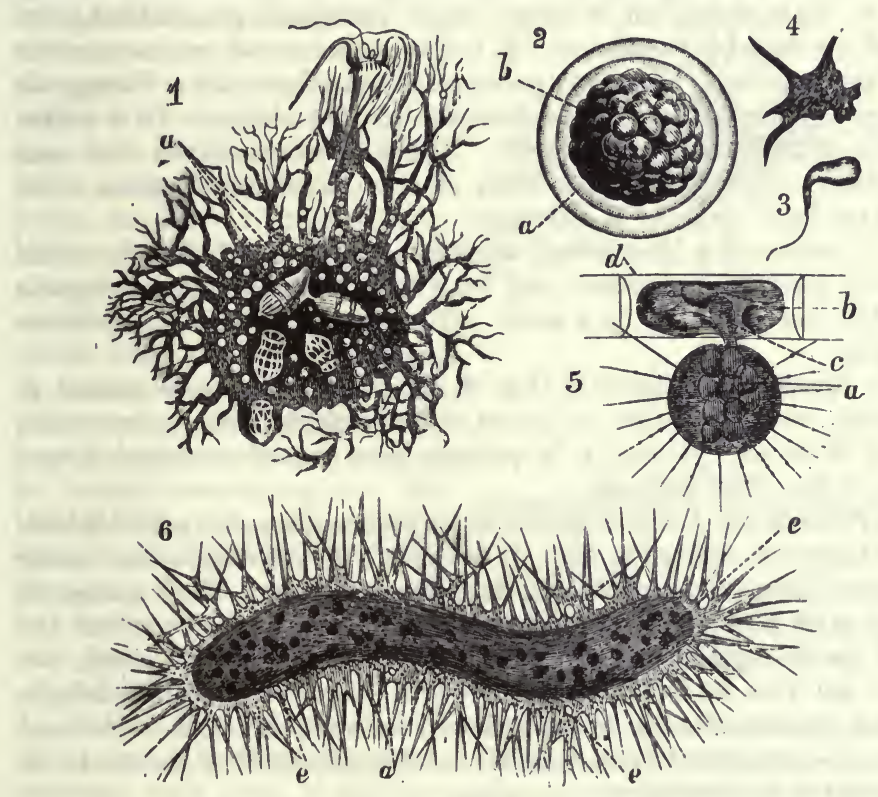

Fio. 6.

1, Protomyxa auranticaca, Haeckel, plasmodium plase. The naked protoplasm shows branched, reticulate processes and numerous non-contractile filaments It is in the act of engnlfing Ceratium. Shells of engulfed Ciliata (Tintinnabula) are embedded deeply in the endoplasm, a. 2, cystic phase of Protomyxa; $a$, transparent cyst-wall; $b$, protoplasm broken up into spores. 3, flagellula phase of Protomyxi. 4, amoebula phase of the same, the form assumed after a short period by the flagellulae. 5, Vampyrella lateritia. Cienk. Actinophrys stage penetrating a cell of Spirogyra, b, by a process of its protoplasm, $c$, and taking up the substance of the Spirogyra cell, some of which is seen within the Vampyrella, $a .6$, large individuals of Vampyrella showing pseudopodia, $e$, and food-particles, $\alpha$. (From Lankester, after Haeckel and Cienkowski.)

materials and these are found with the shrunken protoplasm within the cyst-wall. Occasionally a second membrane is formed around the shrunken protoplasm. The protoplasm divides within the cyst-wall, and the nuclei of the spores this formed are $2 \mu$ in diameter and divide by karyokinesis. From the cyst there escape one, but usually four or five amoebulae, which soon develop radiate pseudopodia and float away in search of their food. In some species (e.g. $V$. gomphonematis) it 
seems certain that several individuals may fuse to form a plasmodium. No contractile vacuoles occur at any stage. The size varies considerably, $20-70 \mu$. They are nearly all freshwater forms, but one species, $V$. gomphonematis, is also marine.

Monadopsis, Klein, is probably a species of Vampyrella.

(I.) Vampyrellidium, Zopf. This genus is parasitic on freshwater Algae, particularly on Lingbya. Two kinds of cysts are formed, the zoocysts with a clear homogeneous membrane, and the hypnocysts with a thicker membrane. In other respects it is closely related to Vampyrella. A nucleus surrounded by a hyaline area is said (Zopf) to occur at every stage.

(I.) Leptophrys, Hertwig and Lesser (Fig. 3), appears to be closely related to Vampyrella, but it forms larger vacuolated plasmodia by the fusion of the amoeboid zoospores. It is also characterised by the presence in the protoplasin of numerous paramylum granules. Like Vampyrella it is found parasitic on various freshwater lower Algae. It is either colourless or tinged with chlorophyll. The cysts are sometimes $0.25 \mathrm{~mm}$. in diameter. They give rise to three or four amoeboid zoospores. No nuclei have been observed at any stage.

(IV.) Monopodium (Haeckelina), Mereschkowsky, is an Actinophrys-like form with hyaline protoplasm and very delicate radiating pseudopodia attached to foreign bodies by a stalk. $0.2 \mathrm{~mm}$. White Sea. Archerina (see p. 33).

(IV.) Nuclearia, Cienkowski (Fig. 8, E), also appears to be related to Vampyrella, but as a nucleus or nuclei and contractile vacuoles have been observed by several authors, it is perhaps more natural to regard it as a member of the order Heliozoa.

(II.) Pseudospora, ${ }^{1}$ Cienkowski, is a small Proteomyxan, $3-4 \mu$, which feeds upon Oedogonium, Spirogyra, etc. It is related to Gymnococcus and other members of Group C in producing flagellate zoospores. These zoospores, provided with one or two flagella and a ninute nucleus, penetrate the bells of the host-plant and give rise to an Actinophrys-like stage, but they do not fuse to form a plasmodium. When they are fully fed the numerous pseudopodia are withdrawn and an anoeboid form is assumed previous to encystment (Fig. 8, B, C). Diplophysalis, Zopf, seens to be closely related to Pseudospora.

(I.), (IV) Myxastrum, Haeckel, was found on the shores of the Canary Islands and is marine. It has a stage with numerous radiating pseudopodia, but forms plasmodia which attrin to $0.5 \mathrm{~mm}$. in diameter. The plasmodium encysts as a whole and the protoplasm forms 100 or more spores which give rise to amoeboid zoospores.

(IV.) Ciliophrys, Cienkowski (Fig. 8, G, H), probably belongs to this group. It is similar to Nuclearia in some respects, but at times it withdraws its radiating pseudopodia, becomes oval in shape, and swims rapidly by means of a long flagellum. Freshwater.

Grovp C.

In this group there is a stage when fine branching and anasto- 
mosing pseudopodia are formed and the affinities seem to be with the Foraminifera. Arachnula has some affinities with Nuclearia and is regarded as a Heliozoon by some authors.

(III.) Protogenes, Haeckel (Fig. 7), is a small spherical organism with very numerous and delicate radiating and anastomosing pseudopodia Neither vacuoles nor nuclei have been observed. Marine.

(III.) Biomyxa, Leidy, is a widespread genus occurring both in fresh and salt water. It passes though a spherical stage with radiating pseudopodia, but afterwards assumes a variety of elongated or outstretched shapes with a few long, isolated, branching and anastomosing pseudopodia. One large or many small nuclei are said to occur (Rliumbler). In $B$. vagans there are mumerous minute contractile (?) vacuoles, but in $B$. (Gymnophrys) cometa there are none. It occurs in swampy sphagnum ground in this country. No definite nuclei have been observed and nothing is known concerning its lifehistory. The genera Gymnophrys,

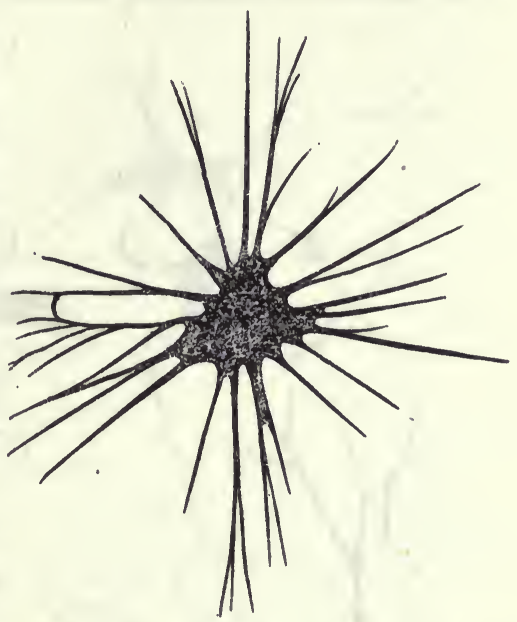

Fic. 7.

Protogenes primordialis, Haeckel, from Schultze's flgure. Cienkowski (Fig. 8, D), and

Penardia, Cash, seem to be allied to Biomyxa. It has been suggested by Archer that Gymnophrys is but a detached portion of a Gromia, and West (27) has found it in a collection containing a large number of specimens of this Foraminifer.

(III.) Arachnula, Cienkowski (Fig. 8, F), also is closely related to Biomyxa, but it forms long strands terminating in branching extremities provided with tufts of delicate anastomosing pseudopodia. Cysts have been described. It is found in fresh and brackish water.

(III.) Pontomyxa, Topsent, is a form closely allied to Biomyxa and Penardia. The body assumes a variety of ribboned or dendritic forms, with numerous or interrupted groups of anastomosing pseudopodia. $P$. pallida from the Mediterranean Sea is colourless, but $P$. flava, like Penardia, is golden yellow in colour. $P$. flava was found in 35-50 metres off the French coast and also in the Mediterranean Sea. The nuclei are said to be very small and reproduction occurs by multiple fission.

(III.) Rhizoplasma, Verworn (26). Spherical or sausage-shaped bodies of an orange-red colour, with numerous anastomosing pseudopodia, 5-10 mm. in diameter when expanded, found in the Red Sea, are placed in this genus. There are 1-3 large transparent vesicular nuclei. The coloured granules circulate in the pseudopodia. 
(III.) Dictyomyxa, Monticelli, is like the preceding genus, but with colourless pseudopodia. On Chaetomorpha crassa at Naples.

Boderia, Wright (Fig. 9), is marine, orange or brown in colour, with a nembranous investment (?), from openings in which protrude three to four long branching pseudopodia. The nucleus or nuclei after a time

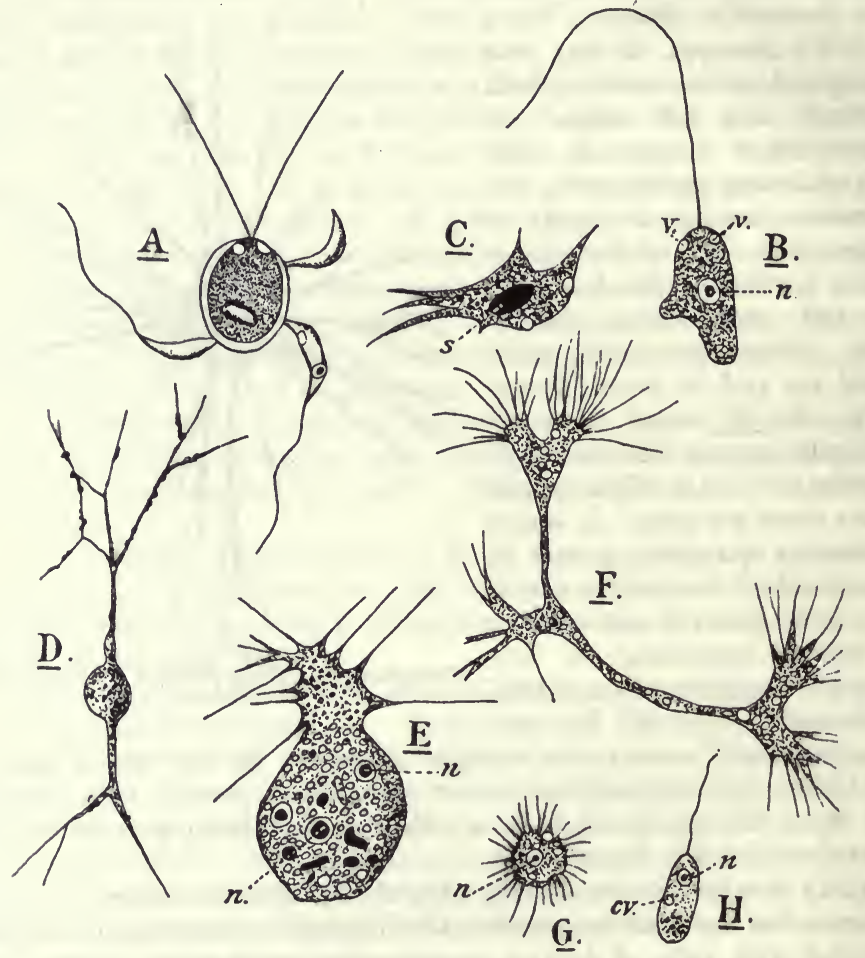

Fia. 8.

A, Colpodella pugnax. Three individuals attacking a Chlanydomonad. B, flagellate zoospore of Pseudospora parasitica; $n$, nucleus; $v$, vacuoles, said to be contractile. C, the amoeboid phase of Pseudospora which succeeds the Actinophrys stage; $s$ is probably a mass of ingested starch-grains. D, Gymnophrys cometu. E, Nuclearia delicatula with three nuclei, $n$. F, Arachnula impatiens. G, Actinophrys stage of Ciliophrys; $n$, nucleus. H, flagellate stage of Ciliophrys infusionuni; c.v, contractile vacuole; $n$, nucleus. (All after Cienkowski.)

disappear, and the protoplasm spreacls out in ragged masses on the slides. A number of naviculoid bodies are formed, from each of which a small amoebula emerges in a few days. Marine. $1-4 \mathrm{~mm}$.

\section{Group D.}

Most of the genera included in this group form plasmodia, and their affinities seem to be with the Mycetozoa. No plasmodium-formation has been found in Aphelidium, Colpodella, Pseudosporidium, and Pseudamphi- 
monas. Zoospores with one or two flagella have been seen in all the genera except Myxodictyum, Bursulla, and Tetramyxa. It is possible that Colpodella is related to the Mastigophora.

(III.) Protomyxa (Fig. 6, 1) was found by Haeckel attached to the shells of Spirula on the coast of the Canary Islands, in the form of orange-yellow flakes consisting of branching and reticular protoplasm nourishing itself by the ingestion of Diatoms and Peridiniae. This is a plasmodium formed by the union of several amoebulae. The plasmodium encysts and gives rise to numerous flagellulae or swarm-spores. The diameter of the cyst is $\cdot 12-2 \mathrm{~mm}$. The flagellulae pass into an amoebula phase, and the amoebulae unite to form the plasmodium.

Myxodictyum, Haeckel, consists of a number of protomyxa-like individuals united by their pseudopodia to form colonies. It is pelagic in habit and was found by Haeckel at Algeciras in Spain. Marine.

(II.) Gymnococcus, Zopf, occurs in Cladophora, Diatoms, and Cylindrospermum. It forms a plasmodium. When fully fed it gives rise to zoocysts, from which three to twelve biflagellate zoospores escape.

(II.) Aphelidium, Zopf, lives in

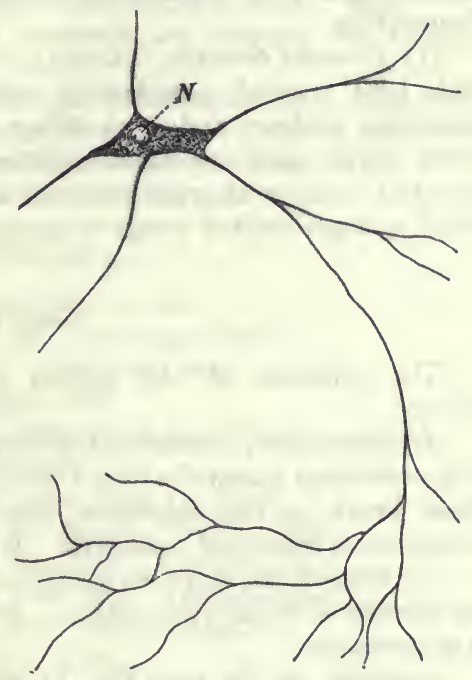

Fia. 9.

Boderia turneri. $N$, nucleus. (After Wright.) the cells of Coleochaeta and in macerations of plant tissues. Hypnocysts furnished with an operculum are formed. A nucleated zoospore with one flagellum has been found in A. lacerans (de Bruyne).

(II.) Protomonas, Cienkowski, has biflagellate zoospores which become amoeboid and unite to form a plasmodium. Freshwater and marine.

(II.) Colpodella, Cienkowski (Fig. 8, A), is possibly allied to Protomonas. The zoospores have only one flagellum, and attack Mastigophora before they become amoeboid. They do not, however, form plasmodia.

(II.) Tetramyxa, Göbel, forms large galls on various water-plants, especially Ruppia.

(II.) Plasmodiophora, Woronin, is the cause of the disease of turnips known as "Fingers and Toes," or "Hanburies" (German, Herniekrankheit). The spores are fuund in damp ground. Each spore gives rise to a minute nucleated amoeboid zoospore with a single flagellum. This penetrates into the cells of the root and loses its flagellum. It increases in size and the nuclei divide. After a sime plasmodium-formation begins by the fusion of neighbouring amoebulae, and the tissues of the host-plant disintegrate. As soon as the plasmodium is formed the nuclei increase rapidly by karyokinesis, but according to Nawaschin (21) there is a perind 
when the plasmodia exhibit no trace of nuclei, the nuclear substance being apparently distributed thronghout the whole plasmodium. Subsequently the plasmodiun breaks up into a great number of minute spherical spores. 1

Pseudamphimonas, de Bruyne, was found on Caulerpa at Naples. The zoospores are biflagellate and extremely amoeboid. They withdraw their flagellae, and two or three have been seen to fuse together to form a plasmodinm.

(I.) Bursulla, Sorokin, is found in horse-dung. A number of amoebulae with long pointed pseudopodia unite to form a plasmodium. The plasmodia contract and form either stalked cysts $(51 \mu)$, the contents of which divide and einerge as eight amoebulae, or they give rise to naked spherical cysts with rosy contents and an outer cortex, from each of which a single stalked zoospore emerges.

\section{Group E.}

The affinities of the genera included in this group are quite obscure.

(I.) Enteromyxa, Cienkowski, forms, by the fusion of amoeboid zoospores, long vermiform plasmodia $(0.5-1 \mathrm{~mm}$.) with short tubercular pseudopodia. These break up into segments, which encyst and give rise to two or seldom more amoeboid zoospores. It feeds on Oscillatoria.

(I.) Endyonema, Zopf, forms cylindrical cysts of considerable length in the threads of filamentous algae. Nuclei are said to occur previous to cyst-formation.

Ectobiella, de Bruyne (Fig. 1), was found in the form of a biflagellate pyriform zoospore. It attacks Licmophora and other diatoms, withdraws the flagella and pushes a pseudopodium into the protoplasn of its prey. When the contents of the diatom are assimilated, the amoeboid organisms wander away and encyst.

Haplococcus, Zopf, is found in the muscles of the pig. Two kinds of cysts are described by Zopf, the zoncysts $(16-22 \mu)$ and the hypnocysts $(25-30 \mu)$. The membrane surrounding the former is thinner in some places than elsewhere, and from them escape six to fifteen amoeboid spores. The further history of the hypnocysts has not been followed.

Pseudosporidium, Zopf, was found by Brass in vegetable infusions. It is amoeboid in form, with short blunt pseudopodia, a nucleus, and a vacuole. The cysts give rise to numerous small flagellate zoospores.

Schizogenes, Pouchet, was found in the haemocoel of freshwater Ostracods and Copepods. It consists of small plastids of hyaline protoplasm, $\cdot 01 \cdot 03 \mathrm{~mm}$. without vacuoles or nucleus, of indefinite forn, and devoid of pseudopodia. It divides into parts, which become new individuals.

Bathybius, Huxley, and Protobathybius, Bessels, are no longer regarded as living organisms. It seems probable that both forms represent a colloid precipitate of calcium sulphate thrown down by the action of alcohol on sea-water (Murray). 


\section{Literature.}

The following recent general works on Protozoology will be found useful to students :-

1. Braun. Animal Parasites of Man. Translated by F. V. Theobald. 1906.

2. Bütschli, $O$. Protozoa. Bronn's Klassen und Ordnungen des Thierreichs.

3. Calkins, G. N. Protozoa. Columbia University Biol. Series. 1901.

4. Cash, J. The British Freshwater Rhizopoda and Heliozoa, vol. i. Ray Society, 1905.

5. Doflein, $F$. Die Protozoen als Parasiten und Krankheitserreger. Jena, 1901.

6. Hartog, M. M. Protozoa. Cambridge Natural History, vol. i., 1906.

7. Lang, A. Lehrbuch der vergleichende Anatomie. Protezoa. 1901.

8. Penard, E. Faune rhizopodique du bassin du Léman. 1902.

The following refer particularly to Proteomyxa :-

9. Beneden, E. van. Q. J. Micr. Sci. xi., 1871, p. 254.

10. Brass. Biol. Studien, i., 1883-4, p. 70. (Pseudosporidium.)

11. de Bruyne, C. Arch. Biol. x., 1890. (Ectobiella, etc.)

12. Cienkowski. Arch. mikr. Anat., 1865, 1876.

13. Dangeard, P. A. Le Botaniste, (2), 1890, p. 33, and (7), 1900, p. 131.

14. Göbel. Flora, No. 28, 1884. (Tetramyxa.)

15. Haeckel, E. Monogr. der Moneren. Jen. Zeits. iv., 1868.

16. - System. Phylog. der Protist. u. Pflanzen. Berlin, 1894.

17. Hongenraad, $H$. K. Arch. Protist. viii., 1907. (Vampyrella.)

18. Mereschkowsky. Arch. mikr. Anat. xvi., 1879. (Monopodium.)

19. Monticelli. Boll. Soc. Napoli, xi., 1897. (Dictyomyxa.)

20. Murray, J. P. R. Soc. London, xxiv., 1876.

21. Nawaschin. Flora, 1899, p. 404. (Plasmodiophora.)

22. Rhumbler, L. Arch. Protist. iii., 1904.

23. Schneider, A. Arch. mikr. Anat. vii., 1878. (Monobia.)

24. Sorokin. Ann. Sci. Nat. Bot. (6) iii., 1876 ; Morph. Jahrb. iv., 1878. (Gloidium.)

25. Topsent, E. Arch. Zool. Expér. (3) i., 1893. (Pontomyxa.)

26. Verworn. Arch. ges. Physiol. lxii., 1896. (Rhizoplesma.)

27. West, G. S. J. Linn. Soc. Zool., 1901, xxviii. p. 308.

28. - l.c., 1903, xxix. p. 108.

29. Woronin. Pringsheim's Jahrbïcher, xi. (Plasmodiophora.)

30. Wright, S. Journ. Anat. Physiol, i., 1867. (Boderia.)

31. Zopf, $W$. Handbuch der Botanik. Edited by A. Schenk. Bd. iii., pt. 2, 1887. 


\section{THE PROTOZOA (continued)}

\section{SECTION B.-THE HELIOZOA ${ }^{1}$}

The term Heliozoa is commonly used to include a number of Protozoa, generally inhabitants of fresh water, with few characters in common except the possession of straight, radial pscudopodia which rarely anastomose, and the absence of anything like a capsular membrane dividing a central portion of the body from a peripheral portion, such as is found

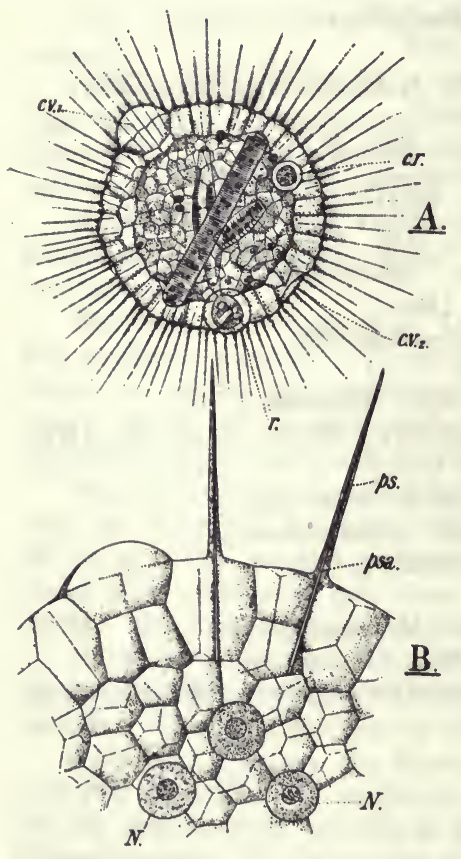
among the Radiolaria. The more highly specialised members of the group have a spheroidal body, which rarely exhibits amoeboid change of shape, divided into a more vacuolated

F1G. 1.

Actinosphaerium Eichhorni, Ehrb. A, a drawing of an individual as seen in optical section; $c . v_{1}$, a contractile vacuole previous to lischarge of its contents ; $c . v_{2}$, the position of a contractile vacuole that has just collapsed; $c . r$, fool-vacuole; $r$, a rotifer in the act of being engulfed in a food-vacuole. $B$, a small portion of the ectoplasm of the same animal very much enlarged; $N$, the nuclei; $p s$, a pseudopodium ; ps. $a$, the axis of the pseudopodium. The axes of the pseudopodia have been recently traced farther into the ectoplasm than is shown in the figure and into closer relation with the nuclei. (After Leidy.)

ectoplasm and a less vacuolated endoplasm, the endoplasm containing one or many nuclei, and sometimes a permanent centrosoma distinct from the nucleus. The pseudopodia are long, slender, and stiff, projecting radially from the surface of the body, and generally consisting of a cortex continuous with the ectoplasm and an axis prolonged into the endoplasm (Fig. 1, ps). In Elaeorhanis, Nuclearia (Fig. 8, E, p. 10), and some others that may be regarded as being on the border-line between the Heliozoa and Group B of the

1 By the late Prof. W. F. R. Weldon, F.R.S., and Prof. S. J. Hickson, F.R.S. 
Proteomyxa (cf. p. 6), no axial rod to the pseudopodium has been discovered. A skeleton may be present or absent; when present it is generally siliceous, though it may be in part chitinous (Actinolophus), or composed of a jelly whose chemical composition is unknown (Heteroplerys), or built up of foreign particles (Elaeorhanis).

Hertwig and Lesser (7), in a memoir which established the main lines of the modern classification of the group, included only those higher forms whose characters have been indicated, giving a conception of the Heliozoa both logical and in many ways convenient; but such a treatment neglects a singularly perfect series of forms, the higher members of which, such as Nuclearia (Fig. 8, E, p. 10), closely resemble undoubted Heliozoa,-while from these we may pass step by step to such forms as Monobia or Vampyrella (Figs. 4 ; 6 (5), pp. 4 and 7), which are probably more nearly allied to the Mycetozoa than to the typical Heliozoa. We have here, in fact, a case such as often occurs in which different types of structure and life-history are connected by a series of intermediate forms so gradual that any attempt to define the limits of either must fail. Under these circumstances, the limits assigned to one or other group in a descriptive classification depend merely on convenience; the only point of importance is to frame the classification in such a way that it shall not disguise the real continuity of the forms described. For this reason, most modern writers, while recognising the great value of the conception formulated by Hertwig and Lesser, have so enlarged it as to include among the Heliozoa a number of transitional genera (p. 6).

For the sake of convenience, the forms that are included in the Heliozoa in this article are those in which one or more definitely formed nuclei are present during the vegetative phases of life, together with those genera which seem to have the closest zoological relation to them although their nuclei are not known. The genera that are sometimes classified with the Heliozoa, mainly on the ground that they have stiff radiating pseudopodia, but which afford some reasons for believing that their nuclei are dissipated during the vegetative phases of life, are placed with the Proteomyxa (see p. 6).

It will be convenient to consider first the structure of the more highly specialised forms to which Hertwig and Lesser proposed that the name Heliozoa should be restricted, and to discuss the transitional genera afterwards.

The characters of the more specialised Heliozoa may be illustrated by describing Actinophrys sol, the common freshwater species already mentioned. The body is spheroidal and minute, rarely exceeding $0.05 \mathrm{~mm}$. in diameter; in a healthy undisturbed individual numerous stiff pseudopodia, each considerably longer than the diameter of the body, radiate from the surface. The body itself is divided into a 


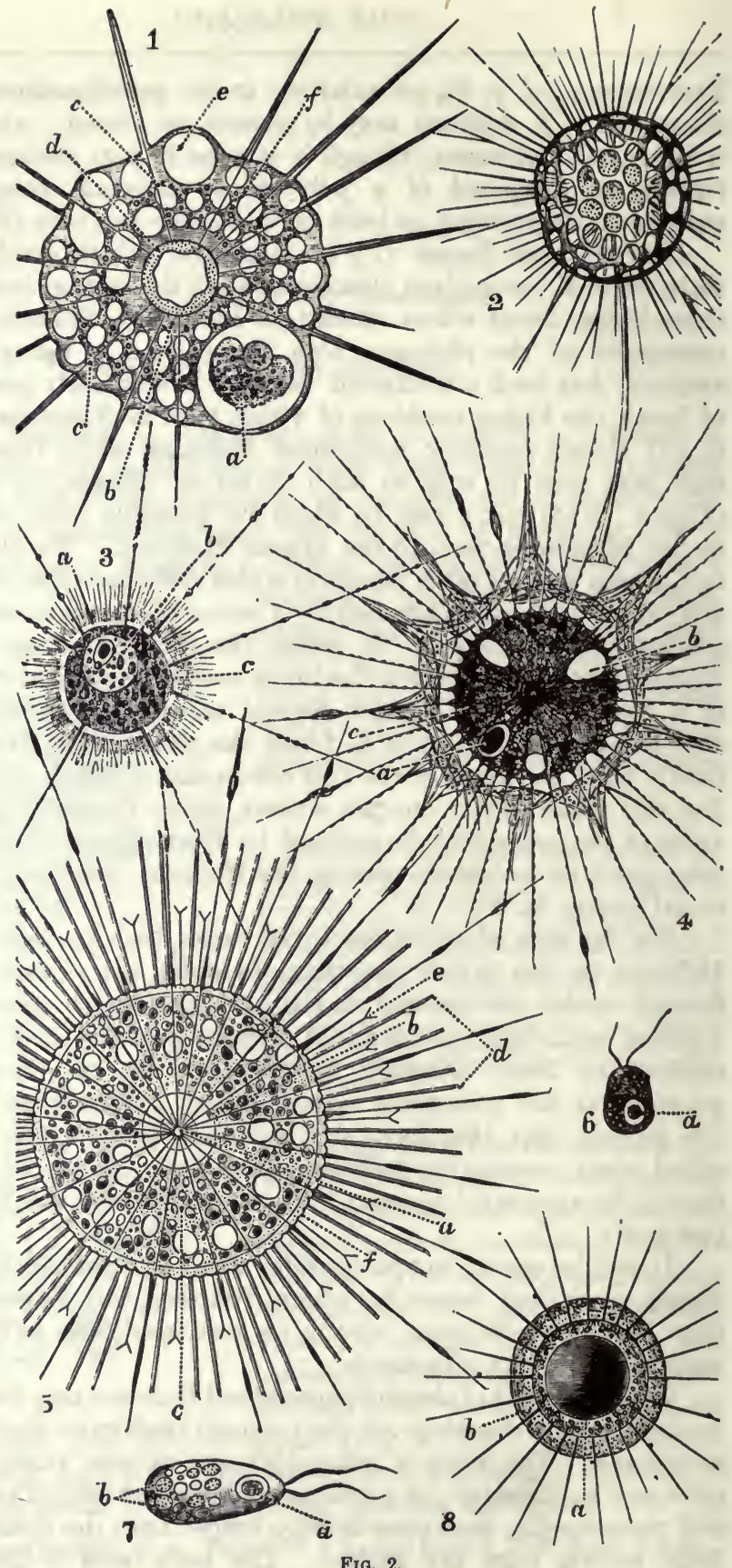

Fig. 2. 
clearer coarsely vacuolated ectoplasm, and a less transparent spongy or feebly vacuolated endoplasm, containing a centrally placed nucleus (Fig. $2(1), d)$. The ectoplasm is normally so crowded with vacuoles that it is reduced to a mere system of septa, and to a thin layer forming the cortex of the radial pseudopodia. During the ingestion of food, however, an aggregation of ectoplasm takes place, forming a short, blunt amoeboid projection by which the food is engulfed, and in which a digestive food-vacuole is formed (Fig. $2(1), a)$. The ectoplasm usually contains a number of bright, highly refringent granules, remarkably uniform in diameter, which are carried from one region to another by streaming movements of the protoplasm; thus they may often be seen streaming to or from the apex of a radial pseudopodium, or towards the apex on one side and away from it on the other. The number of these granules is said to increase with increased nutrition, but their chemical nature is quite unknown.

Fra. 2.

1, Actinophrys sol, Ehrb., $\times 800 ; a$, food-particle lying in a large food-vacuole; $b$, deeplying finely granular protoplasm; $c$, axial flament of a pseudopodium extended inwards to the nucleus ; $d$, the centrally placed nucleus ; $e$, contractile vacuole; $f$, superficial, much-vacuolated protoplasm. 2, Clathrulina elegans, Cienk., $\times 200$. 3, Heterophrys myriopoda, H. and L., $\times 660$; $a$, nucleus ; $b$, clearer protoplasm surrounding the nucleus; $c$, the peculiar felted envelope. 4, Rhaphidiophrys pallida, F. E. Schultze, $\times 430 ; a$, food-particle; $b$, a contractile vacuole (?), the nucleus is probably represented by the circular shaded body lying below $b$; $c$, a food-particle; $d$, the centrosome. The tangentially disposed spicules aro seen arranged in masses at the surface. 5, Acanthocystis turfacea, Carter, $\times 240$; $a$, prodably the centrosome; $b$, clear protoplasm around the centrosome; $c$, more superficial protoplasm with vacuoles and xanthellae; $\iota$, coarser siliceous spicules; $e$, tiner forked siliceous spicules; $f$, finely granular layer of protoplasm. The long pseudopodia stretching beyond the snicules are not lettered. 6 , biflagellate "flagelluls" of Acanthocystis aculeata; $a$, nucleus. 7, Flagellula of Clathrulina elegans ; $a$, nucleus ; $b$, granules of uncertain composition. 8 , Astrodisculus radians, Greeff, $\times 320$; $a$, redcoloured fatty globule; $b$, peripheral homogeneous envelope. (From Lankester, after various authors.)

The endoplasm is rarely vacuolated, and the bright refringent granules are absent from it.

In a normal pseudopodium we can distinguish (1) a cortical layer, and (2) an axial filament. The cortical layer is continuous with the general ectoplasm at the base of each pseudopodium ; it is irregular in thickness, and may by a streaming movement become aggregated into amoeboid droplets of relatively large size during the seizure of prey (Fig. $2(3)$ ). The effect produced upon infusoria, small rotifers, and other ciliated organisms by contact with the pseudopodia is a marked paralysis, which has led many observers, from Ehrenberg onwards, to assume that some poisonous substance is formed by or contained in the cortex; but direct chemical evidence of this is wanting. The axial filament is a clear homogeneous thread, which runs from the apex of a pseudopodium through the substance of the body, to end in a central dilatation in contact with the nuclear membrane. When a pseudopodium is withdrawn, its axial filament disappears, and cannot be demonstrated by staining reagents ; in the living animal it is more easily 
seen at some periods than at others, and may even for a time disappear without retraction of the pseudopodium.

The nucleus is relatively large, with an obvious, doubly-contoured membrane. Within the membrane is a fine reticulum of "linin" threads, on which are small particles of chromatin; there is generally also a single large extra-reticular mass of chromatin, forming a karyosomatic "nucleolus."

The vacuoles are of three kinds: non-contractile and contractile vacuoles which do not contain food-particles, together with digestive vacuoles which contain food. The non-contractile vacuoles form a layer occupying the whole thickness of the ectoplasm; they contain a clear, colourless fluid, in which refringent granules, like those found in the ectoplasm, may often be seen floating, the number of such granules in a single vacuole being sometimes large. A non-contractile vacuole, which contains many granules, sometimes bursts, and the granules are scattered in the surrounding water. There is generally only one contractile vacuole, which rhythmically changes, enlarging slowly until its diameter may be about half that of the body, and then suddenly collapsing; the cycle of dilatation and contraction is completed, at ordinary temperatures, in about one minute (40-100 seconds, Penard [14]). The function of the contractile vacuole is as obscure in this as in other cases. Most observers believe that the fluid, collected during dilatation, is expelled from the body during contraction of the vacuole, so that the whole process is excretory in nature; but while it is difficult towatch an Actinophrys without sharing this opinion, it is equally difficult to demonstrate its truth. The contraction takes place so quickly that it is impossible to be sure whether a rupture of the bodywall occurs or not; and all attempts to show that the collapse of the vacuole is accompanied by a disturbance in the surrounding water, such as would result from the forcible expulsion of its contents, have hitherto failed.

Food-vacuoles are formed in the blunt processes of the ectoplasn already described. When fully formed they contain a clear fluid, surrounding the ingested food-mass, which doubtless contains some solvent in solution, analogous to those demonstrated in the similar vacuoles of amoebae and of ciliata. Formed immediately beneath the surface of the body, the food-vacuole remains throughout its whole existence in the ectoplasm, where the processes of digestion and absorption are completed; a vacuole with a large foodmass may, however, travel into the deeper parts of the ectoplasm. After digestion is completed the residue of the food-mass remains in the vacuole for some time, being ultimately discharged by the bursting of the vacuole at some part of the surface of the body.

The food consists of living organisms, animals and plants. Smaller prey is seized by the blunt ingestive processes alone, with- 
out help from the radial pseudopodia ; a larger creature is seized by a group of radial pseudopodia, which converge round it, generally (always ?) losing their axial filaments, and send out amoeboid processes, which more or less completely engulf the prey. The mass formed by these fused processes and the organism they contain travels towards the body, where it meets and fuses with an ingestive process.

Actinoplurys is capable of performing various rolling or creeping movements on the bottom of the pond, but the creature spends much of its time suspended in the water, where it has a certain power of rising and of sinking, though the way in which this is effected is altogether obscure.

At intervals Actinophrys may withdraw its pseudopodia, the axial filaments of which disappear; it may then secrete a complex cyst of two layers-an outcr, fairly thick transparent layer of gelatinous consistence, within which is a second, thinner layer. After the formation of these layers, the vacuoles disappear, the contractile vacuole being the last to go, and the whole body shrinks. The nucleus now divides mitotically (cf. infra, pp. 25-27), and the cyst divides into two, each of which becomes spherical. Within each of the resulting cysts a third hard, opaque membrane is secreted, and a period of quiescence ensues, after which the walls are ruptured and the creature emerges, new pseudopodia being rapidly formed. This account is based on that given by Schaudinn (17), who says that each daughter-cyst may divide again before entering on a period of quiescence; on the other hand, many observers describe a process of encystment which is not accompanied by any division whatever.

Just as encystment may occur without fission, so fission may, according to Schaudinn, occur without encystment. An individual about to divide in this way withdraws its pseudopodia, and a peculiar mitosis takes place, not accompanied by disappearance of the nuclear membrane or by the formation of centrosomata (infra, p. 28) ; this is followed by fission of the cell-body, and pseudopodia are shortly afterwards emitted.

The processes of fission just described, whether accompanied by encystment or not, are asexual, since there is no previous fusion of individuals or of nuclei. A process of plastogamic fusion, involving the union of a number of individuals (as a rule by the ectoplasm only), without nuclear fusion, frequently occurs. The number of individuals so united is frequently two; but it may be over thirty (Schaudinn). Plastogamic individuals lose their pseudopodia on the surfaces by which they are attached to each other but retain them elsewhere, and the union is not necessarily followed by a period of quiescence. Individuals which have been united in this way for some time may separate without withdrawing those 
pseudopodia which they retained during the plastogamy. Schaudinn thinks it probable that all recorded cases of division without mitosis and without retraction of the pseudopodia are really cases in which plastogamic individuals have been seen to separate.

An observation recently made by Calkins on Paramecium suggests a possible effect of plastogamy. The work of Maupas has shown that, after a certain number of asexual divisions, Paramecium and other Ciliata, when grown in artificial culture-media with a constant supply of food of one kind, exhibit phenomena of degeneration, which quickly lead to the death of the whole culture, unless individuals produced by another zygote are introduced. If such individuals are introduced, plastogamy occurs, which is quickly followed by a complicated sexual (karyogamic) process; and after this the "rejuvenated" culture can enter upon another period of asexual multiplication (cf. Chap. I. Fasc. II. pp. 386, 387). Calkins has, however, shown that a culture which exhibits signs of degeneration may be completely "rejuvenated" by purely chemical stimuli, such as an appropriate change of food, and that if plastogamy alone be allowed to occur, the conjugating individuals being shaken apart before the nuclear changes which precede karyogamy have taken place, these individuals can still go through a further cycle of asexual divisions. Nothing analogous to the phenomena of "senile degeneration" described by Maupas has been observed among the Heliozoa, but it is possible that it may occur, and that the rejuvenescent effect of natural plastogamy is similar to that of the artificial plastogamy observed by Calkins.

Although plastogamy is often followed by a complete separation of individuals, it may be the beginning of a sexual karyogamic process, which has been carefully studied by Schandinn. In this case the mass of individuals, united by ectosarc, sinks to the bottom of the water; the pseudopodia are withdrawn, and a common gelatinous cyst is secreted, like the outer layer of a solitary cyst. Each individual within the gelatinous common cyst secretes a membrane, which is thrown into wrinkles, so that in optical section it looks as if made of spicules joined together. These cysts lie in pairs within the common jelly, the two members of a pair in contact (Fig. 3). The nucleus of each cyst now goes through a mitosis (infra, pp. 25, 27), which results in the extrusion of a single polar body. When the pronuclei of a pair of adjacent cysts have returned to the resting condition, the walls of the cysts break down at the point of contact, the two cell-bodies fuse, their pronuclei also fusing, and the completed zygote becomes spheroidal within the membrane derived from the cyst-walls of the two gametes. After a period of quiescence the nucleus of the zygote divides into two, by a process identical with that observed in asexual cysts, and the division of the nucleus is followed by that 
of the cell-body and of the cyst-wall. On emerging from the cyst, after division, vacuoles and pseudopodia are developed, and the adult condition is assumed.

The majority of the higher Heliozoa resemble Actinophrys in general structure, though their appearance may be greatly altered by the presence of a skeleton or by the formation of a stalk.

The modifications of the cell-body are chiefly those connected with the greater or less development of vacuoles and of various coloured substances. The division into ectoplasm and endoplasm is generally obvious. The ectoplasm usually contains contractile vacuoles, which

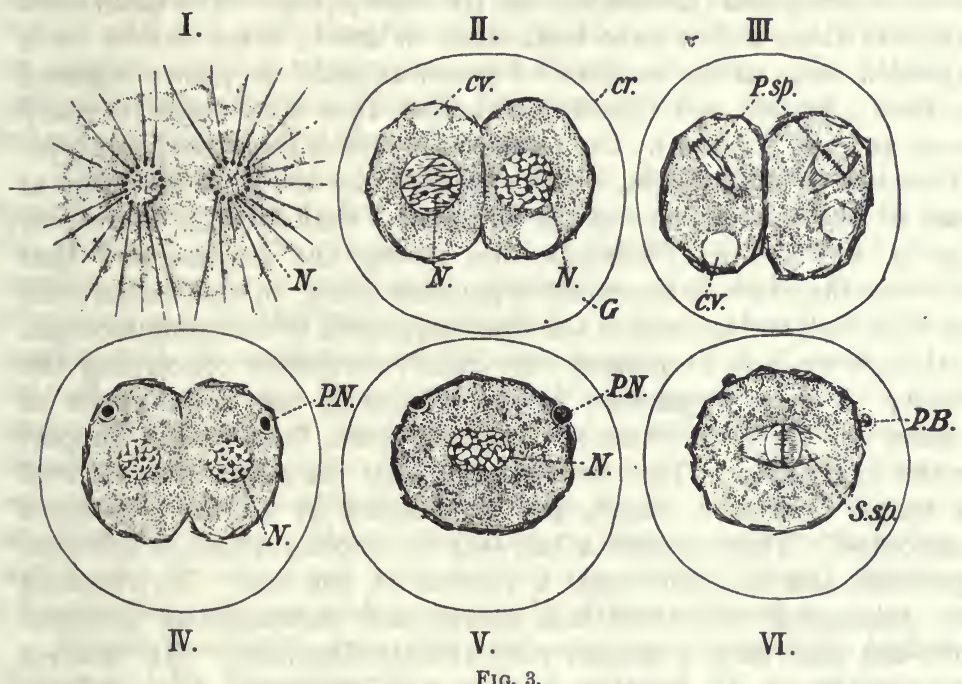

Actinophrys sol. I, two free-swinmung individuals in conjugation. II, the same individuals in an early pliase of encystment. The nuclei are consideribly enlarged. III, forniation of the polar spindles. IV, stige with two reduced nuclei and degenerating polar nuclei. V, the reduced nuclei have fused together and the polar nuclei have reached the periphery. VI, the first segmentation spindle is formed and the polar nuclei are ejected as jolar bodies. cr, cyst membraue; c. $v$, contractile vacuoles; $N$, unclei; $P . N$, polar uuclei ; $P . B$, polar bodies; P.Sp, polar spiudle ; S.Sp, segmentation spinclle. (Áfter Schaudinn.)

may be very numerous (more than 20 in Acanthocystis). In Actinosplucivium the system of non-contractile vacuoles is even more highly developed than in Actinophrys, but in the skelctogenous genera the non-contractile vacuoles are few. The ectoplasm is usually the seat of digestion and assimilation, as it is in Actinophrys ; and usually contains refringent granules, which may be rounded, like those of Actinophrys, or crystalloid (Heterophrys). Perhaps the larger coloured granules which occur either in the ectoplasm or in the endoplasm, or scattered throughout the body, belong to a different category from the refringent granules; large brown granules may occur in the ectoplasm (Pinacocystis), brownish or yellowish bodies may be scattered 
through the whole substance of the body (Pompholyxophrys, Rhaphidiophrys), and in a few forms (Elaeorhanis) a large coloured oil globule is found in the endoplasm. In Actinosphaerium, where digestion and assimilation occur in the endoplasm, that region of the body is crowded with brownish refringent granules, leaving the ectoplasm relatively free. A few of the larger coloured droplets have been described as fatty; but the chemical nature of most of these coloured bodies is quite unknown.

Chlorophyll associated with differentiated chloroplasts is found either in the endoplasm (some varieties of Actinosphaerium Eichhornii) or in the ectoplasm (Rhaphidiophrys, Heterophrys, etc.). The nature and origin of these bodies have been much debated; some writers have regarded them as the remains of green animals or plants ingested as food; Archer and Greeff maintained that they were in many cases, at least, formed by the Heliozoa in which they were observed. There can be little doubt, however, that they are in some cases at least of the same nature as the Xanthellae that occur in Radiolaria (see p. 97) and in Trichosphaerium among the Lobosa, and that they are therefore independent organisms living in association with the Heliozoa, and are not, as has been suggested, of endogenous origin. Although we have at present very little information concerning the history of these organisms in the Heliozon, the observations of Penard on green varieties of Actinosplccerium lend strong support to this suggestion. This author found that the green cells are oval in shape, $7-10 \mu$ in length, and surrounded by a clear gelatinous membrane. They possess a bell-shaped chromatophore, a spherical pyrenoid, and in some cases a vacuole at one end. On crushing the Actinosphaerium, these cells escape, and subsequently protrude first one and then a second very delicate flagellum. He believes the organism to be identical with the Palmellacean Alga Spliaerocystis Schröteri (Chodat). In other cases he has seen a large number of flagellate organisms belonging to the genus Chlamydomonas attached to the surface of an Actinosphaerium, and has shown that they are actively attracted to the host. It is true that at present it has not been proved that the Chlamydomonads actually enter the ectoplasm of the Actinosphaerium and become the xanthellae; but in view of the proof recently published by Keeble and Gamble (10), that the infecting organism of the Turbellarian Convoluta belongs to the Chlamydomonadina, Penard's observation is very suggestive. Awerinzew (1) has recently described the xanthella of Actinosphaerium as Zoochlorella actinosphaerii.

In addition to the xanthellae, other organisms are occasionally found in the ectoplasm of the Heliozoa. Thus, a ciliate infusorian allied to the genus Blepharisma has been found in as many as 30 per cent of the individuals of Rhaphidiophrys viridis found at Bernex, and a rotifer attributed to the genns Monolabis by Archer 
and to the genus Proales by Penard occurs in the ectoplasm of Acanthocystis turfacea. It is probable also that the minute rods that have been found in Acanthocystis turfacea (Leidy) and the corpuscles in A. spinifera, Rhuphidiophrys viridis, and Heterophrys myriopoda may be bacteria.

The structure of the pseudopodia is probably very constant in all the higher forms. In Elaeorhanis, Nuclearia, and Hedriocystis there appears to be no axial filament. In Clathrulina elegans and in Elaeorhanis they are sometimes bifurcated. In a Heliozoon allied to Actinophrys, Crawley (5) has recently observed that the psev topodia are arranged in tufts at the periphery, and may either remain stiff and motionless like the typical pseudopodia of Heliozoa or assume lashing movements like flagella or cilia. In Actinosphaerium arachnoideum, Penard, the pseudopodia are very long, branching, and capable of anastomoses.

The relation of the inner ends of the axial filaments of the typical pseudopodia varies in a remarkable way with variation in the position of the nucleus. In Actinosphaerium, where the number of nuclei is very great (sometimes over 400), the axial fibres end each in the neighbourhood of a nucleus, if not in actual contact with its membrane, so that the relation is here similar to that of Actinophrys. In a great number of genera, however, the centre of the body is occupied by a deeply-staining granule first discovered by Grenacher (6) and now known to behave like a centrosome; to this body the inner ends of the axial filaments are attached (Fig. 6, A). There is never more than a single centrosome, which may be associated with a single eccentrically-placed nucleus (Acanthocystis, etc.) or with many nuclei (Gymnosphaera).

Skeletal investments of several kinds are found among the higher Heliozoa. In Elaeorhanis the body is covered by an agglutination of diatoms, sand-grains, etc., loosely cemented together; in Heterophrys (Fig. $2(3)$ ) the body is surrounded by a finely granular, transparent capsule, of gelatinous consistency and quite unknown composition, soluble in strong acids ; this capsule is separated from the ectoplasm by a considerable space, traversed only by the radial pseudopodia, which emerge through perforations in its substance. The outer surface of the capsule bears delicate radial spines, shorter than the pseudopodia, which are regarded by Penard as being chitinous in composition on the ground that they are soluble in boiling sulphuric acid. In Actinolophus the greater part of the body is naked, except for a short time before encystment; but the stalk, on which the body rests, is a tube of what appears to be chitin, containing one or two thread-like prolongations of the body. The greater number of skeletons are, however, siliceous, the silica being deposited in the form of separate or loosely-articulated plates or spicules (Chalarothoraca) or as a continuous basketwork (Desmo- 
thoraca). In the Chalarothoraca the siliceous particles may be minute and spherical, lying close together and forming one or several layers (Pompholyxophrys), or they may be elongated spicules, or flattened plates. Spicules are of two kinds, the one kind curved and pointed at each end, the other straight, pointed or bifurcate at one end, flattened and expanded at the other. The curved spicules are placed tangentially to the surface of the body, and may be the only skeletal elements present (Rhaphidiophrys), in which case they form a loose investment for the animal, from which groups of - spicules are occasionally carried up the pseudopodia by the

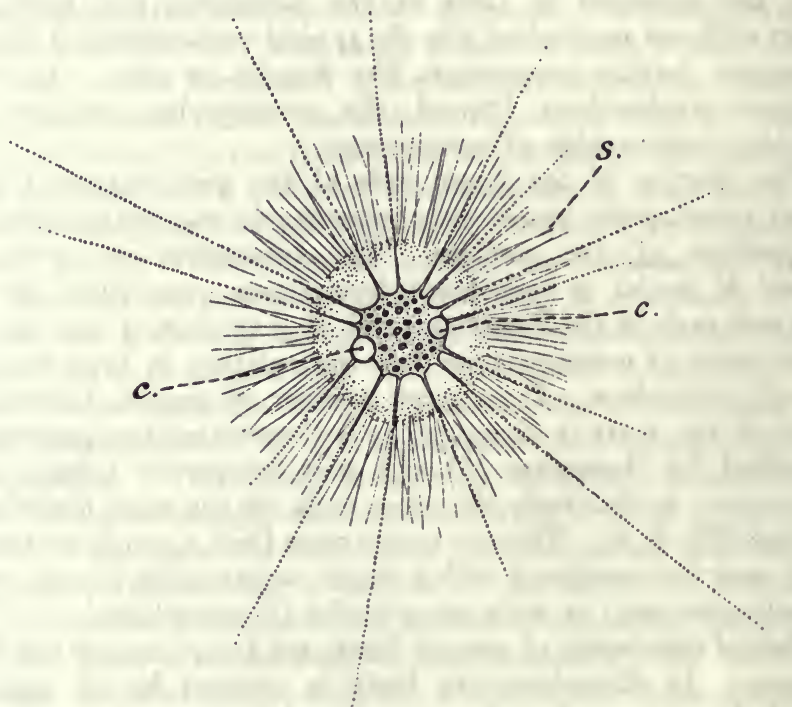

Fig. 4.

Heterophrys Fockei, Archer. c.c, contractile vacuoles. A nucleus is present in the centre of the protoplasm, but is not shown in the figure. 8 , radial chitinous (?) spines surrounding the envelope. Several xanthellae are seen in the protoplasm. (After Hertwig and Lesser.)

streaming movement of the ectosarc (Fig. 2 (4)). In lihaphidocystis some very remarkable funnel-shaped or wine-glass-shaped spicules are found. In Acanthocystis both tangential scales and straight spicules may be present, the latter being radially placed, with their pointed ends outwards. There may be two kinds of these radial spicules, a longer hollow kind with the free extremity bluntly pointed, and a shorter solid kind with the free end forked (Fig. 2 (5)).

Siliccous plates, articulated together by their edges to form a capsule round the body, occur in Pinacocystis and in Pinaciophora. In Pinacocystis the pseudopodia emerge through the spaces between the plates, but in Pinaciophora, according to Greeff, the plates are perforated by fine pores. 
In the Desmothoraca, of which Clathrulina is the best-known genus, the skeleton has the form of a spherical basketwork, the bars of which often show a median ridge on the outer surface, the spaces between the bars being irregularly polygonal with rounded angles (Fig. 2 (2)). This basketwork is supported on a long, hollow siliceous stalk.

The structure of the nucleus and the processes of karyolinesis have been minutely described by R. Hertwig (8) in Actinosphaerium, and his descriptions are in accord with what is known concerning them in the higher Heliozoa generally.

The resting nucleus of Actinosphaerium has a definite membrane continuous with an internal achromatic network whose relation to the chromatin elements is very variable. The whole of the chromutin may be collected into a relatively large mass, supported in a matrix of achromatic substance ("plastin ") and forming a conspicuous "karyosomatic" nucleolus; such a condition of the nucleus may be induced by starvation, or it may appear as a preliminary to division. In well-fed individuals the chromatin spreads through the nucleus in the form of coarse branches or networks.

Nuclear division may be direct, in the formation of buds or swarm-spores, or by karyokinesis. Karyokinesis occurs in the division of the nuclei within the body of the multinucleate forms (e.g. Actinosphaerium) without being followed by division of the body; in forms with a single nucleus it occurs during fission, and during the maturation of the conjugants (gametocytes).

In Actinosphaerium there are three kinds of karyokinesis, that differ from each other in some details of considerable theoretical importance. In the nuclear divisions of the unencysted body no centrosomes are formed, and the spindle is considerably compressed between the two poles. In both the mitoses of the maturation of the gametocytes, centrosomes occur at each pole of the spindle, but in the first (polar) division the chromosomes are larger than in the second (polar) division, and there are some other differences in detail of minor importance. In all three kinds of karyokinesis there are numerous chromosomes (about 150), and both the divisions of the nuclei in the maturation of the gametocytes are of the nature of "equation" divisions, the number of the chromosomes not being reduced.

It may be convenient to describe in greater detail the second polar division of Actinosphaerium as an example of the karyokinesis of the Heliozoan nucleus. At the end of the first polar division, one of the resultant nuclei degenerates and is ultimately ejected with the first polar body, the other remains in the centre of the protoplasm and passes through a short period of rest. At one pole of this resting nucleus there is a clearly-marked centrosome surrounded by a small aster. Antecedent to the second polar division the centrosome diminishes in size (Fig. 5, I, c), and 
subsequently divides into two parts, which travel to opposite poles of the nucleus. The nucleus now begins to increase considerably in size, and is seen to contain several large chromatin bodies which certainly contain both chromatin and plastin derived from the nucleoli (Fig. 5, II). The centrosomes at each pole of the nucleolus are of considerable size and more conspicuons than at any other time in the divisions of the three kinds of karyokinesis. The chromosomes are now formed by a breaking down of the
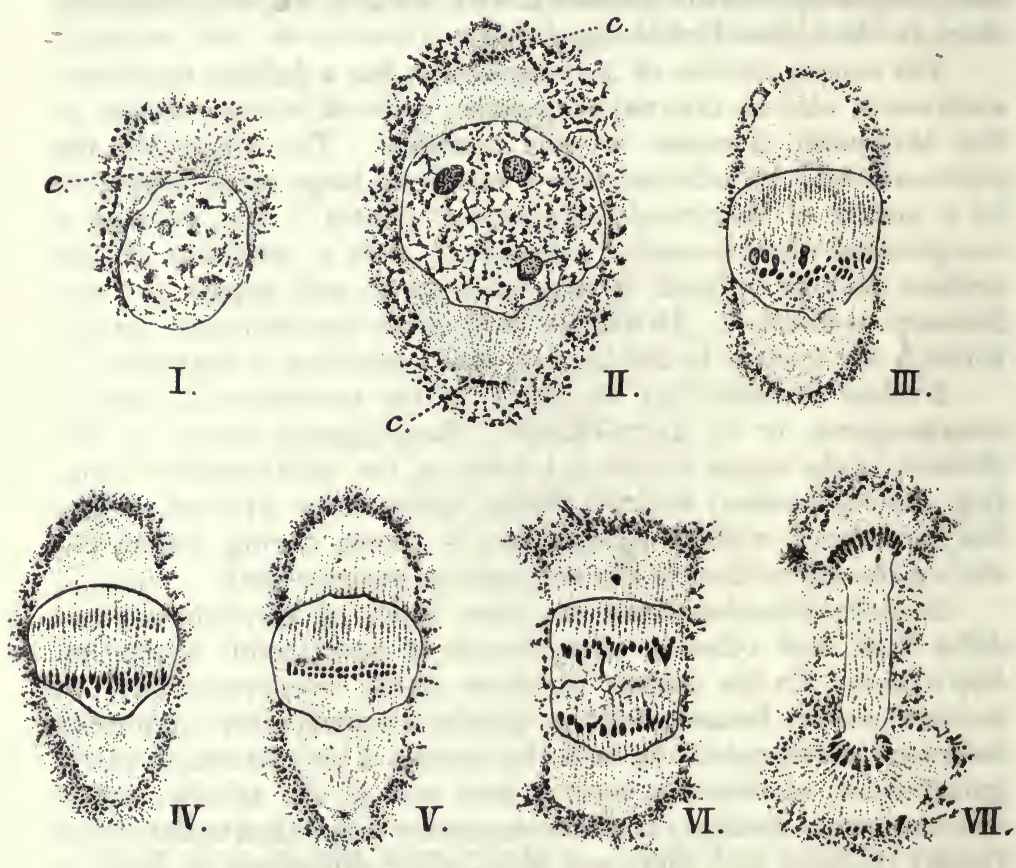

Fid. 5.

Actinosphcerium. Formation of the second polar spindle, 1, the nucleus after the first polar division, the ceutrosome $(c)$ reduced in size previous to the formation of the seconil polar tigure. II, the same nucleus at a later stage with two centrosomes. III, IV, V, VI, VII, stages in the formation of the second polur nucleus. (After R. Hertwig.)

chromatin masses, and gradually assume an equatorial position. They are at first very irregular and angular in shape, but ultimately become rod-shaped, constrict in the middle, and divide transversely. The spindle fibres seem to be formed from the achromatic network, and several plastin remnants remain in the nucleus during the formation of the chromosomes. The chromosomes now travel towards the opposite poles of the spindle in the usual way (Fig. 5, VI), and subsequently become arranged in a fan-shaped manner at the extremities of the now elongated spindle. According 
to Hertwig (8) the chromosomes of the second polar division are only half the size of the chromosomes of the first division, and there is, therefore, a reduction in the mass of the chromosomes, although there is apparently no reduction in their number.

The karyokinesis of the nuclei of the ordinary unencysted Actinospharium differs from that just described principally in the fact that no centrosomes are present. The first sign of commencing division in these nuclei is the accumulation of a clear mass of nearly homogeneous protoplasm at each pole; the nucleus becomes
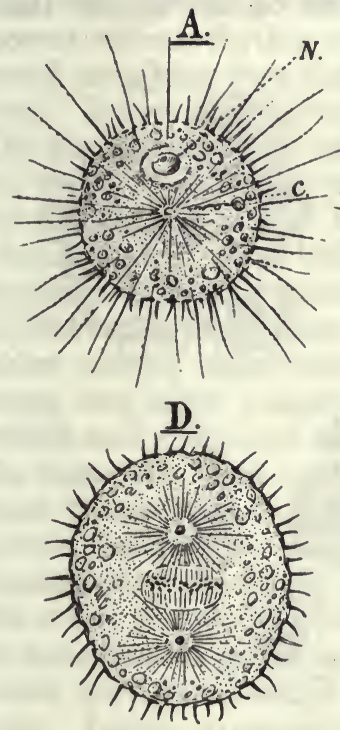

B.
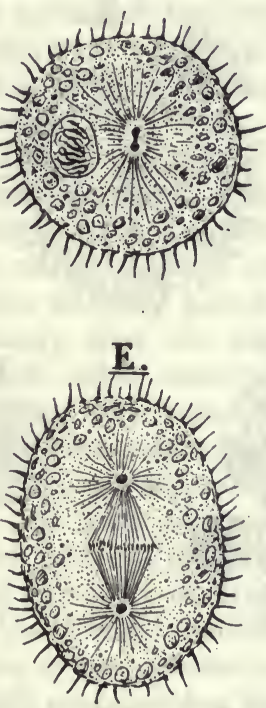

C.
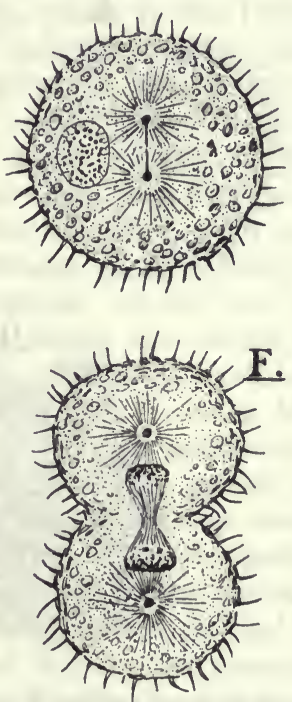

FIG. 6.

A, Accnthocyst is aculeata, H. and $\mathrm{I}_{\text {. }}$, in the living condition, with expanded psendopodia. $N$, the nucleus; $c$, the centrosome. $B, C, D, E, F$, successive stages in the mitoticldivision of the nucleus as seen in preparations. (After Schaudinn.)

flattened so that the diameter which passes through the protoplasmic masses is the shortest, and at each end of this diameter an accumulation of achromatic nuclear substance is formed, giving rise to what Hertwig calls the "polar plates."

In Acanthocystis the nucleus is situated excentrically, and consists of a central deeply-staining body, the "pseudonucleolus," surrounded by an area which certainly contains a linin network but much less chromatin. At the exact centre of the endoplasm there is a small body which exhibits radiating lines which appear to extend outwards and be continuous with the axes of the pseudopodia (Fig. 6). This body, originally described by 
Grenacher (6) as the "Centralkorn," has been proved by Schaudinn to be a true centrosome. It has been discovered to be a permanent of the body in lihaphidiophrys, Actinolophus, Heterophrys, and Sphaerastrum. Before division of the nucleus it divides into two equal parts, which take a position at opposite poles of the endoplasm, each one surrounded by an aster of radiating lines. The nucleus leaves its excentric position and becomes situated in a direct line between the two centrosomes. The nuclear membrane then fades away and a party of numerous small chromosomes occupy a position of an equatorial band on the spindle that
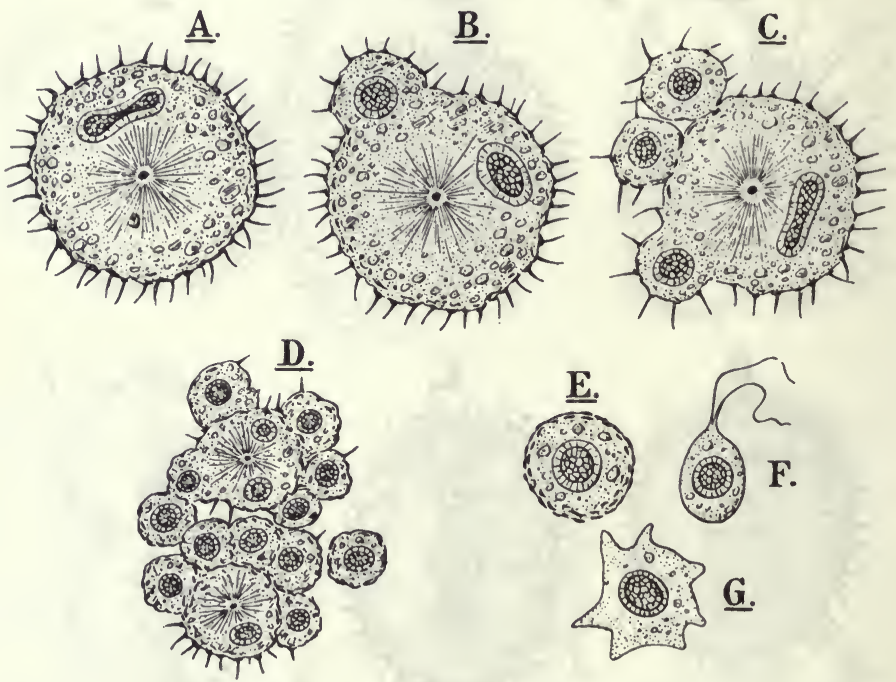

F1G. 7.

A, B, C, direct amitotic division of the nucleus of Acanthocystis aculcata as seen in the process of the formation of buds. 1), a colony of Acanthocystis formed by the gemmation of a single individual. Only two individuals of the colony exhibit a centrosome, and these have been formed by division, with muclear mitosis, of the primary individuals; the others liave been (formed by gemniation without nuclear mitosis. $\mathrm{E}$, a single bud freed from the colony. F, a flagellula. G, an anıeboid spore. (After Schaudinn.)

is formed from the linin of the nucleus. The subsequent phases of the nuclear division resemble those of the typical karyokinesis of the metazoan cell.

In the formation of the buds of Acanthocystis the nucleus divides directly and the centrosome remains unchanged (Fig. 7, A, B). The buds are therefore for a time without any centrosome, but this body is formed afresh in the buds from the nucleus. (See Part I. Fasc. II. Fig. 20, p. 41.)

Reproductive Processes.-Probably all the higher Heliozoa are capable of fission, preceded or not by encystment, although the process has not been observed in all. The division of the nucleus 
is mitotic, and is probably of the type observed in adult Actinosphaerium or of that seen in Acanthocystis, according to the presence or absence of a permanent centrosome.

Budding has been observed in several cases; and the process has lately been described in detail by Schaudinn (19) in Acanthocystis. The nucleus divides directly once or several times, so that the body may contain a considerable number of nuclei; during this process the pseudopodia are not withdrawn, the centrosome and the system of axial filaments remaining unchanged. One of the nuclei resulting from this division remains in the body of the parent without further change; each of the others travels into a small projection from the surface of the body, which is the future bud. Every bud is covered with a layer of spicules derived from the parental skeleton, but it contains no centrosome, nor any trace of radial fibres. The buds so formed may behave very differently in different cases, and there is at present no knowledge of the circumstances which determine their behaviour. A bud may separate from the parent in the condition described, and may divide one or more times, the products of division going through a short resting stage before emitting pseudopodia; or the resting stage may occur immediately after the bud leaves the parent, in which case it does not divide before assuming the adult condition. In these cases there is nothing like "spore-formation"; but a bud may become amoeboid, and creep out of its skeletal investment, either before the skeleton has separated from the parent or immediately afterwards; and such an amoebula may creep about for a day or two, by means of blunt pseudopodia, before it becomes spherical and secretes new spicules; or, division of the nucleus may occur within the bud, so that several amoebulae leave it, instead of one. Lastly, an amoebula, at the moment of leaving the parental skeleton or soon afterwards, may develop two flagella, by means of which it swims for a short time; such "flagellulae" quickly become amoeboid and creep about for a further period as amoebulae, before becoming spherical. None of these buds or spores are known to conjugate, and indeed the origin of sexual spores by an amitotic division would be remarkable; but, however they behave in the meantime, about the fourth or fifth day after emission each of them becomes spheroidal, and secretes a skeleton of small tangential spicules, which are first formed in the immediate neighbourhood of the nucleus, and afterwards travel to the periphery. The centrosome arises from the nucleus (Part I. Fasc. II. Fig. 20, p. 41), and after it is established the axes of the radial pseudopodia appear.

The formation of "swarm-spores" was first described by Cienkowski (4) in Clathrulina; it was more recently discovered by Schaudinn (19) in Acanthocystis; and it may occur in Actinophrys (Penard). 
Sexual (karyogamic) processes have only been observed in Actinophrys and in Actinosphaeriun ; the process in Actinophrys has already been described; the phenomena observed in Actino-

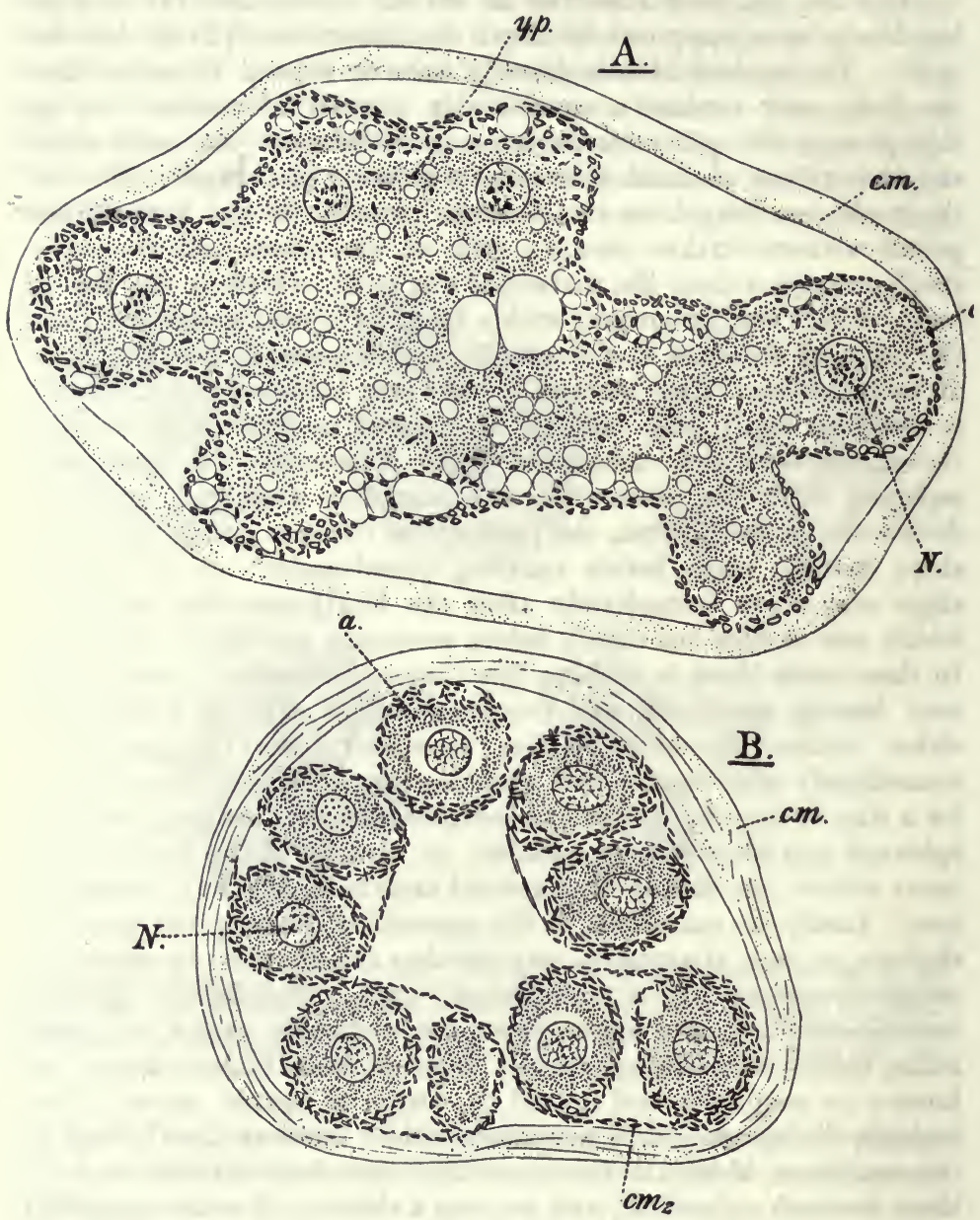

Fia. 8 .

Actinosphaerium. A, a mother-cyst just before it breaks up into primary cysts. The nuclei are considerably reduced in number and the protoplasm contains numerous small oval yolk-plates, y.p. B, the primary cysts have each divided into two secoudary cysts. The sister-cyst of $a$ is not seen in the flgure. $N$, nuclei ; $c . m$, mother-cyst membrane; c. $m_{2}$, cyst inembrane of the first order. (After Brauer.)

sphaerium are in many ways remarkably different. The first indication of approaching karyogamy is the encystment of a single individual. The pseudopodia are withdrawn, their axial filaments 
are absorbed, and the animal sinks to the bottom of the water, where it exhibits considerable amoeboid movement, sometimes giving out slender pointed pseudopodia which have no axial filaments ; food-particles are ejected, and a thick, transparent cyst is formed. This "mother-cyst" is of gelatinous consistence, sticky on the outside, and its substance is deposited in concentric layers. The peripheral vacuoles disappear after encystment, and numerous peculiar oval discs, probably consisting of reserve food-material, appear; these bodies may be called "yolk-plates" (Hertwig). While the yolk-plates are forming, the number of the nuclei diminishes, until not more than one-twentieth of the original number remain. The process by which this reduction is effected is not quite clear; Schneider and more recently Brauer (2) have described a fusion of nuclei during the reduction; and Brauer's figures of this fusion are very convincing; Hertwig, although he considers it not improbable that such a fusion occurs, has never been able to demonstrate it. When the reduction in the number of nuclei is completed, the body divides into as many pieces as there are nuclei, each piece containing a single nucleus. Every result of this division is enclosed in a siliceous "primary cyst," largely formed by rearrangement of scattered spicules secreted before division. The number of primary cysts varies from one to thirty-five; and Smith (20) has recently shown that there is an interesting relation between the number formed and the temperature at which encystment occurs ; at high temperatures the number is smaller and the cysts are larger; at low temperatures the number of cysts is greater and their diameter less. Smith also shows that the quantity of chromatin contained in the nuclei of primary cysts formed at a low temperature is greater than that found in cysts formed at higher temperatures. Shortly after its formation, each primary cyst divides into two; the nucleus behaves in essentially the same way as dividing nuclei in the unencysted form (cf. p. 25); the number of chromosomes is very large, and is estimated by Hertwig at from 130 to 150 . The secondary cysts, formed by the division of each primary cyst, now behave like gametocytes; a centrosome is extruded from the nucleus, and a nuclear division occurs, leading to the extrusion of a first polar body. After the extrusion of the first polar body, the nucleus enters into a resting stage, a single centrosome remaining outside it; a second division now occurs, leading to the formation of a second polar body, which is in turn extruded. The chief points of interest in the formation of the polar bodies are (1) the similarity of the process of formation, so that neither division can be called a "reducing division"; and (2) the very pronounced resting stage which intervenes between them.

After the extrusion of the polar bodies, the two gametes, 
formed from the products of the division of a single primary cyst, fuse again into a single zygote, their pronuclei uniting to form a single fertilised nucleus. After this process is completed, a membranous or gelatinous layer is formed within the siliceous cyst, which Hertwig compares to the yolk-membrane so frequently formed by fertilised ova. A multiplication of nuclei now occurs within the cyst; the creature becomes amoeboid, and emerges. After emergence, individuals with a single nucleus are not very rare, so that the amoeboid young may possibly sometimes divide; but the process has not been observed.

If the foregoing account be correct, we have in Actinosphaerium the only case in the whole animal kingdom in which self-fertilisation is shown to be of normal occurrence. There are, however, several points to be considered before this view can be adopted without qualification. Brauer (2) asserts that the formation of Hertwig's "primary" cysts is preceded by a fusion of nuclei, so that the nucleus of each primary cyst is really formed from two resting nuclei, confirming the view put forward by Anthon Schneider in 1877; and Hertwig admits that there is a considerable body of evidence in favour of this view, though if such a fusion takes place it must be a very rapid process, affecting all the nuclei in the body simultaneously; otherwise its occurrence must have been frequently witnessed by an observer so skilful and patient as Professor Hertwig. The frequent occurrence of plastogamy between adult individuals makes it very possible that all the nuclei in the same body may not be of the same origin; and therefore the formation of the primary cyst-nuclei by the fusion of two others might, in many cases, at least, mean the fusion of nuclei originally produced in different individual bodies (Schaudinn). Such a preliminary fusion of the nuclei of gametocytes, which separate before giving off polar bodies and finally fusing to form a zygote nucleus, has been observed in Spirogyra (cf. Klebahn [11], quoted by Hertwig); and a process of a similar kind-a fusion of gametocyte nuclei before the extrusion of polar bodies, the polar bodies being only given off after division of the fertilised zygoteappears to occur in some desmids (Closterium, Klebahn [11]). If, therefore, we can believe that an individual before encystment has normally exchanged some of its nuclei for those of another individual during an antecedent plastogamy, and that a fusion of nuclei in pairs takes place before the formation of the "primary" cysts, the nuclear history of Actinosphaerium will not be without parallel ; but there is direct evidence that normal encystment may occur without plastogamy, since Hertwig has succeeded in keeping an isolated individual under control through the entire period from "hatching" until the production of normal, fertile cysts. Again, all observers are agreed that plastogamy is not necessarily followed 
by encystment within any definite period, and Hertwig has obtained cysts from individuals in which it had certainly not occurred for several weeks.

\section{CLASS HeliozoA, Haeckel.}

\section{Order 1. Aphrothoraca, Hertwig.}

Heliozoa usually devoid of a skeletal or gelatinous envelope. A membranous envelope, sometimes with siliceous spicules, is only developed during encystment.

Genera-Actinophrys, Ehrb. ca. $50 \mu$. Cosmopolitan in fresh water and probably cosmopolitan in the sea (Fig. 2). Camptonema, Schaud. Numerous small contractile vacuoles and about 50 nuclei. 120-180 $\mu_{\text {. }}$ Marine, Norway. Actinosphaerium, Stein (Fig. 1). Two or more large contractile vacuoles, numerous nuclei. $1 \mathrm{~mm}$. Cosmopolitan in fresh water. Gymnosphaera, Sassaki. Numerous nuclei. Very numerous and very long pseudopodia. $140 \mu$. Pseudopodia up to $800 \mu$ in length. Actinolophus, F. E. Schultze. Body usually pear-sheaped. One nucleus. Pseudopodia long and thin. Sometimes (always ?) with a thin gelatinous membrane perforated by the psendopodia. Attached to a foreign object by a. long hollow stalk. Body $30 \mu$ in diameter. Stalk $100 \mu$ long by $3-4 \mu$ in diameter. Marine. North Sea. The genus Actinosphaeridium, Zacharias, freshwater, Germany, is closely related to Actinolophus. The genera Zooteirea, Wright, an oval form with a contractile stalk, from the Firth of Forth, Estrella, Frenzel, and Phythelius, Frenzel, are imperfectly known. Phythelius is probably an Alga. Nuclearia (see p. 8), Cienkowski, differs from the other Heliozoa in having an anoeboid body and pseudopodia without any definite axis. It is sometimes regarded as a Proteomyxan. Myxodiscus crystalligerus is a form that is doubtfully placed among the Heliozoa. It was found by Prowazek in a sea-water aquarium. The genus Archerina, Lankester, which has been regarded by some authors as a Proteomyxan and by others as a Heliozoon, is now placed by Lankester (12) among the Algae. It is the same genus as Golenkinia (Chodat.), belonging to the Pleurococcaceae, the naked protoplasm surrounding the green organism in many instances observed and figured by Lankester being that of a Vampyrella-like or amoeboid organism symbiotic with or merely crawling on the alga.

\section{Order 2. Chlamydophora, Archer.}

Heliozoa with a soft mucilaginous envelope, but without any solid skeletal elements.

Astrodisculus, Greeff (Fig. 2(8)). Body spherical. Pseudopodia very long and delicate. Several species recently described by Penard. Freshwater. 20-40 $\mu$. The genus Heliophrys, Greeff, is evidently closely related to Astrodisculus, but has also been placed with Heterophrys (see West [21]). The form described by Greeff as Chondropus viridis is regarded by Penard as a peculiar species of Vampyrella. 


\section{Order 3. Chalarothoraca, Hertwig and Lesser.}

Heliozoa with a loose envelope consisting of isolated siliceous or chitinous spicules bound together by a mucilaginous or protoplasmic matrix.

Heterophrys, Archer (Figs. 2 (3) and 4). A granular envelope containing very delicate and indistinct chitinous spicules. One nucleus and one or more contractile vacuoles. $\quad 10-20 \mu$. Freshwater (or marine ?). Sphaerastrum, Greeff. According to Penard (14) this genus represents a species of - Rhaphidiophrys. Elaeorhanis, Greeff. The endoplasm contains a large yellow or brown oil-globule. Envelope with attached sand-grains and diatoms. $50 \mu$. Freshwater. Lithocolla, F. E. Schultze. No definite oil-globule. Envelope with numerous siliceous bodies, for the most part adventitious diatoms, and amorphic grains. Often united together in colonies by a gelatinous matrix. $38-45 \mu$. Freshwater and marine. Lithosphaerella, Frenzel. Envelope covered with several layer's of sandgrains، Freshwater (Argentine) and marine (Mediterranean). All the genera so far mentioned were placed by Schaudinn (18) and others in the Order Chlamydophora, but were transferred to the Order Chalarothoraca by Penard.

The following genera have isolated siliceous skeletal spicules and are regarded as more typical of the Order. Pompholyxophrys, Archer = Hyalolampe, Greeff. Skeleton composed of minute spherical pearls of silex. 40-50 $\mu$. Freshwater. Pinaciophora, Greeff. Skeleton consisting of overlapping circular plates. $50 \mu$. Freshwater. The genus Pinacocystis, H. and L., which is said to be marine, is closely related to Pinaciophora. Rhaphidiophrys, Greeff (Figs. 2 (4) and 9). Skeleton consisting of a number of minute needles, spindles or half-rings arranged loosely, tangentially, and radially in a protoplasmic envelope. This genus contains several species and is widely distributed in fresh water. It is often found in colonial groups. Freshwater and marine ( $A$. pelagica, Ostenfeld [13]). Rhaphidocystis, Penard. Spicules of various forms, but always different from those of Rhaphidiophrys, scattered in a protoplasmic envelope. $\quad 12-20 \mu$. Freshwater. $\quad$ R. simplex $=$ Acanthocystis simplex, Schaudinn. Central Africa. Acanthocystis, Carter (Figs. 2 (5) and 6). The envelope of siliceous spicules apparently continuous, formed of tangential scales apparently touching one another and an armature of radial needles. This genus contains a large number of species, widely distributed in fresh water. Two species, $A$. italica and A. marina (Ostenfeld), are marine. The genera Cienkowskia, Schaudinn, and Wagnerella, Meresch., from the White Sea, differ from the others in the possession of a stalk.

\section{Order 4. Desmothoraca, Hertwig and Lesser.}

Heliozoa provided with a continuous basket-like skeleton perforated by holes.

Clathrulina, Cienkowski (Fig. 2 (2)). Apertures in skeleton relatively large. Provided with a stalk. $70 \mu$. Freshwater. Hedriocystis, H. 
and L. Apertures very small. Provided with a stalk. 20-30 $\mu$. Freshwater. Elaster, Grimn. Apertures very numerous. No stalk. $20 \mu$. Freshwater. Choanocystis, Penard. Apertures provided with long funnelshaped collars. No stalk. $13 \mu$. Freshwater.

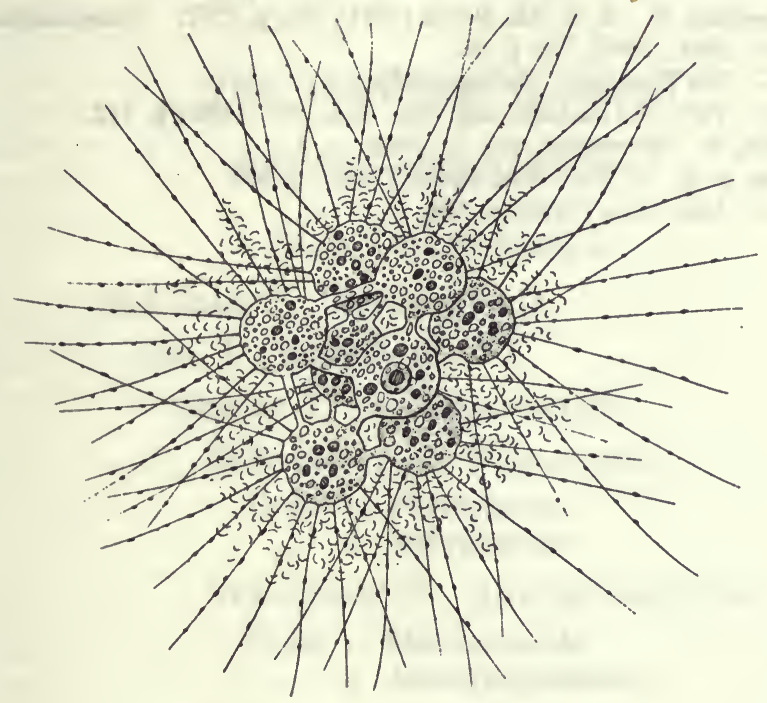

F10. 9.

Rhaphidiophrys elegans. Eight individuals united together by protoplasmic strands and surrounded by a skeleton of half-rings. A nucleus is shown in one individual. (After Hertwig and Lesser.)

\section{LITERATURE.}

A very extensive list of works on Heliozoa will be found in the book by penard (14). The following is a list of some of the principal books and papers referred to in the text :-

1. Awerinzew, S. O zookhlorellakl u Prostyeisshikh (On Zoochlorellae in the Protozoa). Protok. St. P'eterb. Obshch. xxxi. 1, No. 7 (1900), p. 322.

2. Brauer, A. Zeitschr. wiss. Zool. lviii. (1894), p. 189.

3. Bütschli, $O$. Bronn's Thierreich, Protozoa, 1880-82. This contains a bibliography up to the year 1879 .

4. Cienkowski, L. Arch. mikr. Anat. iii. (1867).

5. Crawley, S. P. Ac. Philad. 54 (1902), p. 256.

6. Grenacher, H. Z. wiss. Zool. v. (1869), p. 259.

7. Hertwig, R., and Lesser, E. Arch. mikr. Anat. x. (1874), Supp.

8. Hertwig, R. Abh. k. bayer: Akad. Wiss. xix. (1898).

9. - Fest. von Haeckel (1904).

10. Keeble, F., and Gamble, F. W. Quart. J. Micr. Sci. li. (1907), p. 167.

11. Klebahn, H. Jahrb. wiss. Bot. xxii. (1890), p. 415. 
12. Lankester, E. R. On Archerina, Golenkinia, and Botryococcus. Quart. J. Micr. Sci. lii. (1908), p. 423.

13. Ostenfeld, C. $H$. Meddel. Komm. Havundersög. Kobenhavn.

14. Penard, E. Heliozoaires d'eau douce. Genève (1904).

15. Prowazek, S. von. Arb. Inst. Wien, xii. (1900), p. 294.

16. Schaudinn, F. S. B. Ak. Berlin (1894), (2), p. 1277. (Camptonema.)

17. — Ibid. (1896), (1), p. 83.

18. - Das Tierreich. Heliozoa (1896).

19. - Verh. der deutsch. zool. Ges. Bonn, vi. (1896), p. 113.

20. Smith, G. Biometrica, vol. ii. (1902), p. 3.

21. West, G. S. J. Linn. Soc. xxviii. (1901), p. 308.

22. —— Ibid. xxix. (1903), p. 108. 


\title{
THE PROTOZOA (continued)
}

\author{
SECTION C.-THE MYCETOZOA ${ }^{1}$
}

\section{CLASS MYCETOZOA.}

Sub-Class I. Euplasmodida.

Div. I. Endosporeae.

Cohort I. Amaurosporales.

Sub-Cohort A. Calcarineae.

Order 1. Physaraceae.

"2. Didymiaceae.

Sub-Cohort B. Amaurochaetineae.

Order 1. Stemonitaceae.

"2. Amaurochaetaceae.

Cohort II. Lamprosporales.

Sub-Cohort A. Anemineae.

Order 1. Heterodermaceae.

"2. Liceaceae.

" 3. Tubulinaceae.

" 4. Reticulariaceae.

" 5. Lycogalaceae.

Sub-Cohort B. Calonemineae.

Order 1. Trichiaceae.

"2. Arcyriaceae.

„3. Margaritaceae.

Div. II. Exosporeat.

Order. Ceratiomyzaceae.

SUB-Class II. Sorophora.

Order 1. Guttulinaceae.

"2. Dictyosteliaceae.

1 By J. J. Lister, F.R.S., Fellow of St. John's College Cambrilge. 
THE plasmodial and the spore-bearing phases in the life-history of the Mycetozoa have long been known. Many of the generic names date from the eighteenth century, and Fries enumerated 192 species in 1829 .

By the earlier naturalists these organisms were classed, under the names Myxogastres or NIyxomycetes, with the Gasteromycetous Fungi, to which the sporangia of the Endosporeae present in miniature a considerable superficial resemblance. Although this view of their relationship is now generally abandoned, its influence may be traced in the names "capillitium" and "liypothallus" which are still applied to structures present in the spore-bearing stages of the Mycetozoa.

It was de Bary (1-3) who first worked out (1859-64) the main features of the life-history, showing that the spore hatches out as a naked protoplasmic body which assumes a flagellate form, that this passes after successive divisions into an amoeboid form, and that from the amoebae the large plasmodia arise.

Cienkowski (7) contributed (in 1863) the important observation of the mode of origin of the plasmodia by the fusion of the amoeboid swarm-cells.

De Bary showed how widely different, both morphologically and physiologically, these organisms are, not only from the higher fungi, but from all those included in the vegetable kingdom, and clearly expressed the opinion that they should be regarded as animals.

In the discussion of the relationships of the Mycetozoa which followed the publication of de Bary's work, it was early recognised that some of the simple organisms included in the large and illdefined group of the Monadina present phases comparable with those of the Mycetozoa. Thus Protomonas amyli and P. parasitica, which are parasitic in vegetable tissues containing starch, were found by Cienkowski (8) to begin their development as flagellate swarmcells, and then to become amoeboil, in which stage they take in or envelop starch grains, which they are able to digest. Later they encyst; the protoplasm withdraws from the undigested food and breaks up into a fresh brood of swarm-cells. Moreover, fusion of several individuals may occur in the amoeboid stage prior to encystment. An encysted resting stage is also found in the lifehistory.

With the object of introducing order into the heterogeneous assemblage of organisms which were, at the time of writing, classed as Monadina, Cienkowski proposed (5) to restrict this name to forms which passed through a life-history approximating to that of Protomonas.

The group, as thus limited, was regarded by him (8) as intermediate between animals and plants, and presenting affinities in 
several directions; among others with unicellular algae, the Mycetozoa and such forms as Actinophrys. Of these alliances, that with the algae is the least satisfactorily established by Cienkowski, but that between the "Monadina" and the Mycetozoa has been generally accepted by de Bary and later writers.

Zopf (24) considerably enlarged the "Monadina" of Cienkowski, and in 1887 included them in the Mycetozoa, distinguishing the forms here included, as the Eu-mycetozoa. This course is open to objection on several grounds. The "Monadina" of Zopf appear to be still a very heterogeneous collection of forms, and their inclusion in the Mycetozoa tends to obscure the well-marked features of this group. Further, though the affinity of som? of the Monadina with the Mycetozoa seems probable, others are as closely connected with the Heliozoa, in which class the majority of them are, in fact, included by Bütschli (4). ${ }^{1}$

Hence the limits of the Mycetozoa, as here understood, are the same as those drawn by de Bary. They include (1) the Sorophora of Zopf (the Acrasiae of Van Tieghem); (2) the remainder, and great majority of the species, for which de Bary retained the old name of Myxomycetes. The only objection to retaining this name is that it is generally used as synonymous with Mycetozoa. The term Eu-mycetozoa would have been preferable, but it is used by Zopf to include the Sorophora. Delage and Hérouard have applied the name Euplasmodida to the higher group, a course which avoids all confusion, and emphasises one of the chief characters which distinguishes it from the Sorophora.

In a more recent work (25) Zopf has included the Labyrinthuleae as a sub-order of the Sorophora. He has shown that the singular network described by Cienkowski in Labyrinthula, by which the individuals are united, is pseudopodial in rature, and regards the whole colony as forming a body of the nature of a plasmodium, to which he applies the name thread-plasmodium. There appears to be no evidence, however, that the term plasmodium is any more applicable to the colony of Labyrinthula than it is to those, e.g., of Mikrogromia, or the colonial Radiolaria. The actively parasitic habit, the entirely aquatic life, the defined shape of the members of the colony, and the absence of any proof that it is formed by fusion of individuals, keep Labyrinthula distinct from forms hitherto included in the Sorophora. Penard (20a) has recently extended our knowledge of Chlamydomyxa, showing that the "oat-shaped corpuscles" are not nucleated, and therefore not comparable with the fusiform bodies of Labyrinthula; and also that the contents of the cysts escape as flagellate zoospores. Penard finds a great analogy between this genus and the

1 Whatever position is ultimately assigned to the "Monadina" of Cienkowski and Zopf, it is desirable that this name for them should fall into disuse, for it is now applied in zoological works to the simpler members of the Flagellata, in which the flagellate and not the amoeboid stage is predominant in the life-history. 
Euplasmodida, a view which is by no means shared by the writer of this article. The presence of chlorophyll bodies and the stiff little-branched character of the pseudopodia are altogether foreign to the present group, and here again the plasmodial nature of Chlamydomyxa is far from being established. Both these genera are in the present treatise dealt with separately (pp. 274, 280).

In addition to the remarkable phenomena presented by the plasmodium of the Euplasmodida, the characteristic and unique feature of the Mycetozoa, as a group, is that belonging, as the earlier stages of their life-history show them to do, to the animal stock, and developing their sporophores and sporangia in air, these structures have been differentiated into a series of forms analogous with the sporophores met with among different orders of fungi. So close is the resemblance in many cases, that sporangial forms of each of the three main divisions have been classified among the several orders of fungi: Dictyostelium (Sorophora) among the Mucorinae; Ceratiomyxa (Exosporeae) with the Basidiomycetes Polyporus and Hydnum; and various members of the Endosporeae with the Gasteromycetes.

\section{EUPLASMODIDA.}

\section{The Life-Cycle of the Endosporeae.}

\section{(a) The Swarm-Cell or Zoospore.}

The spores of the Mycetozoa are produced not in water, as are those of the Monadina (except Bursulla), but in air, and they are able to retain their vitality in the dry state for as many as four years, undergoing no apparent change except a collapse of the

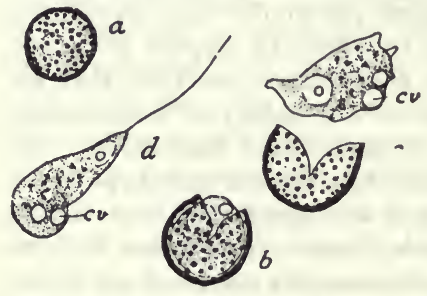

Fra. 1.

The hatching of a spore of Fuligo septica. $\times 1100$. $a$, spore; $b$ and $c$, contents emerging and undergoing annoeboid movements prior to the assumption of the zoospore stage, $d ; c . v$, contractile vacuole. spore owing to the shrinking of the contents on drying. When carried into water, they rapidly swell and resume their original form, which is, in nearly all species, spherical. As they lie in water one or more contractile vacuoles make their appearance in the protoplasmic contents, and after a period varying from a few hours to a day or two, the spore wall is ruptured, and the contents slip out and lie free in the water-a mass of clear protoplasm, containing the nucleus and contractile vacuoles (Fig. 1).

The first movements in the free state are amoeboid, but an elongated shape is soon assumed; and a flagellum, protruded tentatively at first, becomes established at one end. The organism 
which thus enters the swarm-cell or zoospore stage swims free in the water with a peculiar dancing movement produced by the lashing of the flagellum. In this movement it rotates about its own axis, and also moves as though over the surface of a cone, the apex of which is situated at the posterior end of the zoospore (de Bary). It is of an elongated pyriform shape, the narrow ("anterior") end being continued into the flagellum, which is about half to twothirds the length of the body. The thicker ("posterior") end may be evenly rounded, and is then curled somewhat to the side, but is often extended in short pointed pseudopodia (Fig. 2,a). The protoplasm of the anterior part is hyaline, and a layer of hyaline protoplasm invests the rest of the body, the interior of which is granular. The nucleus, with its contained nucleolus, lies in front, at the base of the flagellum, and the contractile vacuole at the posterior end. Non-contractile vacuoles (some of which at least may be food-vacuoles) are also present in the granular protoplasm. The particles of the latter exhibit a change of position within the body, which in the large swarm-cells of Amaurochaete atra recalls the streaming movement characteristic of the plasmodia of the later stage.

Instead of swimming free, the swarm-cells may temporarily assume an attached creeping mode of progression, in which the body is elongated, and the flagellum, extended in front, turns from side to side with movements which appear to be exploratory in purpose. Sometimes the body is contracted and sends out pseudopodia from all parts of the periphery (Fig. 4, c).

Bacteria abound in the wet places among decaying vegetable matter, in which the spores hatch. These are captured by the zoospores by means of the pseudopodia extended from their posterior ends and drawn into the body, where they are digested in vacuoles (Fig. 2) (15). De Bary, to whom this mode of obtaining food by the zoospores was unknown, states $(8$, p. 452$)$ that their nourishment is exclusively saprophytic at this stage. It is impossible to deny that it may be in part saprophytic, and it
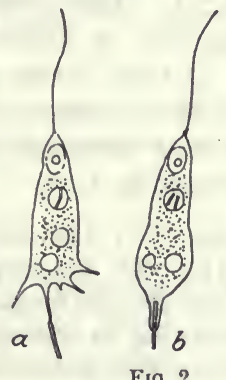

Fra. 2.

Zoospore of Stemonitis fusca, showing successive stages in the ingestion of a bacillus. $\times 800$. In $a$, it is captured by one of the pseudopodia at the hind end ; in $c$, it is enclosed in a digestive vacuole. Another hacillus is contained in an anterior vacuole. (After A. Lister, 15.) appears very probable that it is both holozoic and saprophytic.

The swarm-cells multiply by division. In this process the flagellum is withdrawn, the contractile vacuole disappears, and the body assumes a rounded form. The nucleus, passing to the centre, divides by karyokinesis (Fig. 3), and as the daughter nuclei resulting from this division separate the protoplasm becomes constricted, and division occurs in a plane transverse to the axis of division of the 
nucleus. A contractile vacuole has, meanwhile, appeared in each daughter-cell, at a point remote from the plane of division, and each develops a flagellum after separation is complete (15). It is probable that many generations of swarm-cells produced in this inanner succeed one another during this stage of the life-history.

When the zoospores are treated by Heidenhain's haeinatoxylin method, or with picrocarmine, a reticulum comes into view in the nucleus, and the nucleolus takes a dark stain.

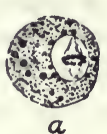

a

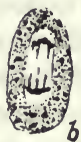

Fig. 3.

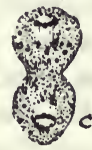

Three stages in the division of zoospore of Reticularia lyenzerilon. $\times$ 1000. (After A. Lister, 17.)

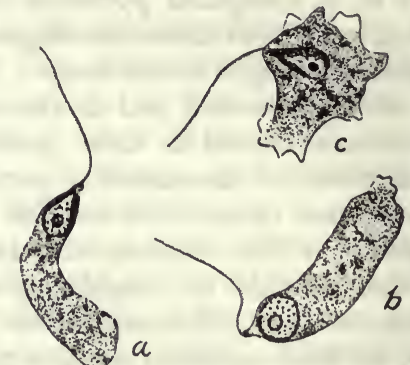

Fro. 4.

Zoospores of Badhemia panicea, stained. $\times 650$.

The nucleus is sometimes round (Fig. 4, $b$ ), but more often it is pyriform, being drawn out towards the base of the flagellum (Fig. 4,a). The protoplasm intervening between the nucleus and the flagellum is differentiated from the rest, and takes a darker stain. It thus forms a more or less bell-shaped investment of the former, the contour of which is most clearly seen in specimens which have assumed an amoeboid shape without retracting the flagellum (Fig. 4, c).

Plenge (21) first callerl attention to this bell-shaped structure; and Jahn (11), who has recently investigated it afresh, considers that it is part of the spindle formed in nuclear division when the zoospore divided, and remaining in connection with the daughter nucleus. Jahn's figures illustrating this point are very clear, but he does not explain how the structure is formed in the zoospore prior to its first division.

In this and also in the succeeding stage a resting phase may intervene between periods of activity. In it the flagellum and pseudopodia are withdrawn, and the protoplasmic body rounding itself into a sphere secretes a hyaline cyst-wall. These cysts are known as microcysts. The formation of microcysts may be readily induced by allowing a cultivation of swarm-cells to dry up, but dryness is not a necessary condition for their production, for they are formed in water, and some are present in almost every cultivation of swarm-cells.

\section{(b) The Amoebula.}

After remaining for a period of uncertain duration in the stage of their life-history in which the dominant form is that of the free- 
swimming flagellate zoospore, the flagellum is permanently withdrawn and the organism passes into the amoeboid stage, which, as we have seen, may be temporarily assumed during the flagellate period. They now creep about, adherent to other objects, emitting blunt pseudopodia, and in this as in the preceding stage they may pass into the condition of microcysts.

Each individual in the amoeboid phase of the life-history is the lineal descendant, through the successive divisions of the flagellulate phase, of a particular spore; but from the amoeboid phase onward the individuality is lost. This results from the remarkable process, first seen by Cienkowski (7), of fusion of the amoebulae to form plasmodia. The amoebulae present in a particular area draw together into groups, becoming endowed, apparently, with the power of mutual attraction, and the groups, once formed, act as centres to which neighbouring amoebulae, scattered through the water, converge. After coming in contact with one another they remain at first visibly distinct, but after a short time a complete

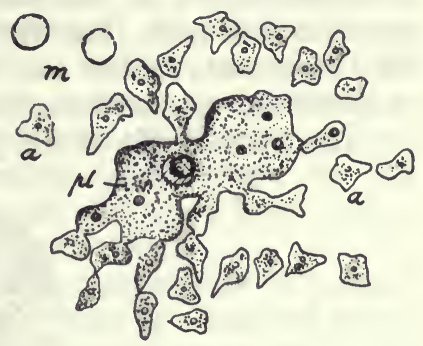

Fro. 5 .

Amoebulae of Didymium difforme uniting to form a plasmodium. $a$, separate amoebulae; $m$, microcysts; $p l$, young plasnodium with ingested iodies $x$ about 320. (After A. Lister, 18.)

fusion of the protoplasm occurs. In this manner, the amoebulae from all sides falling in and fusing in the common mass, the plasmodic are produced.

\section{(c) The Plasmodium.}

The name plasmodium was first applied by Cienkowski in 1862 $(6, p .326)$ to the large expansions of protoplasm which form the dominant phase of the life-history of the Euplasmodida. On his subsequent discovery (7), in 1863 , of their mode of origin by the fusion of amoeboid swarm-cells, Cienkowski stated (p. 421) that such a mode of origin must be included in the definition of a plasmodium.

The question arises whether, in this fusion of amoebulae to form the plasmodia, we have a phenomenon comparable with the conjugation of the gametes of other forms, a view to which the mutual attractiveness with which the amoebulae become endowed appears to offer some support. If the analogy were complete, we should expect that a fusion of nuclei would occur as well as a fusion of the protoplasm of the amoebulae. But the evidence which we have at present as to the behaviour of the nuclei lends no support to this view. As many as eight amoebulae have been watched successively fusing into a common mass, and their eight nuclei have been seen, distinct, in the young plasmodium thus formed $(18$, p. 5). When 
the number of fused amoebulae increases, direct observation of the behaviour of the nuclei is, owing to their small size and the bulk and movements of the protoplasm, increasingly difficult, and soon becomes impossible.

Before describing the plasmodia in detail, it may be briefly stated that they are masses of naked protoplasm of indefinite size, containing numerous small nuclei. As de Bary discovered, they are capable, under certain conditions, of passing into a passive condition known as the Sclerotium, in which the protoplasm is aggregated in cysts (Fig. 8), which together form a mass of hornlike consistency. On the return of favourable conditions the plasmodium resumes the active condition.

The mode of life of the plasmodium differs in different species,

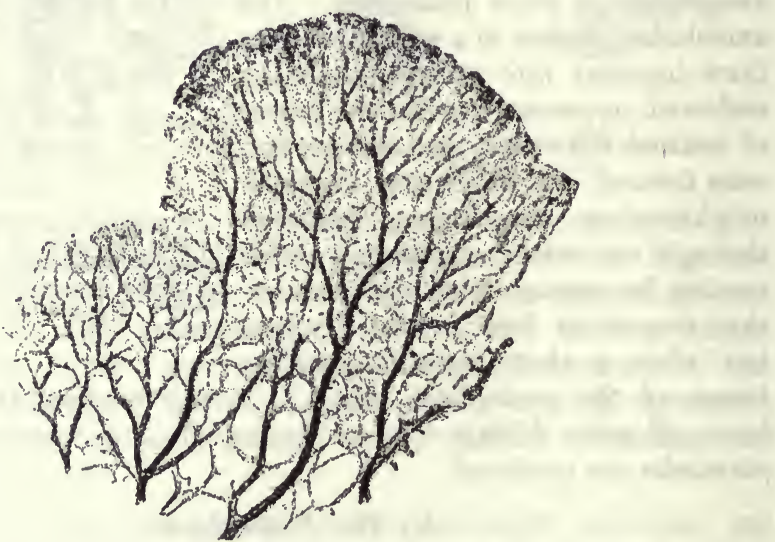

Fia. 6.

Part of a plasmodium of Budhamia utricularis expanded over a slide. $\quad \times 8$.

some (as in most of the Trichiaceae and Arcyriaceae) penetrating the interstices of dead wood, others (as of most species of Craterium and Didymium) living among heaps of decaying leaves, while one species, Badhamia utricularis, feeds on the surface of living fungi which grow from the bark of dead trees.

The plasmodium expands over surrounding objects and moves abont, taking in nourishment. When exposed, it is seen by the naked eye to be traversed by systems of vessel-like thickenings, the main trunks of which divide and subdivide as they approach the periphery, and are in free communication by the anastomosis of their branches (Fig. 6).

The border of the plasmodium in the direction towards which it is moving generally consists of a continuous film of protoplasm, traversed by smaller branches of the system, but in the other parts the film is generally not continuous, being interrupted in the inter- 
spaces between the thickenings. Hence in these regions the plasmodium consists of a reticulum of anastomosing branches, extended over the substratum. The arrangement of the branches closely resembles that of the vessels traversing the mesentery of a mammal, and, before their relation to the spore-bearing stage of the lifehistory was known, the name Mesenterica was, in fact, given to plasmodia of certain forms, under the supposition that they represented a new genus of fungi.

The form and degree of concentration of the plasmodium vary widely according to circumstances. Sometimes it is aggregated in a thick layer on the surface, as after emerging from the interstices of a mass of rotten wood or tan, at other times it is widely expanded in a thin layer of exquisite delicacy. Fries relates how the plasmodium of Diachaea elegans which he had laid in his hat, while collecting, spread within an hour over a great part of the latter in an elegant white network.

By suitable manipulation the plasmodia may readily be induced to spread over glass cover-slips, and may thus be examined microscopically. ${ }^{1}$ When thus seen the vessel-like thickenings are found to be, in fact, streams of moving protoplasm. The flow may be traced from the larger branches through the smaller into the adyancing border of the plasmodium, which becomes swollen and more opaque as the streams pass into it. After a short time the current is seen to slacken, then to stop, and shortly to begin again in the reverse direction, the margin becoming thinner and more transparent as the protoplasm leaves it. In a short time the flow is again reversed, and again directed to the advancing border. Thus a rhythmic flow, towards the margin and away from it, is kept up through the plasmodium - the period in each case being, in healthy conditions, about a minute and a half to two minutes, though its duration is always longer in the direction in which the plasmodium is moving than in the other.

The plasmodium is invested by a thin layer of homogeneous hyaline and colourless protoplasm. Within this the protoplasm is highly granular.

The hyaline layer is exceedingly thin over the greater part of the periphery, but at the advancing boraier it is of considerable breadth. The advance over the substratum occurs chiefly while the flow in the veins is directed towards this border. Under these circumstances the border becomes more and more turgid, and

1 An easy way of making microscopic preparations of living plasmodia is to lay out a number of cover-slips on a plate, sprinkle them with rain-water, aud then to seatter small fragments of sclerotium over them. In a moist atmosphere the encysted protoplasm resumes the active stage in the course of a few hours, and the sinall plasmodia thus arising spread in delicate fan-like expansions over the glass. 'The cover-slips may then be mounted over a hole in wet blotting-paper, on a slide, or in some other manner, ensuring the maintenance of a moist atmosphere. 
small rounded lobes of hyaline substance are seen to start forward, and then to become stationary, as though the surface tension had momentarily been overcome by the pressure from within, and had then been rapidly renewed. It is to be observed that the contents of such a newly-formed lobe are at first not, as might have been expected, the granular protoplasm which flows in the "veins," but they are hyaline, the passage of the granules into the interior of the lobe occurring subsequently.

The material in the "veins" appears to be of highly fluid consistency, the granules moving over one another with great

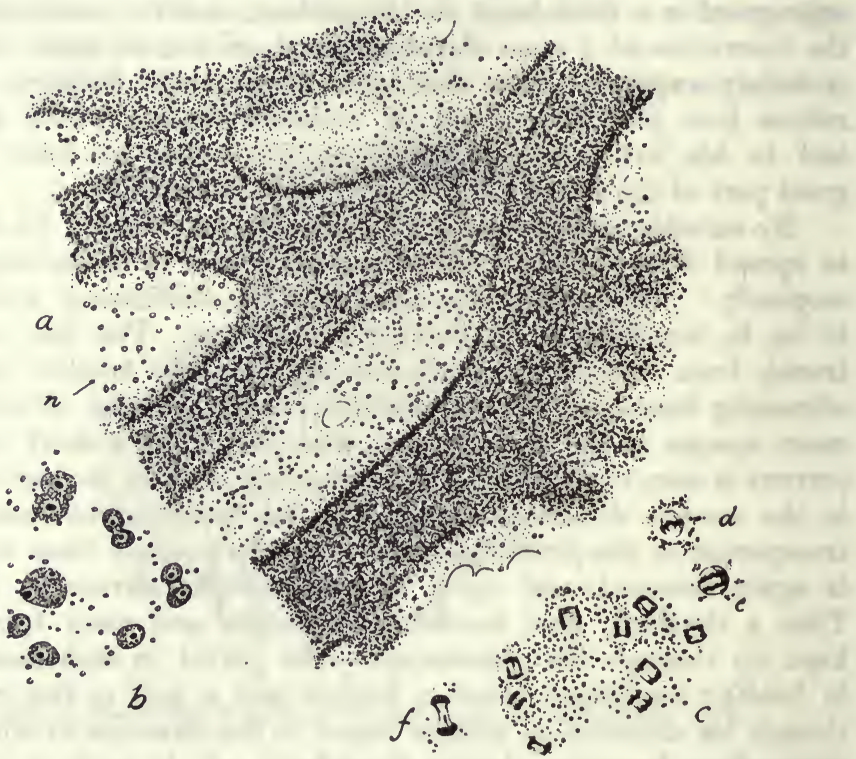

Fia. 7.

u, part of a stained plasmorlium of $B$. utricularis. $n$, nuclei, $\times 110 ; b$, nuclei, $\times 500$. Some are in process of simple division. $c$, part of a plasinodium in whicl the nuclei are in simultaneous division by karyokinesis. $d \cdot f$, other stages in this mode of division. $\times 650$.

freedom. When a small channel is watched it frequently occurs that an ingested sclerotium cyst or other large object blocks a narrow part, and the flow in the channel is temporarily checked. If the object ultimately passes on, its passage is followed by a gush of the protoplasm behind it, at increased velocity, the flow gradually resuming its normal rate. When a vein traversing a continuous portion of the plasmodial film is examined the flow is seen to be rapid at the centre and slower at the sides.

The phenomena presented by the circulation in the veins suggest the view that their contents are passively propelled, as the result of the contraction of the more external part of the plasmodial substance. 
De Bary concluded (2, pp. 43-51) that besides such a positive vis a tergo, due to contraction of the protoplasm in the regions from which the flow occurs, there is evidence of a negative pressure exercised by the plasmodium in the regions towards which the flow is going, and due to its expansion from the previous state of contraction.

While the conclusion appears probable that the streaming movement is due, in part at any rate, to the contraction of the outer portions of the protoplasm, we may bear in mind that such an explanation appears inapplicable to other phenomena, which we should expect to belong to the same category, such as those exhibited by the pseudopodia of the Foraminifera, in which streams of granules course along a filament of extreme tenuity in opposite directions.

When a piece of sclerotium resumes activity on being wetted, it sends out a fan-like expansion over the substratum, and the rhythmic flow is seen to be alternately away from the central mass and back to it; but as the fans extend farther over the substratum, the flow in the several parts of the plasmodium becomes less and less co-ordinated, in proportion as they separate from one another. The several parts separate into distinct plasmodia, and distinct plasmodia fuse with complete freedom.

Reaction of Plasmodia to External Conditions.-Experiments testing the reaction of plasmodia to variations in external conditions have led to some positive results, an interesting account of which is given by Stahl (22).

During the vegetative period of their existence plasmodia move from the drier to the moister parts of their substratum, though at the approach of the spore-producing stage the movement is in the opposite direction, the organism seeking the driest part of its environment whereon to undergo its change into spores. Connected apparently with the favourable influence of a moist atmosphere is the phenomenon, familiar to tanners, of the "flowering of the tanheaps" at the approach of wet weather. This consists of the emergence at the surface of the bright yellow plasmodia of Fuligo septica, commonly known as Flowers of Tan, which abound in the heaps, and, except under such conditions (and at the approach of sporulation), inhabit the deeper parts of the heap.

When water is allowed to flow through the substratum, plasmodia move in a direction opposite to the current, a tendency which may be utilised for the purpose of isolating them for experimental purposes. By arranging strips of filter paper, through which water is flowing, so that their lower ends rest on the mass containing the plasmodia, the latter will crawl up the filter paper, and may thence be transferred, in the same manner, to glass slides. 
The presence of substances suitable for food exercises a strong attraction on plasmodia. When the spreading border touches such a substance the streaming movement is at once quickened in this direction, and the outlying lobes being drawn in, the whole plasmodium is rapidly concentrated on the nutrient material (14).

The contrary effect is seen when harmful substances are brought into their neighbourhood.

The plasmodia of many species are said to shun the light, but this is not the case with all ; that of Badhamia utricularis, for example, will, if a moist atmosphere be maintained, continue to spread over the pilei of the fungi on which it feeds, though these may be exposed to full sunlight.

Nuclei. - The plasmodia are multinucleate from their origin; but from the fact that a minute plasmodium a few millimetres in diameter will grow, when supplied with food, till it is many inches in diameter, and that the nuclei are then as numerous, in a small sample, as they were before the growth had occurred, it is clear that the nuclei increase in number pari passu with the growth of the protoplasm. There is reason to believe that this increase occurs in two ways. (a) A simultaneous division of the nuclei by karyokinesis has been found to be in progress when plasmodia (of Badhamia utricularis, Fig. 7, c-f) are stained (17, p. 541) - a process comparable apparently with the simultaneous division of nuclei which occurs in the vegetative stage of Actinosphaerium. (b) Multiplication by simple division is not easy to establish, where, as in this case, prolonged observation of the nuclei in the living state is rendered difficult by the movement of the plasmodia, but the following observation appears to show that it is of frequent occurrence in their growth:-

A plasmodium of Badhamia utricularis, spreading and feeding on the pilei of the fungus Auriculuria, increased in size about fourfold in fourteen hours; and during this time a small portion of it was removed, smeared on a cover-slip, and fixed every quarter of an hour. On staining the 56 samples so obtained, the nuclei were found to be approximately equally abundant in all, and presented considerable differences in size, but in no case was there any indication of karyokinetic division. Now in the karyokinetic division of nuclei which occurs prior to spore-formation (see p. 52) the process lasts from one to one and a half hours. Assuming the same duration for the karyokinetic division of the nuclei in the growing plasmodium, and bearing in mind that the division in this manner, when observed, was simultaneous, we must conclude that it had not occurred in the fourteen hours during which the observations were made; yet from these observations it appears that in this period the number of the nuclei had increased about fourfold $(18$, p. 9). As a fact, the appearance of the nuclei in various phases of constriction is of common occurrence when stained plasmodia are examined with a 
high power (Fig. 7,b), but the appearance is so similar to that of overlapping nuclei, that without the confirmation afforded by the experiment above described, the conclusion, that in addition to a periodic (?) increase by mitosis, the nuclei multiply by simple division, could hardly have been accepted as secure.

With regard to the distribution of the nuclei, it is to be observed in stained preparations, in which the plasmodium has been suddenly killed, that they appear to be as numerous in proportion to the bulk of the protoplasm in the veins as they are in the film of the plasmodium on either side of them.

In size the nuclei vary from 2.5 to $5 \mu$. In the resting condition they present a well-marked reticulation and a distinct nucleolus.

In mitosis a well-marked spindle is formed, and the chromosomes are rounded and compact. In number the latter appear to be about 8 or 9 , in Trichia (see, however, p. 65). It may be noted that as in other Protozoa the nuclear membrane is maintained until after the separation of the chromosomes to form the daughter nuclei.

Contractile Vacuoles abound in the peripheral layer of the plasmodium, and may be readily seen in the expansions between the channels. They are generally about 7-8 $\mu$ in diameter.

The protoplasm contains abundant granules, of minute size, the nature of which has not been ascertained. In one group of Mycetozoa, the Calcarineae, granules of carbonate of lime abound in the plasmodia. They are not present in other species, and their relation to physiological processes is obscure.

The plasmodia of many species are white, but those of others are yellow, pink, purple, or green, and owe their colour to a fluid pigment scattered in small drops through the protoplasm. In the Calcarineae, the fluid pigment invests the granules of lime.

The Food of Plasmodia.-The plasmodia of the great majority of the Mycetozoa feed on the decaying vegetable matter among which they live. Their mode of nutrition must be regarded as both saprophytic and holozoic, for they are able to absorb nutrient matters in solution (cf. Stahl, 22) as well as to engulf their food. Those living among leaves and under bark are found charged with particles which have been ingested, and the undigested portions are found strewn along the track they have traversed. Badhamia utricularis is exceptional in feeding on living fungi (Stereum, Auricularia, etc.), though it will also live and thrive on the same fungi after they have become dried, if they are wetted again with water.

Experiments have shown that proteids (coagulated albumen, sclerotium cysts), taken in by plasmodia, are digested in vacuoles into which an acid is secreted by the surrounding protoplasm (see the experiments by Miss Greenwood and Miss Saunders, 10), 
although the reaction of the plasmodium as a whole is alkaline (Metschnikoff, 19). Pepsine, the presence of which in plasmodia of Fuligo was shown by Krukenberg (12), is doubtless the agent by which, acting in this acid medium, the digestion is brought about.

Raw starch grains which had been ingested were found to pass unaltered through the plasmodium of Badhamia utricularis, though grains which had been previously swollen in warm water were digested (14).

The plasmodium of this species at any rate has the power of dissolving cellulose. This is evident from the nature of its food, and has also been directly observed (14) when a plasmodium was seen to extend over the hyphac of a mould. The cellular walls of the hyphae were dissolved "like sugar in hot water" as soon as the hyaline border of the plasmodium reached them.

The Sclerotium Condition.-As in the earlier phases of the lifehistory, a passive condition may, as we have seen, be assumed in the plasmodium stage, the protoplasmic mass breaking up into cysts and assuming as a whole a firm consistence. To this condition de Bary gave the name Sclerotium. As it supervenes, the streaming movements gradually cease, foreign bodies are extruded, and the plasmodium becomes separated into distinct

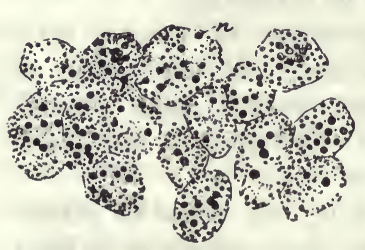

Fia. 8.

Part of a section of the plas. modium of Badhamia utricularis when passing into the sclerotium condition. $\times 310$. $n$, a nucleus. masses, each of which contains 10-20 nuclei, and secretes a membranous cystwall.

The assumption of the sclerotium condition is readily induced by allowing plasmodia to dry, and when so treated they assume a firmer and firmer consistency, until the masses of cysts attain a hard and horn-like condition, in which vitality may be preserved for as many as three years. Sclerotium cysts may, however, be formed in water, but the conditions under which this occurs are obscure. When the dry sclerotia are placed in water the protoplasmic masses absorb or break through the cyst-walls, fuse together, and the active plasmodial condition is resumed. The revival occurs in a few hours.

It is to be noted that the unit represented by the sclerotial cyst is different from the microcyst of the preceding stages, which was uninucleate, and also from the sporangium of the succeeding stage, which is much larger, and contains a much greater number of nuclei.

\section{(d) The Formation of Sporangia.}

The conditions under which plasmodia pass into the succeeding phase, that of spore-production, are in part obscure, but one 
element in this result is the absence of further nourishment. In a cultivation of Badhamia utricularis, after the plasmodium has been supplied with abundant food, and has increased largely in bulk, the formation of sporangia may generally be induced by withholding the supply of fungus, which is the food material of this species. If while food is withheld a suitable substratum, such as clean sticks, is supplied, the plasmodium will generally creep on the sticks and there form into sporangia.

The mode of formation of the sporangia in this species may be described as characteristic of the majority of Mycetozoa, the principal departures from the type being subsequently noticed.

As seen by the naked eye, the plasmodium previously extended in a diffused network over the substratum is seen to become aggregated in lobed masses 0.5 to $1 \mathrm{~mm}$. in diameter, which in this species are grouped closely together, and vary in number from a few to many thousands, in proportion to the size of the plasmodium. These are at first connected by the veins of the plasmodium, and may be seen to expand and contract in accordance with the direction of the streaming movement, which is still maintained. Gradually, however, the veins connecting them diminish, and soon the whole protoplasm is completely segregated into distinct lobes, or young sporangia.

While the formation of the sporangia is in progress, all remaining foreign bodies which have been ingested with food in the plasmodial stage are expelled, and a secretion takes place of a structureless, transparent substance which serves for the support and enclosure of the spores. At the surface of each of the lobed masses, constituting the young sporangia, is thus formed a sporangium wall, which in the mature state is a thin wrinkled membrane, completely investing it. At the constricted base of the sporangium this is continued to the substratum as a slender stalk of varying length (Fig. 9).

While the sporangium wall is secreted on the surface of the sporangium, a similar process occurs along certain tracts throughout the interior, giving rise (in this species) to an anastomosing network of flat bands with broad, thin expansions at the points of

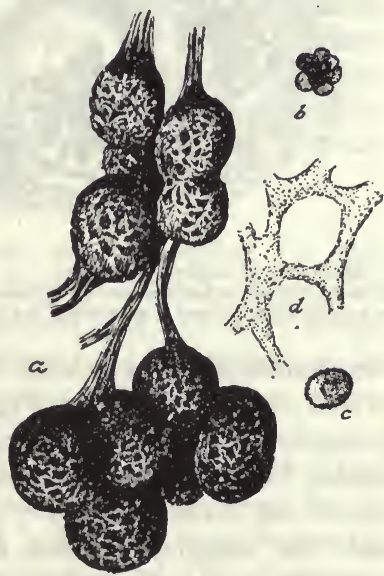

FIa. 9

$a$, a group of sporangia of Badhamia utricularis. $\times 12 . \quad b$, a cluster of spores ; $c$, a single spore; $d$, part of the capillitium containing lime granules. $b$ and $d \times 170$. (After $\mathbf{A}$. Lister, 18.) junction $(d)$. From a superficial resemblance to a structure in Gasteromycetous Fungi, this network traversing the interior of 
the sporangium is known as the capillitium. At the periphery it is continuous with the sporangium wall.

The lime granules, which existed free in the plasmodium, pass out of the protoplasm simultaneously with this secretion. Some are sparsely scattered through the sporangium wall, but the majority are closely packed in the strands of the capillitium, which are white and brittle in consequence (Figs. 9, $d$, and 10).

Until the secretion of sporangium wall and capillitium is complete the protoplasm remains a homogeneous mass, with multitudes of nuclei scattered through it. Their completion is followed by a division of the nuclei by karyokinesis, which occurs

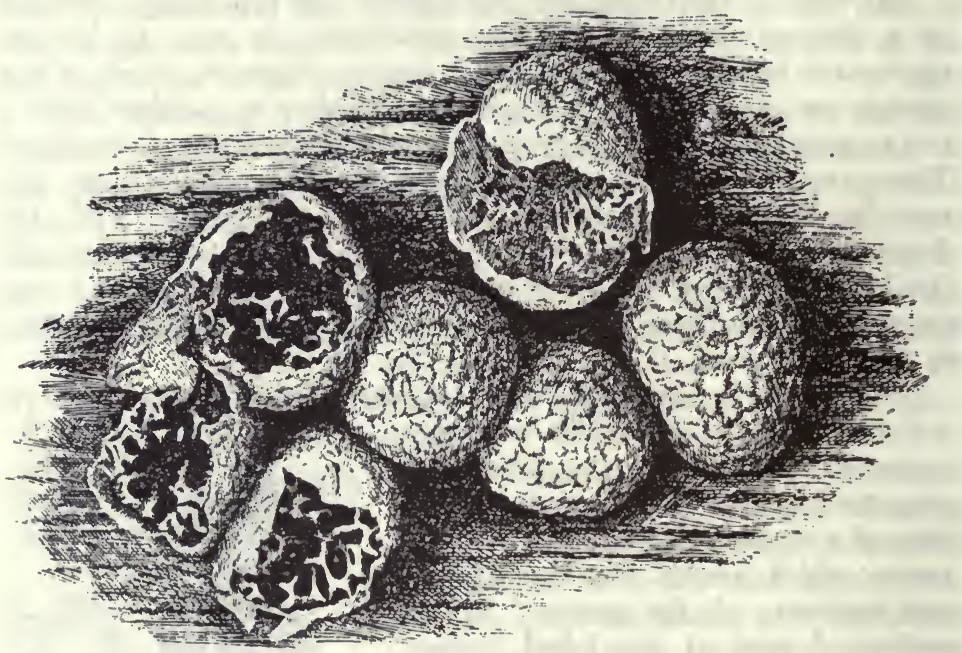

Fia. 10.

Sporangia of Baulhamia panicea. $\times 20$. To the left are three sporangia, the walls of which have ruptured, exposing the spores and capillitium. Three to the right are unopened; above an empty sporangium is seen with the remains of the capillitiun.

simultaneously throughout the sporangium and occupies from one to one and a half hours (Fig. 11). ${ }^{1}$ While this is in progress the protoplasm breaks up into rounded masses which contain some 6-10 nuclei, but they subsequently divide into masses, each containing one of the dividing nuclei ; and as the nuclear division is completed and the daughter nuclei draw apart, a further division of the protoplasm occurs, and each nucleus then occupies a single mass of protoplasm (Fig. 12). These masses are the young spores. They soon secrete a spore-wall which is of a violet-brown colour,

1 This was first observed by Strasburger (23) in Trichia fallax. The observation has been repeated by my father in two other species of Trichia, and in representatives of the genera Comatricha, Physarum, and Badhamia (17), and, since that paper was published, in Reticularia and Arcyria. 
and covered with minute spines or tubercles. The spores are approximately spherical, and 9 to $12 \mu$ in diameter. In several species

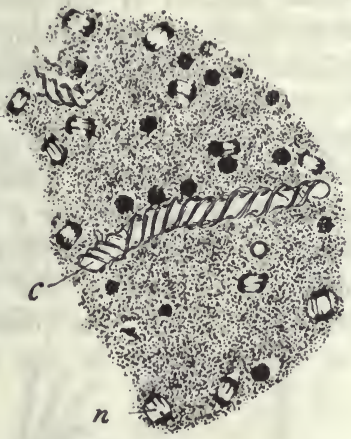

Fio. 11.

Part of a section through a young sporangium of Trichia varia, showlng the division of the nuclei prior to spore-formation. $\times 650$. c, capil. litium thread; $n$, \& nucleus. In several cases the axis of the dividing nucleus is directed towards 118 , and the karyokinetic figure is therefore not displayed. the spore-wall has been found to give the reaction of cellulose.

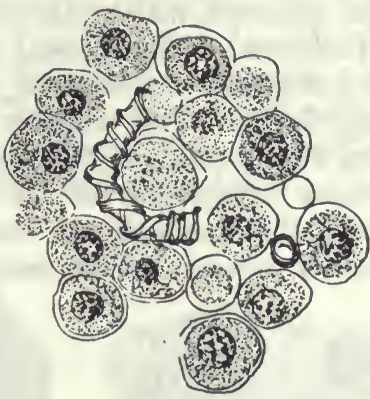

Fia. 12.

Part of a section through a sporangium of Trichia varia after the spores are formed. Capillitium threads are seen in longitudinal and transverse section. $\times 650$.

The ripe sporangium thus consists of a mass of spores, enveloped by the sporangium wall and traversed by a supporting reticulate capillitium, which, like the wall, has a dry membranous character, though charged throughout with white granules of lime. As ripening proceeds the sporangium wall becomes more and more friable, until it breaks and the spores are spread abroad on the lightest currents of air.

Considerable variations of structure are presented by the sporangia of the Mycetozoa. The stalk may be absent altogether, the sporangia being sessile on the substratum (Fig. 13,e). When present it is usually solid, but may be hollow, and sometimes, as in Trichia fallax, may contain cellular elements, which appear to be aborted spores.

In many species the stalk is continued in the interior of the sporangium as a structure known as the columella, which may reach to the apex or terminate short of it. A columella may, however, be present in sessile sporangia, as in species of Chondrioderma (Fig. 13,e).

Stalked sporangia are, at their first formation, sessile, and in the majority of cases the stalk may be regarded as the basal part of the sporangium wall, which has shrunk and fallen in about the base of the sporangium, as the latter has risen above the substratum (Figs. 13, $(l$, and 15,a); but in the Stemonitaceae the stalk, with its 
continuation, the columella, is, as de Bary showed, an axial structure secreted in the interior of the young sporangium (Fig. 14, a-e). In the formation of these sporangia the basal portion of the stalk is formed first and additions are made to the apex as the protoplasm climbs up this axial support. In Stemonitis fusca and splendens the stalked sporangia may attain a height of $20 \mathrm{~mm}$.

In addition to the skeletal or supporting structures of the sporebearing stage above mentioned, another is present in many generathe hypothallus. This consists of a network of strands or a continuous film, formed of the same material as the other supporting structures, extended over the substratum, and forming the base on which the sporangia are inserted (cf. Fig. 13, d). Its presence apparently depends on the occurrence of the secretion, in the later

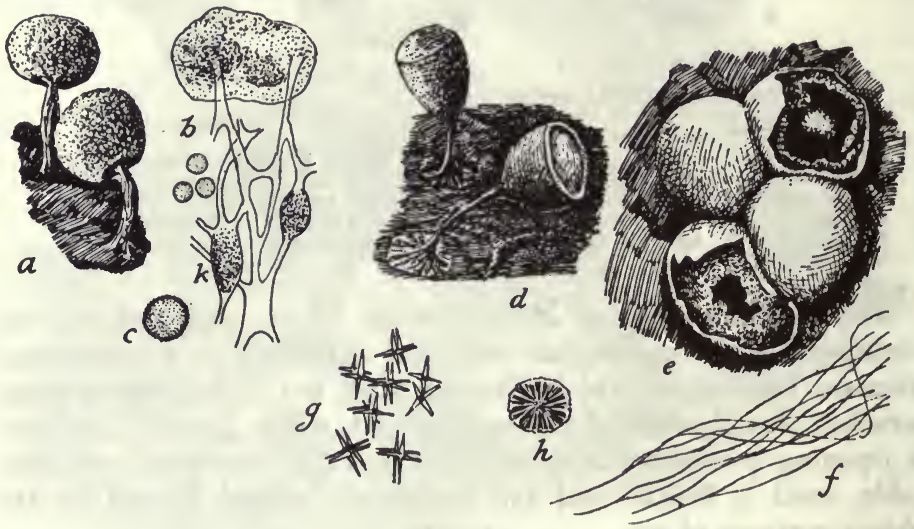

Fia. 13.

a, sporangiz of Physarum nutans, Pers., $\times 15$. ' $b$, piece of sporangium wall, with groups of lime granules, capillitium threads, with lime-knots $(k)$ and spores of Physarum nutans, $\times 210$. $c$, spore of same, $\times 450$. $d$, sporangia of Craterium pedunculatum, Trent, each with a discoidal hypothallus at the base of the stalk, $x$ 17. e, sporangia of Chondrioderma testaceum, Rost., showing the double sporangium wall (outer layer with lime, inner membranous), and in the upper sporangium the columella, $\times 15$. $f$, threads of the capillitium of the same, $\times 280$. $g$, group of crystals of lime from the wall of Spumaria alba, $\times 210$. $h, a$ crystalline disc from the sporangium wall of Lepidoderma tigrinuam, Rost., $\times 210$. (After A. Lister, 18.)

stages of the plasmodial condition, of the substance which dries into the supporting material-its reticular or continuous character corresponding with the state of diffusion of the plasmodium during its formation.

The sporangium wall may consist of two layers as in Chondrioderma (Fig. 13,e), where the outer is densely charged with lime granules, and the inner is membranous and free from lime. In some species of Craterium (Fig. 13,d) the upper portion of the sporangium wall forms a lid, which readily falls away, exposing the contents. In Dictydium (Fig. 14, $f$ ) and Cribraria the wall of the mature sporangium is represented wholly or in part by an open network, through the 
meshes of which the ripe spores escape; and in Comatricha it is evanescent, and disappears soon after the sporangia are ripe.

The capillitium also presents great variation. In the genera forming the Calcarineae the lime may be uniformly distributed through it (Badhamia, Figs. 9 and 10) or collected into lumps ("lime-knots") at the points of junction of the reticulum (Physarum, Fig. 13, b, Fuligo, Craterium). In Chondrioderma (Fig. 13, $e$ and $f$ ), Didymium, and others the lime is only laid down in or on the sporangium wall and the capillitium is free from it. The strands of the capillitium are generally, though not invariably, continuous at the periphery with the sporangium wall, and internally with the colımella, if this structure is present.

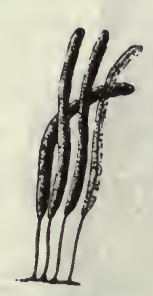

a

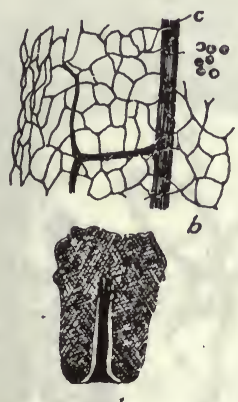

$d$

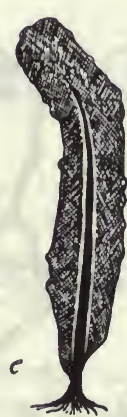

Fig. 14.

$\boldsymbol{\alpha}$, four sporangia of Stemonitis splendens, Rost.; that to the right is represented free from spores and shows the columella extending nearly to the top; $\times 2$. $b$, part of an empty sporangium of $S$. splendens, showing the columella $(c)$ and a branch springing from it and dividing to form the surface network of the capillitium. To the right a group of spores. $d, e$, stages in the development of the sporangia of Stemonitis ferruginea, Ehrenb., showing the development of the columella in the axis of the young sporangium. The space between the columella and the protoplasm is artificial. $f$, empty sporangium of Dictydium umbilicatum, Bchrad., $x$ 30. ( $d$ and $e$ after de Bary, 2 ; the other figures after A. Lister, 18.)

The capillitium attains its most elaborate development in the Arcyriaceae and Trichiaceae (Fig. 15). In the former it consists of an elastic network, attached or not to the base of the sporangium, but free from its sides, and with the strands beset with spines or transverse thickenings, resembling cogs on a wheel (Fig. 15, $f$ ). At maturity the evanescent film of the sporangium wall gives way and the capillitium expands into a long loose tangle, scattering the spores.

In the Trichiaceae the threads of the capillitium have spiral thickenings. In Hemitrichia the threads are united into a network, as in Arcyria, but in Trichia they are usually unbranched and lie free among the spores (Figs. 11, 12, and 15, b). Owing to their spiral sculpture they twist and untwist with varying changes of moisture, and thus subserve the distribution of the spores.

In a large section of genera, the Anemineae, a capillitium is absent. 
In the Physaraceae the lime is aggregated in the sporangium in the form of granules; but in the Didymiaceae, though, as in other Calcarineae, granular in the plasmodium stage, it assumes, when separating from the maturing sporangium, a crystalline form, being deposited on the sporangium wall either in clusters of crystals (Didymium and Spumaria, Fig. 13, g) or in discs with a radiating arrangement (Lepidoderma, Fig. 13, h). It is clear that in this process the lime must be in a state of solution as it passes through the sporangium walls.

The spores vary in diameter from 3-5 $\mu$ (in Tubulina stipitata) to $16-20 \mu$ (in Licea pusilla); and the size is generally approximately uniform in each species. The surface may be smooth, tuberculated,

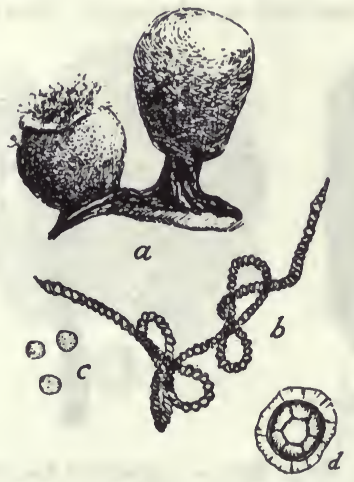

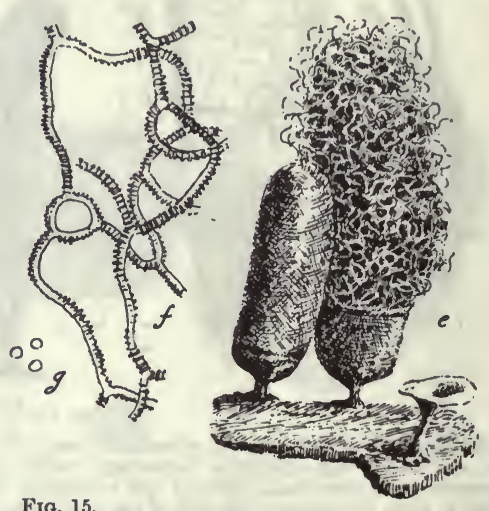

$a$ sporangia of Trichia varia, $\times 15 ; b$, one of the capillitium threads; $c$, spores, $\times 160 ; d$, a spore of Hemitrichia chrysospora, $\times$ nearly $600 ; e$, sporangia of Arcyria incarnata; in one the sporangium wall has broken and the capillitium has expanded, in another the empty base alone remains, $\times 16 ; f$ and $g$, capillitium and spores of $A$. punicea, $\times 160$. ( $a, d$, and $e$, after A. Lister, 18.)

or reticulated (Fig. 15, d); and the sculpture may be absent from one side of a spore, a peculiarity generally associated with the arrangement of the spores in clusters.

Aethalia and Plasmodiocarps.-In several species of Mycetozoa the sporangia, instead of standing apart, are more or less closely fused to form large compound bodies known as Aethalia, which present characteristic features of shape and structure. The identity of the individual sporangia may remain obvious or be entirely lost in the mature aethalia, but in the course of their development their compound nature is usually evident.

In many cases (Fuligo, Fig. 16, Reticularia, Lycogala) the protoplasm withdraws from the peripheral portions of the sporangia, the walls of which collapse in consequence and together form a cortical layer, and a similar withdrawal of protoplasm from the basal 
region often gives rise to a spongy base to the aethalium, to which the name hypothallus has been loosely applied, though the structure is as distinct from the true hypothallus as is any other part of the supporting substance.

Many Mycetozoa forming aethalia are closely allied to species with discrete sporangia. Thus Fuligo is an aethalioid form of
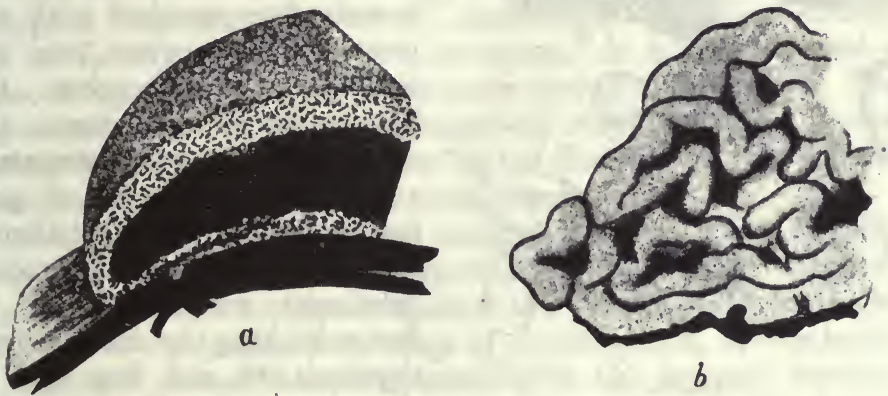

Fia. 16.

Aethalium of Fuligo septica. a, part of a ripe aethalium in section, showing the cortical layer, $x$. $\quad b$, part of a section of the developing aethalium, showing the separate convoluted tubular sporangia of which the aethalium is composed, $x$ about 390 . (After de Bary, 2.)

Physarum, Spumaria of Didymium; and species in which the sporangia are usually distinct may assume an aethalioid form, as in the "confluent" variety of Stemonitis fusca.

In some species the plasmodium does not become rounded off into distinct and symmetrical sporangia in the spore-producing stage, but retains a diffused and lobate form. In other respects maturation proceeds as in ordinary sporangia. These bodies are known as plasmodiocarps (Fig. 17). Aethalia appear to be formed by the fusion of sporangia, while plasmodiocarps are sporangia

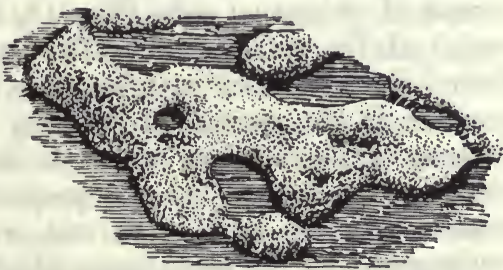

Fia. 17.

The plasmodiocarp form of Didymium effusum. $\times$ 15. (After A. Iister, 18.) incompletely segregated.

Plasmodiocarps are characteristic of some genera (Licea), but frequently occur together with completely-formed sporangia in the same species of others.

\section{The Exosporeae.}

The genus Ceratiomyxa (formerly known as Ceratium), the single: representative of the Exosporeae, differs from the Endosporeae 
in the relation of the spores to the supporting structures, and

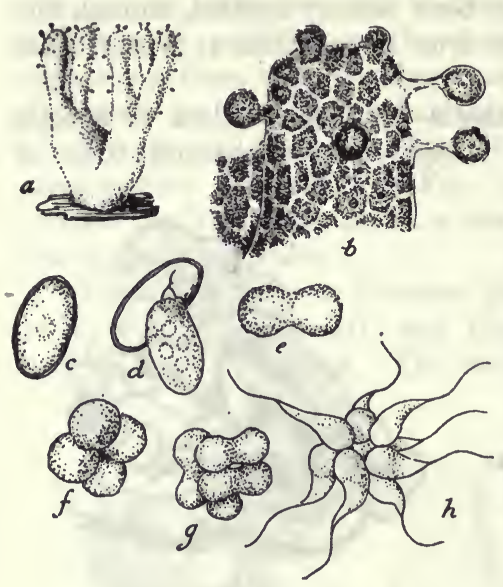

Fia. 18.

Ceratiomyxa mucida, Schroet. $a$, ripe sporophore, $\times 40 ; b$, maturing sporophore, showing the development of the spores, $\times$ about $100 ; c$, ripe spore; $d$, hatching spore; $e-h$, stages in the development of the zoospores, $\times 800$. ( $a$ and $c-h$ after A. Lister, 18; $b$, after Famintzin and Woronin, 9.) in the changes which occur when the spores are hatched (Fig. 18).

The plasmodium inhabits rotten wood and emerges in cushion-like masses, which may become honeycombed with depressions or separate into distinct antler-like branches. On its emergence it assumes the condition of an intimately anastomosing network of protoplasmic strands distributed through an abundant hyaline gelatinous substance, and at first exhibiting the characteristic rhythmic ebb and flow seen in the plasmodium of the Endosporeae. As the definitive shape is assumed, the protoplasm leaves the interior and accumulates at the surface of the mass, at first as a closeset reticulum, and then as a continuous layer investing the gelatinous substance, though with a thin covering of the latter still external to it. The layer of protoplasm then separates into a mosaic of polygonal cells (Fig. 18, b), each occupied by one of the nuclei of the plasmodium. The cells are at first in contact with their fellows at their margins, but they now draw apart, and each projects in the centre of the area which it occupied, beyond the contour of the lobe on which it lies, though still covered by the thin hyaline layer. As the projection increases its base becomes constricted, and finally the cell, or young spore, containing the nucleus and all the protoplasm which occupied the polygonal area, is raised some distance above the general surface, invested by a thin covering, and supported on a slender stalk-both furnished by the investing layer. Each spore now assumes an elliptical shape, secretes a firm colourless wall, and is ready to drop away. ${ }^{1}$

During the later stages of this process the gelatinous material constituting the sporophore dries, and by the time the spores are ripe, forms a shrivelled, white mass of extreme tenuity (Fig. 18, a).

According to Famintzin and Woronin (9), who first described the details of the life-history of Ceratiomyxu, the protoplasm emerges in the morning and the spores are ripe within twenty-four hours.

1 For nuclear changes during spore-formation, cp. p. 66 . 
The spores, which at their formation are uninucleate (Fig. 18, c), are found, on hatching, to contain four bodies which are apparently nuclei (Fig. 18,d), so it would appear that division of the nucleus occurs in the spore stage. When the spores are brought into water the contents emerges, becomes amoeboid, and successively constricted into separate lobes, two, four, and eight in number (Fig. 18, e-g). At the stage when eight lobes are formed each develops a flagellum (Fig. 18, h), and finally, becoming distinct from its fellows, swims off as a zoospore. It is evident that a further division of the nuclei must occur during this process. The zoospore subsequently enters the amoeboid stage, and the amoebae probably fuse to form plasmodia, as in the Endosporeae, though the process has not been followed in Ceratiomyxa.

On comparing the somewhat incomplete details of this lifehistory with those of the Endosporeae, it seems clear that the abundant gelatinous substance in which the protoplasm is contained at the end of the plasmodium stage of Ceratiomyxa is, as Famintzin and Woronin pointed out, comparable with the secreted material which is converted into the supporting structures of the Endosporeae. In Ceratiomyxa the spores, instead of lying in a compact mass, contained in a sporangium, are distributed in a superficial layer, and the sporophore is accordingly disposed so as to offer an extensive surface for their support.

The division of nuclei prior to spore-formation, found wherever the development has been followed in the Endosporeae, has not been seen in Ceratiomyxa, and as this process is frequently met with in other groups of Protozoa, its apparent absence here is remarkable. It is possible that this division is represented by the first of the nuclear divisions occurring within the spore; in which case the spores of Ceratiomyxa would be comparable with the masses into which in the Endosporeae the protoplasm separates about the dividing nuclei before spore-formation, rather than with the spores of that group. If this comparison were established, however, the two following divisions which occur in Ceratiomyxa before the zoospores are formed would remain features peculiar to the genus. ${ }^{1}$

\section{THE SOROPHORA.}

The other group here included with the Mycetozoa, the Sorophora, consists of forms the alliance of which with the Euplasmodida is somewhat remote. They live in decaying vegetables and the dung of herbivorous animals. There is no flagellate stage in the life-history, and it is in the form of amoebulae that the active phase, with grcwth and reproduction by fission, occurs. At the end of this vegetative phase, and only as a pre-

1 Cf. the Postscript at the end of this article. 
liminary step to sporulation, the amoebulae draw towards their fellows in groups, which may be composed of many hundreds of units, but they maintain their individual distinctness and do not fuse to form a true plasmodium as in the Euplasmodida. Sporeproduction occurs in air, at the surface of the substance in which the vegetative phase lias been spent.

In Guttulina, as well as in the members of the Dictyosteliaceae, a remarkable differentiation occurs among the amoebulae forming the pseudoplasmodium, comparable with that characteristic of the
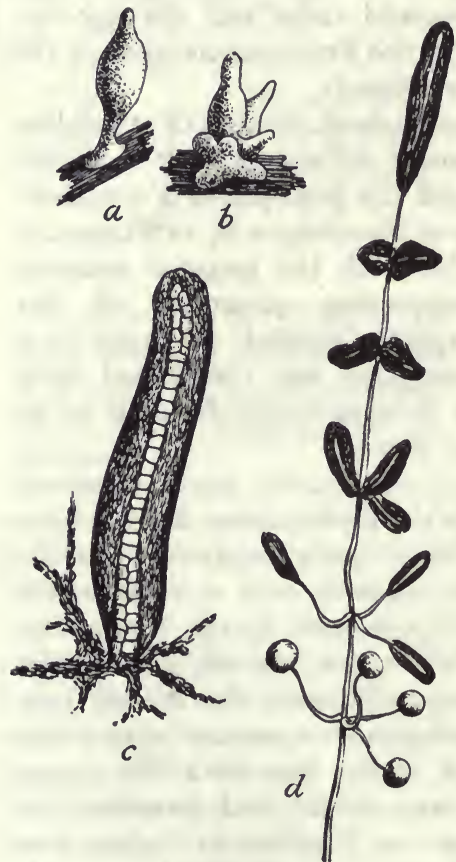

Fia. 19 organisation of the Metazoa. Some of the amoebulae secrete a firm membrane and become joined end to end to form a stalk (Fig. $19, c$ and $d)$, attached below to the substratum, and up this the other amoebulae climb and pass into the cncysted condition at the top as a naked cluster of spores. In Dictyostelium the stalk is long and simple; in Polysphondylium it is branched (Fig. 19, d).

The supporting structures of the Sorophora are evidently of a different nature from those of the Euplasmodida, in which they are not cellular, but formed as secretions of the protoplasm.

It is, of course, possible that the pseudoplasmodia of the Sorophora may represent a stage in the evolution of the true plasmodium, which in the other group is such an important phase of the life-cycle; but it appears more probable that both Euplasmodida and the Sorophora are to be derived from some simple forms with a life-history resembling that of Protomonas or Bursulla among the Proteomyxa.

Two hundred and sixty-five species of the Euplasmodida are simple, $b$, a branched form of sorus, slightly magnified (after Fayod.). $c$ and $d$, Poly. sphondylium violaceum, Brefeld. c, a young sorus, seen in optical section, with a mass of amoebae grouped round the stalk, and others still extended about the base, $\times 110$. $d$, a sorus ajproaching maturity. The stalk has become coinpound. The lowest whorl of secondary sori is complete, those above it are in varying degrees of completeness, $\times 20$. (After I3refeld. From Zopf, 24.)

described in the British Museum Catalogue (18); Zopf (24) enumerated nine species of Sorophora, and Olive (20), more recently, twenty. 
The classificatory characters are mainly derived from the sporangia, the capillitium (when it is present), and the spores. Some species stand apart from their allies with great distinctness. but in many genera examples intermediate in character between the species are of common occurrence, and it is only by large experience of the frequency with which the forms, as they occur in nature, group thiemselves about certain centres that a correct idea of the species can be attained.

The distribution of most species appears to be, so far as it has yet been determined, world-wide in the more humid parts of the temperate and tropical regions of the globe, where woodlands and forests offer conditions favourable to their existence-a fact which is doubtless dependent on the ease with which the minute spores are carried in currents of air.

No Mycetozoa have hitherto been met with in a fossil state, though from the degree of differentiation of the sporangia.we cannot doubt that the group is of high antiquity, and has in past time, as at the present, played an important part in the disintegration of vegetable tissues.

It is remarkable that no parasitic organisms are known to live on Mycetozoa, a fact which Stahl attributes to the readiness with which foreign bodies are cast out by the organisms in the plasmodial stage.

In writing this account of the Mycetozoa constant reference has been made to de Bary's classical work (1-3), to the papers of Cienkowski (5-8), and to Zopf's treatise (24). But I wish especially to acknowledge my obligations to the work of my father, Mr. A. Lister, on their life-history and classification. So far as I have been able to speak of the biological aspects of the group from my own knowledge, it is mainly to the opportunities I have had in following this work that I am indebted. The proof-sheets of this article have been submitted to my father, and I feel that its authority is greatly enhanced when I add, as he allows me to do, that the conclusions are in the main in accordance with his views.

\section{Sub-Class I. Euplasmodida.}

The contents of the spores develop, on hatching, into flagellate zoospores. Amoebulae conipletely fused to forn the plasmodium, which is the dominant phase of the vegetative period.

\section{Division I. Endosporeat.}

Spores developed within sporangia.

Cohort I. amatrosporales.

Spores violet, or violet-brown. 


\section{Sub-Cohort A. 'Calcarineae.}

Sporangia provided with lime.

\section{Order 1. Physaraceae.}

Lime in minute, round granules.

$A$. Capillitium a coarse network charged with lime throughout. Genus-Badhamia, Berk. (Figs. 9 and 10).

$B$. Capillitium a delicate network of threads with vesicular - expansions filled with lime-granules (=lime-knots). a. Sporangia combined into a convolute aethalium. Genus-Fuligo, Haller (Fig. 16). $\beta$. Sporangia single, scattered, or aggregated. $a$, sporangium wall membranous. Genera-Physarum, Pers. (Fig. 13, a). Sporangia subglobose or in the form of plasmodiocarps. Physurella, Peck. Sporangia tubular. $b$, sporangium wall cartilaginous throughout, or at the base only. Genera-Cienkowskia, Rost. Sporangia in the form of plasmodiocarps; capillitium with free hooked branches. Craterium, Trent (Fig. 13, d). Sporangia goblet-shaped or subglobose. Leocarpus, Link. Sporangia ovoid, glossy.

C. Capillitium without lime-knots. Genera-Chondrioderma, Rost. (Fig. 13, e). Sporangium wall of two layers, more or less combined. Trichamphora, Jungh. Sporangium wall of one layer, fragile ; sporangia sancer-shaped.

D. Lime confined to the stalk and columella; sporangium wall membranous. Genus-Diachaea, Fries.

\section{Order 2. Didymiaceae.}

Lime deposited in the form of crystals or crystalline discs on the outer surface of the sporangium wall; capillitium without lime-knots. Genera-Didymium, Schrader (Fig. 17). Lime in crystals; sporangia simple. Spumaria, Pers. (Fig. 13, g). Lime in crystals; sporangia united into an aethalium. Lepidoderma, de Bary. Lime in crystalline discs (Fig. 13,h); sporangia simple.

\section{Sub-Cohort B. Amaurochaetineae.}

Sporangia without deposits of lime ; capillitium dark brown or violet brown.

\section{Order 1. Stemonitaceae.}

Sporangia stalked, the stalk extending within the sporangium as a columella; sporangium wall a single delicate membrane, often evanescent. Genera-Stemonitis, Gleditsch (Fig. 14,a-e). Sporangium wall evanescent ; capillitium springing from all parts of the elongated columella, its ultimate branches forming a superficial net. Comatricha, Preuss. Like Stemonitis, but the branches of the capillitium not forming a superficial net. Enerthenema, Bowman. Sporangium wall evanescent; columella reaching to the apex of the sporangium, where it forms a superficial expansion from which the capillitium springs. Lamproderma, Rost. Sporangium wall somewhat persistent, columella about half the height of the sporangium. 
Clastoderma, Blytt. Sporangium wall partly evanescent, persisting in the form of minute discs, at the tips of the rigid capillitium threads; columella chort or none. Echinostelium, de By. A minute colourless form with long stalks and a sparsely-branched spiny capillitium.

\section{ORDER 2. Amaurochaetaceae.}

Sporangia combined into an aethalium; capillitium of irregular strands and threads, or complex. Genera-Anaurochaete, Rost. Capillitium of irregular branching threads. Brefeldia, Rost. Capillitium of horizontal threads, with many-chambered vesicles.

\section{Cohort II. Lamprosporales.}

Spores variously coloured, never violet.

\section{Sub-Cohort A. Anemineat.}

Capillitium absent, or not forming a system of uniforn threads except in Alwisia.

\section{ORDER 1. Heterodermaceae.}

Sporangium wall membranous, beset with minute round granules, and (except in Lindbladia) foruing a net in the upper part. GeneraLindbladia, Fries. Sporangia sessile, compacted or aethalioid, the wall not forming a net in the upper part. Cribraria, Pers. Sporangia stalked; sporangium wall with thickenings in the form of a delicate persistent net, expanded at the nodes. Dictydium, Schrader (Fig. 14, $f$ ). Sporangia stalked; sporangium wall with thickenings in the form of longitudinal ribs connected by delicate threads.

\section{ORDER 2. Liceaceae.}

Sporangia solitary, sessile or stalked; sporangium wall cartilaginous ; capillitium and columella absent. Genera-Licea, Schrader. Sporangia sessile, globose or in the form of plasmodiocarps. Orcadella, Wingate. Sporangia stalked, furnished with a lid of thinner substance.

\section{ORDER 3. Tubulinaceae.}

Sporangium wall membranous, without granular deposits ; sporangia tubular, compacted together. Genera - Tubulina, Pers. Columella absent. Siphoptychium, Rost. A hollow peeudo-columella is present, connected by tubular extensions with the sporangium wall. Alwisia, Berkeley and Broome. Sporangia stalked; with tubular threads attached to the base and apex of the sporangium wall.

\section{Order 4. Reticulariaceae.}

Aethalia, with the sporangium walls incomplete, perforated, and forming a spurious capillitium. Genera - Dictydiaethalium, Rost. Sporangium walls cap-shaped above and continued down to the base in four to six straight threads. Enteridium, Ehrenberg. Walls of 
convoluted sporangia forming a tissue of interarching bands. Reticularia, Bulliard. Walls of convoluted sporangia forming tubes and folds with numerous anastomosing threads.

\section{Order 5. Lycogalaceae.}

Sporangia forming an aethalium; pseudo-capillitium consisting of branched colourless tubes, the remains of the walls of the fused sporangia. Genus-Lycogala, Micheli.

\section{Sub-Cohort B. Calonemineae.}

Capillitium a system of uniform threads.

\section{Order 1. Trichiaceae.}

Capillitium threads with spiral or annular thickenings. Free or united into an elastic network. Trichia, Haller (Figs. 11, 12, and 15, a-c). Capillitium abundant, threads free, with spiral thickenings. Oligonema, Rost. Capillitium scanty, threads free, with imperfect spiral thickenings. Hemitrichia, Rost. (Fig. 15, d). Capillitium threads combined into a network, with spiral thickenings. Cornuvia, Rost. Sporangia in the form of plasmodiocarps; capillitium threads combined into a network, with annular thickenings.

\section{Order 2. Arcyriaceae.}

Capillitium combined into an elastic network with thickenings in the form of cogs, half-rings, spines, or warts. Genera-Arcyria, Hill (Fig. $15, e-f)$. Sporangia stalked ; sporangium wall evanescent above, persistent and membranous in the lower third. Lachnobolus, Fries. Sporangia sessile, clustered; sporangium wall single, persistent, not thickened with granules. Perichaena, Fries. Sporangia sessile or in the form of plasmodiocarps ; sporangium wall double, at least at the base, the outer layer thickened with angular granules.

\section{Order 3. Margaritaceae.}

Sporangia normally sessile; sporangium wall single, smooth, translucent; capillitium abundant, not consisting of separate threads, nor combined into a net. Genera-Margaritu, Lister. Capillitium profuse, long, coiled, and hair-like. Dianema, Rex. Capillitium of nearly straight threads, without spiral thickenings, attached at both ends to the sporangium walls. Prototrichia, Rost. Capillitium of fasciculate threads, attached above or below to the sporangium wall, and spirally thickened.

\section{Division II. Exosporeae.}

Spores developed on the surface of sporophores.

\section{Order 1. Ceratiomyxaceae.}

Sporophores fragile and evanescent, branched; spores white, borne singly on filiform stalks arising from the areolated sporophore. GenusCeratiomyxa, Schroeter (Fig. 18). 


\section{Sub-Class II. Surophora.}

A flagellate stage is absent from the life-history. The amoebulae become aggregated prior to spore-formation, but do not fuse to form a true plasmodium. In the more highly developed genera some of the aggregated amoebulae are modified to form a stalk on which the remainder are borne after encystment in naked clusters (sori).

\section{Order 1. Guttulinaceae.}

The aggregation of amoelulae, prior to spore-formation, to furm the pseudo-plasmodium, is incomplete in Copromyxa. The amoebulae have the limax form, and the shape of the sori is inclefinite.

Genera-Copromyxa, Zopf (Fig. 19, $a$ and b). Sori wart-like or spindle-shaped, 1-3 mm. high, formed on the surface of the nidus. None of the anoebulae are differentiated to form a stalk. On horse and cow dung. Guttulina, Cienk. Some of the aggregated amoebulae are differentiated to form a short stalk on which the sorus is borne. On decaying wood or horse-dung.

\section{Order 2. Dictyosteliaceae.}

A pseudo-plasmolium is formed prior to spore-formation. Some of the argregated amoebulae are modified to form a stalk. The sori have a definite shape. Amoebulae with short pointed pseudopodia. GeneraDictyostelium, Brefeld. Stalks unbranched, the spores without definite arrangement in the sori. On dung of herbivorous animals. Acrasis, van Tieghem. Spores arranged in rows, like strings of beads, at the ends of the stalks. On beer-yeast. Polysphondylium, Brefeld (Fig. 19, $c$ and $d$ ). Sori globular, on branched stalks, which attain $1 \mathrm{~cm}$. in length. On horse-dung.

\section{POSTSCRIPT.}

Since the foregoing account of the Mycetozoa was written papers have been published, in part of a preliminary character, which appear to throw light on the nuclear history.

In the Endosporeae, Fraulein H. Kränzlin ${ }^{1}$ has described a fusion of the nuclei in pairs, prior to the mitosis which precedes spore-formation, in the young sporangia of Arcyria, and this result is corroborated by Jahn.? The number of chromosomes at this division Jahn believes to be sixteen (" 8 double chromosomes") in Arcyria (at least double that which Jahn found in the division of the zoospore in other genera). In Fuligo Harper ${ }^{3^{-}}$ found the number to be twelve in the mitosis preceding spore-formation.

1 "Zur Entwicklungsgeschichte der Sporangien bei den Trichien und Arcyrien," Arch. f. Protistenkunde, Bd. ix. (1907), p. 170.

2 "Myxomycetenstudien - 6: Kernverschmelzungen und Reduktionsteilungen," Ber. d. deutsch. botan. Gesellschuft, Bi. xxv. (1907), p. 23.

3 "Cell and Nuclear Division in Fuligo varians," Botanical Gazette, vol. xxx. (1900), p. 217. 
These authors suggest that the fusion of nuclei in the young sporangium is a long-deferred karyogamy, separated by the whole of the plasmodium stage, with its many nuclear divisions, from the plastogamy (the fusion of the amoebulae) by which the plasmodium originates. They thus regard the mitosis preceding spore-formation as the one nuclear division in the life-cycle in which the full ("somatic ") number of chromosomes is present.

Jahn (l.c.) and, subsequently, Olive ${ }^{1}$ also state that a fusion of nuclei occurs in Ceratiomyxa prior to the formation of the spores. The fusion is followed by four according to Jahn, by two according to Olive, mitotic divisions, and the ripe spore is four-nucleated (not one-nucleated, as indicated above (Fig. 18, c)).

It would thus appear that there are, at any rate, two mitotic divisions before spore-formation in Ceratiomyxa and only one in the Endosporeae. The spores are thus not strictly homologous in the Endosporeae and Exosporeae. That of Ceratiomyxa is more advanced than the spore of the Endosporeae in that at least two mitotic divisions subsequent to karyogamy have occurred (and the four nuclei thus arising are contained in the spore), but it is less advanced in that no cleavage of the protoplasm about the products of division has taken place.

\section{LITERATURE.}

1. de Bary, A. Die Mycetozoen. Zeits. f. wiss. Zool. vol. x. (1860), p. 88.

2. - Die Mycetozoen. $2^{\circ}$ Auflage, Leipzig, 1864.

3. - Comparative Morphology and Biology of the Fungi, Mycetozoa, and Bacteria. Translation. Oxford, Clarendon Press, 1887.

4. Bütschli, $O$. Protozoa, Abth. $\dot{g}$, Sarcodina. Bronn's Thierreich, Bd. i.

5. Cienkowski, L. Die Psendogonidien. Pringsheim's Jahrbücher, i. p. 371.

6. - Zur Entwickelungsgeschichte der Myxomyceten. Pringsheim's Jahrbuicher, iii. p. 325 (published 1862).

7. — Das Plasmodium. Toid. p. 400 (1863).

8. —_ Beiträge zur Kenntniss der Monaden. Arch. f. mikr. Anat. i. (1865), p. 203.

9. Famintzin, A., and Woronin, M. Ueber zwei neue Formen von Schleimpilzen, Ceratium hydnoides, A. and Sch., and C. porioides, A. and Sch. Mém. de l'Acad. Imp. d. Sciences de St. Pétersbourg, sér. 7, T. 20, No. 3 (1873).

10. Greenwood, M., and Saunders, E. R. On the Rôle of Acid in Protozoan Digestion. Journ. of Physiology, xvi. (1894), p. 441.

11. Jahn, E. Myxomycetenstudien-3. Kerntheilıng u. Geisselbildung bei den Schwärmern von Stemonitis flaccida, Lister. Ber. d. deutschen botanischen Gesellschaft, Jahrg. 1904, Bd. xxii. Heft 2.

12. Krukenberg. Ueber ein peptisches Enzym im Plasmodium der Myxomyceten und in Eidotter vom Huhne. Unters. aus d. physiol. Inst. in Heidelberg, 1878, ii. p. 273.

1 "Cytological Studies in Ceratiomyxa," Trans. Wisconsin Academy of Science, Arts, and Letters, vol. xv. (1907), pt. 2, p. 753 ; and "Evidences of Sexual Reproduction in Slime Moulds," Science (N.S.), vol. xxv. (1907), p. 266. 
13. Lankester, E. R. Article "Protozoa" in Encyclopaedia Britannica, 1891.

14. Lister, A. Notes on the Plasmodium of Badhamia utricularis and Brefeldia maxima. Ann. of Bot. vol. ii. No. 5 (1888).

15. - On the Ingestion of Food Material by the Swarm-Cells of Mycetozoa. Journ. Linn. Soc. (Botany), vol. xxv. (1889), p. 435.

16. - On the Cultivation of Mycetozoa from Spores. Journ. of Botany, Jan. 1901.

17. - On the Division of Nuclei in the Mycetozoa. Journal of the Linnean Soc. (Botany), xxix. (1893).

18. - A Monograph of the Mycetozoa. Brit. Museum Catalogue. London, 1894.

19. Metschnikoff, $E$. Recherches sur la digestion intracellulaire. Annales de l'Institut Pasteur, 1889, p. 25.

20. Olive, E. W. Monograph of the Acrasieae. Proc. Boston Soc. of Nat. History, vol. xxx. No. 6 (1902).

20a. Penard, E. Étude sur la Chlamydomyxa montana. Arch. f. Protisten. kunde, Bd. iv. Heft 2 (1904), p. 296.

21. Plenge, $H$. Ueb. d. Verbindungen zwischen Geissel $u$. Kern bei d. Schwärmerzellen d. Mycetozoen . . . Verh. d. nat.-hist. med. Vereirs zu Heidelberg, N.F. Bd. vi. Heft 3, 1899.

22. Stahl, E. Zur Biologie der Myxomyceten. Bot. Zeitung, Jahrg. 42 (1884), pp. 145, 161, and 187.

23. Strasburger, E. Zur Entwickelungsgeschichte d. Sporangien v. Trichia fallax. Botanische Zeitung, 1884.

24. Zopf, $W$. Die Pilzthiereo der Schleimpilze. Schenk's Handbuch der Botanik, 1887.

25. — Zur Kenntuiss der Labyrinthuleen, einer Familie der Mycetozoa. Beiträge zur Physiologie u. Morphologie niederer Organismen. Heft 2 (1892), p. 36, Leipzig. 


\title{
THE PROTOZOA (continied)
}

\author{
SECTION D. -THE LOBOSA ${ }^{1}$
}

Gymnomyxa (Homokaryota), with lobate or pointed unbranched pseudopodia without an axis and with one or more definite nuclei.

In a large number of the characteristic genera of Lobosa the body consists of a small plastid of protoplasm protruding a few lobate pseudopodia by means of which a slow progression is effected, and exhibiting one nucleus and a contractile vacuole.

In addition to these characteristic forms, however, other genera must be included in the same class in which the body is protected by membranous or rigid shells (Thecamoebida), with radiating and pointed pseudopodia (Trichosphaerium, etc.), with two (Arcella), or numerous nuclei (Pelomyxa), and with no contractile vacuole (Endamoeba, etc.).

In many Lobosa, such as Amoeba terricola (Penard [20]) and others, the superficial protoplasm secretes a membranous envelope through which the pseudopodia may be protruded or particles of food ingested. In Trichosphaerium the envelope is relatively thick, gelatinous in texture, and provided with a series of very delicate radiating spicules, mainly composed of carbonate of magnesia. Spicules similar to these are also found in the ectoplasm of Amoeba pilosa (Cash), in which no true membrane is formed. In Dinamoeba (Leidy) the spicules occur in a hyaline jelly that surrounds the body.

In the Thecamoebida a definite shell is formed through which the pseudopodia cannot penetrate. In this case the pseudopodia can protrude only through a definite and permanent mouth or pore in the shell, which it is convenient to call the pylome (Hartog).

In some forms of Amoeba and in other genera there is often seen an apparent differentiation of the protoplasm into a clear outer layer, called the ectoplasm, and a more granular and more fluid central substance ealled the endoplasin. This appearance is more clearly defined when the protoplasm is very active and several pseudopodia are protruded. In the quiescent stages and conditions of life the ectoplasm usually disappears or becomes extremely attenuated, and in species or forms with only one or two

1 By Prof. S. J. Hickson, M.A., F.R.S. 
pseudopodia it can be clearly observed only on the pseudopodia themselves (Fig. 12, 2). It seems probable, therefore, that in the Lobosa there is no true differentiation of the cytoplasm, and that the appearance known as ectoplasm is only due to the temporary withdrawal of metaplasmic particles from the superficial parts of the active cytoplasm.

At the surface of an Amoeba there may always be seen a dark border which has the appearance of a very thin pellicle. This pellicle may be traced on the sides of the pseudopodia, but fades away towards their extremities, becoming extremely attenuated at the active terminal point. Immediately below this pellicle there is a layer of very hyaline ectoplasm. In carefully prepared sections the hyaline ectoplasm is found to be not strictly homogeneous, but to possess an alveolar structure similar in general characters to that of other forms of protoplasm. At the actual surface there is a single layer of alveoli, in which, as in artificially prepared oil foams, the sides vertical to the surface are parallel, or almost parallel, to each other, giving the appearance of a row of fine vertical striae. It is apparently this marginal alveolar layer which constitutes the pellicle.

The movements of an Amoeba may be best interpreted on the basis of the alveolar hypothesis of the structure of protoplasm.

The protrusion of a pseudopodium begins with a lowering of the surface tension of the marginal alveolar layer over a small area on the surface. This is followed by a flow of endoplasm towards the area of reduced surface tension. It has been suggested that the initial stages are accompanied by a rupture of some of the alveoli at the surface, which liberates a fluid - the enchylema-and that this causes a local diminution of the surface tension. It is possible that the release of enchylema may continue during the whole of the process of the protrusion of a pseudopodium, and stop when the pseudopodium comes to rest. During the active protrusion of a pseudopodium there may be observed a rapid centrifugal flow of endoplasm towards the periphery, called the axial stream. At the apex this stream spreads outwards like a fountain, and is continued as return currents on the surface. Similar fountain-like currents have been observed in the movements of various artificially prepared foams, but in the living protoplasmic pseudopodium the velocity of the return currents diminishes more rapidly and soon comes to rest (Fig. 1). In an Amoeba such as $A$. limax, in which, as a rule, only one pseudopodium is formed, there is a reverse fountain current at the posterior end, the particles at

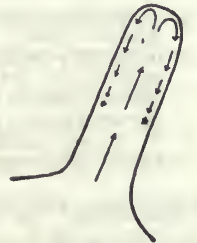

Fia. 1.

Diagram to show the flow of protoplasm in a yrow. ing pseudopodium. (After Buitschli.) the surface flowing towards the axis and joining in the axial stream flowing in $t^{2}$. direction of the advancing pseudopodium. But in 
this case the actual posterior end is not involved in the current, and by the increase of surface tension becomes folded or wrinkled, giving sometimes an appearance of several small pseudopodia (Fig. 12, 2).

In polypodious Amoebae similar reverse currents may be observed in retreating pseudopodia, and in areas of the body that are supplying materials for the axial streams of advancing pseudopodia. 1

Nucleus. - The nucleus of the Lobosa in its resting condition usually exhibits a well-defined membrana limitans. The chromatin is in the form of a number of spherical or irregular particles frequently collected together round the periphery, leaving a more or less clear space in the centre. In some cases a very delicate network of fibrils has been observed, which is regarded as linin (Fig. 2).

One or more nucleoli composed of a substance which differs

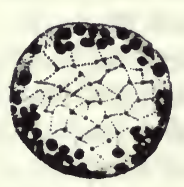

Fra. 2. chemically in some of its reactions from chromatin may or may not be present.

In Paramoeba eilhardi there occurs a deeply staining body in proximity to the nucleus, which was termed by Schaudinn (25), who described it, the "nebenkörper" (Fig. 4, c). This body divides Nuclens of Pelomyxa. previous to the division of the nucleus, and the (After Bott.) two parts take up a position at opposite poles of the spindle. This body is usually regarded as a "nucleolar centrosome." A body corresponding to this has also been found by

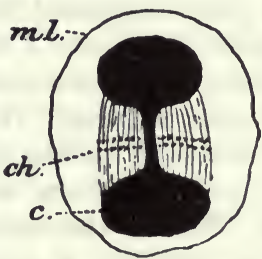

Fia. 3.

Dividing nucleus of Amoeba limax. m.l, the meinbrana linitans of the nucleus; $c$, the nucleolar centrosoine; ch, the chromosomes arranged in an equatorial band. (After Vahlkaupf.)

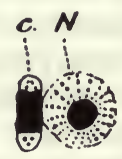

Fro. 4.

The resting nucleus (N) and "nebenkörper" (1:) of l'aramoeba eilhardi. (After Sclıaudinn.)

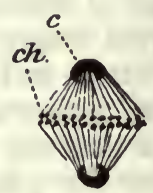

Fia. 5.

The nucleus of the same species dividing. "The "nebenkürper" (c) has divided into two larts, which occupy a position at the foci of the central spindle. $c h$, the chromosomes . arranged in an equatorial band. (After Schaudinn.)

Vahlkampf in the division of the nucleus of Amoeba limax (Fig. 3), ${ }^{2}$ but in this case the nucleolar centrosome lies within the nuclear membrane.

1 The subject of amoeboid novements has of recent years attracted the attention of many observers. The views expressed by Biitschli (Investigations on Microscopic Foams, etc., transl. by Minclin, 1894) have been opposed by Jennings (14), but Jennings' views have been more recently criticised by Rhumbler (23).

2 For a discussion on the nature of these bodies, see Goldschmidt and Popoff, Archiv f. Protist. viii., 1907, p. 321. 
Although the presence of a defined nucleus is regarded as one of the characters of the class, it has been shown that the nuclei sometimes disintegrate and discharge their chromatin into the cytoplasm as scattered granules. This occurs as a result of starvation in Pelomyza (Bott [2]), and as an antecedent to the formation of sexual or reproductive nuclei in Endamoeba.

Chromidia.-In addition to the chromatin contained within the boundaries of the nuclei, there may be present in the cytoplasm of many Lobosa irregular scattered granules or a fine network of a substance which gives the same reactions and is probably of the same nature as chromatin.

In some cases, Difflugia (Fig. 6), the network entirely envelops the nucleus or nuclei, but in others it is separated from the nuclei, Arcella, Cochliopodium, etc. (Figs. 19 and 21 ), by a halo of clear protoplasm.

These granules are called the chromidia, and the network is called the chromidial network (Fig. 6, ch). The chromidia may arise by the migration of particles of chromatin from the nucleus into the cytoplasm or by the disintegration of nuclei, but it seems probable that in some cases they may arise de novo in the cytoplasm.

The fate of the chromidia is varied. They may either give rise to the nuclei of gametes or of swarm-spores (Centropyxis), or they may accumulate in groups and give rise to new nuclei of the

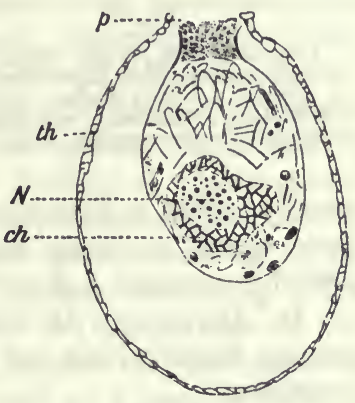

Fic. 6.

Section through Diffugia sp.? showing the nucleus $(N)$ surrounded by the chromidial network (ch). $p$, pylome; th, theca wall. (After ordinary type in the cytoplasm (Arcella, Pelomyxa), in which cases they are called Idiochromidia. Or, on the other hand, they may be associated with the assimilating or vegetative functions of the cytoplasm and disappear when their activity is exhausted, in which case they are called Trophochromidia.

Riefringent and Crystalline Bodies.-In many Lobosa crystalline bodies and vesicles containing a strongly refracting substance or fluid occur in great numbers in the cytoplasm. Very often they are far more numerous and conspicuous during the stages and conditions of life when active feeding is in progress than in conditions of starvation or reproduction. They are usually regarded as of the nature of reserve food materials.

In Amoeba dofleini, Neresheimer (18) found that the crystalline body, proteid in composition, is associated with a trophochromidium which is probably the active principal of its formation. Veley (34) has shown that the refracting bodies of Pelomyxa are proteid in nature.

On the other hand, Zuelzer (35) has described the bodies 
formed by the trophochromidia of Difflugia as carbohydrate in composition, but the crystalline and other bodies of Trichosphaerium,

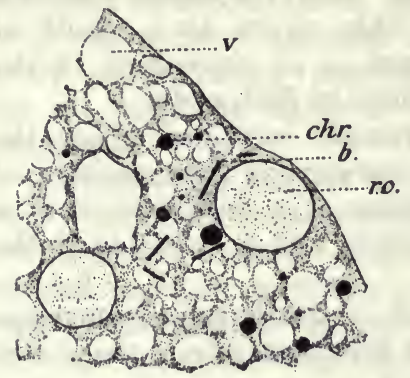

Fig. 7.

A sinall portion of the ectoplasm of Pelomyxa palustris, very highly magnitied. r.c, refringent proteid bodies; $b$, symbiotic bacteria (Cladothrix); chr, scattered cliromidia ; $v$, water vacuoles. (Áfter Bott.) according to Schaudinn, give different reactions.

Vacuoles. - In nearly all the freshwater and marine Lobosa there is at least one contractile vacuole. In Pelomyxa and some of the Thecamoebida, however, contractile vacuoles have not been found. The endoparasitic Amoebida have no contractile vacuoles. In addition to the contractile vacuole numerous non-contractile vacuoles containing a fluid of unknown constitution but probably nearly pure water occur in the endoplasm.

When a particle of food occurs in a non-contractile vacuole it is usually called a food-vacuole, and the fluid in such vacuoles has been shown in some cases to be slightly acid in reaction and probably contains a digestive ferment.

In Arcella and in other Thecamoebida vacuoles containing a gas are found within the shell. These vacuoles serve hydrostatic functions.

Rieproduction-Fission. - Reproduction by fission has been proved to occur as a. normal process in many of the genera of Lobosa. In Amoeba and allied genera the result of fission is a pair of equal-sized daughter
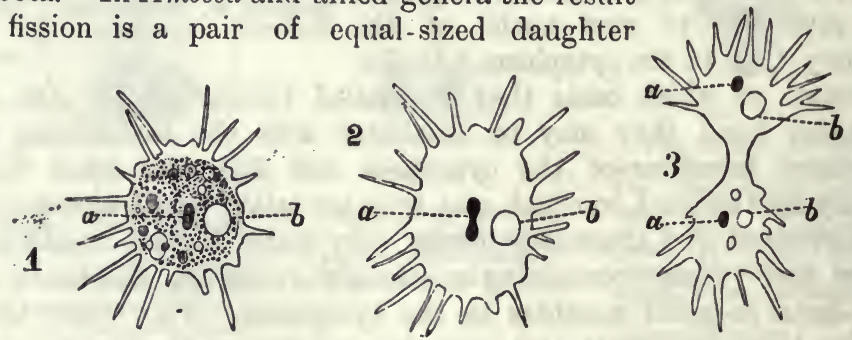

Fig. 8 .

Dactylosphera polypodia, M. Schultze, in three successive stages of division. The stages indicated occupied fifteen minutes. $a$, nucleus; $b$, contractile vacuole. (After F. E. Schultze.)

Amoebae. In Pelomyxa, Trichosphaerium, and probably in other multinucleated Gymnamoebida, fission may be unequal.

In the Thecamoebida one of the individuals of the act of fission retains the shell, and the other sooner or later forms a new shell which is usually larger than that of the parent. 
The process of fission is usually preceded by division of the nucleus, or in the binucleate Amoebae of both nuclei.

In some species (Amoeba binucleata and Paramoeba eilhardi, Schaudinn (Figs. 4 and 5), A. limax, Vahlkampf, and Amoeba proteus, Awerinzew) the division of the nucleus shows some of the characters of ordinary mitosis. The chromatin is collected together into a large number of short chromosomes arranged in an equatorial row. They then divide and travel in two equal parties along faintly stained and nearly parallel lines, supposed to be of the nature of linin, towards the opposite poles of the nucleus, where they unite to form the chromatin network of the daughter nuclei. The threads of the figure do not always converge at the poles to a focal point, and as a general rule it is doubtful whether structures exactly sinilar to the centrosomes of the metazoan nuclei occur. Centrosomes, however, have been described and figured in the division of the nuclei of Pelomyxa (Bott [2], Fig. 11, a).

Notwithstanding the evidence of a primitive kind of mitosis in the division of the nuclei in these and other species, the division of the nucleus of Amoeba crystalligera, of A. hyalina, of Dactylosphaera polypodia, and of Endamoeba coli (Schaudinn) is amitotic.

In Pelomyxa and Trichosphaerium fission usually consists in the pinching off of globules from the body, each containing a few nuclei. These globules rapidly assume the characters of the parent; they increase in size and the number of the nuclei is augmented. This process may be regarded as a case of unequal fission or of gemmation, but it appears to differ from the equal fission of some species of Amoeba in the respect that antecedent division of the nuclei is not an essential preliminary to division of the cytoplasm. In a large multinucleate form of Amoeba proteus, Stolč (31) has found that division of the nuclei may or may not take place before fission; and in some cases some of the nuclei divide and others do not before an act of fission.

Encystment and Spore-Formation.-It is now known that many of the Gymnamoebida periodically undergo a process of encystment in which the pseudopodia are withdrawn, the body becomes more or less spherical, and one or more tough membranes are formed which entirely surround and protect the animal. In some cases these cysts appear to be of the nature of resting cysts (Amoeba limax, Vahlkampf [33]), the organism emerging from the broken cyst-wall with the same characters it possessed previous to cystformation. The formation of resting cysts probably occurs in all the Thecamoebida. In many cases, however, the encystment is accompanied by complicated nuclear changes and divisions followed by division of the cytoplasm, and a swarm of minute spores that are often very different in character from the parent form are hatched out when the cyst-wall breaks down. 
In the case of Endamocba coli (Schaudinn [36]), for example, the uninucleate amoeboid form discharges all foreign bodies from its cytoplasm and becomes surroundes by a clear, soft, jelly-like envelope. Within the cyst-wall it divides into two equal parts each with a single nucleus, and these two parts remain separate for a considerable time. The two nuclei then fragment, their chromatin being scattered in the cytoplasm as isolated chromidia. The two nuclei are now reconstructed, but each reconstructed nucleus is relatively poor in ehromatin. Each of these nuclei now - divides into two by a primitive kind of mitosis; one of them from each half-amoeba is rejected as a polar nucleus and the remaining one divides again. At this stage in the process the protoplasm contracts, the gelatinous membrane disappears, and the cyst is surrounded by a harder membranous wall. The daughter nuclei of this mitosis conjugate reciprocally with the daughter nuclei of the other half-amoeba, and each of the two zygote nuclei thus formed divides twice. The eight nuclei thus formed become the nuclei of eight amoebulae which escape from the cyst.

In Amoeba proteus also, according to Scheel, division of the nucleus and cytoplasm takes place during the encystment, and

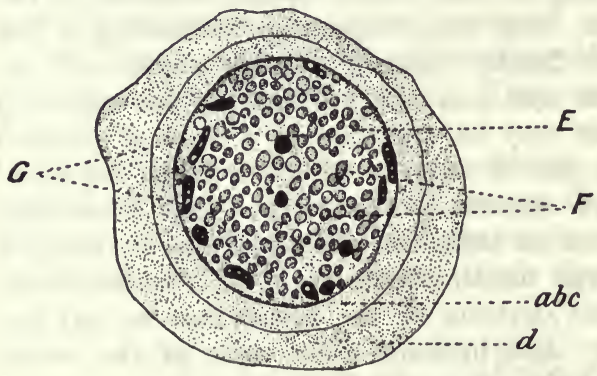

A

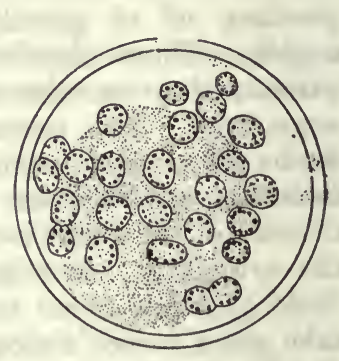

B

A, cyst of $A$ moeba proteus ; $a b c$, cyst-wall ; $d$, gelatinous envelope ; $E, F$, nuclei ; $G$, albuminous bodies. $\times 300$. (After Scheel.) B, cyst of Endamoeba blattae, with 25 nuclei. (After Seluubotz.)

a swarm of small amoebulae emerge from it when the cyst breaks down. In this case, however, there is no evidence that any form of nuclear conjugation takes place during the encystment.

Conjugation.-Although the complete life-history of only a few species of the Lobosa has, at present, been fully worked out, the evidence is accumulating to justify the conclusion that a process of conjugation is an essential condition for the completion of the lifecycle in all forms. The process of conjugation has not yet been observed in Amoeba proteus or in any of its allies. Nuclear conjugation accompanied by fusion of the cytoplasm occurs during encystment in Endamoeba coli. 
In Pelomyxa (Bott [2]) amoeboid isogametes are discharged from the body with a nucleus formed in a manner that suggests that the number of the chromosomes is reduced (infra, p. 76). These gametes conjugate to form a zygote (Fig. 10), which may subsequently encyst.

In Trichosphaerium (Schaudinn [26]) a large number of biflagellate isogametes escape from the cyst and by exogamous conjugation form zygotes which become amoeboid in character.

Biflagellate isospores arise from the cystic stage of Paramoeba eilhardi, but there is no evidence, at present, to show that they conjugate.

In Centropyxis (Schaudinn [27]) heterogametes are formed which have a shell. After conjugation the zygote escapes from the shell and forms a new one like that of the adult individual.

Life-History. - The recent rapid advance in our knowledge of the life-history of Lobosa, due in large measure to the researches of Schaudinn and R. Hertwig, suggests that in all cases the developmental cycle that is passed through is both complicated and varied.

In order to illustrate the general character of these lifehistories, four examples may be taken for description.

Endamoeba coli is found in the upper part of the human large intestine, but unlike Endamoeba histolytica it does not appear to be the cause of or associated with any particular form of disease. It undoubtedly occurs in perfectly normal and healthy hosts.

During the ordinary vegetative life in the intestine it multiplies by simple fission with amitotic division of the nucleus. Occasionally schizogony occurs, when the nucleus divides into eight by successive mitoses and each of these nuclei becomes the nucleus of a daughter amoebula. After a certain period of vegetative life, the normal duration of which has not been estimated, the uninucleated amoebae become encysted, and in that condition are passed into the lower part of the large intestine, and so to the exterior with the faeces. The complicated divisions and the conjugation of the nuclei during and antecedent to complete encystment have already been described. Many of the cysts undoubtedly perish, but the cysts with eight nuclei when swallowed by another host will give rise to eight amoebulae which infest the intestine of the new host. The cysts with more than eight nuclei that are sometimes found in the faeces are, according to Schaudinn, degenerating cysts, and never give rise to active amoebulae.

In Trichosphaerium, a marine rhizopod with peculiar radiate pseudopodia and many nuclei, there are two phases in the life-cycle. In the first phase the gelatinous investment is armed with radiating spicules. It reproduces itself in this phase by simple binary or by multiple fission, the pseudopodia being previously withdrawn. In 
the second phase, in which the radiating spicules do not occur, reproduction may also occur in a manner similar to that of the first phase, but at the conclusion of vegetative growth the pseudopodia are withdrawn, all foreign bodies and excreta are expelled, and a cyst is formed. The nuclei then divide rapidly by repeated mitoses to form an immense number of minute nuclei. These nuclei become the nuclei of minute biflagellate swarm-spores (gametes), which escape from the gelatinous investment of the cyst, and after conjugation give rise to small individuals of the first phase.

In Pelomyxa, a multinucleate freshwater rhizopod (Fig. 14), reproduction is effected by simple or multiple fission during the vegetative

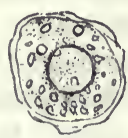

$a$

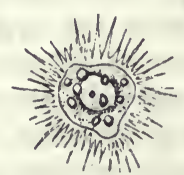

$b$

Fio. 10.

Zygrote of Pelomyxa palustris. a, encysted. $\checkmark$, after escaje from the cyst. (After Butt.) period of life, but at certain times, after a complicated series of nuclear divisions in which a reduction in the number of chromosomes occurs, uninucleated, heliozoan-like swarm-spores escape which conjugate to form a zygote, and this encysts. From the cyst a uninucleated amoebula escapes, which by growth and multiplication of the nucleus gradually assumes the typical Pelomyxa form.

In the preparation of the nuclei for the formation of the gametic nuclei, a considerable part of the chromatin is discharged into the cytoplasm, and from that which remains eight chromosomes are formed on the equatorial band of a central spindle (Fig. 11,a). Two successive divisions take place, the first of which is regarded as a reduction division, and the second as an equation division. 'The chromatin of the four chromosomes of this last division collect together in two lumps, and a transparent globular vacuole appears in their immediate neighbourhood. This vacuole gradually fills with minute granules which rapidly increase in size

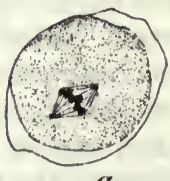

a.

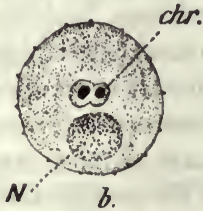

Fic. 11.

Nuclear fornıtion in Pelomyra. a, the spindle of the reduction division with eight chromosomes. $b$, the nucleus $(N)$ of the gamete forming in a clear vacuole. ch, the cliromatin lumps of the last nuclear division. (After Bott.) and gives rise to the nucleus of the gamete (Fig. 11, b). The chromatin lumps at the same time dwindle and eventually disintegrate.

In Centropyxis, one of the Thecamoebida, binary fission occurs by the protrusion and division of the protoplasm preceded by amitotic division of the nucleus. One portion of the divided protoplasm with one nucleus returns to the old shell, the other forms a new shell but of a larger size. It does not seem certain whether 
the individual retained by the old shell is or is not capable of further reproduction, but the occurrence of an immense number of empty shells in cultures of Centropyxis and its allies suggests that it may die after one act of fission. The individual that has formed a new and larger shell, however, certainly divides again, giving rise by a similar process to a daughter individual with a still larger shell. When by these processes of fission the full size is reached, the nueleus degenerates, after giving rise to an expanded chromidial network which, with about two-thirds of the protoplasm, protrudes from the mouth of the shell, is pinched off, and escapes. The remaining one-third of the protoplasm and the degenerate nucleus that remain in the shell probably die.

The escaped protoplasn may give rise to one of two broods of gametes. In one brood (the megagametes) the chromidia give rise to a nucleus and the protoplasm forms a shell; in the other, after a nucleus is formed from the chromidia and a shell is formed as in the first brood, a division into four individuals (the microgametes) takes place, and each of these escapes and forms a small shell. Conjugation takes place between the larger and smaller individual gametes, and the zygote escapes to form a new shell like that of the parent.

\section{Order Gymnamoebida.}

The surface of the body either naked or provided with a thin flexible membrane through which the pseudopodia can be protruded.

Genera Amoeba.-The generic name Amoeba is often applied to any naked amoeboid organism without reference to its subsequent or antecedent history. As our knowledge of the natural history of the simpler Protozoa widens it becomes more evident that the generic name should be used only in a restricted sense. The limits we place upon the use of the generic name can only be regarded as provisional. Further investigations may well prove that the species now included in the genus Amoeba ought to be still further separated into subgeneric or generic groups.

The characters of the genus may be summarised as follows :Solitary Gymnamoebida, with a few short blunt pseudopodia, a single contractile vacuole, and one or more nuclei. No membrane covering the body in the trophic phase of life. Freshwater or marine.

Nine or ten distinct species have been described from fresh water in this country (Cash). They are usually found in the mud at the bottom of ponds or creeping on submerged vegetation. Some of the rarer forms are found in Sphagnum bogs. One of the commonest species is Amoeba proteus (Fig. 12,5), a species capable: of considerable variation in form, but usually exhibiting several. 
digitiform pseudopodia. In this species there may be either one or many nuclei. It may reach a size of $200 \mu$ in diameter. A. guttula (Fig. 12,4) is another very common species of small size, $30 \mu$, which shows slow undulating movements of the ectoplasm but rarely protrudes definite pseudopodia. In Amoeba limax (Fig. 12, 2), which is slug-like in form, the end that is posterior in progression shows a fan-shaped arrangement of short ridges, due probably to the

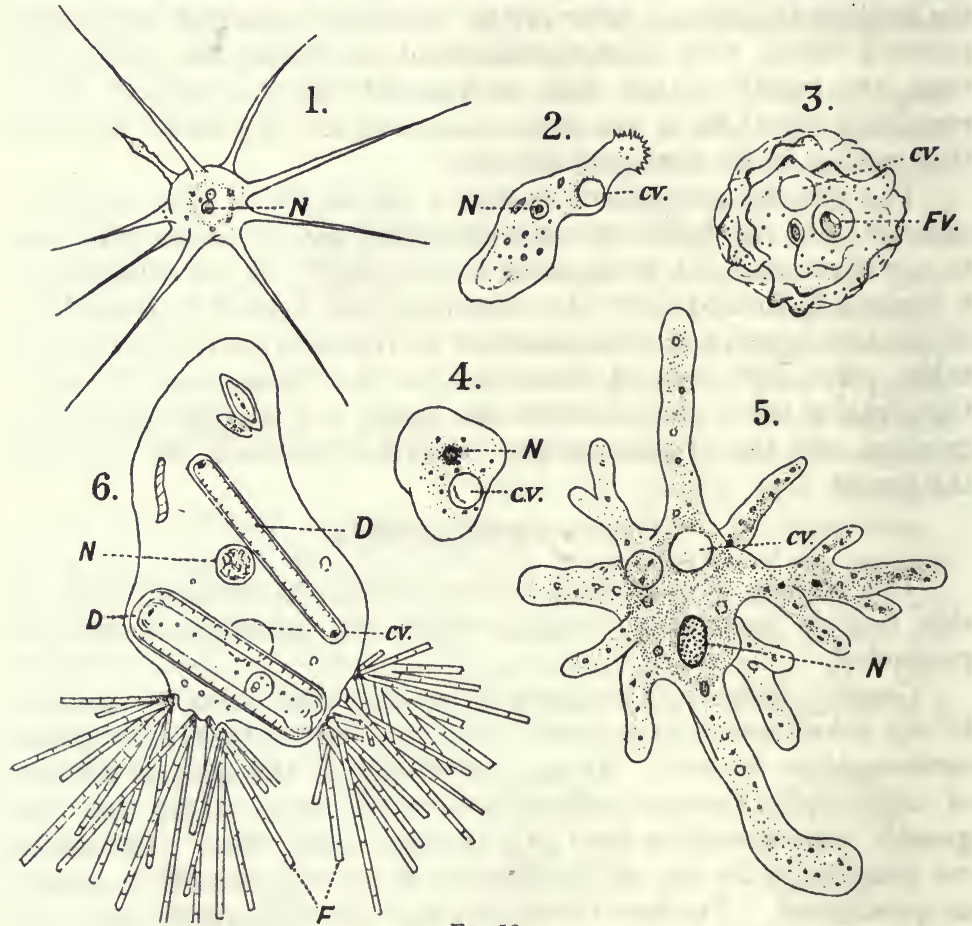

Frg. 12.

Different species of freshwater Gymnamoebida. 1, Dactylosphaera radiosa, $\times 260$. 2, Amoeba limax, $\times 200$. 3, Amoeba verrucosa, $\times 200$. 4, Amoeba guttula, Duj., regarded as a young form of $A$. proteus by Leidy. 5, Amoeba proteus. 6, Amoeba (Uuramoeba) vorax, $\times 130$. N, nucleus; $c . v$, contractile vacuole; $F . v$, food vacuole; $F$, hyphae of a fungus. In Amoeba vorax some of the large diatoms $(D, D)$ upon which it feeds and the approximate positions of the nucleus and contractile vacuole are shown. (1, 2, 3 from Cash ; 4, 5, 6 from Leidy.)

wrinkling of the surface in the vortex of the retreating axial stream (see p. 69).

The marine Amoebae have not yet been carefully recorded. Amoeba crystalligera is often found in marine aquaria, and a species allied to the freshwater $A$. guttula has been found at Woods Hole in America. Amoeba fluida was found in sea-water aquaria in Freiburg by Gruber, and this with two other species were also found by him in the Gulf of Genoa. 
It may be regarded as extremely doubtful whether the forms that the Amoebae present really indicate true differentiation into definite species, or represent the varying influence of certain external conditions acting upon one species, or, again, represent different phases in the life-history of one or more distinct species. Thus it has been observed that when the amoebae found on the surface of decomposing hay infusions are placed upon a slide, broad lobate pseudopodia begin gradually to be extended in various directions and the general form of Amoeba proteus is assumed. After a time, when progression may be induced in one direction, the body becomes elongated and more or less pointed at the anterior end, so that the form becomes similar to that known as $A$. limax. If the water be made very feebly alkaline the amoebae contract into a spherical shape with very short dentate pseudopodia, similar to $A$. guttula, and then protrude long pointed pseudopodia similar to those of Dactylosphaera radiosa. ${ }^{1}$

The forms usually attributed to the genus Ouramoeba, Leidy, have been shown to be Amoebae in which fungal filaments are growing (Poteat [21]). The filaments arise from spores which are always situated in the neighbourhood of the contractile vacuole. It has been suggested that the fungus receives nourishment from the waste products of the amoeba. These filaments have been observed in Amoebae attributed to the species $A$. villosa, $A$. binucleata, and A. proteus.

The life-history of no species of Amoeba has yet been fully worked out, but Calkins (7) has shown that Amoeba proteus normally passes through an early stage when the pseudopodia are relatively long and more pointed and similar to those of $A$. radiosa; and Scheel (29) has proved that the uninucleate condition is succeeded by a multinucleate condition previous to encystment.

Calkins suggests that the life-cycle of Amoeba protens may be somewhat as follows:-The zygote gives rise to a small radiate form, which develops into the uninucleate type-form. This encysts and by schizogony gives rise to uninucleate Amoebae, which develop into the multinucleate type-form. The multinucleate type-form encysts and gives rise to the gametes, which conjugate to form the zygotes.

Paramoeba, Schaudinn. Several radiating pseudopodia. A welldefined chromatin body is present in the cytoplasm close to the nucleus. Swarm-spores with two flagella. $P$. eilhardi was found in a marine aquarium in Berlin. $10-90 \mu$. P. hominis, a human parasite (p. 83).

Dactylosphaera, Hertwig and Lesser (Fig. 12, 1), is distinguished from Amoeba by the numerous rigid pseudopodia, which do not completely retract when at rest. Freshwater. Maximum $120 \mu$.

1 Verworn, General Physiology, English translation, 1899, p. 184 ; and Doflein, F., Archiv Prot. Suppl., 1907, p. 250. 
Lithamoeba, Lankester ${ }^{1}$ (Fig. 13). Body discoid, pseudopodia lobular and hernia-like. A distinct pellicle covering the body, which ruptures for the protrusion of the pseudopodia. Freshwater. Maximum $125 \mu$.

Dinamoeba, Leidy. Pseudopodia long, conical, and acute. Body enveloped in a delicate hyaline jelly bristling with minute spicules. Bogs of New Jersey. $60-160 \mu$.

The following genera were described by Frenzel $(8,9)$ from fresh water in the Argentine Republic: Chromatella, Stylamoeba, Saltonella, and Eikenia.

Centrochlaniys, Claparède and Lachmann. The body covered with a thin, membranous, disc-shaped test through which the pseudopodia pro-
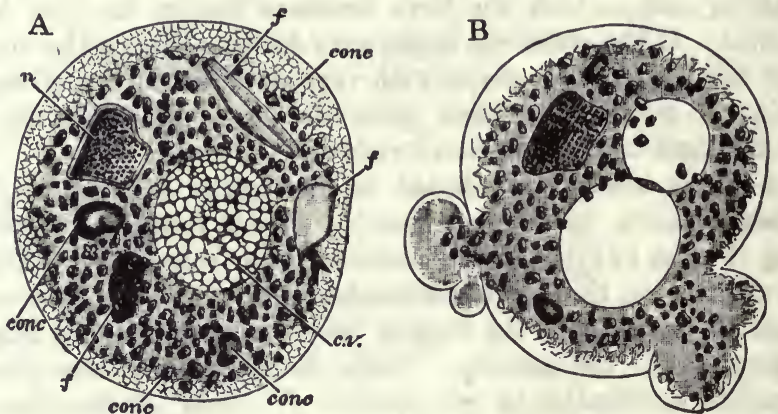

Fia. 13.

Lithamoeba discus, Lank. A, quiescent; B, throwing out pseudopodia. c.v, contractile vacuole, overlying which the vacuolated protoplasm is seen; conc, concretions insoluble in dilute $\mathrm{ICl}$ and dilute $\mathrm{KHO}$, but soluble in strong $\mathrm{HCl} ; f$, food particles ; $n$, nucleus. (After Lankester.)

trude. No definite pylome. A single nucleus and several contractile vacuoles. Freshwater. 40-45 $\mu$.

Amphizonella, Greeff. Probably closely related to Centrochlamys. The body is usually invested by a supple membrane which, under some circumstances, is itself surrounded by a transparent mucilaginous envelope. The pseudopodia are pushed through these membranes and withdrawn again without leaving any definite aperture. It has not been determined whether the position on the test through which the pseudopodia protrude is definitely fixed or varies. These two last-named genera are undoubtedly closely allied to Corycia, Cochliopodium, and other Thecamoebida.

Hyalodiscus, Hertwig and Lesser. The ectoplasm usually very thick, and sometimes exhibiting radiating lines. A creeping movement without pseudopodia frequently ouurs. One or more inconspicuous nuclei. Freshwater. $40-60 \mu$.

Trichosphaerium, Schneider. The structure and life-history of this genus has been fully described by Schaudinn (26). The body is invested by a gelatinous test perforated by many pores for the protrusion 
of long digitate pseudopodia; several nuclei; no contractile vacuoles. Zooxanthellae occur in the protoplasm. Marine.

Pelomyxa, Greeff. A remarkable genus of Gymnamoebida found in the mud of ponds and ditches, and distinguished by the presence of an enormous number of minute nuclei. Several species have been described. $P$. palustris, Greeff, $P$. villosa, Leidy, are frequently found in this country and are probably cosmopolitan. $P$. penardi, Rluunbler (22), was found at Göttingen. P. viridis has only been found in British India.

They vary considerably in size, but when spread out in progression $P$. viridis may attain to a size of 8 $\mathrm{mm}$. in diameter, and the other species to $2 \mathrm{~mm}$.

The form of the animal is like that of an amoeba, and progress is effected by means of numerous blunt lobose, villiform, or sometimes attenuate and anastomosing pseudopodia of very variable form and length. There is neither test nor enveloping membrane.

In the ordinary vegetative con-

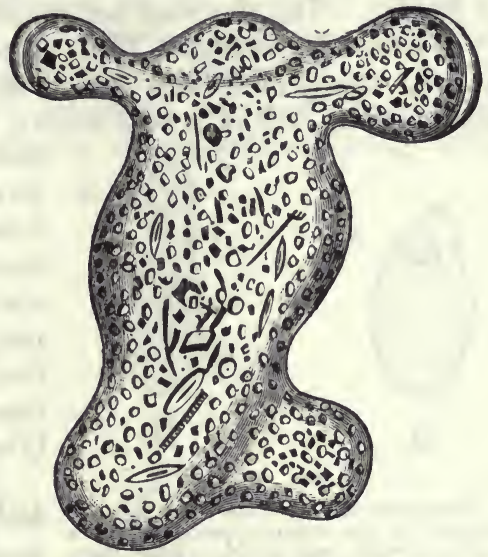

Fia. 14.

Pelomyxa palustris, Greeff. An example with comparatively few food particles. (After Greeff.) dition of Pelomyxa there are very many nuclei. Bourne (3) calculated that in a large specimen of $P$. viridis there may be 10,000 nuclei. In addition to the nuclei there are numerous mimute scattered chromidia (Bott [2]) (Fig. 7). These chromidia may be clearly seen in the ectoplasm. The chromidia are formed by the chromatin discharged from the nuclei, and they never unite to form a chromidial network. In addition to the nuclei and chromidia, the cytoplasm contains refringent bodies of a proteid nature (Veley [34]), numerous symbiotic bacteria, food-vacuoles, and various water-vacuoles, and minute vesicles.

The refringent bodies appear to be waste materials and probably a by-product of metabolism, and are undoubtedly used as the food material of the symbiotic bacteria. They are sometimes ejected from the body, but in general the Pelomyxa relies on the bacteria as scavengers to clear its protoplasm of these bodies. The life-history of the symbiotic bacteria (Cladothrix pelomyxae) has been studied by Veley, who also determined the proteid nature of the refringent bodies by obtaining the characteristic reactions with-(1). Millon's reagent; (2) sugar and sulphuric acid; (3) the xanthoproteic test; and (4) with caustic soda and copper sulphate.

The green vesicles described by Bourne in $P$. viridis appear to be of the same nature as the refringent bodies, but stained with chlorophyll.

The protoplasm of all the species contains a number of vacuoles and vesicles, but none of them appear to be rhythmically contractile. 
Endamoeba. ${ }^{1}$-The species of this genus are parasitic in the intestines of various animals. There is no contractile vacuole, and rarely more than one short pseudopodium is protruded. Endamoeba coli is commonly found in the human intestine. It is often present in perfectly normal health, and is not associated with or the cause of disease. The size does not exceed $50 \mu$.

Endamoeba histolytica is so similar in size and form to $E$. coli in some stages of its life-history that it has been regarded as the same species, but it is now known to have a different life-history

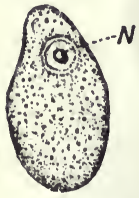

A.

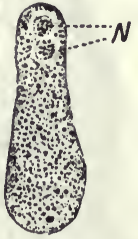

B. and to be the active cause of certain forms of tropical dysentery. It is found not only in the ulcers of the intestinal mucous membrane, but also in abscesses of the liver accompanying the disease. It penetrates the mucous membrane of the intestine and enters the submucosa (Dopter [42]).

FIG. 15.

The life-history of Endamoeba

Endainneba coli. A, a specimen with one nucleus in the resting condition. $\mathbf{B}, \cdot \mathbf{a}$ specimen with two nuclei. (After Casagrandi and Barbagallo.)

histolytica has not yet been fully worked out. It is very similar in size and appearance to $E$. coli, but differs from it in the somewhat indefinite and variable character of having usually a more distinct hyaline ectoplasm. According to Lesage (43) the large cysts, similar to those of $E$. coli, $20 \mu$ in diameter, are never found in this species. In $E$. histolytica the cysts are 3-6 $\mu$ in diameter. During the progress of the disease which it causes it is constantly changing its shape and position, and asexual reproduction proceeds rapidly by simple fission or multiple gemmation. Cyst-formation only begins when healing commences, never in the height of the disease. The encystment is preceded by the rapid discharge of chromidia into the cytoplasm, and then the nucleus degenerates and disappears. The chromidia then collect to form a chromidial network in the ectoplasm, and subsequently spherical bodies, the cysts, each surrounded by a yellowish-brown membrane and containing a portion of the chromidial network, are pinched off (Fig. 16, D). The rest of the life-history las not been followed, but it has been shown that when the cysts are given to cats they cause a dysenteric disease.

Other species of Endamoeba have been described from the human intestines, but it is uncertain at present whether they are or are

1 The account given of Endamoeba coli and E. histolytica is mainly taken from the important memoir of Schaulinn. This memoir is, however, not illustrated. For further information and for figures of Endamoeba coli the reader is referred to the memoir by Casagrandi and Barbagallo (38), and of $E$. histolytica to the memoir of Jürgens (39) and other papers mentioned in the list of literature on p. 92. 
not associated with disease. Endamoeba undulans, Castellani (40), exhibits a peculiar amoeboid form, which occasionally protrudes a single pseudopodium. There is practically no distinction between the ectoplasm and endoplasm. The presence of a peculiar undulating membrane running round one end of the body suggests that the species may have different affinities to the ordinary species of Endamoeba. 25-30 $\mu$. Ceylon. Endamoeba iurai, Ijima (12), has been described from the human intestines in Japan.

The species described under the name Paramoeba hominis by Craig (41) was found in the faeces of patients suffering from

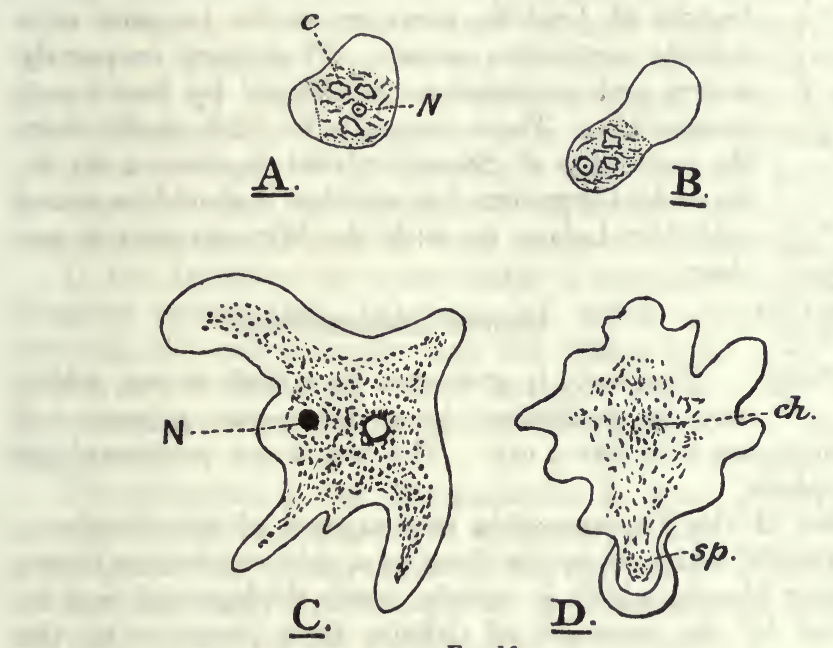

Fra. 16.

Endamoeba histolytica, Schaudinn. A, B, two specimens from a case of dysentery in a cat : $c$, blood corpuscles being dligested; $N$, nucleus. (After Jiirgens.) C, specimen from humau intestine with resting nucleus $\left(N^{\prime}\right)$ and a single non-contractile vacuole. $D$, specimen giving rise by gemination to a spore; ch, chromatin of nuclens in the form of scattered chromidia; $s p$, protoplasm of spore containing some chromidia. (C and $\mathrm{D}$ after Lesage.)

severe diarrhoea in the Philippine Islands, associated with $E$. histolytica and other Protozoa. There appear to be three phases in the life - history: (1) an amoeboid phase, 15-25 $\mu$; (2) a resting cystic stage, $15-20 \mu$; (3) a biflagellate phase, 3-15 $\mu$. Notwithstanding the general resemblance in its life-history to that of the marine Paramoeba eilhardi, it is difficult to believe that this species is rightly placed in the same genus.

Endamoeba blattae is often found in the rectum of the common cockroach. In form it is similar to Amoeba limax, but it seldom pushes out a single pseudopodium and has remarkably clear protoplasm. It may be as much as $80 \mu$ in diameter. Other species probably belonging to the same genus are found in the intestines of mice and in the rectum of the frog. 
It is difficult to determine at present the true nature of many of the amoeboid cells found in the pus and other fluids of pathological conditions, but the following are regarded as parasitic

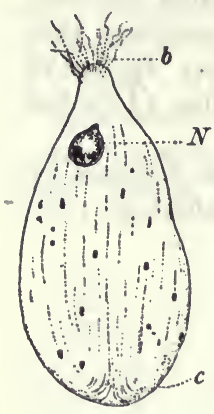

Fig. 17.

Endamoeba blattae. $N$, nucleus ; $b$, bacteria; $c$, at the allterior pole granules are seen arranged in the direction of the protoplasmic currents. (After Schubotz.) organisms: Amoeba urogenitalis, Amoeba kartulisi, Amoeba buccalis:

Leydenia gemmipara is an amoeboid cell originally found by Lieberkiihn in the ascites fluid of malignant tumours. The endoplasm contains numerous fat spherules, remnants of red and white corpuscles, and numerous crystalline bodies. The most remarkable feature of Leydenia, however, is the presence of a definite contractile vacuole. Plastogamy frequently occurs, and reproduction is effected by fission and gemmation. There seems to be little doubt from the researches of Schaudinn that Leydenia is an independent organism, but whether it should be placed with the Lobosa or with the Myxomycetes is not clear.

\section{ORDER Thecamoebida.}

The body is protected by a shell or test, which may be perforated by a hole-the pylome-or widely open on one side like a cap. The test is not perforated by the pseudopodia.

The test of the Thecamoebida is composed of two sheathsan inner sheath, which is in the form of a thin continuous layer; and an outer sheath, which is usually much thicker, and may be strengthened by the secretion of definite hard plates or by the adhesion of coreign materials of various kinds. The chemical constitution of the test is difficult to determine with accuracy, but it appears to consist of an organic matrix usually containing silica in larger or smaller proportions. The inner sheath of the test contains a small proportion or only traces of silica; the plates and prisms of the outer sheath, such as we find in Quadrula and its allies, contain a much larger proportion of silica. The matrix which cements the plates of Quadrula together, and which fastens diatom shells, grains of sand, and other foreign bodies to the test of Difflugia, is an organic substance which also contains a trace of silica. In the plates of Quadrula irregularis calcium appears to take the place of silicon.

There is no evidence of the occurrence of chitin in the tests of any Thecamoebida, but a substance allied to keratin may occur in some cases (Awerinzew [1]).

In the Cochliopodiidae the shell is thin and flexible. It is usually marked by minute punctuations arranged in definite rows or more irregularly distributed. When more highly magnified 
these punctuations appear to be globular in shape, but their precise nature has not yet been determined.

In Quadrula the outer sheath consists of a series of square plates cemented together by the matrix. These plates can be raised to a ligh temperature without destruetion of their form. When boiled for a long time in 10 to 20 per cent KHO, they are dissolved but leave behind a fine granular residue which probably represents the inorganic components of the plates. In Nebela the plates are discoidal, and in other genera irregular in form.

The diatom or desmid shells, the grains of sand or glass, and other foreign bodies that are found fastened to the outer sheath of the test of Difflugia (Fig. 20) and its allies are not adventitiously placed, but are caught and definitely arranged in position by the animal (Rhumbler). There can be little doubt that Difflugia exercises a deliberate choice of the particles it uses for shell purposes, and to a certain extent the character of the foreign particles and their arrangement can be used for racial or specifie distinctions.

In the Arcellidae the outer sheath is composed of hexagonal or irregular prisms (Fig. 18), some of which, situated at regular intervals, are rather longer than the others and project on the surface as round knobs or bosses. The prisms are cemented together by an extremely thin matrix.

The cytoplasm of the Thecamoebida is often arranged in three zones. The cytoplasm of the pseudopodia and of the region of the pylome is usually remarkably hyaline and the granulations extremely fine. In the middle zone it is more

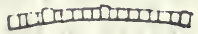

Fig. 18.

Section through the test of Arcella showing tlie hexayonal plates, some of which project at irreurular intervals as sliallow bosses on tlie surface. (After Awerinzew.) coarsely granular, and contains the contractile vacuoles, foodvacuoles, crystalline bodies, excreta, oil-globules, etc. In the zone next to the fundus of the shell is usually found the nucleus or nuclei and the sickle-shaped or more irregularly disposed chromidial network. In the Arcellidae, however, the arrangement is somewhat different from this (p. 86).

The pseudopodia are probably subject to considerable variation in shape and number according to external conditions. In the Difflugiidae there may be only one long finger-like pseudopodium extended to a length double that of the shell, or there may be three or four shorter pseudopodia, or occasionally as many as seven protruded at the same time. In IIeleopera the number of pseudopodia appears to be constantly more rumerous than in other genera of the family.

In some species of Arcellidae and Cochliopodiidae a membranous expansion of the cytoplasm sometimes protrules from the pylome. Very little is known concerning the contractile vacuoles of the Thecamoebida, as the thick opaque test interferes considerably with 
the observation of it in the living animal, but it seems probable that one or more contractile vacuoles are present in all genera.

Nucleus.-For a considerable period in the life-history of Arcella there are two large oval nuclei, from 0.015-0.02 mm. in diameter, which are usually situated some distance apart, near the periphery of the cytoplasm. More rarely three or even four of these relatively large nuclei may be found. These nuclei are derived by the karyokinetic division of the primary single nucleus of the young Arcella. Each nucleus contains a single large (.008 mm.) nucleolus, which apparently consists mainly of chromatin, but is otherwise clear and transparent (Fig. 21).

In other Thecamoebida (Difflugia ${ }^{1}$ and Centropyxis) there is usually only one nucleus during the corresponding phase of the lifehistory, and this exhibits a coarse reticulum of chromatin with numerous nucleoli distributed through it.

The chromidial network of Arcella is in the form of an irregular band or ring at the periphery of the cytoplasm, which sends lobate processes or branches in the direction of the central protoplasm. These processes are sometimes pinched off from the peripheral ring, and appear as isolated patches of the chromidial network in the central cytoplasm.

In Centropyxis the chromidial network is in the form of a thick sickle-shaped band lying in contact with the convex aboral extremity of the body. Sometimes this band envelops the nucleus, but neither in Centropyxis nor in Arcella does the nucleus come into contact with the network, being always surrounded by a halo of clear protoplasm (Fig. 21). In some forms of Difflugia the chromidial network is in contact with the nucleus (Fig. 6); in $D$. globosa and others, however, there is a clear space between the nucleus and the chromidial network as in Centropyxis, but in these cases strands of the chromatin seem to connect the nucleus with the network.

In another phasc of the life-history of Arcella there are numerous nuclei. The number is very variable, from 5 to 39 , but in a great many cases there are about 25 . These secondary nuclei are formed by the concentration of granules of chromatin of the chromidial network, which become rounded off and surrounded by a nuclear membrane. The larger the number of nuclei, the smaller they are. When very numerous these nuclei are not more than 0.009-0.01 mm. in diameter. As the secondary nuclei are formed, the two or three primary nuclei degenerate and disappear.

When a certain number of secondary nuclei have been formed, they divide by karyokinesis. This karyokinesis is a preparation for the process of fission. One half of the nuclei resulting from the karyokinetic division remain at the periphery, the remaining half

1 According to Zuelzer there are $10-30$ nuclei in $D$. urceolata, Carter. 
migrate towards the centre of the protoplasm. It is probably this central party of nuclei that, with their surrounding protoplasm, protrude from the pylome of the shell and give rise to the daughter Arcella in the process of fission. ${ }^{1}$

In Centropyxis (Schaudinn [27]) the formation of secondary nuclei previous to fission does not occur. When fission is about to take place, a considerable portion of the protoplasm protrudes from the pylome, assumes the inverted form of the parent, and develops a shell. The nucleus remains in that part of the protoplasm which at this stage only balf fills the shell of the parent Centropyxis. When the daughter shell is formed the nucleus increases to nearly double its former size, the nucleolus dwindles in size, and numerous minute chromosomes are formed. These changes are followed by the formation of a spindle, the arrangement of the chromosomes in an equatorial plate, and subsequently by nuclear division. One of the nuclei thus formed passes into the daughter individual and the other remains in the parent.

While these changes in the nucleus are taking place, the chromidial network divides into a great number of chromidia, which collect round the two nuclei in equal proportions and pass with them into the resultant individuals.

Encystment.-The formation of resting cysts occurs in Arcella, Centropyxis, Nebela, Difflugia, and probably in all the other Thecamoebida (Martini [16]).

In Centropyxis, Schaudinn found that cysts are formed when external conditions are unfavourable, such as in cases of desiccation, scarcity of food, etc. In such cases the food particles, diatom shells, excreta, a considerable proportion of the water, and any other non-essential contents of the protoplasm, are ejected, while the cytoplasm, with the contained chromidial network and nucleus, contracts into a ball and is surrounded by a cyst-wall.

At the end of encystment the cyst-wall disintegrates, the protoplasm swells up to its former size, and the normal processes of life are continued. It does not seem probable in this case that encystment has any connexion whatever with the sexual process.

In Arcella, however, according to Hertwig (11), a reduction in the number of the nuclei takes place, and it is suggested that the process of conjugation may occur during this period of encystment, in a manner similar to that which occurs in Actinosphaerium. ${ }^{1}$

In Diffugia urceolata (Zuelzer [35]) a process of encystment occurs in the late autumn, and is accompanied by a destruction of a great many of the old nuclei. Before the cysts rupture in the spring the contents break up into a number of uninucleate secondary cysts, but the history of the secondary cysts has not been followed.

1 See Note, p. 93. 
Plastogamy.-A process of the temporary or permanent fusion of two or more individuals has been observed by Schaudinn (27) in Centropyxis, and by Zuelzer (35) in Difflugia urceolata, and probably occurs in other Thecamoebida. In Centropyxis two individuals may join together plastogamically and produce a daughter individual with two nuclei and two chromidial networks, or if three individuals join together they produce a daughter individual with three nuclei and three chromidial networks. In some cases, the daughter individual produced by the plastogamy has an abnormal shell and the two nuclei and chromidial networks fuse together. In other cases, again, only one of the individuals gives rise to a daughter individual, and that is of the normal type.

In Difflugia urceolata a process of plastogamy occurs in which the nuclei and chromidial networks remain passive, when external conditions become unfavourable, but this appears to be antecedent only to disintegration. In the autumn, however, the protoplasm of one of the two participants in a plastogamic union passes into the shell of the other, and more rarely a process of plastogamy occurs in which the nuclei and chromidial network of both individuals are active, but definite fusion of nuclear elements has not been observed. At the end of this plastogamic fusion the empty shell may become firmly fixed to the shell containing the fused individuals, giving rise to the twin-shells so often found in cultures of these creatures (Rhumbler [22]). The meaning of the different forms of plastogamy in the Thecamoebida is not clear, but there is no evidence at present that they represent any phase of the true sexual process.

The only observation of a true conjugation in the order is that

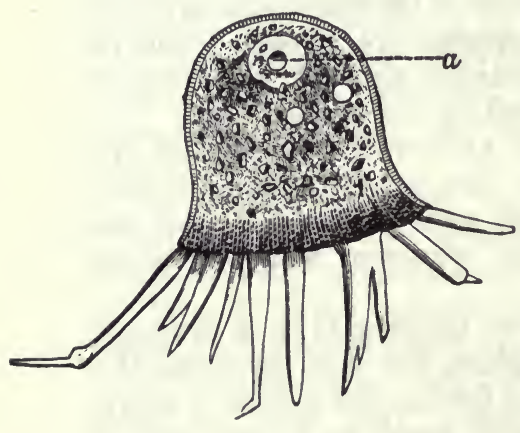

Fig. 19.

Cochliopolium pellucidum, Hert. and Less. $a$, nucleus, surrounded by a halo of clear protoplasm free from the chromidial network. (After Lankester.)

entirely surrounds the body and is perforated by the pseudopodin the definite heterogametes are formed and conjugate (p. 77). ${ }^{1}$

Family Cochliopodidde. Tests usually thin and supple, with a flexible margin, shaped like a cap, limpet shell, or helmet. Pylome widely open.

The genera included in this family have close affinities with some of the Gymnamoebida. The shell is not perforated by the pseudopodia, but in Cochliopodium it often assumes many different shapes according to the conditions of the animal, and in some species usually attributed to the genus (C. actinophorum and C. digitatum) it described by Schaudinn, in which 
apertures being closed again when the pseudopodia are withdrawn. C'ochliopodium, Hert. and Less., then, is the connecting-link between the two orders. In Corycia, Dujardin, the test is supple and menbranous, but the pylome remains open. In Pseudochlamys, Clap. and Lach., the shell is shaped like that of a limpet, but is very flexible, and the margin of the pylome may in the retracted condition be inflected to form a shelf like the velum of a medusa. In Parmulina, Penard, the test is in the shape of a cup or bowl. In Hyalosphenia, Stein, the test is rigid except at its margin.

Family Diffudgitdat. Tests usually globular, or flaskshaped with a narrow pylome. Outer sheath of the test with hard plates, or with adherent foreign particles, or with both.

Difflugia, Leclerc, is a genus which exhibits a great many varieties of form, some of which are very common. The shell is usually flask-shaped, and consists of a tough double membrane to which various foreign bodies, such as diaton shells, sponge spicules, sandgrains, etc., are cemented. The pseudopodia are rarely more than two or three in number, digitiform and blunt, but sometimes frayed at the extremities. Some of the larger varieties are over $0.5 \mathrm{~mm}$. in length.

Centropyxis, Stein ( $=$ Echinopyxis, Clap. and Lach.), is related to Diffugia, but the shell is usually discoidal or oval, with the pylome excentric in position. It is covered irregularly with foreign particles, and sometimes exhibits
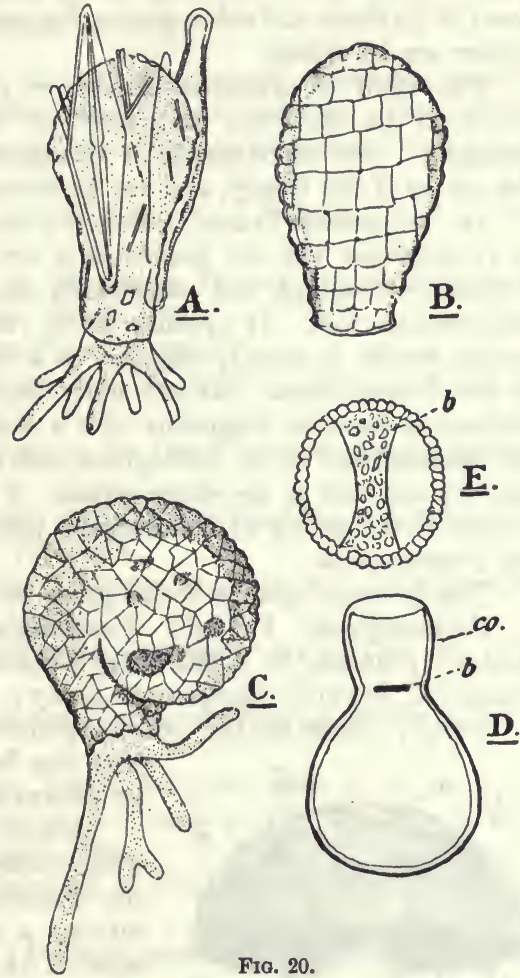

A, Diflugia pyriformis, Perty, with very large diatom shells attaclied to the theca. B, test of Quadrula symmetrica, Wallich. C, Lecquereusia spiralis, Ehr. D, diagram of test of Pontigulasia incisa, Rhumbler, showing the collar (co) and bridge (b). E, view of the bridge $(b)$ of Pontigulasia from above. (A-C after Leidy ; D, E after Penard.) two or three short spines.

Pontigulasia, Rhumbler, and Cucurbitella, Penard, are distinguished by the presence of a short collarette round the pylome. In Pontigulasia (Fig. 20, D and E) a broad flat bridge runs across the base of this collarette and divides the pylome into two apertures. In Lecquereusia, Schlumberger (Fig. 20, C), the shell is cornuate or slightly spirally twisted. The genera Quadrula, Nebela, and Heleopera form shells with siliceous plates and are not usually decorated at all with foreign particles. Quadrula, F. E. 
Schultze, is a common and widely distributed genus, with a shell of variable shape, but distinguished by its regular pavement-like arrangement of square or oblong plates (Fig. 20, B). Nebela, Leidy, is related to Quadrula, but the plates of the shell are round, oval, or even irregular in outline. In some species the shell is strengthened by adherent diatom shells. In all species of this genus particles of "fat" of a pale blue or yellow colour occur normally in the protoplasm. Similar particles also occur in Diffugia and other genera, but are not so constant or characteristic as they are in Nebela.

The shell of Heleopera, Leidy, is provided with square or oblong plates as in Quadrula, but they are usually irregularly or untidily arranged. The pseudopodia of this genus are more numerous than in the others of the family, and are sometimes slightly branched.

In Phryganella, Penarl, the shell is covered with adventitious particles, as in Difflugia, but the pseudopodia are more numerous, more delicate, frequently branched, and occasionally amalgamated at the base to form a membranous web. It appears to be related to Pseudodifflugia, Schlumberger, which is usually regarded as a nember of the Order Gromiidea, of the Foraminifera. As it is quite impossible to draw a definite line of distinction between organisms with a few fine blunt pseudopodia such as are characteristic of the Difflugiidae and those with filamentous branching pseudopodia such as are characteristic of the Gromiidea, there is a group of genera occupying an intermediate position between the Rhizopoda and the Foraminifera.

The principal genera of this group are :

Cryptodiffugia, Penard; Pseudodiffugia, ${ }^{1}$ Schlumb. ; Diaphorodon, 1 Archer; Platoum, ${ }^{1}$ F. E. Schultze; Clypeolina, Penard; Nadinella, Penard; Frenzelina, Penard ; Campascus, ${ }^{1}$ Leidy ; Cyphoderia, ${ }^{1}$ Schlumb.

Family Arcellidae. Shells plano-convex in shape, marked by a

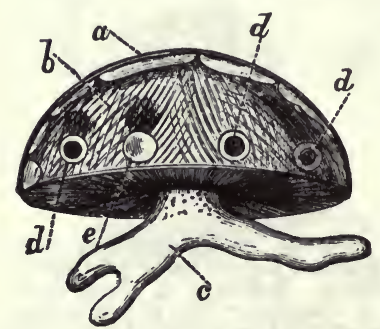

Fio. 21.

Arcella vulgaris, Ehr. $a$, shell: $b$, protoplasm within the shell; $c$, lobose psendopodia; $e$, , one of the
unarginal vacuoles; $d, d$, nuclei surrounded by a halo of clear proto. plasm. (After Lankester.) very fine hexagonal pattern, not supported by adventitious particles.

Arcella, Ehr. This is a common and widely distributed genus. The shells of the common species $A$. vulgaris vary from $80-140 \mu$ in diameter, and like those of most of the species of Arcella are characterised by their brown colour. The flattened side of the shell is usually depressed and perforated at the centre by the pylome, which is less than one-third the diameter of the shell. From the pylome there project three or four, rarely more, digitate pseudopodia. Situated in the ectoplasm, and usually arranged in a circle round the pylome, there is often seen a series of vacuoles, which probably serve a hydrostatic function. They may fuse together to form a single large excentric vacuole, and this may collapse after the manner of a contractile vacuole. 
Arcella is common in bogs and stagnant water, but is occasionally found in clear running water.

Pyxidicula, Ehr., differs from Arcella in having a large gaping pylome. The surface of the shell is ornamented with numerous minute tubercles. 20-50 $\mu$. The genus is comparatively rare and little known.

\section{Literature.}

1. Awerinzew, S. Die Structur und die chemische Zusammensetzung der Geliäuse bei den Siisswasserrhizopoden. Arch. Prot. viii., 1906, p. 95.

2. Bott, $K$. Ueber die Fortpflanzung von Pelomyxa palustris. Arch. Prot. viii., 1906, 1. 120.

3. Bourne, A. (y. On l'elmnyxa viridis 11. sp. Q. J. Mier. Sei. xxxii., 1891, p. 357 .

4. Bütschli, O. Investigations on Microscopic loams and on Protoplasm. Translated by E. A. Minchin. Black, 1894.

5. - Untersuchungen über Structuren. 1898.

6. Calkins, G. N. Marine Protozoa from Woods Hole. U.S. Fish. Comm. Bull. 1901, p. 413.

7. - Evidences of a Sexual Cycle in the Life-History of Amocba protens. Arch. Prot. v., 1904, p. 1.

8. Frenzel, $J$. Untersuchungen über die mikroskopische Fauna Argentiniens. Arch. mikr. Anat. xxxviii., 1891, p. 1.

9. - Ueber einige merkwïrdige Protozoen Argentiniens. Zeitschr, wiss. Zool. liii., 1892, p. 334.

10. Goldschmidt, $R$. Die Chromidien bei Protozoen. Arch. Prot. v., 1904, p. 126.

11. Hertwig, $R$. Ueber Encystirung und Kernvermehrung bei Arcella vulgaris. Fest. Kupffer, 1899.

12. Ijima. New Rhizopod of Man. Annot. Zool. Jap. 1898, p. 85.

13. Jennings, H. S. Contributions to the Study of the Behaviour of the Lower Organisms. Washington, 1904.

14. - The Movements and Reactions of Amoeba. Biol. Centralbl. xxv., 1905, p. 92.

15. Leyden, E., and Schaudinn, F. Leydenia gemmipara. S.-B. Akad. Berlin, vi., 1896.

16. Martini. N. Beobachtungen an Arcella vulgaris. Zeitschr. wiss. Zool. lxxix., 1905, p. 574.

17. Mesnil, Félix. Chromidies et questions connexes. Bull. Inst. Pasteur, iii., 1905, p. 313.

18. Neresheimer, $E$. Ueber vegetative Kernveränderungen bei Amocbr. Arch. Prot. vi., 1905, 1. 147.

19. Penard, $E$. Faune rhizopodiquè du bassin de Léman. 1902.

20. - Amibes it pellicule. Arch. Prot. vi., 1905, p. 296.

21. Poteat, $W$. Leidy's genus Ouramoeba. Science, viii., 1898, p. 778 .

22. Rhumbler, L. Beiträge zur Kenntniss der Rhizopoden. Zeitschr. wiss. Zool. lii., 1891 ; and same journal, lxi., 1896.

23. — Zur Theorie der Oberflächenkräfte der Amoeben. Zeitschr. wiss. Zool. lxxxiii., 1905, p. 1. 
24. Schaudinn, F. Ueber die Theilung von Amocba binucleata. S.-B. Ges. Naturf. Berlin, 1895, p. 130.

25. Ueber den Zengungskreis von Paramoeba eilhardi. S.-B. Ak. Berlin, 1896, 1. 31.

26. Untersuchungen uiber den Generationsweclisel von Trichosphaerium sieboldi. Anliang z. d. Abl. Ak. Berlin, 1899.

27. - Untersuchungen iiber die Fortpflanzung einiger Rhizopoden. Arb. kais. Gesundheitsamte, xix., 1903, p. 547.

2 - - Neuere Forschungen iiber die Befruchtung bei Protozoen. Verh. deutsch. Zool. Ges., 1905, 1. 16.

2. ichcel, C. Beiträge zur Fortpflanzung der Amocben. liest. Kupffer, 1899, p. 569.

30. Schubotz, H. Beiträge zur Kenntniss der Amocba blattae und Amoeba protcus. Arch. Prot. vi., 1905, p. 1.

31. S'tolx, A. Ueber die Teilung des Protoplasmus in melırkernigen Zustande. Arch. Entw. Mech. xix. p. 631.

32. — Plasmodiogonie. Arch. Entw. Mecl. xxi., 1905, p. 111.

33. Vahlkampf, E. Beiträge zur Biologie und Entwickelungsgeschichte von Amoeba limax. Arch. Prot. v., 1905, p. 167.

34. Veley, $V . H$. A Further Study of Pelomyxa. J. Linn. Soc. Zool. xxix., 1905, p. 374.

35. Zuclzer, M. Beiträge zur Kenntniss von Diffugia urceolata, Carter. Arch. Prot. iv., 1904, p. 240.

Some of the more important recent papers on the parasitic Amoebae and Amocliasis.

36. Schaudiun, F. (No. 27.)

This paper contains the most important but unillustrated account of the life-listoiy of Endamocba coli and Endamocba histolytica.

37. Schubcrg, A. Die parasitische Amöben des menschlichen Darmes. Kritische Uebersicht. Centrbl. Bakter. xiii., 1893, pp. 598, 654, and 701.

These papers contain a critical account of the literature of Amoebiasis ul) to the year 1893.

38. Casagrandi, Q., and Barbagallo, B. Entamocba hominis s. Amoeba coli, Lösch. Ann. d'Igiene sperimentale, v., 1897, fasc. i.

This paper contains a full account of Endamocba coli and its occurrence.

39. Jürgens. Zur Kenntniss der Darmamöben und der Amöben-Enteritis. Veröff. a. d. Gebiete Militärsanitätswesens, 1902, Heft 20, p. 110.

This paper contains a good account of Endamocba histolytica.

40. Castellani, A. Protozoa in Human Faeces. Centralbl. Bacter. xxxviii., 1905, p. 66.

41. Craig, C. F. A New Intestinal Parasite of Man, Paramocba hominis. Amer. J. Med. Sci. cxxxii., 1906, p. 214.

42. Dopter, $C$. Sur quelques points relatifs à l'action pathogène de l'Amibe dysenterique. Ann. Inst. Pasteur, xix., 1905, p. 417.

43. Lesage, $A$. Culture de l'Amibe de la dysenterie des pays chauds. Ann. Inst. Pasteur, xix., 1905, p. 9. 
44. Mfugliston, T. C., and Freer, G. D. An Undescribed Form of Ulceration of the Large Intestine, probably of Amoebic Origin. J. Trop. Medicine, viii., 1905 , p. 113.

45. Musgrave, W. E., and Clegg, M. J. Amoebas: their Cultivation and Etiological Significance. J. Inf. Diseases, ii., 1905, p. 334, and Publ. Bureau Govt. Lab. Manila, xviii., 1905, p. 5.

References to the general treatises of Bütschli, Braun, Calkins, Cash, Doflein, Hartog, and Lang will be found on p. 13.

Note. - In a recent paper W. Elpatiewsky (Arch. Prot. x., 1907, p. 441) has shown that Arcella produces small amoeboid gametes (megamoebae and micramoebae) which conjugate and form a zygote. 


\section{THE PROTOZOA (continued)}

\section{SECTION E. -THE' RADIOLARIA ${ }^{1}$}

The Radiolaria are purely marine Gymnomyxa, specialised for pelagic life. The body is usually spherical or conical, and emits radiating thread-like pseudopodia. The cytoplasm is subdivided by a perforated membranous "central capsule" into a central mass and a voluminous mantle. The nucleus, which may be single or multiple, is confined to the intracapsular region, which is also the seat of reproductive changes, the extracapsular mantle being concerned with flotation, feeding, stimulation, and excretion. A siliceous skeleton is usually present, and may take the form of spicules, shells, and tubes in a variety of delicate and exquisite constructions. In one division (Acantharia) the skeleton consists, so far as is known, of strontium sulphate. In most Radiolaria peculiar nucleated yellow corpuscles are found in abundance. They are regarded either as "symbiotic algae" or as Peridinians. Multiplication by fission is known in a few cases; more commonly reproduction by sporeformation has been observed.

\section{Description of Thalassicolla.}

As an introduction to the description of the class the following account of Thalassicolla has been drawn up.

Thalassicolla is a spherical gelatinous Protozoon from 3-5 mm. in diameter. In the warmer waters of the great oceans it occurs in vast swarms that float passively at the surface but also descend into deeper water during the reproductive phase. It ranges for some forty degrees of latitude on either side of the equator, diminishing in numbers towards these limits. It is abundant in the Faroe Channel (Wolfenden, Fowler), and a stray specimen is now and then recorded from our coasts (Delap [40]).

Thalassicolla consists of two parts - a central or medullary region and a thick outer or cortical layer. The two are separated by the central capsule.

The intracapsular mass consists of a large centrally placed nucleus embedded in cytoplasm, heavily laden with concretions,

1 By F. W. Gamble, D.Sc., F.R.S., Manchester University. 
coloured fat, and reserve products. The extracapsular cytoplasm is composed of-(1) a thin, black, fatty assimilative layer or matrix immediately outside the central capsule; (2) a frothy mass of mucilaginous and vacuolated substances secreted by interstitial cytoplasm and forming the so-called "calymma"; and (3) of fine radiating pseudopodia which arise from the matrix and extend freely into the water beyond the gelatinous bubbly layer. The wall of the central capsule is perforated by minute, evenly dis-

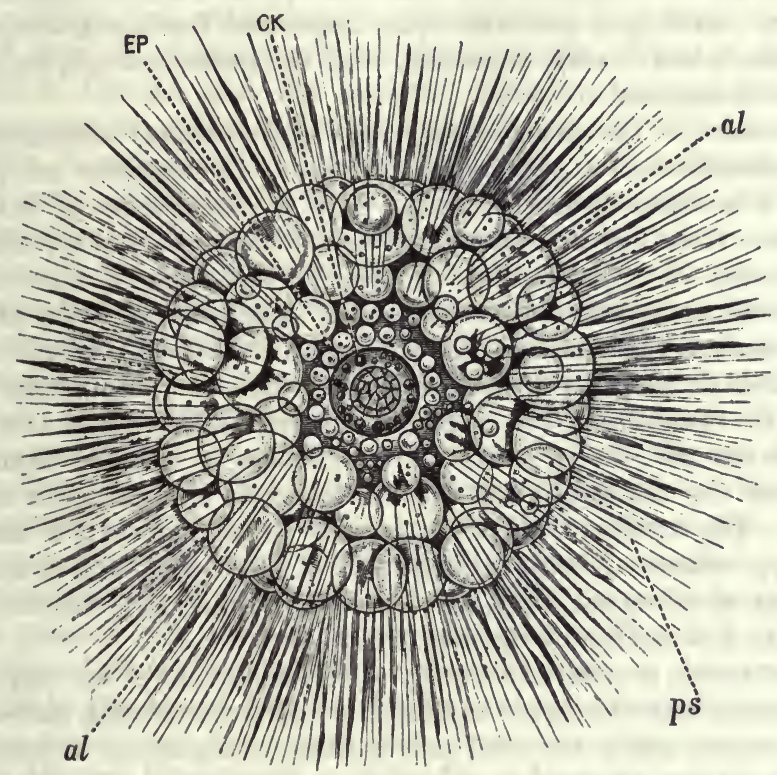

Fio. 1.

Thalassicolla (Thalassophysa) pelagica, Haeckel. $\times 25$. CK, central capsule ; EP, extracajsular protoplasm; al, alveoli, carbonic acid-holding vacuoles in the mucilaginous calyınma secreted by the protoplasmic network; $p s$, pseudopodia. The minute unlettered dots are the "yellow cells." (After Lankester.)

tributed pores, and through these the intra- and extracapsular cytoplasm are continuous.

If the central capsule is shaken out of its calymmal covering and kept under suitable conditions, its contents are capable of regenerating the extracapsular cytoplasm within a week (Verworn [14]). The first sign of this process is the protrusion of new radial pseudopodia, which are completed in twelve hours. The basal ends of these processes form or secrete a layer - the matrix-that invests the central capsule, and their radial extensions secrete the calymma. Finally, vacuoles make their appearance in the jelly and the matrix becomes pigmented. In short, the extracapsular 
protoplasm and its secretions are the product of intracapsular activity. The extracapsular cytoplasm, on the other hand, has no such regenerative power. When detached from the capsule it loses its form, the pseudopodia contract, the vacuoles burst, and the plasma undergoes granular degeneration. For this and other reasons we may speak of the extracapsular cytoplasm as the ectoplasm, and the intracapsular plasma as the endoplasm; for although the pseudopodia are common to both and interconnect them, yet the mass of the calymma is a secretion specialised for contact with the outer world, and performs other important functions, whilst the endoplasm is less directly concerned with the immediate physiological needs of the animal.

Bionomics. - The most remarkable physiological characteristic of Thalassicolla is its paucity of reaction. It possesses no power of active movement, and responds only to two forms of external stimulus - vibration and heat; and to one internal agency, namely, the stimulus of reproduction. Under the influence of wave-action Thalassicollu sinks till a calm stratum is reached, and then after a time ascends to the surface. Towards small variations of temperature it remains as inert as toward all conditions of illumination that have so far been tried; but a long-continued application of temperatures above $30^{\circ} \mathrm{C}$. or below $2^{\circ} \mathrm{C}$. induces a descent from the surface of the sea-water, and this is followed by the death of the animal. The onset of maturity is also correlated with a descent into deep water. During the nutritive phase and under normal variations of vibration, heat, and light, the station of Thalassicolla is at or near the surface of the sea.

This station is ensured for it by the development of the calymma. The mass of this veil is made up of a mucilaginous secretion containing fluid-vacuoles, and is enclosed in a delicate cytoplasmic investment, the quantitative proportion of which is in minimal relation to the bulk of its secretions and vacuolar fluid. By careful observation, weighings, and experiment, Brandt (24) has shown that the vertical movement of Thalassicolla is due to the formation and expulsion of vacuolar fluid. The hydrostatical requirements of the case demand that, for flotation at the surface, the density of this fluid should be that of water saturated with carbonic acid. As the physiological probability is in favour of this conclusion, we may accept Brandt's view as in all likelihood correct. Assuming this, then, the explanation of passive descent and ascent is easy. In calm weather and through a considerable range of temperature the interchange of fluid between the vacuole and the sea is gradual, and the slight wave-motion reinforces the calymma by acting as a stimulant. Thus we may assume the balance of loss and gain, and with it the surface position, are maintained. But the movements of larger or more frequent waves, or the extremes of experimental 
temperature, cause contraction of the calymmal plasma. The pseudopodia are withdrawn, the vacuoles burst, and the animal descends until the calmer zone enables it to reform its calymma and recharge its vacuoles, upon which it ascends. No "contractile vacuoles" are present, but their place is taken by these fluid-spaces in the calymma.

Food.-The food of Thalassicolla consists of Copepods, Diatoms, Infusoria, and probably also of Peridiniae. These organisms adhere to the surface of the Radiolaria by contact with its sticky pseudopodia. They are subsequently enfolded by a plasmic web and carried into the deeper part of the calymma. Here a digestive vacuole is formed, and the ingested organism becomes converted into a granular mass, which is disseminated, by division of the digestive vacuole, throughout the ectoplasm. An accumulation of débris may sometimes be found in the denser layer enveloping the central capsule, and there is little doubt that the products of digestion do not stop here but are carried into the endoplasm, for it is known that a streaming movement occurs along the pseudopodia that connect the inner and outer cytoplasm through pores in the capsular wall. Once inside the capsule, the food material is probably synthesised into the fatty or proteid masses that constitute reserves. The endoplasmic globules of fat are usually coloured with a pigment that varies according to the species of Thalassicolla under consideration. The other reserves take a concretionary form and recall starch grains in their stratified composition, though not in their reactions. They lie in vacuoles filled with a proteid, and are still imperfectly known (Fig. 2, A, Conc.).

Yellow Cells.-The ingestion of solid food is, however, not essential to the life of Thalassicolla for at least several months. If kept in water that has been taken from the open sea and completely filtered, Thalassicolla will live for at least six months without showing retrogressive changes beyond a shrinkage of calymmal volume. Brandt, who has carried out experimental studies on these organisms for many years, states (24) that if comparable batches are maintained in such filtered water in darkness and in light, the illuminated ones alone survive. He infers that Thalassicolla under these conditions lives upon food which is in some way elaborated under the influence of light; and in point of fact such a substance-starch-does exist in the ectoplasm. It occurs both free in the capsular layer and imbedded in the substance of certain corpuscles which are scattered through the calymma and are known as the "yellow cells." The significance of these cells or "zooxanthellae" is, in Brandt's view, a nutritive one.

That these bodies are independent organisms living in association with Thalassicolla and are not part of it was proved by Cienkowski (6). They are spherical structures $.015 \mathrm{~mm}$. in diameter, and consist of 
a cellulose wall, two chloroplasts marked by diatomin or an allied pigment, a pyrenoid, starch of hollow and solid varieties, and a nucleus. During the life of their host the zooxanthellae multiply by transverse fission. After its death they pass into a "palmella state" characterised by a mucilaginous jelly, and from this they often escape as active biflagellated zoospores.

Such zooxanthellae are frequent though not constantly present in Thalassicolla. In T. nucleata they may be plentiful, scarce, or absent. In most species they occur unfailingly; sometimes in the outermost jelly, sometimes in radial masses throughout the calymma, or aggregated round the capsule, but never within it. The adaptation of their host to surface life meets the requirements of the yellow cells for light, oxygen, and no doubt other unascertained demands, with the result that the association has been regarded as one of mutual advantage, as a case of symbiosis.

The more recent work of Famintzin (13) has, however, tended to diminish the importance of the part which, according to Brandt, is played by the abundant starch of the yellow cells in nourishing their host. According to the later writer, the nutrition of Thalassicolla is mainly derived from ingested organisms, and is only aided by the yellow cells in as far as these bodies are digested by the ectoplasm. It is probable, though exact demonstration is as yet wanting, that in some diffusible state exchange of material does take place from zooxanthella to host without involving the death and digestion of the former. Such a relation, however, does not explain the presence of the yellow cells in Radiolaria. ${ }^{1}$

Respiration.-The researches of Vernon (22) have shown that gelatinous or mucilaginous pelagic animals have a high rate of destructive metabolism, and that the amount of oxygen absorbed per unit of dry body-weight is further increased in the smaller animals as compared with the larger members of the same group, and in those of warmer seas as against their cold-water relatives. The maximum relative absorption of oxygen amongst invertebrate planktonic animals is reached, according to Vernon, in the Radiolaria. Collozoum, a near ally of Thalassicolla, has the highest coefficient of all invertebrates, equivalent to forty times that of the frog; and although it is desirable to have further evidence before accepting this startlingly high figure, yet the evidence of other pelagic forms points unmistakably to a very large consumption of oxygen.

The recent work of Pütter (43) has emphasised the singular nature of Protozoon respiration. It has long been known that many of these organisms can live for a time as anaërobes, and it now appears that intramolecular respiration obtains in a great number of cases and to an unexpected extent. Fresh energy is set

${ }^{1}$ For a discussion of the origin of the association see below, p. 129. 
free during the decomposition of reserve materials, and so long as the waste products evolved in this process are removed, respiration will continue in a medium deprived of free oxygen. Such a view enables us to consider the reserve materials of Radiolaria as of respiratory as well as of nutritive significance. It is not improbable that the respiration of the endoplasm (in which these fatty and stratified reserves occur) is of a different character from the more violent exchange which seems to occur in the ectoplasm.

In connection with destructive metabolism we may summarise our view on the nature of excretory processes in Thalassicolla. That carbonic acid and nitrogenous excreta are formed in abundance seems certain from the rapid destruction and regeneration of the calymma and its vacuoles, but there is no accumulation of excretory substances such as occur in most Rhizopods. It is suggested, on the basis of experiments with Turbellaria (Gamble and Keeble [41]), that this absence of excretory matter is due to the activity of the yellow cells, which are attracted to their host chemotactically and from which, by the uric acid or urea therein, they derive their nitrogen. In the same way such a view affords an explanation of the association of zooxanthellae with Radiolaria, and of the apparently concomitant absence of excretory granules. Additional proof of the correctness of this view lies in the fact that such granules occur massively and constantly in one division of the Radiolaria (the Phaeodaria or Tripylaria), and that in this division, and in this only, zooxanthellae are as constantly absent.

Reproduction.-In addition to multiplication by simple fission (25a), Thalassicolla has two true reproductive processes, which, however, never occur in the same individual. These processes concern the formation of spores, which are of two kinds, isospores and heterospores. A given Thalassicolla is, therefore, isosporous or heterosporous.

When the reproductive period ensues, the protoplasm and its contents undergo a metamorphosis, which results in the transformation of the endoplasm into a mass of flagellated spores, in the disintegration of the calymma, and the separation of the sporulating capsule from its envelope. The relatively heavy capsule descends to a depth of $300-400$ metres, its wall bursts, and its spores are liberated. In the case of isospores these bodies are of uniform shape and size (Fig. 2, D); in the case of heterospores (L, M) two varieties occur, of which the larger are not only twice the size of the smaller ones, but possess other distinctive characters which are given below.

The formation of isospores in Thalassicolla nucleata proceeds as follows (Brandt [26]). The nucleus and endoplasm undergo a series of changes. The chromatin, previously coiled up in a thick thread, becomes evenly granular, and the nucleoplasm acquires an 
almost homogeneous and doubly refractive character, and becomes irregular in shape as its membrane disappears. By what appears to be amitotic division the nucleus fragments into a large number of equivalent pieces, each of which behaves as an independent nucleus, and by further division these nuclei become disseminated through the endoplasm. Around each nucleus the cytoplasm condenses to form an ovoid mass, which is differentiated at the nuclear pole into two cilia. Meantime the reserve materials of the endoplasm become subdivided and apportioned, so that each isospore contains a few granules of fat and a crystalloid. These changes may be followed on the accompanying figures (Fig. 2, A-D).

The development of heterospores in Thalassicolla proceeds in a different manner and from distinct individuals. The first step is the formation of a nuclear figure. A clear achromatic vesicle (centrosome, Brandt, 1905) arises in the nucleus and becomes surrounded by granular radiations, upon which the thick bent chromatin threads arrange themselves as in Fig. 2, E. The centrosome now shifts towards the margin of the nucleus, and the more peripheral chromosomes become lumpy and slightly vacuolated. The nuclear wall softens, and through it, at one pole, pass the centrosome and a few apical chromatin granules. Subsequently the nuclear sap escapes over the entire periphery of the nuclcus, together with much of the granular nuclear matrix, into the surrounding endoplasm. The chromatin threads fragment and the fragments become associated with segregated masses of fine nuclear granules to form organised nuclei, which divide mitotically. During this process the nuclei are carried outwards in increasing numbers towards the wall of the central capsule, where they become arranged in columns, until almost the whole of the original nucleoplasm is used up. The most remarkable features of this organising process is that the developing nuclei are of two sizes, which are severally aggregated in the peripheral columns. Meantime the endoplasm and its reserves have been mobilised. The former is converted into cylinders around the mega- or micro-nuclei, and within these cylinders the fat and crystalloids become fragmented and distributed. Finally, by subdivision of these nucleated masses colonies of mega- and micro-spores arise. Both are biciliate, and in comparison with isospores minute, and divided by a groove into a reniform shape. The microspores are from 0.008 to $0.01 \mathrm{~mm}$. in length, the megaspores 0.016 to 0.017 . The microspores have a deeply staining granular nucleus and a cytoplasm free from inclusions except for one or two minute crystalloids. The megaspores, on the other hand, possess a nucleus poor in chromatin, and their cytoplasm is crowded with refringent corpuscles. Both forms of heterospore have the same ciliary mechanism (Fig. 2, L). From one point in the groove two long cilia arise, one of which works 


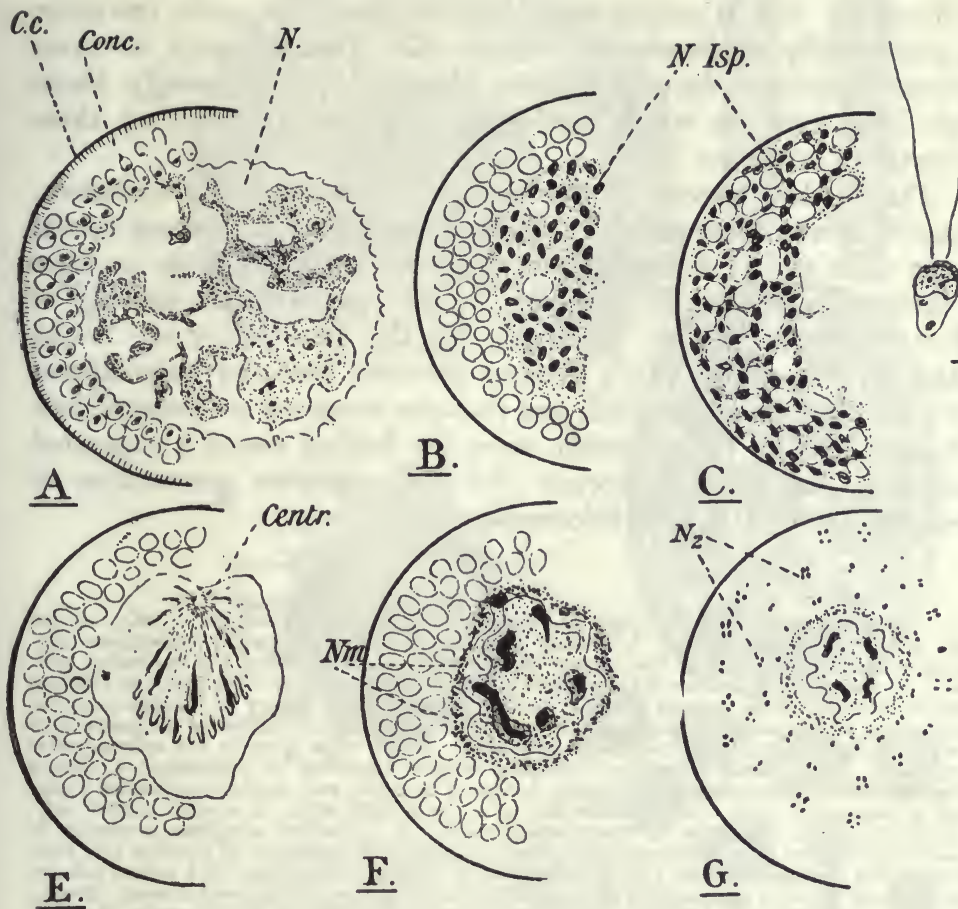

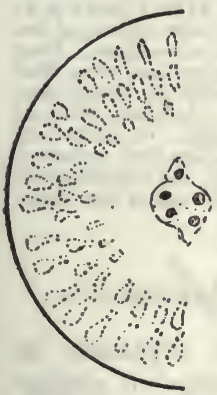

H.

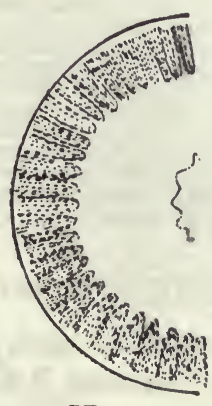

$\underline{\text { K. }}$

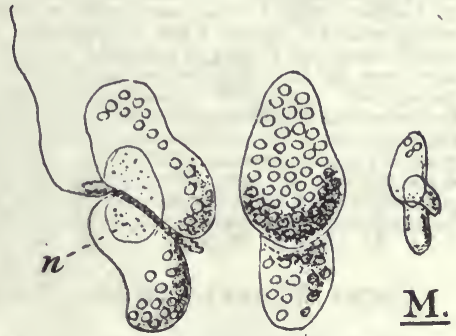

$\underline{I .}$

Fia. 2.

The development of isopores and heterospores in Thalassicolla uucleata. (After Brandt, 1905.) A-C, isospore-formation, $\times 100$. The large nucleus $(N)$ breaking up into spore nuclei $(N$. Isp). $\mathrm{D}$, an isospore $(\times 2000)$; Conc. stratitied concretions lying in proteid vacuoles. $\mathbf{E} \cdot \mathrm{K}$, hotero. spore-formation, $\times 100$. E, nuclear membrane collapsing. Nuclear figure and one intrauuclear centrosome. F, diffusion of nucleoplasm $(\mathrm{Nm})$ outwards. G, organisation of secondary nuclei $\left(N_{2}\right)$. $\quad \mathbf{H}$ and $\mathbf{K}$, segregation of these nuclei to form heterospore nuclei. $I_{1}$, megaspores. This figure shows the two flagella arranged like those of a Dinoflagellate. $\mathbf{M}$, microspores. L, M, $\times 1000$. 
horizontally and is coiled round the body of the spore, the other projects freely outwards and backwards. Consequently, as these minute structures dart or vibrate, they rotate unceasingly about their long axis, the whole mechanism and display recalling those of certain Peridiniae.

The further history of the iso- and heterospores is unknown. Brandt's recent attempts (26) to obtain conjugation between spores of the same and of different individuals have been as futile as those of earlier observers. If, however, we may judge by the analogy of other Protozoa, and in particular by the life-history of Trichosphaerium (Schaudinn [42]), we may presume that the heterospores are male and female gametes, and that the isospores are asexual individuals. But on this question, as on the further one of a suggested alternation between isosporous and heterosporous generations of Thalassicolla, we still lack information.

Fia. 3.

1, central capsule of Thalassicolla nucleata, Huxley, in radial section, $\times 100$; $a$, the large nucleus (Binnenblischen); $b$, proteid vacuoles of the intracapsular protoplasin containing concretions; $c$, wall of the capsule (membranous shell), showing the fine radial porecanals; $d$, chromatin substance of the nucleus. 2,3 , Collozoum inerme, J. Miiller, two different forms of colonies, of the natural size. 4, central capsule from a colony of Collozoum inerme, showing the intracapsular protoplasm and nuclei, broken up into a number of isospores, each of which encloses a crystal of strontium sulphate; $c$, yellow cells lying in the extracapsular protoplasin. 5, a small colony of Collozoum inerme, uragnified 25 diameters; $a$, alveoll (vacuoles) of the extracapsular protoplasm ; $b$, central capsules, each containing besides protoplasm a large oil-ylobule. 6-13, yellow cells of various Radiolaria. 6, normal yellow cell; 7,8 , division with formation of transverse septum; 9 , a modified condition according to Brandt; 10, division of a yellow cell into four; 11 , amoehoid condition of a yellow cell from the body of a tlead Sphaerozoon; 12, a similar cell in process of division; 13, a yellow cell the protoplasm of which is creeping ont of its cellulose envelope. 14, Heliosphrera inermis, Haeck., living example, $\times 400$; $a$, nucleus; $b$, central capsule; $c$, siliceous basket-work skeleton. 15, two isospores of Collozoum inerme, set free from sucli a central capsule as that drawn in 4; each contains a crystal $b$ and a nucleus $a$. 16, two heterospores of Collozoum inerme, of the second kind, viz. devoid of crystals; ancl of two sizes, a megaspore and a microspore. They have been set free from central capsules with contents of a different appearance from that drawn in 4. $a$, nucleus. 17 , Actinommn asterucauthion, Haeck., $\times 260$; one of the Peripylaria. Entire animal in optical section. a, nucleus ; l, wall of the central capsule ; innermost siliceous shell enclosed in the nucleus; $c^{1}$, middle shell lying within the central cajsule; $c^{2}$, outer shell lying in the extracapsular protoplasm. Four radial siliceous spines, holding the three spherical shells together, are seen. The radial fibrillation of the protoplasm and the fine extracapsular pseudopodia are to be noted. 1S, Amphilonche messanensis, Haeck., $\times 200$; one of the Acan. thometrida. Entire animal as seen living. (After Lankester.)

\section{Chief Modifications of Structure in the Radiolaria.}

The Radiolaria may be derived from such an organism as Thalassicolla by-(1) fission and the formation of a colony of similar or dimorphic individuals imbedded in a voluminous communal jelly (Sphaerozoa or polyzoic Radiolaria); (2) by differentiation of the openings of the central capsule from its evenly porose condition (Peripylaria) to a radially segregated oligo-porose type (Acantharia), to a single pore-plate at one pole of the now asymmetrical capsule (Monopylaria), or to a single main aperture and two lateral ones (Tripylaria); (3) by differentiation in the ectoplasm of skeletal spicules and shells of the most diverse forms, which only in the Acantharia invade the endoplasm. 

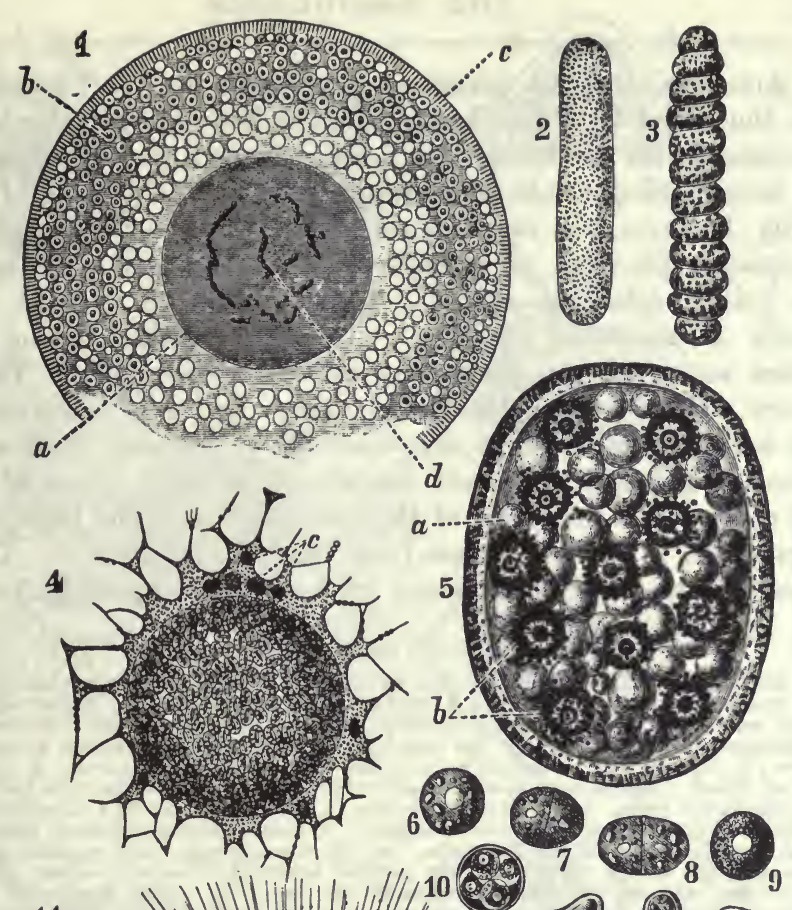

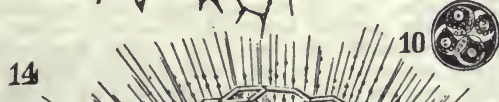

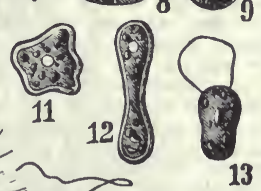

7 1 11 (1) 11

$6 \ldots-14012$

‥

$\approx$ ₹ $=0.015$

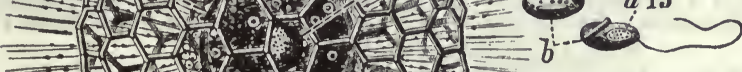

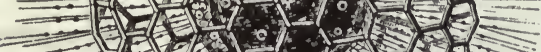

Fo (16)

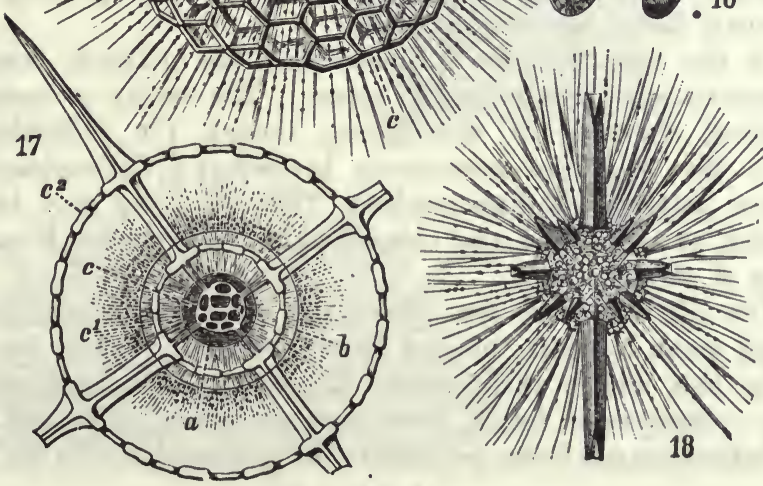

Fic. 3. 
Amongst the most primitive Radiolaria are the Physematiidae and the allied families Thalassicollidae, Thalassophysidae, etc. In all these forms the hydrostatic jelly is so well developed as to give the term Collodaria to the order formed by them. In the first family, however, the vacuoles elsewhere found in the ectoplasm are endoplastic products, no stratified nutritive concretions are found, and yellow cells are absent. The skeleton, if present, consists merely of scattered spicules. These organisms belong to the surface strata of the ocean and are phosphorescent. Their lifehistory falls into well-marked nutritive and reproductive phases. The early nutritive stage was erected by Haeckel into a special genus Actissa, which Brandt has shown to be a phase of growth that occurs in at least two of the five families. The later nutritive stage differs in few characters from that of Illalassicolla. The Physematiidae afterwards pass into an isosporous reproductive phase;

Fic. 4.

1, Lithocircus annularis, Ilertwig; one of the Monopylaria. Whole animal in the living state (optical section). $a$, nucleus; $b$, wall of the central cajsule; $c$, yellow cells; $d$, perforated area of the central capsule (Monopylaria). 2, Cistidium inermine, Hertwig; one of the Monopylaria. Living animal. An example of a Monopylarion destitute of skeleton. $a$, nucleus; $b$, capsule wall ; $c$, yellow cells in the extracapsular protoplasm. 3, Carpocanium diadema, Haeck. ; optical scetion of the beehive-shajell shell to show the form and position of the protoplasmic body. $a$, the tri-lobed nucleus; $b$, the siliceous shell; $c$, oil-globules; $d$, the perforate area (pore-plate) of the central capsulc. 4, (oelodendron gracillimum, Haeck. ; living animal, complete; one of the 'Tripylaria. $a$, the characteristic dark pignent (phaeodium) surrounding the central capsule $b$. The peculiar brauclied siliceous skeleton, consisting of hollow fibres, and the expancled psendopodia are seet. 5, central capsule of one of the Tripylaria, isolated, showing $a$, the uncleus; $b, c$, the inuer and the outer laminae of the capsule wall; $d$, the chief or polar aperture; $e, e$, the two secondary apertures. 6,7, Acanthometron Claparedei, Haeck. 7 shows the animal in ojtical section, so as to exhibit the cliaracteristic meeting of the spines at the central point as in all Acanthometrida; $a$, small nuclei ; $b$, a parasite (Amoebophrya); $c$, wall of the central capsule ; $d$, extracapsular jelly ; e, peculiar intracapsular yellow cells. 8, Spongosphaera streptacantha, Haeck. ; one of the Peripylaria. Siliceous skeleton not quite completely drawn on the riglit side. $a$, the spherical extracapsular shell (compare Fig. $3(17)$ ), supporting very large radial spines which are connected by a spongy network of siliceous tibres. 9. Aulosplucera elegantissima, Haeck.; one of the Phaeodaria. Half of the spherical siliceous skeleton. (Alter Lankester.)

the Thalassicollidae into either isosporous or heterosporous modes of reproduction; and the Thalassophysidae fragment suddenly into hundreds of minute pieces (see pp. 137-8), without passing, so far as is known, into a sporulating phase.

In the next division (Sphaerozoa) the polyzoic condition is characteristic of the nutritive phase. The colony or coenobium is spherical, elongate, or moniliform, though the individuals may retain the primitive homaxonic symmetry (Collosphaeridae) or become flattened (Sphaerozoidae). The skeleton may be absent, spicular, or spheroidal, and the scattered "nuclei" are homogeneous lumps of chromatin.

The life-history of the Sphaerozoa is still incompletely known, though much has been done by Brandt (1885) to follow it. According to this writer three kinds of sexual individuals or colonies occur:-isosporous forms, heterosporous forms produced directly, and heterosporous forms produced after gemmation. In the Sphaero- 

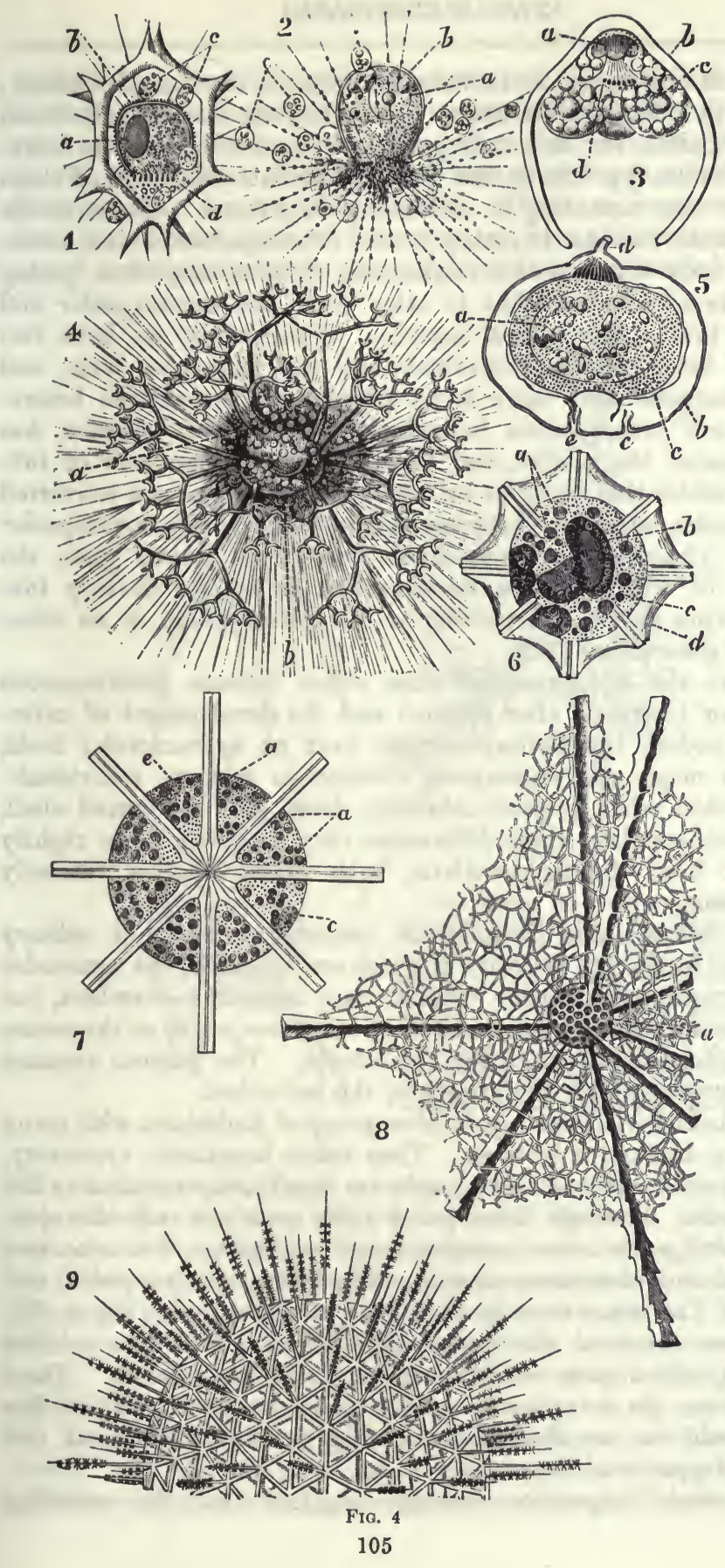
zoidae both megaspores and microspores arise in the same individual ; isospores in different individuals. Moreover, the asexual individuals are not all alike, but in certain genera at least some produce extracapsular bodies (pp. 138-9), and those individuals which bud off these structures are, according to Brandt, young forms. These fertile young forms become in many cases heterosporous - the extracapsular body forming the megaspores, the intracapsulum giving rise to the microspores-but in other cases the extracapsular bud develops into a new central capsule. Consequently we have two forms of heterosporous individuals and one isosporous form, and Brandt suggests that there is an alternation between the heterosporous and homosporous individuals. Famintrin, however, has reinvestigated the matter, and finds, in the vast numbers of fullgrown colonies that occur in autumn at Naples, some are converted into isospores, some into heterospores, and many have extracapsular bodies. These last colonies divide into small winter ones, the majority of which possess extracapsular buds and develop into heterosporous forms. According to Famintzin there is no alternation of generations (13).

Whilst the Sphaerozoidae thus either become heterosporous directly, or indirectly after division and the development of extracapsular bodies, the Collosphaeridae have no extracapsular buds, and their mega- and microspores develop in separate individuals. The skeleton when present takes the form of a perforated shell, but notwithstanding these differences they are held to be rightly separated from the Sphaerellaria, with which Haeckel formerly united them.

The Sphaerellaria include an immense number of solitary chambered forms, the majority of which are spherical, the remainder being elliptical or flattened. Radial bars unite the chambers, but these bars are wholly ectoplasmic, and are never joined at the centre of the endoplasm as in certain Acantharia. The nucleus remains single, but grows with the growth of the individual.

The Acantharia form a primitive group of Radiolaria with many interesting distinctive features. They retain homaxonic symmetry, but the pores of the central capsule are less closely set than in the Spumellaria. Through these pores there pass not only the cytoplasmic bridges between ectoplasm and endoplasm, but also two other radiating structures, namely, stiff pseudopodia (axopodia) and spicules. The latter meet in the centre of the capsule (Fig. 4 (7)), the former surround the centre and alternate with the spicules (Fig. 18), which pass outwards generally in five whorls. These emerge from the ectoplasmic surface at points through which five circles could be inscribed corresponding to the two tropical, two polar, and equatorial lines of the globe.

The whole disposition strongly suggests that the radiating 
spicules have developed by a hardening of the stiff fibre of certain alternate axopodia which formerly met at the centre of the endoplasm as in Heliozoa, to which group this order suggests other points of affinity. The peculiar nature of these spicules is the distinguishing feature of the order. They are composed, in the best investigated cases, of strontium sulphate (Bütschli, 1906), and not of a chitinoid organic acanthin-substance, as Haeckel supposed.
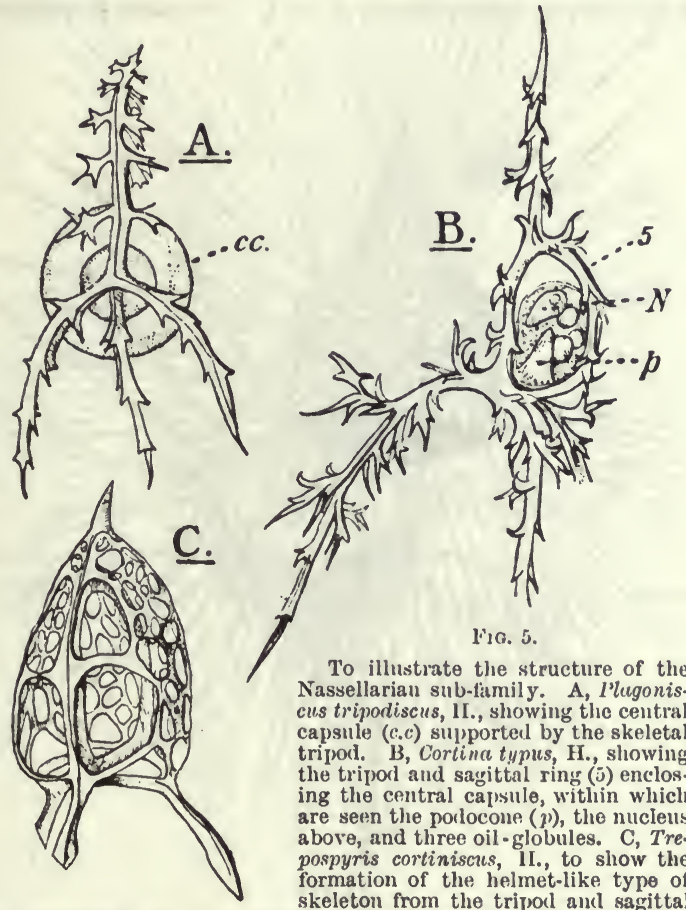

To illistrate the structure of the -tamily. A, l'ugoniscus tripodiscus, 1I., showing the central capsule (c.c) supported by the skeletal tripod. B, Cortina typus, H., showing the tripor and sagittal ring (5) enclos. ing the central cajsule, within which are seen the porlocone $(p)$, the nucleus above, and three oil-globules. C, Trepospyris cortiniscus, 1I., to show the formation of the helmet-like type of skeleton from the tripod and sagittal ring. (After Ilaeckel.)

The nucleus is a multiple structure, and the large body frequently mistaken for a nucleus (Fig. $4(6, b)$ ) is a Suctorian parasite. The Acantharia frequent the upper layers of the ocean (chiefly from the surface down to 300 metres), and are abundant in Arctic and Antarctic seas as well as in the intermediate zones. The yellow cells that in other Radiolaria are confined to the extracapsulum, occur almost exclusively within the central capsule in the Acantharia.

The Monopylaria or Nassellaria include an immense range of forms. In the simplest the central capsule is supported by a siliceous tripod or tetrad spicule, often accompanied by a sagittal ring. 
It contains a peculiar cone of doubtful significance (Fig. $5, \mathrm{~B}, p$ ). The ectoplasm streams out from the capsular pore-plate and forms a dense bubbly mass around this opening. From this point it passes as a thin layer around the capsule, so that the cytoplasm is asymmetrically distributed. These Radiolaria are, in fact, bilaterally symmetrical. Lateral outgrowths from the spicule or sagittal ring give rise to a helmet-like shell or "cephalis," in the upper part

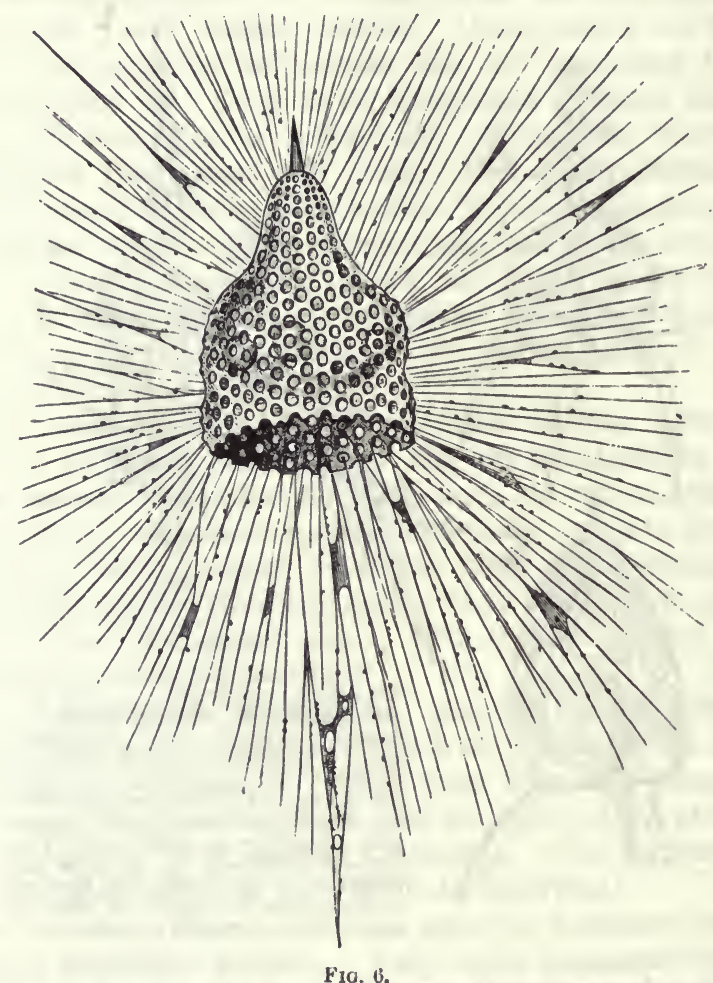

Eucyrtidium cranioides, Haeck., $\times 150$; one of the Monopylaria. Entire animal as seen in the living condition. The central capsule is hidden by the beehive-shaped siliceous shell within/which it is lodged.

of which the central capsule is lodged. The cephalis becomes voluminous and often constricted, producing a vast array of specific, skeletal variety, the whole of which is produced by modification of a single spicule. The nucleus, though often lobed, remains single. Spore-formation is known to occur, but no form of reproduction has been adequately investigated. The bionomics of the group are quite unknown.

The Tripylaria or Phaeodaria form another large group, most 
easily characterised by the brown, greenish-brown, or black accumulation of food material, débris, and resistant "phacodellae" that lie in the oral half of its ectoplasm; and they are also signalised by the mode of distribution of the capsular pores. In the majority of genera the endoplasm communicates with the ectoplasm only by a teat-like operculum and a pair of small lateral conical pores (the so-called astropyle and parapyles). In a few cases two astropyles occur, and in at least one genus (Atlanticella) only a single pore-plate is present. The skeleton varies greatly in structure

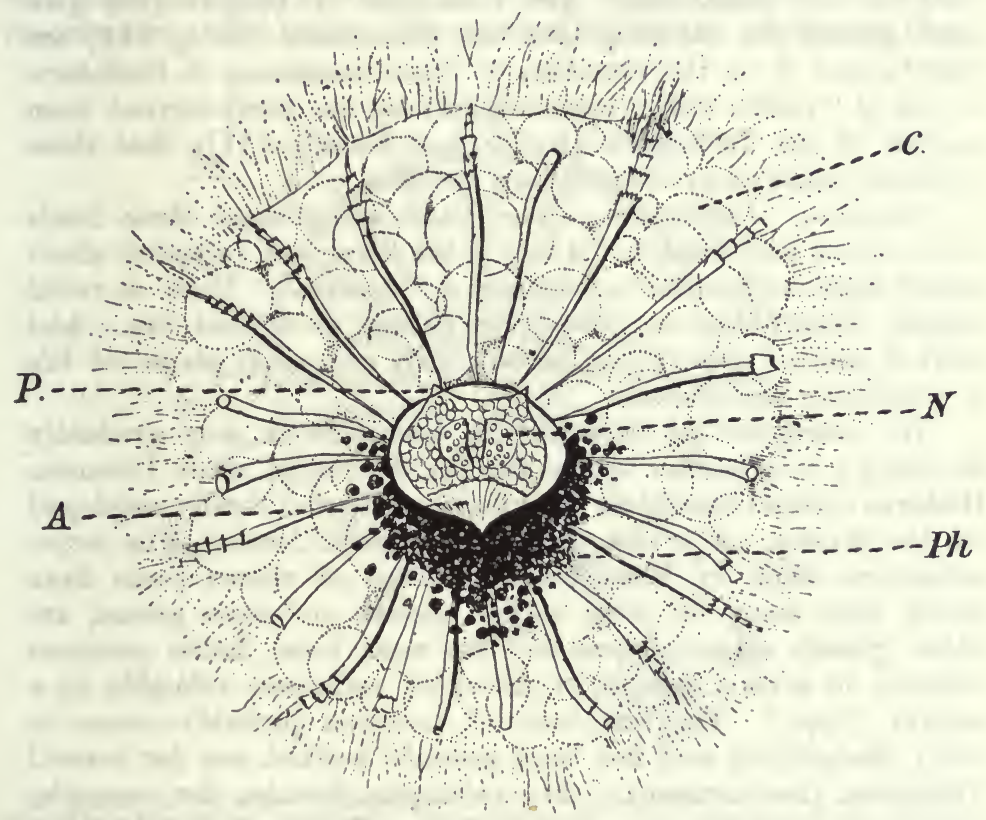

Fia. 7.

Aulactinium actinastrum, H. ; a member of the Phafodaria. (After Haeckel, slightly modified.) $A$, astropyle; $C$, calymma; $N$, double nucleus lying in the endoplasm; $P$, parapyle; $\mathrm{Ph}$, phaeodium.

and configuration. It is usually of a tubular nature, and the hollow cylinders are often subdivided by septa. The basis of these tubes, however, is formed by minute aciculate spicules which are surrounded by a gelatinous sheath, and between this sheath and the surrounding ectoplasmic matrix is a thin membrane, which first becomes silicified. This is followed by deposition of silica in the gelatinous sheath, and in this way complex spicules, often with candelabra-like appendages, are developed. A single or double perforated shell may be present, the surface of which has a peculiar porcellanous appearance and "diatomaceous" structure. In the 
most complex Phaeodaria this shell acquires a bivalvular form and carries many peculiar processes (Fig. 32).

The nucleus is a large, usually single structure, and undergoes a peculiar kind of mitosis accompanied by the formation of a great number of chromosomes. The development and nature of the spores is incompletely known. A characteristic feature of this order is the absence of the yellow cells that occur almost constantly in the other orders. This negative feature appears to be correlated with the presence of that remarkable and still imperfectly analysed complex, the phaeodium. The researches of Borgert (18) give some ground for thinking that the phaeodellae (see p. 119) are excreta, and if so, the retention of these substances in Radiolaria devoid of "yellow cells" lends support to the view, derived from a study of the Turbellaria (Keeble and Gamble [41]), that these symbiotic algae exert a depuratory function.

Vuriation: Dimorplism. - The Radiolaria present three kinds of structural modification. There is the divergent variation about one or more centres that constitutes a "species." There is racial somatic dimorphism in relation to pelagic or abyssal life. And there is gametic dimorphism both in early and adult stages of life in relation to reproduction.

The conception of "species" in Radiolaria is only gradually assuming a form similar to that held in the case of other Protozoa. Hitherto skeletal characters have been mainly and rigidly employed for the erection of a vast number of specific forms. The larger collections made by Plankton expeditions of recent years have shown that many of these earlier species, and even genera, are either growth stages of one and the same form, fission products common to several species, or divergent variations referable to a central "type." The first kind of variation probably occurs in every Radiolarian and has been recently worked out for several Tripylaria (Immermann). In Aulokleptes flosculus, for example, spicules of three kinds can be met with, each one of which was the basis of a separate species in Haeckel's classification. It has been shown, however, by Immermann that the spicules pass through two or more forms before arriving at their definitive stage, and may be arrested at an intermediate stage. Further knowledge of the development of the skeleton will undoubtedly tend to diminish the profusion of species that Hacckel has proposed. But it is not skeletal characters only that are subject to change during growth. Among the Collodaria, in which the spicules are a subordinate feature and in some families entirely absent, the early stages of growth differ so greatly from the later ones as to render their identification a difficult matter and one particularly liable to misinterpretation. Thus the genus Actissa, which Haeckel brought forward as the most primitive of all Radiolaria, has been shown by Brandt (25) to 
be an early stage in species of the two families Thalassophysidae and Physematiidae. Even the presence of developmental stages is not decisive proof that the fertile protoplast or coenobium in question is a final stage in the life-history, since in certain forms ${ }^{1}$ an early and variable reproductive stage is intercalated between the earliest phase and that of full growth. Fission introduces further complexities. The Acantharian genus Litholophus was founded on stages of growth or fission products belonging to other genera; and the division of the Collozoidae by fission leads to minute forms that might easily be mistaken for young stages, although they are reproductive individuals. We are thus led to the conclusion that

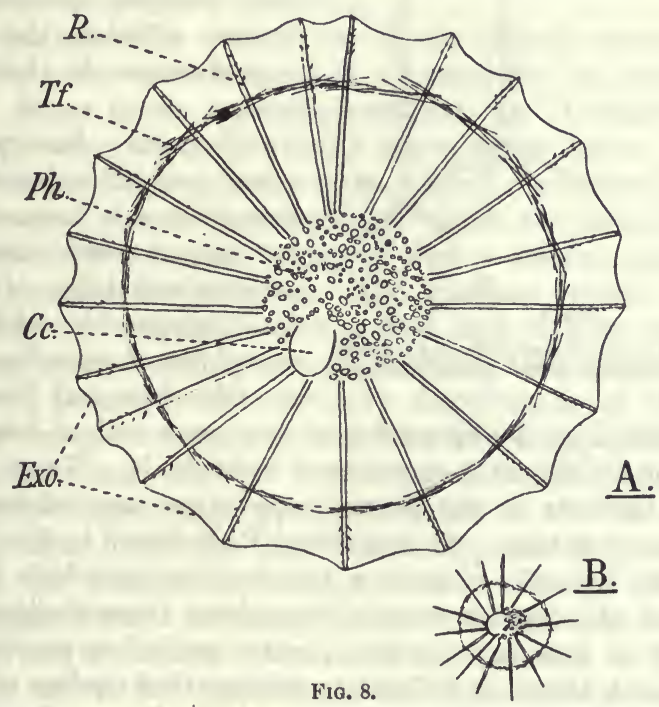

Racial dimorphism in Aulacantha scolymantha, $\times 26$. (After Häcker.) A, deep-sea form ; 13, pelagic form from Naples, 100 fathoms. C.c, central capsule; Exo, ectoplasin; $P h$, phaeodium; $R$, radial spicules; $T f$, tangential spicules.

a knowledge of the life-history is essential to the construction of a permanent classification, and that when this is obtained the species will be groups segregated about their several types.

The dimorphism of Radiolaria is of two kinds: somatic and gametic. Somatic dimorphism is at present known only in few instances. It consists in the development of a small race of a widely ranging species in warmer surface water, and of a large race (usually three times the size of the former) in cold and deep water. Associated with these differences of size there is structural diversity. The spicules of the small race are fewer and simpler, the ectoplasm they support is delicate and limp, often sagging between the

$$
1 \text { E.g. Collosphaera (Fig. 15, A). }
$$


siliceous appendages. The skeleton of the large race ends in more elaborate constructions, and stretches more tightly the tougher, thicker ectoplasm that covers the animal. Such racial dimorphism

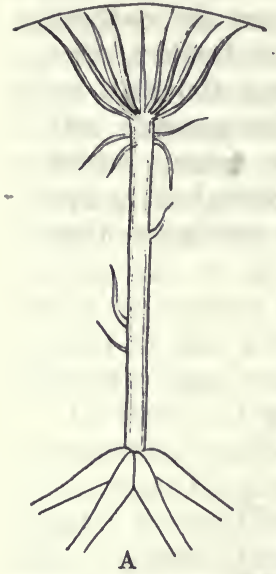

Fra. 9.

Radial spicules of A, abyssal form of Auloscena verticillutus; $\mathrm{B}$, pelagic form. (After Häcker.)

is known in Aulacantha scolymantha (Fig. 8), Circoporus sexfuscinus, in Auloscena verticillatus, and probably will be found more commonly when looked for. Both races are capable of reproduction, and it is improbable that they merge into one another, but it is not known whether the mode of reproduction is the same in both.

Gametic dimorphism is more general and perhaps universal, but is unaccompanied by any known diversity of somatic structure. It is therefore comparable with the dimorphism of such Foraminifera as Discorbina and Truncatulina, and is signalised by the formation of isospores and of heterospores in distinct and differently constituted individuals. These processes involve the contents of the central capsule and are followed by the death of the ectoplasm. An individual Radiolarian is therefore only a phase in the life-cycle of its race, but the changes which lead up to the formation of isospores are so distinct from those that precede the development of heterospores, and involve such deep-seated nuclear transformations, that it is difficult to believe that similar individuals of any one generation can give rise to both forms of spore. On this ground Brandt has been led to formulate the view that isosporous and heterosporous individuals of any one species belong to alternate generations. Direct evidence of this alternation has not been obtained, and therefore the case of the Radiolaria is on a very different footing from the observed alternation in Foraminifera.

Distribution: A, Vertical.- The recently published reports of the German Plankton expeditions, though not yet complete, enable us to picture the vertical distribution of the Radiolaria more accurately than was formerly possible. The older records were derived from surface townettings and from Ehrenberg's researches on Radiolarian deposits at varying depths. They represented the group as occurring at all depths, even on the sea-bottom, and as increasing in variety with depth. The more recent exploring expeditions give a very different result. From them it appears that 
in Atlantic and Antarctic waters-(1) the majority of Radiolaria occur not deeper than 400 metres; that the Collodaria are emphatically surface forms characteristic of the top stratum $(0-50 \mathrm{~m}$.); (2) that in the next stratum below this $(50-400)$ the great development of Radiolarian, as also of diatomaceous, life occurs. Here the majority of Acantharia, many Spumellaria, and many Phaeodaria, e.g. Challengeridae, occur; (3) that in the still deeper water, 400 1000 metres, a still richer Phaeodarian fauna and a few Acantharia are met with, and that beyond this a few remarkable forms range down to 5000 metres. The vertical distribution of the Nassellaria is not yet adequately known, but it probably follows much the same lines as that of the Phaeodaria.

$B$, Horizontal.-The distribution of the class is extremely wide, as is readily understood from their dispersal by the great oceanic currents. Some forms are panplanktonic, e.g. Aulacantha; some are bipolar; many are emphatically warm-water forms; others as characteristically follow cold currents. Such considerations enable us to understand the varying depths at which the same form may occur as its chosen current occupies now a deeper, now a more superficial position in the ocean. The greatest variety of species is met with in equatorial waters, and this fulness extends in diminishing variety north and south for some forty degrees. Then there follows, at least in the northerly direction, as in the case of many other pelagic orders, a barren zone, and finally Arctic waters show a Radiolarian fauna that is rich in individuals though poor in variety, and is apparently greatly inferior to that of Antarctic (Häcker). This mode of distribution explains the comparative poverty of the British Radiolarian fauna. Though the lack of research makes reserve necessary, it seems certain that these waters of the west and north-east coasts of Britain contain only a casual Thalassicolla and a few Acanthometrida, Sphaerellaria, and Phaeodaria, outliers and stragglers of the rich Gulf Stream fauna. The great northern host passes by the Faroes and off the Hebrides, as the lists, pp. 144-151, show, and in those waters the researches of Murray, Fowler, and Wolfenden have revealed a number of interesting forms.

The deposits formed by the accumulation of Radiolarian skeletons constitute a well-known element in the composition of littoral and deep-sea Globigerina ooze and of red clay. They make up certain of the clays, marls, and pumices found in the Miocene deposits of Barbadoes, the Nicobar Islands, and on both sides of the Mediterranean, as at Oran and Tripoli. Siliceous organic rocks of Palaeozoic and of Mesozoic age have been recently discovered in many parts of the world; and microscopical investigations of these rocks have revealed an unsuspected wealth of Radiolaria in them. From the Cambrian age onwards, however, the families and even 
genera appear identical with those now living. Pre-Cambrian Kadiolaria are still doubtful (Hinde [44]). The Sphaerellaria (Polycystina) and Nassellaria are the chief contributors, since the strontium skeletons of the Acantharia are readily soluble, and therefore are unknown in recent deposits or in a fossil state, and the hollow siliceous spicules of the Phaeodaria also appear incapable of resisting decomposition. Many skeletons formerly identified as Radiolarian (such as Dictyota and Mesoscena) are now referred to the Flagellata or to other orders, but the Nassellaria Cyrtoidea form the majority, the Sphaerellaria, Discoidea, and Sphaeroidea the minority, of Jurassic Radiolaria in quartzites and coprolites. In later deposits of Miocene ages this predominance is maintained, but the species found are identical with or closely akin to living forms.

Central Capsule.-The cytoplasm of Radiolaria is distinguished from that of other Protozoa by the great development, specialisation, and delimitation of its ectoplasm. The boundary between this peripheral layer and the central nucleated plasma is almost always a distinct one; and the few cases amongst the Acantharia and Sphaerozoa in which no limiting membrane can be traced, serve to show that this separation is the outcome of more primitive, undifferentiated conditions, which the Radiolaria display in early life, to which they revert during fission, and occasionally retain throughout life.

The central capsule is the sign of this plasmic differentiation, and the mark of a Radiolarian. It consists of a single, or in Phaeodaria of a double, porous membrane of either chitinoid or mucinoid nature. Usually the capsule is of such tenuity as to be visible only after the use of reagents, or, as in Thalassicolla, it may be comparatively thick and areolated by the growth of ridges on its inner surface (Hertwig).

The shape of the capsule is in general correlated with that of the configuration of the animal. In homaxonic Spumellaria and Acantharia it is spherical; in lenticular and discoid forms it is ellipsoidal. In the bilateral Nassellaria it is elongate, and in the Phaeodaria spheroidal; but in the recently discovered spherical Thalassothamnidae it is lobate or branched (Fig. 10). The consistency of the central capsule, however, is not that usually associated with chitinoid structures. It is capable of extension, and in the concentric Sphaeroidea and Discoidea it is lobate and may enclose the inner shells one after another. In the helmetshaped Nassellaria it throws out lobes through the basal plate of the shells. During the processes of fission and sporulation the central capsule in all Radiolaria becomes more or less completely dissolved to allow of the separation or escape of the endoplasmic contents. These phenomena show that the capsule is no per- 
manent excretion, but is composed of a substance capable of adaptation, by growth or dissolution, to changes in the endoplasm.

The walls of this structure are perforated by fine pseudopodia that connect the endoplasm with the exterior in the manner severally characteristic of the Peripylaria, Monopylaria, and Tripylaria (pp. 102-9).

The evenly distributed or segregated pores of the first group admit not only fine plasmic connections, but in Acantharia they also transmit axopodia and radial spicules.

The single pore-plate of the Monopylaria, which, according to

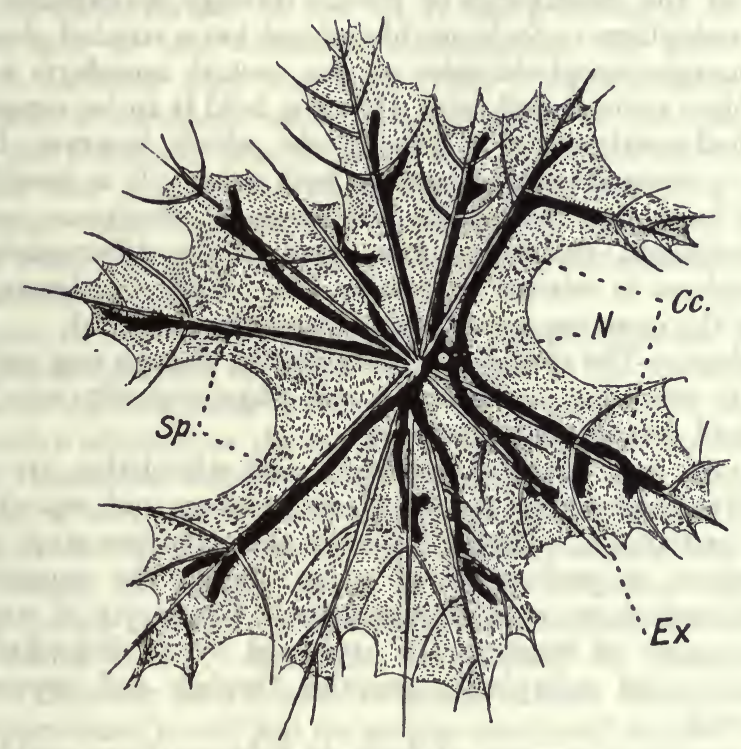

Fic. 10.

Cytocladus spinosus. $\times$ 10. (After Schröder [38].) One of the Peripylaria, to show the branched central capsule $(C . c)$, the radiate single spicule $(S p)$, and the voluminous ectoplasm supported by the spicular rays. It has been recently found off the coast of Japan.

Hertwig, consists of perforated, thickened rods of capsular membrane, is not thoroughly understood. In most Nassellaria the pores are, of course, confined to one plate-like extremity of the capsule, but they may be evenly distributed over the basal plate, confined to a peripheral zone, or to three circles, which in Tridictyopus project peripherally. Associated with this pore-plate is a peculiar, cone-like, fibrillated structure which projects inwards towards the nucleus (Fig. 5, B, p). According to Hertwig this cone is an invagination of the capsular membrane, and the fibrillae are continuations of those that pass through the pore-plate, on their way to join the endoplasm at the apex of the cone. Bütschli, however, 
is inclined to consider the cone as due to the coalescence of axopodia somewhat like those of the Acantharia (9, p. 439).

The central capsule of the Phaeodaria possesses well-marked characteristics in its double nature and the presence and structure of its main opening or astropyle and of its two lateral parapyles. The former consists of a teat-like operculum apparently striated on the inner side owing to the septate character of the subjacent endoplasm. The latter are made up of an inner bulb and an outer cone which opens on a prominence. The endoplasm under the bulb is also radially grouped, and in general it may be said, as evidence of the interchange of plasma through the capsular pores, that the endoplasm in their neighbourhood has a striated character.

The morphological character of the central capsule is a moot point. Most authors, following Hertwig, hold it to be comparable to the shell-membrane of a Thecamoeba, which, however, Dreyer considers is covered by ectoplasm on both sides. It is possible, on the other hand, that the capsule is a basement membrane peculiar to the Radiolaria, and is a consequence of the differentiation of their cytoplasm in relation to pelagic life. Until its development is studied the question cannot be satisfactorily answered.

Cytoplasm.- The cytoplasm of Radiolaria, though one and continuous, is separable anatomically and physiologically into intracapsular and extracapsular portions.

Flotation and dispersal, nutrition and stimulation are offices that devolve chiefly upon the ectoplasm; storage and reproduction upon the endoplasm. During the early and nutritive stage of life the ectoplasm is predominantly active, during the reproductive phase the endoplasm is solely operative. Continuity of structure and community of function are expressed by an interchange of protoplasmic and metaplastic granules through and beyond the capsular wall.

The ectoplasm consists of four chief layers from within outwards :- an assimilative zone of dense protoplasm around the capsule, a thick alveolar layer capable of secreting gelatinous and fluid spheres, an enveloping membrane guarding the animal from contact with its environment, and beyond this a fringe of radiating, contractile pseudopodia. This great development is primarily related to flotation. From Brandt's researches on the hydrostatic function of Radiolaria it is clear that the calymmal gelatinous spheres play the chief part of this office. These spheres he holds are viscous secretions of the ectoplasm and absorb water from without inwards. The specific gravity of the expressed fluid is, however, such as to point to water saturated with carbonic acid, and as we pass from the inner to the outer zones of this alveolar layer, the spheres are found to become more and more vacuolar, until at the surface they are so tense as to collapse at a touch. 
Brandt therefore considers that the outer pseudopodia upon contact with certain stimuli (wave-motion and heat) contract and transmit the stimulus to the subjacent alveolar protoplasm. This in turn contracts and the surface vacuoles collapse. When this process has been continued for a certain time the specific gravity of the animal is raised and a slow descent follows. Equilibrium is again established, the vacuoles are re-formed, and the animal rises again to the surface.

The calymmal spheres do not, however, monopolise the hydrostatic function. The flotation of Radiolaria is determined by extension of its surface as well as by the lowering of its specific gravity, and in this sustentative adaptation the outer pellicle and the skeleton play the chief rôle. The skeleton of the Acantharia is composed of a radiating series of tent-poles upon which the ectoplasm can be raised and tightened by the elastic filaments that pull up the baggy ectoplasm, which upon inflation by vacuolar water expands, and so raises the animal to a higher zone of water; or again contracts, followed by deflation and sinking of the whole mechanism.

Again, in Phaeodaria we have a still more elaborate skeleton, the appendicular parts of which are related to the formation and support of the ectoplastic membrane. In an impressive variety of sustentative adaptations the ectoplasm of Radiolaria deposits silicic acid or strontium sulphate; and the attempt now being made to trace a correlation between the variation of this support, the extent and thickness of the outer membrane, and the density and viscosity of varions tracts of water inhabited by widely varying forms, has already met with some success (Häcker [35]). Racial forms occur. Aulacantha scolymantha, for example, only attains a diameter of $2.3 \mathrm{~mm}$. in warm surface waters; its ectoplastic membrane is soft and its spicules small and simple; whereas in deep, cold water (400-1000 metres) it reaches $7 \mathrm{~mm}$. and consists of a much tougher envelope supported by more numerous spicules. Circoporus sexfuscinus and other Phaeodaria are also dimorphic and exhibit a similar differential relation to the surface and abyssal waters in which they occur.

The ectoplasm rarely contains assimilates or other inclusions. Oil-globules, however, occur in the large Collodaria; pigment (blue, black, brown, or red) in the Thalassicollidae, Sphaeroidea, Discoidea, and some Acantharia; and concretions (probably proteid) in some Thalassicollidae. Yellow cells are generally present in the ectoplasm, and the only large division in which they are unknown is that of the Phaeodaria. In the Acantharia, however, they occur almost constantly in the endoplasm. A further account of these cells is given below.

The myonemes are peculiar modifications of the basal ends of 
certain pseudopodia. They occur exclusively in the Acantharia Acanthometrida, and form circular groups of short, rod-like bodies clustered round each of the radial spicules (Fig. 11). Upon careful examination they are found to connect the ectoplasm with the pseudopodial covering of the spicule and to possess a high degree of contractility. Their form varies accordingly. When expanded the myonemes appear as homogeneous threads 006 to $.013 \mathrm{~mm}$. long. When contracted they not only become shorter (·012-02) and thicker, but exhibit in many cases a very distinct cross-striping. They are, in fact, muscular structures comparable

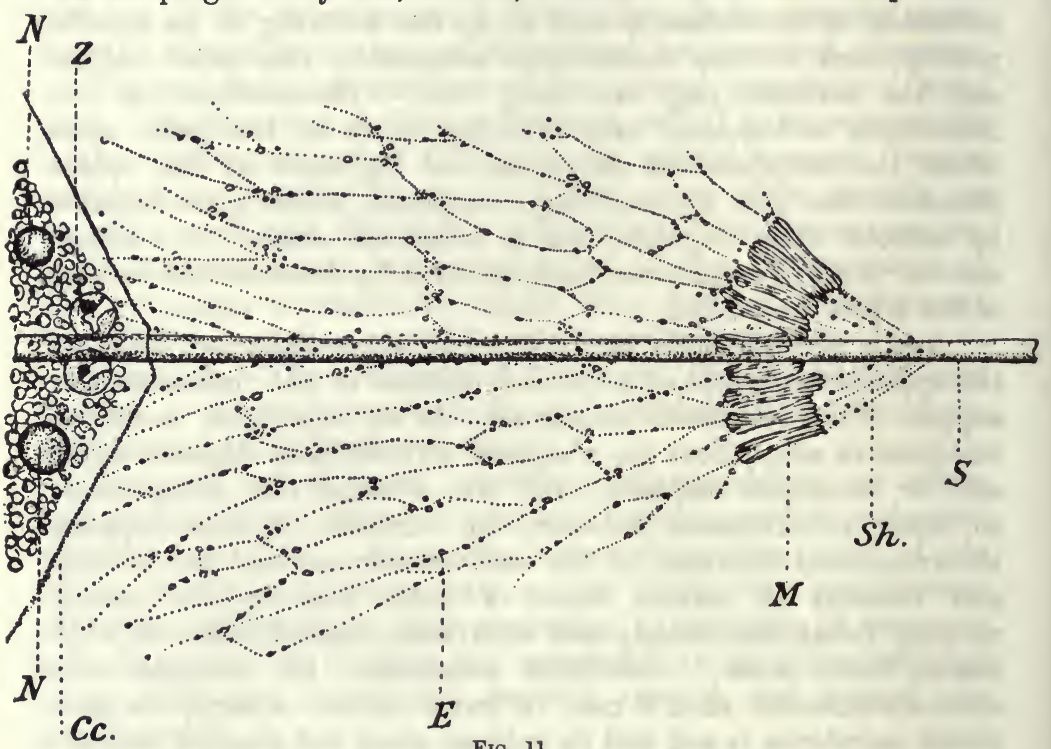

Fio. 11.

Portion of a living specimen of Acanthometron pellucidum, one of the Acantharia, $\times 900$ (after Schewiakoft), to show endoplasm and ectoplasin. The latter consists of vacuolated cytoplasm $(K)$ slung up to the rod $(S)$ by striated myonemes $(M)$, which are inserted into the sheath $(S h)$ around the rod. In the endoplasm two nuclei $(N)$ and zooxanthellae $(Z)$ are seen.

with the contractile fibrillae of Gregarines and Infusoria (Schewiakoff [33]), and they serve to raise or lower the hydrostatic, ectoplasmic apparatus of these Radiolaria, and so to facilitate their ascent or descent. :

Another cytoplasmic modification of the Acantharia may here be mentioned, namely, the axopodia. They consist of contractile pseudopodia that radiate from near the centre of the endoplasm to the periphery of the animal, and possess an axial fibre around which an unceasing cyclosis of granules takes place. These axopodia differ from the ordinary pseudopodia of the Acantharia not only in their deeper origin but also in their more limited 
numbers and cyclical arrangement, and they resemble the peculiar pseudopodia of such Heliozoa as Acunthocystis in all points except in not arising from a centrosome. The peculiar cytoplasmic threads that compose the so-called flagellum of the Discoidea are also in all probability of a similar nature. This flagellum consists of immobile pseudopodia fused into a tapering mass which projects freely at one point of the ectoplasm, and its component pseudopodia, unlike those of the surrounding calymma, can be traced almost to the centre of the endoplasm. They appear to spring from the nucleus.

A peculiar accumulation occurs in the extracapsulum of the Tripylaria, to which the name phaeodium is given. It consists of a greenish or brownish mass concentrated about the main aperture of the central capsule, but extends around the capsule for a third of its extent. So constant and characteristic is this coloured mass that the term Phaeodaria is frequently used as an alternative to Tripylaria.

The constituents of the phaeodium in Aulacantha are variouspartly extrinsic, partly intrinsic. To the former class belong diatoms and the débris of other vegetal organisms, small Radiolaria, and Crustacea. Most of these undoubtedly represent food material; the diatoms, however, may be symbiotic. The characteristic elements of the phaeodium are, however, the phaeodellae, which consist of spherical or ellipsoidal corpuscles which vary from less than $1 \mu$ to $20 \mu$ in diameter. These corpuscles occur singly or in masses. They appear homogeneous, granular, or striated, and vary in colour from a hyaline transparency through yellow-brown, light and dark green, to black. They may be free from inclusions or contain both blackish particles of varying size and refractive granular spheres and rods. Towards reagents they show great refractoriness, and do not give a uric acid reaction (Borgert).

About the nature of these phaeodellae, opinion has long been divided. Haeckel maintained that they were symbiotic algae, other zoologists that they were food particles. The recent researches of Borgert on Aulucantha have suggested another explanation. Borgert has pointed out the resemblance of certain granules formed in the endoplasm in the neighbourhood of the astropyle to these phaeodellae, and he regards these corpuscles as excretory products of the endoplasm that pass out through the capsule and accumulate in the surrounding ectoplasm. Recent work on the brilliantly coloured algoid structures in bathybial Challengeridae and Concharidae have shown that probably both assimilation and excretion are carried on in the phaeodium (36).

Endoplasm. - The endoplasm is the site of storage and of reproductive changes. It consists of a granular streaming cytoplasm often highly vacuolated, and stratified radially and 
concentrically. Imbedded in it are fatty and proteid reserves, pigment, crystalline structures, and one or more nuclei. Oilglobules are generally present in the Spumellaria and Nassellaria, fatty granules in the Phaeodaria. The fat may be colourless or coloured red, yellow, brown, or blue. The pigment is often closely associated with the oil-globules, and occurs in Thalassophysa on the peripheral surface of the globules. The crystalline

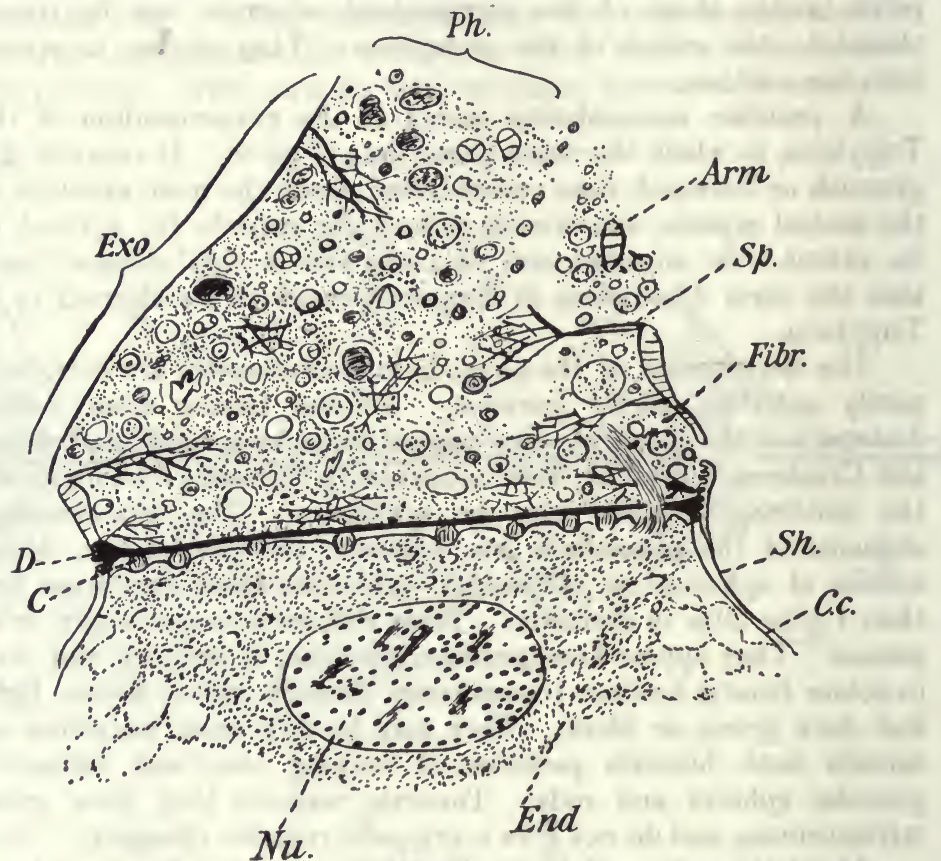

:Fua. 12.

-Portion of a section through l'lanktonetta atlantiva, Borg., one of the Pliaeodaria, to show the phaeodium $\left(P^{\prime} h\right)$ filling up the ectoplasm $(E x)_{0}, \times 30$. (After Fowler.) Cf. Fig. 29 for whole animal. The black horizontal line is the "diaphragm" or ectocapsular membrane, that is perforated by a single bundle of fibres $(F i b v)$, if not also by the snialler sinilar structures $(C)$. In the upper ectoplasmic half of the figure the complex phaeodium is seen together with branches $(S p)$ of the arms. In the lower half the delicate central cajsule (C.c.) surrounds thejendoplasin $(E u d)$ and nucleus $(N u)$, and is itself enclosed in a shell $(S /)$ that forms a float.

structures are of two kinds: (a) small whetstone-shaped bodies probably of albuminous nature; and $(b)$ large rhombic structures indestructible at a red heat. The latter, regarded by Brandt as excretory, are in all probability crystals of strontium sulphate (Bütschli). With this exception the contents of the endoplasm may be regarded as reserve material destined partly for the metabolism of the animal itself, but more especially for the provisioning of the spores, into which, the endoplasm breaks up.

Nucleus. - The nucleus of the Radiolaria is still very im- 
perfectly investigated, and the following statement can only be regarded as a provisional account of its coarser features. The two chief phases of life are signalised by distinctive characters in the nucleoplasm. In the vegetative phase it consists of a single large vesicular structure, or of a few derived from this by mitotic division, or of many equivalent, amitotically produced, small nuclei. In only a few cases are chromidia or other nuclear derivatives as yet known to occur in this phase (Collosphatera, Siphonosphaera, and Aulacantha), and there is no separation of somatic and germinal nucleoplasm. The Radiolaria are, in fact, homokaryota. Nevertheless, at the advent of the sporulating phase, the nucleus displays new characters. Either it becomes differentiated and divides into spore nuclei ; or it fragments partly into chromidia and plasma, which recombine to form the spore nuclei, and partly into a residue which perishes with the parental exuviae. In this process we can detect a certain analogy with the extrusion of nucleoplasm during the formation of the spores in the Heliozoa. But since the fate of the Radiolarian spores is unknown, a just comparison of the two cases is at present impossible.

The nucleus lies wholly in the endoplasm, and no chromidia or other nuclear products have yet been recognised in the extracapsulum; but the axopodia which radiate from the neighbourhood of the nucleus in certain Nassellaria, the similar fibrillae that run from the nucleus outwards to form the flagellum of the Discoidea, are indications of the paths along which the nucleus probably exerts its influence upon the ectoplasm, and vice versa. Further evidence of this perinuclear sphere of influence is found in the apparently porous character of the nuclear membrane (Physematium, I'halassolampe, and certain Sphaeroidea) and the radial arrangement of its peripheral plasma.

The characters of the nucleus vary according as to whether it is a single or multiple structure. The Collodaria, Sphaeroidea, Nassellaria, and Phaeodaria are generally mononuclear : the Sphaerozoa and Acantharia, polynuclear forms. In the first group the nucleus is vesicular and differentiated into membrane, sap, chromatin, and achromatin. In the second the nuclei are without a distinct membrane, and, in the vegetative stage, homogeneous; their origin from the spore or zygote nucleus has been traced in no single instance.

One or two special forms of nucleus may be referred to. Among the Phaeodaria the majority possess a nucleus such as that shown in Fig. 15, A, together, in some cases (Aulacantha scolymantha), with chromatin particles. scattered through the endoplasm. The Tuscaroridae, however, are peculiar in having (Figs. 13 and 30) an elongate nucleus, with a loop of chromatin enclosed by the nuclear sap. 
Among the recently discovered and reinvestigated Spumellarian families, Thalassothamnidae and Orosphaeridae, a totally new type of nucleus has been found (Schröder and Häcker). It

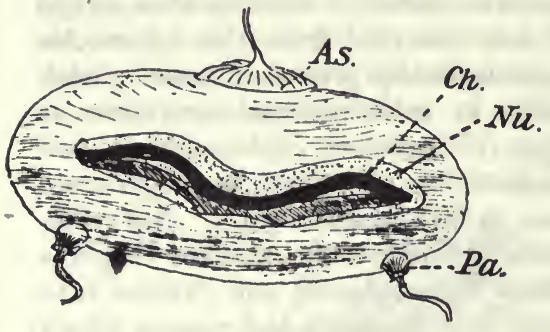

Fic. 13. consists of a discoid structure ( $1 \mathrm{~mm}$. diam.) enveloped by a crenate membrane, and is composed of a thin cortical substance and a central mass of very distinct nucleoplasm, the cortical and medullary substances being separated apparently by a membrane (Fig. 14). The central nucleoplasm contains segre-

The central capsule and nucleus of Tuscarora nationalis. (After Borgert.) As, the astropyle; $P a$, the two parapylae; $N u$, the nucleus with its chromatin band $(C h) . \times 45$.

tical layer, on the other hand, is densely chromatised. The most striking feature of this nucleus is perhaps the presence of lenticular bodies at intervals along the junction of its two component layers, or in one genus (Orosphaera) just outside it. These contain large compact lumps of chromatin imbedded in a less densely staining medium. In addition to this central nucleus, scattered, chromatin-like granules (Fig. 14, s) occur in the endoplasm, and in Orosphaera these peripheral granules are unmistakable nuclei of a simple character.

The shape and size of the nucleus often undergo considerable change during growth. It remains vesicular, large, and spherical,

\section{Fic. 14.}

Portion of a section throngh the hranched cential capsule of Thalassothamnus. (After Häcker.) The centre of the capsule with its nuclens $(N)$, endo. plasm, and inclusions are shown. The stratified concretions $(s)$ stain with haematoxylin, and are probably chromidial structures. In Orosphnera (a genus which, according to Hicker, is closely allied to 'Thulassothamnus) these peripheral nucleoplasmic structures are capable of division. The nucleus $(N)$ shows well the division into crenate membrane, peripheral chromatic layer, and the central, mainly achromatic substance in which groups of staining granules occur. Large lenticular bodies (l) of unknown significance occur also.

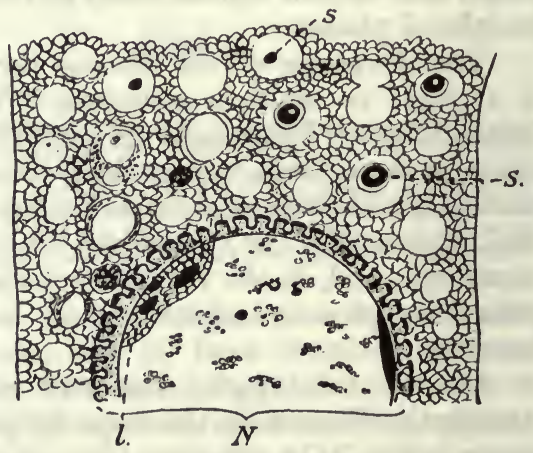

and more or less chromatised in the Thalassicollidac and Physematiidae; but in the Thalassophysidae it becomes papillose, elongate, and serpentiform, its plasma not only differentiates into inner and outer substances, but the spherical or thread-like 
chromatin accumulates at its periphery (Fig. 21, A, B). In the Sphaeroidea the nucleus becomes tubercular and follows the growth of the central capsule, as this encloses successive shells. In the simpler forms of Nassellaria the vesicular nucleus remains elliptical, but in the Cyrtoidea, in which it lies near the apex of the shell, it sends lobes (Fig. 4, 3) into the adjoining lappets.

The multinucleate Radiolaria offer other distinctive characters. In the Sphaerozoa each individual of the colony possesses a gradually increasing number of structureless, singly refracting nuclei, which multiply by direct fission, and have rather the appearance of nuclear fragments than of true nuclei. In the case of Collosphaerc and Siphonosphaera, scattered chromidia (not associated as far as is known with reproduction) occur as well. In Acantharia the multiple nuclei have apparently a membrane and nucleoli, the multinucleate condition is constant, and the distinction drawn by Haeckel between such forms and oligo- or mononucleate Acantharia is a mistaken one due to the presence of a parasitic Amoebophrya (Acinetaria), which was mistaken for a nucleus (Part I. p. 423, Fig. 90). More difficult to account for is the careful description by Hertwig of a temporary nuclear condition discovered by him in a species of Acanthometron and of Amplilonche. In the comparatively few nuclei of young specimens, Hertwig found that the membrane became invaginated on its peripheral side, whilst the massive nucleolus showed differentiation into two parts. The neck of the infolded membrane became radiately arranged, and its deeper portion creased into circular folds lying one over another. After a time these appearances vanished and the nuclei resumed their simple spherical form. The phenomenon may be one of internal budding.

The advent of sporulation is prefaced and accompanied by changes in the nucleus. These changes, however, are but imperfectly known (p. 139). Vesicular nuclei shrink, their membrane gives way, and the altered chromatin and enclosed nucleoplasm either flows out into the endoplasm or gives rise to a nuclear figure and then disperses (see above, pp. 99-100, for Thalassicolla). From the fragmented material spore-nuclei arise. By the former method isospore-, by the latter heterospore-, nuclei develop. In the Sphaerozoa, however, the homogeneous scattered nuclei remain undifferentiated during the formation of isospores, and only exhibit a change from a singly to a doubly refractive property; but previous to the development of heterospores their nuclei become modified into chromatic and achromatic portions, which are further differentiated in the mega- and microspores.

In the Phaeodaria the ellipsoidal nucleus is usually a single large structure, but two or three nuclei may be present. It consists (Fig. 14) of a membrane containing a linin network. The chromatin is massed at the centre, and from this point radiating 
strands, threads, and lumps run outwards towards the periphery. In addition to these chromatised elements, threads and granules of another substance, the so-called paranuclein of Borgert (18), are present. Nucleoli are absent.

The phenomena of nuclear division in this group have been carefully studied by Borgert $(17,18)$ and Karawiew (16) in Aulacantha scolymantha, but only mitotic division has been fully described. Direct division of the nucleus without elongation is known, but only a preliminary account has as yet been published. -The behaviour of the nucleus during sporulation is unknown. Nuclear mitosis in Aulacantha exhibits five phases. In the first or spirem stage, the linin threads form a dense coil, along which the chromatin becomes arranged in a moniliform fashion; a few remnants, together with the paranuclein, lie scattered through the nucleoplasm. The coil is in all probability never a continuous thread, and no distinct centrosomes appear at this or any subsequent phase. The next stage consists of two events. The threads of chromatin become cut up into varying lengths, and split longitudinally so as to form rows of chromatin globules on either side of the linin threads. The second event is the condensation of these globules into thick short lengths of double chromosomes. The pairs so formed are unequal in size and different in form, some being spherical, some elongated or rod-like, but the members of a pair are alike. Amongst these the paranuclein granules lie in isolated heaps. The next or third stage is characterised by a second longitudinal splitting of the chromosomes in a plane at right angles to the first. The fission products separate, elongate, and become thinner and twisted, leading up to the fourth stage or second spirem phase, which is so far different from the first in that the chromatin elements are obviously discontinuous, and the nucleus as a whole has now become flattened, discoidal, and bent, in consequence of the loss of its membrane, so that it presents, in side view, a somewhat triangular outline, the apex directed towards the astropyle. The large mass of chromosomes is now organised on either side of a median transverse plane passing at right angles to the flattened nucleus. The position of this plane is occupied by a mass of short chromatin elements and débris, between which paranuclein granules occur. The chromosomes are aggregated on each side of this central mass, which prevents them from being continuous from one side of the nucleus to the other, and are more densely crowded near the centre. The whole flattened structure stretches out until on the aboral side it touches the central capsule. The fifth phase is signalised by the appearance of the equatorial plate. The origin of this structure has not been described, but the chromosomes now arrange themselves in close relation to it, and become heaped up in parallel series, though still maintaining marked 
differences of length and thickness. The plate becomes twisted sigmoidally and divides parallel to its surface, the two columns
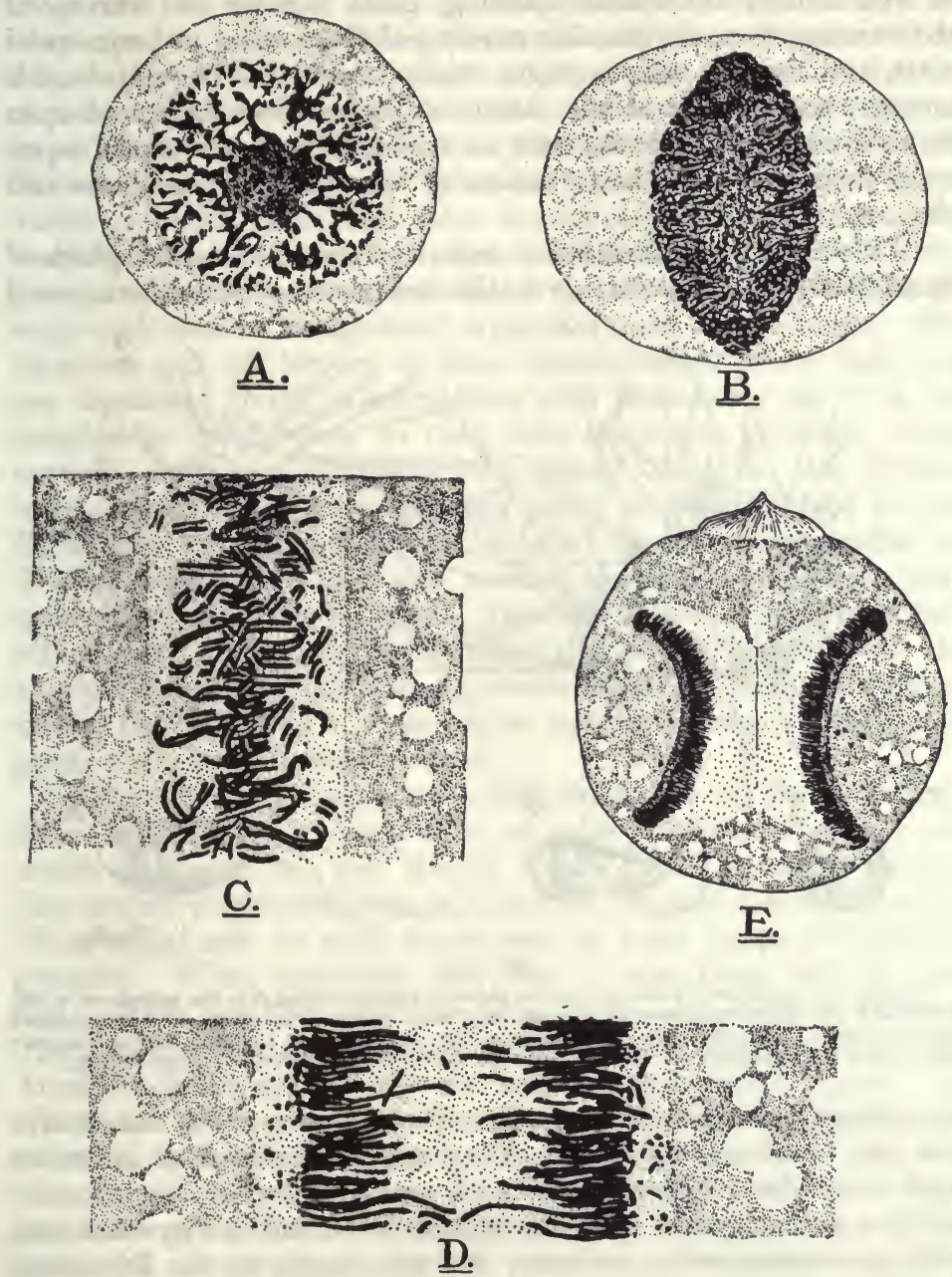

Fia. 15.

Mitosis in Aulacantha scolymantha. (After Borgert [18].) A, central capsule and resting nucleus showing distribution of chromatin. B, second spirem stage showing conmencing separation of the chromatin. C, portion of sigmoidally curved nucleus showing the equatorial plate, and the chromosomes definitely arranged about the middle line. D, separation of the two rows of chromosomes and of the two daughter plates into which the equatorial plate has divided. E, central capsule showing the withdrawal of the daughter plates and commencing reconstitution of the nuclei. $A, B$, and $E \times 150, C$ and $D \times 900$.

of chromosomes move apart and organise two daughter nuclei (Fig. 15). 
These complex mitotic phenomena offer many peculiarities, some of which are discussed by Borgert. The absence of a spindle and of centrosomes, the double splitting, great number and variety of the chromosomes, the peculiar twisting of the nucleus and equatorial plate, and the two spirem stages render this form of karyokinesis unique; and in spite of the labour which has been bestowed upon its analysis, several points, such as the origin and fate of the equatorial membrane and the formation of the daughter nuclei, are still obscure.

Yellow Cells.-Zooxanthellae occur commonly in the ectoplasm of Spumellaria and Nassellaria; in the endoplasm of Acantharia; and

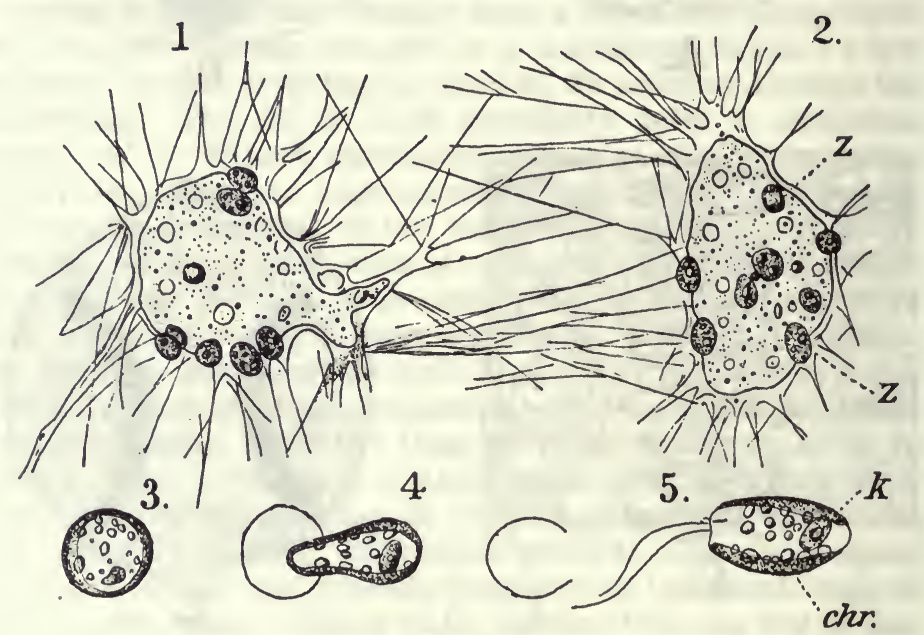

Fio. 16.

1 and 2, two specimens of Collozoum inerme, showing zooxanthellae $(Z)$ in the ectoplasm, $\times 100$. 3,4 , and 5 are magnified views of a single xanthella, showing its escape as a biflagellated organism from the cyst which it forms during the palmella state $(\times 330) . K$, the nucleus ; $c h r$, the two chromatophores; the inclusions are hollow, amyloid grains. (After Brandt.)

are absent from the Pliaeodaria. Their occurrence is facultative and not absolutely constant. They are very abundant in orders with a well-developed calymma such as the Collodaria (both monozoic and polyzoic), less so in the Sphaerellaria, and in Physematiidae with no extracapsular vacuoles, and are absent in the Discoidea. Similarly, zooxanthellae increase in number with the increase of size of the animal or coenobium in which they occur. Young colonies of Collozoum up to 50 or 100 members contain few or no zooxanthellae, older ones become impregnated with them.

The zooxanthellae of the Spumellaria are similar in structure and behaviour to those of Thalassicolla (pp. 97-8). They are usually spherical organisms with a single apparently homogeneous nucleus, capable of assimilating carbon and of forming sheaths of a singly 
refractive amyloid substance around a clear centre. In the Collodaria they vary from 015 to $.025 \mathrm{~mm}$. in diameter; in the Sphaerellaria from .005 to $.01 \mathrm{~mm}$. In the Nassellaria the zooxanthellae are very small in some Cyrtoidea (Eucecryphalus); very large in others (Eucyrtidium, Dictyopodium). A cellulose wall is present and encloses cytoplasm which contains two chromatophores impregnated by chlorophyll and diatomin. In addition to the scattered hollow vesicular, singly refractive structures that react to iodine by a violet or bluish-violet tint, other doubly refractory granules occur, and these are unaffected by iodine. After the death of the ectoplasm in which these zooxanthellae live, they pass into a palmella stage and issue as biflagellated organisms upon a free stage. The structure and life-history of these zooxanthellae prove that they are organisms living in association with Radiolaria, but it is not possible to assign them to their true systematic position. Most authors, following Bütschli, have placed them in the Cryptomonadinae, a small heterogeneous group of simple algae; but, as Schaudinn has pointed out in his work on the zooxanthellae of Trichosphaerium (42), it is also possible that these organisms have quite other affinities. Brandt (10a) and Klebs (46) have drawn attention to the similarity between the flagellated stage of the xanthellae and the Peridinian Exuviaella marina. Further investigation of the behaviour of these yellow cells is necessary before their position can be accurately defined.

Yellow Cells of Acantharia.-The xanthellae of the Acantharia differ in many ways from those of other Radiolaria. They are mainly intracapsular, and always naked cells. In some families they assume a spherical form, in others an irregular amoeboid shape. These cells pass by easy transitions to mere heaps of pigment granules. When numerous they vary in size from 006 to 008 $\mathrm{mm}$. When few they attain a much larger size, 015 to $03 \mathrm{~mm}$. The latter, which are found in Acanthoniidae, Lithopteridae, and Amphilonchidae, are probably the largest zooxanthellae known. In Acanthonia tetracopa and other members of the same family, besides the usual intracapsular mass of zooxanthellae, a few occur now and then in the extracapsulum. In Dorataspis and Actinomma large amoeboid zooxanthellae occur regularly in this position. These structures are almost constant in Acantharia, but they are absent in young specimens and in the few species taken in deep water.

The observations of Brandt $(10 a)$ on the finer structure of the Acantharian zooxanthellae suggest that they have acquired a much closer association with these Radiolaria than have those of Spumellaria with their host, and that the older view of their nature was nearer to the true significance of the association than the modern one that regards the zooxanthellae as merely immigrant algae. Haeckel and Hertwig regarded them as pigment cells formed by the segregation 
of the scattered granules and vesicles about so many nucleated centres in the endoplasm, and therefore as integral parts of the Radiolarian, acting the part of storing reserve material. Brandt has shown that their structure, though not suggesting this view of their origin, serves to explain the connection between the pigment granules, pigmented granular heaps, and definite yellow cells. Starting from the last, with its single nucleus, plates of diatomin, numerous amyloid vesicles and refractive granules, Brandt finds other xanthellae with multiple minute nuclei, and by fragmentation of these yellow cells he accounts - for the presence of the isolated yellow granules, each of which, he affirms, is a living corpuscle and possesses a very small nucleus $(10 a$, p. 237). This degeneration of the zooxanthellar nucleus into a heap of chromatin granules, associated with the breaking up of the

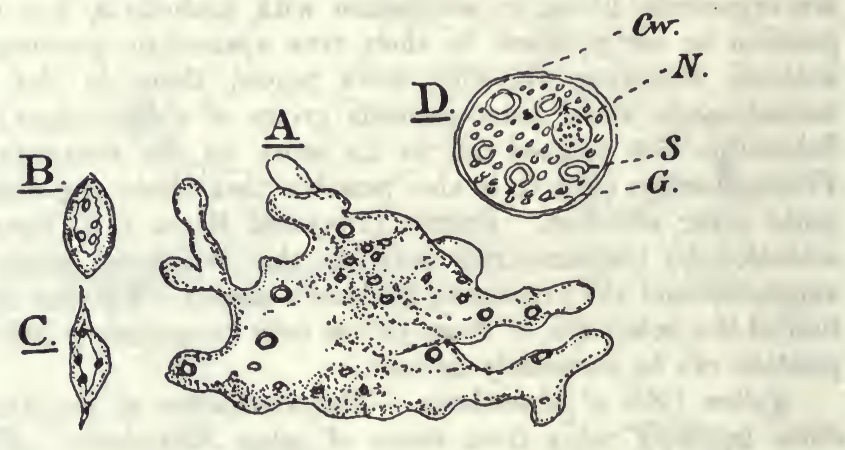

Fig. 17.

A-C, yellow cells (zooxanthellae) of Acantharia. (After Brandt.) A, large amoeboid cell from Acanthonia tetracopa. B, C, spindle-shaped zooxanthellae (A.tetracopa). D, single xanthella of Thalassophysa sanguinolenta, to show its cell-wall (C.w), hollow, singly refractive inclusions that stain bluish violet with iodine. $G$, doubly refractive granules unaffected by iodine. $\times 1000$.

cell, is probably not to be explained through digestion of the yellow cells by the Acantharian, but as a consequence of the intimate association between the two structures. Unlike the zooxanthellae of the Spumellaria, which live, divide, and sporulate after the death and dissemination of their host, those of the Acantharia lose their power of independent existence, and when the endoplasm in which they occur becomes transformed into isospores or heterospores they too pass into these spores in the form of granules and starch grains. Thus the flagellated heterospores of Xiphacantha alata (Fig. 26, A) contain a mass of yellow granules, besides an amyloid body (staining blue with iodine), which is constantly present in the iso- and heterospores of this species. It is therefore possible that the yellow cells of Acantharia pass from mother to offspring, and it is certain that amyloid deposits are so transmitted. The zooxanthellae of Acantharia, therefore, once they have entered the Radiolarian, 
never leave it. They become assimilating granules, apparently incapable of independent life, and are transmitted from parent to offspring. In the former conclusion we have a remarkable parallel to the history of the green cells (zoochlorellae) in Convoluta roscoffensis (Keeble and Gamble [41]).

The nature of these interesting zooxanthellae is not satisfactorily settled, but the scanty evidence points to affinities quite distinct from those of the other Radiolaria. In the absence of a knowledge of the life-history, Brandt's view is as likely as any other, but it is by no means certain that all the Acantharian zooxanthellae are of similar parentage. This view is that the zooxanthellae of Acanthoniidae and Dorataspidae are isolated spindles of Labyrinthula vitellina or of some allied species, and Brandt (10a, p. 239) points out the agreement between the two structures in their shape, size, colouring, and nuclei.

The association between Radiolaria and the zooxanthellae is usually regarded as a symbiosis, i.e. one of mutual advantage. It is, however, clear from the foregoing description that no single formula will cover the important facts-(1) that we have degrees of intimacy that have grown up between the two organisms; and (2) that the last term in the series of association is one to which symbiosis in any but the widest sense of that term is inapplicable.

The origin of the association is probably to be traced to the hunger for nitrogen on the part of the zooxanthellae; to the minimal quantity of inorganic nitrogenous food-stuffs in the warmer seas (Johnstone [45]); and to the convergent adaptation of Radiolaria and zooxanthellae to life at or near the surface of the ocean. This pelagic and insolated station is attained by the Radiolaria through the evolution of calymmal structures in which nitrogen is in all probability abundantly present. These swarms of inert mucilaginous Radiolarian capsules and colonies are therefore in every way suitable media for the nutrition of the zooxanthellae. Attracted in all probability chemotactically by the nitrogenous stores in the mucilage, the zooxanthellae enter the ectoplasm and then divide and assimilate. Protected by their cellulose envelope, they can at first resist the digestive enzymes of their host; ultimately, however, their nucleus becomes degenerate, and with this change the protective wall, whose formation it governs, becomes weakened. In this way some of the daughter-cells of the primary zooxanthellae become food for their host (Famintzin). The Radiolarian, which in its early stages fed on Peridinians, Infusoria, and small Crustacea, ceases to ingest solid food and relies upon the reserves it has accumulated or upon the secondary xanthellae for its supplies. Meanwhile, its nitrogenous metabolism, and possibly its intramolecular respiration, is maintained by the xanthellae, which are removing the waste nitrogenous substances. In confirma- 
tion of this statement reference may be made to the Phaeodaria. This group of Radiolaria possesses no zooxanthellae, and might therefore be expected to show some accumulation of excretory granules. This appears to be the case, for the complex phaeodium is made up of refractory, insoluble vesicles which are generally held to be excretory substances. The association of diatoms with Radiolaria has probably a similar significance.

Finally, when the endoplasm sporulates the dying ectoplasm serves as a medium in which the zooxanthellae rapidly divide and - issue as naked biflagellated spores upon a new, free existence. In the case of the Acantharia, which are also mainly epiplanktonic or surface organisms, the zooxanthellae are naked cells, almost exclusively confined to the central portion of the Radiolarian. Whether they develop from antecedent zooxanthellae that occur in the spores of Acantharia or infect it from sea-water, or whether both modes of origin obtain, is at present unknown. The apparent absence of xanthellae from young Acantharia makes the first suggestion unlikely. Within the central capsule they divide, multiply, and assimilate. Certain of them fragment into particles, the process being initiated by nuclear fragmentation, so that the zooxanthellae are no longer cells but mere chromatised, pigmented corpuscles, associated with free granules of starch or amyloid substances. There is no evidence to show whether in this or in the earlier coherent stage the xanthellae are digested by the Acantharian. They become in the last event mere assimilative corpuscles, and when the endoplasm sporulates the whole of the zooxanthellae, with their associated starch, pass into the bodies of the flagellated spores, and are probably used up as food by the developing zygote. Throughout this series we see that, in opposition to the idea of mutual benefit, the animal is the predominant partner. The association is one beginning with myxophytism and leading to a case of parasitism, in which the zooxanthellae are the host and the Radiolarian the parasite.

Skeleton.-The skeleton of the Radiolaria has developed in each of the great sub-classes into a complexity of form and variety of detail that are found in no other group of animals. So characteristic are the skeletal products that it is usually possible from them alone to recognise broadly the systematic position of the organism that produced them. So complex and diverse a tracery seems utterly beyond the needs of simple Protozoa living under apparently similar conditions of pelagic life; and though attempts have been made to explain this manifold skeletal development in terms of cytoplasmic structure, its variety still evades a biological treatment. Recent investigation has, however, done something to reduce this variety to a few plans, and to attach a biological meaning to some of its elaborations. These results justify the hope that, as we come to 
regard the skeleton as a response to the varying media, stresses, and strains that fall upon the cytoplasm from within and from without, that then its utilitarian character will be more completely recognised, and its variety found to be explicable in terms of composition, mode of deposition, and the need of response to widely varying combinations of stimuli that occur in the apparently monotonous sea. Two very different substances compose the greater part, and probably the whole, of these skeletal structures. In the Spumellaria and Nassellaria pure silica is present; in the Phaeodaria the silex is mixed with organic substance; but in the Acantharia a substance is present which, from the time when it was first described by Johannes Müller to the present, has given rise to differences of interpretation. Müller, relying on the indestructible nature of the Acantharian skeleton when heated, regarded it as siliceous. Haeckel found that it was apparently destroyed by heat, and regarded it in the main as an organic horny substance which he called acanthin. Schewiakoff (33) tested its properties and attempted a quantitative analysis, the result of which went to show that the so-called acanthin was a complex silicate. Quite recently Bütschli (39) has reinvestigated the skeleton of Antarctic and of some Mediterranean Acantharia, and has proved that in these cases it is composed of strontium sulphate.

The diverse forms of Radiolarian skeletons are largely founded upon developments of scattered aciculate and tetrahedral spicules. Dreyer has indeed attempted to trace the evolution of the skeleton (1) in the Acartharia to an axopodial type derived from the hardening of the axis that runs down the peculiar radiating pseudopodia of this sub-class; and (2) in other Radiolaria to the modifications of a tetrad spicule, which in turn he traces to the deposition of silica at the intersecting planes of adjacent cytoplasmic vacuoles or alveoles; but the absence of a knowledge of the development of the skeleton rendered this attempit suggestive rather than convincing, and there are many forms of skeleton which it is difficult to assign to any conceivable modification of the tetrahedral type. In the present state of our knowledge it must be admitted that the vacuolated cytoplasm has the power of depositing its silica in the form of perforate or imperforate shells, plates, and processes, so that in addition to the spicules there is often a great development of siliceous matter, the form of which cannot be referred to the alveolar structure of protoplasm.

In form as in composition the skeleton of the Acantharia is sharply marked off from that of other Radiolaria. With few exceptions, it consists of twenty rods united in various ways : (1) by apposition and also by adcentral processes; (2) by fusion of all or of opposite pairs, at the centre of the endoplasm. These radii are disposed so as to emerge from the spherical cytoplasm along five 
circles, which may be compared to the equatorial, the two circumpolar, and the two tropical circles of the globe (Müller's law). In a few cases two radii mark the vertical axis, and the other eighteen are disposed in three circles-an equatorial one, and the other two respectively $45^{\circ}$ above and below it (Brandt's law); whilst in the apparently primitive Astrolophidae the spines vary in number and

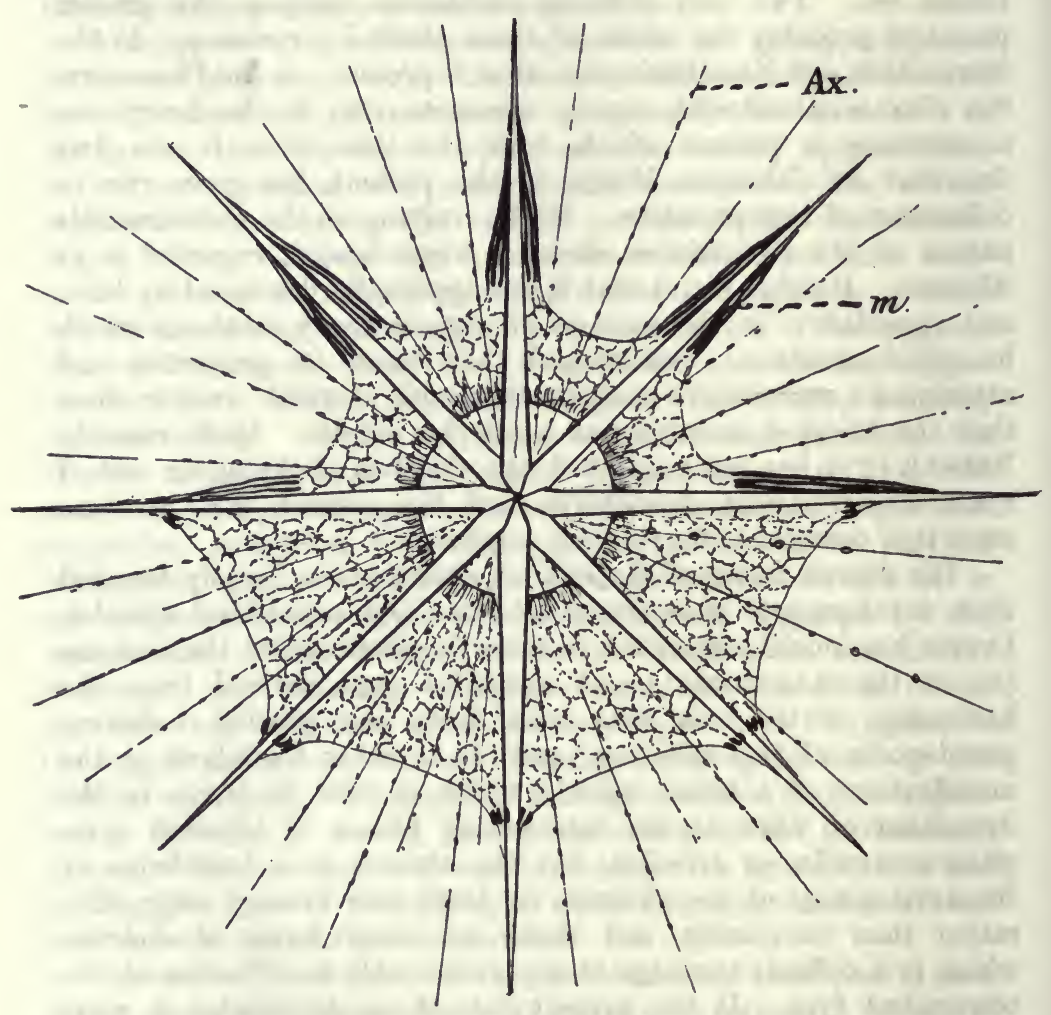

Fra. 18.

Acanthonia tetracopa in its two extreme phases of expansion and contraction, one half of the animal being drawn in each case. The relation of the inyonemes to the ectoplasm, and their insertion into sheaths around the radial spines, is also seen (cf. Fig. 11). The full number (20) of spines is not indicated. (After Schewiakoff.) $\times 170$.

possess no regular arrangement beyond their radial disposition. This loose order is repeated in the early development of the Acanthoniidae. The young of this family possess ten loose rods arranged crosswise, which subsequently become divided at the centre of the capsule into the typical twenty radii. In the Acanthochiasmidae the distal portion of each radius gives off tangential processes which unite with those of adjoining spines and so form a perforated 
shell. By repetition of the process farther along the radii a second and succeeding concentric shell may arise. In the most modified case (Sphaerocapsidae) the lattice alone is present, but the place of the radii is shown by twenty large pores distributed according to Müller's law.

In the Spumellaria the skeleton is either absent, spicular, or shelly. Both spicules and perforated shells are often present simultaneously, and have evidently developed independently in two of the main subdivisions-Sphaerozoa and Sphaerellaria. But whilst in the former the shell is single, in the latter it often becomes multiple, interconnected by radial bars, and flowers out into a wealth of appendicular growths that characterise this vast group, which numbers two-fifths of the known Radiolaria. The Sphaeroidea retain the homaxonial form; the Discoidea have only the first or first and second chambers spherical, and farther outwards become flattened and often cruciform, the arms of the cross being irequently divided into a large number of chambers, into all of which the endoplasm and its associated pigmented oil-globules may pass. Other modifications are mentioned in the conspectus (pp. 144-145). In the Nassellaria, the Radiolarian skeleton develops into its richest expression of geometric form. Its simplest types consist of a single or multiple ring and of a tripod or tetrad (see Fig. 5), and from these a helmet-shaped perforated shell has arisen, apparently by lateral extensions of the simpler plan. Such a cephalis may be simple or divided both sagittally and transversely by one or more constrictions, and in exceptional cases a spherical shell may be developed. The most interesting feature of this group is that the whole of its variety can be traced fairly confidently to the modifications of a single element which Bütschli (8) believes to be a ring and Dreyer (15) a tetrad spicule.

The skeleton of the Phaeodaria has followed another line of evolution. It consists essentially of minute aciculate spicules imbedded in a gelatinous matrix. Between these a jelly-like substance is secreted ; the inner layer of this matrix becomes silicified to form a tube, the eavity of which is often subdivided by one or more septa; or the intermediate jelly may also become silicified as a porous plate or shell of porcellanous texture. Commencing with the Phaeocystina, in which the skeleton is absent or composed merely of isolated radial and tangential spicules, the formation of a lattice-shell has come about in several ways. The simplest mode is that seen in the Aulosphaeridae, in which the tangential spicules unite to form an open peripheral network. To this a second shell may be added by the formation of a reticulum immediately outside the central capsule (Cannosphaeridae). If the outer shell is absent, a condition found in the Castanellidae is obtained. In these Phaeodaria the single shell is composed of two conjoined membranes 
imbedded in a porcellanous impregnation throughout which minute aciculate spicules occur. It is provided with an oral opening on the end of a projecting and often spiny peristome. Again, this inner shell may assume a bivalvular form (Fig. 32), and then carries a number of complex appendages. Some of these are branching hollow species, terminating in anchor-like expansions; others constitute the "galea" and "rhizocanna" (see Fig. 32, p. 151).

Biological Significance of the Skeleton. - The results of recent investigation point to the conclusion that the chief skeletal function is a hydrostatic one and is effected by stretching or folding the superficial ectoplasm. The older conception of the skeleton as projecting freely beyond the cytoplasm has been shown to be a mistaken one in many instances, and it is probable that the skeleton is during life covered by the outermost delicate plasmic layer in all Radiolaria. Between the characters of this layer and the development of the supporting rods a definite relation holds for certain forms. A few widely varying Radiolaria are dimorphic, a small pelagic variety and a larger abyssal form being readily and apparently rightly distinguishable (Aulacantha scolymantha, Auloscena and Sagosphaera-species). In these cases the surface-form possesses a delicate ectoplasmic layer, and the supporting rods are simpler and shorter, whereas in the bathybial variety the outermost cytoplasm is dense, more voluminous, and usually more stiffly supported by verticillate skeletal projections. 'The graceful and elaborate skeletal appendages of other Phaeodaria are probably to be explained not as a means of catching food, but as a support for the ectoplasm; and the whole plan and construction of the tubular skeleton in these forms is no doubt related intimately to the pressures that fall upon this limiting layer.

In connection with this sustentative function of the Phaeodarian skeleton, the mode of formation of its tubular systems offers some features of special interest. The most general mode is that indicated at the close of the last section (p. 133), and in this method minute needle-like spicules form the contre around which tubular developments of silica take place. But in addition to this intrinsic centre, many Phaeodaria have adopted extrinsic objects, and around these as catalysators, the tubular silica is deposited. Like other Radiolaria, but to a greater extent, the Phaeodaria ingest quantities of foreign bodies, with which their phaeodium is distended. Amongst these ingesta, diatoms and Radiolarian skeletons are abundant. From Phaeodaria, in which such gatherings are casual, we can trace a series leading to forms in which diatom-selection becomes a regular habit, associated directly with the formation of a radial skeleton. Thus Aulographis pandorn and Auloceros arborescens from the Atlantic and Indian Oceans contain in their phaeodia frustules of Ihizosolenia, and spicules of many species of Aulacanthids picked up apparently in 
a casual manner, and probably serving to increase the extent of exposed surface. Cannosphaera from Antarctic seas possesses a hollow skcleton the tubes of which are almost filled with masses of the diatom Corethron; and finally, in Aulokleptes (Fig 19) and Aulodendron the diatoms are planted radially in the ectoplasm, surrounded by a mucilage, and finally incorporated into the walls of a hollow radial tube, the lamellae of which are laid down from within outwards, and the top of which is moulded into the form severally characteristic of the species (Immermann, Häcker).

The biological significance of the varieties of Nassellarian spicule and of the scattered Spumellarian spicules and lattice-shells is at present quite obscure, but the skeleton of the Acantharia offers perhaps the clearest case of functional significance to be found in the whole group (Dreyer, Brandt, Popowsky). The twenty radial spokes of the Acanthometrea serve as so many tent-poles for the insertion of the myonemes (Figs. 11

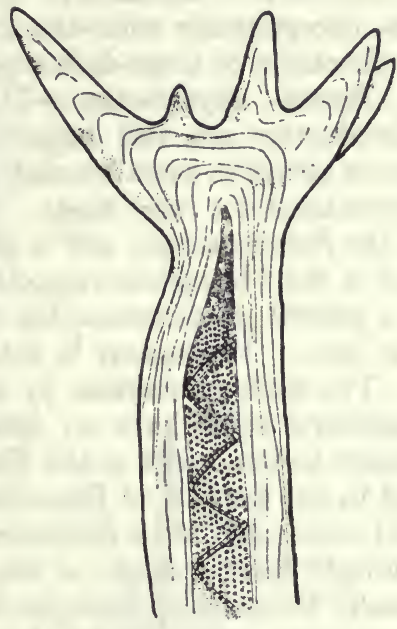

Fia. 19.

Spicule from Aulokleptes flosculus formed around a dividing diatom Rhizosolenia. (After Immermann.) $\times 55$. and 18) that hoist the calymmal cones. This action, combined with absorption of water into the vacuoles, causes a swelling of the cytoplasm and brings the animal towards the surface; whereas relaxation of the myonemes and contraction of the calymma depresses it beyond the reach of wave-action. The skeleton of this subdivision is, however, related to hydrostatic ends in another way. The definite arrangement of the twenty spines according to what is known as Müller's law (p. 132) has recently been correlated with flotation and dispersal. Brandt has shown that the distribution of the radii in five alternate and superposed circles, each of four spicules, is such as to expose them freely and without overlapping to the viscosity and resistance of the water. The absence of vertical or axial spines is also intelligible, since they would increase the weight of the body without giving additional buoyancy. Moreover, the shape as well as the arrangement of the spines assist the Acantharia in their flotation and dispersal. Like all other Radiolaria, these are dependent on currents and drift for their dissemination. In order to utilise this horizontal force, the radial spokes are frequently provided with four flanges or blades, which serve the double purpose of encountering 
sustentative and propulsive forces. When these blades are wanting and the spines are merely flattened, they are set in each of the three circles, so as to turn the flat edge somewhat differently to the water, the equatorial ones lying flat on the water, the tropical ones turned half over, and the polar spines set on edge. By this means the amount of resistance to the water in every direction is increased. The exceptionally wide distribution of the Acanthometrida is some confirmation of these deductions.

Fission-Reproduction.-The phenomena of multiplication and of reproduction are still imperfectly known. Binary or multiple fission occurs in some Spumellaria, Acanthometrida, and Phaeodaria. Gemmation is a rarer mode. It produces the extracapsular bodies of the Sphaerozoidae, and is found in one species of the Acantharia and of the Phaeodaria respectively. The development of zoospores is a general phenomenon, but has been followed in detail only in a few cases. Plastogamy is unknown.

The mode of increase by fission is probably restricted to those Radiolaria which have no spicules or a lax and osculate skeleton. Binary fission occurs in the Thalassicollidae, some Acanthometridae, and in two families of Phaeodaria. Division both of the Sphaerozoid colony and of its component individuals takes place at intervals. Multiple fission occurs in the Thalassophysidae. The process is usually initiated by changes in the endoplasm and nucleus, and a long interval may follow before any corresponding alterations occur in the ectoplasm (Phaeodaria).

In the Acanthometrida (Acanthoniidae) binary, quatenary; and multiple fission are said to occur (Popowsky). The former process is illustrated in Fig. 20. The skeletal rods separate at their central ends into two bundles, the nuclei segregate into two groups, the central capsule divides, and ultimately fission takes place. The fission-products are, however, asymmetrical, and Fig. 20 shows how the new radii are developed and how the rods are swung into position, probably by contraction of the myonemes inserted into them, until the whole arrangement is brought into conformity with Müller's law.

Fission in the Phaeodaria is carried out in several ways. Aulacantha scolymantha is the best-known example of the direct process. In this Radiolarian the large single nucleus divides either by mitosis or amitotically; the endoplasm segregates round the daughter nuclei ; the central capsule, after disappearing for a time, re-forms about the two masses. Lastly, the phaeodial complex, the calymma and spicular skeleton are subdivided each into two groups, and the whole organism divides into two. In the Phacodaria, which possess a shell, one or more modifications of the process are found. The helmet-shaped Challengeridae, for example, undergo fission within the shell. One half of the organism now escapes 
through the oral aperture and develops into a new individual (Borgert [21a], p. 100).

The most remarkable case of multiple fission occurs in the Thalassophysidae, and constitutes the only known means of increase

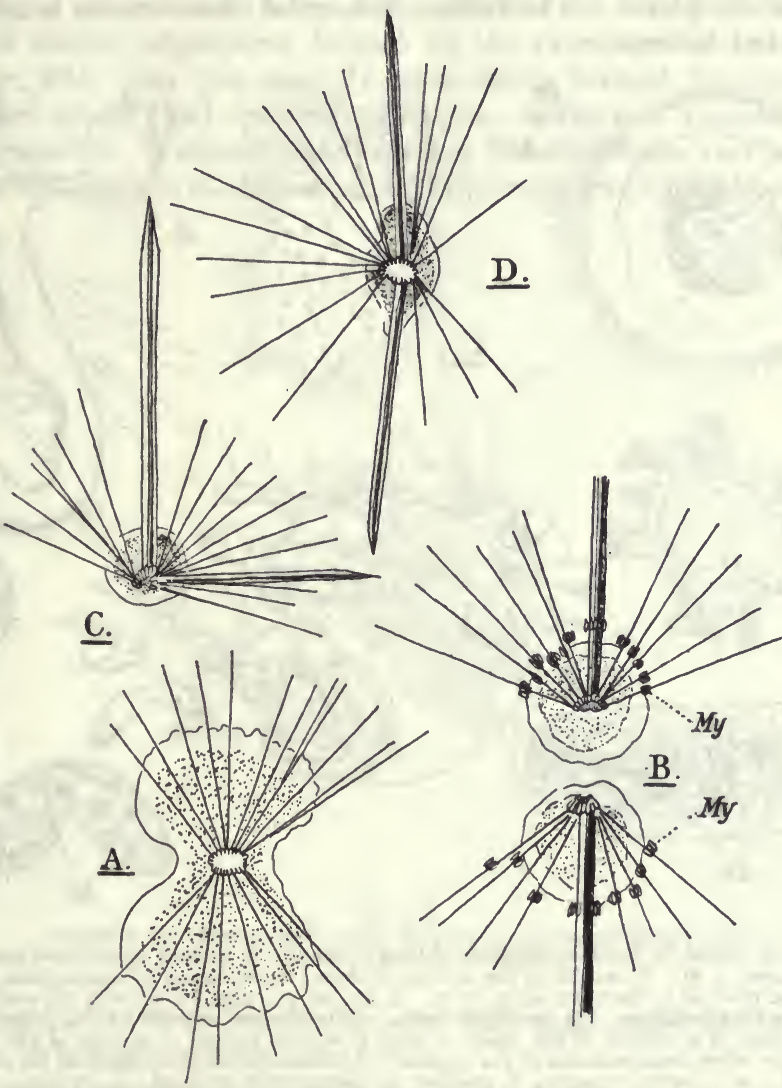

F10. 20 .

Iliustrating fission in the Acanthometrida. (After Popowsky.) A, Acanthometron bifidum about to divide. The spicules are arranged in two bundles. The central capsule has disappeared. The ectoplasm is a mere hyaline border round the granular endoplasm, $\times 150$. $\mathrm{B}$, fission of Amphilonche atluntica. My, the myonemes, $\times 150$. C, regeneration of the same; formation of a directive large spicule, $\times 150$. D, spicules reassuming their characteristic arrangeineut, $\times 150$.

in this family. Fig. 21 illustrates the process, which has been investigated by Brandt (25). The central capsule and nucleus become irregular-branching, vermicular, or radiating structures. The oil-globules and their associated pigment granules become disseminated through the endoplasm. Then the nucleoplasm breaks up into a vast number of minute homogeneous corpuscles, followed 
by rapid division of the capsule and endoplasm. The ectoplasm fragments and the products are disseminated through the water. Each minute product consists of several nuclei lying in a pigmented, oily fragment of endoplasm and supported by a portion of the original ectoplasm. The further history of these bodies is unfortunately not known.

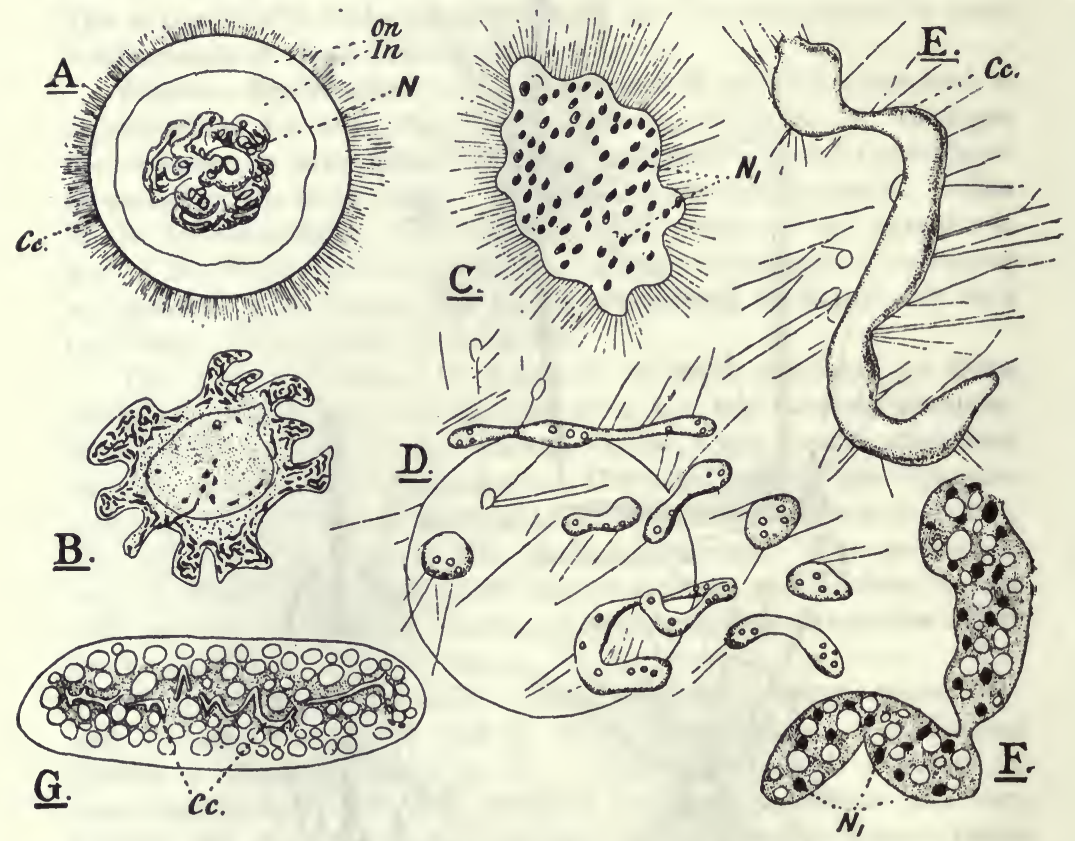

Fig. 21.

Multiple flssion in Thalassophysidae. (After Brandt.) A, central capsule and nucleus of Th. spiculosa, $\times 40$. B, section of the nucleus to show the two zones of nucle(uplasm and the vermicular nucleoli in the outer layer, $\times 66$. C, Th. pelagica about to divide: the nucleus has undergone fragmentation. 1), multiple fission of the central capsule of Th. pelagica. $\mathbf{E}$, enlarged view of a portion of the same, $\times 200$. F, stained portion of capsule of the same to show nuclei before fragnentation of the capsule. G, division of central cupsule of Th. sanguinolenta, $\times 7$. C.c, central capsule; $N$, nucleus of vegetative individual ; $N_{1}$, nucleus of fragmenting individual ; $O n, I n$, outer and inner zones of endoplasn.

The separation of a portion of the Radiolarian organism as a bud is a rare phenomenon, of which the "extracapsular bodies" of the Sphaerozoidae offer the best example. These structures occur in small colonies of Collozoum inerme, C. rudiosum, C. fulvum, and of Sphaerozoum neapolitanum. They consist of a lobate, highly refractive, cytoplasmic mass, containing a group of modified nuclei .ranged about a grape-shaped mass of fat, and are loosely attached to the colonial jelly (Fig. 22). These extracapsular bodies are budded off from the endoplasm of certain members of the colony in which they occur, and are at first uninuclear. According to Brandt's 
account (10) these bodies have a twofold significance. Either they become additional members of the parental colony and develop central capsules, or they become megaspores and the small parental endoplasm develops microspores. In his later work Brandt lays additional stress on the latter fate. He has not only seen the beanshaped active megaspores formed by the extracapsular bodies, but $(26$, p. 264) also the mass of microspores formed by the small capsules which had budded off these bodies: a proterogynous arrangement. It should be added that Brandt affirms very strongly the juvenile nature of these small budding colonies; whilst Famintzin,

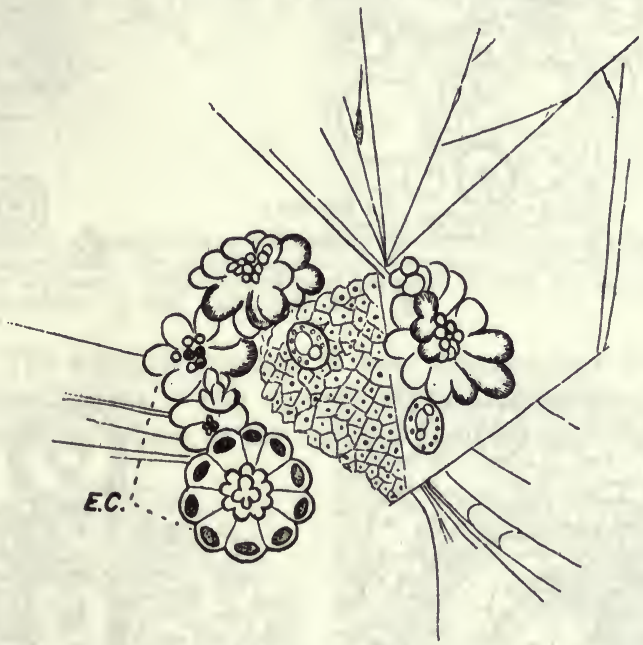

Fra. 22.

Collozoum sp. Portion of a colony showing extracapsular bodies (E.C). $\times$ 100. (After Brandt.)

working in the same locality, asserts that their small size is due to fission of full-grown coenobia (13).

Spore-Formation.-Flagellated spores occur in the four main divisions of the Radiolaria, but their exact nature is only known in some Collodaria and some Acantharia, and it is in the former order that their formation has been traced. The process is described for Thalassicolla on pp. 99-102.

Isospores. - The development of isospores in the Sphaerozoa takes place in colonies distinct from those that produce heterospores. After a vegetative life of several months these colonies exhibit characteristic changes (Fig. 25). The nuclei become ranged in a single or double row just beneath the capsular membrane. Without becoming obviously differentiated, these lumps of chromatin divide directly and acquire a doubly refractive character. Hundreds of 
minute crystals arise in the endoplasm, a few larger ones also in certain Collosphaeridae. The single oil-globule of each capsule becomes very rapidly subdivided into as many minute vesicles as there are nuclei, and in association with this process a blue pigment develops
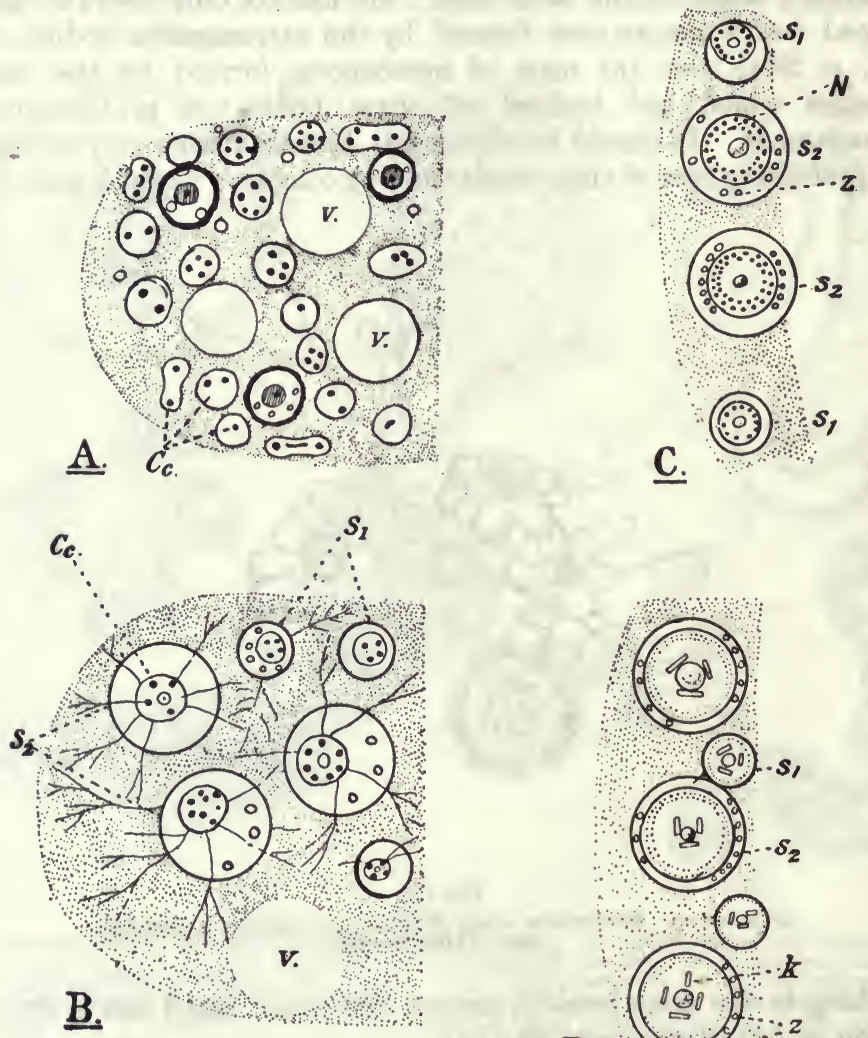

Fia. 23.

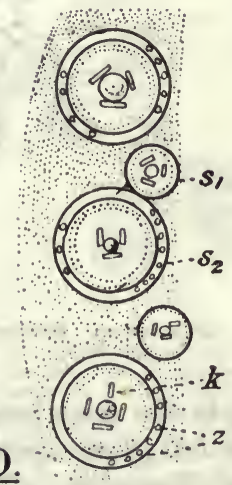

Collosphaera huxleyi. Optical sections of different growth-stages to illustrate $(\mathrm{A}, \mathrm{B})$ dimorphism $\left(S_{1}, S_{2}\right)$ in early and later stages, and $(C, D)$ the formation of isospores. A, young actively dividing colouy (the young reproductive phase of 13randt, comparable with the formation of extracapsular bodies in Sphaerozoidae). Many individuals are naked central capsules with one or more nuclei; others have a shell $\left(s_{1}\right)$ and are larger and already provided with zooxanthellae $(z)$. B, later vegetative phase. The naked cajsules have now secreted a large shell $\left(S_{2}\right)$, and a marked dimorphism has resulted. C, part. of a full-grow colony about to sporulate. The formation of isospores is indicated by the grouping of the nuclei. $D$, later stage in isospore-formation showing the crystals aggregated about the oil-globule. $\times 75$. (After Brandt.)

in Myxosphaera coerulea and Collosphaera huxleyi. Numerous vacuoles arise in the centre of the capsule, each with a central granule, until a number equivalent to that of the nuclei has been formed. Meantime these nuclei, which have become very numerous, are evenly 
disseminated through the endoplasm forming the centres about each of which a crystal, an oil-vesicle, a vacuole, and granule are

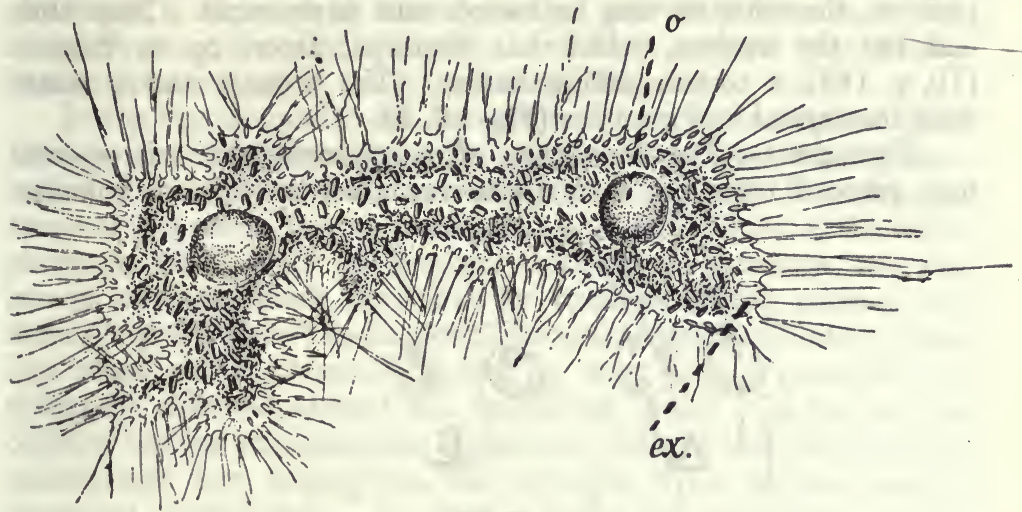

Fra. 24.

Portion of a colony of Sphaerozoum neapolitanum about to form isospores. The spicules and "yellow cells" are omitted. The central capsule has disappeared, snd only a thin peripheral ectoplasmic layer is present. Minute crystals are scsttered through the endoplasm, and two oil-globules (o) are shown. $\times 300$. (After Brandt.)

clustered. The whole endoplasm is now transformed into a mass of biflagellated spores. The central capsule suddenly disappears, and the ectoplasm, which in the interval has undergone contraction

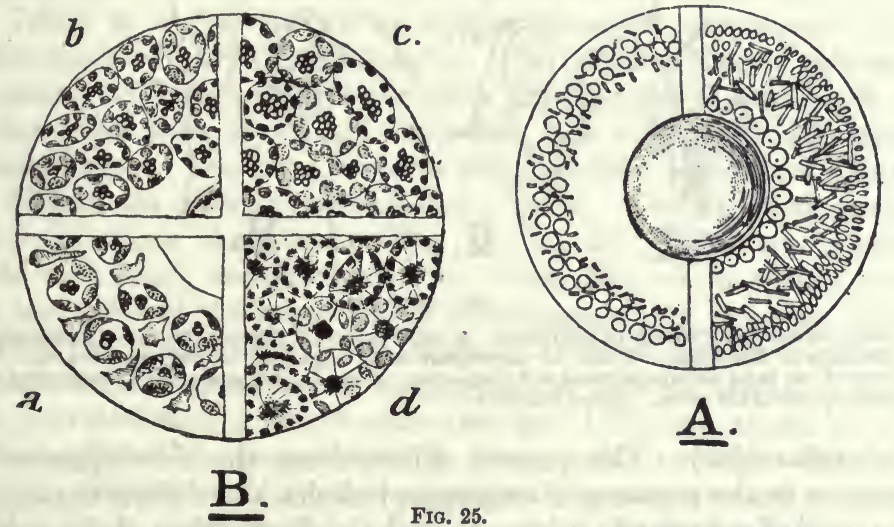

A, formation of isospores in Collozoum inerme. Two stages are shown on opposite sides of a central capsule. On the left side the nuclei and crystals are aggregated peripherally, but the central oil-globule is intact. On the right the nuclei are smaller and more numerous and the oil-globule is breaking down. B, formation of heterospores in the same shown by quadrants. $a$, early stage ; several grouped, modifled nuclei and fat-granules; between the groups are undifferentiated nuclei and endoplasin; $b, c$, and $d$ are later stages.

and degeneration, breaks to pieces. The colony descends and the isospores swarm out, leaving (in the Collosphaeridae) the large crystals and the greater part of the pigment behind. Each is a 
conical structure (.012 mm. long). From its pointed end spring the two cilia, one of which is usually held in a somewhat horizontal position, the other curving backwards and downwards. Near this end lies the nucleus, which has acquired, according to Brandt (10, p. 163), a certain differentiation. The broader end is filled with the crystal and granules (Fig. 26, E).

Heterospores. - The formation of megaspores and microspores may proceed from the same (Sphaerozoidae) or separate colonies

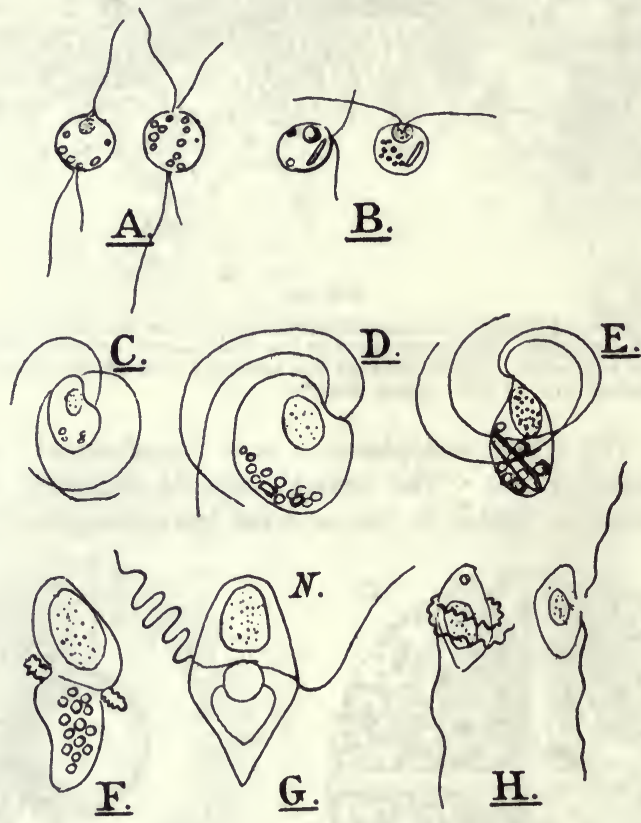

Fig. 26.

Isospores and heterospores of Radiolaria. A, heterospores of Xiphacantha alata (Acantharia). $\mathbf{B}$, isospores of the same. $\mathbf{C}$ and $\mathrm{D}$, microspore and megaspore of Collozoum inerme. $\mathbf{E}$, isospore of the same showing crystal and inclusions. F and $G$, megaspores of Sphaerozoum sp. $\mathbf{H}$, microspores of the same. (After Brundt.)

(Collosphaeridae). The process differs from the development of isospores in the presence of segregated nuclei, the differentiation in the nuclei of achromatic substance, and the dimorphism of the megaand micro-nuclei. In the Collosphaeridae the full-grown vegetative colony shows the first traces of heterospore-formation by the segregation of its homogeneous nuclei into groups of 2,4 , or 8 . This arrangement is temporary, and very soon the nuclei are found arranged in several layers, each nucleus being now clearly composed of a highly refractive and achromatic ground-substance, in which are imbedded thread-like masses of chromatin. 
According to the colony under consideration so will these nuclei belong either to the microspore or megaspore. In the former the chromatin is disposed in stout granules and thick strands, in the latter in much smaller quantity. In other respects the colony behaves precisely as in the formation of isospores.

In the Sphaerozoidae the formation of heterospores takes place both in small, apparently young, colonies that bear extracapsular bodies and also from full-grown vegetative colonies. In both cases many of the nuclei become segregated and differentiated, the endoplasm in which they lie acquires distinctive characters, and the groups so formed are separated by undifferentiated plasma and nuclei (Fig. 25, B). The oil-globule becomes subdivided into a grapelike mass, which ultimately splits up into minute granules, and these are collected around the specialised nuclei. In the case of colonies bearing extracapsular bodies the whole of this bud becomes transformed into megaspores, the contents of the central capsule becoming microspores. In older colonies the endoplasm is converted into a vast number of portions, in each of which the differentiated nuclei are aggregated. These nuclei are, however, not all of one kind. Each collection is either meganucleate or micronucleate, and accordingly stains feebly or strongly. The contents of the capsule now becomes resolved into biflagellated megaspores and microspores, the ectoplasm degenerates and collapses, the central capsule deliquesces, and the spores become disseminated.

Little is as yet known as to the formation of isospores and heterospores in other Radiolaria. In Acanthochiasma rubescens (Acantharia) Brandt records the early development of two kinds of bodies-one with crystalloid inclusions, the other with lobulated masses of fat. The same observer has described the active spores of Xiphacantha alata and Acanthometra sicula. Two kinds of spores occur in these Acantharia (Fig. 26, A, B). Both are minute (.004 $\mathrm{mm}$. long), and provided with three cilia, which spring from the two poles of the spheroidal or pear-shaped body, but they differ in that the spores of any one individual either contain a minute crystal and few granules or many granules but, no crystal. Both are provided with a starch-grain (see pp. 128), and traces of the yellow cells of the parent occur in the granular variety. It seems highly probable, therefore, that crystal-bearing isospores and granular heterospores occur in this sub-class as in the Spumellaria; but although the results of more recent expeditions have extended very largely the number of Acantharia in which the early development of spores has been shown to occur, the free spores have not been again noticed; nor do we possess any exact observations on the flagellated bodies that have occasionally been seen in Nassellaria and Phaeodaria. 


\section{CLASSIFICATION. ${ }^{1}$}

\section{CLASS RADIOLARIA.}

\section{Sub-Class I. Peripylaria (Spumellaria).}

Central capsule homaxonic, uniformly perforated by numerous similar and extremely small pores. Skeleton siliceous. Extra-capsulum voluminous (except in Physematiidae).

\section{Order 1. Collodaria.}

Large monozoic forms not forming a true coenobium. Skeleton absent or spicular.

Family 1. Physematiddar. Large vacuoles confined to the endoplasm. No stratified concretions in the latter. No pigment. Few "yellow cells." Nucleus spherical, with smooth membrane and a few rounded nucleoli. Selected forns :-Physematium mülleri, H. ; Thalassolampe margarodes, H., Mediterranean and Canary Islands; Lampoxanthium murrayanum, Fowl., Faroe Channel. The genus Actissa of Haeckel is an early stage of growth of some species of this family.

Family 2. Thalassophysidae. Large vacuoles extracapsular. Structure similar to that of the Thalassicollidae, but nuclear membrane usually tubercular or papillary. Reproduction by rapid and peculiar fragmentation (Fig. 21). Spores unknown. Selected forms:-Thalassiosolen atlanticus, Wolf. (28); Thalassophysa pelagica, H. (Fig. 1), Faroe Channel ; T. sanguinolenta, H. ; T. papillosa, H., Mediterranean and Canary Islands (often deformed by ingested Coccolithophoridae). For further account of this family see Brandt (25).

Family 3. Thalassicollidae. Nuclear membrame smooth and spherical. Stratified concretions present in the endoplasm. Multiplication by binary fission, by isospores, and by heterospores (see Fig. 2; Brandt [25, 25a, and 26]). Selected forms :-Thalassicolla nucleata, Hux., Valencia Harbour, Faroe Channel, and cosnopolitan; T. spumida, H., Canary Islands ; T. pellucida, H., cosmopolitan.

Family 4. Thalassothamidae, Häcker (37). Skeleton in the form of a large single radiate spiculum or of a double spiculum. Central capsule sometimes spherical, characteristically lobed or branched. Nucleus complex. Nuclear membrane crenate (Fig. 14). Endoplasm with stratified inclusions. Selected fornı:- Thalassothamnus ramosus, Häck., Antarctic ; Cytocladus spinosus, Schröder (Fig. 10), Japan Seas (38).

Family 5. Orosphaeridae. Protoplasm organised as in the preceding faniily. Skeleton a perforated shell with branched and thorny spines. Orosphaera, H., deep water of mid-Atlantic. This family has been removed by Häcker (37) from the Phaeodaria, with which group Haeckel associated it ; but if the presence of a phaeodiun, astropyle, and parapyles is confirmed, its systematic position will have to be revised.

1 The number of geuera and species in this class is so large that only a selection can be referred to here. North Atlantic forms have been chiefly selected. 
Colonial forms.

\section{Order 2. Sphaerozoa.}

Family 1. Sphaerozoidae. Both mega- and microspores developed in the same individual. A lattice-shell absent. Selected forms:Collozoum inerme, Norway (Figs. 3, s, and 25) ; C. pelagicum, Shetlands ; Sphaeroroum ovodimare, Faroe Channel.

Family 2. Collosphaeridae. Mega- and microspores in separate individuals. Skeleton, when present, takes the form of a lattice-shell with or without associated spicules. Selected forms:-Collosphaera huxleyi, Mediterranean (Fig. 23) ; C'hoenicosphaera murrayann, Shetlands.

This order is treated fully by Brandt in his Monograph (10) and (22).

\section{ORder 3. Sphaerellaria.}

Sub-Order 1. Sphaeroidea. Central capsule and shell (or shells) spherical. Selected forms :-Hexalonche philosophica, H., Faroe Channel ; Hexacontium enthacanthium, Jörg. ; H. pachydermum, Jörg., North Sea ; Hexadoras borealis, Clev., North Seи ; Echinomma leptodermum, Jörg., Norway and Sweden ; Rhizoplegma boreale, Clev., Norway.

Sub-Order 2. Prunoidea. Central capsule and shell elliptical or cylindrical ; often with transverse constrictions. Selected form :-Prunocarpus datura, H., Faroe Channel.

Sub-Order 3. Discoidea. Central capsule and shell discoid or lenticular; often much flattened. Selected forms:-Trochodiscus heliodes, Cler., North Sea ; T. echiniscus, H.; Lethodiscus microporus, H.; Astrosestrum acanthastrum, H. ; Spongodiscus favus, Ehr., Faroe Channel.

Sob-Order 4. Larcoidea. With lentelliptical central capsule and shell. Selected forms:-Lithelius minor, North Sea; L. arburescens, H., Faroe Channel ; Phorticium pylonium, H., Norway and Sweden.

Sub-Order 5. Sphakropylidea. With basal or basal and apical pylome (large opening to the shell). See Dreyer (15).

\section{Sub-Class II. Acantharia.}

Radiolaria in which the skeleton is composed neither of the so-called horny acanthin nor of silica, but (in many cases) of strontium sulphate. The central capsule is perforated uniformly or in networks and segregated pores. The skeleton has the form of spicules radiating from a central point within the capsule (Acanthonetrida). Rarely a fenestrated extracapsular skeleton is added (Acanthophractida).

\section{Order 1. Acanthometrida.}

Sub-Order 1. Actinelidda. With 10-201 radial or diametral spines not arranged according to Müller's Law (p. 132).

Family 1. Astrolophidae. Spines radiating from a common centre. Genus 1. Actinelius. All spines of equal length and similar shape. $A$. purpureus, H., Mediterranean. Genus 2. Astrolophus. Spines of unequal length. 
It is probable that further investigation of the Actineliida will clear up the anomalies that at present attach to their isolated position. They are regarded by Haeckel as the ancestral stock of the whole Radiolaria. The family Litholophidae which he associated with them is now regarded as composed of growth-stages of the genus Acanthonia.

Family 2. Acanthochiasmidae. With ten or sixteen diametral spines irregularly arranged. Genus Acanthochiasma. With ten spines, $A$. fusiforme, found near Plymouth and in the North Sea. A. cruciata, A. krohnii, generally distributed in the Atlantic.

- Sub-Order 2. Acanthonilda. With twenty spines arranged in four zones of five spines to each (Müller's Law).

Family 1. Acanthometridae. Spícules circular in transverse section. Genera-Acanthometron; proximal end of spines without flange ; A. pellucidum, N. and E. Scotland. Phyllostaurus, with flange; $P h$. quadrifolius, abundant in North Atlantic.

Family 2. Zygacanthidae. Spines compressed and double-edged, lanceolate in section. Genus-Zygacantha, without flange at base of spines; Z. septentrionalis, North Atlantic.

Famili 3. Acanthoniddae. Spines cruciform in cross section. Genus-Acanthonia; $A$. mülleri, N. Scotland and North Sea; $A$. ligurina, W. coast of Greenland; Acanthonidium; A. echinoides, North Sea, Faroes and Norway ; A. pallidum, N. and E. coasts of Scotland.

Family 4. Amphilonchidae. Two opposite spines much larger than the rest. Genus-Amphilonche. A. belonoides, generally distributed in the Atlantic. For the exotic family Lithopteridae, see Haeckel's Monograph (11).

\section{Order 2. Acanthophractida.}

Sob-Order 1. Sphaerophracta. With twenty radial spines of equal size. Shell spherical.

Family 1. Sphaerocapsidae. Shell composed of very numerous small plates each with a single pore. Genera-1. Sphaerocapsa. Sph. cruciata, Faroes, North Atlantic. 2. Astrocapsa. A. tritonis and $A$. coronata, Faroes and North Atlantic. 3. Porocapsa. P. murrayana. 4. Cannocapsa. C. osculata, Faroe Channel and North Atlantic.

Family 2. Dorataspidae. Shell composed of the meeting branches of two to four apophyses given off by the twenty radial spines. Seventeen genera are known, mostly from equatorial or southern waters.

Family 3. Phractopeltidae. Shell double; the inner one enclosed by the central capsule. No genera known from northern waters.

Sub-Order 2. Prunophracta. Two or six spines much larger than the rest. Shell not spherical.

Family 1. Belonaspidae. Shell ellipsoidal. Two enlarged spines. The genus Platnaspis occurs in North Atlantic and Mediterranean.

Family 2. Hexalaspidae. Shell lentelliptical. Six enlarged spines. The genus Hexaconus is known from the North Atlantic.

Family 3. Diploconidae. Shell diploconical with two opposite large funnels (the sheaths of the two enlarged spines). Pseudopodia con- 
fined to the two polar apertures. The genus Diploconus is known from the Mediterranean.

\section{Sub-Class III. Monopylaria (Nassellaria).}

Radiolaria with monaxonic central capsule that bears at one pole a porous plate forming the base of an inwardly directed cone.

\section{Sub-Legion 1. Plectellaria.}

Without a coinplete lattice-shell.

Order 1. Plectordea. Skeleton a basal tripod (Fig. 5). Selected forms :-Plagiacantha arachnoides, Clap., W. coast of Norway, North Sea ; Plagiocarpa procyrtella, H., North Atlantic, Iceland; Hexaplagia arctica, H., Greenland ; Polyplagia novenaria, H., Faroe Channel, North Atlantic; Plectophora arachnoides, $\mathrm{H}$., and $\mathrm{Pl}$. novena, $\mathrm{H}$., North Atlantic and Faroe Channel, North Sea.

Order 2. Stephordea. Skeleton a sagittal ring, and usually no tripod. Selected forms:-Lithocircus annularis, Müll.; Cortiniscus typicus, H. ; Eucoronis nephrospyris, H. ; all cosmopolitan.

\section{Sub-Legron 2. Cyrtellaria.}

Skeleton a complete lattice-shell (cephalis).

Order 1. SpYrordea. Cephalis bilocular with cephalic construction. Almost exclusively southern forms.

Order 2. Botryoidea. Cephalis multilocular. Selected forms :Botryocampe inflata, Ehr., cosmopolitan ; Phormobotrys hexathalomia, H., Mediterranean.

Order 3. Cyrtordea. Cephalis single, without constrictions or lobes. Selected forms:-Tridictyopus elegans, Hert., Mediterranean ; Cornutella clathrata, Ehr., cosmopolitan ; Cyrtocalpis obliqua, H., cosmopolitan ; Lithomelissa thoracites, H., cosmopolitan ; L. setosa, H., Norway ; Eucecryphalus gegenbauri, H., cosmopolitan ; Carpocanium diadema, H., cosmopolitan ; Dictyocephalus ocellatus, H., Faroe Channel ; Dictyophimus clevei, Jörg., Norway; Theoconus ariadnes, H., cosmopolitan ; Cladoscenium tricolpium, Norway ; Clathrocyclas craspedota, Norway.

\section{Sub-Class IV. Tripylaria (Phaeodaria).}

Radiolaria in which the central capsule is double and usually possesses a chief aperture (astropyle) and two accessory apertures (parapyles). A dense resistant pigment (phaeodium), probably of excretory nature, accumulates in the extracapsulum. The skeleton is siliceous and often made up of hollow tubes.

\section{ORDER 1. Phaeocystina:}

The skeleton consists of isolated spicules.

Family 1. Aulacanthidae. Skeleton of tangential needles and radial hollow rods. Selected forms:-Aulacantha scolymantha, H., Hebrides, 
Faroe Channel, Shetlands ; Aulographis zctesios, Borg. ; A. furcellata, Wolf., Faroe Channel ; Au. tetrancistra, H., Norway ; Aulodendron boreale, Wolf., Faroe Channel.

\section{Order 2. Phaeosphaeria.}

Skeleton composed of an extracapsular shell or of two concentric shells separated by the extracapsulum. Outer shell usually spherical.

Family 1. Sagosphaeridae. Outer shell a lattice-work with triangular or areolar meshes. Selected forms :-Sagena ternaria, H. ; Sagosphaera trigonilla, H., cosmopolitan ; Sagenoarium sp., Jörg, Norway.

Family 2. Aulosphaeridae. An outer lattice-shell alone present, the hollow bars of which contain septa. Selected forms:-Aulosphaera flexuosa, H., Faroe Channel ; Auloscena verticillatus, H., Norway ; Aulotractus fusulus, H., Faroe Channel, Hebrides.

Family 3. Cannosphamidae. Inner and outer lattice-shells present, interconnected by radii. Cannosphaera antarctica, H., bipolar form.

Family 4. Porospathidae. Inner shell alone present, composed of two finely grained membranes; elliptical

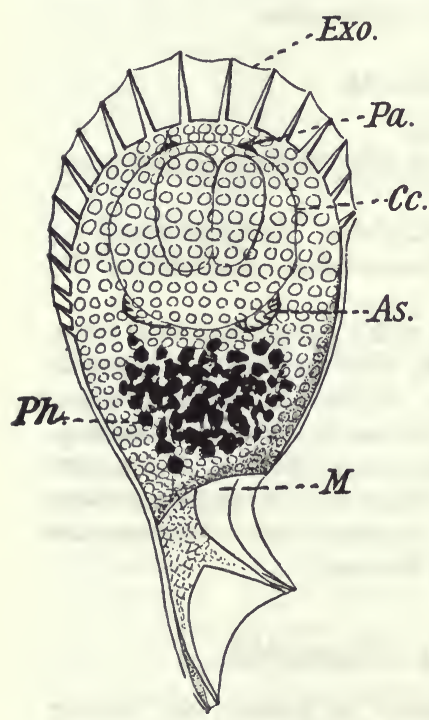

Fig. 27.

Challengeron armatum, Borg. $\times 225$. The mouth $(M)$ of the perforated shell is surrounded by processes, and its aboral surface bears a crest $(E x o)$. The central capsule possesses two astropyles $(A s)$, two parapyles, and two nuclei. The hrown phaeodellae $(P h)$ are shown. (From \& living specimon, after Borgert.)

shell. Euphysetta nathorsti; tiara, H., Faroe Channel. $B$. Small forms $(0 \cdot 8-\cdot 3 \mathrm{~mm}$.), with hooded primary shell provided

\section{Order 3. Phaeogromia.}

A single simple shell present, variable in shape, but always provided with a projecting peristome.

Family 1. Challengeridae. Shell monaxonic, composed of two layers which exhibit an extremely fine diatomaceous graining. Peristome toothed. Selected forms :-Lithogromia silicea, H., Faroe Channel; Protocystis tritonis, H., Faroe Channel, Shetlands, North Sea; Pr. tridens, H., Norway and Sweden; Pr. harstoni, Murray, Norway ; Pr. xiphodon, H., Earoe Channel; Challengeron trioden, balfouri, golfense, johannis, armatum (Fig. 27) ; Cadium melo, Clev.; Pharyngella gastrula, H. ; Entocannula hirsuta, H. ; Faroe Channel.

Family 2. Medosetridae. Primary shell alveolar. Peristome with articulated feet. A secondary shell may be developed in relation to the phaeodium.

A. Small forms (averaging $0.1 \mathrm{~mm}$ in diam.), with primary shell and few radial spines. Phaeodium in primary Clev., North Sea, Scotland; Medusettc 
with six long radial spines. Phaeodium still in the primary shell. Gazelletta, Fowler.

C. Large forms, with conical shell, completely filled by central

Fig. 28.

Atlanticella craspedota, Borgert. In this newly discovered family of Phaeo. daria the central capsule (C.c) is a large inflated 4. lubate structure. The skeleton consists of a me. dian hollow part (M.Sh) and of four pendent septate arms $(S p)$. The black area is the phaeodium $(P h)$. $\times$ 50. (After Borgert.)

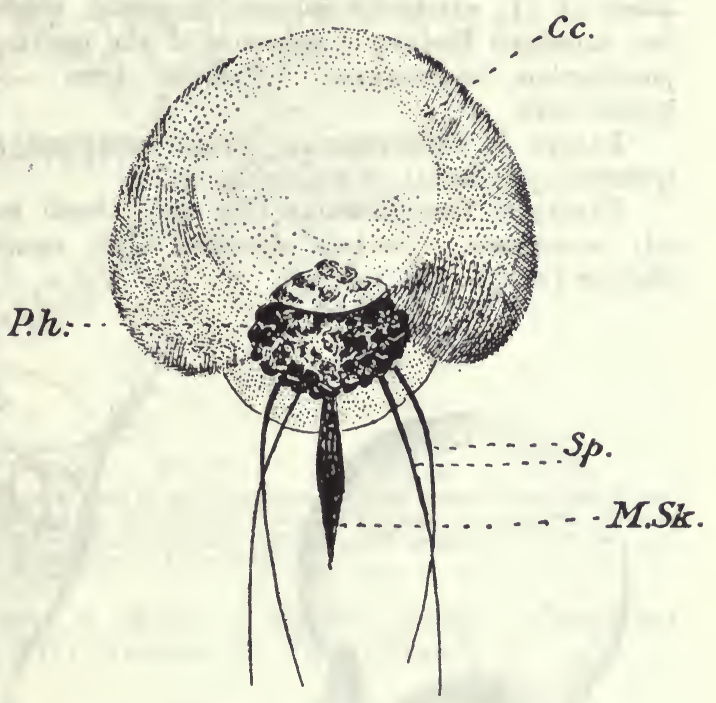

capsule, which is converted into a swim-bladder. A diaphragm, perforated (Häcker [37]) by several astropyles and parapyles, separates endoplasm from ectoplasm (Fig. 12). Phaeodium outside primary

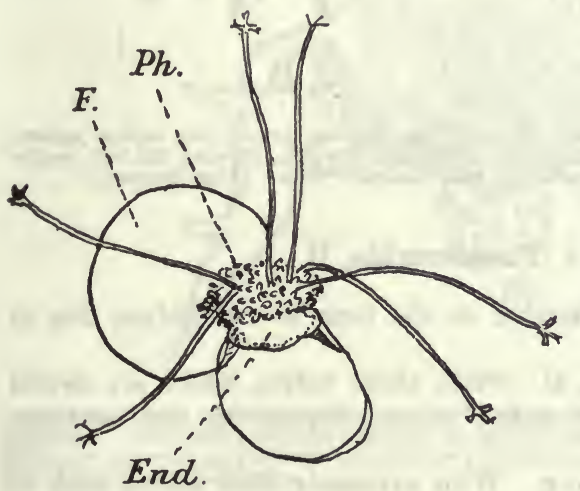

Fia. 29.

Planktonetta atlantica, Borgert. (After Fowler.) $\times 66$. The entire animal is shown as seen in a preserved specimen. One pair of arms is omitted. The central capsule (End) is invested by a skeletal membrane and forms a float. The arms are embedded in the phaeodium $(P h)$ and attached to this is the outer shell $(F)$, comparable with that of Medusetta and Atlanti. cella. A section through this animal is seen at Fig. 12, p. 120.

shell, with intra-phaeodial skeleton. A float present. Planktonetta atlantica, Borg., Faroe Channel $(29,37)$.

$D$. Large forms, without primary shell. Central capsule a swimbladder. Diaphragm and phaeodial skeleton as in preceding sub-family. Secondary shell projecting over peristome. No float. Nationaletta. 
$E$. Mid-sized forms, without primary shell. Secondary shell with four arms. Atlanticella. (Fig. 28.) Borgert (21).

Family 3. Castanelidide. Primary shell two-layered and composed of (1) extremely delicate tangential siliceous needles; (2) the two conjoined limiting membranes of the two layers, united by (3) a porcellanous impregnation. Selected form:-Castanidium apsteini, bipolar (36).

Family 4. Circoporidae. Shell composed as in Fanily 3, but spherical, polyhedral, or inultipolar (36).

Family 5. Tuscaroridae (Fig. 30). Shell rarely spherical, generally monaxonic. Nucleus elongated with sigmoid chromatin band. (Borgert $[21 a]$.)

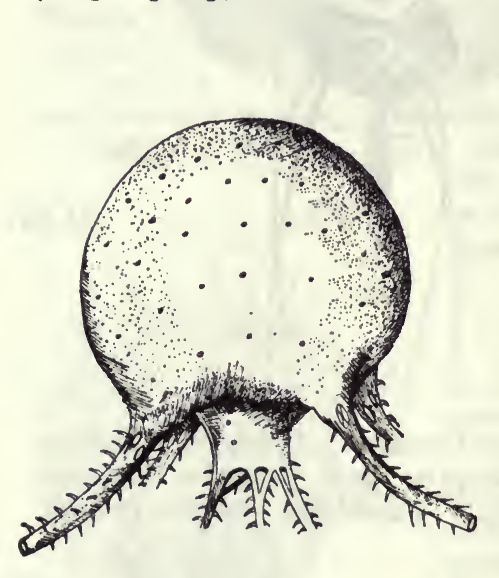

A.

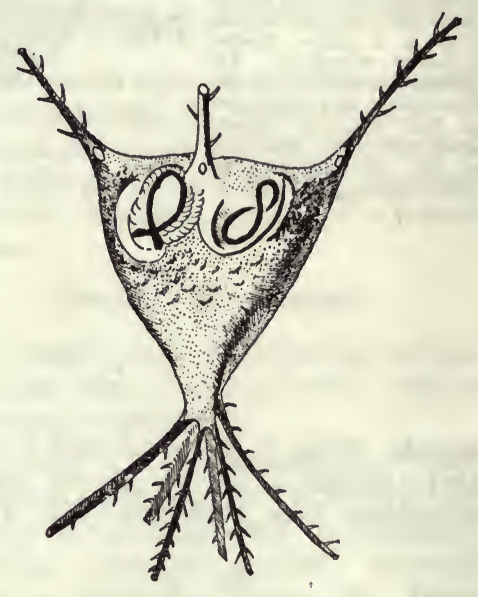

B.

Tnscaroridae. A, Tuscarusa globosa, Borgert, showing the peristomial hollow spines ; the rest are broken off. $\times 39$. B, Tuscarora nationalis, Borgert, showing the two centrai capsules in the shell. Each capsule contains a bent nucleus. $\times 24$. (After Borgert.)

\section{Order 4. Phaeoconchia, H.}

Central portion of the skeleton in the form of two valves, free or hinged together.

Family 1. Concharidae, H. With thick valves, which are devoid of an apical cupola and of radial tubes. Equatorial and southern forms.

Family 2. Coelodendridae. With extremely thin valves, each of which bears a cupola and tubular processes. Coelodendron ramosissimum, Faroe Channel and cosmopolitan.

Family 3. Comlographidae. Each cupola provided with a hollow process (rhizocianna), which communicates with the cupola by a paired or unpaired frenulum. Radial tubes strongly develuped, sometimes forming an outer bivalved mantle. The largest and most complex 


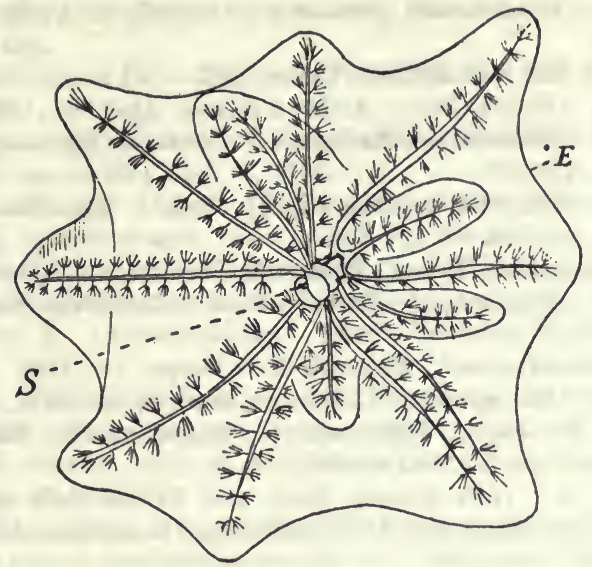

Fia. 31.

Coelothamnus davidoffi, Bütschli; one of the Phaeodaria. Entire animal drawn from a dead specinien. $\times 4$. Sixteen radii spring from the bivalve shell (S) which encloses the central capsule. The ectoplasm $(E)$ is shown investing the skeleton which supports it on the anchorlike extremities of its tufted appendages. (After Biitschli.)

Radiolaria (20-30 mm. in diam.). Selected forms : - Coeloplegma murrayanum, H. (Fig. 32) ; C. tritonis, H., Faroe Channel.

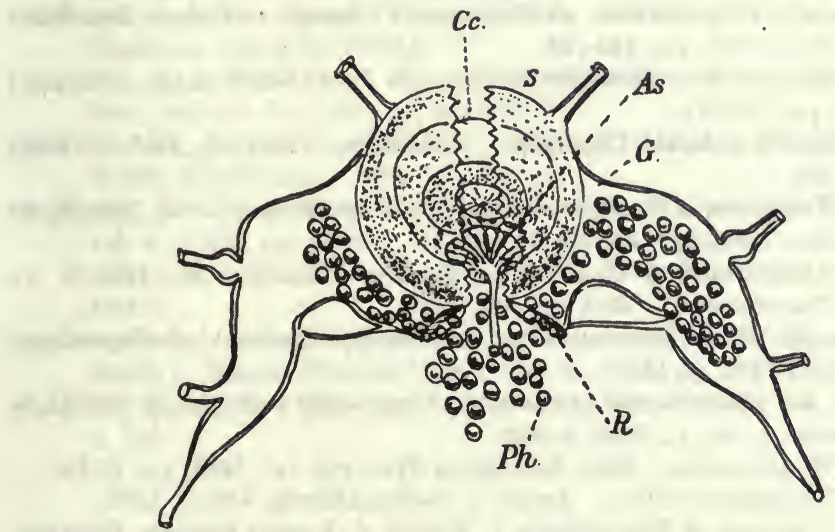

Fro. 32.

Central capsule and adjacent structures of Coeloplegma murrayanum, $\mathrm{H}$.; one of the Coelographidae. The bivalve shell $(S)$ supports the hollow-branched galea $(i)$, in which the phaeodellae are seen emerging through the aperture $(k)$ of the nasal tube (rhizocanna). The astropyle $(A s)$ is drawn out into a tube.

\section{LITERATURE.}

1. Ehrenberg, Ch. G. Monatsberichte d. Berliner Akad. 1844-73.

2. - (Fossil Species.) Abhandl. d. k. Akad. Berlin, 1872, pp. 131-397.

3. Huxley, T. H. (Thalassicolla.) Annals and Mag. Nat. Hist. vol. viii., 1851, pp. 433-442. 
4. Müller, J. (Fundamental Treatise.) Abhandl. d. Berliner Akad. 1858, pp. 1-62.

5. Haeckel, E. Die Radiolarien. Berlin, 1862.

6. Cienkowski. (Yellow Cells.) Archiv f. mikros. Anat. vii., 1871, pp. 372-381.

7. Hertwig, R. (Structure of Radiolaria.) Jenaische Denkschriften, vol. ii., 1879, pp. 129-277.

8. Bütschli, O. (Skeleton of Nassellaria.) Zeit. f. wiss. Zool. vol. xxxvi., 1881, pp. 485-540.

9. - (Monograph.) Bronn's Thierreich, Protozoa, vol. i., 1885, pp. 332-478.

10. Brandt, K. (Sphaerozoa.) Fauna v. Flora d. Golfes von Neapel, vol. xiii., 1885.

10a. - (Zooxanthellae.) Mittheil. Stat. Neapel, iv., 1883.

11. Haeckel, E. (Monograph.) Challenger Reports, vol. xviii., 1887.

12. Lankester, E. Ray. Radiolaria in Encyclopaedia Britannica, Art. "Protozoa," pp. 20-23 of reprint.

13. Famintzin, A. (Life-History, Food, and Yellow Cells of Sphaerozoa.) Mémoires de l'Acad. Sci. St. Pétersbourg, 7 th series, vol. xxxvi. No. 16, 1889, p. 21.

14. Verworn. (Thalassicolla.) Pflïger's Archiv f. Pliysiologie, vol. li., 1891, p. 118.

14a. - (Hydrostatics.) Ibid. vol. liii., 1893, pp. 140-155.

15. Dreyer, F. (Evolution of Radiolarian Skeleton.) Jenaische Zeit. f. Naturwiss. vol. xxvi., 1892, 1p. 204-468.

16. Karawiew. (Fission in Aulacantha.) Mém. Soc. Natur. Kiew. vol. xv., 1896.

17. Borgert, A. (Reproduction of Tripylaria.) Annals and Mag. Nat. Hist. (6), xviii., 1896, pp. 422-426.

18. - (Fission in Aulacantha.) Spengel's Zool. Jahrb. Anat. vol. xiv., 1900, pp. 203-274.

19. - (North Atlantic Tripylaria.) Nordisches Plankton, Lief. i., 1901, pp. 1.52.

20. - (Tripylaria of the German Plankton Expedition.) Zool. Jahr. Syst. vol. xix., 1904, pp. 733-760.

21. - (Atlanticellida.) Ergeb. Plankton-Expedition, vol. iii., 1906.

21a. - (Tuscaroridae.) Ibid. vol. iii., 1906.

22. Vernon, H. M. (Respiration in Collozoum.) Journal of Physiology, vol. xxi., 1897 , p. 443.

23. Brandt, $K$. (Bionomics of Acantharia.) Ergebnisse d. deutschen PlanktonExpedition, vol. i., 1892, p. 338.

24. - (Hydrostatics.) Zool. Jahrbücher Syst. vol. ix., 1895, pp. 27-74.

25. — (Thalassophysidae.) Archiv f. Protistenkunde, vol. i., 1902.

25a. - (Division of Thalassicolla.) Mitteil. d. Vereins Schlesw.-Holstein. Aerzte, 12. Heft, 1890.

26. - (Thalassicollidae.) Ibid. vol. vi., 1905, pp. 245-271.

27. — (Classification of Sphaerozoa.) Zool. Jahrb. Suppl. vol. viii., 1905, pp. 311-352.

28. Wolfenden, $R . N$. (Radiolaria of Faroe Channel and Shetlands.) Journal Marine Biol. Assoc. N.S. vol. vi., 1902, No. 3. Trans. Linn. Soc. vol. x., pt. 4, 1905.

29. Fuwler, G. H. (Planktonetta.) Quart. Journ. Mic. Sci. (2), vol. xlvii., 1903, pp. 133-143. 
30. Fowler, G. H. (Gazelletta.) Quart. Journ. Mic. Sci. (2), vol. xlviii., 1904, pp. 483-488.

31. - (Radiolaria of Faroe Channel.) Proc. Zool. Soc. 1896-98, pp. 991, 523, 1016.

31a. Popowsky, A. (North Atlantic Acantharia.) Nordisches Plankton, Lief. iii., 1905, pp. 43-69 ; Lief. v., 1906.

32. - (Acantharia.) Ergeb. Plankton-Expedition, 1904; Appendix in Archiv f. Protistenkunde, vol. v., 1905, pp. 339-357.

33. Schewiakoff, $W$. (Skeleton, Myonemes, and Flotation of Acantharia.) Mémoires de l'Acad. des Sci. St. Pétersbourg, vol. xii., 1902, No. 10.

34. Immermann, $F$. (Aulacanthidae.) Ergeb. Plankton-Expedition, vol. iii., 1904.

35. Häcker, $V$. (Biological Significance of Tripylarian Skeleton.) Jenaische Zeitschrift f. Naturwiss. vol. xxxix., 1905, pp. 581-648 ; Zeit. f. wiss. Zool. vol. lxxxiii., 1905, pp. 336-375 ; Archiv f. Protistenkunde, vol. ix., 1907, pp. 139-169.

36. - (Challengeridae, Tuscaroridae, Circoporidae of the Valdivia Expedition.) Archiv f. Protistenkunde, vol. viii., 1906; and Verhandl. deutsch. zool. Gesellschaft, vol. xiv., 1906, pp. 122-156.

37. - (Thalasssothamnidae, Medusettidae.) Zool. Anzeiger, vol. xxx., 1906, No. 26, pp. 878-895 (16 figs.).

38. Schröter, O. (Cytocladus.) Zool. Anzeiger, vol. xxx., 1906, pp. 448 and 587.

39. Bütschli, G. (Strontium Sulphate in Skeleton of Acantharia, etc.) Zool. Anzeiger, vol. xxx., 1906, No. 24, pp. 784-789.

40. Delap, $M$. and $C$. (Irish Thalassicollidae.) Scientific Investigations, Irish Fisheries, 1905 (vii.) [1906].

41. Kecble, F., and Gamble, F. W. (Green Cells of Convoluta.) Quart. Journ. Micr. Sci. vol. li., 1907, pp. 167-219.

42. Schaudinn, F. (Trichosphaerium.) Abhandl. d. kgl. preuss. Akad. Wiss. Berlin, Supplement, 1899.

43. Pütter, E. (Respiration of Protozoa.) Zeit. f. allgemeine Physiologie, vol. v., 1905, pp. 566s612. Ibid. vol. vii. pp. 46-53.

44. Hinde, J. G. (F'ossil Radiolaria.) Quart. Journ. Geol. Soc. vol. lv. pp. 38-64.

45. Johnstone, J. (Summary of Recent Work on Marine Nitrogenous FoodStuffs.) Science Progress (N.S.), vol. ii., 1907, pp. 191-210.

46. Klebs, G. (Yellow Cells and Peridinians.) Bot. Zeitung, vol. xlii., 1884, p. 721. 
THE PROTOZOA (continued)

SECTION F.-THE MASTIGOPHORA ${ }^{1}$

CLASS MASTIGOPHORA.

Sub-Class I. Lissoflagkllata.

Order 1. Monadidea.

Tribe 1. Pantostomatina.

Sub-Tribe 1. Holomastigoda.

"2. Rhizomastigoda.

Tribe 2. Protomastigina.

Sub-Tribe 1. Monomastigoda.

2. Paramastigoda.

"3. Heteromastigoda.

"4. Isomastigoda.

Tribe 3. Polymastigina.

Sub-Tribe 1. Trimastigina.

Order 2. Euglenoidea.

"2. Monostomatina.

" 3. Distomatina.

"4. Lophomonadina.

Tribe 1. Euglenina.

"2. Astasiina.

"3. Peranemina.

Order 3. Chromomonadidea.

Tribe 1. Chloromonadina.

"2. Chrysomonadina.

"3. Cryptomonadina.

Sub-Class II. Choanoflaghllata.

Order 1. Craspedomonadina.

2. Phalansteriina.

Sub-Class III. Phytoflagel.lata.

Order 1. Chlamydomonadina.

"2. Volvocina.

Sub-Class IV. Dinoflagellata.

Tribe 1. Gymnodiniaceae.

"2. Prorocentraceae.

,3. Peridiniaceae.

Sub-Class V. Cystoflagellata.

Sub-Class VI. S Silicoflagellata.

1 By Arthur Willey, F.R.S., and Prof. S. J. Hickson, F.R.S. 
THE unicellular organisms which are associated in the class Mastigophora or Flagellata in the wide sense, comprise a very heterogeneous assemblage of forms, having in common the possession of certain characteristic traits of organisation (a single nucleus, one or more contractile vacuoles, one or more flagella), and further united together phyletically by the occurrence of transitional or annectant types.

Our knowledge of the group dates back to the time of Anton Leeuwenhoek, at the beginning of the eighteenth century, while the foundation of the modern system may be safely attributed to the labours of Christian Gottfried Ehrenberg during the early part of last century (1830-1838).

From the most general point of view the peculiar biological interest of the Mastigophora rests upon the fact that, in this more than in any other class of Protista, the formal distinctions which are commonly drawn between the animal and vegetable kingdoms vanish. It was formerly a question whether such and such an order of Mastigophora should be reckoned among the unicellular Algae or among the Protozoa, but this controversy is now practically over, and biological disquisitions upon the group are equally at home and equally necessary in zoological and botanical treatises and journals.

When an organism possesses a green colour, due to the presence of chloroplasts stained with chlorophyll, has a cell-wall that gives the chemical reactions of cellulose, and is devoid of a mouth for the ingestion of solid food, it is usually regarded as a plant. When, on the other hand, an organism bears no chlorophyll, has no cellwall, or has a cell-wall that does not give the cellulose reaction, and possesses a mouth for the ingestion of solid food, it is usually regarded as an animal.

If it were possible to divide the Mastigophora into two divisions, one containing all those forms provided with a mouth and devoid of chlorophyll and a cellulose cell-wall ; and the other containing all those forms without a mouth, bearing chlorophyll and surrounded by a cellulose cell-wall, then the former division could be assigned to the animal kingdom and the latter to the vegetable kingdom. Such a division would, however, be thoroughly unscientific and unnatural. It could only be made by deliberately ignoring obvious genetic relationships. Moreover, such a division would leave out of account a number of organisms-particularly Monadidea-which fail to fulfil all the conditions for their admission into either of the divisions.

It is not by the study of any one stage of the life-history of these organisms that it is possible to arrive at any clear conception of the best distinction that can be drawn between the animal and vegetable kingdoms.

The study of the whole life-history of some of the lower 
animals and plants, however, suggests a line of distinction which is perhaps more in accordance with a natural system of classification.

In the life-history of Ulothrix, one of the Ulotrichaceae, an example of an organism that is universally regarded as a plant, we find two forms of cells. There are the cells of the filamentous thallus, protected by a cell-wall, containing chlorophyll, and, under favourable conditions, growing and increasing in number by fission; and there are the cells provided with two or four flagella that escape from their cellulose investments and eventually conjugate to form a motionless zygospore.

If we compare this with the life-history of such a form as Mastigella, one of the Mastigophora that is universally regarded as an animal, we find that during the phase of life when growth and repeated multiplication by fission occurs the organism is actively moving about by means of its flagellum or its pseudopodia, and that the gametes that it gives rise to are also active and flagellate. Any period in the life-history of Mastigella when active movements cease is not, as in the case of Ulothrix, a period of vegetative growth.

If we regard, then, as marks of distinction between an animal and a plant (1) that the stage of growth and reproduction of somatic cells by fission is marked by a period of active mobiity in the former, and of stability in the latter; and (2) that the flagellate cells of the latter do not grow and divide by fission, but conjugate and give rise immediately to a sedentary zygospore, whereas in the former the flagellate cells may grow and divide by fission, we represent a consideration which has had considerable weight in determining the action of zoologists in including the Mastigophora in the animal kingdom. But the boundary thus drawn, even if it is the best that can be drawn, is itself subject to some exceptions.

In some of the Chlamydomonadina we find, for example, that flagellate individuals similar in general characters to the gametes form a gelatinous investment, withdraw their flagella, grow and divide repeatedly by fission. It is difficult to distinguish this phase of life (the "palmella-stage," as it is called) from a true plant under the terms of our definition. The close relation of the Chlamydomonadina to the Chromomonadina, however, is so clear that to separate them by placing one order in the vegetable kingdom and the other in the animal kingdom on this ground alone would be absurd.

The life-history of the Chlamydomonadina seems to support very strongly the view that some of the families of the lower Algae have sprung from a flagellate ancestry, but it does not justify the assumption that the vegetable kingdom as a whole owes its origin to the class Mastigophora. ${ }^{1}$

1 See Blackman and Tausley (2), and West (22, pp. 32 et seq.). 
It is principally in respect of their modes of nutrition that the Mastigophora appear to betray the mixed animal and vegetable properties, so that as a class they have come to be regarded as mixotrophic micro-organisms. The four possible methods of alimentation-holozoic, parasitic, saprophytic, and holophytic-are all to be met with among the members of this protean series, either separately or in combination. When a single species can vary its metabolism in adaptation to its immediate environment, for example, according as it is exposed to or deprived of the influence of light, it is said to be mixotrophic in the strict sense of the term (Pfeffer).

It is not always easy to assert positively in what manner food is conveyed into the protoplast (protoplasmic body of the cell), but it is certain that holozoic nutrition is often associated in the same species with saprophytic (Monadidea), saprophytic with parasitic, saprophytic with holophytic (Euglenoidea), and, more rarely, holozoic with holophytic (Chromulina). Sometimes three methods are found in combination-holozoic, saprophytic, and holophytic (Ochromonas). It may be stated as a general rule that all Lissoflagellata (i.e. true Flagellata in the restricted sense) are capable of saprophytic nutrition, that is to say, of absorbing nutriment from putrescent substances in an aqueous medium, but that this source of food is usually accessory to some other essential means of nourishment. Where saprophytism is the sole condition of existence, as in the case of the Astasiina, there is reason to regard it as a secondary state derived, in the particular instance quoted, from a condition of holophytism.

The parasitic forms may be described broadly as falling into three categories : ectoparasites (Costia, Stylochrysalis, Silicoflagellata); endoparasites (species of Hexamitus, Megastoma, Tetramitus, Trichomastix, Trichomonas, Trichonymphidae); and haematozoa (Trypanosoma, Herpetomonas).

The non-parasitic Mastigophora are either free-swimming or sessile, solitary or colonial in habit.

Some species are capable of temporary fixation by means of a protoplasmic stalk either of pseudopodial (e.g. Oicomonas sp., Fig. 5 (31)) or of flagellar (e.g. Bodo sp.) origin. Some solitary free forms are closely related to solitary fixed forms (e.g. Euglena and Ascoglena), and many free-swimming colonial genera have sessile representatives(e.g. Dinobryon and Hyalobryon).

The form of association of individuals in the colonies varies. within limits, and there is a great amount of parallelism in this respect between members of different orders. An entire colony or coenobium may attain to a certain degree of individuation, which is. most marked in the Volvocina, but is not wanting in other groups, as is evident from the co-ordinated movements which they execute and from the fact that the whole coenobium may undergo binary- 
fission (Uroglena). On the other hand, the units often retain a facultative independence, and the coenobium may then undergo dissociation (Synura).

The principal forms of association of individuals are the following :-1. Linear aggregates, e.g. Hirmidium, Chlorodesmus, Ceratium; 2. Rosettes, e.g. Bicosoeca socialis, Cyclonexis annularis, Gonium pectorale; 3. Plates, e.g. Proterospongia, Platydorina; 4. Spherical aggregates, e.g. Sphaeroeca, Uroglena, Volvox; 5. Dendroid associations, e.g. Dinobryon, Hyalobryon, Poteriodendron, Anthophysa, Rhipidodendron, Dendromonas, Phalansterium.

Of the above colonial assemblages it is to be remarked that the dendroid form is the most polymorphic in actual appearance. As for transitional forms, it is not difficult to construct a series, while analogies are stupefying in their abundance. Thus a biserial linear aggregate like Chlorodesmus in comparison with a rosette like Cyclonexis is absolutely paralleled by species of the pelagic Ascidian, Salpa. A transition from a rosette to a plate is afforded by Gonium, and from a rosette to a spherical aggregate by the volvocine genus Stephanosphaera, in which the units are arranged in a rosette though surrounded by a common gelatinous envelope.

The Mastigophora as a class may be defined broadly as uninucleated Protista which perform their movements by means of one, two, or several flagella, usually arising at or near the anterior end, i.e. the end which is directed forwards during locomotion. The typical motion of the flagellum has been described as one of circumduction (Delage), by which the cell is drawn along at the same time that it rotates about its axis. The flagellum of a typical Flagellate Infusorian is therefore a tractellum, as opposed to the tail of a spermatozoan, which is a pulsellum. ${ }^{1}$ It acts, however, as a pulsellum in exceptional cases among the Monadidea, and with the Choanoflagellata when they quit their attachment in order to effect change of position.

According to the number, position, and proportions of the flagella we recognise monomastigote forms, with a single porrect flagellum; paramastigote, with one or two small accessory flagella at the base of the main one; isomastigote, with from two to four equal flagella; heteromastigote, with divergent flagella, one directed forwards or transversely, the other directed backwards; polymastigote, with more than four flagella; to which may be added holomastigote forms, with numerous flagella distributed over the entire surface of the cell. The disposition of the flagella has a distinct systematic importance, but of much more limited application than was formerly

- I The terms "tractellum" and "pulselium" were suggested by Prof. Lankester. In some elongate metabolic species (Astasiina) the tractellum is directed straight forwards, and only the apical portion of it executes rapid vibrations, drawing the body slong without rotation. 
supposed, since the phenomenon of parallelism is as strikingly displayed in this respect as in the manner of formation of colonies.

The heteromastigote condition merits particular notice since it characterises an entire sub-class (Dinoflagellata), where the one flagellum is transverse, usually lying in an annular depression, while the other is longitudinal and is also partially protected by a groove, but extends backwards freely (Fig. 10). This is a special manifestation of the heteromastigote condition, but equally interesting examples occur in many families of Lissoflagellata, where the anterior flagellum is normally directed forwards (tractellum) and the posterior flagellum which arises from the body of the cell close to the former is trailed behind. The posterior flagellum in these cases exerts a directive and modifying influence upon the movements of the Infusorian, serving also as an anchor and sometimes as a spring promoting a rapid jerking movement of leaps and bounds like the tail of a Podurid.

The posterior flagellum of heteromastigote Mastigophora may be aptly described as a gubernaculum (Fig. $7(10)$ ) and referred to by that term.

The flagellum is usually so extremely attenuated that it is very difficult to discover any structure in it, but as its base may often be traced from the surface through the ectoplasm to the endoplasm, it seems probable that it consists of an axial filament derived from the endoplasm and a delicate cortical sheath derived from the ectoplasm. It is interesting to note that in the Rhizomastigoda there is an endoplastic axial filament in the pseudopodia (Fig. 1). It is impossible to draw any morphological line of distinction between a flagellum and a cilium, and in the Lophomonadina, for example, the vibratile processes have been interpreted as flagella by those who regard this group as belonging to the Mastigophora and as cilia by those who regard it as a family of Infusoria. Since the discovery that

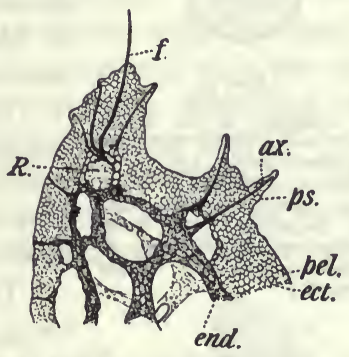

Fig. 1.

Mastigella vitrea, Goldschmidt. Anterior end of an active individual. ax, axial strand from the endoplasm (end) extending into the ectoplasm $(e c t)$ of the pseudopodia $(p s) ; f$, flagel. lum; pel, pellicle; $R$, flagellar reserlum; pel, pellicle; $R$, flagellar reser-
voir. $\times 650$. (After Goldschmidt.) the equatorial groove of the Dinoflagellata (p. 182) is not ciliated, it is usually regarded as a character of the class that true cilia do not occur; and if the vibratile processes of the Polymastigina are true flagella, the only exception to this is to be found in the aberrant genera Pteridomonas, Maupasia, and Monomastix (pp. 164 and 170). As a rule, there seems to be no connection between the base of the flagellum and the nucleus, but such a connection can be traced 
in the genera Mastigamoeba and Mastigina, recalling the relation of the axial filament to the nucleus in certain Heliozoa (p. 23).

At the base of the axial filament there is sometimes found a minute granule, with peculiar staining properties, known as the blepharoblast (Fig. 2, b), and closely associated with this there is in the Trypanosomata ${ }^{1}$ a small detached

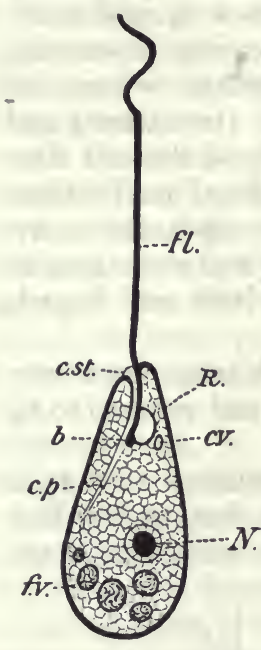

Fia, 2.

Diagram of the structure of Copromonas. $b$, blepharoblast; c.p, cytopharynx; c.st, cytostome ; $c . v$, contractile vacuole ; $f$, flagellum; $f . v$, foodvacuoles; $N$, nucleus; $R$, flagellar reservoir. (After Dobell.) portion of the nucleus known as the "kinetonucleus."

At the base of the flagellum there is often found a special vacuole into which the contractile vacuoles may or may not open (Figs. 1 and 2). This is the flagellar reservoir. In some forms (Trichomonas and Trypanosomata) a delicate undulating membrane is found at one side of the flagellum (Fig. 2, p. 195).

Besides the flagellate movements there are two other important ways by which locomotion can be effected by certain species, namely, by amoeboid and by so-called metabolic or euglenoid changes of shape, the former resulting in the protrusion of pseudopodia, and the latter involving alternate protraction and contraction of the body, as may be observed in many worms (Fig. 5 (28)).

The possibility of executing amoeboid and metabolic movements depends largely upon the nature of the integument or pellicle which protects the protoplast from the surrounding fluid medium.

There are three principal kinds of integument, with many degrees of differentiation:-

1. Periplast.-This is an integral portion of the protoplast, from which it is never separated and with which it divides. In naked cells, such as Mastigamoeba, it appears as a simple ectoplasm covered by a very thin pellicle (Fig. 1, pel), or as an alveolar layer of protoplasm (Multicilia). In most cases there is a more or less welldefined pellicle or plasmatic membrane, which may be distinguished under the name of proteid-membrane. This achieves its highest development in the Euglenoidea, where it often presents a spirally striated structure and resists decomposition (Fig. $5(16,17))$.

2. Perisarc. - The perisarc does not, as a rule, form an integral part of the protoplast, and does not usually divide with it, so that after the division of the protoplast one of the fission-products issues

1 For a discussion of the relations of these structures compare Dobell (3), Minchin (13), Moore (14), T.urtman and von Prowazek.(5). 
from the perisarc as a naked cell. The protoplast is never completely adherent to its perisarc, but is capable of more or less independent movement within it, and recedes from it upon the formation of the resting-stage, and also in consequence of plasmolysis. Its chemical composition is based upon a gelatinous substance of carbohydrate nature, and in Dinobryon Klebs has found that the perisare gives the typical cellulose reaction.

The periplast is always present in Lissoflagellates, but the perisarc is a secondary formation secreted by the protoplast through the periplast, and may or may not be present.

The perisarc may occur as a capsule closely investing the cell with an apical opening for the flagellum, as in Chrysococcus and Trachelomonas. In the Chrysomonadine genera Synura, Mallomonas, Hymenomonas, and Microglena the protoplast is closely adherent to the perisarc, which here tends in the direction of a true cell-wall and is called a cuticle. In Hymenomonas by exception the perisarc divides with the cell.

The most familiar form in which the perisarc is developed is that of a cupule, as in the calyptoblastic Hydroids. Well-known examples of cupule-formation are presented by the genera Bicosoeca, Poteriodendron, Salpingoeca (Fig. $7(6,7)$ ), Dinobryon, etc. Some genera secrete a stalk only, without a cupule, of which Anthophysa and Cephalothamnion are among the best-known examples.

3. Cell-Wall.-This stands in intimate relation with the protoplast, as in Algae and higher plants, so that the cell-body has no independent movement, apart from the automatic streaming of granules. The cell-wall may (Dinoflagellata) or may not (Volvocaceae and Coccolithophoridae) divide with the protoplast. Its chemical composition resembles that of the perisarc, and in the Dinoflagellata consists of cellulose. In the Coccolithophoridae the cellwall is built up of several shells composed of calcium carbonate.

Nucleus. - The nucleus of the Mastigophora shows many varieties of intimate structure. In some cases the chromatin is distributed in the form of a simple chromatic network (Herpetomonas), in others (Bodo, Copromonas, Fig. 2) the chromatin is present in the form of a central lump or mass. In Euglena there is within the nuclear membrane separate chromatin masses, and in addition a substance which has been variously interpreted, but is usually known as the 
"nucleolar centrosome." In Noctiluca (Fig. 3) an archoplasmic body situated outside the membrane accompanies the nucleus and gives rise to the achromatic spindle of the mitotic figure. Mitotic division of the nucleus has been described in a large number of cases taken from all the principal divisions of the group, but it is certain that in some cases nuclear division occurs by amitosis (Copromonas and others, Dobell [3]). Nuclear reduction in the formation of the gametes has been observed in some cases (Trichomonas, Bodo, Hexamitus, Copromonas, and others).

Notwithstanding the great variety of structure and mode of division of the nuclei in the Mastigophora, there is no evidence that in any case a division of the nuclear substance takes place into a somatic nucleus and sexual nucleus, comparable with the meganucleus and micro-nucleus of the Infusoria (Heterokaryota). The separation of the kineto-nucleus from the main nucleus in the Trypanosomata may suggest that in this case there is a delegation of special functions in connection with the flagellum to a detached portion of the nucleus; but apart from this all the Mastigophora are in the strictest sense Homokaryota (Hickson).

The life-history of the organisms comprised by the class Mastigophora shows so many varieties that no general principles can be laid down in this place. The life-histories of several forms are described in the account given of the various subdivisions of the group. The great advance in our knowledge of these forms that has been made during the past few years suggests that a process of gametogenesis followed by conjugation of the gametes occurs in the life-histories of all the orders.

The Mastigophora are an important component of the microplankton of oceanic and lacustrine waters. The Dinoflagellata together with the Algae of the natural order Bacillariaceae, to which the tormer appear to be more or less closely related, are said to constitute the bulk of the primary food-supply (Urnahrung) of the sea [Schütt].

It is customary, in the more recent treatises, to employ the term Flagellata in a restricted sense, equivalent to the Lissoflagellata of Lankester, with the inclusion of the Choanoflagellata. In this sense also the term Euflagellata has been employed, and the flagellate members of the freshwater plankton comprise Euflagellate, Dinoflagellate, and Phytoflagellate ${ }^{1}$ forms. The marine plankton comprises in addition the Cystoflagellata and the Coccolithophoridae.

It is in order to avoid possible confusion that the term Mastigophora, introduced by Diesing in 1866, is employed to designate the entire group of flagellate organisms.

\footnotetext{
1 Sometimes the Phytoflagellata are comprehended within the Euflagellata, but this tends to misapprehension.
} 
The six sub-classes of Mastigophora may be tabulated as follows :-

$$
\begin{aligned}
& \left.\begin{array}{l}
\text { Sub-Class 1. Lissoflagellata } \\
\text { 2. Choanoflagellata }
\end{array}\right\} \text { Euflagellata. } \\
& \text { 3. Phytoflagellata (Volvocaceae). } \\
& \text { 4. Dinoflagellata (Peridiniales). } \\
& \text { 5. Cystoflagellata. } \\
& \text { 6. Silicoflagellata. }
\end{aligned}
$$

The Euflagellata are defined as Protozoa which possess a sharply defined, uninuclear sarcode, whose periplast is either a simple ectoplasm or a definite pellicle. During the greater portion of their life they are in motion, or at least capable of motion. They have a definite anterior end, from which one, two, or many flagella arise, and they possess one contractile vacuole or several. Reproduction takes place by simple longitudinal fission, ${ }^{1}$ generally in the flagellate condition, sometimes in a resting condition. It seems probable that most of the Euflagellata are capable of forming resistent cysts, usually called sporocysts.

The occurrence of a process of conjugation was asserted by Dallinger and Drysdale and others of the earlier observers, but some doubt was thrown upon the accuracy of these statements by Bütschli and Senn. In recent years, however, the formation of definite gametes and a process of conjugation have been proved to. occur in Mastigella by Goldschmidt (4), in Pseudospora by Robertson (18), in Monas and Bodo by von Prowazek (16), in Copromas by Dobell (3), and in Trypanosoma and other forms by Schaudinn (19). There seems to be little doubt, therefore, that conjugation is a normal process in the life-history of all the Euflagellata.

\section{Sub-Class I. Lissoflagellata, Lankester.}

The members of this sub-class are distinguished from the other Euflagellata by the absence of a collar. The sub-class is divided into the three orders:
1. Monadidea.
2. Euglenoidea.
3. Chromomonadidea.

\section{ORDER 1. Monadidea, Bütschli.}

The Monadidea comprise the least differentiated forms of Mastigophora, and include genera that exhibit affinities with the Proteomyxa (Multicilia, Pseudospora, p. 8), with the Lobosa (Rhizo-

1 Cases of true transverse fission are very rare among the Lisso- and Choanoflagellates; e.g. Oxyrrhis, Stylochrysalis, Phalansterium. 
mastigoda), and possibly also with the Heliozoa (Dimorpha). They are colourless Flagellata with one to an indefinite number of flagella, a simple vacuole system, and usually a single nucleus. Their nutrition may be holozoic, parasitic, or saprophytic, but probably never holophytic.

\section{Tribe 1. Pantostomatina, Seun.}

Solid food may be ingested at all points in an amoeboid fashion.

Sub-Tribe 1. Holomastigoda, Lauterborn. With polyaxonic body, flagella scattered all over the surface, pseudopodial ingestion of food, locomotion rotatory, defaecation at all points.

Multicilia, Cienkowski ; M. marina, Cienk., with one nucleus; $M$. lacustris, Lauterborn, plurinuclear, the only instance of the kind among Mastigophora. The genus Grassia, Fisch., closely allied to Multicilia, is found in the alimentary canal of the frog and in the blood of Hyla.

Sub-Tribe 2. Rhizomastigoda, F. E. Schultze. With one or two flagella, natant and amoeboid or heliozooid phases. The flagella persist through the amoeboid or heliozooid phase. The monomastigote and dimastigote genera present a parallel series, and in addition there is an aberrant genus, Pteridomonas, Penard, in which there is a circlet of 8-12 cilia, which can be rolled inwards like a watch-spring and then bent outwards, exerting a

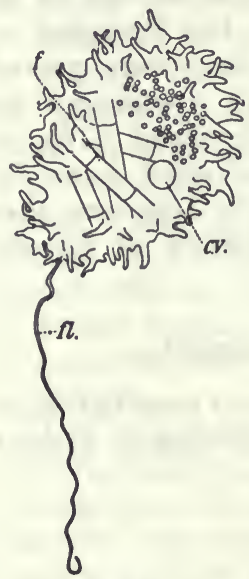

Fro. 4.

Mastigella vitrea, Goldschmidt. One of the Rhizo. mastigoda. Active form. c.v, contractile vacuole; $f$ portions of flamentous algae ingested as fond : $\lambda_{\text {, }}$ flagellum. $\times 250$. (After Goldschmidt.) jerking action by which the animal hops backwards. These cilia surround the base of the single main flagellum.

In the genus Mastigamoeba the flagellum arises directly from the nucleus. The genus Mastigina (Frenzel) is closely related to Mastigamoeba, but the body is covered with a thick pellicle. The position of Mastigella, Frenzel (Fig. 4), is more difficult to determine, as there may be one or more flagella which are quite independent of the nucleus. In Mastigamoeba schulzei (Frenzel) and Mastigina setosa (Gollschmidt) the body is thickly beset with long rigid bristles which have the general appearance of cilia, but seem to have the same nature as the adhesive granules (Klebkörner) with which the pellicle and superficial ectoplasm of several species of the three genera are provided. It is possible that they are of the same nature as the spicules of the ectoplasm found in several of the Lobosa (Trichosphaerium, etc.) and some of the Heliozoa (Heterophrys) (cf. pp. 23, 68).

The life-history of Mastigella vitrina has recently been fully investigated by Goldschmidt (4). During the vegetative life of this animal a series of binary fissions occur which are preceded by a withdrawal of the pseudopodia and flagellum and a mitotic 
division of the nucleus. The number of chromosomes seen in these mitotic divisions is about 40 , and there are no centrosomata at the poles of the spindle. The sexual reproduction is preceded by the formation of mega- and microgametocytes. In the early stages the gametocytes cannot be distinguished from the ordinary vegetative individuals except as regards the microscopic character of the nuclei. A number of minute granules of chromatin (chromidia or sporetia of Goldschmidt) are extruded from the nucleus, increase in number and size, and give rise to the nuclei of the numerous gametes. The cytoplasm of the gametes is formed by a differentiation of clear protoplasm around each nucleus. In the case of the formation of the megagametes at least one mitotic division of the nucleus occurs, which has been interpreted to be a polar division. A similar polar division of the nucleus probably takes place also in the formation of the microgametes. The elements of these nuclei are so small that it has not been proved that a definite reduction in the number of the chromosomes occurs. In both kinds of gametocytes an encystment accompanied by withdrawal of the pseudopodia and flagella occurs, but the microgametocyte encysts sooner than the megagametocyte. The gametes escape from the gametocytes and conjugate to form a zygote. The megagametes are about 3-6 $\mu$ in diameter and are provided with a single flagellum $15-18 \mu$ in length. The microgametes are 2-8 $\mu$ in diameter and are also provided with a flagellum. The zygote is a minute active monad, which divides several times by simple fission and then grows and assumes the general characters of the genus.

The principal genera are :-Amoeboid and monomastigote : Mastigamoeba, Schulze; Mastigella, Frenzel ; Mastigina, Frenzel. Amoeboid and limastigote : Cercobodo, Kent = Dimastigamoeba, Blochmann, and some of the species attributed to the genus Cercomonas (Fig. $5(32,33)$ ). Heliozooid and monomastigote: Actinomonas, Kent. Heliozooid and dinastigote: Dimorpha, Gruber.

Tribe 2. Protomastigina (sensu stricto).

Solid food is ingested at a fixed point near the base of the flagellum.

Sub-Tribe 1. Monomastigoda. ${ }^{1}$ A. Flagellum directed forwards, a. Oicomonas, Kent (Fig. $5(29,30,31)$ ). Ingestion of food at base of flagellum by means of a protuberant vacuole (vacuolar ingestion) which subsequently migrates to the posterior end. $\beta$. Leptomonas, Kent. Rodshaped or fusiform, parasitic in intestine of insects. B. Flagellum directed backwards. a. Ancyromonas, Kent. The single flagellum arises at anterior end, but is bent backwards and serves as an anchor or

1 This sub-tribe comprises the Cercomonadina of Saville Kent or the Oicomonadaceae of Senn. From the work of Klebs and others it seems necessary to reject the genus Cercomonas, since the confusion surrounding it cannot be lightened. 


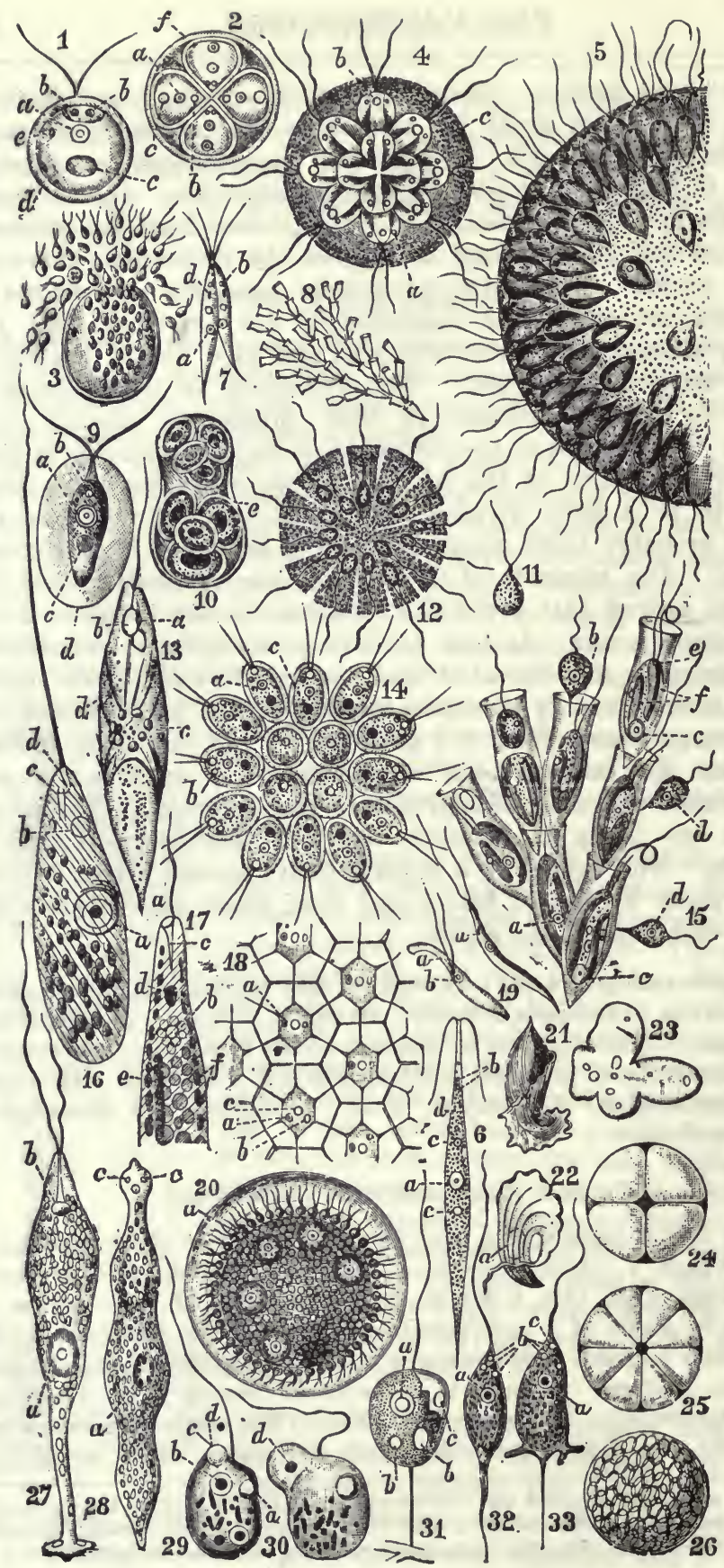

Fra. 5. 
gubernaculum as in Bodo. Marine. $\beta$. Phyllomonas, Klebs. A triangular, contorted, foliaceous monad with the flagellar pole directed backwards in locomotion; the flagellum acts therefore as a pulsellum. Stagnant water. C. Sessile, calyptoblastic genera. a. Codonoeca, Clark, cunstructs a pedunculate, ribbed, colourless theca in which it resides freely. Freshwater and marine. $\beta$. Platytheca, Stein, constructs a membranous encrusting theca.

The Family Trypanomorphidae, containing the single genus Trypanomorpha, Woodcock, belongs to this sub-tribe. A full description of this form is given in Section G, p. 193.

Fia. 5.

1, Chlamydomonas pulvisculus, Ehrb. ; one of the Phytoflagellata ; free-swimming Individual ; $a$, nuclens; $b b$, contractile vacuoles; $c$, pyrenoid; $d$, cellulose investment ; $e$, stigma (eye-spot). 2, resting-stage of the same with fourfold division of the cell-contents; letters as before. 3, apyst that has been formed by the conjugation of gametes and is now liberating a large number of minute biflagellate zooids. 4, Syncrypta volvox, Ehrb.; one of the Chrysomonadina. A colony enclosed by a mucilaginous test $(c) . \quad a$, stigma; $b$, vacuole. 5 , Uroglena volvox, Ehrb.; one of the Chrysomonadina. Half of a large colony. 6, Chlorogonium euchlorum, IChrb. ; one of the Phytoflagellata ; $a$, nucleus ; $b$, contractile vacuoles ; $c$, pyrenoids'; (?) $d$, eyespot. 7, the same species, showing conjugation of the gametes. 8 , a colony of Dinobryon sertuluria, Ehrb. ; one of the Chrysomonadina loricata, $\times 200.9$, Sphcierella palustris, Girod (= Haematocoecus palustris); one of the Chlamydomonadina; ordinary individual with widely separated test, to which it is attached by delicate strands of protoplasm, not shown in the figure; $a$, nucleus ; $b$, contractile vacuole; $c$, pyrenoid. 10, dividing resting-stage of the same. 11, \& gamete of the same. 12, Phalansterium consociatum, Cienk. ; one of the Choanoflagellata, $\times 325$. Disk-like colony. 13, Euglena viridis, Ehrb. ; one of the Euglenina, $\times 300$; $a$, pigment spot; $b$, flagellar reservoir; $c$, paramylum granules; $d$, chromatophores. 14, Gonium pectorale, O. F. M.; one of the Volvocina ; colony seen from the flat side, $\times 300 ; a$, nucleus; $b$, contractile vacuole; c, pyrenoid. 15, Dinobryon sertularia, Ehrb.; one of the Chrysomonadina loricata ; $a$, nucleus; $b$, contractile vacuole ; $c$, paramyluin, (?) nucleus ; $d$, free colourless flagellates probably not belonging to Dinobryon; e, stigma; $f$, chromatophores. 16, Paranema trichophorum, Ehrb.; one of the Paranemina, $\times 140 ; a$, nucleus ; $b$, contractile vacuoles; $c$, pharyngeal apparstus; $d$, mouth. 17, anterior end of Euglena acus, Ehrb., in profle ; $a$, mouth ; $b$, contractile vacuoles; $c$, pharynx; $d$, eye-spot; $e$, paramylum bodies; $f$, chromatophores. 18, part of the surface of Volvox globator, L., showing intercellular connective fibrils; $a$, nucleus ; $b$, contractile vacuole; $c$, pyrenoid. 19 , two antherozooids (= microganietes) of Volvox globator. 20, ripe asexually produced daughter individual of Volvox minor, Stein, still enclosed in the cyst of the parthenogonidium; $a$, young parthenogonidia. 21 and 22, Undulina ranarum, E. R. L. (see Fig. 1, p. 194). 23-26, reproduction of Bodo caudatus, Duj.; one of the Heteromastigoda, according to Dallinger and Drysdale. 23, fusion of several individuals (plasmodiuin). 24, eneysted fusion-product dividing into four. 25, later into eight. 26 , cyst filled with swarn-spores. 27, Astasia tenax, O. F. M. ; one of the Astasiina, $\times 440$ Individual with two flagella and strongly contracting hinder end of the body; $a$, nucleus; $b$, flagellar reservoir. 28, the same devoid of flagella. 29, Oicomonas termo, Ehrb.; one of the Protomastigina, $\times 440 ; a$, nucleus ; $b$, contractile vacuole; $c$, food-ingesting vacuole; $d$, food-particle. 30, the food-particle has now been ingested by the vaciole. 31, Oicomonas mutabilis, Kent, with adherent stalk; $a$, nucleus $; b$, contractile vacuole; $c$, food-particle in food. vacuole. 32, 33, Cercobodo (Cercomonas) crassicauda, Duj., showing two conditions of the pseudopodium-producing tail; $a$, nucleus; $b$, contractile vacuoles; $c$, mouth. (After Lankester and various authors.)

Sub-Tribe 2. Paramastigoda. Solitary or colonial forms with one long flagellum and one (rarely two) short accessory flagellum near its base; vacuolar digestion at the anterior end. A. Solitary genera. Monas, Stein ; Sterromonas, Kent; Physomonas, Kent. Freshwater. B. Colonial genera. Cephalothamnium, Stein; Anthophysa, Bory (Fig. $7(12,13))$. Freshwater.

Sub-Tribe 3. Heteromastigoda. Solitary, colonial, free or attached forms with at least two flagella of different kinds, of which one is directed forwards and another is directed backwards, acting as a gubernaculum or steering flagellum in the free forms or as a stalk of attachment in the fixed forms. A. Free solitary and naked genera (Bodonina, Bütschli) ; Bodo (Fig. 7 (10)), Ehrenberg-freshwater and marine ; 
Pleuromnnas, Perty ; Phyllomitus, Stein ; Colponema, Stein ; Rhynchomonas, Klebs, Oxyrrhis (Fig. 10 (2)), Duj.-marine. Bodo can execute characteristic jumping movements by means of the gubernaculum. It captures its food (bacteria and infusoria) and sucks out the protoplasmic contents by means of a rostral process (rostral ingrestion). According to Dallinger and Drysdale a process of plasmodium-formation occurs in this genus, followed by encystment and subsequent division of the protoplasmic contents into numerous swarm-spores (Fig. 5 (23-26)). In Oxyrrhis there is a large oral funnel and a rudimentary pharynx similar to that of the Euglenoidea. This genus is said to divide transversely instead of longitudinally as in all other Heteromastigoda. The genus Costia (Leclerq) with three flagella, reposing in a groove when at rest, may belong to this sub-tribe (see p. 157). B. Sedentary and usually colonial forms, protected by a cup-shaped or closed theca and attached to the base of it by the gubernaculum. At the anterior extremity there is a plate-like expansion of the ectoplasm (the peristome). (Bikoecina, Stein); Bicosoeca, Clark, solitary or in rosettes; (B. socialis, Lauterborn). Peristome thin and membranous. Freshwater and marine. Poteriodendron, Stein, fixed, "dinobrycil" association of stalked thecate individuals; peristome thick, proboscis-like.

The Bikoecina appear to suggest a transition from the Lissoflagellata to the Choanoflagellata in virtue of their peristome, which is perhaps comparable to the collar.

The Family Trypanosomatidae, containing the blood-parasites Trypanophis, Trypanoplasma, and Trypanosoma, belong to this sub-tribe. The family is fully described in Section G, p. 193.

Sub-Tribe 4. IsomastigodA. Monaxonic boly with two equal flagella at the anterior end. A. Solitary (Amphimonadina). Amphimonas, Duj. ; Streptomonas, Klebs ; Diplomita, Kent. Freshwater. B. Colonial (Spongomonadina). Numerous individuals united "in a common jelly or in branched gelatinous tubes, the end of each of which is inhabited by a single and distinct individual." 1 Spongomonas, Stein ; Cladomonas, Stein; Rhipidodendron, Stein. Diplomita (Kent) is now regarded as an individual of Spongomonas living isolated in the theca of a Bicosoeca. All freshwater.

An interesting Protozoon which is known by the name of Pseudospora volvocis, Cienkowski, and was placed by Bütschli in the tribe Isomastigoda, is found parasitic upon Volvox. According to Robertson (18), it has three forms, each from 12 to $30 \mu$ in diameter. $A$, an amoeboid form; B, a pear-shaped flagellate form, with two flagella at one end ; $\mathrm{C}$, a spherical Actinophrys-like form. In each of these forms there is a single definite nucleus containing a centrally placed karyosome surrounded by clear nucleoplasm. The amoeboid form feeds by ingesting individuals of the Volvox colony, and it gives rise to the flagellate form, which swims away and attacks another colony. Reproduction of the amoeboid form occurs

1 Lankester, E. R., Ency. Brit., 9th Ed., Art. "Protozoa." 
accompanied by a definite mitotic division of the nucleus. Alternation of the amoeboid and flagellate forms with reproduction by fission continues for about eighteen days, and then gametogenesis sets in. The gametes are minute (1-2 $\mu$ in length) uniflagellate organisms, and soon after their escape they conjugate in pairs to form the zygotes. Gametogenesis occurs in the amoeboid form, without encystment or withdrawal of the pseudopodia, and the number of gametes formed by a single individual may exceed one hundred. After a time the zygotes withdraw their flagella, assume a spherical shape, and then creep into a Volvox individual. Gametogenesis may also occur in the radial form, but it has not been observed in the flagellate form.

It does not seem to be certain that the species described belongs to the same genus as others that have been attributed to Pseudospora (see p. 8), but the description of its life-history given by Miss Robertson proves that it is not a Proteomyxan, but is correctly placed with the Mastigophora.

\section{Tribe 3. Polymastigina.}

With more than two flagella (exclusive of Multicilia).

Sub-Tribe 1. Trimastigina. Three flagella. Trimastix, Kent; Dallingeria, Kent ; Elvirea, Parona. Costia necatrix, Henneguy, is a flagellate ectoparasite of the trout which cannot live in infusions, but requires very pure water. It is the only flagellate ectoparasite known which cannot live apart froin its host. It penetrates into the epidermis of the fry, frequently causing a mortal disease. The adult fishes are immune, being protected from the parasite by their scales.

Sub-Tribe 2. Monostomatisia. ${ }^{1}$ Four (rarely six) flagella, one mouth-spot or oral groove, unilateral, asymmetrical. Tetramitus, Perty (Fig. 7 (14)); Collodictyon, Carter; Trichomonas, Donné; Trichomastix, Blochmann ; Monocercomonas, Grassi ; Megastoma, Grassi. Megastoma entericum, parasitic in man and domestic animals, is regarded as intermediate between the Tetramitina and the Distomatina (Klebs), having a unilateral mouth as in Tetramitus and sextuple flagella as in Hexamitus. Trichomonas, Donné. T. intestinalis is found in the intestine of mice. "It is pear-shaped with three flagella springing from the blunt end, and an undulating membrane with a thickened border passing in a spiral manner round the body and terminating in a free flagellum "(Wenyon).

Sub-Tribe 3. Distomatina, Klebs, 1892. Body bilateral but not symmetrical, since the two mouth-spots (oral grooves) are placed on opposite surfaces of the body; flagella arranged in pairs. Principally found in stagnant water. Gyromonas, Seligo, 4 flagella; Trigonomonas, Klebs, 6 flagella ; Trepomonas, Duj., 8 flagella ; Hexamitus, Duj. (Fig. 7 (5)), and Urophagus, Klebs, with 6 or 8 flagella, of which two or three pairs are anterior and the fourth pair are gubernacula (Schleppgeisseln). The two last-named genera are, alone among Flagellata, characterised by

1 Including the Tetramitina, with a wider significance. 
forming, as products of metabolism, glycogen-like bodies (Klebs). $H$. muris is found in the intestine of mice (Wenyon). Lamblia intestinalis is found in the intestines of various mammals, and is not infrequently parasitic in man. It is not thought to be pathogenic. Spironema, Klebs. Polymastigote; flagella arising in pairs at the margins of the spiral mouth-grooves.

Sub-Tribe 4. Lophomonadina, exclusively parasitic in the rectum of insects. This sub-tribe is regarded by some authors as having closer affinities with the Ciliata. It has already been described under the heading Family Trichonymphidae in Fasc. II. p. 417 of this Treatise. The genus Maupasia (Schewiakoff) has the anterior part of the body covered with cilia, but at the posterior end it bears a long flagellum. By some authors it is regarded as a Polymastigine flagellate, but its affinities seem to be with the Ciliata. Freshwater. Hawaii. Monomastix, Roux, is another genus with a polar flagellum and cilia in longitudiual rows. There are said to be two meganuclei and two micronuclei. This genus should also be included in the Ciliata.

\section{ORdER 2. Euglenoidea.}

The second order of Lissoflagellata comprises the most highly organised members of the sub-class. This high degree of specialisation is indicated by the structure of the pharyngeal armature of the tribe Peranemina, which consists of two converging rods; which can be protruded from the base of the oral funnel.

With regard to the nutrition of Mastigophora as a class, to which allusion has already been made, it is necessary, even from a purely systematic standpoint, to consider (1) the nature of the food; (2) the mechanism of ingestion; (3) the products of metabolism. In holozoic nutrition the food consists of bacteria, other monads, swarm-spores of Algae, starch, and the like. The modes of ingestion by which these food-bodies are conveyed into the protoplast of the feeding organism are of five principal kinds, namely, pseudopodial ingestion (Pantostomatina); vacuolar ingestion (Monomastigoda, Paramastigoda, Isomastigoda, Choanoflagellata); rostral or suctorial ingestion (Heteromastigoda); stomatic ingestion, by which the food sinks into the protoplasm through one (Monostomatina) or two (Distomatina) points of least resistance situated in one or two depressions (oral grooves) below the insertion of the flagella; pharyngeal ingestion (Peranemina).

Not only does the mode of feeding distinguish the Peranemina from all other Flagellata, but they are further distinguished by their well-narked, spirally striated periplast or cuticula. In the Euglenoidea the periplast is generally a striated, resistent proteidmembrane.

The vacuole-system of the Euglenoidea consists of a non-contractile or feebly contractile reservoir provided with an excurrent 
canal opening at the apex of the cell, and one or many accessory contractile vacuoles discharging into the reservoir (Fig. 5(17)). A similar kind of compound vacuole-system is also met with among the Peridiniales (Fig. 12).

The products of metabolism which occur in the Euglenoidea consist of fatty oil and paramylum, a substance allied to starch, but not giving the typical starch-reaction. It is interesting to note that the saprophytic Euglenoids of the tribe Astasiina, which are destitute of chlorophyll, none the less produce paramylum.

The Euglenoidea include holozoic, holophytic, saprophytic, and mixotrophic species, and one of the most characteristic properties which they have in common is the formation of paramylum as the principal product of metabolism.

This order presents a series of forms analogous to the Monadidea in regard to the distribution of the flagella: monomastigote forms (Euglena, Peranema, Astasia); paramastigote (Distigma, Sphenomonas, Tropidoscyphus); isomastigote (Eutreptiu); and, finally, heteromastigote forms (Heteronema, Dinema, Anisonema).

The Euglenoidea are divisible into two sections and three tribes.

A. Without special pharyngeal apparatus.

\section{Tribe 1. Euglenina.}

Holophytic. A red stigma or eye-spot close to the vacuole is present, and green chromatophores.

Euglena, Ehrb. (Fig. 5 (13,-17)); Colacium, Ehrb.; Lepocinclis, Perty ; Trachelomonas, Ehrb.; Eutreptia, Perty ; Ascoglena, Stein ; Cryptoglena, Ehrb. Trachelomonas is sometimes found in the sea; the others are freshwater forms.

\section{Tribe 2. Astasina.}

Saprophytic, without chlorophyll. Astasia (Fig. 5 (27, 28)), Duj. ; Distigma, Ehrb.; Sphenomonas, Stein; Menoidium, Perty ; Rhabdomonas, Fresenius; Atractonema, Stein. All freshwater forms.

B. With special pharyngeal apparatus.

Holozoic.

\section{Tribe 3. Paranemina.}

a. With one flagellum. Paranema (Fig. 5 (16)), Duj.; Euglenopsis, Klebs ; Urceolus, Meresch.; Petalomonas, Stein ; Scytomonas, Stein. All found in fresh water, but Euglenopsis flourishes in vegetable infusions. Copromonas, Dobell (3), parasitic in intestine of frogs.

$\beta$. With two flagella. Heteronema, Duj.; freshwater and marine. Dinema, Perty ; stagnant freshwater. Zygoselmis, Duj.; freshwater. Tropidoscyphus, Stein ; freshwater; Anisonema, Duj. ; freshwater Entosiphon, Duj.; marine and freshwater. 
One of the commonest of the Euglenoidea is Euglena viridis, a species which is frequently found in shallow ditches and puddles, giving the water a green tint or forming a green scum on its surface. The free-swimming individuals are about $0.1 \mathrm{~mm}$. in length, provided with a single flagellum arising just in front of a short funnel-shaped cytostome at the pointed anterior end of the body. Opening into the cytostome funnel there is a small reservoir, which itself receives the fluids discharged by a system of minute contractile vacuoles. The chlorophyll is present in the form of numerous minute chloroplasts, and the paramylum in the form of many minute plates. At the base of the flagellum there is a red eye-spot composed of numerous granules of "haematochrome." There is a single nucleus. An important phase in the life-history is the resting stage. The individuals swarm to the surface of the water, where they form the green scum. Each indiviaual in the scum loses its flagellum, and, secreting a gelatinous substance which joins with that of its neighbour's to form a continuous jelly, encysts.

Division of the nucleus and cell-substance takes place during the resting stage at night. The mitotic changes commence about two hours after dark and are completed in five hours. The nucleus has in the resting stage a centrally placed "nucleolocentrosome." This becomes dumb-bell-shaped and then elongates in mitosis. The chromosomes become parallel to this body, and eventually form an equatorial ring round it. In this position they undergo longitudinal splitting (Keuten).

Euglena undergoes several successive divisions under the same cyst-membrane, forming quadrants, octants, etc., but all result from successive longitudinal division, unlike the ciliate infusorian Colpoda, which produces similar clusters resulting from successive crossdivision. Again, in the Volvocines the clusters arise by alternate longitudinal and transverse division (Klebs).

Thus, in the case of Euglena and Copromonas, division takes place after the loss or withdrawal of the flagellum, but in the allied Astasiina division takes place during the motile phase.

Euglena gracilis occurs in both green and colourless conditions, so that, employing Pfeffer's terminology, it may be at one time autotrophic (holophytic), at another time heterotrophic (saprophytic), the two conditions being connected by a mixotrophic transition.

An important contribution to the life-history of the Euglenoidea. has recently been made by Dobell (3). In Copromonas subtilis, from the intestine of the common frog and toad, reproduction is effected by simple longitudinal fission accompanied by amitotic division of the nucleus. After a period of from two to six days a considerable number of individuals are found to be conjugating. All the 
individuals appear to be facultative gametes and there is no soxual differentiation. During the conjugation the nucleus of each of the conjugants divides at least once, one of the daughter nuclei thus produced, being a polar nucleus, degenerates in the cytoplasm and is lost. The remaining nucleus of each conjugant fuses with its fellow to form the nucleus of the zygote. It should be stated that after the first division of the nuclei of the conjugants small granules of chromatin are protruded from the central chromatin mass and are lost in the cytoplasm (heteropolar division). The zygote behaves exactly like an ordinary individual and divides soon after it is formed by longitudinal fission in the ordinary manner.

\section{Order 3. Chromomonadidea.}

This is the first of the groups of Mastigophora that are regarded by many authors as belonging to the vegetable kingdom; for, although there is an active free-swimming stage of life, the method of nutrition appears to be in all cases holophytic. In the Chloromonadina, which may be regarded as in many respects intermediate between this order and the other Lissoflagellata, there is a funnelshaped depression at the base of the flagellum; but this does not serve the purposes of a mouth, but is an excretory duct of the contractile vacuole reservoir. In the other tribes of the order even this vestige of the Lissoflagellate mouth is lost. The Chloromonadina also resemble the Euglenoids in having the chlorophyll scattered through the endoplasm in minute chloroplasts. "No process of conjugation has yet been observed in this order. Among the Chrysomonadina, Chrysamoeba has the ordinary form of a flagellate organism when it is actively swimming, but when it comes to rest it protrudes delicate radiating pseudopodia and resembles a Mastigamoeba.

Chromulina rosanoffi, according to Woronin (23), forms a scum of encysted individuals at the surface of ponds in Finland. This gives rise to the flagellate swarm-spores which after a time penetrate the cells of Spirogyra and again encyst. In Dinobryon the individuals are attached to the base of an open receptacle. They usually occur in dense spreading free-swimming colonies (Fig. 5 (8)). Reproduction is by fission or by the formation of spherical cysts which escape from the receptacle and start new Dinobryoid colonies. Syncrypta (Fig. 5 (4)) forms globular colonies invested by a mucilaginous test through which the flagella protrude. Uroglenu also forms globular colonies, but the flagellate individuals are at the periphery and the centre is filled with mucilage.

The genera comprised in this order are freshwater in habit, except the Coccolithophoridae, which are exclusively marine.

The order is divided into three tribes:- 


\section{Tribe 1. Chloromonadina, Klebs.}

The body is naked, the periplast consisting of a smooth non-resistent membrane formed by a thick layer of ectoplasm, in place of the integument of the Euglenoids. The chloroplasts are generally numerous and the vacuole-system is compound, resembling that of the Euglenoids. The product of metabolism is neither starch nor paramylum, but fatty oil. There is a funnel-shaped depression at the base of the flagellum corresponding with the cytostome, but not used for the ingestion of food. Stein.

Genera-Vacuolaria, Cienkowski ; Coelomonas, Stein; Raphidomonas,

Tribi 2. Chrysomonadina, Bütschli.

The members of this tribe resemble the Protomastigina, with the addition of chromatophores which carry a yellowish-brown pigment called chrysochrome, allied to diatomin. The chrysochrome-plates are usually two in number, placed right and left. They do not contain pyrenoids and do not manufacture starch. There is a red stigma (eye-spot). The products of metabolism are fatty oil and a refringent soluble proteid called leucosin (Klebs).

Nutrition is generally holophytic; there is no mouth ; generally two flagella.

The tribe is divided by Klebs into three sections or sub-tribes :-

\section{A. Chrysomonadina nuda.}

Chrysamoeba, Klebs; Chromulina, Cienkowski ; Ochromonas, Vyssotzki; Stylochrysalis, Stein. The last-named is attached to colonies of Eudorina.

\section{B. Chryomonadina loricata.}

Dinobryon, Ehrenberg (Fig. 5 (8, 15)) ; Hyalobryon, Chrysopyxis, Ehrb.; Chrysococcus, Klebs ; Cyclonexis, Senn.

The researches of Lohmann (11) have shown that the family Coccolithophoridae must be included in this group.

The members of this family are extremely minute organisms, of which the largest species are only $25-50 \mu$ in diameter, found in the plankton of the sea and characterised by the possession of a theca composed of minute calcareous shells which have long been familiar to zoologists under the names "coccoliths" and "rhabdoliths."

The organism bears one flagellum or two equal flagella, a single nucleus, two (rarely one) large green or brown chromatophores, each containing a drop of a substance which appears to be oil (Fig. 6, D), and in many cases a vacuole situated near the base of the flagellum. The body is surrounded by a soft membrane which supports the theca of calcareous shells. The shape of the shells that compose the theca shows immense variety in the family. Two 
kinds have been distinguished, those that are imperforate (discoliths, lopadoliths, calyptroliths), and those that have a central perforation (Fig. 6, B) (placoliths and rhabdoliths). The significance of the perforation in the placoliths and rhabdoliths is not clear, but there is no evidence at present that it transmits protoplasmic processes from the ectoplasm.

When the theca is once formed it is never increased in size by the addition of new shells, but when the growth of the organism

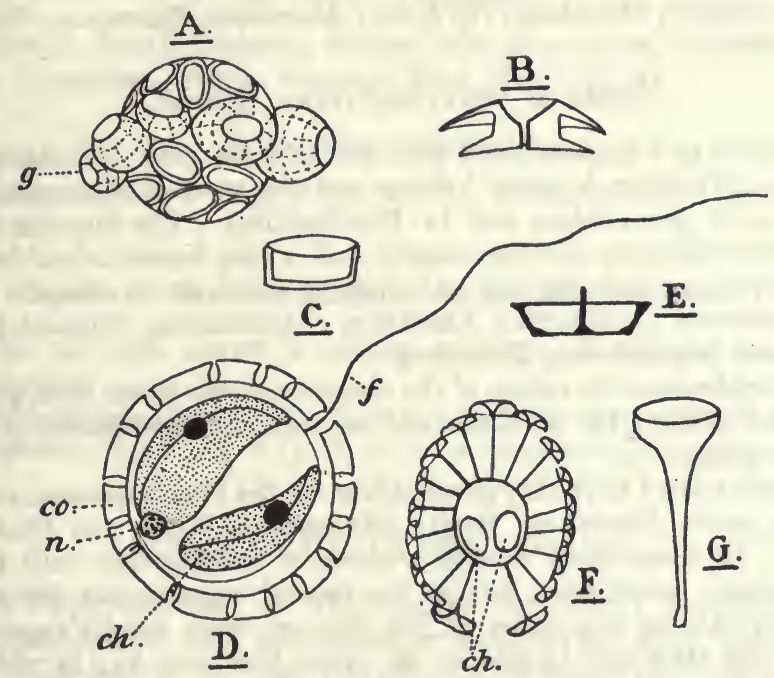

Fro. 6.

To illustrate the structure of the Coccolithophoridae, A, Scyphosphaera apsteini, Lohmann, $\times 2000$, g, a girdle of peculiar enlarged coccoliths. B, optical vertical section of an example of a perfurated coccolith of Coccolithopora leptopora, M. and B. C, side-view of a simple collarshaped imperforate coccolith of Calyptrosphaera oblonga, Lohmann. D, vertical section of Pontosphaera haeckelii, Loh.; co, the sheath of coccoliths ; ch, the two chromatophores, each containing a highly refractive globule; $f$, the flagellum; $n$, the nucleus. E, side-view of one of the coccoliths of the same species. F, Discosphaera tubifer, M. and B. ; ch, chromatophores. G, trumpet-shaped projection from the coccolith of Discosphaera tubifer, $\times 2000$. (After Lohinann and Murray and Blackman.)

requires it, the theca is cast off as a whole and a new one formed in its place.

Reproduction is usually effected by simultaneous longitudinal fission of the theca and protoplasm, but occasionally large thecae are found containing two individuals, indicating that fission of the protoplasm may precede division of the theca or the formation of two thecae.

No evidence has yet been obtained of the formation of gametes.

The Coccolithophoridae are exclusively marine, but are found everywhere except in pure polar waters. They reach their greatest numbers at a few fathoms from the surface. 
Sub-Family Syracosphaerinae. Pontosphaera (Fig. 6, D), Scyphosphaera (Fig. 6, A), Syracosphaera, and Calyptrosphaera-all described by Lohmann.

Sub-Family Coccolithophorinae, Lohmann. Coccolithopora, Loh.; Umbilicosphaera, Loh. ; Discosphaera, Haeck. (Fig. 6, F) ; Rhabdosphaera, Haeck.

\section{Chrysomonadina membranata.}

Mallomonas, Perty ; Synura, Ehrenberg ; Syncrypta, Ehrenberg (Fig. 5 (4)); Uroglena, Ehrenberg (Fig. 5 (5)); Microglena, Ehrenberg; Hymenomonas, Stein.

Tribe 3. Cryptomonadina, Bütschli.

Coloured or colourless forms with one to three green chromatophores or none. Nutrition is never holozoic and the product of metabolism is starch, as in green Algae and in Dinoflagellata. The anterior end is more or less obliquely truncate, usually with a deep frontal infundibulum ${ }^{1}$ like a peristome, from the side or bottom of which the two flagella arise.

Cryptomonas (holophytic), Ehrenberg ; Cyathomonas, Fromentel ; and Chilomonas (saprophytic), Ehrenberg.

In Cryptomonas the colour of the chromatophores varies from green to brown and yellow; two are dorsal and one ventral. Cyathomonas possesses no chloroplasts.

Closely related to the Cryptomonadina are the Phaeocapsaceae, containing the genera Phaeococcus, Borzi ; Phaeosphaera, West; and Stichogloea, Chodat. In these forms a large number of non-flagellate cells form a mucilaginous investment; but as the asexual reproduction takes place principally during this phase of life, they are more usually regarded as algae. The same may be said of the genus Hydrurus, Ag., in which the cells are enclosed in a tough cylindrical mucilaginous envelope.

\section{Sub-Class II. Choanoflagellata, Saville Kent.}

The Choanoflagellata are frequently regarded as constituting a subdivision of the Protomastigina, a proceeding which is in accordance with their affinities, though such is the singularity of their form that it seems quite as appropriate to preserve their independence as to merge them into a larger group. There are no permanently free-swimming species, all are either sessile or pedunculate, solitary or colonial. They can, however, quit their attachment temporarily and swim about with the collar directed backwards. The collar may be defined as a special development of the peristome surrounding the single flagellum which acts as a pulsellum in locomotion. The collar is a contractile protoplasmic process comparable in some respects to an undulating membrane.

The organism feeds by means of vacuolar ingestion, the food

1 Flagellar fundus. See also under Dinoflagellata, p. 187. 
particles being carried down on the outer surface of the collar, at the base of which they sink into the body of the cell.

Several of the genera are found both in the sea and in fresh water.

There are two orders of Choanoflagellata :-

\section{Order 1. Craspedomonadina, Stein.}

A. NUda, Lankester.

Monosiga, Kent ; Diplosiga, Frenzel (with two collars, one within the other); Hirmidium, Perty ; Codosiga, Kent (Fig. $7(3,4))$.

B. Loricata, Lankester.

Salpingoeca, Clark (Fig. $7(1,6,7))$; Polyoeca, Kent; Sphaeroecu, Lauterborn.

\section{OrDER 2. Phalansteriina (= Gelatinigera, Lankester).}

"The cell-units secrete a copious gelatinous investment and form large colonies."

Phalansterium, Cienkowski (Fig. 5 (12)), with inconspicuous collars; Proterospongia, Kent (Fig. 7 (15)), with conspicuous collars.

\section{Sub-Class III. Phytoflagellata.}

The Phytoflagellata or Volvocaceae are clearly related to the Chromomonadidea, and some authors include this order in the subclass. Now that it has been definitely ascertained that conjugation does occur in many of the Euflagellata, the formation of a zygote by the copulation of two gametes is a feature that does not distinguish the Phytoflagellata from the other sub-classes of the Mastigophora. Moreover, although in Copromonas and some other Monadidea the conjugating individuals cannot be distinguished from the asexual individuals, definite micro- and megagametes are formed in the lifehistory of Mastigella, Trypanosoma, and others. The phenomenon of gametogenesis therefore is not a distinguishing character of the sub-class. The Phytoflagellata, however, exhibit a much more definite approximation to a purely vegetable structure than any of the Euflagellata, and it may be convenient to keep them together for the present in a separate sub-class.

The sub-class includes solitary and colonial forms, and the body of the cell-unit is enclosed by a firm cell-wall which sometimes takes the form of a bivalvate shell (Phacolus). In the colonial forms the cell-units are embedded in a gelatinous matrix. There is no indication of pharynx, nutrition being holophytic except in the case of Polytoma, which is a colourless, saprophytic Chlamydomonad. 


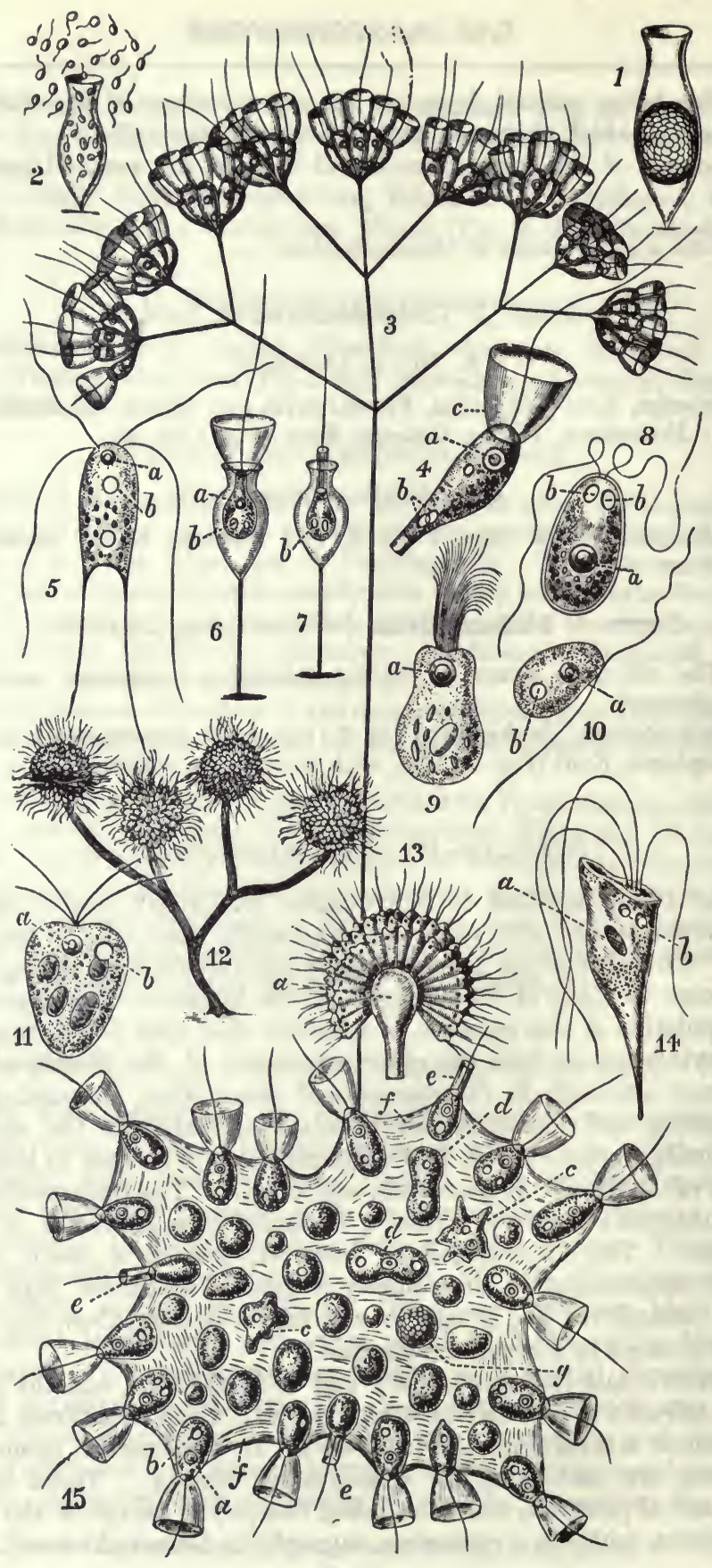

Fig. 7. 
There is usually a single large green chloroplast enclosing one or more pyrenoids, and the product of metabolism is starch. The vacuole-system consists, as a general rule, of two alternately contracting vacuoles. There is a red stigma at or near the flagellar basis. There are never less than two equal flagella, rarely four as in Carteric and Pyramidomonas.

The Phytoflagellata are freshwater in habit.

Among the organisms which are closely related to the Phytoflagellata, but which are regarded in this volume as being just over the border-line between the animal and vegetable kingdoms, we may include the families Pleurococcaceae, Hydrodictyaceae, Protococcaceae, and Palmellaceae. The genera Pleurococcus, Menegh., and Trochiscia, Kützing, belonging to the Pleurococcaceae, have a more definite cell-wall and a more pronounced

FIG. 7.

1. Salpingoeca fusiformis, Kent; one of the Craspedomonadina. The protoplasmic body is drawn together within the goblet-shaped cell, and divided into numerous spores. $\times 1500$. 2 , escape of the spores of the same as monomastigote swarm-spnres. 3, Coclosiga umbellata, Tatem; one of the Craspedomonadina. Adult colony formed by dichotomous growth. $\times 625$. 4 , a single zooid of the same, $\times 1250$. $a$, nucleus; $b$, contractile vacuole; $c$, the collar. 5 , Hexamitus infatus, Duj.; one of the Polymastigina, $\times 650$. Normal adult showing $(a)$ nucleus and $(b)$ contractile vacuole. 6, 7, Salpingoecr urceolata, Kent; one of the Craspedomonadina. 6, with collar extended; 7 , with collar retracted within the stalked cupule. 8, Polytoma uvella, Miull. ; one of the Chlainydomonadina, $\times 800 ; a$, nucleus : $b$, contractile vacuoles. 9 , Lophomonas blattarum, Stein; one of the Polymastigina. 10, Bodo lens; one of the Heteromastigoda, $\times 800$; $a$, nucleus; $b$, contractile vacuole; the wavy filament is a flagellum, the straight one is the ginbernaculum. 11, Tetramitus sulcatus, Duj.; one of the Polymastigina, $\times 430$; $a$, nucleus ; $b$, contractile vacuoles. 12, Anthophysa vegetans, O. F. M. ; one of the Paramastigoda, $\times 300$. A typical, erect, shortly-branching colony stock with four terminal monad clusters. 13, monad cluster in same optical section $(x 800)$, showing the relation of the individual monads to the stem (a). 14, Tetramitus rostratus, Perty, $\times 1000 ; a$, nucleus; $b$, contractile vacnole. 15, Proterospongia haecheli, Kent; one of the Phalansteriina, $\times 800$. A social colony of about forty flagellate zooids. $a$, nucleus; $b$, contractile vacuole; $c$, amoebiform zooid sunk within the comnion test; $d$, similar zooid multiplying by transverse fission; $e$, normal zooids with their collars retracted; $f$, hyaline mucilaginous common test or zoothecium; $g$, individual contracted and dividing into minute flagellate spores (microgametes), comparable to the spermatozoa of a sponge. (After Lankester and various authors.)

vegetative phase of life than Sphaerella, but in other respects are closely related to it. The genus Hydrodictyon, Roth, forms a net-like coenobium which floats at the surface of the water, and Pediastrum, Meyen, which is also placed in the family Hydrodictyaceae, a flat plate-like coenobium of cells that is protected by a thick and ornamented cell-wall. Among the Protococcaceae such genera as Botryococcus, Kützing; Tetracoccus, West; Ineffigiata, West, are probably closely related to some ancestral form allied to Sphaerella; but in some of the other genera, such as Selenastrum, Reinsch ; Ankistrodesmus, Corda ; Dactylococcus, Nägeli, in which the cells are elongated and spindle-shaped; and in Archerina, Lankester ${ }^{1}$; and Chodatella, Lemmermann, in which the cell-walls are provided with long, stiff, bristle-like processes, there is a more pronounced diversion from the Chlamydomonadine ancestry.

The family Palmellaceae has diverged from the same ancestry by the development of a conspicuous envelope of mucilage, but it contains some

1 The genera Golenkinia, Chodat, Richteriella, Lemmermann, and Phythelius, Frenzel, are probably the sanie as Archerina (see p. 33). 
of the most primitive of the Chlorophyceous Algae. The principal generi are Palmella, Lyngbye; Palmodactylon, Nägeli ; Sphaerocystis, Chodat; Schizochlamys, A. Br. ; Tetraspora, Link ; Apiocystis, Nägeli ; Gloeocystis, Nägeli ; and Palmodictyon, Kützing.

\section{ORDER 1. Chlamydomonadina.}

Solitary forms in the flagellate phase.

In Chlamydomonas, which may be taken as an example of this order, there are two flagella in the free-swimming stage, the body is enclosed in a cellulose investment, there are two small contractile vacuoles at the anterior end, a stigma (eye-spot), a single nucleus, and one or more pyrenoids. Two individuals may conjugate and form a zygote. The zygote encysts, the flagella being lost, and the protoplasmic contents divide into as many as sixty-four cells (Fig. 5 (3)); these cells escape as flagellate individuals similar in general characters to the gametes, but instead of conjugating they form a gelatinous investment, lose their flagella, and divide repeatedly (the "palmella-stage"). From the gelatinous investment of the colony that is thus formed the flagellate gametes ultimately escape. Reproduction may also occur by the formation of a resting cyst and the division of the cell-contents into two, four (Fig. 5 (2)), or eight cells, which escape in a form like the parent.

The introduction into the life-history of this genus of a nonflagellate "palmella-stage" during which growth and reproduction take place has suggested that Chlamydomonas "is the phylogenetic starting-point of the various lines of Chlorophyceous descent" (Blackman and Tansley). That there is a strong resemblance between the swarm-spores of many Algae and flagellate forms such as Chlamydomonas cannot be denied, but the conclusion that all the green Algae are descended from a flagellate ancestry is not universallv accepted (see West [22], p. 33).

Sphaerella, Sommerfeldt, 1824, is probably the correct generic name for a very abundant organism found in rain-pools, water-butts, etc., that is sometimes called Haematococcus, Agardh ; Chlamydococcus, Braun; or Protococcus, Huxley and Martin. The individuals may become brick-red owing to the presence of "Haenatochromin," and give rise to the phenomena known as "red rain" and "red snow." The structure and life-history of this organism are very similar to that of Chlamydomonas. The infecting organism which forms the green cells in the Turbellarian worm Convoluta roscoffensis is, according to Keeble and Gamble (7), a Chlamydomonad allied to Carteria.

The principal genera are :

Carteria, Diesing, with four flagella; Chlamydomonas, Ehrenberg; Sphaerella, Sommerfeldt (Fig. $5(9,10))$; Haematococcus, Agardh; Polytoma, Ehrenberg (Fig. 7 (8)); Chlorogonium, Ehrenberg (Fig̀. 5 (6)); Pyramimonas, Schmarda. 


\section{ORDER 2. V̇olvocina.}

Individuals biflagellate, arranged in colonies called "coenobia," of definite forms, with a gelatinous matrix. Reproduction takes place by the cleavage of certain individuals (cells) of the colony called the gonidia. There are two kinds of gonidia-the parthenogonidia or asexual forms, and the gametogonidia or sexual forms. The gametogonidia consist of the oogonidia or female gametes and the antherogonidia or spermatozooids. These conjugate to form the zygotes.

The volvocine colony is physiologically an individual organism, exhibiting histological differentiation and correlated locomotor activities of the constituent cells. In Eudurina the cells are differentiated into male and female, the male cells arising from the anterior quartet, the remainder becoming female. In Volvox the reproductive cells, both parthenogonidia and gametogonidia, are limited to a few of the cells which compose the coenobium. In Pleodorina the parthenogonidia are confined to the posterior hemisphere (Fig. 9).

Protoplasmic intercellular connections between the cells (individuals composing the colony) only occur in the genus Volvox, in apparent correlation with the high degree of individuation attained by this form. Each cell or "coenocyte" is contained within its own capsule, which is separated from neighbouring capsules by a radial cell-wall. The sarcode is separated from the cell-walls by a wide space which is occupied by the gelatinous matrix, and protoplasmic processes radiate through the matrix and traverse the cell-walls (Fig. 5 (18)).

The coenobium of Volvox is a sphere ronsisting of a single layer of cells surrounding a central cavity, and thus presents a superficial analogy to the blastula-stage in the embryonic development of Metazoa. The presence of flagella, eye-spots, and contractile vacuoles attest its animal properties, while the presence of chromatophores, pyrenoids, and starch granules proclaim . its vegetable affinities.

The sphere comprises two differently constituted hemispheres. The trophic hemisphere is that which is directed forwards during locomotion, and the component cells are distinguished by the brighter development of the eye-spots. The other hemisphere is the generative hemisphere, in which the oogonidia, antherogonidia, and parthenogonidia are chiefly formed.

Locomotion is rotatory, i.e. forward progression accompanied by rotation about the main axis either to the right or to the left, though sinistral rotation is more frequent than dextral.

In Volvox globator, L., the average number of cells in a mature coenobium is 10,000 , the actual numbers ranging from a minimum 
of 1500 to a maximum of 22,000 . In $V$. aureus, Ehrb., the number of cells varies from 200 to 4400 . In a third species, $V$. tertius, Meyer, intercellular protoplasmic threads are only present in young unhatched colonies, not in the adult condition.

The form of the coenobium varies in the different genera.

Gonium, Müller (Fig. 5 (14)); cells 4-16, arranged in a squarish plate with flagella upon one face only; envelope closely adherent. Stephanosphaera, Cohn; cells 4-8, arranged in a rounded plate with flagella upon une face only; envelope swollen; oval or spherical. Eudorina, Ehrb. ; coenobium ellipsoidal or spherical ; cells 16-64, similar, not crowded nor reaching towards centre. Pandorina, Bory; coenobium ellipsoidal or

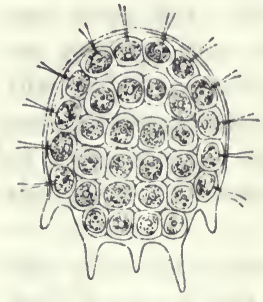

A.

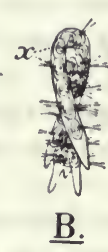

Fic. 8.

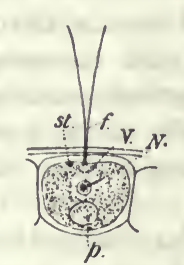

c. spherical ; cells 16-32, similar, crowded, reaching towards centre; outer membrane or sheath of coerobium showing characteristic concentric stratification. Platydorina, Kofoid (Fig. 8); coenobium horseshoe. shaped, flat, one cell deep, with 3-5 prolongations of the gelatinous matrix at the posterior end; cells 16 or 32 ;

Platydorina crutata, Kofoid. A plate-like Volrocine colony. The two surfaces of the colony are alike. The aspect of the adjacent cells alternates, so that the pole bearing the flagella and stigma of one cell is turned in the opposite direction to that of its im. mediate neighbours, $\mathrm{B}, x$. A, front view of the colony ; $B$, side view ; $C$, a single cell showing, $f$, the flagella ; $V$, the vacuoles; st, the stigna; $N$, the nucleus; and p), the pyrenvid. (After Kofoid.) Platydurina has been observed by its discoverer (Kofoid, 1900) repeatedly during five years, but sexual reproduction has not been seen in this genus. All the cells are gonidial, each capable of dividing to form a daughter coenobium. The daughter colonies acquire the adult form and torsion before escaping from the materual matrix, which then undergoes disintegration. Pleodorina, Shaw (Fig. 9); coenobium ellipsoidal ; cells 32, arranged in 5 circles, 4 in each polar circle, 8 at the equator, and 8 in each intervening tract. Vegetative cells always 4 at the anterior pole. Gonidial cells twice as large as the vegetative.

\section{Sub-Class IV. Dinoflagellata.}

The Dinoflagellata or Peridiniales, formerly called Cilioflagellata under an erroneous impression concerning the nature of the transverse flagellum, are heteromastigote fornıs usually possessing a complete cellulose membrane or cuirass which is never silicified. The chromatophores are predominantly brownish coloured with a 
pigment known as peridinin. Reproduction takes place by oblique tission (Fig. 11) and by swarm-spores. ${ }^{1}$ There are two flagella generally lodged in grooves, of which one traverses the latitude of the body and the other the longitude. The former is ealled the cmnulus or girdle and the transverse flagellum plies within it (Fig. $10(3)$ ). The longitudinal groove is the sulcus harbouring the longitudinal flagellum.

As already indicated, the Dinoflagellata constitute a very important component of the freshwater and marine plankton, the same generic forms occurring in both media. Moreover, they play an important part in the physiology of oceanic life as a whole.

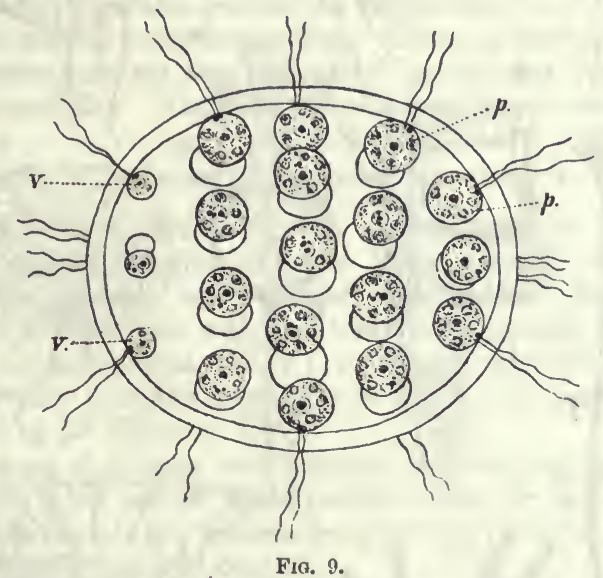

Pleolorina illinoisensis, Kofoil. Colony of thirty-two cells. The four suall cells at the anterior pole are the vegetative cells $(v v)$, the remainder are facultative parthenogonidia, 2?. $\times 300$. (After Kofoid.)

\section{Tribe 1. Gymyodiniacene.}

There is no cuiruss, but the grooves are present. The transverse groove may be semiannular in extent and subcentral in position, with the longitudinal fissure straight and nearly at right angles to it on the ventral side (Hemilinium, Fig. $10(1)$ ) ; or the transverse groove may form a complete ring subterminal in position passing into the ventral longitudinal fissure, the anterior or prae-annular portion being much smaller than the posterior and presenting the appearance of a rostrum (Amphidinium); again, the annulus may be complete and occupy approximately the equator of the cell, and the sulcus straight (Gymnodinium); finally, both annulus and suleus may have a spiral twist (Spirodinium, etc.).

1 Zeilerbaner (24) has described a process of the fusion of the protoplasm of two indiviluals of Cerctium hirundinella which he regards as conjugation, but as the further history of the zygote (?) has not been traced, it nay be only of the nature of plastoganic union such as we find in the Lobosa and Heliozoa. 
The genera thus fall into two groups :-

A. Annulus and sulcus simple, at right angles to one another, decussating at one point, from which the two flagella take their origin.

Gymnodinium, Stein. Freshwater and marine. Hemidinium, Stein (Fig. $10(1)$ ). Freshwater. Amphidinium, Clap. and Lach.

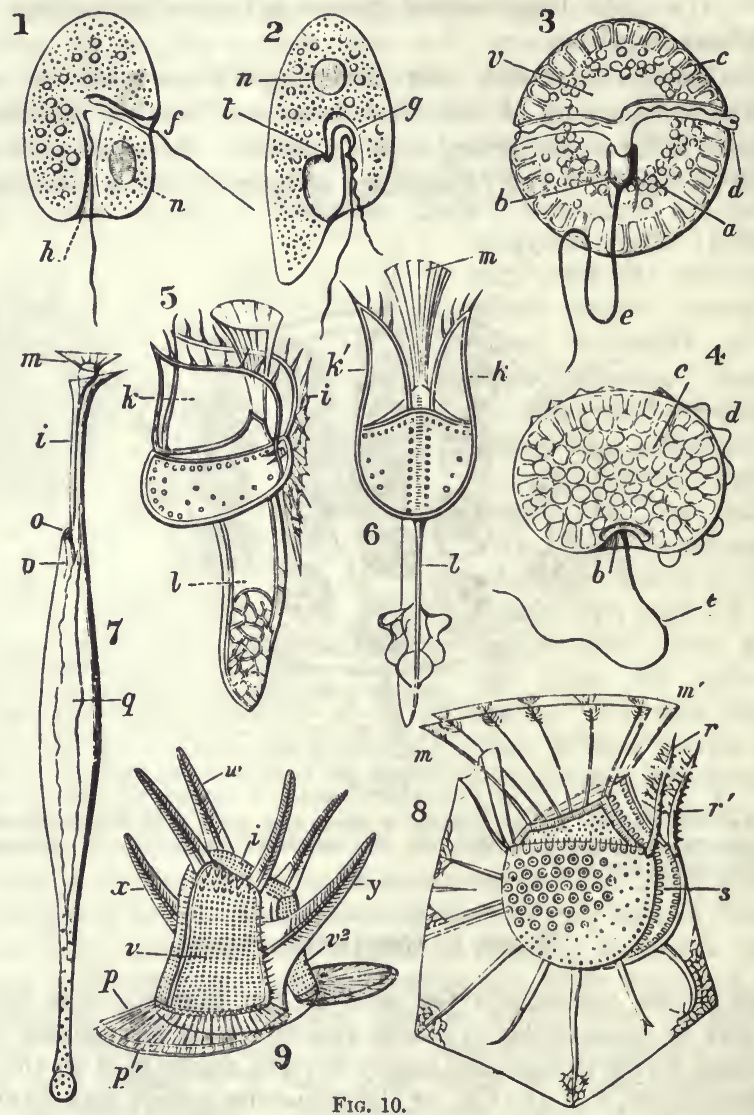

1, Diagram of Hemiclinium, one of the Dinoflagellata; $n$, nucleus; $f$, Hagellum of the transverse groove; $h$, flagellum of the vertical groove. 2, diagrain of Oxyrrhis, one of the Heteromastigoda (to compare with the preceding); $n$, nucleus; $g$, the deep fossa or pit in which the two flagella are affixed : $t$, the origin of the flagellum, which corresponds with that of transverse groove of Dinoflagellata. 3, Glenodinium cinctum, Ehrb., one of the Peridiniaceae; $u$, amyloid granules; $b$, eye-spot; $c$, chromatophores; $d$, flagellum of the transverse groov $\theta$; $e$, flagellum of the vertical groove; $v$, vacuole. 4 , the saine seen from the hinder pole. 5, cuticle of Histioneis cymbalaria, IStein, from the Atlantic; $i$, ventral process ; $k$, cuticular collar; $l$, posterior process. 6 , the same seen from the dorsal surface; $m$, cephalic funnel (epitheca). 7, cuticle of Amphisolenia globifera, Stein, from the Atlantic, seen froin the left side; $m$, epitheca; 0 , the fundus from which the sulcus proceeds to the sub-terminal annulus ; $p$, pharynx; $q$, the shrunken protoplasin. 8, cuticle of Ornithocercus magnificus, Stein, from the Atlantic ; $m, m^{\prime}$, the epitheca ; $r, r^{\prime}$, the two large ribs of the cuticular collar; $s$, the two rows of cuticular teeth. 9, cuticle of Ceratocorys horrida, Stein, from the Southern Ocean ; $p, p^{\prime}$, borders of the annulus expanded into a rim; $w, x, y$, vlumose spines of the left margin of the snlcus. -(After Lankester and various anthors.) 
B. Annulus spiral with a single pitch, sulcus slightly (Spirodinium) or markedly (Pouchetia) spiral, decussating the annulus at both ends. The transverse flagellum arises at the anterior end of the annular spire, the longitudinal flagellun at the posterior end of the sulcar spire.

Spirodinium, Schütt; Cochlodinium, Schïtt ; Pouchetia, Schütt. All marine.

Pouchetia resembles Cochlodinium, but is distinguished by the possession of a complicated stigmatic apparatus consisting of a red or black pigmented body with one or more large refractive lens-like spherules adjoining it.

The interesting genus Polykrikos, Bütschli, consists of two, four, or rarely eight individuals united together into a colonial organisation (Kofoid [10a]). It is also peculiar in the possession, of nettling organs, and is said to present holozoic nutrition. Coasts of Europe and California.

All Gymnodiniaceae may be naked or enclosed temporarily in a gelatinons membrane. The tribe includes marine and fresliwater species.

\section{Tribe 2. Prorocentracene.}

Carapace bivalve, perforated with numerous pores, without annular plates and without annulus, the two halves meeting directly like the edges of two opposed watch-glasses; longitudinal flagellum has the character of a tractellum with the transverse flagellun vibrating about its base; chromatophores yellow; contractile vacuoles represented by pusulae opening into the groove from which the flagella arise at the anterior end of the cell-body.

At the time of division each daughter-cell receives one parent valve and forms the other anew. The Prorocentraceae are entirely marine.

Lotsy (12) regards this tribe as being probably similar in some respects to the ancestors of the Diatomaceae.

Exuviaella, Cienkowski, rounded in front and behind. Prorocentrum, Ehrenberg, heart-shaped, flattened, pointed behind, with rostral prolongation of one of the valves at the anterior or flagellar end.

\section{Tribe 3. Perininiaceae.}

These are characterised by the possession of a multitabulate cellulose carapace or cuirass, each valve being composed of at least two plates which are frequently areolated, and, in addition, there are three or inore annular and sulcar plates. The longitudinal flagellum plies in the sulcus; the transverse flagellum arises at the junction of sulcus and annulus and vibrates in the latter groove (Fig. 12).

The cellulose membrane which constitutes the carapace or cell-wall of the Peridiniaceae is perforated by minute pores and is generally provided with processes which may take the form of horns, spines, or aliform expansions.

Multiplication takes place by oblique longitudinal (rarely transverse) division, each daughter-cell receiving half of the parent carapace, that is to say, half of each valve, and regenerating the other half. Resting sporocysts are enclosed in a gelatinous membrane, and it may be noted 
that the endogrenous formation of swarm-spores results in the production of gymnodiniform young.

Chromatophores, indefinite in number, may be green, reddish yellow, or absent. The reddish-yellow variety of chlorophyll has been named peridinin (Schïtt). The colour of the chromatophores turus green at death owing to the solubility of the peridinin. Many genera comprise both coloured and colourless species, but the latter are furnished with lencoplasts. Otlier plastids ilescribed as fat-forming bodies or lipoplasts are also met with.

- The vacuole-system consists of saccules and pusules discharging into the depression from which the flacrella arise.

The excrescences of the carapace serve as floats for these pelagic organisms and occur as linear (Ceratium, Fig. 11) or foliaceous

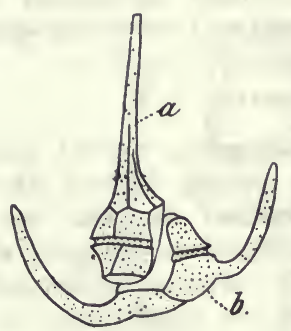

EIG, 11.

Ceratinimertos. Dnesal view shortly after fission, the two daughter individuals still attached to each other. ", the: anterio inclivilual protecteil by the ereater part of tlis: parent's epitlicea; $b$, the pos: terior individual protecter] by the greater part of the hypo. theca. (After scliiitt.)

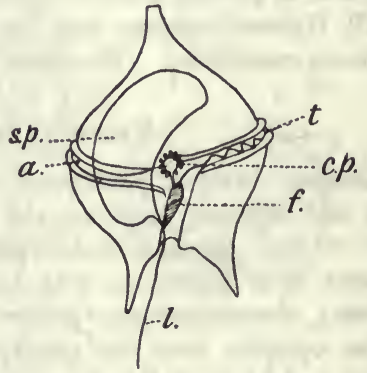

Fic. 12.

Perilinium divergens. Ventral view showing the vacuole-systent. c.p, the small collector-pusule surrounded by a rosette of still sinaller pusules which open into it; s.p, the large sac-pusule or reservoir; both opening into the fundus $(f)$, from which both the transverse flagellum $(t)$ lying in the annulus $(e)$ and the longitulinal flagellum (l) arise. (After Scliitt.)

(Ornithocercus, Fig. $10(8)$ ) expansions. At the anterior or apical end of the cell there is an apical pore which is frequently closed by a perforated plate resembling a nuadreporic plate (e..j. Blepharocystu). The sulcus is ventral, but there is no plane of symmetry.

Some species of Cerctium and Peridinium are found in freshwater lakes, but the other genera appear to be exclusively marine.

In respect of individnal numbers the principal habitat of the Peridiniaceae is in the cold waters of the North Sea, Baltic, and North Atlantic. In point of specific divergence the southern waters are richer. Individual variation is often excessive, and seasonal dimorphism has also been noterl.

Genera and species are determined by the form of the body and by the characters of the cuirass.

The Perilliniacene are divided into four families as follows:-

Family 1. Gutivoniniddae, intermediate between Gymnodiniaceae and Peridiniacene. Cuiriss soft, membranous, consisting of two structureless 
valves with an annulus between them. Glenodinium pulvisculus, Ehrb. (Fig. 10 (3 and 4)).

Family 2. Ptychodiscidae. Body lens-shapel, valves perforate, annulus soft, membranous. Ptychodiscus nocticula, Stein.

FaMily 3. Ceratidiae. The typical genera are the well-known forms of Ceratium and Peridinium. The valves of the cuirass are described as the epitheca and the hypotheca respectively. The former carries the apical pore and the latter the sulcus. But the sulcus sometimes extends heyond its decussation with the annulus up the ventral side of the epitheca to the apex of the cell, e.g. in Steiniella, Schütt, and Gonyaulax, Diesing; or the sulcus may be short, extending equidistantly on either side of the annulus as in Protoceratium, Bergh.

In the genus Ceratium we meet with two-, three-, four-, and fivehurned varieties. The chromatophores of the freshwater species of the genus are green, of the marine species yellowish to brownish in colour.

Some Ceratiidae are spherical, as Blepharocysta, Ehrb., in which the annulus and sulcus are only indicated by the arrangement of the plates. Closely allied to Blepharocysta is the genus Podolampas, St., which has a peridinioid form of body though a different tabulation. Others are fusiform like the remarkable genus Oxytoxum, Stein. In Ceratocorys horrida, Stein (Fig. $10(9)$ ), the borders of the annulus are expanded like the rim of a hat, while the left sulcar margin is expanded into a wing bearing long plumose spines. Pyrophacus is an oyster-shaped Ceratian in which sporulation has been observed by Schuitt. The new genera Heterodinium, Murrayella, Acanthodinium have recently been described by Kofoid.

Family 4. Dinophysidae. The shell is divided by a sagittal suture into two lateral subequal portions. The epitheca is flattened and much smaller than the hypotheca. The borders of the annulus are funnelshaped, and minute brown-coloured corpuscles called Phaeosomata often occur in the space between the two superimposed funnels. The right sulcar border is inconspicuous, but the left borler may be monstrously developed into wings and spines (e.g. Ornithocercus, Fig. 10 (8)).

In Amphisolenia (Fig. 10 (7)) the epitheca is excessively reduced, consisting of two minute plates united together by a sagittal suture. The disproportionately large hypotheca in this genus consists of two elongated plates likewise united by a sagittal suture. The sulcus of Amphisolenia (Fig. 10 (7)) proceeds from the subterminal annulus along the neck of the cell for a distance equal to about one-quarter of the length of the body, terminating at a rather deep pit, representing the depression from which the flagella arise in other forms. This depression nay be conveniently distinguished by the term flagellar fundus or simply the fundus. ${ }^{1}$

In Amphisolenia the protoplasmic contents of the cuirass consist of a nucleus, a moniliform chromatin reticulum, and several ellipsoidal plasmosomes of anyloid character. A pusule situated near the nucleus opens by a slender canal intn the flagellar pore, and one or more accessory pusules may lie near it in the cytoplasm.

1 The German term is "Geisselspalte." It is not a true pharyngeal pit although it strongly resembles one. 
Other genera of Dinophysidae are Phalacroma, St. ; Dinophysis, Ehrb. ; Histioneis, St. (Fig. $10(5,6)$ ), Citharistès, St.; Triposolenia, Kofoid-San Diego region of the Pacific.

\section{Sub-Class V. Cystoflagellata.}

There are only three genera in this sub-class, and of these Noctiluca has long been known as a widely distributed organism that is often the principal cause of the phosphorescence of the surface of the sea. The other two genera are little known.

Noctiluca possesses a sub-spherical body with bilateral.symmetry, the median plane of symmetry being determined by an elongated groove on the ventral side called the peristome (Fig. 15 (5)), at the bottom of which is the mouth. The nutrition is holozoic, and the mouth leads directly into the central part of the protoplasm, from whence trabeculae, exhibiting in life a streaming of the granules, ridiate outwards towards the periphery. In certain regions the trabeculae are concentrated in the form of dense groups of fibrillae giving rise to a fibrillar plexus. One such plexus arises from the posterior end $(f)$ of the central protoplasm, and is inserted along a thickened linear area of the integument behind the peristome called the bacillary organ, "Staborgan" (Fig. $15(5, c))$.

The integument consists of a resistent ectoplasm, a dense reticulate layer of alveolar protoplasm. The striated proboscislike tentacle which arises in the middle line at the anterior end of the peristome, and constitutes one of the most notable features of its organisation, has a length equal to half the diameter of the sphere. It is a flattened contractile organ, convex on its outer side and concave on the inner adoral side. The protoplasmic trabeculae which traverse the tentacle are so disposed as to produce a striatel structure comparable to that of striped muscle-fibres.

Other peristomial organs are the dentiform process; the flagellum, which is borne upon or near a protuberance termed the lip; and lastly the mouth. The tooth ${ }^{1}$ and the lip are placed asymmetrically upon the right wall of the peristome. The mouth occupies the posterior two-thirds of the fundus of the peristome, which is deepest behind and becomes progressively shallower in front. In front of the mouth, that is to say, in the anterior third of the peristome, are the lips, with the flagellum, the tooth, and the tentacle. The flagellum lies well within the peristome and requires practised observation for its discovery. ${ }^{2}$ It resembles the typical flagellum of Mastigophora, namely, a filament of uniform thickness from base to apex. The tentacle can be extruded far beyond the confines of the peristome, but it can also be retracted, rolled up, and so escape superficial observation.

\footnotetext{
1 The tooth is a protoplasmic organ. 2 It was discovered by Krohn in 1852.
} 
The nucleus is lodged within the central protoplasm, and presents during life a transparent, homogeneous appearance.

The ingested food is enclosed in food-vacuoles, which are sometimes so large as to occupy the greater portion of the body. No contractile vacuole has been observed. The products of metabolism consist of albuminoid and fatty granules.

Neither the slow contractions of the tentacle nor the rapid vibrations of the cilium are sufficient to impart movements of progression to the inert body of Noctiluca, which merely drifts with the rest of the plankton, kept afloat by its own buoyancy. The
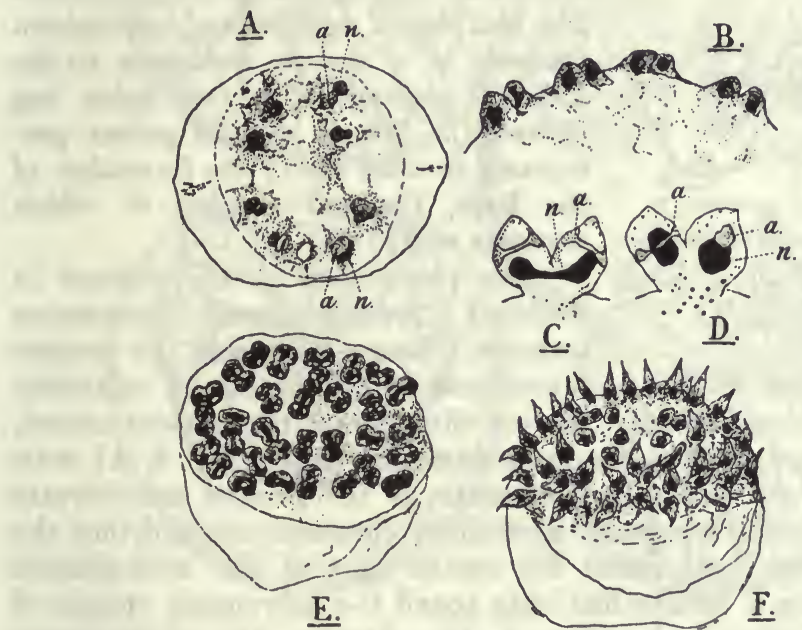

C.
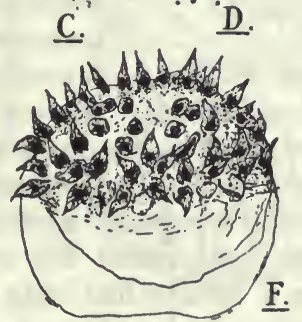

Fio. 13.

Sporulation by blastogenesis in Noctiluca miliaris, Sur. A, surface view of the germinal disc, showing the nuclei that give rise to the nuclei of the spores. Each nucleus $(n)$ is accom. panied by an archoplasmic body (a). B, cleavage-products (buds) in side view. C, D, buds in process of division. The archoplasmic body $(a)$ is seen to have divided before the nucleus $(n)$. $\mathrm{E}$ and $\mathrm{F}$, later stages of blastogenesis. (After Doflein.)

phosphorescence of Noctiluca is the manifestation of its response to mechanical, electrical, thermal, and chemical stimuli. According to the observations of Quatrefages (quoted by Watase), "the light emitted from the whole body, or any of its parts, is composed of a vast number of instantaneous scintillations."

The life-history of Noctiluca comprises the phenomena of simple longitudinal fission (Fig. 15), resting-phase, conjugation, and blastogenesis. The transition of an ordinary individual into the resting condition does not involve the formation of a protective cystmembrane, but simply the degeneration of the peristome and its annexes.

When two individuals come together for the purpose of con- 
jugation, they attach themselves at the peristomial region and gradually fuse together to form a zygote having twice the normal volume. The fusion of the nuclei of the conjugants has been observed directly, under the microscope, by Cienkowski and later by Plate.

It seems likely, although still awaiting demonstration, that the production of swarm-spores (zoospores) by exogenous budding depends upon previous conjugation.

- The production of buds is limited to a particular area of the sphere, namely, the area corresponding with the peristomial region

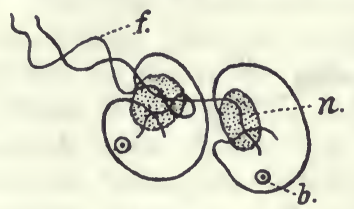

Fia. 14.

Two ripe spores of Noctiluce miliaris, showing $n$, nucleus; $f$, flagellum; $b$, the body interpreted to be a blepharoblast or a centrosome. (After Ishikawa.) where the central protoplasm is massed. The cleavage of nucleus and protoplasm proceeds in a manner analogous to the discoidal cleavage of a yolk-laden egg (Bütschli). Nearly all the parent protoplasm is used up in the formation of the buds, the full number of which amounts to 512 .

The phenomena of karyokinesis in

Noctiluca present some interesting

features. There is outside the nuclear niembrane, but in the neighbourhood of the nucleus, a relatively large archoplasmic body. Before division of the nucleus occurs, this body elongates to assume a dumb-bell shape (Fig. 3, A), with an aster at each end. The chromatin of the nucleus concentrates into a number of elongated moniliform chromosomes, and then the nucleus warps itself round the central part of the archoplasmic body, forming a spindle-like body round the achromatic spindle of the archoplasmic body (Fig. 3, B). Finally, the chromosomes divide into two parties, which travel to the opposite poles of the spindle, and then both nucleus and archoplasm divide transversely.

The buds project from the surface of the body, but remain attached to it until all have attained a certain sizc, and until each has acquired its flagellum, which represents the cilium of the adult Noctiluca.

The detached free-swimming buds have a dinoflagellate appearance, and it may be broadly stated that the blastogenesis of Noctiluca results in the formation of gymnodiniform young (Fig. 14). The growth of the young into the adult condition has not been observed.

The sub-class contains only three genera: Noctiluca, Suriray, 0.3-1.25 mm., probably cosmopolitan; Leptodiscus, Hertwig, 0.6-1.5 mm.; and Craspedotella, Kofoid (8), 0.15-0.18 mm.-E. Pacific. Craspedotella has a strong resemblance to a craspedote medusa in form, being beli-shaped and having a distinct velum at the margin. 


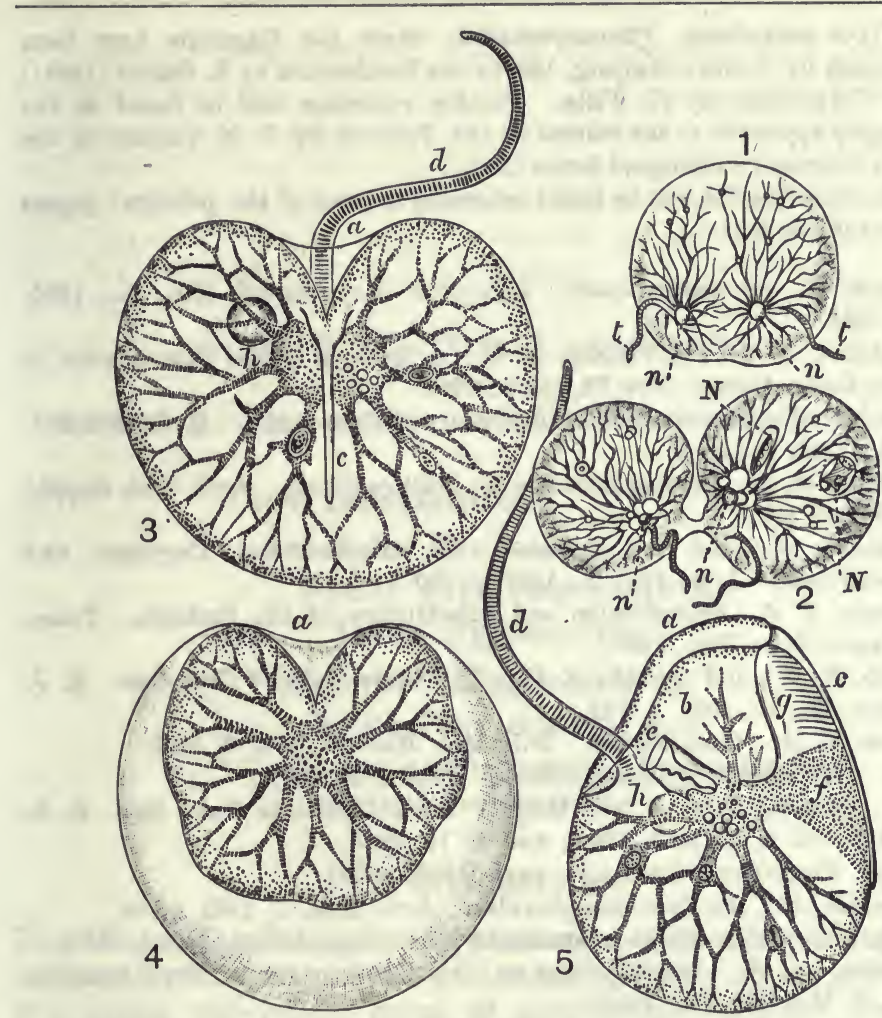

Fis. 15.

Noctiluce milicuris, Suriray. 1, 2, two stages in the longitudinal fission; $n$, nucleus ; $\wedge$, food-particles; $t$, tentacle. 3, aboral view; $a$, entrance to the peristome; $c$, the bacillary organ; $l$, the tentacle; $h$, the nucleus. 4 , the animal acted upon by iodine solution, showing the protoplasm like the "primordial utricle " of a vegetable cell shrunk away from the cuirass. 5 , lateral view, showing (a) the entrance to the jeristome in which $b$ is placed ; $c$, the bacillary organ; $l$, the tentacle; $e$, the mouth and pharynx, in which the flagellum is situated; $f$, broad plexus of fibrillae passing from the central protoplasm to the bacillary organs; $h$, nucleus. After Lankester.)

\section{Sub-Class VI. Silicoflagellata.}

This division of the Mastigophora affords an apparent transition from the Flagellata to the Radiolaria. It is monotypic, comprising the single species Distephanus speculum, Stöhr, which is parasitic upon or commensal with Radiolaria, and while possessing a flagellum, has also a fenestrated siliceous skeleton.

\section{LITERATURE.}

Since the publication of Bütschli's treatise on the Mastigophora in Bronn's Klassen und Ordnungen des Thicrreichs, 13d. i. Abth. 2, 1885, this class of Protozoa has received the fullest general treatment in the pages of Engler and 
Prantl's Die natïrlichen Pflanzenfamilien, where the Flagellata have been written upon by G. Senn (Leipzig, 1900) ; the Peridiniales by F. Schütt (1896); and the Volvocaceae by N. Wille. Further references will be found in the bibliography appended to the volume on the Protozoa by G. N. Calkins in the Columbia University Biological Series (1901).

In the following list will be found references to some of the principal papers mentioned in the text :-

1. Apstein, C. Pyrocystis lunula. Lab. inter. Meeresforsch. Kiel, viii., 1906, p. 263.

2. Blackman, F. F., and Tansley, A. G. A Revision of the Classification of the Green Algae. New Phytol. i., 1902.

3. Dobell, C. C. Structure and Life-History of Copromonas. Q. J. Micr. Sci. lii., 1908, p. 75.

4. Goldschmidt, R. Lebensgeschichte der Mastigamöben. Arch. Prot. Suppl., 1907, r. 83.

5. Hartman, M., and von Prowazek, S. Blepharoblast, Caryosom und Centrosom. Arch. Prot. x., 1907, p. 307.

6. Hickson, S. J. Reproduction and Life-History of the Protozoa. Trans. Manch. Micr. Soc., 1900.

7. Keeble, F. W., and Gamble, F. W. The Green Cells of Convoluta. Q. J. Micr. Sci. li., 1907, p. 167.

8. Kofoid, C. A. Craspedotella. Bull. Mus. Harvard, xlvi. 9, 1905.

9. — New Species of Dinoflagellates. Ibid. 1. 6, 1907.

10. Dinoflagellata of San Diego. Univ. California Pub. Zool. ii. 8, 1906 ; iii. 6, 7, and 8, 1906 ; and iii. $13,1907$.

10c. — Polykrikos. Zool. Anz. xxxi., 1907, p. 291.

11. Lohmann, H. Die Coccolithophoridae. Arch. Prot. i., 1902, p. 89.

12. Lotsy, J. $P$. Vortrïge über botanische Stammesgeschichte. Jena, 1907.

13. Minchin, A. E. Investigations on the Development of Trypanosomes. Q. J. Micr. Sci. lii., 1908.

14. Moore, J. E. S. The Cytology of the Trypanosomes. Ann. Trop. Med. i., 1907.

15. Murray, G., and Blackman, V. H. The Nature of the Coccospheres and Rhabdospheres. Phil. Trans. vol. cxc., 1898, p. 427.

16. Prowazek, S. von. Flagellatenstudien. Arch. Prot. ii., 1903, p. 195.

17. Untersuchungen uber einige parasitische Flagellaten. Arb. k. Gesundheitsamte, xxi., 1904, p. 1.

18. Robertson, MI, Psendospora volvocis. Q. J. Micr. Sci. xlix., 1905, p. 213.

19. Schaudinn, F. Generations- und Wirtswechsel bei Trypanosoma. Arb. k. Gesundheitsamte, xix., 1902, p. 169.

20. Untersuchungen iiber die Fortpflanzung einiger Rhizopoden. Ibid. 1903 , p. 547.

21. Wenyon, C. MI. Observations on the Protozoa in the Intestines of Mice. Arch. Prot. Suppl., 1907, p. 169.

22. West, G. S. A T'reatise on the British Freshwater Algae. Cambridge, 1904.

23. Woronin. Chromophyton rosanofii. Bot. Ztg., 1880.

24. Zederbauer, $E$. Geschlechtliche u. ungeschlechtliche Fortpflanzung von Ceratiun. Ber. d. D. bot. Gesell. xxii. 1, 1904. 


\title{
THE PROTOZOA (continued)
}

SECTION G. - THE HAEMOFLAGELLATES AND ALLIED FORMS ${ }^{1}$

\author{
Order Lissoflagellata. ${ }^{2}$ \\ Sub-Order MONADINA. \\ Family Trypanomorphidae. \\ Genus Trypanomorpha. \\ Sub-Order HETEROMASTIGINA. \\ Family Trypanosomatidae. \\ Genera Trypanophis, Trypanoplasma, \\ and Trypanosoma.
}

\section{INTRODUCTORY.}

The Haemoflagellates, or Trypanosomes, although possessing in common a uniform type of organisation, probably do not all belong to a single, well-defined group of monophyletic origin. They are preferably regarded as an assemblage of forms which have sprung from two quite different stocks, the resemblances exhibited being due to convergence, brought about by the acquirement of similar adaptations in response to their similar and highly specialised mode of life. They are entirely parasitic, their characteristic habitat being the blood of a Vertebrate; and, as is well known, certain of them are the cause of severe, often fatal illness.

The Haemoflagellates possess either one or two flagella. When there are two, they originate close together, at or near the anterior end of the borly. One is free and directed forwards; the other turns back and is attached for the greater part of its length to the side of the body, by means of an undulating membrane, ultimately terminating in a free portion directed posteriorly. Thus a Heteromastigine condition is found. When only one flagellum is present

1 By H. M. Woollcock, D.Sc. (Lond.), Assistant to the University Professor of Protozoology.

2 The classification of the Flagellates here made use of differs somewhat from that adopted in the account of the Mastigophora. The position of the Trypanosomes accorling to that scheme will be seen on reference to pp. $167,168$. 
it is invariably attached in this manuer, but the flagellum is probably not homologous in all these cases. In certain Trypanosomes which are to be derived from a Monadine ancestor, it is, of course, the single flagellum that is represented, with the free part lirected anteriorly ; other forms, however, are rather to be looked upon as derived from a Heteromastigine ancestor, the flagellum that persists being the trailing, posteriorly directed one (the so-called "Schleppgeissel ")." There are two nuclear bodies, one, the trophonucleus, regulating the trophic life of the cell, the other, the kinetonucleus, directing its locomotor activities.

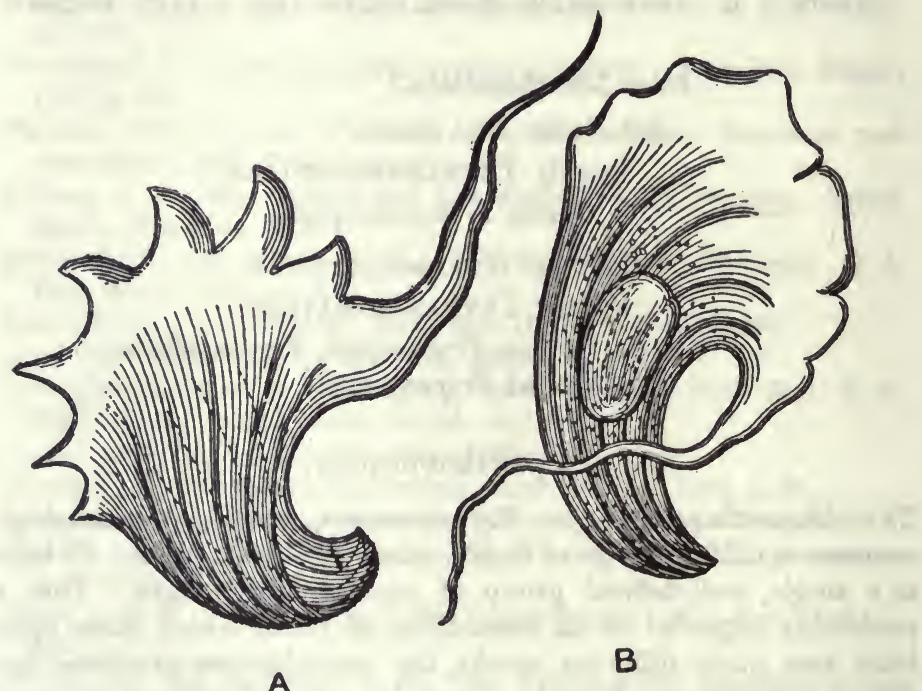

Fig. 1.

"Unululinu rencerum," Lankester, 1sil. In B the nucleus is shown.

The most general method of reproduction is by binary, longitudinal fission; but multiple division or segmentation is also met with. As regards the life-cycle of the parasites, only little is as yet known in most cases. From the results of the most recent researches, however, it certainly appears probable that, apart from various blood-sucking Invertebrates which may (mechanically) transmit a given parasite, there is, in general, a true alternate host for each form; one, that is, in which definite phases of the lifecycle-including, most likely, sexual conjugation-are normally undergone. Further knowledge on this subject is greatly needed.

Historical. - The first observation of a Trypanosome is probably to be ascribed to Valentin, who, in 1841, announced his discovery

1 This flagellum is also termed the gubernaculum (see p. 159). 
of Amoeba-like parasites in the blood of a trout. In the two or three years following, Remak, Berg, and others recorded the occurrence of Haematozoa which were undoubtedly Trypanosomes in different fishes. The parasite of frogs was first seen by Gluge (1842), and in July 1843 Mayer described and figured certain corkscrew-like and amoeboid organisms from the blood of the same animal, which he termed variously Amoeba rotatoria and Paramoecium costatum or loricatum. A few months later (November) Gruby also published (24) an account of this parasite, to which he gave the new generic name of Trypanosoma. 'The same form was subsequently described and figured by Lankester (30) in 1871, who, unaware of Gruby's work, called it Undulina ranarum; this author was the first to indicate the presence of a nucleus in the organism (Fig. 1, B). The well-known parasite of rats was discovered by Lewis, in India, in 1878, and was afterwards named Herpetomonas lewisi by Kent. ${ }^{1}$ It is to Mitrophanow (1883 to 1884) and Danilewsky (1885 to 1889), however, that we owe the first serious attempts to study the comparative anatomy of these Haematozoa. The work of the latter researcher in particular is deserving of recognition, especially when the primitive state of knowledge in regard to blood-technique in those days is borne in mind. Some of Danilewsky's figures of a Trypanosome of birds are reproduced in Fig. 2.

Trypanosomes were first met with in cases of disease by Griffith Evans, who, in 1880 , found them in the blood of horses suffering from Surra in India. The organisms were thought by him to be Spirilla. Steel rediscovered the same form a few years later and took a similar view of its affinities, naming it Spirochacta
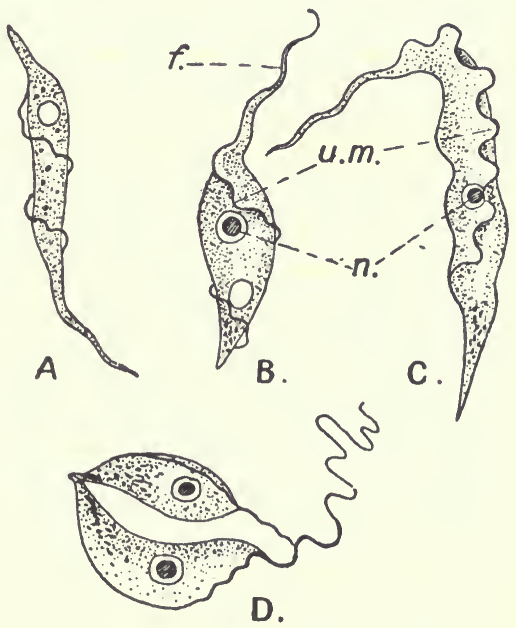

Fig, 2.

A-C, different forms of Tryponosoma sanguinis avium, Danilewsky. D, the same parasite dividing longitudinally. $n$, nucleus; $u . m$, undulating mem. brane; $f$, flagellum. (After Danilewsky.)

evansi. In 1894 Bruce found the celebrated South African parasite (T. brucii) in the blood of cattle and horses laid low with Nagana, or Tsetse-fly disease; and this worker subsequently demonstrated, in a brilliant manner, the essential part played by the fly in transmitting the parasite. Bruce's discovery may be said to have jnaugurated a rapid increase in the number of known forms, the

2 This form is now placed in the genus Tiypcenosoma. 
knowledge of which has in many cases thrown light upon the etiology of maladies previously obscure. Thus, two characteristic diseases, Dourine, which afflicts horses and mules in Northern Africa and the Mediterranean littoral, and Mal de Caderas of horses in South America, were next shown to be caused by different Trypanosomes; and since then many other varieties of trypanosomosis have been described, chiefly from Africa, the home of the dreaded Tsetse-fly.

- Prominent among them, unfortunately, is human trypanosomosis. The credit for first recognising a Trypanosome in human blood, and describing it as such, must undoubtedly be assigned to Nepveu (1898). The parasites were not definitely associated with disease, however, till 1901, when they were seen in the blood of a European in Senegambia suffering from intermittent fever. Forde first found the organisms, but was uncertain of their nature; he showed then to Dutton, who recognised them as Trypanosomes, and gave this form the name of Trypanosoma gambiense. A year later (1902) Castellani discovered what has been shown to be the same parasite in the cerebro-spinal fluid of patients suffering from sleeping-sickness in Uganda, and it has since been conclusively proved by Bruce and Nabarro that this organism is the true cause of that terrible disease.

More important, however, from the standpoint of Protozoology, than these interesting medicaldiscoveries have been the investigations by Brumpt, Laveran and Mesnil, Léger, Minchin, Schaudinn, the Sergents, and others during the last few years upon numerous other, mostly " tolerated" forms; to their researches, indeed, we owe most of our knowledge at the present time, relating to the life-cycle of the Haemoflagellates. And it is fitting, here, to pay a tribute to the value of the characteristic stain first made known by Romanowsky, and its subsequent modifications (e.g. those of Giemsa, Laveran, Leishman, etc.), without which, it is safe to say, this progress would have been impossible.

\section{Occurrence and Transmission; Habitat and Effects oN Host.}

\section{(a) Occurrence and Transmission.}

Trypanosomes are harboured by members of all the chief classes of Vertebrates, with the exception of Cyclostomes. Mammals, birds, and fishes furnish, however, by far the greater number of hosts. Fewer parasites have been described from Amphibia, and up till now only from frogs ; while, among Reptiles, their occurrence has only been observed in two or three instances. Data with regard to the frequency with which individual species are to be met with, in any 
particular kind of host, are as yet somewhat scanty. In one or two cases, however, the parasites are known to be fairly common. Irypanosoma lewisi, for example, occurs in a considerable pcrcentage of sewer-rats throughout the world, having accompanied these rodents in their ubiquitous migrations; the proportion of hosts infected varies usually from 10 to 40 per cent.

In considering the occurrence of Trypanosomes in Mammals careful distinction must be drawn between true or natural hosts and strange or casual ones. In the former case, by reason of the long-existing association between host and parasite, a condition of mutual toleration has been reached, which, in ordinary circumstances, enables a proper balance to be maintained on both sides. On the other hand, when a Trypanosome gains an entry into animals which have never been previously liable, by their distribution, to its invasion, and which are consequently unaccustomed and unadapted to the organism, it usually produces markedly harmful effects. Such a state of affairs has resulted, for example, from the march of civilisation into the "hinterlands" of the various Colonies, where man, together with the numerous domestic animals which accompany him, has been brought into proximity to big game, etc., and what is equally important, into the zone of the blood-sucking insects which prey upon the same.

Very many of the common domestic Mammals can be successfully infected (either in an accidental way or else artificially) with different "pathogenic" Trypanosomes, to which they succumb more or less readily ; they cannot be regarded, however, as natural hosts of those Trypanosomes. In considering disease-causing forms, the more narrowly the original source of the parasite concerned is defined, the closer do we get to the true Vertebrate host or hosts. In the case of the Nagana parasite, it has been shown that such are almost certainly to be found among buffaloes and various Antilopidae (e.g. the gnu, "koodoo," etc.), while, again, the native host of T. equinum, of Mal de Caderas in South America, is most probably the capybara. It may be said undoubtedly, with regard to the many lethal Trypanosomes now known, that there is, in each case, some indigenous wild animal tolerant of that particulau form, which serves as a latent source of supply to strange Mammals coming into the vicinity.

Transmission.-In the transmission of the parasites from one Vertebrate individual to another, a blood-sucking Invertebrate is almost invariably concerned. ${ }^{1}$ In the case of all Trypanosomes of

1 Trypanosoma equiperdum, the cause of Dourine or horse-syphilis, is conveyed by the act of coitus ; and it is quite meertain whether this parasite is ever transmitted naturally by an insect. Moreover, Koch has recently bronght forward evilence (29, Schluss-Bericht) which, he thinks, tends to show that the human prasite ( $T$. gambiense) can also be transmitted by sexual intercourse. 
warm-blooded Vertebrates for which the transmitting agent is known, this is an insect, generally a member of the Diptera; in that of Trypanosomes of cold-blooded Vertebrates the same rôle is usually played by an Ichthyobdellid leech (Piscine forms), but possibly now and again by an Ixodes (some Amphibian or Reptilian forms).

'The actual relation between the parasite and the transmitting Invertebrate has long been questioned, and there are still some very important instances in which the real state of affairs is not certain. But it would seem, from the results of recent work, that in most cases some Invertebrate or other acts as a true alternate host. Thus, so far as leeches are concerned in connection with the Trypanosomes of fishes, the investigations of Léger (50), Brumpt (10-12), and Keysselitz (27) have made it elear that the parasites not only live quite normally, but undergo a lefinite evolution in particular organs of leeches which have fed on infected tish. Frequently this further development can only proceed, at least to its full extent, in a certain leech to the exclusion of others (e.g. in a IIemiclepsis and not in a Piscicolu, or vice versu); this restriction points distinctly to the leech in question being a specific natural host. Again, according to the celebrated researches of Schaudinn (75) on all Avian Trypanosome, Trypanommplia (Trypanosoma) noctuae, a species of gnat (Culer ${ }^{2}$ ) provides the alternate host, in which a complex part of the life-cycle takes place. It is interesting to note that, as might be expected, there is a regular periodicity in the infectivity of the gnat; that is, it can only transmit the infection after such and such an interval has elapsed since the meal when it became itself infected. Coming, lastly, to the Mammalian forms, Prowazek (68) has described phases of development of 7 . levisi in the rat-louse (Haematopinus sp.), and considers that this insect serves as a true Invertebrate host; though he was not able to prove the actual transmission of the parasites back to the rat by means of it. ${ }^{1}$

Interest and discussion has mostly centred, however, upon the part played by the transmitters of the lethal Trypanosomes, and it is only quite recently that any light can be said to have been thrown upon the subject.

It has for some time been generally recognised that, in many cases at any rate, a particular biting-fly is chiefly responsible for the spread of a particular parasite in an infective district. In such cases, a striking coincidence usually exists between the area over which a certain trypanosomosis is prevalent and the zone of distribution of a certain fly. Thus, of two well-known African Trypanosomes, one, T. brucii, the cause of Nagana

1 This has been effected, however, by earlier observers (Rabinowitseh and Kempner) by means of Heas, which are possibly the "right" insects. 
or Tsetse-fly disease in South-East Africa, is conveyed by Glossina morsitans 1 (Fig. 3, A and B), while the other, T. gambiense, the cause of sleepingsickness, has for its carrier in Uganda another Tsetse-fly, G. palpalis.

Working upon this knowledge, many investigators have at one time or another performed series of experiments with a view to finding out whether any developmental cycle is undergone by the parasites while in the fly, and whether definite periods of infectivity occur, on the analogy of the malarial parasites in mosquitoes. The earlier results obtained secmed to indicate that the rôle of the fly was purely mechanical-the insect acting merely like an artificial inoculating tube. Bruce, in the course of his pioneer work in Zululand, found that the flies could, with
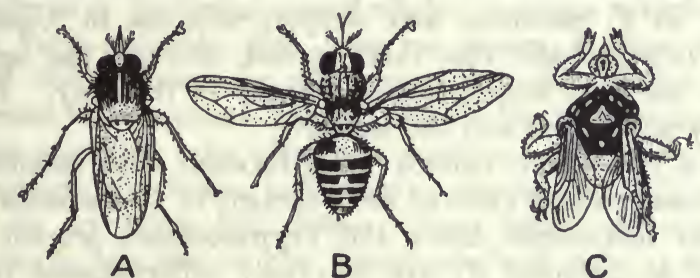

C

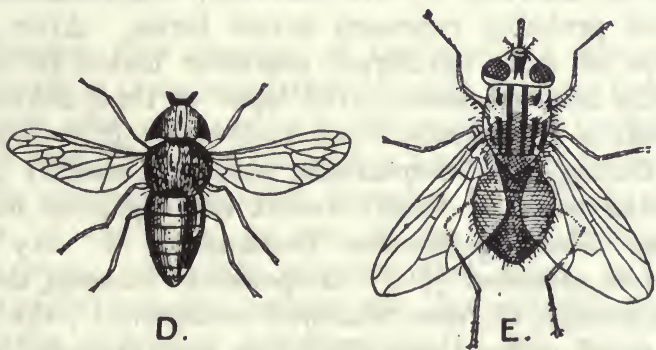

Fro. 3.

Various bloorl-sncking flies. A and B, Glossince morsitans (transmits Trypunosome, brucii, of Nagana), $\times 2$; C, Hippobosal rufipes (thought to transmit T. theileri, the cause of " bile-sickness"), $\times 1 \frac{1}{2} ;$ D, Tabanus lineola (probably conveys the Surra parasite, $T$. evansi), $\times 1 \frac{1}{2}$ E, stomoxys crlcitrans (suspected in connection with $T$. equinum, of Mal de Caderas), $\times 2 \frac{1}{2}$. (A ani 13 from Lav. and Mesn., after Bruce; $\mathbf{C}$ after L. and M. ; D and $\mathbf{E}$ after Salmon and Stiles.)

varying success, infect a healthy animal if allowed to bite it up to fortyeight hours after being themselves fed on an infected one, but not afterwarls. Similarly, Bruce, Nabarro, and Greig (8) ascertained that $G$. pulpalis could give rise to an infection eight, twenty-four, or forty-eight hours after feeding, but after two days they could no longer obtain a successful inoculation. Moreover, some experiments extended over two months gave no sign of any periodicity of infection. Nevertheless, these workers found that the Trypanosomes could at all events live and retain their mobility in the stomach of the fly up to seventy-one hours.

Similar results were obtained by Minchin, Gray, and Tulloch. In their interesting report (59) these authors state that they could find no evidence of a fly becoming infectious at any particular period after

1 'This parasite is also conveyerl, in different districts, by' $(i$. pallidipes and G. fuscere. 
being fed, experiments being carried out up to an interval of twenty-two days. An additional and significant fact remarked upon by them is that only the first animal which the experimental fly was allowed to stab became infected; if the insect was removed before its meal was completed and immediately placed on another animal, this latter did not become infected. That is to say, after a fly had been allowed to, as it were, clean its proboscis from the Trypanosomes renuining in it since its previous meal (on an infected animal), it was no longer infections.

- These facts make it certain that Trypanosomes can be and are conveyed by Tsetse-flies in a purely direct and mechanical manner; and so far as $T$. gambiense and sleeping-sickness in Uganda are concerned, it is probable that their spread, through the agency of G. palpalis, has been largely if not entirely in this way. But this does not by any means end the matter.

Minchin, Gray, and Tulloch bring forward observations which point to a commencing cycle of development of $T$. gambiense ${ }^{1}$ in the fly. Up to forty-eight hours the Trypanosomes present in the stomach of an infected fly are markedly differentiated into two types, which probably represent sexual forms. After forty-eight hours a type of more indifferent character makes its appearance, which usually bccomes scanty with lapse of time, till at ninety-six hours scarcely a Trypanosome can be found. It is interesting to note that during this interval the parasites steadily increase in size. Coming next to Koch's recent investigations on behalf of the German Sleeping-Sickness Commission, a very important observation is recorded (29). A species of Glossina, distinct from G. palpalis, namely, G. fusca, was bred in captivity; the individuals born and reared under these conditions were regarded as certainly free from Trypanosomes. ${ }^{2}$ Several of these flies were fed on rats infected with $T$. gambiense. They were examined from ten to twelve days later, and after this long interval were found to be infected with those parasites. Moreover, individuals of another Tsetse-fly, G. tachinoides, similarly fed, were also found to contain T. gambiense after the same lengthy interval.

Still more recently Stuhlmann (80), in bis description of $G$. fusca, has published some extremely interesting notes on the relation of $T$. brucii to this fly. Using reared flies, considered to be certainly free from infection, Stuhlmann was able to infect about 80 to 90

1 The case of $T$. gambiense in Glossina palpalis is unfortunately complicated by the occurrence in the same species of fly of other Trypanosomes, distinguished by Novy (61) as "fly-Trypanosomes." One of these, T. grayi, at any rate is entirely different from $T$. gambiense ; and it is highly probable that some of the observers (e.g. (Yray and Tulloch [23], Koch [28]), who first described what they regarded as developmental phases of $T$. gambiense, were dealing in reality with $T$. grayi.

2 This is on the assumption, of course, that the parasites were not inherited; but most authorities seem to be agreed that hereditary transmission of Trypanosomes by Tsetse-flies does not take place. 
per cent, and in from two to four days was able to observe various developmental phases of the parasites. This further development continued on the flies being fed upon healthy animals, but only in about 10 per cent of the individuals; in the rest it gradually disappeared. This percentage, it is instructive to observe, was about the same as that of the Tsetses (G. fusca) found to be infected with $T$. brucii (in all probability) in nature.

It will be seen that it is impossible to draw any certain conclusious from the present position of the problem. Nevertheless, there is good reason to suppose that, for a given lethal Trypanosome, there is a particular insect which is a true alternate host. ${ }^{1}$ It seems very probable that here, as among leeches, there are right and wrong hosts for the parasites; that while the complete normal development, culminating in the transfer back to the Vertebrate, can only take place in a certain species of fly, attempts at development which are, to a varying degree, partially successful may go on in other biting-flies; these latter, however, being able to act in relation to the Vertebrate host only as mechanical carriers.

Before leaving this question of the mode of transmission of Trypanosomes, it is to be noted that Minchin has put forward (57) an entirely new view with regard to the method of infection. His idea is based especially upon the highly interesting discovery made by him of the occurrence of cysts, doubtless for external dissemination by way of the anus, in one of the "fly-Trypanosomes," Trypanosoma grayi. Minchin suggests that there may be two varieties of cyclical infection among the Haemoflagellates; in the one, the parasite undergoes cyst-formation in the insect, resulting in a contaminative infection of the Vertebrate, by means of its food or drink; in the other, distinguished as the inoculative type, the infection takes place through the proboscis of the fly (as, for example, in the malarial parasites). Up to the present, however, $T$. grayi remains the only known form in the case of which infection is most probably of the first type. ${ }^{2}$ From what has been learnt so far of the development of other Trypanosomes - whether in leeches or in insects-the distribution of the parasites in the body (see under "Habitat") points at any rate to inoculative infection of the Vertebrate. The possibility of the occurrence of both modes in any one Trypanosome is not, so far as is known, excluded; but there is, as yet, no definite evidence in favour of this.

1 For further remarks bearing on this point, see pp. 230-231, 261.

2 Although the Vertebrate host of $T$. grayi has not been actually demonstrated, both Minchin and others have made an important observation in connection with the hiology of the Tsetse-Hy, which-taken in conjunction with the non-occurrence of liereditary infection-seems to show that it is inpossible for the parasites to be merely fly-Trypanosomes. This is to the effect that the 'Tsetses, unlike mosquitoes, feed only on blool, never on foul or decaying matter of any kincl. 


\section{(b) Habitat and Effects on Host.}

1. Relution to the Invertelrate Host.-Schaudinn, in his work on the parasites of an owl (Athene noctua) (l.c.), has described in full the distribution and course of development of Trypanosomes in the body of a gnat (Culex pipiens). Although, as is pointed out below (see under "Life-Cycle"), it is now disputed how far Schaudinn's description actually relates to Avian Trypanosomes, the great interest excited by this anthor's work renders a brief abstract of his account necessary.

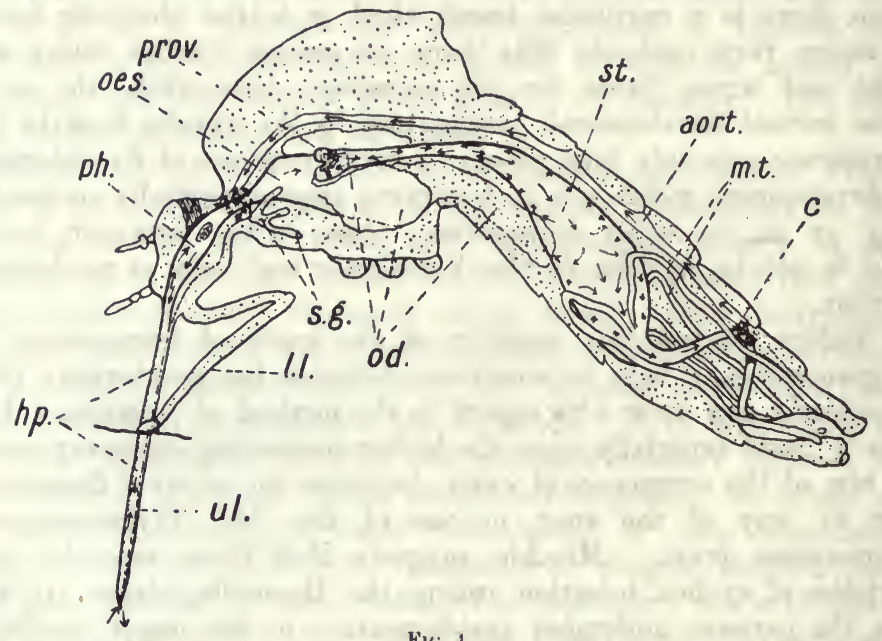

Fis: 4.

Dia:ramnatical longitudinal section through ciulex pipiens to show the distribution of thes parasites. The arrows indicate the direction of their movement, the clusters of stars the placest of agglomeration. $\quad . l$, upper lip; $l . l$, lower lip; $h p$, hypopliarynx ; $p h$, pharynx ; s.g, salivary gland; ors, oesophagus ; 0.1 , oesophageal diverticula (gas reservoirs); prov, proventriculus: t $t$, stomach; m.t, Malpighian tubes; $e$, junction of ileum and colon; a.ort, aorta. (After Schauliun.)

The distribution of the parasites ${ }^{1}$ is intimately connected with the process of digestion. Towards the end of the digestion of the imbibed blood, the Trypanosomes, after a period of multiplication, enter upon a resting phase, and are found either attached to or between the epitheliak cells. After a second meal another multiplicative period occurs, and the parasites gradually collect in the anterior part of the stomach, where the nutriment remains longest unabsorbed. Here (Fig. 4, prov) the organisms begin to cluster in large numbers, being able to penetrate the delicatc surface of the layer of in raginated oesophageal epithelium in this region. Finally, there is an enormous accumulation of the Trypanosomes at this place, all arrangerl in rows and in a resting condition. The next inflow

1 This sunmary relates to the first of the two parasites described by Schaulinn, Tryprenomoriplia (Tiypannsoma) nimtune. 
of blood drives this mass before it, in the form of a rolled-up ball, until it reaches the junction of the ileum and colon (Fig. 4, c), the narrowest point of the intestine. The wall here is very thin and easily ruptured, and most of the Trypanosomes pass through it, into the vascular lacunae around, whence they are carried to the heart. Finally, the parasites become arrested in the sinus surrounding the pumping-organ of the pharynx, where they continue to multiply and collect again into agglomeratel masses, which press on the wall of the pharynx in this region. By the end of the third digestive period, these clumps of Trypanosomes have broken through, and partly block up the lumen; and in the next biting act they are forcibly ejected into the blood of the owl. Thus the parasites cannot leave the gnat until the fourth meal, including that which effected their entry, has taken place ; and Schaudinn found that the shortest time elapsing between entrance and exit was seven or eight days, when the insects were maintained at the optimum temperature for digestion.

An interesting discovery is the occurrence of true hereditary infection. After breaking through the wall of the colon, a few of the Trypanosomes, usually females, instead of being carried forwards, may pass to the ovarian follicles, penetrate into the young eggs, and so infect a succeeding generation.

According to Prowazek (l.c.), the behaviour of Tryponosmmu lewisi in Haematopinus and its passage through the louse resembles in the main the account above summarised. Such differences as there are stand in close relation, on the one hand, to the somewhat different mode of feeding and of absorption of nutriment in the louse, and on the other hand to the fact that $T$. leuisi appears to be more resistant to "external" influences.

With regard to other Mammalian Trypanosomes, the evidence so far available is mostly to the effect that they are confined entirely to the alimentary canal, and never occur in other organs of the insect. Concerning T. gambiense in G. palpalis, Minchin, Gray, and Tulloch (l.c.) remark that these parasites were found only in the mid-gut, and never passed either backwards into the proctodaeum or forwards into the proventriculus. ${ }^{1}$ According to Stuhlmann (l.c.), T. brucii is apparently much more at home in G. fusce (which may prove to be its true specific host), being met with in different forms from the hind-gut (colon) to the proboscis. But this author also emphasises the fact that the 'Trypanosomes were never observed anywhere else, and, particularly, never in the salivary glands. The only positive observation of the occurrence of Trypanosomes in the salivary glands which need be taken into account is the recent. statement made by Koch (29) that, of the different types which

1 Gray and Tulloch (l.c.) stated that they had olserved $T$. gambiense in the salivary glands, but Minchin has since shown that what they took to be salivary glands was in reality proventriculus ; moreover, they may have been dealing, not with T. gainbiense, hut with one of the other parasites in this fly. 
he noticed in Glossinue (sp. not given), one which from its entire agreement with 7 . gambiense was to be identified with that form occurred in two instances in the salivary glands. If this observation be corroborated, its importance is, of course, very great.

Several important facts have been litely brought forward by lirumpt (10-12), which tend to show that the habitat of Piscine Trypanosomes in leeches is also restricted to the alimentary canal. ${ }^{1}$ Three types of behaviour can be distinguished. (a) The parasites develop solely in the stomach and never pass into the intestine or into the sheath of the proboscis. At the moment when the leech sucks the blood of another fish, the Trypanosomes pass into the latter directly, by way of the proboscis. This mode is exemplified by $T$. remaki of the pike. (II) The development begins in the stomach and is continued in the intestine, where the parasites may remain for a long while. From the intestine the Trypanosomes pass back into the stomach, to gain at length the proboscis-sheath. 'T. granulosum of the eel is an example of this type. In the third variety $(c)$ the development goes on in the stomach, but the parnsites succeed in passing finally into the proboscis-sheath; ex.: I. dunilewskyi of the carp. In the case of certain marine forms ('T'. raiue and T. scyllii), whose development goes on in Pontobdella, Brumpt found the parasites in the stomach and intestine, but could not ascertain how they got back into the fish. Miss Robertson, however, has lately described (72) various developmental phases of a Trypanosome which she regards as identical with $T$. raiae, and states that small, slender forms do migrate up into the proboscis : it is probably these which serve to infect the Vertebrate.

2. Relution to the Virtebrute Host.-Once an entrance into the blood is effected, the parasites pass rapidly into the general circulation, and are thus carried to all parts of the body. In considering the distribution and numerical abundance or otherwise of the Trypanosomes in any given individual, it is necessary to bear in mind whether they are in a tolerant host or in an unaccustomed one. Dealing with the former case first, the trend of olservation points to their being usually rather scarce, sometimes very rare. The reason for this scarcity is probably the fact that multiplicative phases are very rarely met with, at all events in the general circulation. Except for a short period at the beginning of the infection, multiplication appears to be largely in abeyance; this has been well shown by Laveran and Mesnil (37) in the case of $T$. lewisi of the rat. The parasites are often more numerous in the spleen, bone-marrow, kidneys, and liver than elsewhere; and it has been found that multiplication goes on rather more actively in the capillaries of these organs. One very important point may be

1 Brumpt has recently noted (14), however, cases of hereditary infection of leeches, with both Trypanosoma and Trypanoplasma. 
conveniently mentioned here, namely, that hereditary infection of the Vertebrate host is not known to occur in the case of most of the great classes. Moreover, in Mammals, whether tolerant or unaccustomed hosts, the parasites appear to be, as a general rule, unable to traverse the (uninjured) placenta. Pricolo has recently stated, however (67), that he has found 1'. duttoni in the foetus of an infected mouse, and thinks this a case of true hereditary infection.

The Trypanosomes in the active, motile form are always free in the blood-plasma (intercorpuscular). It is very uncertain whether the parasites ever come into relation with the blood-corpuscles. According to Schaudinn's investigation on two Avian forms, one, namely, Trypanomorpha (Trypanosomu) nocture, becomes in certain phases attached to a red blood-corpuscle (ectocorpuscular), while at other times it penecrates inside the corpuscle (endocorpuscular) and eventually destroys it. The other form, Trypanosoma (Spirochaet $\iota$ ) ziemanni, apparently draws up into itself the white corpuscle (leucocyte) to which it becomes attached. It must be admitted, however, that some doubt exists as to these alleged occurrences. ${ }^{1}$ In addition there are two or three very positive statements of observations showing that other Trypanosomes, including Mammalian forms, may come into relation with the red corpuscles; see Buffard and Schneider (16) with regard to T. equiperdum, and Voges (85) with regard to $T$. equinum. On the other hand, Prowazek (68) could find neither an ecto- nor an endocorpuscular condition in 7 . lewisi, and considers that the habitat of this parasite is restricted to the plasma.

Considering now the Trypanosomes in an unaccustomed Mammalian host, for which they are lethal, the parasites may either remain infrequent or rare - sometimes, indeed, being umnoticed until shortly before death-or they may soon become numerous and go on increasing (Fig. 5). In the latter case the disease is acute and rapidly fatal ; in the former it is more chronic and lasts much longer, often several months.

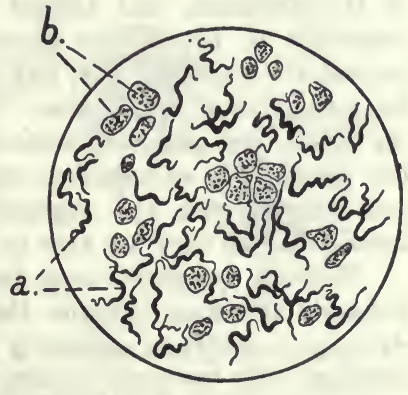

FIG. 5 .

Trypectosome erfuiperelum (of Dourine), in the blood of a rat eight days after inoculation. $a$, parasites; $b$, bloodcorpuscles. (After Doflein.)

There is often considerable varia-

bility with regard to the appearance and number of the parasites in

1 It is said that Schaudinn has mistaken two distinct Haemosporidian parasites, a Halteridium and a Leucocytozoon, for resting-phases of these other Haematozoa (see under "Life-Cycle"). 
the blood at any moment. Occasionally and at irregular intervals, evidently following upon a period of multiplication, the Trypanosomes may be fairly numerous, their appearance frequently coinciding with an access of fever. At other times, they seem to vanish almost entirely from the peripheral circulation; for what reason, however, is not certain. Some authorities attribute it to the rise in temperature, as being unfavourable to the parasites; others think it is due to the more potent operation of chemical and physiological defensive agencies of the host at a higher temperature. However this may be, it has long been known that certain of the organisms situated, probably, in some internal, more favourable part of the body can survive and give rise later to a fresh succession of parasites in the blood. ${ }^{1}$

The main features of the illness show a general agreement, whichever varisty of trypanosomosis is considered; one symptom may be, of course, more marked than another in a particular type. The pathogenic effects are chiefly referable to disorganisation either of the circulatory or of the nervous system, or of both combined.

Fever always occurs, at some time or other, during the course of the nualady. Its manifestation is extremely irregular, both in character and in time of occurrence, and it is, therefore, usually readily distinguishable from malarial fever. There is, particularly in chronic cases, marked and progressive anaemia and emaciation, leading to pronounced enfeeblement, which is, in fact, the most characteristic symptom of naturally occurring trypanosomosis. A common feature is the occurrence of oedeniatons fwellings in various parts, chiefly in the neighbourhood of the genitals, of the abdomen, and around the eyes. The parasites are often more numerous in the bloody serosities bordering these places than in the general circulation. This fact is of great importance in connection with the transmission of Dourine. In this disease the parasites are rare in the blood, but generally numerous in the immediate neighbourhood of the oedematous excoriations on the penis, so that, in coitus, they come into contact with the vaginal mucous membrane of a healthy mare, through which they are able to pass.

Nervous symptoms may be only slightly noticeable (e.g. a dull and lethargic tendency towards the close of the illness), or they may be strongly in evidence, especially in Dourine, Mal de Caderas, and sleepingsickness. In the two former, more or less general paralysis of the posterior part of the body frequently sets in ; Mal de Caderas of horses in South America is, indeed, often called "hip-paraplegria." In sleepingsickness the Trypanosomes penetrate into the cerebro-spinal canal, and can usually be found upon centrifugalising a sufficient quantity of the

1 Holmes (Journ. Comp. Pathol. xvii., 1904) and, more recently, Salvin-Moore and Breinl (Ann. Trop. Med. i., 1907) consider that these resistant forms, for which the latter propose the term "latent bodies," are represented by certain of the amoeboid involution-forms described by Bradford and Plimmer, Laveran and Mesnil, and others (cf. p. 222). 
fluid; they have also been seen, in post-mortem examination, in the lateral ventricles of the brain. It is this invasion by the parasites of the nervous system that marks the transition of the case from one of "Trypanosoma-fever" (while the parasites are confined to the blood) to one of sleeping-sickness. The results of the change are soon apparent in the onset of lassitude, tremor, and the other associated nervous synptoms which characterise this dreadful malady.

Death from trypanosomosis is due either to weakness and emaciation (in chronic cases), or to blocking of the cerebral capillaries by the parasites (where these are abundant and the disease consequently acute and rapid), or to the disorganisation of the nervous system (paraplegic and sleepingsickness fornis). Laveran and Mesnil have expressed the opinion that sone factor in aldition to the presence of the parasites themselvesespecially when these are rare-is requisite to explain the severe effects produced, and suggest that the Trypanosomes secrete a toxine. Neither they nor other investigators have, so far, been able to discover traces of any such substance. In post-mortem examination, the most obvious pathological feature is hypertrophy of the spleen, which may be very pronounced. The lymphatic glands in the neck, inguinal region, etc., are often greatly swollen and contain numerous parasites.

The spleen and lymphatic glands are undoubtedly the organs which react most strongly to the parasites, and their enlarged condition is, probably, to a great extent due to enhanced activity in elaborating blood-corpuscles and leucocytes to cope with the enemy. Ingestion and dissolution of the Trypanosomes by phagocytes has frequently been observed (Fig. 6). It is very likely also that the haematopoetic organs secrete some chemical or physiological substance which exerts a harmiful action on the parasites, causing them to undergo involution and assume weird-looking "amoeboid" and "plasmodial" forms.

\section{Comparative Morphology.}

Trypanosomes vary greatly with regard to size; even in one and the same species this variation is often noticeable, especially 
under different conditions of life. The well-known Trypunus(mu⿰ rotatorium of frogs (Fig. 8, A and B) is, taking it all in all, one of the largest forms so far described. Its length ${ }^{1}$ varies from 40 to $60 \mu$, while its greatest width dorso-rentrally ${ }^{2}$ is from 8 to $30 \mu$; in the
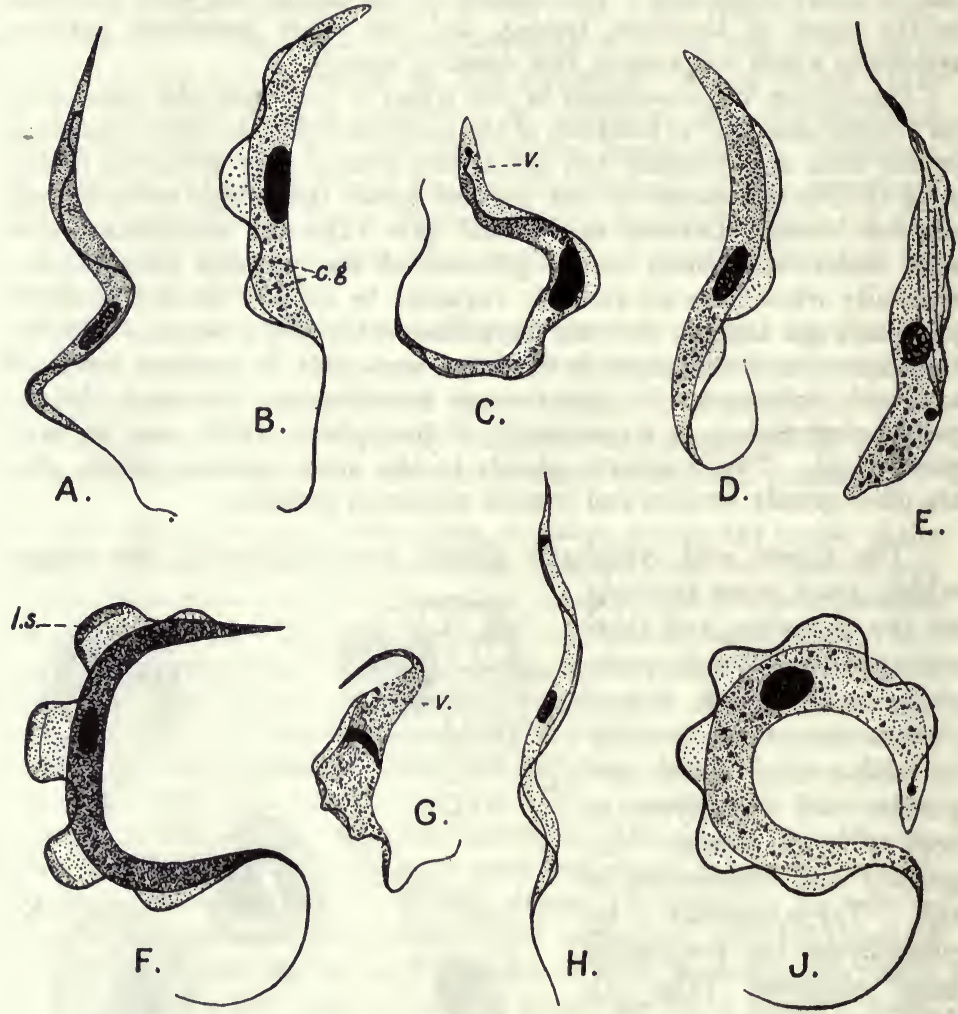

E.

Fic. 7.

Representative Mammalial, Avian, and Reptilian Trypanosomes. A, Trypanosoma lewisi, after Bradf. and Plim.; B, T. brucii, after Lav, and Mesn., $\times 2000$; C, T. gambiense (blood, T.fever), after Bruce and Nabarro; D, T. equinum, after L. and M., $\times 2000$; E, Trupanomorpha (Trypanosoma) noctuce, after Schaud. ; F, Trypanosoma avium, after L. and M. ; G, T. hannae, after Hanna ; H, T. (Spirochacta) ziemanni, after Schaud. ; J, T. damonicue, after L. and M., $\times 2000, \quad . g$, chromatoid grains; $v$, vacuolo; $l . s$, longitudinal striation.

very wide individuals breadth is gained more or less at the expense of length. Conversely, the human parasite, T. gambiense (Fig. 7, c), is one of the smallest forms, its average size being about 21 to $23 \mu$ by $1 \frac{1}{2}$ to $2 \mu$. The majority of Mammalian Trypanosomes

1 The length is always inclusive of the flagellum, unless otherwise stated.

2 Adopting Léger's convention, by which the convex side, bearing the undulating membrane, is distinguished as dorsal ; the measurements of width always include the undulating membrane. 
are fairly uniform in size (Fig. 7, A-D), the chief exceptions being T. theileri (Fig. 33), which is much larger than the rest, varying from 30 to $65 \mu$ in length; and $T$. nanum, which is correspondingly minute, being only about $14 \mu$ long. The Piscine forms, on the other hand, though possessing an equally great range, exhibit a more regular gradation. Starting with relatively small types, like 1'. remaki, var. parva, with a medium length of $30 \mu$, parasites of all sizes are to be met with up to T. granulosum (Fig. 8, K) and T. raiae (Fig. 38, B), which are among the longest Trypanosomes known, attaining a length of $80 \mu$.

There is equally great diversity of appearance. Typically the body is elongated and spindle-shaped; it is generally more or less curved or falciform, and tends to be slightly compressed laterally. It may be, however, anything from extremely slender or vermiform (Figs. 8, $\mathrm{K} ; 34$ ) to thick-set and stumpy (Figs. 8, A ; 35). Moreover, apart from the fact that a full-grown adult, ready to divide, is in many cases much broader than a young adult (cf. T. lewisi, Fig. 20, B), considerable polymorphism also sometimes occurs (e.g. T. rotatorium, Figs. 8, A, B ; 37). Again, there can be little or no doubt that, in some instances at any rate, sexual differentiation is expressed by more or less pronounced differences in appearance. In fact, from one reason and another, it is often practically impossible to define any one type within hard and fast limits, either of shape or size.

In the biflagellate, Heteromastigine forms (Trypanoplasma and Trypanophis), the anterior extremity of the body is that, of course, from which spring the two flagella. With regard, however, to the correct orientation in the uniflagellate Trypanosomes (the genus Trypanosoma sens. lat.) considerable uncertainty exists. For the present, ${ }^{1}$ in order to avoid confusion, the two ends may be designated as flagellate or flagellar, and non-flagellate or aflagellar respectively. On the whole, the flagellar extremity is fairly uniform and nearly always more or less tapering; but the nonflagellate end presents great variation, being, as Laveran and Mesnil point out, particularly plastic. On the one hand, it may be blunt and even rounded off at the tip, as in certain individuals of $T$. brucii (Fig. 7, B), T. equiperdum (Fig. 32, D), and in a Trypanosome of Senegambian birds (Fig. 35); on the other hand, it may be very long and attenuated, as in T. hannae (Fig. 7, G), occasionally simulating a true flagellum to a remarkable degree, this being the case in T. polyplectri. Between these two extremes all manner of intermediate conditions are to be found. An instance which well illustrates the great variability in one and the same form is seen in

1 The whole question is so closely bound up with that of the phylogeny of the group that its consideration is best deferred until the two can be discussed together (see below, p. 246). 


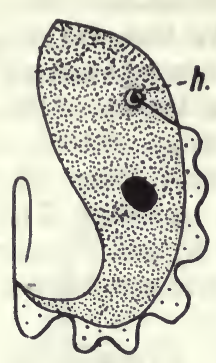

A.

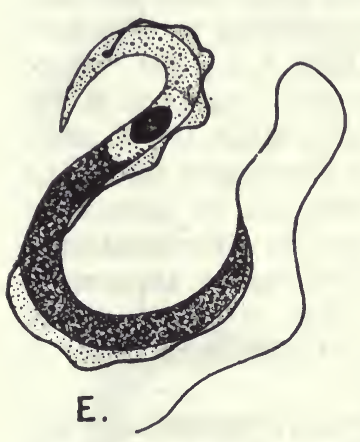

B.
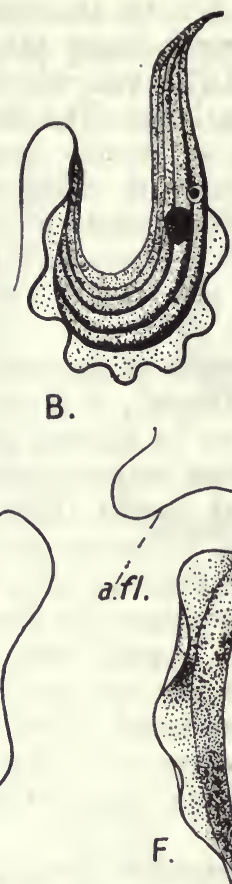

F.
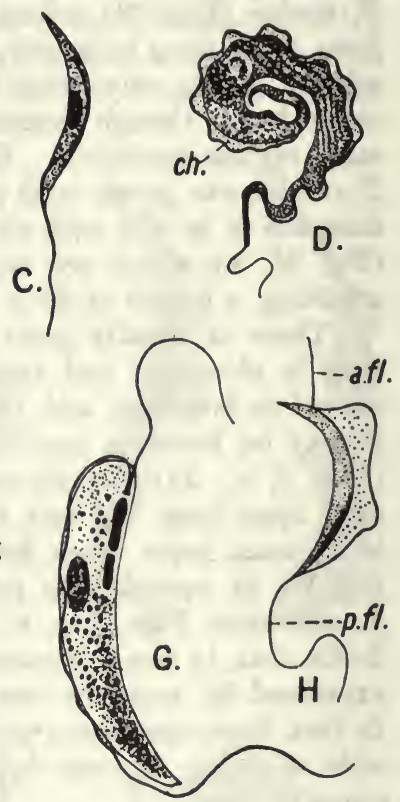
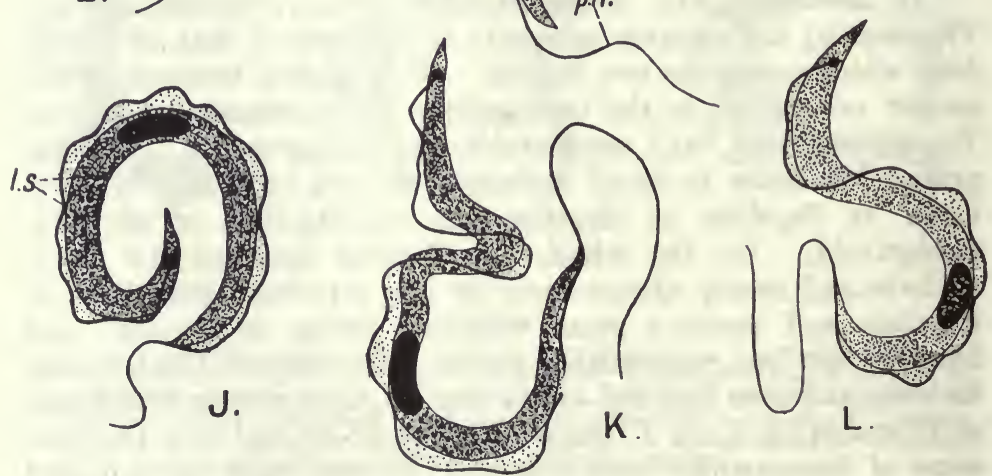

F10. 8.

Representative Amphibian and Piscine Trypanosomes. A and B, Trypanosoma rotatorium, after Lav. and Mesn., $\times 1400 ; C, T$. inopinatum, after Sergent, $\times 1000 ; D, T$. karyozeukton, after Dutt. and Todd, $\times 1000$; E, T. nelspruitense, after L. and M., $\times 2000$; F and G, Trypanoplasma borreli (living and stained), after Léger; H, T. cyprini, after Plehn; J, Trypanosoma soleae after L. and M. $\times 2000 ; \mathbf{K}, T$. granulosum, after L. and M. ; L, T. remaki, var. magna, after L. and M., $\times 2000$. $h$, clear zone or halo around kinetonucleus; ch, chain of chromatic rodlets running between the two nuclei; $a . f$, anterior flagellum; $p . f$, posterior flagellum ; $l . s$, longitudinal striations or myonemes; $v$, cytoplasmic vacuole.

T. lewisi. Usually this parasite has a sharp, pointed aflagellar end (Fig. 7, A); but in many of the individuals found in rats which 
have been recently infected (e.g. five or six days previously) this extremity is enormously drawn out and tapering like a whip (Fig. 9). In such forms the flagellum is often very short.

The two flagella, in Trypanoplasma and Trypanophis, are inserted into the body close to the anterior end (Fig. 8, F, G). They are quite separate from each other, and while one (that most anteriorly situated) is entirely free and directed forwards, the other at once turns backwards and is attached to the convex (dorsal) side of the body for the greater part of its length. This latter flagellum terminates in a shorter or longer free portion.

The comparative degree of development of the two flagella in different cases is worth pointing out, since it is very instructive in a phylogenetic connection. Starting with Bodo lacertae, from a type similar to which the biflagellate forms may be derived, both flagella are of about equal total length, and the trailing one does not reach the posterior limit of the body. In Trypanophis grobbeni (Fig. 30) the posterior flagellum is more developed than the anterior one, and attached to the side of the body, but its free termination is very short. In Trypanoplasma borreli the anterior flagellum and the free portion of the posterior one are of equal length. Lastly, in T. cyprini the former is much shorter than the latter, and shows signs of reduction. From this condition to its disappearance is but a small step.

In all other Trypanosomes there is only one flagellum, which is invariably attached to the body in the same manner as the posterior one of the biflagellate forms. The point of origin of the flagellum is generally near the nonflagellate end, but may vary considerably. Although there is usually a free continuation of the flagellum, it may be short or lacking (cf. Fig. 34).
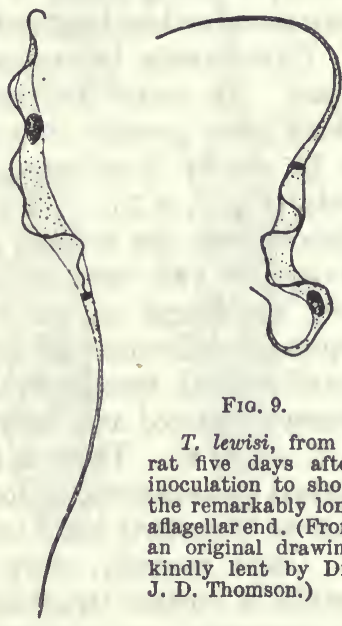

Fia. 9.

T. lewisi, from a rat five days after inoculation to show the remarkably long aflagellar end. (Froni an original drawing kindly lent by Dr. J. D. Thomson.)

Along the dorsal side runs a characteristic fin-like expansion of the body, the undulating membrane This always begins proximally at the place where the attached flagellum emerges from the body; and its free edge is really constituted by the latter, which forms a flagellar border, more or less sinuous in outline. The membrane may be only narrow, and chiefly discernible by its well-marked border (Figs. 7, A, G; 8, c), or it may be well developed and sometimes thrown into broad folds or pleats (Figs. 7, F ; 8, A, B). Distally the membrane thins away concurrently with the body. 
Minute Structure.-The body appears to lack any distinct limiting membrane or cuticle. A differentiation of the peripheral cytoplasm in the form of an ectoplasmic layer, the so-called "periplast," has only been definitely described in a few cases (Prowazek [68], Wasielewsky and Senn [86]). Nevertheless, it is probable that in most Trypanosomes there is such a layer, although it may be, in some forms, only poorly developed around the body generally. The undulating membrane, however, is certainly largely, if not entirely an ectoplasmic development. This is usually much clearer and more hyaline in appearance than the general cytoplasm. The latter is finely granular or alveolar in character, though its exact degree of coarseness and density varies in different forms, sometimes even in different parts of the same individual. The cytoplasm of male forms is in general clearer and less granular than that of female ones. The cytoplasm in T. mega and T. karyozeukton is rather unusual in structure. In the third of the body on the aflagellar side of the nucleus, it is very loose and spongy; in the other two-thirds, it is arranged in alternating light and dark, densely-staining bands ("hyaloplasm" and "spongioplasm"), running more or less longitudinally.

Cytoplasmic inclusions of one kind or another are often to be found. In many Trypanosomes, deeply-staining granules occur, which vary greatly in number and size. These granules appear to be chiefly distributed, as a rule, in the flagellate half of the body (Fig. 7, B, D). They are of a chromatoid nature, and probably derived from the nucleus (see Lignières [54]). In Trypanophis there are one or two rows of highly refractive, yellowish inclusions running the length of the body (Fig. 30). It is thought that these represent collections of fatty or oily substances. In certain forms, a well-defined, usually oval vacuole is often, though not constantly present, situated at a varying distance from the aflagellar end (Figs. $7, \mathrm{C}, \mathrm{G} ; 8, \mathrm{~F})$. There is no reason to doubt that this vacuole is a normal cell-constituent, for it has been observed in parasites in their natural (tolerant) hosts under quite normal conditions.

Until recently, very little was known with regard to the details of nuclear structure. A Trypanosome was merely described as possessing an unmistakable nucleus, and also a small deeplystaining element of uncertain significance, situated at the root of the flagellum, and termed variously "blepharoplast," centrosome, or micronucleus. It is to Schaudinn that we are indebted for the revelation of the essential nuclear nature of the latter organella, its intimate connection with the larger nucleus and the complexity and differentiation which the whole nuclear apparatus may exhibit. Since then several workers have brought forward observations relating to one point or another, which, taken altogether, suggest strongly that the nuclear organisation of Trypano- 
somes in general is based upon a plan fundamentally similar to that described by Schaudinn in the case of his Avian parasite, Trypanomorpha noctuae. The development and ultimate constitution of the nuclear apparatus in this type are as follows :-

The account may be commenced with the condition found in an indifferent ookinete or individual which will become an indifferent (nonsexual) Trypanosome. Here a single, large compound or double nucleus is present, consisting of an external portion and of an internal, central

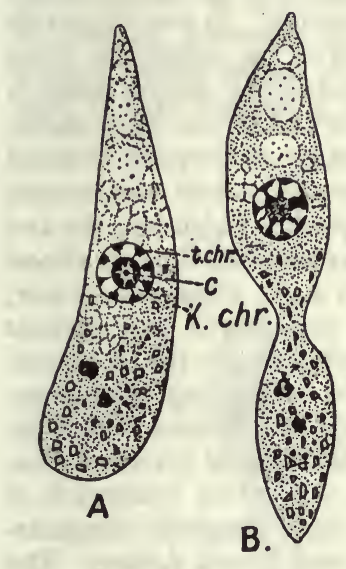

Fia. 10

Development of an indifferent Try. panosome from an ookinete of indifferent character. (After Schaudinn.) t.chr, trophonuclear chromosome; $K$.chr, kinetonuclear chromosome; $c$, centrosomic granule; $a . s$, first axial spindle; $a . s^{2}, a . s^{3}$, second and third spindle ; $t$, trophonucleus ; $k$, kinetonucleus; $k . c$, kinetonuclear centro. some; t.c, trophonuclear centrosome : $m$, myonemes ; $f . b$, flagellar border of undulating membrane (third axial spindle); $c^{3}$, its proximal centrosoine.
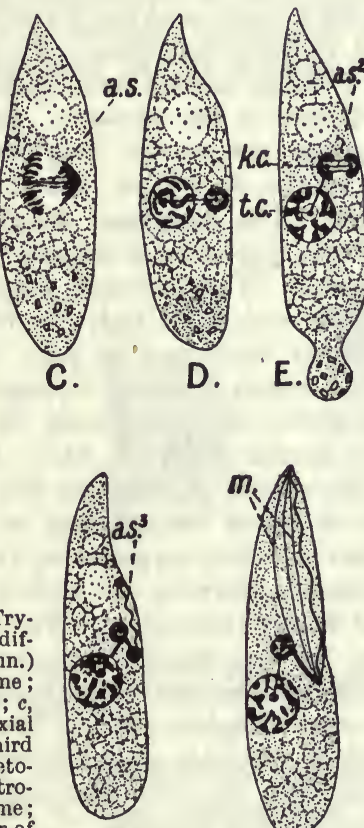

$\mathrm{F}$.

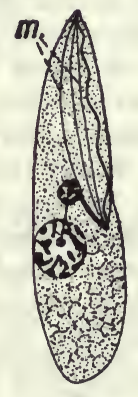

G.

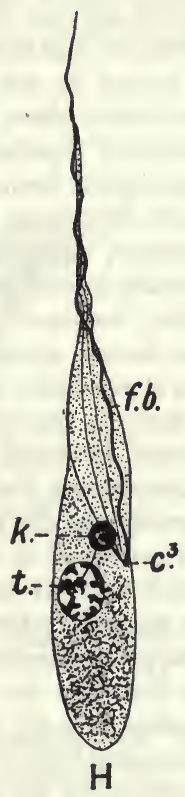

portion (Fig. 10, A). The former has eight distinct, peripherally situated chromosomes; the latter also has eight separate chromosomes. In the centre of all is a well-marked centrosomic granule (c). The first change takes place by the inner body becoming amoeboid and giving up its material to the outer, surrounding part (B). The result is that the eight chromatic elements of the former become united, by the aid of the plastinoid basis present, with those of the latter, leaving the above-mentioned grain in the middle. This granule divides in a dumb-bell-like manner, producing a small axial spindle (c, a.s.), around which the eight compound chromosomes arrange themselves. These next split, and the halves pass to either end, forming a diaster which is markedly heteropolar. The 
right (or dorsal) half is perceptibly smaller, but denser and more deeply staining than the other. In this manner, therefore, two distinct nuclear bodies are formed, of different size and character. They remain connected together by a fine achromatic thread, representing the original central spindle, which ends in a small granule near the centre of each. The larger nucleus, lying nearer the middle of the body, rapidly reconstitutes itself and enters upon a resting-phase. This nucleus regulates the trophic functions of the cell, for which reason we have proposed (3) for it the name trophonucleus.

Meanwhile, the other, smaller nucleus proceeds to give rise to the characteristic locomotor apparatus of the Trypanosome. It passes forwards slightly and takes up a position at the periphery of the endoplasm, lying indeed against the limiting ectoplasm. Its centrosome divides again in a similar manner, forming another axial spindle $\left(\mathrm{E}, a . s^{2}\right)$ at right angles, as before, to the length of the parasite. Another heteropolar division next takes place, giving rise to two daughter-nuclei; these also remain connected together by the drawn-out central spindle, which join the two centrosomic granules. The peripheral daughter-nucleus, situated almost in the ectoplasm, forms yet another spindle ( $\left.\mathrm{F}, a . s^{3}\right)$, whose axis is now, however, longitudinal. This assumes large proportions and spreads forward to the anterior end of the body, the whole lying in the ectoplasm, which becones greatly devcloped to form the undulating menibrane. The central spindle becomes excentric in position and sinuous in outline, and strengthens, or rather itself constitutes, the free edge of the membrane, forming a flagellar border to it $(\mathrm{H}, f . b)$. A supporting framework is formed hy eight myonemes, representing the eight elongated daughterchromosomes, four of which are arranged on each lateral surface. The flagellar spindle does not stop on reaching the anterior end of the body, but continues to elongate, drawing out with it the undulating membrane, which narrows and finally thins away. The myonemes then unite with the spindle to form the free flagellum, the centrosome at the distal end disappearing as such, but that at the basal or proximal end persisting (c). By this time the other daughter-nucleus has become rounded off as the kinetonucleus $(k)$, which regulates all the kinetic activities of the parasite ; it remains connected with the locomotor apparatus by a delicate thread, representing the second axial spindle. ${ }^{1}$

According to Prowazek's recent investigations, the same type of nuclear structure is also shown by two Mammalian forms, T. lewisi and $T$. brucii; indeed, it is maintained that the system of axial spindles produced by successive divisions of the karyocentrosome

1 Two other points bearing on the view that the flagellum represents the greatly elongated axial spindle of a nuclear division may be noted. In Trypanosoma johnstoni, where there is no free portion of the flagellum, this terminates (at the limit of the cytoplasm) in a small deeply-staining granule (Fig. 34), perhaps comparable to the distal centrosome of such a spindle. Again, Miss Robertson (72) sums up her account of the origin of the flagella in the developnient of the flagellate form from the rounded, aflagellar type in the case of her leech-Trypanosome by saying, "the two flagella appear to be developed from a pair of arrested mitotic figures developed out of the distal of the two segments into which the original kinetonucleus divides." 
is even more elaborate in the former parasite than in the case just described. Further evidence in support of Schaudinn's view of the intimate relation and correspondence between the two nuclear organellae is furnished by Léger (50), who has observed, in "ookinetes" of T. barbatulae (see under "Life-Cycle"), heteropolar division of a single large nucleus, doubtless leading to the formation of tropho- and kinetonucleus; and by Bradford and Plimmer (6), who have observed the latter element ("micronucleus") ${ }^{1}$ given off from the larger nucleus in $T$. brucii. Perhaps the most striking confirmation of the essential nuclear character of the kinetonucleus is afforded, however, by a comparison of this organella in Trypanoplasma borreli, where it is particularly large, and like a nucleus; in fact, it was originally regarded as the nucleus (trophonucleus) of
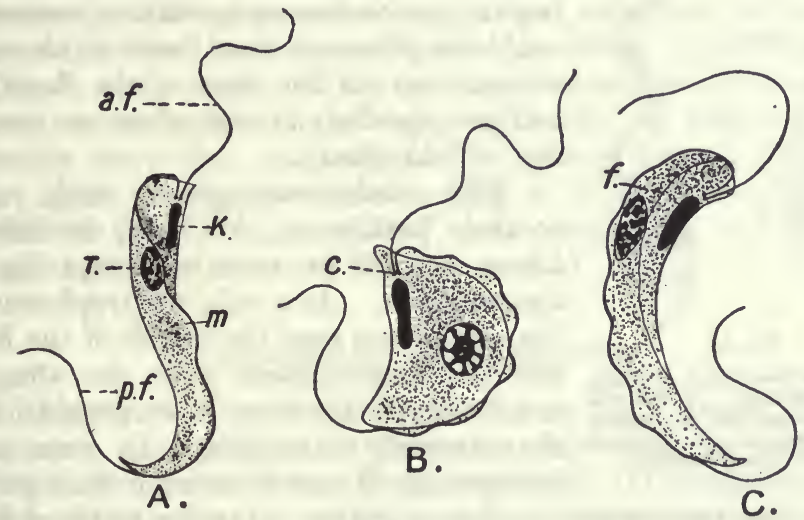

Fro. 11.

Trypanoplasma borreli, Lav. and Mesn. a.f, anterior flagellum ; $p . f$, posterior flagellum ; $m$, undulating membrane; $T$, trophonucleus; $K$, kinetonucleus ; $f$, fibril (myoneme) ; $c$, centrosomic granule at base of flagellum. (After Léger.)

this parasite. Moreover, in addition to the kinetonucleus, and immediately in front of it, two centrosomic granules can be distinctly seen, one at the base of each flagellum (shown clearly in Léger's figures, Fig. 11, B, c). In Trypanosoma also, in many cases, the root of the flagellum is not actually connected with the kinetonucleus, but terminates before reaching it in an unmistakable granule, which 'we have found to stain much darker than the flagellum. Lastly, in this connection the writer has been very kindly permitted by Prof. Minchin to make use of two figures of his (about to be published) of Trypanosoma grayi undergoing division, in which centrosomic granules, associated with the kinetonuclei, are clearly

1 It is necessary to point out that the kinetonucleus is not a "microuucleus," in the sense in which this term is always used as applying to that body in the Infusoria. In the Trypanosomes both nuclei are equally essential and functional, in somatic life as well as in sexual reproduction. 
shown (Fig. 12). An additional feature of interest is the presence of a well-developed axial spindle, still connecting the two trophonuclei (which have divided last), and ending in a granule inside each, which is doubtless the trophonuclear centrosome. In other cases (e.g. T. remaki, Trypanoplasma borreli, Trypanophis) as well, a large, distinct granule has been described in the centre of the nucleus, which very probably represents the trophonuclear centrosome (karyocentrosome). To sum up, the above facts leave little reason to doubt (a) that the kinetonucleus of a Trypanosome is not merely an extranuclear centrosome,${ }^{1}$ but a true nucleus, homologous with and equivalent to the trophonucleus, the two being specialised for different functions; and $(b)$, that distinct centrosomes are associated

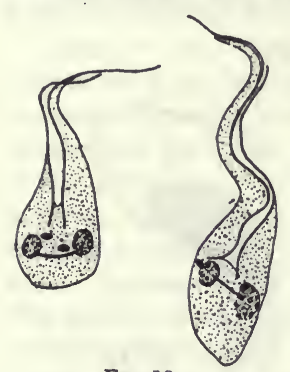

Fro. 12.

:Trypanosoma grayi, dividing. (After Minchin.) In the left. hand drawing the two centrosomic granules are seen one above each kinetonucleus. with both nuclei, the trophonucleus possessing an intranuclear one, while in connection with the kinetonucleus there is an extranuclear one (at the base of the flagellum) and perhaps also an intranuclear one (according to Schaudinn).

Both nuclei vary greatly with regard to their position in the body, in different forms, as will be seen on comparing the figures given. As a rule, the trophonucleus lies somewhere near the middle of the body, and the kinetonucleus near the aflagellar end, being farther from it in proportion as the extremity is tapering. In some cases, however, at all events during certain periods of life, the two nuclei lie close together centrally, at times being actually in contact (cf. T. inopinatum, Fig. 8, c ; T. "transvaaliense," Fig. 33 ; T. rotatorium, Fig. 8, B; and T. lewisi, young forms, Fig. $21, \mathrm{E})$. The trophonucleus is generally ovoid in shape, the longer axis being longitudinal, but in the Trypanosome described by Dutton and Todd from Senegambian birds, and also in T. hannce, the long axis is transverse to that of the body (Figs. $7, \mathrm{G} ; 35$ ). As regards its minute structure, the trophonucleus appears generally to consist of an aggregation of chromatin grains embedded in a plastin-like matrix. No mention is usually made of a nuclear membrane or reticulum. In his account of Trypanoplasma borreli, it may be noted, Léger (49) has described eight dumb-bell-shaped chromosomes. An unusual appearance of the trophonucleus has been observed in one or two instances ( $T$. brucii, Stuhlmann and Miss Robertson; T. raiae, in the leech, Miss Robertson). In these,

1 Salvin-Moore and Breinl, in their account of the "cytology of the Trypanosomes" (Ann. Trop. Med., Liverpool, 1907), continue so to regard this organella, in spite of all the evidence to the contrary, much of which (e.g. that furnished by Trypanoplasma) they entirely overlook. 
this organella is very much elongated, and the chromatin is arranged in the form of a ladder of parallel rods or pairs of granules (chromosomes ?). There is not much to note with regard to the kinetonucleus. In a solitary instance, namely, T. equinum, it is extremely minute and difficult to distinguish ; it appears as a dot-like thickening at the root of the flagellum (Fig. 7, D). In this case, the organella has apparently become reduced.

The occurrence of prominent myonemes in the undulating membrane of Trypanomorpha, and their nuclear origin (as "mantlefibrils"), has been already described. According to Prowazek (l.c.), a similar development occurs in both T. lewisi and T. brucii; here the myonemes lie in the general ectoplasm of the body, four on each side, but they are very delicate and difficult to make out. In two or three other parasites longitudinal striations', comparable to muscle-fibrillae, have been observed; nothing is known, however, about their origin. Thus in Trypanoplasma borreli there are two, one on each side of the body, which start in front and run backwards more than half-way, finally joining ventrally (Fig. 11, C, f). Again, in Trypanosoma soleae (Fig. 8, J), the ribbed forms of T. rotatorium (Fig. 37, A), and in T. avium (according to Novy and M'Neal), myoneme striations are well marked.

\section{Biological Considerations.}

A. Movement.-In general, Trypanosomes are extremely active organisms. According to the manner in which they are produced, two kinds of movement can be distinguished: (1) displacement of the body, usually rapid ; and (2) creeping and pushing movements, by means of flexion, extension and contraction of the body, etc. The latter kind are brought about, in all probability, by the superficial myonemes mentioned above ; they are, in fact, often comparable to " euglenoid" movements (cf. the flexion movements of sporozoites). In all such cases, it is important to note, the non-flagellate end moves first.

In active movements of displacement, the flagellar extremity generally leads the way. The motion may be very rapid and relatively considerable, as in $T$. lewisi ; or sluggish and inconsiderable, as in T. brucii, whose powers of active displacement appear slight or else little used. There is some difference of opinion as to whether the undulating membrane or the flagellum plays the principal part in this locomotion. The flagellum doubtless acts to a certain extent as a tractellum, especially in cases of very rapid movement. In Trypanoplasma, in which, of course, the anterior end goes first, the principal organella concerned, according to Léger (l.c.), is the undulating membrane, whose rapid vibrations produce quickly succeeding waves, running backwards. The oscillations 
may be continued into the posterior flagellum, which then acts as a pulsellum; Léger thinks, however, that this flagellum. functions chiefly as a rudder (Schleppgeissel). The anterior flagellum is not greatly, if at all, concerned in the movement.

All Trypanosomes undergo, more or less continually, a vibratile or undulatory motion of the membrane, which may take place in either direction. Among the elongated Piscine forms, movements of contortion are much in evidence, the body being frequently coited up on itself. In many Trypanosomes, again, especially the more spirochaetiform ones, the membrane appears spirally wound round the body, this being due to a more or less pronounced torsion of the latter, which gives the animals a corkscrew-like motion.

B. Aggloneration.-This characteristic phenomenon of Trypanosomes occurs chiefly or only upon the advent of unfavourable biological conditions in the surrounding medium. In the normal blood or other humour of Vertebrate hosts agglomeration has only, been observed in one or two cases, when it has been termed autoagglomeration. Agglomeration is readily brought about artificially in various ways; e.g. when drawn blood containing the parasites is kept for some time at a low temperature; when sera of other animals, especially of animals which have been once or twice inoculated with the particular Trypanosome, are added to fresh blood; or by the addition of chemical solutions. ${ }^{1}$

Agglomeration generally commences by two Trypanosomes coming together and joining (Fig. 14, A); and the union may sometimes remain only binary. In most cases, however, the agglomeration progresses rapidly, a number of parasites collecting round a common centre and forming a multiple union or rosette (Figs. 13 ; 14, B). Such a cluster or rosette is known as a primary agglomeration, and may consist of as many as a hundred individuals ; sometimes the rosettes themselves become grouped together to form large tangled masses. In a natural (as opposed to an artificial) medium, agglomeration of a particular form of Trypanosome takes place, typically, by the same extremity. In Trypanomorpha noctuae, according to Schaudinn, this is the flagellate (anterior) end; i.e. the parasites unite with the flagella pointing towards the centre (Fig. 13). In Trypanosoma, on the other hand, the union is by means of the aflagellar end. ${ }^{2}$

1 For fuller details the reader is referred to the works of Laveran and Mesnil $(37,43)$, Lignières (54), Thiroux (83), and others.

2 In artificial cultures, clnsters are frequently observed in which the arrangement of the parasites is not constant, even in the same species; that is to say, sometimes the Trypanosomes have their flagella at the periphery, while at others the flagella are centrally directed. It appears, however, that two entirely different processes are concerned. In some cases, at any rate, those clusters which have the flagella pointing centrally are instances not of agglomeration, but of rapid successive division, where the parasites remain more or less in contact and form large colonies. This has been well brought out by Novy and M'Neal (62. 63), Thiroux, and others. 
This peculiar feature of Trypanosomes differs in one or two important respects from the somewhat similar phenomenon of agglutination in Bacteria. The Trypanosomes do not in the slightest lose their mobility during the process. Each individual continues active movements, its flagellum lashing away at the periphery, and appears to be making strenuous endeavours to escape. Again, a rosette is able to become disagglomerated; a bacterial cluster or agglutination, on the other hand, is never dissolved. Disagglomeration is apparently in consequence of the retention of the power of movement by the parasites. Sometimes all the individuals, apparently quite unaltered niorphologically,

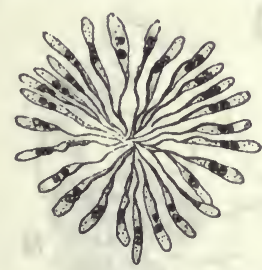

Fra. 13.

Agglomerated cluster of male forms of Trypunomor. pha noctucue in the intestine of the gnat. (After Schaudinn.)

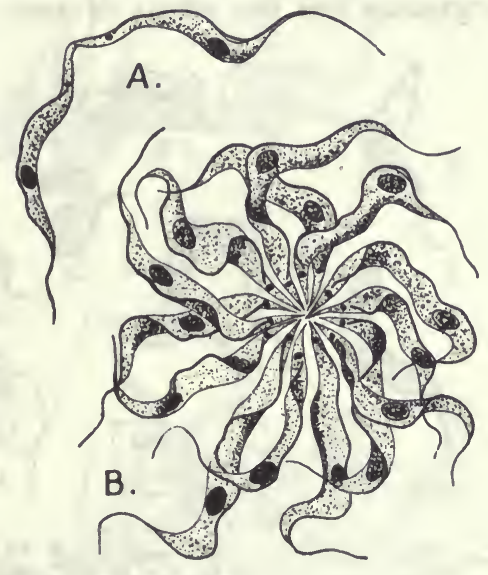

Fig. 14.

A, binary union or agglomeration of $T$. lewisi. B, primary rosette of the sane parasite. (After L. and M.)

become thus dispersed. At other times disagglomeration is only partial, a certain number of the more feeble Trypanosomes remaining together and slowly dying. If the agglomerating serum is very powerful, however, or if the biological conditions remain unfavourable, the rosette does not break up and the parasites at length die off.

The significance of the process has yet to be ascertained. By some it is regarded as a purely involuntary proceeding on the part of the parasites, and brought about more or less mechanically. A suggestion put forward by Lignières (54) is not without interest, particularly when the recent work of Calkins on the essential meaning of fertilisation is borne in mind. This author considers it quite probable that, as a result of the close intimacy, a molecular interchange goes on between the associated individuals, which may have a stimulating or recuperative value.

C. Abnormal and In 'ution Forms.-Involution and degenerative phases of Trypanosomes have received attention and acquired an 
importance altogether undeserved, owing chiefly to the fact that many of these parasites have been studied, so far, only in strange and unaccustomed hosts-hosts to which they are unadapted, and for which they, on their part, prove markedly pathogenic.

Trypanosomes appear to be, in most cases, able to support, for a longer or shorter period, unfavourable conditions of environment, whether due to the reaction of the host itself or to the transference of the parasites to a strange medium. Sooner or later, however, the organisms feel the effects of such changed circumstances and

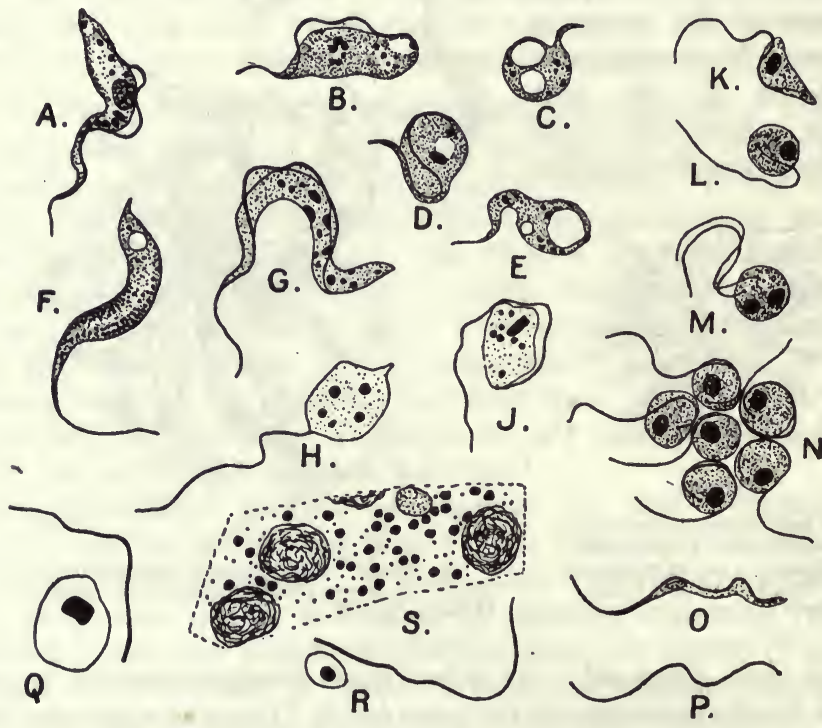

Fia. 15.

Involution and degeneration forms of different Trypanosomes. A-E, T. gambiense (A, C, and $\mathbf{F}$ after Bruce and Nabarro; $\mathbf{B}$ and $\mathbf{D}$ after Castellani). $\mathbf{F}, \mathrm{K}-\mathrm{P}, \mathrm{T}$. brucii (F after $\mathrm{Br}$, and

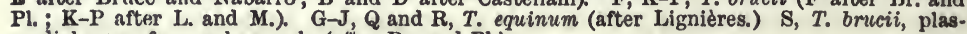
modial mass, from spleen pulp (after Br. and Pl.).

become markedly altered. The strange forms and appearances frequently described are probably for the most part ${ }^{1}$ abnormal ; i.e. they do not represent phases in the typical life-cycle, but are varying stages in a process of degeneration. Nevertheless, it by no means follows that the parasites rapidly die off. On the contrary, many of these involution-forms, on entering the blood of a fresh host, are able to infect it, though they may even have been kept for some time in artificial surroundings.

The course which involution takes varies in different cases, but the process generally follows one or another of three lines, which 
may occasionally be met with in combination in any given abnormal form. (a) Chromatolysis. Here there is either a more or less complete loss by the nucleus (usually the trophonucleus) of its chromatic constituents, which pass _out into the cytoplasm leaving only a faintly staining plastinoid basis (Fig. 15, A); or else direct fragmentation of the nucleus occurs $(F-J)_{0}^{1}$ (b) Vacuolisation. The frequent presence of a vacuole in many Trypanosomes, which is

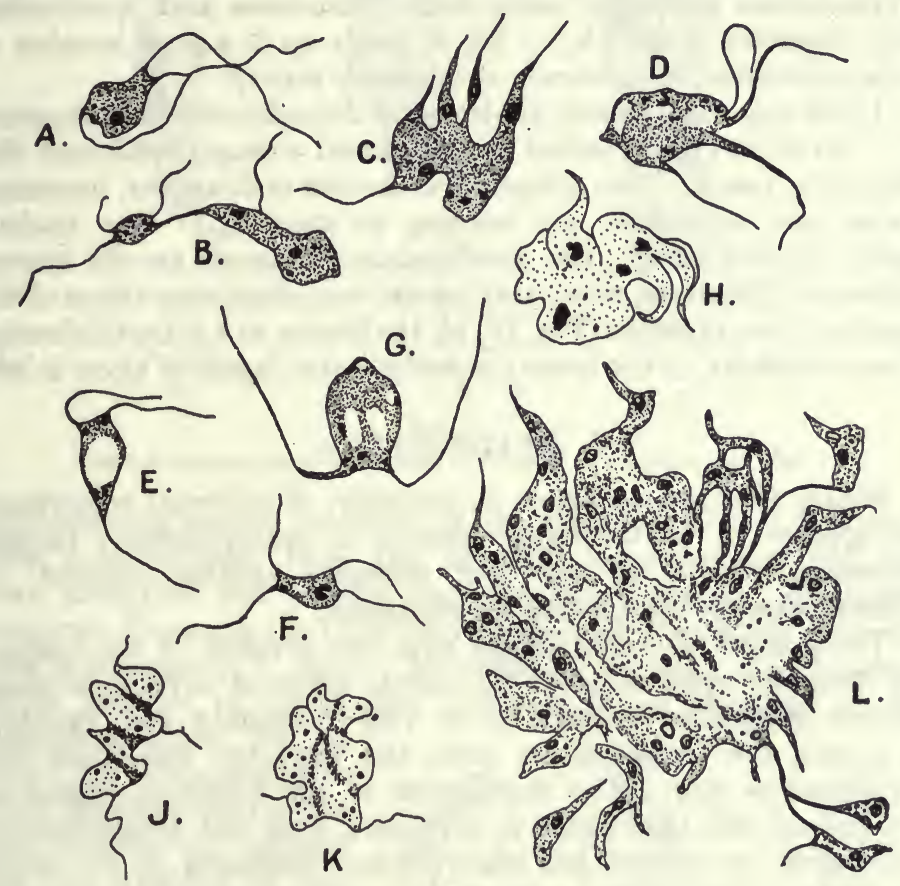

Fig. 16.

Involution and degeneration forms (continued). A-C, T. brucii, after Br. and Pl. ; D-G, T. gambiense, after Castellani ; H, T. brucii, after Martini ; J, K, $T$. equinum, after Voges ; $L, T$. brucii, aggiomeration-cluster, commencing to form a plasmodium, after $\mathrm{Br}$. and $\mathrm{Pl}$.

probably to be regarded as a normal cell-organella, has been mentioned above. The first indication of abnormality in this direction is perhaps afforded when the vacuole increases very greatly in size (Figs. $15, \mathrm{E} ; 16, \mathrm{E})$. Other, irregular ones may appear in the cytoplasm, when the involution becomes pronounced in character (Figs. 15, C; 16, G). (c) Change of form. This is, from the weird forms often resulting, the most obvious line of involution. Alteration in shape is generally accompanied by an increasing loss of mobility. In the

1 In certain of these cases it is possible that something in the nature of cliromidial formation may be going on, leading to nuclear readjustment. 
case of single forms the body becomes stumpy (Fig. 15, C-E), losing almost entirely its trypaniform shape, and ends by being ovoid or like a ball $(\mathrm{C}, \mathrm{H}, \mathrm{L}) ;^{\mathrm{l}}$ the flagellum is limp and inactive and partially coiled up (J). In other cases, quite irregular multiplication occurs, accompanied by incomplete cytoplasmic division, leading to the formation of distorted multinucleate and multiflagellate bodies (Fig. 16, A-G). Lastly, various fusion-forms may be met with, masses of Trypanosomes gradually losing their distinctness and constituting large plasmodia (Figs. 16, L; 15, s), made up of a great number of nuclei embedded in a common cytoplasmic matrix.

If the organisms remain subjected to the unfavourable influences, or if involution has reached too advanced a stage, death and disintegration result. The cytoplasm is the first to disappear, becoming hyaline and colourless, and refusing to stain up. The nucleus rapidly follows suit. The most resistant elements are the kinetonucleus and flagellum, which may persist long after other traces of the organism have vanished (Fig. 15, P), the former as a little thickening at one extremity of the latter; sometimes the flagellum alone is left.

\section{Multiplication.}

Binary longitudinal fission is, probably, of universal occurrence, and appears to be the usual method of multiplication; though Trypanosoma lewisi, at any rate, possesses another method in addition, namely, rosette-like segmentation.

The process of fission begins with the division of the nuclear and locomotor apparatus, but the actual order of division of these different organellae appears to be very inconstant and variable. As a rule, the kinetonucleus leads the way, but sometimes the trophonucleus may. The duplication of the flagellum begins at its proximal end, that which is in relation with the kinetonucleus. Until lately the process has always been considered as an actual longitudinal splitting of the flagellum, following upon the separation of the two daughter-kinetonuclei. Now and again examples are met with in which the duplication of the flagella has taken place before the kinetonucleus has divided. It seems probable that it is really the division of the kinetonuclear centrosome which is the essential prelude to the division of the locomotor apparatus. This flagellar splitting has been described either as extending to the distal end of the undulating membrane, after which the two halves separate (Fig. 17, C), or as being practically limited to the root-

1 It is here, if anywhere, that there might be a possibility of regarding as involutionforms phases which really belong to the normal life-cycle; e.g. rounded-off, resting phases (cf. the "resistant forms" of Holmes and others). In such, however, the flagellum would doubtless be absent, while the nuclear elements and cytoplasin would be as usual ; in fact, the parasites might well show a resemblance to the LeishmanDonovan bodies (cf. pp. 255 et seq.). 
portion, which becomes thickened and then divides, one half breaking away as a new, short flagellum, whose further growth is basal and centrifugal (Fig. 20, D). Schandinn found, however, that in

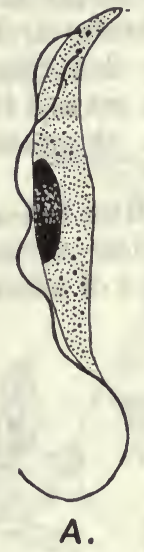

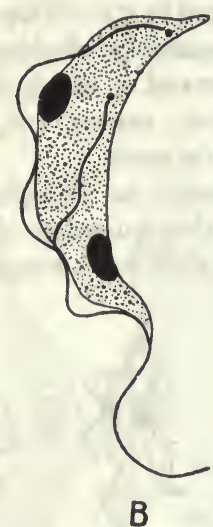

Fig. 17 .

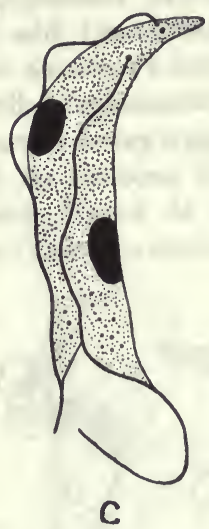

C

Stages in binary longitudinal fission of T. brucii. (After Lav. and Mesn.)

Trypanomorpha noctuae the whole of the flagellum, etc., is developed quite independently from the daughter-kinetonucleus, and laid down alongside and parallel with the old locomotor apparatus; moreover, Prowazek (l.c.) and also M'Neal (56) maintain that the same is the case in T. lewisi. Nevertheless, in many cases it seems hardly possible to doubt that there is actual splitting of the flagellum; where, for instance, the two new flagella of the proximal part of the body appear to actually join and continue as one, yet undivided flagellum (as seen in Fig. 17, A and B). Again, even where a daughter-flagellum is separate from the main one, the course of the two is often so exactly parallel that their origin by longitudinal fission is highly

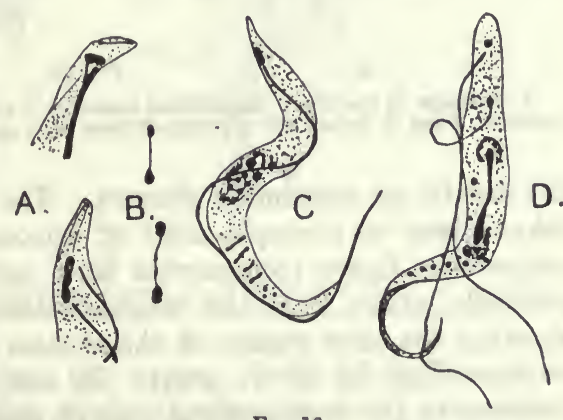

Fio. 18.

Details in the nuclear division of $T$. brucii. (After Prowazek.) probable.

So far, we have not much knowledge with regard to the cytological details of nuclear division. Prowazek has given a description of the process in $T$. brucii. The kinetonucleus becomes thickened and spindle-like (Fig. 18, A). Subsequently it becomes 
dumb-bell-shaped, after which the two halves become farther separated, remaining connected only by a short thread (в). The chromatin of the trophonucleus is arranged in eight rather elongated chromosomes, which next begin to divide in a similar dumb-bell-like manner (Fig. 18, c). The trophonuclear karyosome (karyocentrosome) has frequently divided by this time; but in one instance Prowazek observed it much drawn out and functioning as an intranuclear division centre (D), the chromatin having become aggregated around its ends.

In her account of $T$. raiae in Pontobdella Miss Robertson (l.c.) has gone at length into the question of nuclear division. The kinetonucleus appears to divide by a simple kind of mitosis though
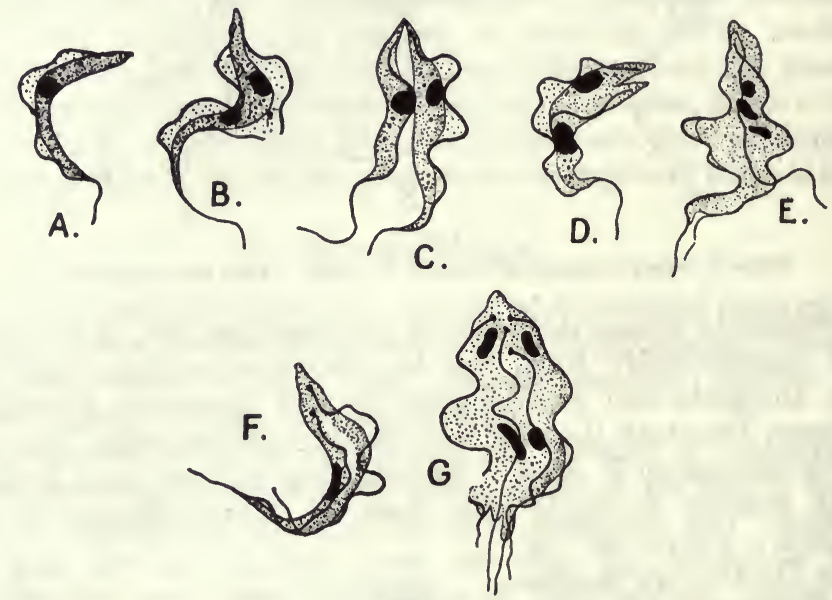

Fig. 19.

$\mathbf{A}-\mathbf{D}$, stages in the binary longitudinal fission of $T$. equinum; $\mathbf{E}$, multiple fission in the same parasite; $F$ and $G$, binary and multiple division in $T$. equiperdum. (After Lignières.)

the details are extremely obscure. The trophonuclear division also takes place by a simple kind of mitosis, but shows a well-defined achromatic figure (comparable to a series of axial fibrils). This probably arises from the trophonuclear centrosome. The figures showing the later phases of the process convey quite the same idea as does Fig. 12 of $T$. grayi. In fact, this case also appears to conform to the same general plan as those above described.

The division of the cytoplasm takes place last. In the great majority of forms this is equal or sub-equal, and the two resulting daughter-Trypanosomes are of approximately the same size (Figs. $17 ; 19, \mathrm{c})$. Although the cytoplasmic fission usually begins at the flagellar end, it may start at the opposite extremity (cf. Fig. 19, D). In some instances (Fig. 19, $\mathrm{E}$ and $\mathrm{G}$ ) the longitudinal fission is 
multiple, the original individual giving rise, simultaneously, to three or four descendants.

T. lewisi differs from most Trypanosomes in that the.cytoplasm generally ${ }^{1}$ divides in a very unequal manner (Fig. 20). Indeed, the process is more comparable to budding, since the larger or parent individual may produce, successively, more than one
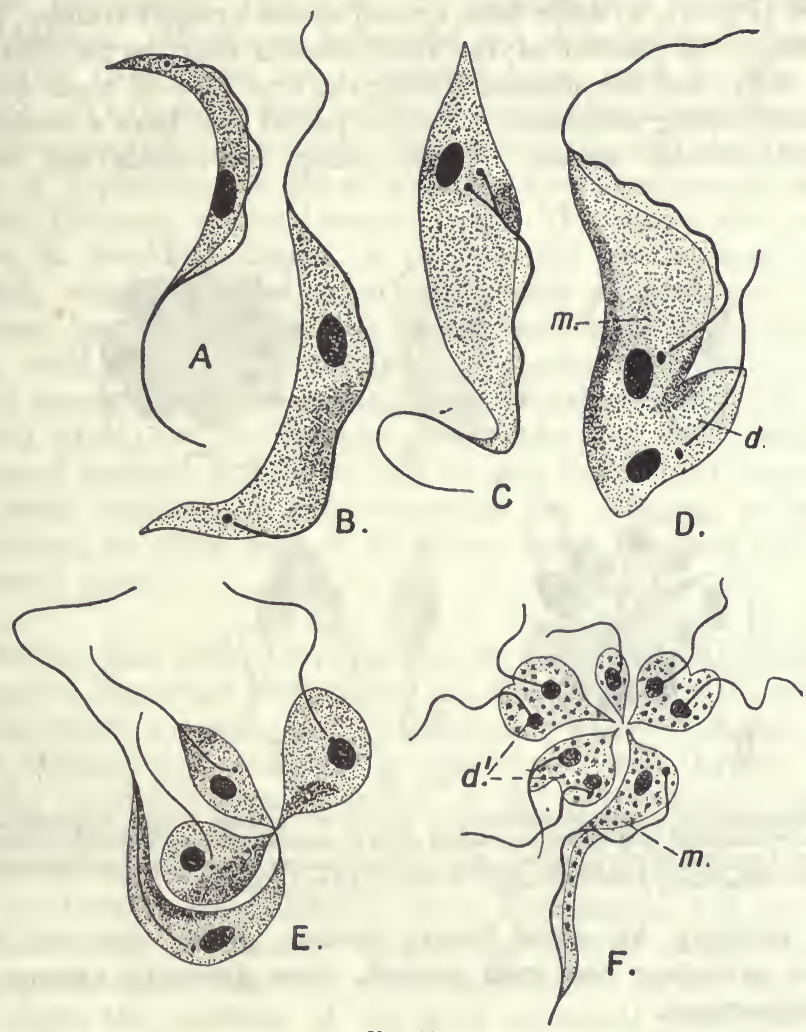

FIG. 20.

Unequal division in T. lewisi. $m$, parent-indivilual ; $l$, daughter-individual ; $d$, danghterindividual dividing. $\times 2000$. (A-E after Lav. and Mesil. ; F atter Wasielewsky and senn.)

daughter-individual; moreover, the progeny may themselves subdivide before separating, the whole family remaining connected together by the non-flagellate end (Fig. 20, $\mathrm{E}$ and $\mathrm{F}$ ). In this type of division, it may be noted, the kinetonucleus comes to lie alongside the trophonucleus, or even passes to the other side of it (i.e. nearer the flagellar end). This method of division forms, as it were, a

1 Swingle (81) has recently found that $T$. lewisi may also divide by equal binary fission ; and in such cases the two flagella may lie on opposite silles of the body. 
transition between binary fission and the other characteristic method of $T$. lewisi, namely, segmentation or rosette-formation (Fig. 21). The chief difference is that, in the latter, no parent-individual is recognisable, the segmentation being equal and giving rise to a rosette of equal daughter-Trypanosomes.

The small parasites resulting from either of these modes of division (Fig. 21, E) differ from typical adults by their stumpy, pyriform shape, the position of the kinetonucleus near the flagellar end of the body, and the absence, during the first part of their youth, of an undulating membrane. At this period they have a somewhat Herpetomonas-like aspect. These young individuals can them-

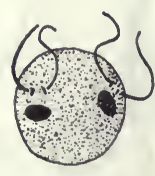

A

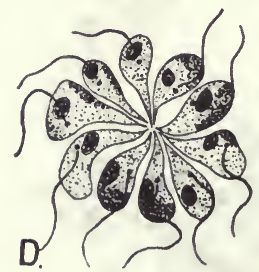

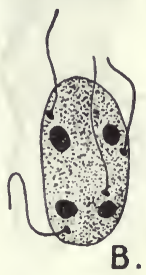

B.

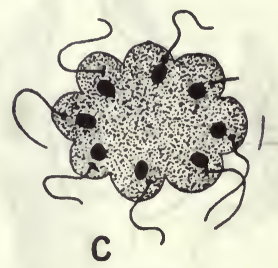

C

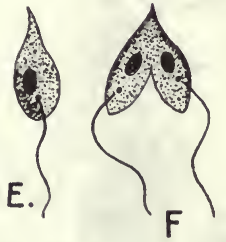

F'ic, 21.

A-D, segmentation (rosette-formation) in $T$. lewisi; in C nuclear division has finished and the danghter-nuclei (of hoth kinds) have taken up a superficial position, while the cytoplasm has become lobulated at the periphery, prior to the formation of the danghter-Trypanosones. E, daughter-individual; F, one dividing. $\times 1750$. (After L. and M.)

selves multiply by equal binary fission, giving rise to little fusiform parasites; and, with growth, these gradually assume the adult appearance.

\section{The Life-Cycle of Trypanosomes}

It may be safely said that this remains, even to-day, one of the most difficult and most debated questions among the whole of the Protozoa, in spite of the amount of work, of one kind or another, which has been contributed to the subject during the last few years. When the present writer compiled his Review of the Haemoflagellates (3) some years ago, Schaudinn's remarkable observations had been, to all appearance, amply corroborated in various directions by the testimony of the Sergents ( $7 T$ ), Billet $(4,5)$, Brumpt (9), Léger (50), and Rogers (94); in short, the 
whole trend of research pointed at the time to a very complex life-cycle of the Haemoflagellates and to in close comnection with the Haemosporidia. Since then, however, owing in a great micasure to the work of Novy and M'Neal on the Trypanosomes of birds (62) and of mosquitoes (63), the results obtained by Schaudinn have become, to a large extent, discredited; these authors maintaining that they are capable of a quite different interpretation. Moreover, influenced by their work on Insectan Flagellates, Novy and his colleagues have gone to the other extreme and expressed their belief not merely that Haemoflagellates and Haemosporidia sire entirely distinct, but also that the Trypanosomes of Vertebrates do not undergo any true development or part of their life-cycle in the Insectan host. This latter view, at all events, is, we think, shown to be incorrect by the most recent research, which, as above mentioned, seems all in favour of an alternate, Invertebrate host, one of the most important indications being with regard to the specificity of the latter-a point of the utmost consequence in its bearing upon investigations of this kind. Leaving aside for the moment a consideration of Schaudinn's celebrated memoir, it will be best to give first a brief account of the results obtained in this connection by different prominent researchers, to other aspects of whose work reference has been previously made.

Dealing first with Trypanosomes of cold-blooded Vertebrates, the earliest important observations are those of Léger (50), relating to Tinprenoplesma rarium and Trimpenosma burbutulue of the loach. Le'ger distinguishes ordinary ("indifferent") and larger, more granular (probably female) forms of the Tr!ypunoplasma in the blood of the fish. When a leech (IIrmiclepsis sp.) was allowed to snck blood containing only these parasites, which thereupon passed into its stomach, the indifferent forms degenerated. and perished, while the female ones became massive and showed nuclear changes, preparatory, Léger thinks, to a sexulul process. At any rate, after some days, the intestine of the leech contained numerous little narrow Trypanoplisms, of which some, very filiform, perhaps represented male forms, while others possessed a kind of beak or rostrum in place of the anterior flagellum, which made them resemble Trypanosomes. The development of Trypanosoma barbutulae in another leech (P'iscicolı) showed a certain amount of igreement with that described by Schaudinn in the case of his Avian Trypanosome in the gnat (Culex). Eighteen hours after the leech had fed on blood containing exclusively $T$. barbatulae, pyriform bodies lacking a flagellum ("ookinetes") were found in the intestinal contents. Some of these had a single large nucleus (i.e. a compound nucleus); others had two nuclei, one smaller than 
the other. Four days later the intestine contained numerous Trypanosmes which could be readily distinguished as belonging to one of Schaudinn's three types-namely, indifferent, male, or female. The male forms are very elongated and slender, provided with a minute rostrum at the aflagellar end, and with a well-developecl flagellum at the opposite extremity, which renders them extremely active; they also creep or crawl with the rostrum in front. Their cytoplasm is very clear and usually lacks granulations. Female forms, on the contrary, are large and broad, with deeply staining, usually granular cytoplasm; the flagellum is only feebly developed and the movement is sluggish. The indifferent individuals occupy in most respects an intermediate position between the other two types. A point of importance is that the kinetonucleus frequently lies in about the middle of the body, and may be close to the trophonucleus. There can be no doubt, it may be here remarked, that these different sets of forms are of regular occurrence in, at any rate, certain Trypanosomes. Since Schaudinn first described them several observers have recognised them, in some instances in the Vertebrate host, but always more sharply differentiated in the Invertebrate. In general, the three types show the same characteristics as noticed in the case of $T$. barbatulae. The indifferent forms, Léger states, underwent active multiplication, by equal fission; those females which divided did so very unequally, by a process somewhat like budding. The manner and form in which the parasites passed back into the fish were not ascertained.

In his valuable contributions on the behaviour of Piscine Trypanosomes in leeches, Brumpt (11) has noted developmental phases of $T$. granulosum of the eel in Hemiclepsis. Some hours after arrival in the stomac ${ }^{\circ}$ of the leech, all the parasites become pyriform, and by the position of the kinetonucleus close to the trophonucleus recall Léger's Crithidia-type (see below). By active multiplication, an enormous number of little forms are produced, which by the end of forty-eight hours have nearly all passed into the intestine. Here they rapidly become elongated, assuming a Herpetomonad-form, which may be retained for several months. Some, however, by the end of seventy-two hours, have given rise to true Trypanosoma-forms, with typical undulating membrane, which pass forwards towards the stomach, and may be found accumulated in the foremost stomach-coeca and in the proboscis-sheath by the fifth day. These are the forms which are inoculated into the eel, becoming by simple elongation ordinary $T$. granulosum again.

Miss Robertson has published (72) some interesting observations on a Trypanosome met with in Pontobdella muricata, which she regards as $T$. raiale. This view is rendered extremely probable from the fact that Brumpt (10) has found that T. raiae does develop in Pontobdella. According to both authors the earliest phases occurring 
are rounded forms with both nuclei but no locomotor apparatuscomparable to ookinetes, in short (cf. T. barbutulue above). These individuals, says Miss Robertson, which divide in this condition fitirly actively, gradually disappear from the crop and are found only in the intestine. Here they develop a locomotor apparatus, but persist for some time in a Crithidict-like form; they are of varying size and may be very small. Later on, these individuals take on a more or less typical Trypanosome-like, or, as we have previously termed it, trypaniform character, with the kinetonucleus in the aflagellar half of the body, though its actual position varies greatly. These trypaniform individuals are of two main types, which appear, however, to be connected by intermediate grades. One kind is relatively very broad, with a relatively small kinetonucleus, but usually with a fairly long flagellum. The other type is a long slender Trypanosome, with a large kinetonucleus, but the free flagellum is not, as a rule, very long. The constitution of the trophonucleus presents an unusual condition; it is very much drawn out, and the chromatin is arranged in a number of transverse rods or bars (perhaps comparable to chromosomes) arranged more or less parallel, like a ladder (cf. author's note on T. brucii above, p. 216). About the middle of digestion, these Trypanosomes occur chiefly in the intestine, but also in the crop, often in large numbers. At a later period, a still more slender, practically thread-like form is developed, which is met with chiefly in the proboscis, though also, apparently, in the intestine. This type, which differs rather from the last, appears to die off if it remains in the leech, and taking this in conjunction with the occurrence of these individuals in the proboscis, the inference is that this is the form in which the parasites are inoculated into the fish. At the close of digestion, a number of very small forms are always to be seen, either in a rounded (probably resting) condition or in a very early Crithidial phase. These seem to be persistent forms, through which the leech retains the infection.

Miss Robertson discusses the likelihood of the two contrasting trypaniform types above described representing male and female individuals, but for several reasons hesitates to accept this view. However this may be, it is more probable that conjugation itself takes place soon after the transfer of the parasites from one host to the other, i.e. after the arrival in the Invertebrate; and that the ookinete form is the immediate result of the process. This is suggested by Léger's work on 'T. barbatulue, as well as by Keysselitz's account of the life-cycle of Trypanoplasma borreli (2i). It is also regarded by Prowazek (68) as being the case in 'T. lewisi, in the louse.

According to Keysselitz, male and female gametes can be readily recognised in the blood of the fish (carp), the conjugation 
taking place in the leech, after various regulatory or maturation processes have been undergone. The copulae give rise to the three general types, distinguished principally by nuclear differences.

In the case of T. lewisi, Prowazek states that soon after reaching the mid-gut of the louse, the parasites undergo reduction of the nuclear apparatus, by which the number of chromosomes is sitid to be reduced from sixteen to four. The gametocytes (parentindividuals of the gametes) are not strikingly differentiated from one another, but in the formation of the microgamete from the male form, the body becomes diminished in size, its nucleus (trophonucleus) very elongated and at first spirally twisted, then band-like, while also the cytoplasm stains differently from that of the female element (megagamete).

Coming now to what is known of the development of Mam. malian Trypanosomes in Tsetse-flies (Glossinae), we have first to mention the knowledge obtained by Minchin, Gray, and Tulloch (59) with regard to $T$. gambiense in $G$. palpalis. This, unfortumately, is largely of a negative character, owing in all probability (as we bave seen earlier) to this species of fly not being the correct alternate host, but one in which the attempts of the parasite to continue its life-history are, for some reason, unsuccessful. Nevertheless, the important observation that the types already recognised as male and female in the blood of the Vertebrate at first greatly predominate, with, moreover, a much more marked differentiation of sexual characters and without any forms intermediate in type, is also strongly in favour of the idea that conjugation occurs, in general, soon after the arrival of the Trypanosomes in the insect.

No mention is made by Stuhlmann (80), in his highly interesting account of $T$. brucii in $G$. fusca, of the occurrence of any similar phases, or of anything in the nature of ookinetes, at the beginning of the infection. The first individuals found by this investigator were of the indifferent type, occurring in large numbers in the hinder part of the gut, two to four days after infection of the fly. It seems probable, however, that Stuhlmann missed some early essential phases of the development, since, as said above, Léger found ookinetes of $T$. barbatulae eighteen hours after feeding, while Minchin and his collaborators say that the sexual forms were best developed after about twenty-four hours, while by the end of fortyeight hours a type of more indifferent character was making its appearance. According to Stuhlmann, the indifferent parasites apparently spread forwards through the nid-gut, but usually pass right forward only when the flies are fed again (from an uninfected animal). By this means the presence of the Trypanosomes in the proventriculus was obtained, and in the "long" form, quite similar 
to the type occurring in the proventriculus and oesophagus of freshly caught "wild" flies.

This type manifestly corresponds to Miss Robertson's-very slender forms in the front part of the gut and proboscis of the leech ; the agreement extends to the ladder-like arrangement of the chromatin (chromosomes?) of the trophonucleus. Whereas, however, in $T$. raiae, it is these forms, or their derivatives, which appear destined to return to the fish, Stuhlmann found, in the proboscis of freshly caught Tsetses, little Crithidial forms ("small" forms), with the kinetonucleus alongside, or on the flagellar side of the trophonucleus. ${ }^{1}$ Stuhlmann regards these individuals, which he was unable to obtain in artificially infected flies, as representing the phase in which T. brucii is transmitted to the Vertebrate; though he states that the long forms seem to degenerate in the proventriculus after a time (as well as the small ones). In no case, unfortunately, was he able to actually infect a Vertebrate by means of either kind, which suggests that there is some other, as yet unknown, factor or condition concerned in this perplexing question.

Stuhlmann describes and figures certain phases found in one case in the proventriculus of an artificially infected fly, which he thinks are perhaps indicative of conjugation. In all the stages figured, the cytoplasmic body of the parasite is single; the nuclear and locomotor organellae, on the other hand, show different conditions from single to double. Of course, here as in so many other cases, it is entirely a matter of the sequence in which the figures should be taken. Stuhlmann's chief reason for his interpretation is that, in what he regards as the earlier stages of union, the flagella lie on opposite sides of the body ; whereas, in the usual mode of division, the two flagella lie on the same side of the body. Still, Stuhlmann himself agrees that the condition may be only one of an unusual mode of division; and this seems the more likely explanation, for such a mode of division has been observed in $T$. lewisi. ${ }^{2}$

That the course of a Trypanosome life-cycle may take, however, a quite different direction from that outlined in the above instances is proved unmistakably by Minchin's valuable investigations on Trypanosoma grayi (57 and 58), which led him to the unexpected discovery of the encystment of this form in the proctodaeum of $G$. palpalis.

Minchin recognises three well-marked types of this Trypanosome in the fly. (a) The ordinary type, having a multiplicative function, and probably giving rise to the swarm of parasites often found. It is usually of large size, and shows great variability, especially in the position of the kinetonucleus. While generally a little in front (i.e. on the flagellar side) of the trophonucleus, it may be alongside,

1 For an account of the proboscis-forms recently described by Roubaud, see Postscript, p. 261. 2 Cf. footnote, p. 225. 
or even behind it (i.e. nearly terminal at the aflagellar end), though it is not often in the last position. Minchin thinks this last form most nearly represents that in which $T$. grayi occurs in its Vertebrate (probably Avian) host. The second type (b) is constituted by slender, - often greatly elongated individuals, with well-developed undulating membrane and flagellum. Minchin was at first inclined to regard these as male forms; but from their occurrence in one case in remarkable numbers in the proctodaeum, to the exclusion almost entirely of any other kind, he has since thought this view to be unlikely. The primary habitat of the slender type is the proctodaeum, from which region it may extend forward through the intestine and stomach of the fly. (c) Small, very narrow forms, of a typical Herpetomonas-like structure, practically lacking any

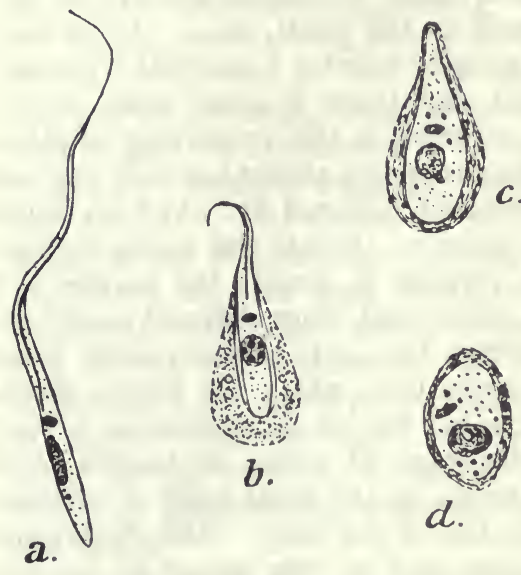

F19. 22.

Encystment of the narrow, Herpetomonad form of Trypanosoma grayi. (After Minchin.) undulating membrane (Fig. $22, a)$, which stain more faintly and appear much more delicate than parasites of type (b); the kinetonucleus is often relatively large. These individuals were found in the proctodaeum, and, rarely, in the linder intestine; they are apparently derived from young forms of the indifferent type, produced by rapid multiplication in the hinder part of the intestine.

It is this Herpetomonad type which undergoes encystment. In cyst-formation the flagellum becomes shortened and at the same time apparently thickened. The cyst begins to appear as a layer of substance, probably of a slimy or mucoid nature (cf. Prowazek's "Schleimcysten" in the case of Herpetomonas muscae-domesticae [69]), which forms a cap at the aflagellar end (Fig. 22, b). These two processes continuse until, on the one hand, the flagellum is completely retracted, and, on the other hand, the body is enveloped in a pear-shaped cyst $(c)$, which is at first incomplete at the pointed end. The flagellum appears next to become retracted into a pinkstaining vacuole (cf. the opposite process in the formation of the flagellar phase of Leishmania (Piroplasma) donovani); finally, the flagellar vacuole fades away, the cyst meanwhile closing up. Eventually there results an oval or circular cyst, containing hyaline cytoplasm and the two chief nuclear masses $(d)$. In this gurise, 
presumably, T. grayi passes into the outer world, to be swallowed subsequently by its alternate host. ${ }^{1}$

Comparing $T$. grayi with $T$. brucii, an essential point of contrast is at once noticed. In the first-named, the small, Herpetomonad forms, which have the function of propagating the infection to a fresh host, occur mainly in the proctodaeum and leave the fly per anum. In the latter, on the contrary, the small, Crithidial forms, which are compared by Minchin with those of $T$. grayi just mentioned, were found almost exclusively in the proboscis; moreover, no Trypanosomes of any kind were seen in the hindermost part of the gut (proctodaeum). Hence the propagation of $T$. brucii would appear to be just as certainly by the inoculative method as that of T. grayi is by the contaminative one. Further, just as there is at present no evidence of contaminative infection in $T$. brucii, so there is none of inoculative infection in T. grayi; for although Minchin says that the slender type, which he also thinks is a propagative form, was met with farther forward than the Herpetomonad type, it was not met with farther forward than the stomach. And this is as far as our knowledge goes up to the present.

\section{Schaudinn's Work on Haematozoa of the Little Owl.}

There remains for consideration the remarkable research of the late Fritz Schaudinn on certain parasites of Athene noctua and Culex pipiens, namely, Trypanomorpha (Trypanosoma) noctuae and "Trypanosoma" (Leucocytozoon, Spirochaeta) ziemanni. Exigencies of space preclude a detailed account of this work, only the main outlines. of which can be given here, but a full description will be found in the writer's article on the Haemoflagellates (3).

Taking first Trypanomorpha noctuae, the life-cycle may be conveniently commenced with the motile copula or ookinete resulting from conjugation in the stomach of the gnat. While the nuclear fusion of the two sets of elements (kinetic and trophic) derived from the original gametes is being completed, leading to a single, large, compound nucleus, the ookinete is getting rid of unnecessary material, such as the pigment-grains and reduction-nuclei left over in the cytoplasm (Fig. 10, A, B). Even in the ookinete stage, Schaudinn recognises the three types of individual, indifferent, male, and female, distinguishable by differences in the size of the nuclei relative to the cytoplasm, and by the varying appearance of the latter.

The development of an indifferent Trypanosome has been described above (p. 213). When formed, a period of active movement and multiplication sets in, succeeded later by a resting condition. The

1 The reasons for considering that this parasite is not merely a "fly-parasite" have been given on p. 201 . 
parasites now become gregariniform, and strongly recall the similar phase described by Léger $(48,51)$ in certain Herpetomonads. The Trypanosome bores into an epithelial cell of the stomach by means of its flagellum, which is reduced to a short, rod-like organella. Binary fission may go on, often leading to the formation of a dense layer of attached parasites. On the parasites again becoming trypaniform, the flagellar apparatus is reconstituted by the kinetonucleus. This alternation of resting and active periods is limited. Eventually the indifferent Trypanosomes may pass into the blood

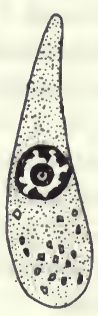

A.

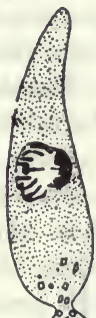

(a)

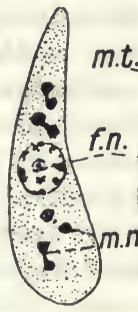

c.

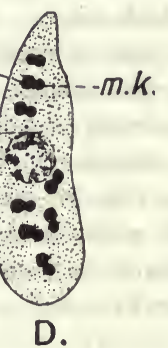

D.

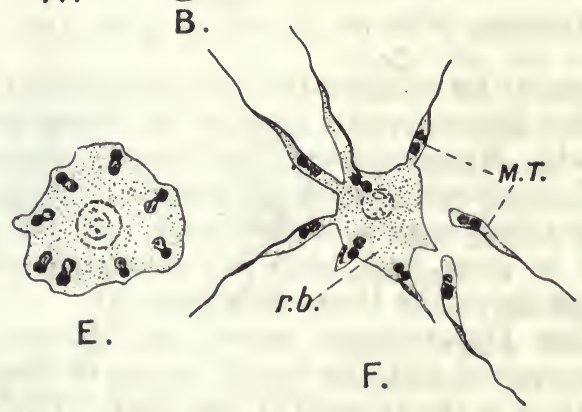

Fin. 23.

Development of micro'rametocyte and male Trypanosomes from an ookinete of male character. (After sclianlinu.) m.n, male nuclei ; f. $n$, (iewenerating female nuelens; $m . t$, male trophonucleus; $m . k$, male kinetomcleus ; $M . T$, mal. Trypanosome; r.b, resillual body.

of the owl; or they may apparently become sexual forms, male or female ; or clse, during a period of hunger, they die off.

In the development of an ookinete of male character, or microgametocyte, there is an early separation of the nuclear constituents into two halves, male and femaie. The female portion consists of a large, loose nucleus (Fig. 23, C and $\mathrm{D}, f . n$ ), which gradually degenerates and disappears. The male portion, on the other hand, gives rise to eight little double-nuclei $(\mathrm{C}$ and $\mathrm{D}, m . n)$, each consisting of trophic and kinetic portions. The microgametocyte now becomes rounded, the cight double-nuclei take up a peripheral position (E), and the cytoplasm opposite each grows out as a little prominence. As 
these elongate, each accompanied by a double-nucleus, they take on a trypaniform appearance, which is completed by the development of a flagellum. Finally, the eight little male Trypanosomes (F, M.T $)$, which are homologous with microgametes, break away from the central resiluum. These forms are apparently incapable of further development in any way and soon die off. Schaudinn accounts for this by the condition of the trophonucleus, which, he says, has undergone reduction.

The early stages in the formation of a female Trypanosome are similar to those in the case of a microgametocyte. Here, however, it is the eight small doublenuclei, representing the male constituents, which degenerate, leaving the large female nucleus to become differentiated and give rise to the locomotor apparatus in the same way as in an indifferent form (Fig. 24, c). In the females the flagellum, etc., is poorly developed, and the movements of the parasites are slow and feeble. These Trypanosomes seem unable to divide. They grow to a large size, and store up a considerable amount of reserve-nutriment in the cytoplasm. These forms are the most resistant to external in-

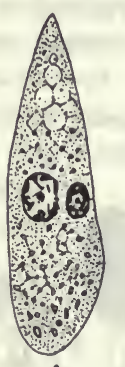

A.

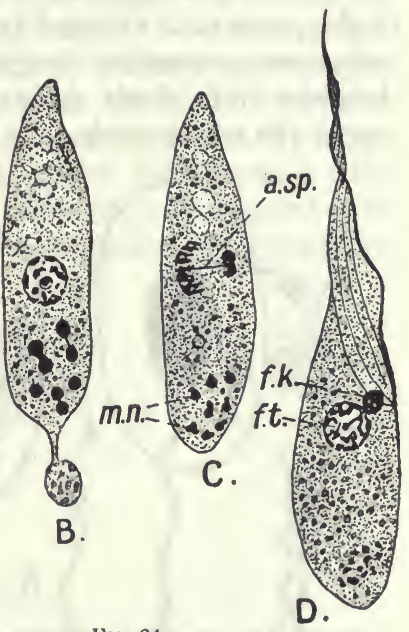

F'Ict. 24.

Development of a female Trypanosome fron an ookinete of female character. (After Schandinn.) $m . n$, degenerating male nuclei ; $a . s p$, first axial spindle of female nucleus; f.t, female trophonucleus; $f . k$, female kinetonncleus.

fluences, and can survive

long hunger-periods, in a gregariniform, resting condition. ${ }^{1}$ With the advent of fresh blood into the stomach of the gnat, the female forms undergo a process of parthenogenesis, consisting of nuclear reduction and a kind of self-fertilisation. Thus rejuvenated, they are able to give rise to a fresh succession of Trypanosomes of all three types.

The Behaviour and Development of the T'rypanosomes in the Blood of the Oul. - All the Trypanosomes met with in the bird can be recognised as belonging to one of the three categories observed in the gnat. On entering the blood, the small indifferent forms at once

1 Accorling to Schaudimn, these gregariniform females can bring alout hereditary infection, remaining dormant in the ovaries until the eggs are laid and the larvae develop. 
attach themselves to the red blood-corpuscles (Fig. 25, A and s), and begin a period of rest and growth. The locomotor apparatus disappears and the two nuclei come close together. The form of the parasite is now quite that of a young Halteridium, a well-known malarial parasite of birds, and, moreover, in twenty-four hours the first pigment-grains appear in the cytoplasm (c). By this time the parasite has greatly increased in size. It becomes vermiform and active, reconstitutes its flagellum, etc., and leaves the host-cell (D), usially in the night-time, becoming once more a typical Trypanomorpha (E). This alternation of attachment and growth with active movement in the plasma is repeated for six days, until the full size of the parasite is attained ( $\mathrm{F}$ and $\mathrm{G}$ ). The adult Trypanosome then unclergoes successive longitudinal divisions, until the resulting daughter-individuals have reached a minimum size, when they repeat the whole cycle. It is worth noting that Schaudinn never

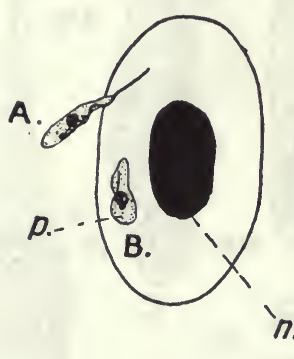

'n.

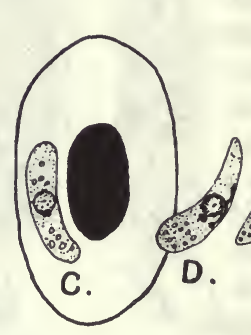

FIG. 25.

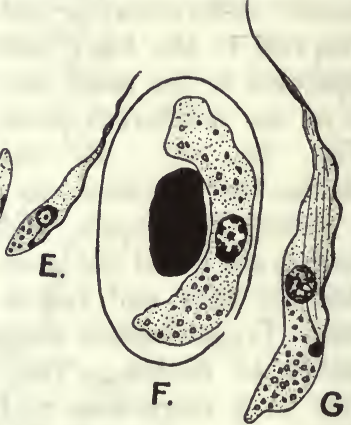

G

Staces in the growth of an indifferent Trypanosome in the blood of the owl. $n$, nucleus of red blood-corpuscle; $p$, young ectocorpuscular parasite. (After Schaudinn.)

observed any multiplication of the parasites in the gregariniform (Halterilium) condition, by schizogony, such as is met with in other Haemosporidia.

Microgametocytes (male forms) arise from very young indifferent Trypanosomes. Each gives rise to eight small, slender microgametes, in the same way as do the corresponding forms in the gnat. The microgametes are very specialised organisms. The trophonucleus (in a reduced condition) forms a long thread, on which four chromosomes are strung at intervals. There is no free flagellum at the anterior end, but the body has a whip-like tail posteriorly.

The full-grown megagametocytes are lar'ge female Trypanosomes, which are no longer able to assume the trypaniform condition, but remain enclosed by the pallid and disorganised host-cell which they were last able to penetrate. In other words, they are identical with the female gametocytes of Halteridium. Maturation 
and fertilisation do not take place until the sexual forms are transferred to the gnat. The process in its main outlines has been previously described by MacCallum in another species of Halteridium. 1 Schuudinn adds that, as soon as the parasites leave the warmblooded host, the megagametocytes become rounded off, rupture the delicate envelope still surrounding them, and then undergo a series of reduction-divisions, after which they are ready to be fertilised. The zygote develops into one of the three kinds of ookinete with which this description began.

Leucocytozoon ("T'rypanosoma") ziemanni.-Even more surprising are the data put forward by Schaudinn in the case of the other parasite (or set of parasites) upon which he worked. Just as a species of Halterilium is regarded as ontogenetically related to T'rypanomorpha noctuae, so Leucocytozoon ziemunni, a parasite of the white corpuscles and erythroblasts, is said to be intimately connected with what has been hitherto taken for a species of the genus Spirochucta, a wellknown bacterial type. Far from being, however, a true member of the Bacteria, this species at any rate was regarded by Schaudinn as possessing all the fundamental characteristics of a Trypanosome (see Fig. 7, $\mathrm{H}$ ).

The plan of the life-cycle is fundamentally similar to that just summarised, the same sets of forms being described. Two or three distinguishing features may be noticed. The indifferent Trypanosomes are extremely spirochaetiform (Fig. 26, A-D); after longitudinal fission, the two daughter-individuals remain attached end-to-end ( $\mathrm{B}$ and $\mathrm{C}$ ), the resemblance to a Spirochaete being thereby accentuated. ${ }^{2}$ The resting-phases, little pear-shaped forms with two nuclear elements (E and F), are very Piroplasmalike and strongly recall the

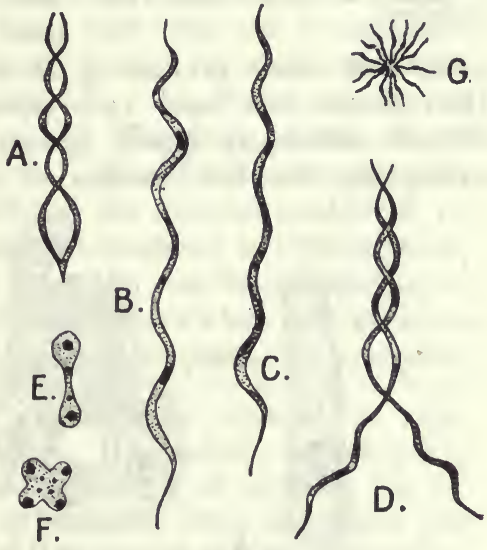

-FIG. 26.

A-D, formation and fission of spirochaetiform "couples" in "Trypanosoma" (Spirochacta) ziemanni; E, F, resting-pliases of same; G, agglomerated cluster of very minute forms. (After Schaudinn.) Leishman-Donovan bodies. On the other hand, the gametocytes (in the blood of the owl) are very large and broad, and distinctly trypaniform, even possessing

1 Sec the account of the Sporozon, by Minchin, in this treatise (Vol. I. Part II.).

3 According to Novy, I'Neal and Torrey (64), Töpfer has recently cultivated a true Spirochnete (i.e. a Bacterium) from the owl, which possesses also minute restingforms. Hence Schandinu's spirochaetiform "Trypanosoma" miy have been really this same spirochueta. 
well-marked myonemes. Prior to gamete-formation, both gametocytes come into relation with the leucocytes, in an unusual manner (see under "Habitat," p. 205), and lose all trace of locomotor organellac.

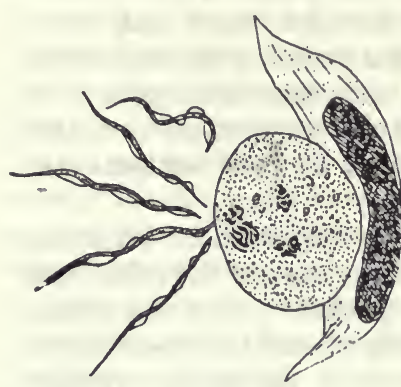

FiG. 27.

Fertilisation of a megagamete by a microgamete. The trophic and kinetic feinale promuclei are seen on the left. Near the middle lie the two relluctionnuclei. The remains of the host-cell together with the cast-off envelope of the parasite are on the right. (After Schaudinn.) error, and there is nothing to show that this was eliminated. The opinion has been very generally expressed that Schaudinn did not sufficiently guard against the liability of confusing and mixing up the life-histories of entirely distinct parasites. In
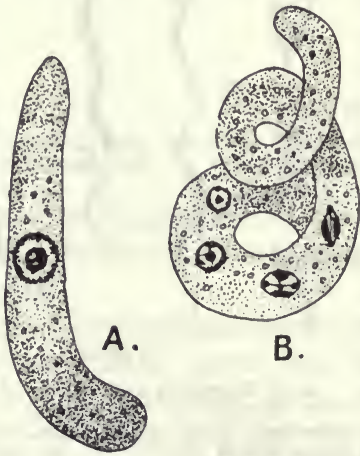

Fit, 28 .

Growtlı aud metamorphosis of an inlifferent ookinete ; in $\mathrm{C}$ nuclear multiplication is well advancetl. (After Schaudinn.)

the first place, it is said that in the species of owl used at least four separate Haematozoa occurred: two free parasites, namely, a Trypanosome and a Spirochaete ("Trypanosoma" ziemanni); and two intracellular ones, a Halteridium and a Leucocytozoon. It may be at once admitted that this is quite possible. At any rate, the entire subject is reopened and camnot be settled definitely until 
the life-cycle of some or of all the parasites concerned has been reinvestigated. (See Note below.)

While preserving an open mind upon the matter, the writer would point out that, if no indubitable confirmation of Schaudinn's far-reaching conclusions can be said to have been furnished, the merely negative evidence adduced by Novy and his colleagues is by no means sufficient proof of their erroneousness. Because the injection of cultures of certain Trypanosomes in artificial media, into birds, was not followed by the appearance of Cytozoa in the blood, these workers aprarently conclude (l.c.) that there is no connection whatever between these two groups of Haematozoa. And this comprehensive generalisation is put forward, although in nearly all cases they failed to obtain even a Trypanosome-infection by this means, apart altogether from the question whether the particular form with which they did once succeed had itself an intracellular phase!

We will admit that the cultivation-method, which is of undoubted use in other ways, may not be without value in studying the lifehistory. In certain cases, for example, the behaviour of the parasites on their arrival in the culture-medium may to some extent indicate or suggest what happens when they pass into the Invertebrate host, because of the general similarity of the physical conditions, etc., to which they are at first subjected. An illustration of this is afforded by the development of the Flagellate phases of the Leishman-Donovan bodies in cultures. Nevertheless, we certainly think that the value (in this respect) of the cultural method of research is limited, and that great caution is necessary in drawing inferences as to a parasite's life-history from the results obtained by it. We dissent entirely from the American authors when they maintain that the culture-medium is, for all practical purposes, the equivalent

Note.-The present writer has always been reluctant to think Schaudinn made such a series of nistakes. It has al ways seemed to him that this author's celebrated work on the Coccilia of Lithobius has not been taken into account sufficiently by those who lave maintained that he was hopelessly wrong in the case of the parasites of the Little Owl.

It is with the grentest pleasure, therefore, that on the point of publication of this article, the writer is able to add that after a most arduous investigation on the Haemato\%or of the common chaffinch (Fringilla coelebs), he has at length obtained the first definite and unmistakable evidence, of which he is aware, in favour of one of Schaudinn's conclusions. Here, there is only room to say that, as a result of his observations, he has now little doubt that a Hulteridium parasitic in the chaffinch becomes actually, in certain phases, a little Trypanosome; in other words, that the Halteritium and the Trypanosome which occur in this birl are ontorenetically comnected (vide Q.J. Micr. Sci. liii. p. 339, Feb. 1909). Hence the writer feels reassured with regarl to the truth of the corresponding part of Schaudinn's work. 
of the medium in the Insectan host; on the contrary, we consider that the former, whatever indications it may furnish, cannot replace altogether the latter.

It seems to us that Novy and M'Neal entirely fail to appreciate the intimate and specific relations of Protozoan parasites to their hosts, and the remarkable degree to which their biology is adapted to the same. The Sporozoa in their entirety illustrate this, so do other parasitic Protozoa, and there is no reason to suppose the Haemoflagellates are different. We agree fully with Brumpt that the chemical and physiological medium of a particular Invertebrate is essential for the adequate development of all such phases of the life-history of a Trypanosome as may be undergone outside the Vertebrate host. And the various researches above summarised, which go to show that there are right and wrong hosts for the parasites, and that only certain "ripe" phases, the outcome of the sojourn in the right host, can reinfect the Vertebrate host successfully, afford strong support to this view.

Another criticism put forward by Novy and M'Neal and others is that the Flagellate phases found in the mosquitoes (Culex), which Schaudinn regarded as belonging to Trypanomorpha of the Little Owl, were in all likelihood purely Insectan parasites, of a Herpetomonad or Crithidial type, which had nothing to do with the blood forms. Before discussing this view it is necessary to consider briefly the subject of these Insectan Flagellates, one which is also of very great importance because of its bearing upon the phylogeny and derivation of the Trypanosomes.

\section{The Insectan Flagellates: The Evolution and Phylogeny of Trypanosomes.}

\section{(a) The Insectan Filugellates.}

Several of the earlier workers have commented upon the occurrence of Flagellates in mosquitoes. Thus in 1898 Ross observed parasites which he has recently (74) compared with Léger's genus Crithidia in Anoplieles, larva, pupa, and imago. A similar parasite was found by Christophers in 1901, occurring in swarms in Anopheles and Culex. Durham, again, the year before, had noticed numerous "Trypanosomes" in a Stegomyia which had fed upon a bat. The first serious contributions, however, to our knowledge of the Flagellates parasitic in Insects are Léger's researches $(47,48,51,52), 1902-1904$, on certain Herpetomonadine forms.

Besides the genus Herpetomonas, Léger has distinguished another type of form, which he has termed Crithidia. Both types show, in general, an alternation of monadine (flagellate) phases with gregariniform (resting, non-flagellate) ones. In the latter condition, the parasites occur as small, rounded, pear-shaped, or 
even oblong bodies, attached, often in great numbers, to the epithelial cells. The flagellum is either absent or reduced to a short rostrum, serving for attachment (Fig. 29, D and G). The two nuclei (tropho- and kinetonucleus) lie close together, usually near the base of the cell. In this phase, the general resemblance to the Leishman-Donovan bodies may be quite marked. The distinction between the two generic types is based upon the form and size of the monadine phase. In Herpetomonas the body is very elongated and slender, often acicular, the posterior end usually

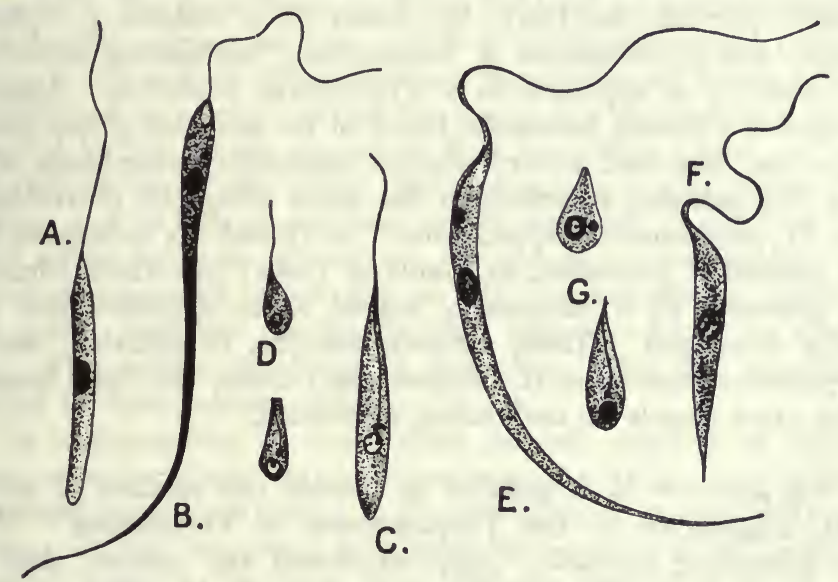

FiG. 29.

A, C, Herpetomonas (Crithidia) minuta; D, attached (gregariniform) phases of same; B, $H$. gracilis, Léger ; E, F, H. subulata, Léger; G, attached phases of same. (After Léger.) $\times 1800$.

tapering away finely (Fig. 29, B and E). In Crithidia, on the other hand, it is much shorter and wider, of a pyriform shape; the hinder end is never drawn out, but terminates bluntly in a rounded or an obtuse manner. The parasite Herpetomonas (Crithidia) minuta, Léger, appears to be intermediate, however, between these two types, some individuals approximating to a Herpetomonad form (A), others to a Crithidial one (Fig. 29, C). As a matter of fact, the classificatory distinctions between these various Insectan Flagellates cannot be regarded as at all settled.

In many forms of Herpetomonas (e.g. H. muscae-domesticae, ${ }^{1} H$. jaculum, or $H$. gracilis (B)), the kinetonucleus is situated near the

$1 H$. muscae-domesticae is included here as a typical uniflagellate Herpetomonad. Prowazek (69) described this form as possessing a pair of flagella, parallel to and counected with one another; he considered this parasite to be a bipolar type (on the lines of Schaudinn's "Urhaemoflagellate") in which the body has been bent up so that the two ends have come together and united, the flagella alone remaining distinct. Léger observer no signs of two flagella in non-dividing individuals, either of this species or others; and the same is true of the describers of the numerous other Herpetomonads. 
anterior end ; the flagellum is not attached to the side of the body at all but straightway becomes free, and there is no sign of an undulating membrane. These forms are mostly parasitic in Invertebrates which do not suck blood. In $H$. subulatu, however, which is parasitic in the digestive-tube of Tabanus and Huematopota, predatory on cattle and horses, the kinetonucleus lies much farther from the anterior end, and may be almost opposite the trophonucleus (Fig. 29, F). The flagellum, which has been, as it were, drawn back with it, is in most individuals attached for the proximal part of its length to the anterior part of the body, by means of a delicate cytoplasmic border, which constitutes a rudimentary undulating membrane. Thus there is an approach to a trypaniform condition. Again, in the case of Crithidia fasciculata, found in the intestine of mosquitoes, Léger has described a very distinct undulating membrane, which gives the parasite, especially in the more elongated individuals, a very Trypanosome-like appearance. Novy and his colleagues have also studied $C$. fasciculata, as found in Culex; but while admitting the presence of a membrane, regard it as imperfect and only poorly developed. These authors describe, in addition, another Herpetomonadine type, $H$. (Trypanosoma) culicis, the long forms of which show clearly an undulating membrane.

We are now in a position to discuss the relation (if any) of these Flagellates to the Trypanosomes of Vertebrates. When first describing Crithidia, Léger expressed the opinion that this parasite was very likely only a stage in the development of a Haemoflagellate; further, in his notes on $H$. subulata (52) he added the remark that the same was probably true of many of these Herpetomonad or Crithidial forms found in biting Insects, though this would not apply, of course, to those species occurring in nonbiting Insects (such as Musca, Sarcophaga, etc.). Moreover, Schaudinn himself (l.c.) comments on the great similarity between (what he took to be) the phases of Trypanomorpha noctuae in Culex and those of Léger's Crithidia.

Quite the opposite view is held by Novy and M'Neal, who, after first (62) regarding the Flagellates found by Schaudinn in mosquitoes as being simply "cultural" forms, of no real significance in the life-history, in their later paper (63) consider it much more likely that the Insectan parasites are entirely distinct from the Trypanosomes in the blood. (They look upon the parasites found in leeches, however, as "cultural" forms of Piscine Trypanosomes.) A similar opinion is expressed by Ross, who points out that he found Crithidia in the mosquitoes (larvae and pupae) before they fed on blood, and thinks the parasites were in the first place swallowed by the larvae.

In a very interesting note Patton has recently (65) described 
stages in a Herpetomonas of Culex pipiens, whose life-cycle would seem in some respects to conform to the scheme suggested by Ross. In its monadine, determinative form, the parasite appears to be a typical Herpetomonas, with no indications of an undulating membrane. All the phases observed, Patton states, exhibit great similarity with those of Piroplasma donovani (see pp. 256 et seq.). Here it may be pointed out that in the larvae the parasites resembled the LeishmanDonovan bodies as they occur in human tissues; in the nymphs, stages corresponding to the developmental forms of the LeishmanDunovan bodies (in cultures, or in the bed-bug), i.e. pear-shaped forms with flagella, were numerous; while in adult mosquitoes (mid- and hind-gut) there were fully developed Herpetomonad forms. Patton thinks these are passed out into the water, and in some guise or other ingested by the larvae, the cycle thus beginning again. (He has privately informed the writer that the parasites may encyst in the rectum, and be thus passed out to the exterior, to give rise to the small round forms in the larva.) Patton also notes the occurrence of a Herpetomonad, which has an obvious undulating membrane, and which possesses similar rounded aflagellar forms, in a waterbug. The author concludes by regarding these two parasites as limited to their Insectan hosts.

In endeavouring to draw some general conclusions from the above opposing ideas, we are, it seems to the writer, greatly helped by comparing what is known in the case of other groups of Trypanosomes. In the first place, as regards those met with in Tsetse-flies, some of which, at any rate, were formerly considered to be solely fly-parasites, there appears to be no escape from the conclusion that, on the contrary, all the forms are blood-parasites. In our opinion the utmost weight is to be attached to this conclusion. In addition, we have the Trypanosomes of leeches, which are generally agreed to belong to different Piscine forms. On these grounds alone, then, it appears justifiable to suppose that Avian Trypanosomes are to be found in mosquitoes, and not at all improbable that some at least of the phases so clearly described by Schaudinn from mosquitoes which had fed on infected owls, did indeed appertain to Trypanomorpha noctuae.

Again, to consider the subject from the Insectan standpoint, so far as the writer can see, Novy and his colleagues have by no means proved that their Flagellates in wild mosquitoes are not, in some cases at any rate, phases of Trypanosomes of birds (or other Vertebrates). For instance, the Trypanosoma (Herpetomonas) culicis described by these authors-with various forms of which they compare certain phases of Trypanomorpha-is quite as probably a blood-parasite as a purely Insectan form; indeed, the possibility of this being so is admitted by its describers. Moreover, they remark on the resemblance between the genera Herpetomonas, Crithidia, and 
Trypanosoma, especially when the "cultural" forms of the last-named are compared with those of the other two (or with what Novy and M'Neal regard as their equivalents-the Insectan forms). In the case of the Trypanosomes, there is the same relative position of the two nuclei, either close together, or the kinetonucleus even on the flagellar side of the trophonucleus; while certain of them show no sign of an undulating membrane, but have a typically Herpetomonad facies. Novy and M'Neal, in fact, would include all these types in the genus Trypanosoma.

Further, we may point out that according to the view which these authors themselves hold regarding the origin of the bloodTrypanosomes, it is most natural to suppose that they are to be met with, quite at home, in an Insectan host. The American workers say that parasitism in the living blood is to be looked upon as the result of previous adaptation to the more or less digested blood (in the Invertebrate). (As will be seen later, we agree with this view, where certain Insects are the Invertebrate hosts.) Now, in this course of evolution of certain blood-Trypanosomes, it may be reasonably inferred that at one stage the parasites still remain connected with the Invertebrate after having gained a footing in the Vertebrate (say a bird). The question would seem to be, rather, which if any blood-forms so descended have lost the ability to live (and develop) in their Invertebrate host-a course which would probably greatly restrict their opportunities for dispersal. (In this connection the case of the Leishman-Donovan bodies is most instructive ; cf. pp. 258, 259.)

Hence, summing up, there can be little doubt that certain of these parasites of mosquitoes, especially those with trypaniform characters, are connected with some Vertebrate host, just as are those of other blood-sucking Invertebrates. At the same time, it is also probable that some of the (typical) Herpetomonads found (e.g. those occurring in larvae, such as Patton's form, also certain forms described by the Sergents) are simply and primarily parasites of the Insect. Lastly, it is, of course, possible that such a parasite may have developed a trypaniform condition as an adaptation to the food of a sanguivorous Insect; without, however, having become able to live in the Vertebrate host; but so far no example of sucb a case is definitely known. And this brings us to the subject of the derivation of the Trypanosomes.

\section{(b) Evolution and Phylogeny.}

It must be fully recognised that any views which can be at present advanced upon this interesting, but very puzzling topic are at best little more than speculations. Formerly (l.c.), the writer inclined to the idea that all Haemoflagellates are to be derived from 
forms originally parasitic in Invertebrates; in other words, the Invertebrate was regarded as the primary host, the Vertebrate as the secondary or intermediate one. We now think this view was probably, to a considerable extent, wrong; in this we have been mainly influenced, on the one hand, by the intestinal Trypanoplasmata, and on the other, by the case of T. grayi. As above remarked, it seems evident that a Vertebrate is the primary host of this latter parasite; and the same would follow, by inference, for the other (Mammalian) Trypanosomes transmitted by Tsetse-flies. Moreover, the writer thinks he did not allow sufficient weight to the fact that the Invertebrates which harbour Trypanosomes are, with but few exceptions, blood-suckers. For these reasons we are now inclined to consider most of the Invertebrates concerned (e.g. leeches, many biting-flies, etc.) as the secondary, intermediate hosts of various Vertebrate parasites (probably all the Piscine and Amphibian ones, many, but perhaps not all the Mammalian ones, and perhaps some Avian ones).

The only important ${ }^{1}$ exceptions are among Insects; and here it is quite likely that we have both primary and secondary hosts. Besides the Tsetses, Tabanids, etc., the common house-fly and related genera, in which Herpetomonads (e.g. H. muscae-domesticae, $H$. sarcophagae, etc.) occur, ought apparently also to be placed in the category of secondary hosts. For Prowazek (l.c.) states that, according to Brauer, the latter flies are probably descended from blood-sucking ones; in which case their parasites may very well be descended from haemal forms, which are now, perforce, restricted to the Invertebrate host. On the other hand, there are several instances of the parasites occurring either in nonsanguivorous Insects or in forms that only rarely suck blood, which are, we think, more likely cases of primary parasitism. Among these, for example, are $H$. bombycis, in Bombyx mori; $H$. gracilis, in larvae of Tanypus; Crithidia campanulata, in larvae of Chironomus plumosus. Lastly, we have the mosquitoes and their parasites, both of imago and larva.. The latter is, of course, aquatic, and the imago is by no means limited to blood for nutriment. Having regard also to the illustrative series of transitional forms between the monadine type and the trypaniform one, made known by Léger and others, it appears to us that here as well the Insect is the primary host of the various Flagellates concerned, and that where these are connected with a Vertebrate host the latter is to be regarded as the secondary, intermediate one. This wonld apply chiefly to certain parasites (e.g. Trypanomorpha) of birds, though not necessarily, it is to be noted, to all.

Many authorities (such as Laveran and Mesnil, Lühe, Novy and

1 Herpetomonas bütschlii from a Nematode (Trilobus) and the curious Trypano. phis from Siphonophores do not appear to have any bearing upon this question. 
$M^{\prime}$ Neal) have maintained the view that the Invertebrate is the primary host in all cases. Minchin, however, has always considered the Vertebrate as the principal host; and in his latest memoir on the Trypanosomes of Tsetse-flies (58), proofs of which he very kindly allowed the writer to see, he regards all Trypanosomes as descended from an intestinal Vertebrate form, and indicates the lines upon which the evolution may be supposed to have advanced. This ancestral form produced resistant cysts for dispersal, and thus contaminative infection was brought about. (It would be extremely interesting to ascertain whether the intestinal Trypanoplasmata known (see p. 249) have such a cyst-formation.) The next stage in evolution is when the parasite has penetrated the intestinal wall, and come into relation with the circulatory system. Until it came into relation with a blood-sucking Insect, this type would have to pass back into the alimentary canal for dissemination. So far, we have no evidence of an existing instance of this stage. Subsequently, the blood-parasite became adapted to an Insectan host, in the gut of which it encysted, reinfection of the Vertebrate being by the contaminative method. 'T. grayi in all probability furnishes an example of this type. Lastly, the parasite is thoroughly adapted to the biology of the Insect and passes forwards to the front part of the alimentary canal: infection of the Vertebrate is now by the inoculative method. This may possibly be combined in some cases with the contaminative mode, but probably in most encystment no longer takes place, being unnecessary (e.g. the lethal Trypanosomes, Piscine forms, etc.).

Of course, in those cases where, as above remarked, the Vertebrate is probably the secondary host, there is no reason to suppose that, as a rule, the parasites leave the circulatory system.

Phylogeny.-As stated at the beginning of this article, the Trypanosomes, as a whole, are to be regarded as including two entirely distinct families, in one of which (the Monadine type) the attached flagellum becomes free at the true anterior end, and in the other (the Heteromastigine type) at the true posterior end. The former type is derived by the progressive migration backwards of the kinetonucleus towards the posterior (aflagellar) end, in the manner well illustrated by Léger's series of Herpetomonadine forms (see Fig. 29). The latter type is derivable from a Trypanoplasmatine ancestor-itself in turn doubtless to be derived from a Bodo-like form-by the loss of the anterior free flagellum $;^{1}$ so that the nonflagellate extremity is the true anterior one.

The writer is unable, owing to limits of space, to enter fully

1 A comparison of the different degree of development of the flagella in various forms is instructive as illustrating the manner in which the Trypanoplasmatine condition may have resulted from that found in Bodo, and its further evolution. 
here into the reasons for and against this diphyletic view, which was first put forward by Léger (49). A complete discussion will be found in his Review of the Haemoflagellates (pp. 270-278). Lühe, in his account of the Haematozoa in Mense's Handbuch der Tropenkrankheiten (2), has adopted it, though on somewhat different lines from those taken by us. Minchin, also, has expressed the opinion (Brit. Med. Journ., 1907, ii. p. 1320) that Trypanosomes are most likely diphyletic. On the other hand, many authorities, including Laveran and Mesnil, hold the view that all Trypanosomes are descended from Herpetomonadine ancestors, basing their opinion on the resemblance to a Herpetomonad shown by many Trypanosomes in cultures, and by young individuals of T. lewisi (cf. Fig. 20). In many cases, at any rate, we regard this phase-as we have previously said - rather as a "pseudo-Herpetomonadine" condition ; and in such cases do not attribute to it the phylogenetic importance which is done by some, but consider it to be probably capable of explanation on other grounds (see l.c.). A fact which seems to us of considerable significance is that Trypanoplasmatine forms are known to occur in the digestive tract of fishes, e.g. "Trypanoplasma" intestinalis in Box boops, and "T." rentriculi in Cyclopterus lumpus; moreover, another Heteromastigine parasite (Bodo lacertae) is found in a lizard. On the other hand, no indubitable Herpetomonad has yet been described from the alimentary canal of a Vertebrate, which we may assume to have been the original habitat of the primitively Vertebrate parasites.

Hence, all things considered, we come to the general conclusion that the Trypanosomes which have the Vertebrate for their primary host are Heteromastigine forms; those derived from primitively Invertebrate parasites, on the other hand, are probably Monadine forms. Endeavouring to use this view practically, for purposes of classification, or, at any rate, of convenient partition of the Trypanosomes, we have as follows:- The parasites of fishes belong to the Heteromastigine type; this can be said with some degree of confidence, in spite of the "Crithidial" forms assumed by the parasites in leeches. Probably the same is true also of most forms of cold-blooded Vertebrates. Of the Avian ones, on the contrary, some at any rate (e.g. those of the type of Trypanomorpha noctuae) are Herpetomonadine forms. Among Mammalian parasites the various lethal Trypanosomes (e.g. T. brucii, etc.) are to be regarded as Heteromastigine forms. We will only mention in passing that certain movements of these forms in the living blood (cf. p. 217) suggest very forcibly that the aflagellar end is the true anterior extremity. Of the other known (accustomed) parasites of Mammals, whose number has considerably increased of late, it is quite possible that some (e.g. those of bats, which may have, perhaps, mosquitoes as their alternate hosts) are Herpetomonadine forms. 


\section{Systematic.}

The reasons for the division of the Trypanosomes into two distinct families have been alluded to in the previous section. Besides the fundamental diagnostic characters, namely, the true orientation of the body and the biological features associated therewith, it is quite likely that important differences in regard to the life-cycle will become evident as our knowledge increases.

\section{SUb-Order Monadina.}

Family TryPanomorPhidae, Woodcock. - Haemoflagellates derived from a uniflagellate, Herpetomonadine form, in which the point of insertion of the flagellum into the body has travelled backwards from the anterior end for a considerable distance, the flagellum itself having become, concurrently, attached to the body for part of its length by means of an undulating membrane. At present only one genus is distinguished.

Genus Trypanomorpha, Woodcock. With the characters of the family. The genus was founded for Schaudinn's Avian parasite, Trypanosoma (Halteridium) noctuae (Celli and San Felice), ${ }^{1}$ from Athene noctua and Culex pipiens. As above mentioned, it is probable that other Avian forms, and perhaps some Mammalian ones, will be found to agree with this generic type; at present, however, it is not possible to say which with any certainty, and hence they are retained under the heading "Trypanosoma."

Reference has been made to the possibility of Léger's Crithidia fasciculata from Anopheles maculipennis, and other Insectan parasites which show marked trypaniform characters, being also really Haemoflagellates. In such a case the genus Trypanomorpha may prove to be synonymous with Crithidia; if so, the latter name will take priority. Lühe, it is to be noted, in his account of the Haematozoa (l.c.), regards all the Trypanosomes of Mammalia as belonging to the Herpetomonadine type, and has proposed the new generic name Trypanozoon for these forms.

\section{Sub-Order Heteromastigina.}

Family Trypanosomatidae, Doflein. - Flagellates, with but few exceptions haemal parasites, derived from a biflagellate, Bodolike type, in which the posteriorly directed (trailing) flagellum is always present and attached to the side of the body by an undulating membrane, of which it constitutes the thickened border.

1 Schaudinn placed this form in the genus Trypanosoma. We incline, however, to the view that the type-species of that genus ( $T$. rotatorium) is a Heteromastigine type, and therefore restrict that genus to snch forms. 
The other, the anterior flagellum, may or may not persist. At least three genera known so far.

Genus Trypanoplasma, Laveran and Mesnil. The anteriorflagellum is present.

Type-species, T. borreli, Lav. and Mesn. (Fig. 11). Length of body 20-22 $\mu$, of free flagella 13-15 $\mu$, breadth $3 \frac{1}{2}-4 \frac{1}{2} \mu$. Parasitic in Leuciscus erythrophthalmus, rudd, and Phoxinus laevis, minnow. Other species are $T$. cyprini, from the carp, and T. varium, a rather larger forn, from the loach.

Genus Trypanophis, Keysselitz. The anterior flagellum is present. The free part of the posterior flagellum is short, and the undulating membrane is straight and relatively narrow. The species for which this genus was founded is parasitic in certain Siphonophores, and almost certainly not a haemal form.

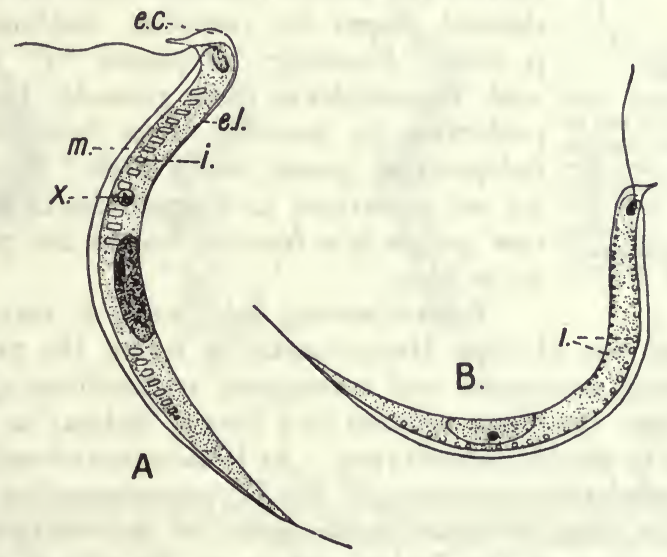

FiG. 30.

Trypanophis grobbeni (Poche). e.c, ectoplasmic cap; e.l, delicate ectoplasmic layer, thin. ning out posteriorly; $i$, inclusions in the cytoplasm; $x$, nuclear body of uncertain origin and significance. (After Keysselitz.)

Type-species, T. grobbeni (Poche). Average length 60-65 $\mu$, width about $4 \mu$. From Cucubalus kochii, Halistemma tergestinum, Monophyes gracilis, Gulf of Trieste. Apparently the same parasite has also been observed in Abyla pentagona, Gulf of Naples. The organisms are to be found in all the ramifications of the coelenteron, from the digestive-cavity of the gastrozoids to the radial canals of the nedusoid buds. Nothing is known with regard to the transmission from one Siphonophoran colony to another.

Great interest attaches to certain Trypanoplpmmatine parasites recently described from the alimentary canal of fishes. In their general morphology and the possession of an undulating membrane they agree closely with Trypanoplasma, and their describers have included them in this genus, as T. intestinalis, Léger, and T. ven- 
triculi, Keysselitz. So far as those points are concerned, however, they agree also with the above-mentioned genus Trypanophis (cf. Figs. 30 and 31). Indeed, Léger, in his account of T. intestinulis,

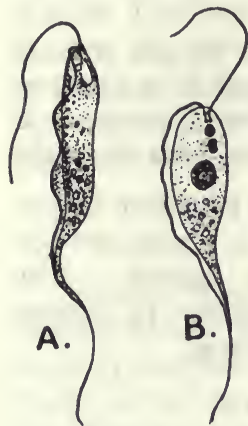

FIG. 31.

"Trupanoplasma" in testinalis. In $\mathbf{A}$ a row of spherules is seen running down the side near the undulating-membrane (cf. Tryponophis); in B the kinetonucleus is domble. (After an original draw. ing kindly lent by Prof. Léger.) commenting on the great resemblance of this parasite to Trypanophis, suggested that the latter form might be included in Trypanoplasma. We consider that Trypanophis grobbeni, on account of its curious habitat and somewhat peculiar nature, should certainly be kept distinct. Moreover, as regards the intestinal Trypanoplasmatine forms, the fact that they are most likely not haemal parasites renders it very probable that their life-cycle differs in many ways from that of the blood-Trypanoplasmata (cf. the hypothetical stages in evolution outlined above, p. 246). Formerly, we placed " $T$." intestinalis with T'rypanophis on these grounds; but it seems preferable to consider it as belonging to an independent genus, along with " $T$." ventriculi. As we are averse to the practice of instituting new genera in a treatise, we do not propose to do so here.

Before leaving this point, it may be noted that, in the case of these Heteromastigine forms, the presence of an undulating membrane and consequent trypaniform appearance does not bear the same relation to a haemal habitat as seems to be the case in the Monadine types. As Doflein has already pointed out, ${ }^{1}$ the undulating-membrane, in the Trypanoplasmatine parasites, has doubtless been developed as the result of the contiguity of the trailing flagellum of the Bodonine type to the side of the body; a quite different origin from that in the other section. Hence this condition is more or less independent of the habitat of these forms.

Genus Trypanosoma, Gruby (principal synonyms: ${ }^{2}$ Undulina, Lank., 1871 ; Herpetomonas, Kent, 1880, but only in part, since the type-species is $H$. muscae-domesticae; Paramoecioides, Grassi, 1881 ; Haematomonas, Mitrophan., 1883; Trypanomonas, Danil., 1885 , for young forms). There is no anterior flagellum. The point of insertion of the attached (posterior) flagellum into the body, and, consequently, the commencement of the membrane, may be anywhere in the anterior half of the body, but is usually near the extremity. ${ }^{3}$

${ }_{2}^{2}$ For remarks on the synonymy of this genus, readers are referred to the writer's previous article (p. 287).

3 The type-species is $T$. rotatorium, Mayer, of frogs. At present, unfortunately, this parasite cannot with certainty be included in the above diagnosis, owing to its 
The sub-classification of this genus, or rather the grouping and arrangement of the numerous Trypanosomes at present included in it, is a question of great difficulty and one in regard to which hardly anything has been done as yet. ${ }^{1}$ This is chiefly owing to the fact that so little is still known of the life-history of most that hitherto any attempt to group the parasites has been dependent upon their adult morphology. This is not a very satisfactory criterion, since, as we have seen, on the one hand, the differences in this respect between different forms may be very slight; and on the other, a particular parasite may itself vary very greatly at different times and under different conditions (see under "Morphology"). Moreover, it may very well be that as more life-histories come to be revealed, some of the forms at present placed for convenience in

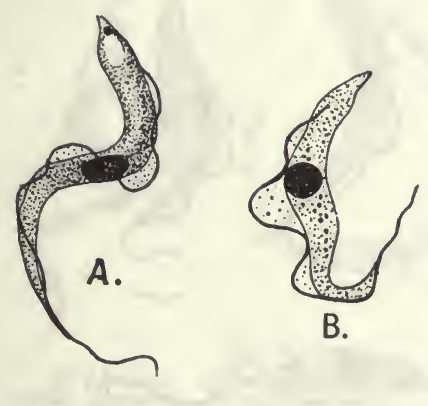

FIG. 32.
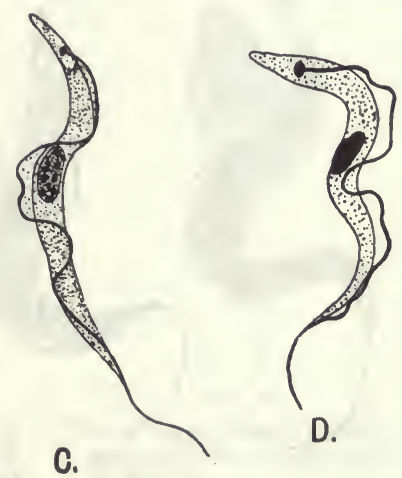

D.

A, Trypanosoma gambiense (from the blood), after Bruce and Labarro; B, T. equinum, after Ligniè'es; C, T. evansi, from an original drawing; $D, T$. equiperdum, after Lign.

the genus Trypanosoma will have to be transferred to new ones (as an example may be mentioned $T$. grayi).

For the present, at any rate, a very useful aid towards distinguishing different species is furnished by the biological relations of the parasites. For it may be assumed that here, as is usual among the Sporozoa, a particular species is, in general, restricted either to one particular host, or, at most, to a few allied ones. Difficulty arises in considering the Mammalian forms, many of which have never been observed in the true, natural hosts, but only

unusual shape, position of kinetonucleus, etc. The occurrence, however, of an allied form in Hyla, which is evidently intermediate between $T$. rotatorium and the more typical, fusiform species, suggests that the former also belongs to the Heteromastigine section.

1 Koch, however, has attempted a classification of the Mammalian forms, which he arranges in two groups, the first including such different forms as $T$. levvisi and the large $T$. theileri of cattle; the other, most of the lethal forms, which he considers are not distinct species. This arrangement is very artificial and has nothing to recommend it. 
in variaus unaccustomed animals, for which they are more or less lethal. The important immunisation experiments first carried out by Laveran and Mesnil, and since then by other workers, have shown, however, that several of the parasites causing the different trypanosomoses now known are distinct species.

A full description of all the known forms and their characteristics is impossible within the limits of this article. It must suffice to mention some of the more important and better-known parasites, arranged under the different classes of Vetebrate hosts; for fuller details regarding them, reference should be made to the writer's previous account, or to Nabarro's revised edition of Laveran and Mesnil's treatise, which is most useful in
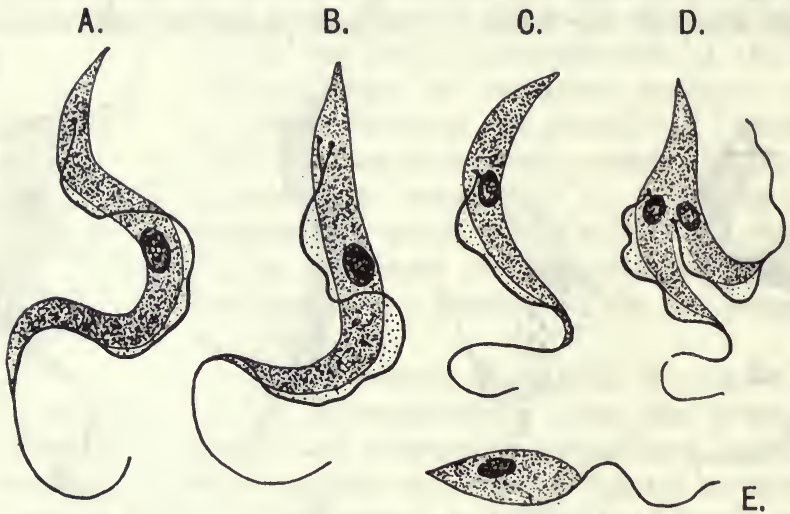

Fig. 33.

A and B, T. theileri; C-E, T. "transvaaliense." $\times$ 1250. (After L. and M.)

this connection. A list of known hosts and their Trypanosomes is given at the end of this chapter.

(a) Parasitic in Mammals. Trypanosoma lewisi, Kent, the common natural parasite of rats (Figs. 7, A ; 20, A). Length ${ }^{1} 24-25 \mu$, breadth $1 \frac{1}{2}-2 \mu$. This species is characterised by its narrow and pointed aflagellar extremity, and by the position of the trophonucleus in the flagellar half or third of the body. The cytoplasm is usually free from gramules. $T$. brucii, Plimmer and Bradford. Length 28-30 $\mu$, breadth 2-2 $\frac{1}{2} \mu$. The anterior end is usually bluntly rounded (Figs. 7, B ; 17, A). The cytoplasm often contains granules in the posterior half. Natural hosts probably various Antilopidae (e.g. gnu, "koodoo," etc.), and buffaloes. The cause of Nagana or Tsetse-fly disease in cattle, horses, etc., in South Africa. T. gambiense, Dutton (Syn. T. ugundense, Castell). Length 21-23 $\mu$, breadth $1 \frac{1}{2}-2 \mu$. This species (Fig. 32, A), according to its average size, is one of the smallest known. The cause of human trypanosomosis in West and

1 The dimensions given are intended to indicate the average size of the parasite in each case, but can only be considered as approximate. The length is inclusive of the flagellum, unless otherwise stated. 
Central Africa. The earlier stages of the disease, when the parasites are confined to the blood, are known as Trypanosoma-fever; the later ones, after the organisms have penetrated into the cerebro-spinal canal, constitute the deadly malady of sleeping-sickness. The true, natural host is unknown. T. equinum, Voges (Syn. T. elmassiani, Lign.). Length 22-25 $\mu$, width $1 \frac{1}{2}-2 \mu$. Distinguished from all other forms by the ninute size of the kinetonucleus (Fig. 32, B). Hydrochoerus capybara is almost certainly a natural host. Other well-known lethal parasites are : T. evansi (Steel), of Surra in horses in Indo-Burmah (Fig. 32, c) ; T. equiperdum, Doflein (Syn. $T$. rougeti, Lav.), the cause of Dourine in horses, transmitted naturully by coitus (Fig. 32, D) ; T. theileri, Laveran, a very large form, often surpassing $50 \mu$ in length, which causes "bile-sickness" of cattle in the Transvaal (T. transvaaliense, Lav., with the kinetonucleus near the middle of the

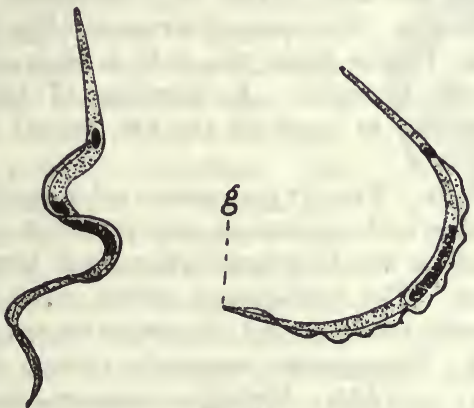

Fig. 34.

T. johnstoni. $g$, deeply-staining granule at distal extremity of flagellar border. $\times 1500$. (After Dutton and Todd.)

body (Fig. 33, C-E), has been sliown to be, in all probability, only a phase of $T$. theileri); and T. dimorphon, Dutt. and Todd, which gives rise to a trypanosomosis of horses in Senegambia.

(b) Parasitic in birds. T. avium, Danil., Lav. emend., probably the form to which Danilewsky's original investigations related, occurring in owls and, according to Novy and $\mathrm{M}^{\prime} \mathrm{Neal}$, in various other birds. Length $35-45 \mu$ (Fig. 7, F). T. johnstoni, Dutt. and Todd. Length 36-38 $\mu$, width $1 \frac{1}{2} \mu$. This parasite is so slender as almost to justify the description spirochaetiform (Fig. 34). From Estrelda. The opposite extreme of form is seen in a Trypanosome, T. hannae, Pittaluga, originally described by Hanna (25) from an Indian pigeon (Fig. 7, G); this is relatively very broad, and has, moreover, a long, attenuated aflagellar extremity, the latter character being not unusual in bird-Trypanosomes. On the other
A Trypanosome from Senegambian birds. $\times 1500$. (After D. and T.)

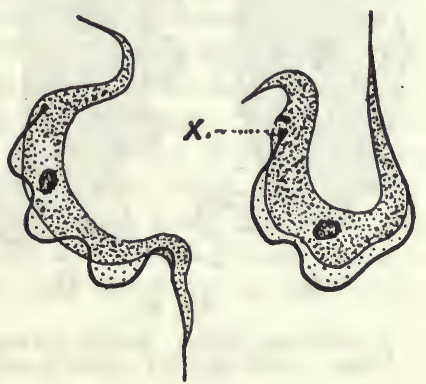

Fra. 36 .

T. paddae. At $x$ the base of the flagellum is thickened prior to division. $\operatorname{lum}_{\times 1200 \text {. (After Thiroux.) }}$ 
hand, Dutton and Todd have described a wide form from Seneganibian birds, which has this end bluntly rounded, giving the parasite a stumpy appearance (Fig. 35). It is interesting to note that this Trspanosome occurred in the same birds (Estrelda) in which the very different $T$. johnstoni was found. T. paddae (Fig. 36), from the Java sparrow, has been studied by Thiroux (83), and apparently lents itself to cultivation and inoculation into other birds as readily as do many Mammalian forms. Finally, there is the remarkable parusite, "T." (Spirochaeta) ziemanni, described by Schaudinn. If this form is really a Trypanosome, it certainly belongs to the Heteromastigine section, and may for the present be placed in the genus Trypanosoma. But it may be, after all, a true Spirochaete, and belong to the Bacteria (cf. footnote, p. 237).

(c) Reptilian forms. Scarcely any Trypanosomes have been observed in Reptiles. The only one which has been figured is T. damoniae, Lav. and Mesn. Length $32 \mu$, breadth $4 \mu$. The general structure (Fig. 7, J) presents nothing unusual. As in Piscine forms, the body is often rolled up on itself. From Damonia reevesii, a tortoise. Another form (1. boueti), lately described from a lizard, is said to resemble the flat, smooth type of $T$. rotatorium (below).

(d) Parasitic in Amphibian hosts. The Trypanosomes of frogs show a remarkable variation in form, size, and appearance, and it is not at all certain, in some cases, how far this is due to polymorphism, and how far to distinct species being concerned. The type-species of the genus is $T$. rotatorium (Mayer). (Synn. Amoeba rotatoria and Paramsecium costatum or loricatum, Mayer, July 1843 ; Trypanosoma sanguinis, Gruby, Nov.

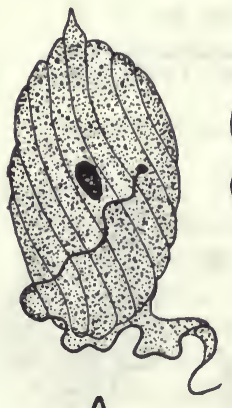

A.

Fia. 37.

T. rotatorium (Mayer). Ribbed and smooth forms. $\times 1000$ (approx.). (After L. and M.)
1843 ; Undulina ranarum, Lank., 1871.) Laveran and Mesnil have worked on this form and distinguish two principal types, one having the surface of the body thrown into parallel ridges (Figs. $8, \mathrm{~B} ; 37, \mathrm{~A})$, the other having a smooth, regular surface (Figs. $8, \Delta$; $37, .8)$. The parasites are very large, being $40-60 \mu$ in length, by from $5-40 \mu$ in width; the two dimensions vary more or less inversely. The great variation in shape of the body and of the anterior end is seen from the figures. The kinetonucleus is usually situated some distance from the non-flagellate or anterior extremity, and may be quite close to the trophonucleus; sometimes, however, it is fairly near the end. Chiefly for this reason, Franca and Athias (22) split up the species into two, T. costatum or loricatum (Mayer), with the kinetonucleus near the centre, and $T$. rotatorium, with it near the end. As the position of this organella is very variable and intermediate stages occur, we do not think anything is gained by doing this, at present. Similarly, the validity of two new species which Franca and Athias 
create, namely, $T$. undulans and $T$. elegans, is somewhat doubtful. Dutton and Todd have described two very long forms from Gambian frogs, which they have named $T$. mega and T. karyozeukton; these forns exhilit peculiarities in the cytoplasm (see p. 212), and in the latter parasite a chain of chromatic granules runs from one nucleus to the other (Fig. 8, D). A type which is certainly distinct is $T$. inopinatum, Sergent, from the edible frog. This parasite (Fig. $8, c)$ is slender (25-30 $\mu$ by $3 \mu$ ), and resembles a Mammalian or Piscine form. Another well-characterised species is $T$. nelspruitense, Lav., in which the body is very verniform and the free flagellum very long (Fig. 8, E).

(e) Forms parasitic in fishes. Trypanosomes occur very frequently in fishes, and a great many species hare been descriled. T. remuki, Lav. and Mesn. This parasite occupies about the same position among Piscine Trypanosomes as does $T$. leurisi among Mammalian ones. It is a slender form, with tapering, pointed extremities. The trophonucleus is in the posterior half of the body. Parasitic in Esox lucius, the pike. Laveran and Mesnil have distinguished two varieties, based

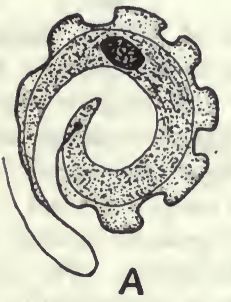

A

Fis. 38.

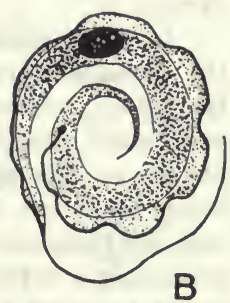

A, T. scyllii; B, T. raiae. $\times 1200$. (After $\mathrm{L}_{\mathrm{s}}$ and M.)

upon the considerable differences in size met with, namely, var. parta, medium length $30 \mu$, of free flagellum 10-12 $\mu$, with breadth $1 \frac{1}{2}-2 \mu$; and var. magna (Fig. 8, L), minimum length $45 \mu$, of which 17-20 $\mu$ is for the flagellum, and breadth $2-2 \frac{1}{2} \mu$. T. cobitis and $T$. carassii (Mitrophanow) were among the first Piscine forms to be described, and probably correspond to niany of those seen by Danilewsky. T. granulosum of the eel is a remarkably long, eel-like form (Fig. 8, $\mathrm{x}$ ), $70-80 \mu$ by $2 \frac{1}{2}-3 \mu$. The kinetonucleus is relatively very large, as is often the case in Piscine furms, and close to the anterior end, which is sharply acute. Several forms have been observed in flat-fish, certain of which (e.g. T. flesi, Lebailly) belong to a different type, being relatively wide, with only a short flagellum. From Elasmobranchs, two very large forms ( $T$. scyllii and T. raiae) have been described by Laveran and Mesnil; these attain a length of $70-80 \mu$, and usually have the body coiled up on itself (Fig. 38).

\section{APPENDIX.}

\section{(A) The "Leishman-Donovay-Wright" Bodieg.}

Although these remarkable bodies have not been shown yet to possess an actual trypaniform structure, the fact that they are known to give rise to Flagellate phases of very Herpetomonadine character points so conclusively to their connection with that type of parasitic Flagellate, and is of such importance as proving that a parasitic Flagellate 
can and does become intracellular in the Vertebrate host, that a brief consideration of them is essential to the completeness of this article.

The Leishman-Donovan bodies are constantly found in certain tropical fevers (such as Dum-dum fever, Kala-Azar), particularly prevalent throughout Inclo-Burmah, of which they are now generally admitted to be the cause. These parasites were discovered by Leishman in 1900, but before his first account of them was published (91) they were also seen independently by Donovan. Moreover, organisms very similar to these parasites (indeed, morphologically, the two kinds are hardly distinguishable) are found in varions sores or ulcers (knuwn as Delhi boil, Oriental sore, "bouton d'Alep"), to which people in different parts of the tropics are liable. The latter were first clearly recoynised and described by Wright (97).

In the former type of disease, there is a geueral systemic infection, the parasites spreading to all parts of the body, and being met with in the spleen, where they are usually very abundant, liver, bone-marrow, and (more rarely) in the peripheral circulation. The latter type of discase, on the other hand, is one of localised infection, the organisms being restricted to the neighbourhood of the skin lesions; and in this case the parasites never seem to become distributed throughout the body, producing a systemic infection. For this reason, though the organisus in the two cases seem to be undoubtedly closely related, they are probably specitically distinct. In the Vertebrate host, the parasites are generally intracellular. Free forms are met with, doubtless liberated by the break-up of the host-cells, but these probably soon invade fresh cells. Leislıman's form is parasitic in large uninuclear lencocytes (Fig. 39, II), and especially in cells of the rascular endothelium, which are often packed with the little bodies, becoming greatly distended (as macrophages). According to both Donovan (88) and Laveran and Mesnil (90), the parasites also occur in the red blood-corpuscles. Wright's form occurs in the ulcer cells, and in large migratory corpuscles (phagocytes) of the granulationtissue.

The parasites themselves are very minute, and usunlly ovoid or pyriform in shape, the latter being perhaps the more typical. The splenic form is somewhat smaller than the localised type, being $3 \frac{1}{2}-4 \mu$ in length by $1 \frac{1}{2}-2 \mu$ in width $(39, I)$, while Wright's furm is about $4 \mu$ by $3 \mu(39$, III $)$. The cytoplasm is finely granular and fairly uniform in character; but sometimes it is vacuolated. The most interesting point about the morphology is the fact that two clironatic bodies, of very unequal size, are invariably to be recognised. The larger nuclear body, which corresponds to the trophonucleus of an ordinary Haemoflagellate, is usually round or oval ; the smaller one, representing a kinetonucleus, has the form either of a little rod or of a round grain, and stains very deeply. The two nuclei are generally quite separate, but sometimes they appear to be connected. The organisms multiply in two ways: (a) by binary fission, and (b) by multiple division or segmentation. The chief stages in the first method are well known (Fig. 39, I, b) ; they offer great resemblance to the corresponding process in Piroplasma. Multiple division has not yet been so satisfactorily made out. It appears to conform more 
or less to the radial or rosette type of multiplication (I, c), enlarged rounded parasites, with a varying number of nuclei (up to about 10) uniformly arranged near the periphery, having been often noticed. The details are, however, rather differently described by different workers.

Our knowledge of any further development undergone by these parasites is limited at present to the Leishman-Donovan bodies, and is due in the first instance to Rogers (94). Rogers cultivated the parasites

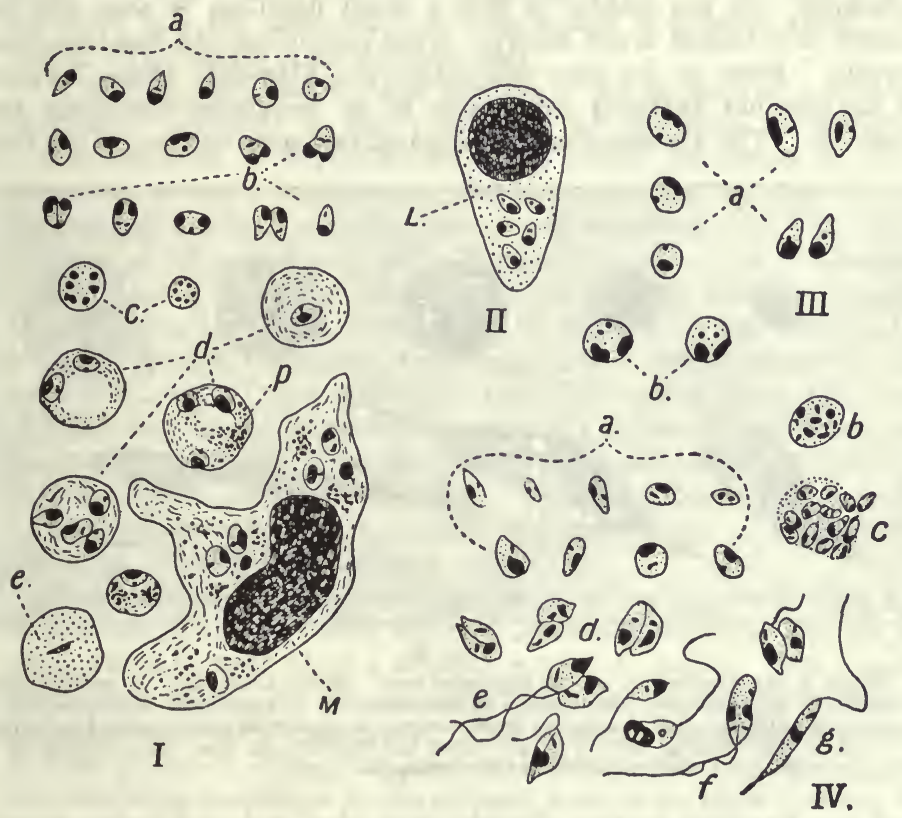

Frg. 39.

I, Leishmania (Piroplusma) donovani (Lav. and Mesn.). a, typical pear-shaped or oval forms ; $b$, various stages in binary fission ; $c$, nuclear division, preparatory to multiple fission; $d$, endocorpuscular forms in red blood-corpuscles ( $p$, rigment grains); $e$, bacillary form of the parasite in a corpuscle; $I I$, large macrophareal cell with many parasites. (After Donovan.) II, Uni. nuclear lencocyte (I.) containing several parasites. (After L. and M.) III, L. (I'., Helcosoma) tropica (Wright). $\quad u$, single individuals; $b$, dividing forms. (From Mesnil, mostly after Wright.) IV, L. (P.) domncuni in cultures of different ages. $a$, ordinary forms of varying size; $b, c$, stages in multiple division ; $e, f$, and $g$, flagellate forms. (After Rogers.)

in citrated blood, at a lower temperature, and made the astonishing discovery that Flagellate forms were developed from them. This result has since been fully corroborated and further details ascertained by Christophers (87), Leishman and Statham (92), and others. Different stages in the process are seen in Fig. 39, IV, $d-g$; and Fig. 40. The parasites increase greatly in size and become vacuolated (this is probably due to the artificial medium in which they are). Multiplication by binary fission takes place, and with successive generations the shape of the body alters ; from being pyrifurm it passes through a fusiform condition, and finally becomes elongated and slender. Meanwhile, in many of these phases, a 
flagellum has made its appearance; when this is fully developed the parasite quite resembles an ordinary Herpetomonas.

The origin of the flagellum is interesting. A distinctive vacuole-like structure arises near the end which will become the flagellar end, in close connection with the kinetonucleus-a point, probably, of importance. This vacuole increases and suddenly is ruptured, some of its contents being extruded to the exterior as a tuft or fringe of pink-staining substance. In the middle of this, a small flagellum is seen, but how exactly it is formed is not known. Once constituted, the flagellum grows rapidly. Even in the most fully-developed Flagellate phases, however, in no case has anything comparable to an undulating-membrane been observed. The kinetonucleus is comparatively near one end of the body,

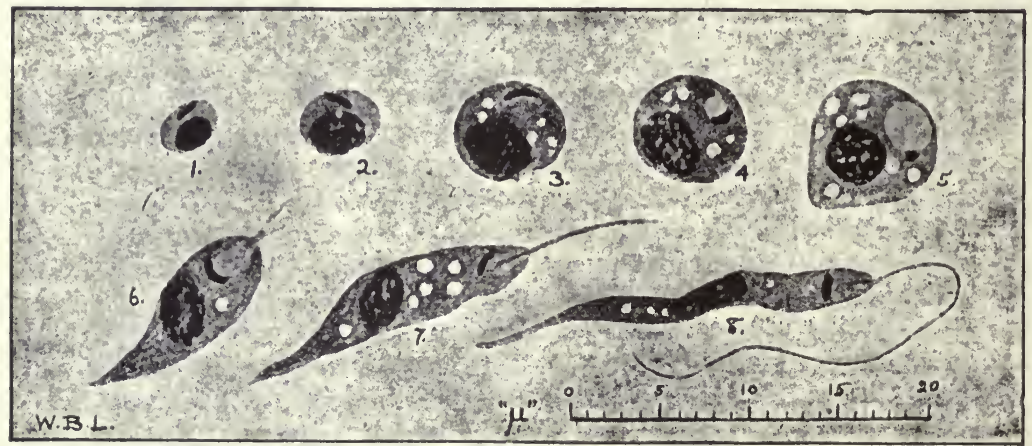

FI0. 40.

Stages in the development of the flagellated form. (From Leishman.) 1, ordinary spleen parasite $; 2,3$, growth and vacuolisation in cultivation ; 4, 5, appearance and growth of the special "flagellar vacuole," close to the kinetonucleus; 6 , rupture of this vacuole and protrusion of a tuft of pink-staining threads; 7 , growth of the flagellum, its base being inserted in the collapsed vacuole; 8 , acquirement of the Herpetomonad form.

and the flagellum springs directly from that end, not being actually connected, apparently, with the former organella.

Another remarkable process observed in these developmental forms in cultures is unequal longitudinal fission. Very thin, sickle-like ("spirillar") portions of the body are split off from one side of a parent-individual. More than one of these thread-like forms may be successively cut off. The unusual feature of the process is that neither the two principal nuclear elements nor the flagellum take part in it. Subsequently, these fission forms seem to give rise to very slender flagellar ones. To what extent this represents a normal (natural) mode of multiplication is uncertain.

No other stages have been observed in cultures, and the organisms degenerate and ultimately die off. The above facts demonstrated, however, that the Leishman-Donovan bodies can undergo important changes outside the human host, and rendered it probable that they do so naturally, though whether in the free condition or in an alternate host was, until lately, quite unknown. The superficial position of the localised form (Wright's type) points very strongly to infection by means of some biting Insect, and it is natural to infer that the same holds also for the 
splenic type, when its occurrence in the circulation is borne in mind. Here, again, Rogers gave the lead. This worker, finding that the parasites developed flagellar stages most readily in an acid medium, suggested (95)that the stomach of some blood-sucking Insect (such as a flea or bug) was probably the place where the above described extra-corporeal phases of the parasite's existence would be found to occur. This has been recently proved to be the case by Patton (93), who has found the Flagellate phases in the bed-bug (Cimex rotundatus [macrocephalus]). It is most probable, therefore, that the infection of human beings is brought about hy this Insect, which serves as an alternate host.

The systematic position and affinities of this parasite have been much discussed. Leishman at first considered the organisms as representing involution-forms of Trypanosomes, being largely influenced by the two chromatin masses; in this view he was supported by Marchand and Ledingham. Later, he went farther and suggested that they perhaps represented an actual stage in a Trypanosome life-cycle. Laveran and Mesnil, taking more into account the general form and very suggestive binary fission, thought a new species of Piroplasma was concerned, and named the bodies Piroplasma donorani; in this view Donovan and others have concurred. Other authorities (e.g. Christophers, Ross, and Wright) thought they saw in the parasite an entirely different kind of Sporozoan. Ross called the splenic type Leishmania, and a little later, Wright termed the ulcer-form Helcosoma tropicum. Recently, Rogers has placed the Leishman-Donovan form in the genus Herpetomonas, on account of the similarity in the Flagellate-phase.

It is probably best to regard the parasites as generically new forms; in this case the splenic form becomes Leishmania donorani and the ulcertype, which is most likely a separate species, L. tropica. The organisms are closely related, on the one hand, with the Herpetomonads, and on the other with the Piroplasmata. With regard to the parasites possessing, at some period or other, a trypaniform structure, the complete absence of an undulating-membrane in the cultural forms is no proof that one is not present under certain conditions in Nature. For, as already noted, many Trypanosomes, when "cultivated," may have a very slight indication of a membrane or none at all. Nevertheless, it is by no means improbable that these parasites have remained solely Herpetomonad forms and have not developed the characteristics of a Trypanosome. The fact that the Flagellate-phase is only known to occur in the Invertebrate host, points very strongly to this being the original primary host. In this connection the Herpetomonas Iately described by Patton from Culex pipiens (to which reference has been made above) is very interesting, because of the occurrence of resting-phases resembling the Leishman bodies. Leishmania may well be a similar form which, parasitic in a sanguivorous Insect, has become adapted to the Vertebrate host in its resting, gregariniform phase, and perhaps never develops a trypaniform condition, or even an active flagellar phase therein. Turning to the other side, there can be little doubt that the Piroplasmata are intimately connected with the Leishman-Donovan-Wright bodies. The general agreement of the intracellular forms as regards appearance and binary fission has been noted 
above. In addition, there is the most important point that some species of Piroplasma are stated to show, at certain times, the same characteristic nuclear dimorphism. Schaudinn was the first to notice this, in the case of $P$.canis; and he was confirmed by Kossel and Weber. Since then additional observations to the same effect are recorded by other workers (e.g. Liihe) for various species. This being so, the Piroplasmata also are most probably to be derived from Flagellate forms. ${ }^{1}$

-(B) A word or two, lastly, with reference to the supposed connection of the spirochatue with the Trypanosomes. Besides the instance of Trypanosoma (spirochaeta) ziemanni, Schaudinn, in his great memoir (l.c.), was inclined to consider that other Spirochactae (e.g. S. obermeieri of relapsing fever) were also only phases in the life-cycle of other HaemoHagellates. Subsequently, however, as a consequence of his investigations on Spirochaeta plicatilis, the type-species, and other forms, he relinquished this view, finding that the latter were of a totally different nature, and should rather be placed with the Bacteria. Much has since been written with regard to the nature and affinities of the various Spirochaetae. We do not propose to go into the general question here, as the preponderance of opinion is decidedly agrainst these organisms belonging to the Protozoa. It is only necessary to mention one or two forms which have been definitely referred to the Trypanosomes. Certes, in 1882, described a parasite from the digestive tube, including the crystalline style, of oysters, which he named Trypanosoma ballianii. A few years ago Laveran and Mesnil (99) re-examined this organism, and came to the conclusion that it was not a Trypanosome but a Bacterium, allied to Spirochaeta. Other workers who have recently observed this form also agree that its structure shows none of the essential features of a Trypanosome, but, on the contrary, greatly resembles that of a true Spirochaete. Perrin, it may be noted, has endeavoured (100) to connect it with Schaudinn's bipolar "Urhaemoflagellate." This idea has received no support, and indeed Perrin's whole paper is most unconvincing. Another, much more important example is that of the remarkable spirochaetiform parasite first described by Schaulinn and Hoffmann (103) in cases of syphilis, and which is now considered to be most likely the cause of that disease. Schaudiun found (102) that this organism differs in many ways from an ordinary Spirochceta, and placed it in a new genus Treponema as T. pallidum. In a recent memoir (101), Krzysztalowicz and Siedlecki have given a detailed account of this organism, and state that they have observed distinct trypaniform stages in its life-cycle. For this reason they consider it to be allied to the Trypanosomes and place it actually in the genus Trypanosoma, as T. luis. This view lacks, as yet, corroboration, and so here, as in the case of Schaudinn's research, the question must

I Since this was written we are able to add that confirmation of this view is forthcoming. In a most important note, Miyajima (Philipp. J. Sci. ser. B, ii. 1907, p. 83) describes the devclopment of Flagellate-phases in cultures of a Piroplasma (cf. parvum) of cattle in Japal. In seventy-two hours, forms with well-developed undulating-membrane were numerous. The author seems to have carefully guarded against the possibility of this highly-interesting occurrence being due to undetected Trypanosomes present in the blood. 
be left unsettled. There is one point, however, which may not be without significance, namely, the considerable resemblance between the biology of this parasite in relation to its host (i.e. as regards mode of infection, habitat, connection with the lesions, etc.) and that of Trypanosoma equiperdum, the cause of Dourine or "horse-syphilis" (cf. above, pp. 197, 206).

\section{POSTSCRIPT.}

As this article goes to press, a most interesting note by Roubaud (C.R. Ac. Sci., 24th Feb. 1908, p. 423) comes to hand. This worker has been investigating the relation between certain lethal Trypanosomes (T. gambiense, T. brucii, T. dimorphon, and others) and Glossina palpalis, and finds that the parasites undergo important changes as soon as they arrive in the proboscis of the Tsetse-fly. The kinetonucleus passes to the middle of the body, the undulating-membrane disappears, the flagellum becomes short and thickened, and the parasites quickly attach themselves to the wall of the proboscis by the flagellar end. The whole process may be accomplished, indeed, in five minutes. Moreover, active multiplication goes on, and after a time an immense number of attached Trypanosomes are present throughout the entire proboscis, often grouped in masses or colonies. This "temporary culture" (culture d'attente) persists for two days in the case of T. brucii, and longer-up to five to six days - in the other forms.

This remarkable developinent is apparently specific for Glossinae; it only occurs in a small number, and is doubtless due to the influence of special properties of the salivary fluid. As Rouband remarks, it probably affords an explanation of the selective rôle played by the Tsetses in the propagation of different trypanosomoses in Africa. Roubaud, however, considers that these forms found in the proboscis are the only ones capable of giving rise to an infection in a Vertebrate after the lapse of twenty-four hours. This is going too far, in view of the facts now known with regard to the length of time Trypanosomes may live and develop in the digestive tract of Glossinae (cf. pp. 200, 230). It is noteworthy that Roubaud was unable to obtain a successful inoculation from a proboscis so infected. Moreover, the repeated failures of investigators to infect animals from flies after forty-eight hours (cf. pp. 199, 200) seem to show that the later-developed "proboscis-forms" at all events are not infective, since they may reasonably be supposed to have been present in some of the many experiments tried. On the other hand, there is an important observation made by Bruce when working on T. brucii, to which Minchin $(58$, p. 210$)$ has drawn attention, showing that "wild" flies, caught while feeding on a healthy animal, could infect another animal on which they were subsequently fed. This certainly points to the presence of some developmental phases in the Insect other than Roubaud's proboscisforms; the proboscis had been presumably "cleaned" by the first biteon the uninfected animal on which the fly was caught. And this view is entirely borne out ly Stuhlmann's recent research, summarised in the body of this article. 


\section{LIST OF KNOWN (NATURAL) HOSTS OF TRYPANO- SOMES AND ALLIED FORMS.}

[In the compilation of this list, Nabarro's edition of Laveran and Mesnil's Treatise has been of considerable service to the writer.]

\section{Mammalia.}

? Bovidae (Indian, indigenous)

? $B$. (various, African)

Buffaloes

Catoblepas gnu, gnu, "wildebeeste" .

? Cattle (" hill," India)

Cavia cobaya, guinea-pig

Cricetus frumentarius (arvalis), hamster

Hydrochoerus capybara, capybara

Lepus cuniculus, rabbit . .

Meles taxus, badger

Miniopterus schreibersii, bat

Mus decumanus, sewer-rat .

M. rattus, black rat, $M$. rufescens

M. sylvaticus, field rat

M. musculus, nouse

M. niveiventer, rat (Indian)

Myotis murinus, a bat

Myoxus avellanarius, M. glis, dormice

Nesokia (Mus) giganteus, bandicoot .

Phyllostoma sp. See under Stegomyia Pipistrellus pipistrellus, bat

Pteropus medius, a bat

Sciurus palmarum, squirrel (Indian) . Spermophilus guttatus, S. musivus, spermophile

Strepsiceros capensis, "koodoo"

Talpa europaea, mole

Tragelaphus scriptus sylvaticus, "bushbuck"
Trypanosoma eransi (Steel).

$T$. theileri, Lav., and $T$ ' transvaaliense, Lav. [most probably $=7$. theileri].

T. brucii, Bradford and Plimmer.

T. brucii.

T. himalayanum, Lingard (syn.'.T. lingardi, Blanchard) [perhaps = T. theileri].

A Trypanosome [possibly a Trypanoplasma], Kunstler, 1883.

T. rabinowitschi, Brumpt (syn. Trypanozoon criceti, Lühe).

T. equinum, Voges.

T. cuniculi, Blanchard.

$T$. pestanai, Bettencourt and França.

A Trypanosome [Dionisi, 1899].

$T$. lewisi (Kent); T. longocaudense, Lingard [probably $=T$. lewisi].

T. lewisi (Kent).

T. sp. [lewisi ?], Gros, 1845.

T. duttoni, Thiroux ; T. musculi [syn. T. d. ?], Kendall.

T. longocaudense, Lingard [probably $=T$. lewisi].

T. nicolleorum, Sergent, E. and E. [perhaps syn. T'. vespertilionis, Battaglia].

T. blanchardi, Brumpt (syn. T'. myoxi, Blanchard).

T. bandicotti, Lingard.

$T$. sp. (compared with $T$. nicolleorum), Petrie.

A Trypanosome [Donovan, in Lav. and Mesn., 1904].

T. (Trypanozoon) indicum, Lühe.

A Trypanosome [Chalachnikov, 1888].

T. brucii, Br. and $\mathrm{Pl}$.

T. talpae, Nabarro.

T. brucii. 
Vespertilio kuhli, bat.

V. noctula, bat .

Vesperugo nattereri, $V$. pipistrellus ("pipistrelle"), $V$. serotinus, bats
7'. nicolleorum, Sergent, E. and E. ; T. vespertilionis, Serg. [both perhaps synn. T. vespertilionis, Battaglia].

T. vespertilionis, Battaglia.

T. dionisii, Bettencourt and França [perhaps syn. $T$. vespertilionis, Battaglia].

Various Trypanosomes which have been given distinct names have been lately described from certain Equidae and Bovidae in different regions of Africa, as the cause of more or less pronounced trypanosomosis. It is probable that some of these, at any rate, are really forms of other better-known African parasites. They are mentioned here, in order to complete an enumeration of species, for purposes of reference. They are $T$. cazalboui, Lav. ; $T$. congolense, Broden; $T$. nanum, Lav. (an extremely small form); $T$. pecaudi, Lav. ; $T$. soudanense, Lav.; $T$. suis, Ochmann; and $T$. vivax, Ziemann. The true (natural) hosts are uncertain.

Aves.

Agelaius phoeniceus, red-winged blackbird

Alcyon sp., kingfisher (Cameroon)

Asturinula monogrammica, hawk (Congo State)

Athene noctua, little owl

\section{A. brama, owl (Madras)}

Butco lineatus, red-shouldered hawk . Bycanistes buccinator, trumpeter hornbill

Chelidon urbica, house-martin

Colaptus auratus, "flicker"

Columba sp., pigeon (Indian)

Coracias garrula, roller-bird

Corvus sp., crow or raven (Indian)

Crane.

Crithagra sp., "millet-eater"

Cyanocitta cristata, blue jay

Dryobates villosus, hairy woodpecker .

Egret .

Emberiza citrinella, yellow-hammer .

Estrelda estrelda, "millet-eater"

Fringilla (Carduclis) carduelis, goldfinch

F. coelebs, chaffinch

Goat-sucker
T. avium (type L. and M.), [Novy and M'Neal, 1905].

A Trypanosome [Ziemann, 1905].

A Trypanosome [Dutton, Todd and Tobey, 1907].

Trypanomorpha (Trypanosoma) noctuae (Schaud.); also Trypanosoma [Spirochacta ?] ziemanni (Lav.).

A Trypanosome [Donovan, in Lav. and Mesn., 1904].

T. mesnili, Novy and M'Neal.

A Trypanosome [Dutton, Todd and Tobey, 1906].

A Trypanosome [Petrie, 1905].

T. avium (type Lav. and Mesn.).

T. hannae, Pittaluga.

T. "avium," Danilewsky.

A Trypanosome [Hanna, 1903].

A Trypanosome? [Gros, 1845].

$T$. sp. [Dutton and Todd, 1903].

$T$. avium (type L. and M.); also $T$. sp. [Novy and M'Neal, 1905].

$T$. sp. incert. [Novy and $\mathbf{M}^{\top} \mathrm{Neal}$, 1905].

T. sp., perhaps avium [Cerqueira, 1906].

A Trypanosome [Petrie, 1905].

T. johnstoni, Dutton and Todd.

T. sp. [Sergent, E. and E., 1904].

A Trypanosome [Ziemann, 1898 ; also Petrie, 1905].

A Trypanosome? [Gros, 1845]. 
Harporhynchus rufus, brown thrasher

Hirundo rustica, swallow

Icterus galbula, Baltimore oriole. Laniarius cruentus, shrike (African) . Linota (Acanthis) rufescens, redpoll. Melospiza fasciata, song-sparrow .

Merula migratoria, robin (American) . M. merula, blackbird .

Milvus govinda, kite (Indian)

Neophron percnopterus, vulture (African) Nicticorax gardenia (Brazil)

Padda oryzivora, Java sparrow

Passer domesticus, sparrow .

Passerine birds, many (except Corvus and Pica)

Polyplectrum germani, pheasant (Annam)

Scolephagus carolinus, rusty blackbird Sialia sialis, bluebird . . . Spinus tristis, goldfinch (American) . Sylvia atricapilla, black-cap warbler . Syrnium aluco, tawny owl .

\section{Tachyphornus ornata .}

Treron calva, dove (Angola)

Turdus musicus, song-thrush

Troglodytes aedon, house-wren .

Zenaidura macroura, mourning-dove .
A Trypanosome [Novy and $M$ Neal, 1905].

T. mathisi, Serg., E. and E. ; a Trypanosome (T. $m$. ?) [Petrie, 1905].

T. avium (type L. and M.).

A Trypanosome [Neave, 1906].

$T$. sp. [original observation].

$T$. avium (type L. and M.).

T. avium (type L. and M.).

A Trypanosome [Petrie, 1905].

A Trypanosome [Donovan, in Thiroux, 1905].

A Trypanosome [Neave, 1906].

$T$. sp., perhaps avium [Aragao, in Cerqueira, 1906].

T. paddac, Thironx.

T. avium (type $\mathrm{I}_{\text {. and }} \mathrm{M}$.).

Trypanosomes [Sjübring, in $N$. and M'Neal, 1905].

T. polyplectri, Vassa].

T. sp., Novy and M'Neal.

T. avium (type L. and M.).

T. laverani, Novy and M'Neal.

$T$. sp. [Sergent, E. and E., 1904].

T. avium, Danil., emend. Lav. ; also "T." [Leucocytozöon] zicmanni (Lav.).

A Trypanosome [Cerqueira, 1906].

A Trypanosome [Wellman, 1905].

A Trypanosome [Petrie, 1905].

A 'Trypanosome [N.' and M'N., 1905].

$T$. avium (type L. and M.).

\section{REPTII.IA.}

Crocodile (Uganda)

Crocodilus cataphractus? (Congo)

Damonia reevesii, tortoise .

Gecko.

Mabuia raddonii, a lizard (French Guinea)

Python

Snake (unspec., Gambia)

Tortoise (Indian-Emys or Kachuga tectum)

Tortoise (unspec., Gambia)
A Trypanosome [Minchin, Gray and Tulloch, 1906].

A Trypanosome [Dutt., Todd and Tob., 1907].

T. damoniae, Lav. and Mesn.

A Trypanosome [Gehrke, 1903].

T. boucti, Martin.

"T." pythonis, Robertson [really a Haemogregarine].

A Trypanosome [Dutton and Todd, 1903].

A Trypanosome [Simond, in L. and M., 1904].

A Trypanosome [Dutton and Todd, 1903]. 


\section{Амрнinia.}

Bufo vulgaris and viridis, toads .

$B$. reticulatus (Somaliland).

Diemyctulus viridescens (American newt)

Hyla arborea and $H$. viridis, tree-frogs

H. lateristriga (?), Brazil

Rana angolensis (Transvaal)

$R$. esculenta, edible frog
Frogs (unspec., Gambia)

T. rotatorium (Mayer).

$T$. somalense, Brumpt.

A Trypanosome [Tobey, 1906].

T. mega and T. karyozeukton, Dutton and Todd.

T. rotatorium (Mayer); T'. sp. [?], Lav. and Mesn.

$T$. borreli, Marchoux and Salimbeni.

T. nelspruitense, Lav.

$T$. rotatorium (Mayer). (Syn. $T$. loricatum or costatum (Mayer) and T. rotatorium (Mayer), França and Athias ; T. r. var. nana, Sergent, E. and E.; T. inopinatum, Sergent, E. and E. ; T. elegans and T. undulans, F. and A. [doubtful species].)
R. temporaria
R. t. (?) (Hong Kong) .
$R$. theileri (Transvaal)
R. trinodis (?) and other sp. (Gambia)
T. rotatorium (Mayer).
T'. belli, Nabarro.
T. nelspruitense, Lav.
T. rotatorium (Mayer).

\section{Pisces.}

\section{$(T p l .=$ Trypanoplasma. $)$}

Abramis brama, bream

Acerina cernua, pope .

Anguilla vulgaris, eel

Bageus bayard, bagara (Nile)

Barbus carnaticus (India) .

B. fluviatilis, barbel .

Blennius pholis, blenny

Bothus rhombus (Rhombus laevis), brill

Box boops.

Callionymus dracunculus

Carassius auratus, goldfish

C. vulgaris, Prussian carp .

Clarias (Silurus) clarias, a Silurid (Cochin-China).

C. angolensis (Congo State)

Cobitis barbatula, loach
T. abramis, Lav. and Mesn.; Tpl. abramidis, Brumpt.

T. acerinae, Brumpt; a Trypanoplasm [Keysselitz, 1906].

T. granulosum, Lav. and Mesn.

A Trypanosome [Neave, 1906].

A Trypanosome [lingard, 1904].

T. barbi, Brumpt; Tpl. barbi, Brumpt.

T. delagei, Brumpt and Lebailly.

$T$. bothi, Lebailly.

$T p l$. [?] intestinalis, Léger.

$T$. callionymi, Brumpt and Lebailly.

T. danilewskyi, Lav. and Mesn.

T. carassii (Mitrophan.). (Syn. Haematomonas c., Mitr.; T. piscium and $T$. fusiforme piscium, Danilewsky.)

T. clariac, Montel.

A Trypanosome [Dutton, Todd and Tobey, 1906].

$T$. barbatulae, Léger; $T p l$. varium, Léger. 
C. fossilis .

Cottus bubalis

C. gobio, river bull-head

Cyclopterus lumpus, lump-fish

Cyprinus carpio, carp.

Esox lucius, pike

Gobio fluviatilis, gudgeon .

G. giuris (India)

Gobius niger, goby

Leuciscus (Scardinius), erythrophthalmus, rudd or red-eye

$L$. idus, $L$. cephalus, $L$. rutilus, roaches

L. spp.

Limanda platessoides.

Lota vulgaris

Macrodon malabaricus (Brazil) .

Macrones seenghala, M.tengara,Silurids (India)

M. cavasius (India)

Mugil sp., noke (Nile)

Ophiocephalus striatus, Silurid (India)

Perca fluviatilis, perch

Phoxinus laevis, minnow

Platophrys laternae

Pleuronectes flesus (Flesus vulgaris), flounder

P. platessa (Platessa vulgaris), plaice .

Polypterus sp., dabib (Nile) . .

Raia clavata, $R$. macrorhynchus, $\boldsymbol{R}$. mosaica, and $R$. punctata, rays

R. microcellata.

Rhandia queler (Brazil) . .

Saccobranchus fossilis, a Silurid .

Salmo fario, trout

Scyllium canicula, S. stellare, dogfish
T. cobitis (Mitroph.). (Syn. Haematomonas c., Mitr.; T. piscium and T. fusiforme, Danilewsky.)

$T$. cotti, Brumpt and Lebailly.

T. langeroni, Brumpt; Tpl. guernei, Brumpt.

Tpl. [?] ventriculi, Keysselitz.

$T$. danilewskyi, Lav. and Mesn.; Tpl. cyprini, Plehn.

T. remaki, Lav. and Mesn.; Tpl. sp. [Minchin, 1908].

T. clegans, Brumpt.

$T$. sp. [Castellani and Willey, 1905].

$T$. gobii, Brumpt and Lebailly.

$T p l$. borreli, Lav. and Mesn.; $T$. scardinii, Brumpt.

A Trypanosome and Trypanoplasm [Keysselitz, 1906]. [Probably $\boldsymbol{T}_{p l}$. borreli and $T$. leucisci.]

T. leucisci, Brumpt.

T. limandae, Brumpt and Lebailly.

A Trypanosome and Trypanoplasm [Keysselitz, 1906].

T. macrodonis, Botello.

A Trypanosome [Lingard, 1899].

A Trypanosome [Castellani and Willey, 1905].

A Trypanosome [Neave, 1906].

A Trypanosome [Lingard, 1899].

$T$. percae, Brumpt; also a Trypanoplasm [Keysselitz, 1906].

T. danilewskyi (?), Lav. and Mesn.; T. phoxini, Brumpt; Tpl.borreli, Lav. and Mesn.

T. laternae, Lebailly.

T. flesi, Lebailly (syn. T. pleuronectidium, Robertson).

T. platessae, Lebailly (syn. T. pleuronectidium, Roberston).

A Trypanosome [Neave, 1906].

T. raiac, Lav. and Mesn.

A Trypanosome [Robertson, 1906].

T. rhamdiae, Botello.

T. saccobranchi, Castellani and Willey. Tpl. truttae, Brumpt. [Valentin, in 1841, observed a Haematozoan, which was probably either a Trypanosome or a Trypanoplasm.]

T. scyllii, Lav. and Mesn. 
Silurus glanis

Solea vulgaris, sole

Squalius (Lenciscus) cephalus, chub

Synodontis schal, gargur (Nile) .

Tinca tinca, tench

Trichogaster fasciatus (India)
A Trypanosome [Keysselitz, 1906].

T. soleae, Lav. and Mesn.

T. squalii, Brumpt.

A Trypanosoine [Neave, '1906].

T. tincae, Lav. and Mesn. ; a Trypanoplasm [Keysselitz, 1906].

A Trypanosome [Lingard, 1899].

\section{INSECTA.}

Anopheles maculipennis

A. m. (larvae)

Anopheles sp., mosquitoes (India)

Bombyx mori, silkworm

Chironomus plumosus

Cimex rotundatus, bed-bug (India)

Culex fatigans

C. pipiens .

Dasyphora pratorum .

Glossina fusca

G. morsitans and G. pallidipes

G. palpalis

\section{G. tachinoides}

Haematopinus spinulosus, rat-louse

Haematopota italica

Hippobosca rufipes, (?) H. maculata

Homalomyia scalaris .

Melophagus ovinus, sheep-louse

Musca domestica .

Nepa cinere

Pollenia rudis

Pulex sp., fleas

Sarcophaga haemorrhoidalis, blow-fly

Stegomyia fasciata

S. $f$. (an individual which had fed on a bat, Phyllostoma)

Stomoxys calcitrans

Tabanus glaucopsis
Crithidia fasciculata, Léger.

A Herpetomonad (cf. with $H$. jaculum)

[Sergent, E. and E., 1906].

Herpetomonads (said to resemble Leger's Crithidia) [Ross, 1898; Christophers, 1901, and others].

Herpetomonas bombycis, Levaditi.

Crithidia campanulata, Léger.

Leishmania (Piroplasma) donovani.

Herpetomonads [Ross, 1898 ; Christophers, 1901 ; Patton, 1907].

Trypanomorpha noctuae (Schaud.); Crithidia fasciculata; "Trypano. soma" (Herpetomonas) culicis, N., $\mathrm{M} ' \mathrm{~N}$., and Torrey ; $H$. algeriense, Sergent, E. and E. ; $H$. sp., indet. [Patton, 1907].

$H$. lesnei, Lèger.

$T$. brucii; perhaps $T$. gambiense.

(?) T. brucii.

T. grayi, Novy ; T. tullochii, Minchin ;

(?) T. dimorphon, Dutt. and Todd.

(?) T. brucii ; (?) T. gambiense.

(?) T. lewisi.

(?) H. subulata, Léger.

T. theileri (probably).

$H$. (cf. muscae-domesticae) [Léger].

"Trypanosome-like parasites" [Pfeiffer, 1905].

H. muscae-domesticae, Burnett.

H. jaculum, Léger.

$H$. (cf. m..d.) [Léger].

T. lewisi (probably); a Herpetomonad [Balfour, 1906].

H. sarcophagae, Prowazek.

H. algeriense, Sergent, E. and E.

A "Trypanosome" [Durham, 1900].

(?) T. equinum; a Herpetomonad [Gray, 1906].

H. subulata, Léger. 
T. lineola and other sp.

T. tergestinus

Tanypus sp.

Theicomyxa fusca

"Water-bug" (India)
(?) T. evansi.

Herpetomonas (Crithidia) minuta, Léger.

$H$. gracilis, Léger.

$H$. (cf. $m_{\text {.- }} d$.) [Léger].

A Herpetomonad [Patton, 1907].

\section{Arachinida.}

Rhipicephalus sanguineus, llog-tick “T." christophersi, N., M'N., and (India)

Torrey.

\section{HiRUdINEA.}

Calobdella punctata . . . . T. cotti and T. soleae [Brumpt].

Helobdella algira . . . . T'. inopinatum [Billet].

Hemiclepsis marginata . . . Tpl. varium [Léger].

H. sp.

T. abramis, acerinac, barbi, danilewoskyi, granulosum, percae, phoxini, remaki, squalii; perhaps also ' $T$ '. barbatulae (?), langeroni, leucisci, scardinii [Brumpt]. Tpl. abramidis [Brumpt].

Piscicola sp.

T. barbatulae [Léger]; Tpl. borreii, barbi, guernei, (?) truttae [Brumpt].

$P$. geometra

Pontobdella muricata. $T p l$. borreli; also other Try panoplasms [Keysselitz].

P. sp. . . . . . . . T. scyllii [Brumpt].

\section{SiphoNOPHORA.}

Abyla pentagona, Cucubalus kochii,

Trypanophis grobleni (Poche).

Halistemma tergestinum, and Monophyes gracilis

\section{LI'TERATURE.}

I. Relating to the Trypanusomes.

A. Comprehensive works.

1. Laveran, A., and Mesnil, $F$. Trypanosomes et trypanosomiases. Paris (Masson et Cie.), 1904. An English edition, translated and considerably enlarged and brought up to date by D. Nabarro, has lately been published (London, Baillière, Tindall and Cox, 1907, 581 pp., 81 text-figg.).

2. Lïhe, $\boldsymbol{M}$. Die im Blute schmarotzenden Protozoen. In Mense's Handbuch der Tropenkrankheiten, vol. iii. pt. i. (Leipzig, J. A. Barth, 1906), pp. 69 268, 3 pls., text-figg.

3. Woodcock, H. M. The Haemoflagellates. Q.J. Micr. Sci. I., 1906, pp. 151-331, 65 text-figg. 
B. List of the more important memoirs cited in the text. (N.B. Full references to the cxisting literature are given in each of the above works.)

4. Billet, $A$. Culture d'un Trypanosome de la grenouille chez une Hirudinée: relation autogénique possible de ce Trypanosome avec une Hémogrégarine. C.R. Ac. Sci. cxxxix. p. 574, 1904.

5. _ Sur le Trypanosoma inopinatum . . . et sa relation possible avec les Drepanidium. C.R. Soc. Biol. lvii. p. 161, 16 figg., 1904.

6. Bralford, J. IR., and I'limmer, $H$. G. The Trypanosoma brucii, the Organism found in Nagana or the T'setse-fly Disease. Q.J. Micr. Sci. xlv. p. 449, 2 pls., 1902.

7. Bruce, $D$. Reports on the Tsetse-fly Disease or Nagana. Ubombo, Zululand, 1895 and 1896 ; London, 1897 and 1903.

8. - Nabarro, D., and Greig, E. D. [Reports on Sleeping-Sickness and various Animal Trypanosomoses in Uganda.] Roy. Soc. Conm., 1903-1907.

9. Brum,t, $E$. Contribution ì l'étude de l'évolution des Hémogrégarines et des Trypanosomes. C.R. Soc. Biol. Ivii. p. 165, 1904.

10. - Sur quelques espèces nouvelles de Trypanosomes parasites des poissons l'eau clouce; leur mode d'évolution. Op. cit. lx. p. 160, 1906.

11. Mode de transmission et évolution des Trypanosomes des poissons; description de quel ques espèces de 'Trypanoplasmes des poissons d'eau douce ; Trypanosome d'un crapaud africain. T.c. p. 162, 1906.

12. - Expériences relatives an mode de transmission des Trypanosomes et des Trypanoplasmes par les Hirudinées. Op. cit. 1xi. p. 77, 1906.

13. — Rôle pathogène et mode de transmission du Trypanosoma inopinatum, Ed. et Et. Sergent. Mode d'inoculation d'autres Trypanosomes. T.c. p. 167, 1906.

14. - De l'hérédité des infections à Trypanosomes et Trypanoplasmes chez les hôtes internédiares. Op. cit. lxiii. p. 176, 1907.

15. — and Lebailly, C. Description de quelques nouvelles espèces de Trypanosomes et d'Hémogrégrarines parasites des Téléostéens marins. C.R. Ac. Sci. cxxxix. p. 613, 1904.

16. Buffurl, NI., and Schneider, G. Le Trypanosome de la Dourine. Arch. Parasitol. iii. p. 124, pls., 1900.

17. Castellani, A. Trypanosoma and Sleeping-Sickness. Reports S.S. Comm. Roy. Soc. i. and ii., 1903.

18. Dunileusky, _- Recherches sur la parasitologie comparée du sang des oiscanx. Wharkotr, 1888-1889.

19. Zur Parisitologie des Blutes. Biol. Centrlbl. v. p. 529 (1885).

20. Dutton, $E$. Note on a T'rypanosoma occurring in the Blood of Man. Brit. Mled. Journ., 1902, ii. p. 881,1 fig.

21. - and 'odd, J. L. First Report of the Trypanosomosis Expedition to Senegambia (1902). Mem. Livpl. Sch. Trop. Med. No. 11, 1903.

22. França, C., and Athias, C. Recherches sur les Trypanosomes des Amphibiens : I. Les 'l'rypanosomes de la Rana esculenta. Arch. Inst. R. Bact., Lisbonne, i., 1906.

23. Gray, A. C., and Tulloch, F. M. The Multiplication of the Trypanosorna gambiense in the Alimentary Canal of Glossina palpalis. Rep. S.S. Comm. Roy. Soc. No. 6, p. 282, 1 pl., 1905.

24. Gruby. Recherches et observations sur une nouvelle espèce d'Hématozoaire (T'rypanosoma sanguinis). C.R. Ac. Sci. xvii. p. 1134, 1843; and Ann. Sci. Nat. (3), i. p. 105, 7 figg., 1844. 
25. Hanna, $W$. Trypanosoma in Birds in India. Q.J. Micr. Sci. xlvii. p. 433, 1 pl., 1903.

26. Keysselitz, G. Ueber Trypanophis grobbeni (Trypanosoma g., Poche). Arch. Protistenk. iii. p. 367, 3 figg., 1904.

27. — Generations- und Wirthswechsel von T'rypanoplasma borreli, Lav. ct Mesn. Arch. Protistenk. vii. p. 1, text-figg., 1906.

28. Koch, R. Vorläufige Mittheilungen über die Ergebnisse meiner Forschungsreise nach Ostafrika. Deutsch. med. Wochenschr., 1905, p. 1865, text-figg.

29. - Ueber den bisherigen Verlauf der deutschen Expedition zur Erforschung der Schlafkrankheit in Ostafrika. Op. cit. 1906, Appendix, p. 51; also 1907, p. 49. Schluss-Bericht. Op. cit. 1907, p. 1889.

30. Lankester, $E$. $R$. On Undulina, the type of a New Group of Infusoria. Q.J. Micr. Sci. xi. p. 387, 4 figg., 1871.

31. The Sleeping-Sickness. Quart. Rev., July 1904, p. 113, 7 figg.

32. Laveran, A. Sur un nouveau Trypanosome des Bovidés. C.R. Ac. Sci. cxxxiv. p. 512, 1902.

33. —- Au sujet de deux Trypanosomes des Bovidés du Transvaal. Op. cit. cxxxv. p. 717, 5 figg., 1902.

34. - Sur un 'Trypanosome d'une chouette. C.R. Soc. Biol. lv. p. 528, 2 figg., 1903.

35. —_ Contribution à l'étude de Haemamoeba ziemanni. T.c. p. 620, 7 figg., 1903.

36. - Sur une nouveau Trypanosome d'une grenouille. Op. cit. lvii. p. 158, 2 figg., 1904.

37. — and Mesnil, $F$. Recherches morphologiques et expérimentales sur le Trypanosome des rats, Tr. lewisi (Kent). Ann. Inst. Pasteur, xv. p. 673, 2 pls., 1901.

38. — and - Sur les Flagedlés à membrane ondulante des poissons (genus Trypanosoma, Gruby, et Trypanoplasma, n. gen.). C.R. Ac. Sci. cxxxiii. p. $670,1901$.

39. - Sur la structure du Trypanosome des grenouilles et sur l'extension du genre Trypanosoma, Gruby. C.R. Soc. Biol. liii. 1. 678, 3 figg., 1901.

40. - and - Sur les Hématozoaires des poissons marins. C.R. Ac. Sci. cxxxv. p. 567, 1902.

41. — and - Sur quelques Protozoaires parasites d'une tortue d'Asie (Damonia reevesii). T.c. p. 609, 14 figg., 1902.

42. - and - Des Trypanosomes des poissons. Arch. Protistenk. i. p. 475, 15 figg., 1902.

43. — and Recherches morphologiques et expérimentales sur lo Trypanosome du Nagana ou maladie de la mouche tsé-tsé. Ann. Inst. Pasteur, xvi. p. 1, 13 figg., 1902.

44. - and - Sur un Trypanosome d'Afrique pathogène pour les Equidés, T. dimorphon, Dutton et Todd. C.R. Ac. Sci. cxxxviii. p. 732, 7 figg., 1904.

45. Lebailly, C. Sur quelques Hémoflagellés des 'Téléostéens marins. Op. cit. cxxxix. p. 576, 1904.

46. Léger, $L$. Sur la structure et la mode de multiplication des Flagellés du genre Herpetomonas, Kent. C.R. Ac. Sci. cxxxiv. p. 781, 7 figg., 1902. 
47. Léger, L. Sur un Flagellé parasite de l'Anopheles maculipennis. C.R. Soc. Biol. liv. p. 354, 10 figg., 1902.

48. - Sur quelques Cercomonadines nouvelles ou peu connues parasites de l'intestin des Insectes. Arch. Protistenk. ii. p. 180, 4 figg., 1903.

49. - Sur la morphologie du Trypanoplasma des vairons, et sur la structure et les affinités des Trypanoplasmes. C.R. Ac. Sci. cxxxviii. pp. 834, 856, 5 figg., 1904.

50. - Sur les Hémoflagellés du Cobitis barbatula, L. ; Trypanosma barbatulae, n. sp.; it Trypanoplasma varium, n. sp. C.R. Soc. Biol. Ivii. pp. 344, 345, 1904.

51. __ Sur un nouveau Flagellé parasite des Tabanids. T.c. p. 613, 6 figg., 1904.

52. - Sur les affinités de l'Herpetomonas subulata et la phylogénie des Trypanosomes. T.c. p. 615, 1904.

53. — Sur la presence d'un Trypanoplasma intestinal chez les poissons. Op. cit. lviii. p. 511, 1905.

54. Lignières, J. Coutribution à l'étude de la trypanosomose des Équidés Sud-Anéricains connue sous le nom de Mal de Caderas (Trypanosoma elmassiani). Rec. Méd. Vét. Bull. et Mém. (8), x. pp. 51, 109, 164, 2 pls., 1903.

55. Lingard, A. A new Species of Trypanosome found in the Blood of Rats (India), etc. J. Trop. Vet. Sci. i. p. 5, 1 pl., 1906.

56. $M^{\prime} N e a l, W . J$. On the Life-History of T. lewisi and T. brucii. J. Inf. Diseases, i., Nov. 1904.

57. Minchin, E. A. On the Occurrence of Encystation in Trypanosoma grayi, Novy, etc. P. Roy. Soc. lxxix. B, p. 35, text-figg., 1907.

58. — Investigations on the Development of Trypanosomes in Tsetse-flies, etc. Q.J. Micr. Sci. lii. p. 159, 6 pls., 1908.

59. - Gray, A. C., and Tulloch, F. M. Glossina palpalis in its Relation to Trypanosoma gambiense and other Trypanosomes. P. Roy. Soc. Ixxviii. B, p. 242,3 pls., 1906.

60. Mitrophanow, … Beiträge zur Kenntniss der Hämatozoen. Biol. Centrlbl. iii. p. 35,2 figg., 1883.

61. Novy, F. G. The Trypanosomes of Tsetse-flies. J. Inf. Diseases, iii. p. 394, 3 pls., 1906.

62. - and $M \Gamma^{\prime} N^{\top} e a l, W . J$. On the Trypanosomes of Birds. Op. cit. ii. p. 256, 11 pls., 1905.

63. — and - On the Cultivation of Trypanosoma brucii. Op. cit. i. p. 1, 1904.

64. - - and Torrey, H. N. The Trypanosomes of Mosquitoes and other Insects. Op. cit. iv. p. 223,7 pls., 1907.

65. Patton, $W . S$. Preliminary Note on the Life-Cycle of a Species of Herpetomonas found in Culex pipiens. B.M.J., 1907, ii. (July 13th).

66. Plehn, M. Trypanoplasma cyprini, n. sp. Arch. Protistenk. ii. p. 175, 1 pl., 1903.

67. Pricolo, A. Le Trypanosome de la souris. Cycle de développement des Trypanosomes chez le fotus. Centralbl. Bakt., Abt. 1, xlii. Orig. p. 231, 1906.

68. Provvazek, S. Studien. über Säugethiertrypanosomen. Arb. kais. Gesundhtsa. xxii. p. 1, 6 pls., 1905.

69. — Die Entwickelung von Herpetomonas, einen mit den Trypanosomen verwandten Flagellaten. Op. cit. xx. p. 440, text-figg., 1904. 
70. Rabinouitsch, L., and Kcmpner, $W$. Beiträge zur Kenntniss der Blutprarasiten, speciell der Rattentrypanosonen. Zeitschr. Hyg. xxx. p. 251,1 pl., 1899.

71. Robertson, M. Notes on Certain Blood-inhabiting Protozoa. Proc. Physic. Soc. Edinb. xvi. p. 232, 2 pls., 1906.

72. - Studies on a Trypanosome found in the Alimentary Canal of Pontobdella muricata. Op. cit. xvii. p. 83, 4 pls., 1907.

73. Roger's, $L$. The Transmission of the Trypanosoma evansi in India by Horseflies, etc. Proc. Roy. Soc. 1xviii. p. 163, 1901. Also see B.M.J., 1904, ii. p. 1454.

74. Ross, li. Notes on the Parasites of Mosquitoes found in India between 1895 and 1899. Journ. Hyg. vi. p. 101, 1906.

75. Schaudinn, $F$. Generations- und Wirthswechsel bei Trypanosoma und Spirochacta. Arb. kais. Gesundhtsa. xx. p. 387, text-figg., 1904.

76. Sergent, $E$. and $E$. Sur un Trypanosome nouveau parasite de la grenouille verte. C.R. Soc. Biol. lvi. p. 123, 1 fig., 1904.

77. - Hémanibes des oiseaux et moustiques. Générations alternantés de Schaudinn. Op. cit. lviii. p. 57, 1905.

78. - Sur des Trypanosomes des chauves-souris. T.c. 1. 53, 2 figg., 1905.

79. _ Sur un Flagellé nouveau de l'intestin des Culex et des Stegomyia, Herpetomonas algeriense. Op. cit. 1x. p. 291, 1906.

80. Stuhlmann, $F$. Beiträge zur Kenntniss der Tsetsefliegen (Gl. fusca and $G l$. tachinoides). Arb. kais. Gesundhtsa. xxvi. p. 83, 4 pls., 1907.

81. Swingle, L. D. Some Studies on Trypanosoma lewisi. Trans. Amer. Mier. Soc. xxvii. p. 111,1 pl., 1907.

82. Thiroux, —. Sur un nouveau Trypanosome des oiseaux. C.R. Ac. Sci. exxxix. p. 145, 5 figg., 1904.

83. _ Recherches morphologiques et expérimentales sur les Trypanosoma paddae. Ann. Inst. Pasteur, xix. p. 65, 1 pl., 1905.

84. — Recherches... sur Trypanosoma duttoni, Thiroux. T.c. p. 564, 1 pl., 1905.

85. Voges, O. Mlal de Caderas. Zeitschr. Hyg. xxxix. p. 323, 1 pl., 1902.

86. Wasielewsky and Senn, G. Beiträge zur Kenntniss der Flagellaten des Rattenblutes. Op. cit. xxxiii. p. 444, 3 pls., 1900.

\section{Relating to the "Leishman-Donovan-IVright" Bodies.}

87. Christophers, S. R. Reports on a Parasite found in Persons suffering from Enlargement of the Spleen in India. Sci. Mem. India, Nos. 8, 11, 15, 1904-1905.

88. Donovan, C. Human Piroplasmosis. Lancet, 1904, ii. p. 744, 1 pl.

89. James, S. P. Oriental or Delhi Sore. Sci. Mem. India, No. 13, 1905.

90. Laveran, A., and Mesnil, F. Sur un Protozoaire nouveau (Piroplasma donovani, Lav. et Mesu.), etc. C.R. Ac. Sci. cxxxvii. p. 957, 17 figg., 1903 ; and op. cit. cxxxviii. p. 187, 1904.

91. Leishman, $W$. On the Possibility of the Occurrence of Trypanosomosis in India. Brit. Med. Journ. 1903, i. p. 1252, 2 figg.; see also op. cit., 1904 , i. p. 303.

92. - and Statham. The Development of the Leishman Body in Cultivation. Journ. Army Med. Corps, iv. p. 321, 1 pl. 2 figg., 1905. 
93. Patton, W. S. Prelim. Report on the Development of the LeishmanDonovan Body in the Bed-Bug. Sci. Mem. India, No. 27, 1907.

94. Rogers, $L$. On the Development of Flagellated Organisms ... from the Spleen Protozoic Parasites of Kala-Azar. Q.J. Micr. Sci. xlviii. p. 367, 1 pl., 1904.

95. — Further Work on the Development of the Herpetomonas of KalaAzar . . . from the Leishman-Donovan Bodies. Proc. Roy. Soc. Ixxvii. B, p. 284, pl. 7, 1906 ; see also Lancet, 1905, i. p. 1484.

96. Ross, R. A New Parasite of Man. Thompson-Yates Lah. Rep. (5), 2, p. 79, 1 pl., 1904.

97. Wright, J. $/ T$. Protozoa in a Case of Tropical Ulcer (Delhi Sore). Journ. Med. Restarch, Boston, x. p. 472, 4 pls., 1903.

C. Relating to the Spirochaetae.

98. Certes, $A$. Note sur les parasites et les commensaux de l'huître. Biol. Soc. Zool. France, vii. p. 347,1 pl., 1882 ; see also op. cit. xvi. pp. 95 and $130,1891$.

99. Laveran, A., and Mesnil, $F$. Sur la nature bacterienne du prétendu Trypanosome des huîtres, "T." balbianii. C.R. Soc. Biol. liii. P. 883, 1901.

00. Perrin, W. S. Researches upon the Life-History of "Trypanosoma" balbianii (Certes). Arch. Protistenkunde, Jena, vii. p. 131, 2 pls., 1906.

01. Kraysatalowicz, $F$, and Siedlecki, $M$. Contribution à l'étude de la structure et du cycle évolutif de Spirochaeta pallida, Schaud. Bull. Ac. Cracovie, 1905 , p. 713, 1 pl.

02. Schaudinn, $F$. Zur Kenntniss der Spirochaeta pallida. Deutseh. med. Wochenschr. No. 42, 1905, p. 1665 ; see also t.c. p. 1728 (gen. Treponema proposed).

03. — and Hoffmann, E. Vorläufiger Bericht neber das Vorkonmen von Spirochaeten in syphilitischen lirankheitsproducten. Arb. kais. Gesundhtsa. xxii. p. 527, 1905. 


\section{APPENDIX A. ${ }^{1}$}

\section{CHLAMYDOMYXA.}

THIs genus is represented by two species. C. labyrinthuloides was discovered by Archer in pools in moorland country in Ireland and described by him in 1875 (1). It has subsequently been investigated by Geddes (2) in material supplied by Archer; and by Hieronymus (3), who found it in the Riesengebirge and elsewhere in Germany. C.montana was first described by Lankester (5) and obtained by him in Sphagnum swamps in Switzerland, and has since been investigated by Penard (6).

Two main phases of the life-history are in many respects well known-a free active stage, with pseudopodia more or less extended, and a (much commoner) encysted stage; and we now have evidence, though it is still incomplete, of stages of multiplication by fission and of sporeformation.

Chlamydomyxa unites in a remarkable manner the holophytic and holozoic nodes of nutrition. The protoplasmic body is crowded with chromatophores, by means of which it is able to increase largely in size in the encysted state; but it is also able, in its active phase, to engulf and to digest animal and vegetable organisms.

The body consists of hyaline protoplasm containing nuclei, chromatophores, and small refracting bodies - the "oat-shaped corpuscles" of Lankester. In the encysted condition it may form a globular inass, measuring, when fully grown, 60-90 $\mu$ in diameter in C. labyrinthuloides, the cysts of $C$. montrna being a little smaller.

The nuclei (Fig. 1, $a, b$, and $d$ ) vary from 1.5 to $3 \mu$ in diameter. They are generally evenly distributed through the protoplasm, and they increase in number with its growth. In the large cysts of $C$. labyrinthuloides there may be as many as 32 or more ; in C. montana, according to Penard, 100 or more. They contain a nucleolus or group of nucleoli at the centre, and there are indications of a nuclear reticulum at the periphery. Their mode of division is, according to Hieronymus, intermediate between mitosis and amitosis. In life they are usually hidden by the chromatophores, and thus escaped the notice of the earlier observers.

The chromatophores are oval bodies varying in size up to $3 \mu(C$. mon$\tan a)$ and $5.5 \mu$ (C. labyrinthuloides, Fig. 1, d). They consist of coloured

1 By J. J. Lister, M.A., F.R.S., Fellow of St. John's College, Cambridge. 
and colourless tracts, which are apparently differently distributed in the two species. The colour varies from grass-green to olive-green, yellow, and brown, and is dependent on the presence, in varying proportions, of chlorophyll and of a yellow-brown colouring matter (? diatonin). They
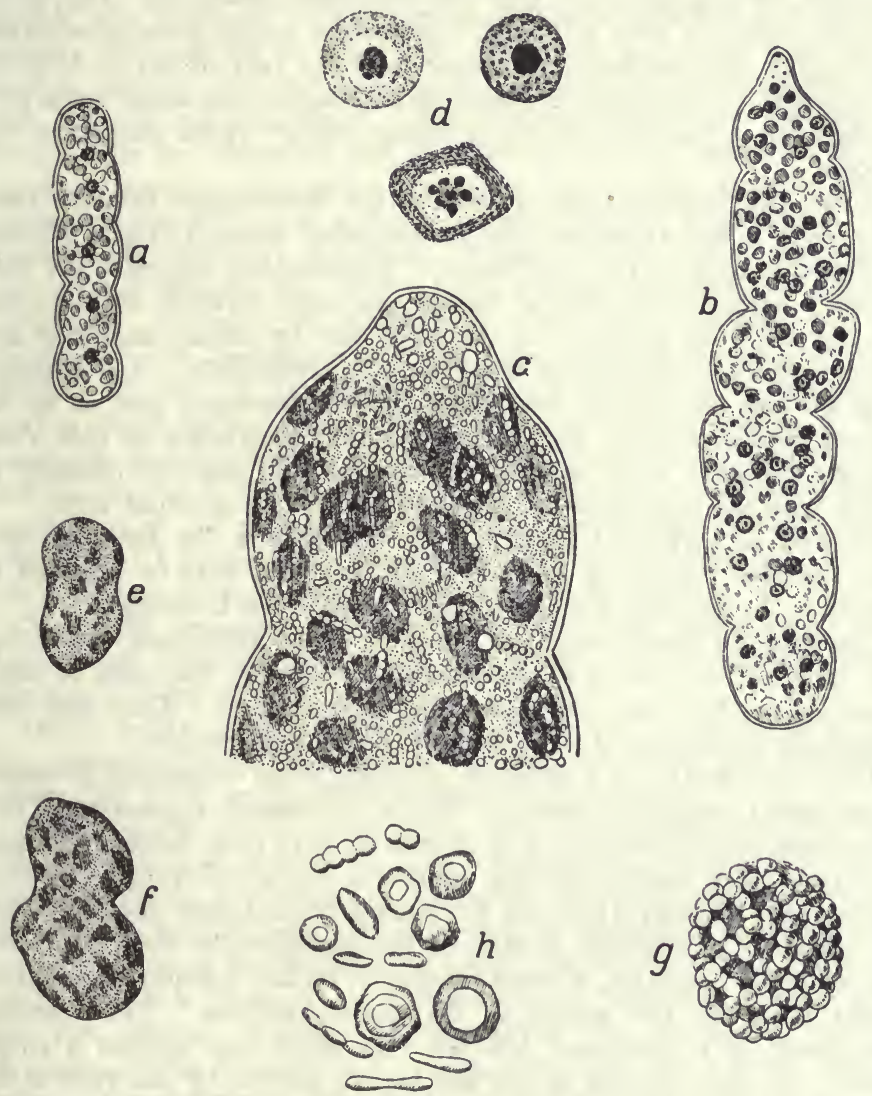

Fia. 1.

Chlamydonnyxa labyrinthuloides. $a$ and $b$, cysts from leaf-cells of Sphagnum, constricted by the characteristic annular bands of the latter, from stained preparations showing the chromato. phores and nuclei, $\times 620$. c, end of a living cyst, treated with weak methylene blue solution. The chromatophores are shaded. The nuclei are not seen. $\times 5000$. $d$, nuclei highly magnified; $e, f$, living chromatophores; $g$, chromatophore after treatment with Flemming's fluid and fuchsin; $h$, oat-shaped corpuscles; $e-h \times$ about 10,000. (After Hieronymus.)

appear to multiply by binary fission (Fig. $1, f)$. The absence of a cellulose envelope and of a nucleus, as well as other characters of the chromatophores, prevent their being regarded as symbiotic algae. As a degeneration product, and especially under the influence of bright sunlight, the colouring matter breaks down, producing a red or brown fatty substance (lipochrome) which accumulates in drops in the interior of the cysts, and, 
by its colour, reveals the presence of Chlamydomyxa when it is present in abundance on the vegetation of a pool.

The oat-shaped corpuscles ("spindles" of Archer, "plyssodes" of Hieronymus) are shining, highly-refracting bodies, homogeneous or faintly laminated, of a pale bluish tint and semifluid consistence (Fig. 1, h). They are round or oval in shape, but become longer (oat-shaped) when drawn out on the pseudopodial filaments. They vary in size up to about $2 \mu$ in length. As regards composition, Hieronymus identifies them with phloroglucin, a member of the aromatic series which occurs in the Fucaceae.

When Chlamydomyxa was discovered the resemblance between these bodies, held in the expanded, stiff pseudopodial network (Fig. $3(2)$ ) and the nucleated units of the associations of Labyrinthula, suggested the view that they might be of similar nature, although nothing of a nuclear character could be revealed in the corpuscles by stains, and they are, moreover, much smaller than the units of Labyrinthula. The evidence which we now have as to the nuclei of Chlamydomyxa, and as to the chemical nature of these bodies, prevents our acceptance of this view. They are probably to be regarcled as reserve food nuaterial (possibly in relation with the metabolism of cellulose) stored in a granular form.

Crystals of oxalate of lime, furmed doubtless in the katabolic processes, are also present in the cell-fluids, and they may be crowded in vacuoles of the encysted animal, to be expelled when it emerges.

The cysts of Chlamydomyxa are found in great abundance within the large cells of the leaves of Sphagnum, or between the cells of other aquatic plants (Hypmum, Eriocaulon, cotton-grass, etc.). They may also be found on the surface of these and other submerged bodies.

They are invested by a cellulose envelope, often consisting of several laminae added one within another, and the investment appears to be of is plastic consistency, expanding with growth so as to cover large protrusions of the cyst which extend through apertures in the cell-wall, and it may close in about portions which are withdrawn from deeper recesses of the plant tissue. Considerable growth of the protoplasmic body may occur in the encysted condition, a result dependent on the holophytic nutrition brought about by the agency of the chromatophores. The youngest cysts found in a Sphagnum leaf are very small and contain a single nucleus. As they increase in size and become limited hy the walls of the elongated leaf-cells they grow in length (Fig. 1, $a$ and $b$ ). The cysts may finally break through the wall of the cell and project in lobate prominences to the exterior. The activities of the encysted organism do not, however, result in uniform growth, for many cysts have shrunken contents, and have formed a fresh wall separate from the original one, and in the space between the envelopes gronps of the red oil-globules referred to above may lie, discharged before the inner wall was secreted. Moreover, the contents of a cyst may undergo division within the envelope into two or more parts, and each part then forms a wall of its own.

When the cysts are fully grown and favourable conditions occur, an aperture is formed in the envelope, presumably by the solvent action of the protoplasm on the cellulose, and the contents emerge in the free state. 
The accounts of the behaviour of the organism in the free state differ considcrably and are not easy to reconcile.

In C. labyrinthuloides, as described by Archer (cp. his figure in the Q.J.M.S. vol. xv. Plate vi., from which Fig. 3 (2) is taken), the protoplasmic body was still partially contained in the cyst. Extending throngh the aperture, it was produced into a dendriform system of branches, diminishing in thickness. From the ends and sides of the branches filiform hyaline pseudopodia of small but uniform thickness reach far out into the water. The chromatophores are not seen in relation with the filaments, but these are plentifully beset with the oatshaped corpuscles. The latter are drawn out in the direction of the filament, and slowly travel along it in one direction or the other. The filaments are sparingly branched; whether or not they anastomose, observers are not agreed. They have a "stiff but flexille" (Penard) consistency. Lankester is inclined to regard the filaments as "inert products of the metamorphosis" of the protoplasm, over which a "delicate varnish" of hyaloplasm extends, investing the corpuscles and carrying them along in its flow. Yet the whole system of these remarkalle pseudopodia can be rapidly withdrawn into the general mass when the animal is disturbed. Hieronymus describes a peculiar fibrous arrangement of the protoplasm even in the encysted state, which may be in relation with the peculiar characters of the extended filaments (Fig. 1, c. Note the linear arrangement of the oat-shaped corpuscles).

Contractile vacuoles abound in the extended protoplasmic body. Their period probably varies with its activity. In C. montana Penard finds it to be very slow.

In the active condition Chlamydomyxa is able to engulf and digest algae, desmids, Peridinidae, etc., and outlying masses of protoplasm may be seen (Fig. 3 (2)) accumulated about such food-bodies.

The accounts of the active phase of $C$. montana agree, on the whole, with Archer's observations of C. labyrinthuloides, except that in the former species the protoplasm, on emerging, completely quits the old cystwall and lies free in the water as a mass of constantly changing shape. It may be more or less splerical or drawn out into a ribbon, attaining a length of $300 \mu$ (Penarl). A definite hyaline ectoplasm is also present. (Cp. the figures of this species given by Lankester, Q.J.M.S. vol. xxxix. Plates xiv. and xv.) In it, moreover, the yellow colouring matter of the chromatophores usually predominates over the green.

According to nost observers, the free state of the organism would appear to encl, after lasting at least "several hours," by the withdrawal of the extended protoplasm and the re-encystment of the whole animal. Hieronymus differs considerably from other observers in his account of the free state. He lias also seen the contents emerge from a cyst of C. labyrinthuloides, assume an irregular amoeboid form, and ingest food "auf thierische Weise"; but it is remarkable tliat he has never, during the twelve years over which his observations have extended, seen the long filamentary pseudopodia protruled in the manner which has, in both species, attracted attention. The nearest approach to such filaments which he has seen were those of a small specimen suspended free in the 
water and emitting long pseudopodia on all sides (3; Plate ii. Fig. 25). After ingesting food the animals were found by Hieronymins to encyst on the surface of plants, and he statcs that division of the nuclei follows the encystment. But in the majority of cases a different process was observed to follow the emergence from the encysted state. The proto.plasm puts out short pseudopodia and divides up forthwith, by successive bipartition or by simultaneous division, into sumall uninucleate amoebae, the products of division being equal in number to the nuclei contained in the original cyst. The division into the ultimate products is usually complete in a few minutes from the emergence of the protoplasm. The small amoebae so found may creep about and ingest small algae or bacteria before passing into the encysted form. While this is the usual course, Hieronymus describes cases in which the process of division ceased after one or two partitions hal occurred, and was followed by a stage of feeding and subsequent encystment. Further evidence of such cases would be desirable, and it seems possible that two separate phases of the life-history may liave been here confused; but it is clear that the fission of the multinucleate body into uninucleate products represents a phase of reproduction comparable with that which occurs in many other protozoan life-histories, and of which we had no previous evidence in Chlamydomyxa.

Spore-Formation.-The process of spore-formation has been most fully observed by Penard in $C$. montana, ${ }^{1}$ but stages of it have been seen by Archer and Hieronymus in C. labyrinthuloides. The contents of an encysted form are segregated by simultaneous fission into a number (20 to 40 ) of equal (Fig. 2, a) (? sometimes only sub-equal (Fig. 2, b)) divisions. These are at first continuous with their neighbours by protoplasmic strands (3; Plate i. Fig. 7), but later they separate into bodies which become spherical and each secretes a cellulose wall. They are liberated by the opening of the cyst (in a manner not observed). Penard finds that these secondary cysts, or spores (Fig. 2, c), as we may call them, measure in $C$. montana $18 \mu$ in diameter, and that each contains two nuclei lying opposite one another in a meridian of the sphere. ${ }^{2}$ In some cases the contents of the spores were found to have emerged as naked masses of protoplasm, containing the chromatophores and refracting corpuscles characteristic of the species. Each acquired a flagellum (or two flagella?) about equal to the body in length (Fig. 2, d), and for some moments ("pour quelques instants") was actively motile. Some of these flagellate bodies appeared to possess one nucleus, others two or even three, and there was an indication of their fusion in pairs ("lorsque les petits flagellates viennent à se rencontrer, ils peuvent se fusionner en un seul," p. 331). Some continued to show a slow movement for twentyfour hours, but ultimately they died under the cover-slip.

It would be premature at present to make any dogmatic statement as

1 It was only for a few days that Penard succeeded in observing this stage in the life-history. It occurred in March, in the neighbourhood of Geneva.

2 Penard's account of the subsequent history of these boclies is of great interest, but, owing to the sparseness of his material and the rapidity of some of the events, he was unfortunately not able to observe the stages with pecision. With this reserve, an outline of his results is here given. 
to the course of the life-history of Chlamydomyxa. The observations of Penard suggest that the flagellulate bodies hatching from the spores are gametes, which proceed to conjugate with one another, though the existence of two nuclei in the spones requires explanation. If this is the case, we have, as in Trichosphaerium and many other Protozoa, a life-cycle in which a sexual phase recurs in a series of generations reproducing by fission.

With regard to the affinities of Chlamydomyxa, we have seen that the resemblance to Labyrinthula turns out to be in part at least misleading.
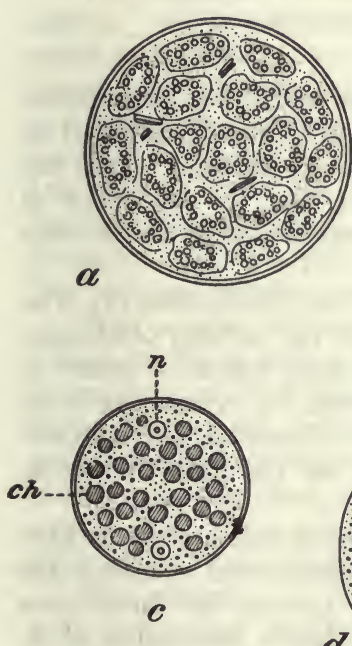

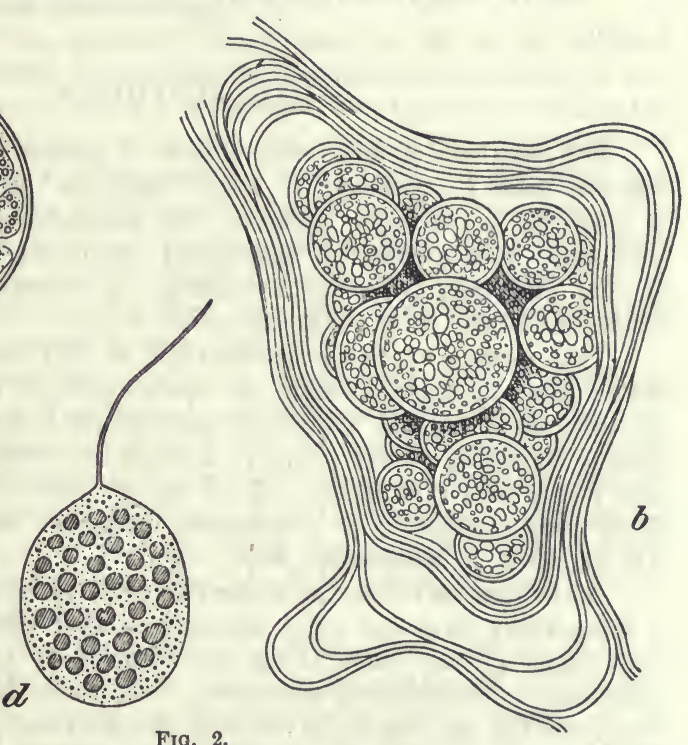

Fia. 2.

Chlamylomyxa. $a$, Early stage of spore-formation in $C$. montano. The contents of a cyst have become divided up into young spores; $b$, a cyst of $C$. labyrinthuloides, with mature spores, $\times 200 ; c$, a single spore of $C$. montana, showing two nuclei; l, flagellate body hatched from a spore. ( $a, c$, and $d$ after Penard ; $b$ after Archer.)

IVe are unable to agree with Penard that it is allied to the Mycetozoa, for there is no evidence that the protoplasnic masses are plasmodia in the true sense of the term. It appears that the most satisfactory position to assign to it, in the present preliminary stage of our knowledge of lifehistories, is as an isolated rhizopod, containing chromatophores, which may be provisionally placed in the neighbourhood of the freshwater forms with filose pseudopodia which, in this work, are included in the Order Gromiidea of the Foraminifera (see p. 283). In the possession of many nuclei it resembles Trichosphaerium among the Rhizopoda Lobosa.

\section{LiteratURE.}

1. Archer, $W$. On Chlamydomyxa labyrinthuloides, nov. gen. et sp., a New Freshwater Sarcodic Organism. Quart. Journ. Micr. Sci. N.S. xv. (1875), p. 107. 
2. Gideles, 1. Obscrvations on the Resting State of Chlanydomyxe lahyrinthuloilles, Archer. Ibicl. xxii. (1882), p. 30.

3. Hieronynus, G. Zur lienntniss von Chlamydomyxa labyrinth̄uloides, Archer. Hedwigia, Bd. xxxvii. (1898), p. 1.

4. Jenkinson, $J . W$. Abstract and Review of the above paper by Hieronymus. Quart. Journ. Micr. Sci. N.S. xlii. (1899), p. 89.

5. Lanlicstcr, E. Ray. Chlamydomyxa montana, n. sp., one of the Protozoa Gymnomyxa. Quart. Journ. Micr. Sci. xxxix. (1896), p. 233.

6.-Pencirl, E. Etude sur la Chlamydomyxa montana. Arch. f. Protistenkunde, Br. iv. Heft 2 (1904), p. 296.

\section{LABYRINTHULA.}

The members of this genus consist of associations of nucleated protoplasmic units ("amoebae" of Zopf, "spindles" of Cienkowski) joined in a network of sparingly branched and anastomosing threacls. They are met with in a diffuse or aggregated condition, and, as the result of drying, the units pass into a condition of encystment, from which they hatch out in the furm of the amochoid units.

Two marine species were described in 1867 by Cienkowski (1), who found them on algae growing on wooden piles in the harbour of Odessa : L. vitellinu, Cienk., in which the protoplasmic units contain a yellow or orange colouring matter; and L. macrocystis, Cienk., in which the units are larger and colomless. Zopf (4) in 1892 described a freshwater form very similar to $L$. macrocystis, parasitic on the alga Vaucheria. He named it $L$. cienkowskii, Zopf.

In the marine forms the system of connecting threads appears to have a remarkably firm and rigid consistency, and Cienkowski describes the movement of the units along the threads, as though the latter were peculiarly differentiated structures; but from Zopf's description of $L$. cienkmoshii it can hardly be doubted that they are pseudopodial in nature. Zopf observed them to be slowly protruded from a mass of units, and to be withdrawn, to move slowly from side to side, and to fuse with their neighbours. He also describes the passage of fool-granules along them.

The units are without a limiting membrane and contain a single nucleus, with a nucleolus. When drawn out in the expanded condition of the organisin they are generally spindle-shaped (Fig. 3 (3)), but they may present processes in three directions (Fig. 3 (4)). In the aggregated condition the units are round or oval. Those of $L$. macrocystis measure 18-25 $\mu$ in long diameter, those of $L$. vitellina and $L$. cienkouskii about $12 \mu$. The protoplasm is granular, and in L. vitellina contains a yellow or or:mge fatty pigment, soluble in alcohol. A small vacuole is usually present, but it is not stated that it is contractile.

The whole organism, or a part of it, is often found in the aggregated condition (Fig. $3(5)$ ), and the marine species may thus form masses measuring a millimetre or so in diameter. The main aggregate is described by Cienkowski as invested, in L. vitellina, by a "cortical substance" (neither protoplasmic nor of the nature of cellulose) through 
which the filaments are protruded, but this was not seen in the peripheral aggregates of this species, nor at all in the active condition of the other species.

Labyrinthula is actively parasitic on the algae which it infests, breaking down the contents of the cells into a granular mass.

As the result of drying, the organism passes into a condition of encystment. The units became closely aggregated and each secretes a cystwall, which is double in $L$. cienkowskii. A firm common envelope may now be formed (in L. macrocystis, Fig. 3 (5), but not in other species) in which the encysted units are embedded.

The behaviour of the encysted unit appears to vary in the different species. In $L$. cienkowskii Zopf describes and figures the emergence of a single mass from the cyst. In the other species, Cienkowski found that the contents divided into four within the cyst (Fig. $3(6$ and 7$))$. Zopf observed the protrusion of one or two long pointed pseudopodia, on hatching, and the final emergence of the protoplasmic mass from the cyst, which was left empty. From the fact that on one occasion three enipty cases were found with three units in their neighbourhood, and that these were in comnection by their pseudopodia, Zopf concludes that the hatched units join with one another to start a fresh association.

Zopf regards the association of units of Labyrinthula as representing a stage in the formation of a plasmodium intermediate between the true plasmodium of the Euplasmodida (cf. p. 43), in which there is a complete fusion between the protoplasmic bodies of the uniting amoebulae, and the pseudoplasmodium of the Sorophora, in which the amoebulae, aggregating before spore-formation, come into apposition but maintain their distinctness (p. 60). This intermediate form he would distinguish as a Threadplasmodium (Fadenplasmodium).

The propriety of this view seems far from clear. We are familiar with many cases among Protozoa in which an association of individuals, a colonial organism, is formed by the successive multiplication of the units, whose offspring remain in connection by protoplasmic processes (Colonial Radiolaria, Volvox, Mikrogromia), and the higher animals and plants are often regarded as such colonial organisms, in modified forms.

That an increase in the number of units in the associations of Labyrinthula occurs by binary fission of the units is abundantly clear. It is true that it appears probable, from Zopf's observation above quoted, that a fusion may occur in Labyrinthula (thongh it was not actually observed) between the pseudopodia of individuals recently emerged from the encysted state; but a parallel to this process may be found in the fusion of the protoplasmic masses emerging from the cysts of the sclerotial condition of the Mycetozoa on revival of activity (cp. p. 50). There are fair grounds for regarding the fusion of the amoebulae by which the Mycetozoan plasmodium takes its origin (in the Euplasmodida) as a part, at any rate the plastogamic part, of a sexual union of which the final, karyogamic, stage is deferred. It would not be suggested that the fusion after the sclerotial stage is a repetition of this process in the Mycetozoa, and we may well hesitate, in the present 


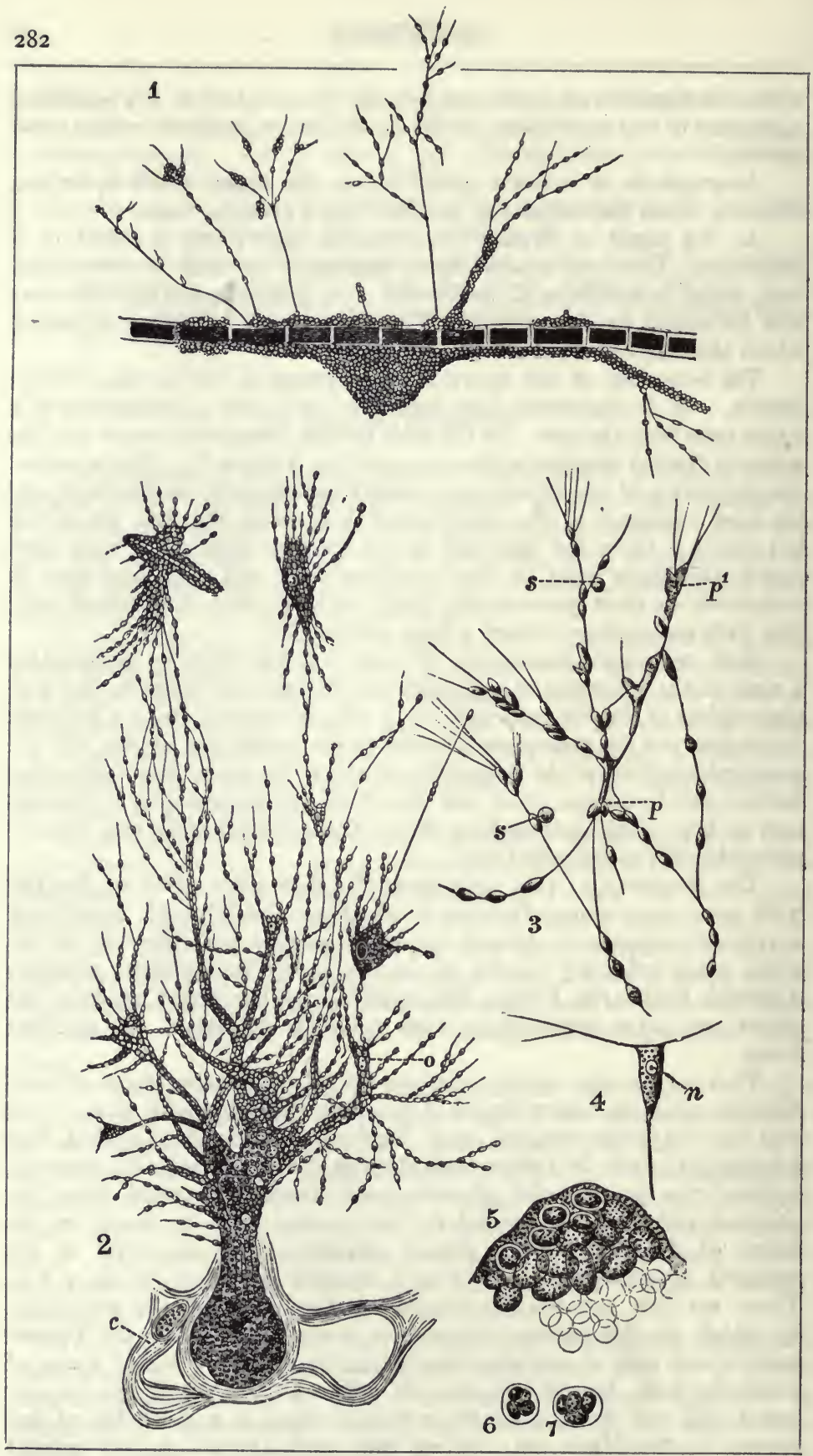

Fig. 3. 
fragmentary state of our knowledge of Labyrinthula, to accept the conclusion that the (inferred) fusion between the pseudopodia after encystment represents this important event in its life-history.

We are therefore inclined to regard Labyrinthula as a colonial organism of which the units remain in connection by their pseudopodia. As the result of drying they may pass into the encysted stage, in which they are isolated from their fellows by the cyst-walls. It appears probable, from Zopf's observation, that, on resuming activity, they may again unite with their fellows to form a colony. Other stages of the life-history are at present unknown to us.

With Labyrinthula Zopf associates the genus Diplophrys (Archer), Cienk. The species named Diplophrys stercorea by Cienkowski (2) is a colonial organism, with simple thread-like pseudopodia, living on horsedung. It can hardly belong to the same genus as Diplophrys Archeri (Barker), with ramifying pseudopodia and a distinct though membranous

\section{Fio. 3.}

2. Chlamyilomyxa labyrinthuloides, Archer. The animal in the free state partially emerged from the many-layered cyst. A small encysted inass is seen at $c$ between the envelopes of the latter. At 0 and elsewhere in the main body of the protolulasm, as well as in outlying portions, ingested fool particles are shown. The oat-shaped corpuscles are seen on the stiff extended filanents. $\times$ about 150. (From Lankester, after Archer.) 1 and 3, Iabyrinthula vitellina, Cienk. 1, a colony crawling upon an alga. The units are partly aggregated, partly extended ont the network of stiff' extended pseudopodia. $\times$ about 120. 3, part of the network, $\times$ about 250. At $p$ and $p^{1}$ several units have fused into a common mass; $s, s$, units which have assumed the spherical shape and are sfationary. 4-7, Labyrinthula macroeystis, Cienk. 4, a single unit giving out three pseudopodia; $n$, its nucleus; $\times 320$. 5, a group of encysterl units invested in a tough secretion, $\times$ about $250 ; 6$ and 7 , encysted units the contents of which have divided into four, $x$ about 320. (From Lankester, after Cienkowski.)

test. Both forms, together with Labyrinthula and Chlamydomyxa, may provisionally be regarded as related in one direction to outlying members of the Gromiidea, here included in the Foraminifera, and in others to the Heliozon and the Proteomyxa. The grounds for regarding the two latter genera as especially related have vanished in the light of fuller knowledge.

\section{LITERATURE.}

1. Cienkowski. Ueber den Bau u. Entwickelung der Labyrinthuleen. Areh. f. mikr. Anat. Bd. iii. (1867), p. 274.

2. - Ueber einige Rhizopoden und verwandten Organismen. Ibid. Bd. xii. (1876), p. 44.

3. Lankester, E. R. Article "Protozoa" (Class Labyrinthulidae). Encyclopaedia Britannica, 1891.

4. Zopf, W. Zur Kenntniss d. Labyrinthuleen, eine Fam. d. Mycetozoen. Beitr. zur Phys. u. Morphologie niederer Organismen, Heft 2 (1892), p. 36, and Heft 4 (1894), p. 60 . Leipzig. 


\section{APPENDIX B.}

\section{THE XENOPHYOPHORIDAE, F. E. Schultze.}

Tне organisms that are now included in this family were formerly regarded as Porifera, and several of them were described in 1889 by Haeckel in the "Challenger" volume xxiii., on the deep-sea Keratosa. ${ }^{1}$ In the year 1892, Goös (1) described "a peculiar arenaceous Foraminifer from the American tropical Pacific" as Neusina ayassizii, which Hanitsch in the following year proved to be identical with Haeckel's deep-sea Keratose sponge Stannophyllum zonarium. We are inclebted to Schultze (2) for an exhaustive treatise on these genera, and the more definite proof that they are not sponges, but probably related to the Foraminifera. They are spherical or disc-shaped (Psammetta), fan-shaped (Stannophyllum, Fig. 1), or dendritic (Stannoma) bodies of about $20 \mathrm{~mm}$., more or less, in

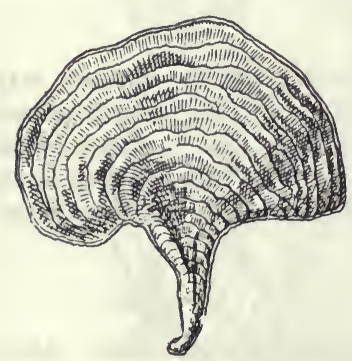

Fig. 1.

Stunnophyllım zonarium, Haeck. $\times$ 3.. (After Schultze.) diameter or height, and of a fibrous, spongy texture. They have been found at depths of from 550 fithoms to 3000 fatloms in the Indian, Atlantic, and Pacific Oceans.

They consist of a plexus of thin-walled tubes, some of wlich open on the surface, and the meshes of the plexus contain a large number of foreign bodies (xenophya), such as the shells of Radiolaria, Foraminifera, spicules of sponges, and grains of sand.

The tubes contain either a large number of dark olive-brown bodies, the sterkomata, or else a multinucleated plasmodium containing numerous clear solid bodies called the granellae. The sterkomata are remarkably resistant to strong acids and alkalis, and they often contain fragments of radiolarian and foraminiferan shells. They are regarded by Schultze as of the nature of the frecal balls such as are found in other Foraminifera (Gromia, Saccamina, etc.). The tubes containing the sterkomata (Sterkomarium) are probably continuous with the tubes containing the granellae (granellarium). The granellae are about 1-2 $\mu$ in diameter, and are mainly composed of barium sulphate. The nuclei which occur in the plasmodium of the granellarium are very numerous, and usually scattered 
irregularly among the granellae. In some cases (Fig. 3) aggregations of nuclei with an investing portion of the protoplasm become separated from

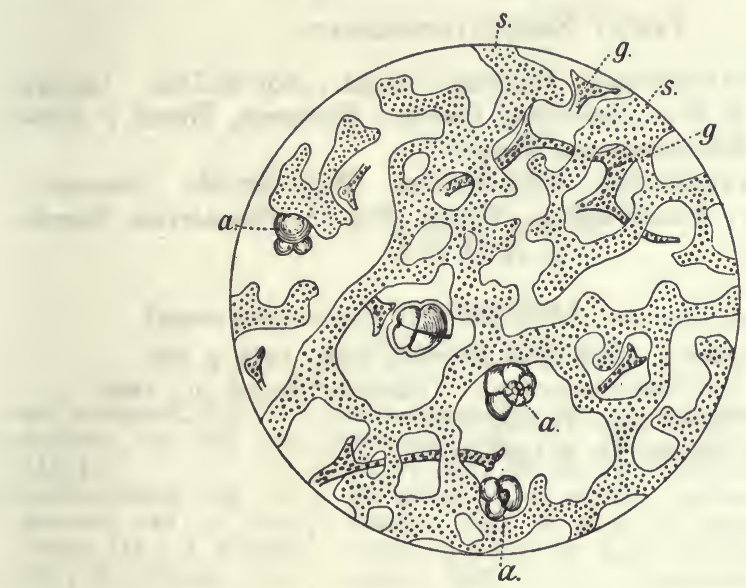

FIg. 2.

Section through the middle layer of Psammina globigerina, Haeckel, showing the plexus of tubes containing a multinucleated plasmodium. At $a, a$ are seen some of the foreign bodies (xenophya) associated with the organism; $s, s$, tubes of the sterkoniarium; $g, g$, tubes of the granellarium. (After Schultze.)

the plasmodium, and these break up into swarnı-spores, which Schultze regards as possibly gametes.

In the family Stannomidae there are found, in addition to the tubes. already mentioned, many fine skeletal fibres called the "linellae," which form a plexus in the interstices of the other parts of the organisms.

In the absence of any information concerning the early stages of development, or of the character of the pseudopodia in the nembers of this family, it is difficult to assign to them their proper systematic position. The Foraminiferan genus Polytrema and some of its allies have the same habit of incorporating into their substances sponge spicules and other foreign bodies, and they also lose at an early stage of development the external evidence of the chambered condition, and assume dendritic forms. Moreover, in Polytrema we find, in addition to the calcareous skeleton, a system of

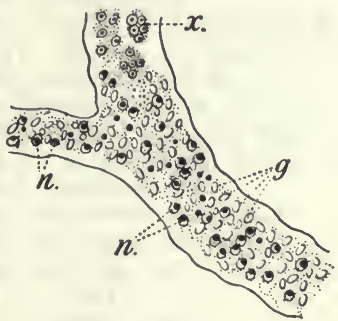

Fig. 3.

Diagram of the granellar region of a Xenophyophorid, showing the nuclei, $n, n$, and granellae, $g, g$, of barium sulphate. At $x$ clusters of nuclei and associated protoplasnı are breaking up into spores. horny or chitinous tubes which have some resemblance to the tubes of the Xenophyophoridae. In the absence of a calcareous skeleton the family differs from all the higher and more differentiated fimilies of Foraminifera, but nerertheless the affinities of the family are greater with this class than with any other Protozoa. 
The Xenophyophoridae may therefore provisionally be placed in the Class Foraminifera.

\section{FaMiLY XeNophyophoridae.}

Sub-Family Psamminidar. Without linellae. Not flexible. Genera -Psammetta, F. E. S. ; Psammina, Haeck.; Cerelasma, Haeck. ; Holopsamma, Carter; Psammopemma, Marshall.

Sub-Family Stannomida. With linellae. Body flexible. GeneraStannoma, Haeck. ; Stannophyllum, Haeck. (Fig. 1); Stannarium, Haeck.

\section{Literature.}

1. Goës. Neusina agassizi. Bull. Mus. Harvard, xxiii., 1892, p. 195.

2. Schultze, F. E. Die Xenophyophoren. "Valdivia" Exped. xi., 1905.

3. —_ "Siboga" Exped. Mon. iv. bis, 1906.

4. - Bull. Mus. Harvard, li. 6, 1907. 


\section{N DEX}

Figures given in thick type refer to the systematic position.

$f$. refers to an illustration.

Abyla pentagona, 249

Acantharia, 94, 102, 106, 113,145

Acanthochiasma, $146 ; A$. cruciata, 146 ; A. fusiforme, $145 ; A$. krohnii, 145 ; A. rubescens, 143

Acanthochiasmidae, 146

A canthocystis, 21, 24, 28, $29,34,119$; A. aculeata, $16 f ., 27 f$., $28 f$. ; $A$. italica, 34 ; A. marina, 34 ; $A$. simplex, 34 ; $A$. spinifera, 23 ; $A$. turfacea, 23

Acanthodinium, 187

A canthometra sicula, 143

Acanthometrida, 113, 145

Acanthometron, 123, 146 ; A. bifidum, $137 f . ; A$. Claparedei,105f.; A. pellucidum, $118 f$., 146

Acanthonia, 146 ; A. ligurina, 146 ; $A$. mülleri, 146 ; A. tetracopa, 127, $128 f ., 132 f$.

Acanthonidium, 146 ; $A$. echinoides, 146 ; A. pal. lidum, 146

Acanthoniidae, 146

Acanthophractida, 146

Acrasiae, 39

Acrasis, 65

Actineliida, 145

Actinelius, 145 ; A. pur. pureus, 145

Actinolophus, 15, 23, 28, 33

Actinomma, 127 ; A. aster. acanthion, $103 f$.

Actinomonas, 165

Actinophrys, 18, 19, 21, $29,30,33,39 ; A$. sol, $15,16 f ., 21 f$.
Actinosphaeridium, 33 $23,25,26 f$. $29,30,32$, $33,48,86,87$; A. arach noideum, 23 ; A. Eich. horni, $14 f_{\text {., }} 22 ; A$. im. patiens, $10 f$.

Actissa, 104, 110, 144

Aethalia, $56^{\circ}$

akaryote, 1, 2

Alwisia, 63

Amaurochaetaceae, 63

Amaurochacte, 63 ; A. atra, 41

Amaurochaetineae, 62

Amaurosporales, 61

Amoeba, 2, 69, 77; $A$. binucleata, 73, 79; $A$. buccalis, 84 ; A. crystalligera, 73,$78 ; A$. dofleini, 71; A. fluida, 78; A. guttula, $78 f$. 79 ; A. hyalina, 73 ; $A$. kartulisi, 84 ; $A$. limax, 69, $70,73,77 f ., 79,83 ; A$. pilosa, 68 ; A. proteus, 73, 74f., 77, 78f., 79 ; A. rudiosa, 79 ; A. rotatoria, 195 ; A. terricola, 68 ; A. urogenitalis, 84 ; $A$. verrucosa, $78 f$; $A$. villosa, 79 ; $A$. vorax, $78 f$.

Amoeba (marine forms), 78 A moebophrya, $105 f$. 123

Amoebulae in Heliozoa, 29 ; in Lobosa, 76, 77; in Mycetozoa, 42, 59; in Proteomyxa, 4, 5

Amphidinium, 183

Amphilonche, 123, 146 ; $A$. atlantica, $137 f, ; A$. belonoides, 146 ; A. mes. sanensis, $103 f$.
Amphilonchidae, 146

Amphimonas, 168

Amphisolenia, 187 ; $A$. globifera, $184 f$.

Amphizonella, 80

Ancyromonas, 165

Anemineae, 55, 63

Anisonema, 171

Ankistrodesmus, 179

Anopheles, 240 ; A. maculipennis, 248

Anthophysa, 158, 161, 167 ; A. vegetans, $178 \mathrm{f}$.

Aphelidium, 3, 10, 11 ; A. lacerans, 11

Aphrothoraca, 33

A piocystis, 180

Apstein, 192

Arachnula, 9

Arcella, 68, 71, 72, $85 f ., 86$ 87,$90 ; A$. vulgaris, $90 f$.

Arcellidae, 85, 90

Archer, 22, 274, 277, 279

Arcyria, 52 n., 55, 64, 65 ; $A$. incarnata, $56 f$.; $A$. punicea, $56 f$.

Arcyriaceae, 44, 55, 64

Ascoglena, 157, 171

Astasia, 171 ; $A$. tenax, $166 f$.

Astasiina, 171

Astrocapsa, 146 ; $A$. coronata, 146; A.tritonis, 146

Astrodisculus, 33 ; $A$. radians, $16 f$.

Astrolophidae, 145

Astrolophus, 145

A strosestrum acanthastrum, 145

Athene noctua, 202, 233

Athias, 254, 269

Atlanticella, 109, 150;A.

craspedota, 149

Atractonema, 171 
A ulacantha, 113, 119, 121 ; | Brass, 13

A. scolymantha, $111 f$., $112,117,121,124$, $125 f, 136,147$

Anlacanthidae, 147

Aulactinium actinastrum, $109 \mathrm{f}$.

A ulolendron boreale, 148

A ulographisfurcellata, 148;

A. tetrancistra, $148 ; A$.

żetesios, 148

A ulokleptcs flosculus, 110, $135 f$.

A uloscena verticillatus, 112 $f ., 148$

Aulosphacra elegantissima,

$105 \mathrm{f} . ;$ A. Hexwosa, 148

A ulotractus fusulus, 148

A uricularic, 49

Awerinzew, 35, 91

Badhamia, 52 n., 55, 62 ; B. panicea, $42 f$., $52 f$.;

B. utricularis, $44 f_{\text {. }}, 46$

$f ., 48,49,50 f$., $51 f$.

Barbagallo, B., 92

de Bary, 38, 61, 66

Basidiomycetes, 40

Bathybius, 12

Belonaspidae, 146

Beneden, E. van, 2, 13

Berg, 195

Bicosoece, 161, 168 ; socialis, 158, 168

Bikoecina, 168

Billet, 226, 269

Biomyica, 2, 3, 9; B. cometa, 9 ; B. reuguns, 9

Bionomics in Thalassicolla, 96

Blackman, 180, 192

Blepharisma, 22

Blepharocysta, 186, 187

Boderia, 10 ; B. tarneri, $11 \mathrm{f}$.

Bollo, 157, 161, 162, 163, 167,246 ; B. caudatus, $166 \mathrm{f}$. ; B. lacertne, 211, 247 ; B. lens, $178 f$.

Boclonina, 167

Borgert, 110, 119, 124, 152

Botryocampe inflata, 147

Botryococcus, 179

Botryoidea, 147

Bott, 91

Bourne, 81, 91

Box boops, 247

Bradford, 206 n., 269

Brandt, 96, 97, 104, 106, $110,112,127,129,152$
Brauer, 31, 32, 35, 245

Braun, 13

Brefelelia, 63

Breinl, $206 n$.

Bruce, 195, 199, 269

Brumpt, 196, 198, 204, 226, 228, 269

de Bruyne, 4, 13

Buffard, 205, 269

Bursulla, 3, 11, 12, 40, 60

Biitschli, 5, 6 n., 13, 35, $39,66,70,91,115,131$, 151,190

\section{Cadium melo, 148}

Calcarineae, 49, 55, 62

Calkins, G. N., 13, 20, 79, 91, 219

Calonemineae, 64

Calymma, 95

Calyptrosphaera, $176 ; C$. oblonga, $175 \mathrm{f}$.

Campascus, 90

Camptonema, 33

Cannocapsa, 146 ; C. osculata, 146

Cannosphaera antarctica, 148

capillitium, 52,55

Carpocanium diadema, 105 f., 147

Carteria, 178, 180

Casagramili, Q., 92

Cash, J., 13, 78

Castanelliclae, 150

Castanidium apsteini, 150

Castellani, A., 92, 196, 269

Caulerpa, 12

Central capsule in Radiolaria, 114

Centralkorn, 28

Centrochlamys, 80

Centropyxis, 71, 75, 76, 77, $86,87,88,89$

Cephalothamnion, 161, 167

Ceratiidae, 187

Ceratiomyxa, 40, 57, 59, 64,66 ; C. mucida, $58 f$.

Ceratiomyxaceae, 64

Cerativm, 158, 186, 187 ; C. hirundinella, 183 n. ; C. tripos, $186 f$.

Ceratocorys horrida, $184 f$., 187

Cercobodo, 165 ; C. crassicanda, $166 \mathrm{f}$.

Cercomonces, 165

Cerelasma, 286

Certes, 273
C'haetomorpha crassa, 10

Chalarothoraca, 23, 34

Challengerillae, 113, 148

Challengeron armatum, 148 $f$. ; C. balfouri, 148 ; C'. golfense, 148 ; C. johannis, 148 ; C. trioden, 148

Chilomonas, 176

Chironomus plumosus, 245

Chlamydococeus, 180

Chlamydomonalina, 180

Chlamydomonas, 22, 180 ;

C. pulvisculus, $166 \mathrm{f}$.

Chlamyclomyxa, 39, 274 ;

C. labyrinthuloides, 274 , $275 f_{\text {., }} 279 f_{\text {. }}, 282 f_{\text {. }}$; C. montuna, 274, 277, $278,279 \mathrm{f}$.

Chlamydophora, 33

Chlorodlesmus, 158

Chlorogonium, 180 ; $C$. euchlorum, $166 \mathrm{f}$.

Chloromonaclina, 174

Choanocystis, 35

Choanotlagellata, 176

Chodat, 22

Chodatella, 179

Chondrioulerma, 53, 54, 55, 62 ; C. testacerm, $54 f$.

Chondropus viridis, 33

Christoplers, 257, 272

Chromatella, 80

Chromiclia, in Heliozon, 18 ; in Lobosa, 71 ; in Proteomyxa, 1 ; in Radio. laria, 121

Chromononadiclea, 173

Chromulina, 157, 174; $C$. rosanofi, 173

Chrysamoeba, 173, 174

Chrysococcus, 161, 174

Clirysomoualina, 174; C. loricata, 174 ; C. menbranata, 176 ; C. nuda, 174

Chrysopyxis, 174

Cienkowski, on Chlamy. domyxa, 280 ; on Heliozoa, 29,35 ; on Labyrinthula, 283; on Mastigophora, 190 ; on Mycetozoa, $38,43,61,66$; on Protozoa, 4, $10 f$., 13 ; on Radiolaria, 97,152

Cienkozvskia, Heliozoa, 34 ; Mycetozoa, $6 \mathbf{2}$

Ciliophrys, 4, 8, $10 \mathrm{f}$. ; $C$. infusionum, $10 \mathrm{f}$.

Cimex rotundatus, 259

Circoporidae, 150 
Circoporius sexfuscinus, 112,117

Cistidium inerme, $105 f$.

Cladomonas, 168

Cladophora, 11

Cladoscenium tricolpium, 147

Cladothrix, $72 f . ; C$. pelomyxae, 81

Classification of Haemoflagellates, 248 ; of Heliozoa, 33 ; of Lobosa,Gymnamoebida, 77 ; Thecamoebida, 84 ; of Mastigophora, 154; of Mycetozoa, 61 ; of Proteomyxa, 6 ; of Radio. laria, 144 ; of Xenophyophoridae, 286

Clastoderma, 63

Clathrocyclas craspedota, 147

Clathrulina, 25, 29, 34 ; C. elegans, $16 f$., 23

Closterium, 32

Clypeolina, 90

Coccolithophorinae, 176

Coccolithopora, 176 ; leptopora, $175 \mathrm{f}$.

coccoliths, 174

Cochliopocliidae, 84, 85, 88

Cochliopodium, 71, 80, 88 ;

C. actinophorum, 88;

C. digitatum, $88 ; C$. pellucidum, $88 \mathrm{f}$.

Cochlodiniuin, 185

Codonoeca, 167

Codosiga, 177 ; C. um. bellata, $178 f$.

Coelodendridae, 150

Coelodendron ramosissimum, 150 ; C. gracillimum, $105 f$.

Coelographidae, 150

Coelomonas, 174

Coeloplegma murrayanum, $151 f_{\text {. }}$; C. tritonis, 151 Coelothammus davidoff, $151 \mathrm{f}$.

Coenobia, 181

Colacium, 171

Coleochaeta, 11

Collodaria, 113

Collodictyon, 169

Collosphaera, 111 n., 121, 123 ; C. huxleyi, $140 \mathrm{f}$. ; C. murrayana, 145

Collosphaeridae, 104, 145

Collozoum, 98, $139 \mathrm{f}$; $C$. fulvum, 138 ; C. inerme, 103 f., 126 f., 138,
$141 f_{.,} 142 f_{.,} 145 ; C . \mid$ polypodia, $72 f_{.}, 73$; pelagicum, 145 ; C. D. radiosa, $78 f ., 79$ radiosum, 138 Damonia reevesii, 254

Colpodella, 3, 5, 10, 11 ; Dangeard, P. A., 3, 13 C. pugnax, $10 \mathrm{f}$.

Colponema, 168

columella, 53

Comatricha, 52 n., 55, 62

Concharidae, 150

Contractile vacuoles in Heliozoa, 18 ; in Lobosa, 85 ; in Mastigophora, 160 ; in Mycetozoa, 49; in Proteomyxa, 3 ; in Radiolaria, absent in Thalassicalla, 97

Convoluta, 22 ; C. roscoffensis, 129, 180

Copromonas, $160 \mathrm{f}$., 161, $162,163,171,172,177$; C. subtilis, 172

Copromyxa, 65 ; C. protea, $60 f$.

Cornutella clathrata, 147

Cornuvia, 64

Cortina typus, $107 \mathrm{f}$.

Cortiniscus typicus, 146

Corycia, 80, 89

Costia, 157, 168; C. necatrix, 169

Craig, C. F., 92

Craspedomonadina, 177 ; C. loricata, 177

Craspedotella, 190

Craterium, 44, 54, 55, 62 ; C. pedunculatum, $54 f$.

Crawley, 23, 35

Cribraria, 54, 63

Crithidia, 228, 240, $241 f$. ; C. campanulata, 245 ; C. fasciculata, 242, 248

Cryptoglena, 171

Cryptomonadina, 176

Cucubalus kochii, 249

Cucurbitella, 89

Culex pipiens, $202 f$., 233, 243

Cyathomonas, 176

Cyclonexis annularis, 158

Cyclopterus lumpus, 247

Cylindrospermum, 11

Cyphoderia, 90

Cyrtocalpis obliqua, 147

Cyrtoidea, 114, 147

Cystoflagellata, 188

Cytocladus spinosus, 115 $f ., 144$

Dactylococcus, 179

Dactylosphaera, 79
Danilewsky, 195, 255, 269

Delage, 39

Delap, 153

Dendromonas, 158

Desmothoraca, 23, 25, 34

Diachaea, $62 ; D$. elegans, 45

Dianema, 64

Diaphorodon, 90

Diatoms, 11

Dictydiaethalium, 63

Dictydium, 54,$63 ; D$. umbilicatum, $55 \mathrm{f}$.

Dictyocephalus ocellatus, 147

Dictyomyxa, 10

Dictyophimus clevei, 147

Dictyopodium, 127

Dictyosteliaceae, 60, 65

Dictyostelium, 60, 65

Dictyota, 114

Didymiaceae, 56,62

Didymium, 44, 55, 56, 62 ; $D$. difforme, $43 f$.; $D$. effusum, $57 \mathrm{f}$.

Difflugia, $71 f ., 72,84$, $85,86,89 ; D$. globosa, 86 ; D. pyriformis, $89 f$.; D. urceolata, $86,87,88$

Difflugiidae, 89

Dimastigamoeba, 165

Dimorpha, 164, 165

Dinamoeba, 68, 80

Dinema, 171

Dinobryon, 157, 158, 161, 173,$174 ; D$. sertularia, $165 f$.

Dinoflagellata, 182

Dinophysidae, 187

Dinophysis, 187

Diploconidae, 146

Diploconus, 146

Diplomita, 168

Diplophrys, 283 ; $D$. Archeri, 283; D. stercorea, 283

Diplophysalis, 4, 5, 8

Diplosiga, 177

Discoidea, 145

Discorbina, 112

Discosphaera, 176 ; D. tabifer, $175 f$.

Distephanus speculum, 191

Distigma, 171

Distomatina, 169

Dobell, 160 n., 163, 172, 192 
Doflein, F., 13

Donovall, 256, 272

Dopter, 82, 92

Dorataspiclae, 146

Dorataspis, 127

Dourine, 196, 197, 206

Dreyer, 116, 131, 152

Dunı-dum fever, 256

Durham, H., 240

Dutton, 196, 255, 269

Echinomna leptodermum, 145

Echinostelinm, 63

Eitobiella, $3 \mathrm{f}$., 12

Ehrenberg, 17, 112, 151, 155

Eikenia, 80

Elaeorhanis, 14, 22, 23, 34

Elaster, 35

Elpatiewsky, W., $93 n$.

Enchylema, 69

Endamoeba, 68, 71, 82 ; E. blattae, 74 f., 83, $84 f . ; E$. coli, 73, 74, T5, $82 f$. ; E. histolytica, $75,82,83 f$. F . iurai,

83 ; $E$. undulans, 83

Eudosporeae, 40, 57

Endyonema, 2, 3, 5, 12

Enerthenema, 62

Eingler, 191

Enteridium, 63

Enteromyxa, 3, 12

Entocannula hirsuta, 148

Entosiphon, 171

Essox lucius, 255

E'strella, 33

E'ucecryphalus, 128

Encoronis nephrospyris, 146

Eucyrtidium, 127 ; $E$. cranioides, $108 \mathrm{f}$.

Eudorina, 181, 182

Éuglena, 157, 161, 171 ; E. acus, 166 f.; $E$. gracilis, $172 ; E$. viridis, $166 f ., 172$

Englenina, 171

Euglenoidea, 170

Euglenopsis, 171

En-niycetozoa, 39

Euplasmodida, 39, 40, 43

Euphysetta nathorsti, 148

Entreptia, 171

Evans, G., 195

Exosporeae, 40, 57, 64

Exuviaella, 186 ; marina, 127

Fanintzin, 58, 66, 98, 129, 152
"Fingersand Toes" disease, 3

Flagellata (Mastigophora), 155

flagellulae, 4, 5, 29

Flovers of Tan, 47

Forde, 196

Fowler, 113, 152

Fraulęa, 254, 269

Frenzel, 91

Frenzelina, 90

Fuligo, 50, 55, 56, 57, 62, $65 ; \quad F$. scptica, $40 f$., $47,57 \mathrm{f}$.

Gamble, 22, 35, 99, 110, $129,153,180,192$

Gametocytes, 25

Gasteromycetes, 40

Gazelletta, 149

Geddes, 274, 280

Giemsa, 196

Glaeocystis, 180

Glenodiniidae, 186

Glenodinium cinctum, 184 f. ; G. pulvisculus, 187

Gloidium, 2, 3, 5, $6 f$.

Glossina, 200 ; $G$. fusca, 199 n., 200, 201; $G$. morsitans, 199 ; G. pallidipes, 199 n.; G. palpalis, 199, 200, 231 ; $G$. tachinoides, 200

Gluge, 195

Goebel, 13

Goës, 284, 286

Goldschmidt, R., 91, 163, 164,192

Golenkinia, 33, 179

Gomphonema, 7

Gonizm, 158, 182 ; G. pectorale, $158,166 f$.

Gonyaulax, 187

Grassia, 164

Gray, 199, 200, 203, 230, 269

Greeff, 22, 24

Greenwood, 49, 66

Greig, 199

Grenacher, 23, 28, 35

Gruber, 78

Gruby, 269

Gubernaculum, 159, 194

Guttulina, 60, 65

Guttulinaceae, 65

Gymnamoebida, 77

Gymnococcus, 3, 5, 11

Gymnodinium, 183, 184

Gymnophrys, 2, 3, 9 ; G. cometa, $10 \mathrm{f}$.
Gymnosphaera, 23, 33

Gyromonas, 169

Häcker, 113, 117, 122, 153

Haeckel, 1, 13, 104, 110, $119,127,131,152,284$

IIaeckelina, 8

Haematococcus, $180 ; H$. palustris, $166 f$.

Haematononas, 250

Haematopinus, 198, 203

Haematopota, 242, 258

Haemoflagellates, 193 ; biological considerations, 217 ; classification, 248 ; comparative morphology, 207 ; evolution anl phylogeny, 240 ; habitat, 196; historical, 194; Leishman - Donovall Wright bodies, 255 ; lifecycle, 226 ; list of hosts, 162 ; literature, 268 ; multiplication, 222

Halistemma tergestinum, 249

Halteridium, 236, 248

Hanburies, 3

Hanna, 270

Haplococcus, 3, 12

Harper, 65

Hartmann, 159 n., 192

Hartog, E., 13, 68

Hedriocystis, 23, 34

Helcosoma tropicum, 259

Heleopera, 85, 90

Heliophrys, 33

Heliosphaera inermis, $103 f$.

Heliozoa, 14 ; classification, 33 ; food, 18 ; karyokinesis, 25 ; literature, 35 ; nucleus, 25 ; reprodnction, 28 ; skeletal investments, 23 ; struc. ture, 15

Hemiclepsis, 227

Hemidinivem, 183, 184

Hemitrichia, 55, 64; $H$. chrysospora, $56 \mathrm{f}$.

Hérouard, 39

Herpetomonas, 157, 161, $226,240,241,250$; $H$. biitschlii, 245 n.; 11 . bombycis, 245 ; H. culicis, 242 ; $H$. gracilis, 24], 245 ; II. jaculum, 241 ; II. levvisi, 195; II. minuta, 241 ; H. muscaeInnesticae, 232, 241 ; II. sarcophagae, $245 ; H$. subuluta, $241 f$. 
Hertwig; on Heliozoa, 15, 24 f., 25, 27, 31, 32, 35 ; on Lobosa, 75, 87, 91; on Ralliolaria, 114, $115,123,127,152$

Heterodermaceae, 63

Heterodivium, 187

Heteromastigina, 248

Heteroinastigoda, 167

lieteromastigote, 158

Heterophrys, 21，22，28, 34, $164 ;$ H. Fockei, 24 $f$. ; II. myriopoda, $16 f$., 23

Hexacontium enthacanthium, 145 ; $H$. pachydermum, 145

Hexaconus, 146

II exadoras borealis, 145

Hexalaspidae, 146

Hexalonche philosophica, 145

Hexamitus, 157, 162, 169 ; $H$. inflatus, $178 \mathrm{f} . ; H$. muris, 170

Hexaplagia arctica, 147

Hickson, 162, 192, 194 n.

Hieronymus, 274, 276, 280

Hinde, 153

hip-paraplegia, 206

Hippobosca rufipes, $199 \mathrm{f}$.

Hirmidium, 158, 177

Histioneis, 188 ; H. cymbalaria, $184 f$.

Hofinann, 273

Holmes, $206 n$.

Holomastigoda, 164

holornastigote, 158

Holopsamma, 286

Homokaryota, 68

Hoogenraad, 13

Hosts of Haemoflagellates, list of, 262-268

Huxley, 151

Hyalobryon, 157, 158, 174

Hyalodiscus, 80

Hyalolampe, 34

Hydnum, 40

IIydrochoerus 252

Hydrodictyon, 179

IIydrurus, 176

Hymenomonas, 161, 176

liypnocysts, 4

hypothallus, 54

Idiochromidia, 71

Ijima, 91

Immermaun, 110, 153

Ineffigiata, 179

isomastigote, 158
Jahn, $42,65,66$

James, 272

Jenkinson, 280

Jennings, 70, 91

Johnstone, 129, 153

Jürgens, 92

Kala-Azar, 256

Karawiew, 123, 152

Karyokinesis, in Actinosphaerium, 25 ; in Lobosa, 73 ; in Mastigoplora, 161 (Noctiluca, 190); in Mycetozoa, $46 f ., 48$, 65 ; in Proteomyxa, 2 ; in Radiolaria, 126 ; in a Trypanosome, 213

Keeble, 22, 35, 99, 110, $129,153,180,192$

Kempner, 198, 272

Kent, 195

Keuten, 172

Keysselitz, 198, 229, 270

Klebalın, 35

Klebs, 127, 153, 161, 165, 172

Koch, 200, 203, $251 n_{\bullet}$, 270

Kofoid, 182, 187, 192

Kränzlin, 65

Krohn, 188 .

Krukenberg, 50, 66

Krzysztalowicz, 273

Labyrinthula, $39, \quad 276$, 280 ; L. cienkoroskii, 280 ; L. macrocystis, $280,282 f$. ; L. vitellina, $129,280,282 f$.

Labyrinthuleae, 39

Lachnobolus, 64

Lamblia intestinalis, 170

Lampoxanthium miurrayanum, 144

Lamproderma, 62

Lamprosporales, 63

Lailg, A., 13

Lankester, on Chlamydomyxa, 274, 277, 280 ; on Haenoflagellates, 195 , 270 ; on Heliozoa, 17, 36 ; on Labyrinethula, 283 ; on Lobosa, 80 ; on Mastigophora, $158 n$., $162,168 n$. ; on Myce. tozoa, 67 ; on Radiolaria. 152

Lareoidea, 145

Laveran, 196, 204, $206 n$., 207, 218 n., 245, 252, $268,270,272,273$
Lebailly, 270

Lecquererusia, 89 ; $\quad L$. spiralis, $89 \mathrm{f}$.

Leeuwenhoek, 155

Léger, 196, 198, 215, 217 n., 226, 229, 230, $234,240,242,245,247$, 257 n., 258, 270, 271

Leidy, 23

Leishman, 196, 257, 272

Leishmania donovani, 232 256, $257 f$. ; L. tropica, $257 f$. 259

Leishman-Donovan-Wright bodies, 255

Leocarpus, 62

Lepidoderma, 56, 62 ; $L$. tigrinuum, $54 \mathrm{f}$.

Lepocinclis, 171

Leptodiscus, 190

Leptomonas, 165

Leptophrys, 2, 3, 4 f., 5, 8

Lesage, 82, 92

Lesser, 15, $24 f$.

Lethodiscus microporus, 145

Lenciscus erythrophthalmus, 249

Lewis, 195

Leyden, 91

Leydenia, 84 ; L. gemmipara, 84

Licea, 63 ; L. pusilla, 56

Liceaceae, 63

Lieberkiihn, 84

Life-history of Chlamydomyxa, 274 ; of Haemoflagellates, 226 ; of Heliozoa, 15 ; of Lobosa, 75 ; of Mastigophora, 155 , $162,164,172,180,189$; of Mycetozoa, 40, 58, 59 ; of Proteoniyxa, 3 ; of Radiolaria, 104, 111

Lignières, $218 n_{\bullet}, 219,271$

lime-knots, 55

Lindbladia, 63

Lingard, 271

Lingbya, 2, 8

linin, 18

Lister, A., 61, 67

Literature, of Chlamydomyxa, 279 ; of Hæmoflagellates, 263 ; of Heliozoa, 35 ; of Labyrinthula, 283 ; of Lobosa, 91 ; of Mastigophora, 191 ; of Mycetozoa, 66 ; of Proteomyxa, 13 ; of Radiolaria, 151 ; of Xenophyophoridae, 286 
Lithamocba, 80 ; L. discus, $80 \mathrm{f}$.

Lithelius arborescens, 145 ; L. minor, 145

Lithocircus anutaris, 105 $f$., 146

\section{Lithocolla, 34}

Lithogromia silicea, 148

Litholophus, 111

Lithomelissa setosa, 147 ;

- L. thoracites, 147

Lithosphccerella, 34

Lobosa, 68 ; cliromidia, 71 ; classification, 77 ; literature, 91 ; nucleus, 70 ; reproluction, 72

Lohn1amn, 174, 192

I.ophomonadina, 170

Lophomonas blattarum, $178 \mathrm{f}$.

Lotsy, 192

Lülue, 245, 247, 268

Lycogala, 56, 64

Lycogalaceae, 64

\section{Mallomonces, 161, 176}

Margarita, 64

Margaritaceae, 64

Martini, 91

Mastigamoeba, 160, 164 ; M. schulzei, 164

Mastigella, 156, 163, 164, 177 ; M. vitraea, $159 \mathrm{f}$., $164 \mathrm{f}$. ; $M$. vitrina, 164

Mastigina, 160, 164; $M$. setosa, 164

Mastigophora, 11, 155 ; classification, 163 ; halit, 157 ; literature, 191; nucleus, 161 ; nutrition, 157 ; structure, 158

Maupas, 20

Maupasia, 159, 170

Mayer, 195

M'Neal, 217, 218 n., 223, $227,240,242,244,245$, 253,271

Medusetta tiara, 148

Medusettidae, 148

Megastoma, 157, $169 ; M$. entericum, 169

Menoidium, 171

Mereschkowsky, 13

Mesenterica, 44

Mesnil, 91, 196, 201, 206, 207, 218 n., 245, 252, 272,273

Mesoscena, 114

Metschnikoff, 50, 67

nicrocysts, 42

Microglena, 161, 176
Mikrogromia, 39, 281

Minchin, 159 n., 192, 196, 199, 200, 201, 203 n., $230,231,233,246,271$

Mitrophanow, 195, 271

Monadina, 38, 248

Monadineae, 5, $6 n$.

Monadopsis, 8

Monas, 166, 167

Monera, 1

Monobia, $3,5 f ., 6,15$

Monocercoinonas, 169

Monolabis, 22

Monomastigorla, 165

Mouomastigote, 158

Monomastix, 159, 170

Monophyes gracilis, 249

Monopodium, 8

IIonopylaria, 103, 107

Monostomatina, 169

Monticelli, 13

Moore, $159 n$., 192, $206 n$.

Mucorinae, 40

Mugliston, T. C., 93

Miiller, Johannes, 131, 151

Multicilia, 160, 163, 164 ; M. lacustris, 164

Murray, J., 13, 113, 192

Murrayella, 187

Musgrave, W. E., 93

Mycetozor, 37 ; classification, 61 ; life-cycle, 42 ; literature, 66

Myxastrum, 3, 5, 8

Mysodictyum, 11

Myxodiscus crystalligerus, 33

Mysosphaera coerulea, 140

Nabarro, 199, 252

Nadinella, 90

Nagana 195, 197, 198

Nassellaria, 107, 113, 114

Nationaletta, 149

Nawaschin, 11, 13

Nebela, 85, 87, 90

nebenkörper, 70

Nejveu, 196

Neresheimer, 71, 91

Neusina agassizii, 284

Noctiluca, 162, 188, 190 ; N. miliaris, $161 \quad f ., 189$ $f_{\text {. }}, 190 f_{.}, 191 f$.

Novy, 200 n., 217, 218 n., $227,239,240,242,244$, $245,253,271$

Nivclearia, 8, 9, 14, 15, 23, 33 ; $N$. delicatula, $10 f$.

Nuclei in Chlamydomyxa, 274 ; in Haemoflagellates, 194,212 ; in Heliozoa,
18; in Actinosphaerium, 25,32 ; in Labyrinthula, 280 ; in Lobosa, 70,86 ; in Mastigoplıora, 161 ; in Mycetozoa, 48, 59 ; in Proteomyxa, 2 ; in Radiolaria, 94, 107, 110, 120

Oat-shaped corpuscles, 39 , 274

Ochromonas, 157, 174

Oedogonium, 8

Oicomonas, 157, 165; 0 . mutabilis, 166 f. ; 0 . terno, $166 f$.

Oligonema, 64

Olive, $60,66,67$

Orcadella, 63

Ornithocercus, 186, 187 ; O. magnificus, $184 \mathrm{f}$.

Orosphaera, 122, 144

Orosphaeridae, 144

Ostenfeld, 36

Ouramoeba, 78 f., 79

Oxyrrhis, 163 n., 168, $184 \mathrm{f}$.

Oxytoxum, 187

Palmella, 180

Palmella stage in Zooxanthellae, 98 ; in Flagellates, 156

Palmodactylon, 180

Palmodictyon, 180

Panulorina, 182

Pantostomatina, 164

Paramastigoda, 167

paramastigote, 158

Paramecium, 20 ; I'. cos. tatum, 195 ; 1 '. lorica. tum, 195

Paramoeba, 79 ; P. eillarili, $70 f_{.}, 73,75,79,83$; P. hominis, 79, 83

Paramoecoides, 250

Paranema, $171 ; P$. trichophorum, $166 f$.

Paranemina, 171

Parmulina, 89

Patton, 242, 259, 271, 273

Pediastrum, 179

Pelomyxa, 2, 68, $70 \mathrm{f}$., 71, 73, 75, $76 f ., 81 ; P$. palustris, $72 \mathrm{f} ., 76 \mathrm{f}$, $81 f_{\text {: }}: P$. penardi, 81 ; I'. villosa, 81 ; $P$. viridis, 81

Penard, E., 13, 22, 23, 33, $36,39,67,91,274,277$, 278,280

Penardia, 3, 9 
I'erichuena, 64

Peridiniaceae, 185

I'eridinium, $186187 ; P$. divergens, $186 f$.

Peripylaria, 102

Perrin, 273

P'etalomonas, 171

Pliaeoconchia, 150

P'haeocystina, 147

Pliacorlaria, 108, 113

Phaeogromia, 148

J'hreosplucera, 176

Phaeosphaeria, 148

Phalacroma, 188

Phalansteriina, 177

Phalcusterium, 158, $163 n$. 177 ; $P$. consociatum, 166 $f . ; P$. volvocis, 168

Pharyngella gastrula, 148

Phormobotrys hexathalonia, 147

Phorticium pylonium, 145

Phractopeltidae, 146

Phryganella, 90

Phyllomitus, 167

Phyllomonas, 167

Phyllostaninus, 146; $P$. quadrifolius, 146

Pliysaraceae, 56, 62

l'hysarella, 62

Physarum, 52 n., 55, 62 ; $P$. mutans, $54 f$.

Physematiidae, 104, 144

Physematium, 121; $P$. mülleri, 144

I'hysomonas, 167

I'hythelius, 33

Phytoflagellata, 177

l'inaciophorce, 24, 34

Pinacocystis, 21, 24, 34

I'iroplasma, 256; $P$. donovani, 243, $257 f$., 259

Plagiacantha arachnoides, 147

Plagiocarpa procyrtella, 147

Playoniscus tripodiscus, $107 f$.

l'lanktonetta atlantica, $120 f$., $149 f$.

I'lasmodiocarps, 56

Plasmodiophora, 2, 3, 4 n., 5,11

I'lasmodium, in Labyrinthula, 282 ; in Lobosa, 72 ; in Mycetozoa, 43, 57 ; in Proteomyxa,3

plasson, 2

plastogamic fusion, in Heliozoa, 19; in Lobosa, 88
Plate, 190

Platnaspis, 146

Platoum, 90

Platydorina, 158, $182 ; P$. cauluta, $182 f$.

Platythecu, 167

Plectellaria, 147

Plectoillea, 147

Plectophora arachnoides, $147 ; I^{\prime}$. novena, 147

Plehn, 271

Plenge, 42, 67

Pleodorina, 181, $182 ; P$. illinoisensis, $183 \mathrm{f}$.

Pleurococcus, 179

Pleuromonas, 167

Plimmer, 206 n., 269

l'odolampas, 187

I'olykrikos, 185

Polymastigina, 169

polymastigote, 158

Polyoeca, 177

Polyplagia novenaria, 147

Polyporus, 40

P'olysphondylium, 60, 65 ; P. violaceum, $60 \mathrm{f}$.

Polytoma, 177 ; $P$. urella, $178 f$.

Pompholyxophrys, 22, 24, 34

Pontigulasia, 89 ; P. incisa, $89 \mathrm{f}$.

Pontobdella, 204, 224, 228

Pontomyxa, 9; P. flava, 9 ; $P$. pallicla, 9

Pontosphaera, 176 ; haeckelii, $175 \mathrm{f}$.

Popowsky, 136, 153

Porocapsa, 146 ; P. murrayana, 146

Porospathiclae, 148

Poteat, W., 91

Poteriodendron, 158, 161, 168

Pouchetia, 185

Prantl, 192

Pricolo, 171

Proales, 23

Prorocentraceae, 185

I'rotanoeba, 2, 5, 6

Proteomyxa, 1

1'roterospongia, 158, 177 ; $P$. haeckelii, $178 f$.

Protobathybius, 12

Protoceratium, 187

Protococcus, 180

Protocystis harstoni, 148 ; $P$. tridens, 148 ; $P$. tritonis, $148 ; P$.xiphodon, 148
Protogenes, 5, 9 ; P. primordialis, $9 f$.

Protomastigina, 165

Protomonas, 11, $60 ; P$. amyli, 38 ; $P$. parasitica, 38

Protomyxa, 3, 4, 7 f., 11 ; $P$. parasitica, $10 f$.

Prototrichia, 64

von Prowazek, on Haenoflagellates, 198, 205, 212 , 223, 229, 231 n., 232, 257 n., 271 ; on Heliozoa, 35 ; on Mastigophora, 159 n., 163, 192 ; on Proteomyxa, $4 n$.

Prunocarpus datura, 145

Prunoilea, 145

Prunophracta, 146

Psammetta, 286

Psammina globigerina, $285 f$.

I'samminidae, 286

Psanmopemma, 286

P'seudamphimonas, 11, 12

Pseudochlamys, 89

Psendoclifflugia, 90

Pseudopodia, of Heliozoa, 23 ; of Lobosa, 85 ; of Nastigophora, 160 ; of Proteomyxa, 3; of Radiolaria, 96, 106

Pseudospora, 3, 5, 8, 163

Pseudosporidium, 10, 12

Ptychodiscidac, 187

Ptychodiscus nocticula, 187

pulselluni, $158 n$.

Piitter, 98, 153

Pyramidomonas (=Pyramimonas), 179, 180

Pyrophacus, 187

Pyxidicula, 91

Quadrula, 84, 85, 89 ; Q. irregularis, 84

Quatrefages, 189

Rahinowitscl, 198, 272

Radiolaria, 94 ; biononics, 96 ; central capsule, 114 ; classification, 144 ; cytoplasm, 116 ; distribution, 112 ; food, 97 ; literature, 151 ; nuclens, 120 ; reproduction, 136 ; skeleton, 130 ; variation in, 110 ; yellow cells, 126

Raphidomonas, 174

Remak, 195

Reproduction, in Chlamydomyxa, 275 ; in Hae- 
moflagellates, 222 ; in Heliozoa, 19, 28 ; in Lobosa, 72 ; in Mastigophora, 156 ; in $\mathrm{My}$ cetozoa, 41 ; in Proteomyxa, 4.; in Radiolaria, 99,136

Reticularic, 52 n., 56, 64 ; $R$. lycoperilon, $42 f$.

Reticulariaceae, 63

rhabdoliths, 174

Rhabdomonas, 171

Rhabdosphaera, 176

Rhaphidiophrys, 22, 24, 28,34 ; $R$. elegans, $35 f$. ; $R$. pallida, $16 \mathrm{f}$; $R$. viridis, 22,23

Rhaphidocystis, 24, 34

Rhipidodendron, 158, 168

Rhizonnastigorla, 164

Rhizoplasma, 9

Rhizoplegma boireale, 145

Rhumbler, 5, 6 n., 13, 70, 85,91

Rhynchomonas, 168

Robertson, 163, 169, 192, 204, 214 n., 216 n., 224, $228,229,272$

Rogers, 226, 257, 2خ2, 273

Romanowsky, $196^{\circ}$

Roubaud, $203 n$., $231 n$.

Ross, 240, 242, 272

Ruppia, 3

\section{Sagena ternaria, 148}

vigenoarinm, 148

S'crgosphaera trigonilla, 148

Sagosphaericlae, 148

Sulpingoece, 161, 177 ; S. fusiformis, $178 t ; s$. urceolata, $178 \%$.

Saltonella, 80

Sannclers, 49

Schaudinn, on Haemotlagellates, 196, 198, 202, $205,212,217,226,235$, 241 n., 248 n., 258, 272, 273 ; on Heliozor, 27, $29,32,34,36$; on Lobosa, 70, 75, 87, 88, 92 ; on Mastigophora, 163,192 ; on Proteomyxa, $6 n$.; on Ralio. laria, 153

Scheel, 74, 92

Schewiakoff, 118, 131, 153

Schizochlamys, 180

Schizogenes, 12

Schleimcysten, 232

Schleppgeissel, 194, 218
Schneider, $5 f$., 13, 31, 32, 205, 269

Schröder, 122, 153

Schuberg, A., 92

Schubotz, 92

Schultze, 285, 286

Schitt, 187, 192

Sclerotium, 44, 50

Scyphosphaera, 176 ; apsteini, $175 \mathrm{f}$.

Scytomonas, 171

Selenastrum, 179

Senn, 192, 212, 272

Sergent, ] 96, 226, 272

Siedlecki, 273

Siphonosphaera, 121, 123

Siphoptychivem, 63

Smith, 31, 36

Sorokin, 13

Sorophora, 39, 59, 65

Sphaerastrum, 28, 34

Sphaerella, 179, 180 ; $S$. palustris, $166 f$.

Sphaerellaria, 106, 113, 114

Sphaerocapsa, 146 ; S. cruciata, 146

Sphaerocapsidae, 146

Sphaerocystis, 180 ; $S$. Schröteri, 22

Sphaerocca, 158, 177

Sphaeroidea, 145

Sphaerophracta, 146

Sphaeropylidea, 145

Sphaerozoa, 102, 104, 145

Sphaerozoidac, 104, 145

Sphaerozoum neapolitanum, $138,141 f$. ; S. ovodimare, 145

Sphenomonas, 171

Spirilla, 195

Spirochaetc evansi, 195

Spirodinium, 183, 185

Spirogyra, 7, 8, 32

Spiroidea, 147

Spironema, 170

Spirula, 11

Spongodiscus favus, 145

Spongomonas, 168

Spongosphaera streptacan. tha, $105 f$.

Sporangia, 50

Spores in Mycetozoa, 53 ; in Proteomyxa, 4

Sporophore, 58

Spumaria, 56, 57, 62 ; S. $a l b a, 54 f$.

Spumellaria, 113

Staborgan, 188

Stahl, 61, 67

Stannarium, 286
Stannoma, 284, 286

Stannomida, 286

Stannophyllum, 284, 286 ;

S. zonarium, $284 f$.

Statham, 257, 272

Steel, 195

Stegomyia, 240

Steiniella, 187

Stemonitaceae, 53, 62

Stemonitis, 62 ; S. ferruginea, $55 f_{0}$; S. fusca, $41 f$., 57 ; S. splendens, $55 f$.

Stephanosphaera, 158, 182

Stephoidea, $\mathbf{1 4 7}$

Stereum, 49

Sterromonas, 167

Stichogloea, 176

Stolě, A., 92

Stomoxys calcitrans, $199 \mathrm{f}$.

Strasburger, $52 n ., 67$

Streptomonas, 168

Stuhlmann, 200, 203, 216, $230,231,272$

Stylamoeba, 80

Stylochrysalis, 157, 163 n., 174

Swingle, 225 n., 272

Symbiotic Algae (Peridinians), 94

Syncrypta, 173, 176 ; $S$. volvox, $166 f$.

Synura, 157, 161, 176

Syracosphaera, 176

Syracosphaerinae, 176

Tabanus, 242 ; T. lineola, $199 f$.

Tansley, 180, 192

Tanypus, 245

Tetramitus, 157, 169 ; $T$. rostratus, $178 f . ; T$. snlcatus, $178 f$.

Tetramyxa, 2, 3, 11

Tetraspora, 180

Thalassicolla, 94, 113, 114, 123 ; $T$. pelagica, $95 \mathrm{f}$. ; T. pellucilla, 144; $T$. nucleata, 98, 99, $101 f$., $103 f$., 144 ; $T$. spumida, 144

Thalassicollidae, 104, 144

Thalassiosolen atlanticus, 144

Thalassolampe, $121 ; T$. margarodes, 144

Thalassophysa, $120 ; T$. papillosa, 144 ; $T$. pela. gica, $138 \mathrm{f}$., 144; $T$. sanguinolenta, $128 f$., 
$138 f ., 144 ; T$. spiculosa, $138 f$.

Thalassophysiltae, 104, 144

Thalassothamniclae, 144

Thalassothamnus, $122 f$. ; T. ramosus, 144

Thecamoebida, 68, 84

Theoconus ariadnes, 147

Thiroux, $218 n$., 239, 272

Thread-plasmodium, 39 , 281

Todd, 255

Töpfer, $237 n$.

Topsent, 13

Torrey, $237 n$.

Trachelomonas, 161, 171

tractellum, $158 n$.

Trepomonas, 169

Trepospyris cortiniscus, $107 \mathrm{f}$.

Trichamphora, 62

Trichia, 52 n., 64; $T$. fallax, 52 n., $53 ; T$. varia, $53 f$. $56 f$.

Trichiaceae, 44, 55, 64

Trichomastix, 157, 169

Trichomonas, 157, 160, 162,$169 ; T$. intestinalis, 169

Trichosphaerium, 22, 68, $72,73,75,80,102,127$

Tridictyopus, 115 ; T. elegans, 147

Trigonomonas, 169

Trimastigina, 169

Trimastix, 169

Triposolema, 188

Tripylaria, 102, 108, 147

Trochiscia, 179

Trochodiscus echiniscus, 145 ; $T$. heliodes, 145

Trophochromidia, 71

Trophonucleus, 214

Tropidoscyphus, 171

Truncatulina, 112

Trypanomonas, 250

T'rypanomorpha, 167, 198, 238, 248

Trypanomorphidae, 167, 248

Trypanophis, 168, 209, $211,213,216,249 ; T$. grobbeni, 211, $249 f$., 250

Trypanoplasma, 168, 209, $211,217,249$; T. borreli, $210 f ., 211,215 f_{\text {. }}, 216$, 217,249 ; T. cyprini, $210 \mathrm{f}$., 211, 249 ; $T$. intestinalis, 247, 249, $250 \mathrm{f} . ; \mathrm{T}$. ventriculi,
247, 249; T. varium, 249

Trypanosona, 157, 163, $168,177,248,250 ; T$. avium, $208 f_{\text {., }} 217,253$; T. brurbatulae, 215, 227, 228, 230 ; $T$. boneti, 254 ; T. brucii, 195, $199 \mathrm{f}$., $200,201,203,208 f$., 209, 214, 215, 216, 217, $220 f_{\text {. }}, 221 f_{\text {. }}, 223 f_{\text {., }}$, 231,233 ; T. carctssii, 255 ; T. cobitis, 255 ; $T$. costutum, $254 ; T$. damoniae, $208 f$., 254 ; $T$. danilewskyi, $204 ; T$. dimorphon, 253 ; $T$. duttoni, $205 ; T$. elegans, 255 ; $T$. elmassiani, 253 ; T. equinum, 197, $199 \mathrm{f}$., $205,208 f$., 217, $220 f$., $221 f_{\text {., }} 224 f_{\text {. }}, 251 f_{\text {., }}$ 253 ; T. equiperdum, 197 n., $205 f_{\text {. }}, 209$, $224 f_{\text {. }}, 251 f_{\text {. }}, 253$; $T$. evansi, $199 f ., 253 ; T$. flesi, 255 ; T. gambiense, 196, 197 n., 199, 200, 203, $208 f_{\text {. }}, 220 f ., 221$ $f ., 230,251 f ., 252 ; T$. granulosum, 204, 209, $210 \mathrm{f}$. 228,255 ; $T$. grayi, 200 n., 201, 215, $216 f$., $224 f$., 231, $232 f$., 233, 245, 246, 251; $T$. hannae, $208 f$., 209, 216, 253,254 ; $T$. inopinatum, $210 f$., 216, 255 ; $T$. johnstoni, $214 \quad n$., $253 f$., 254 ; $T$. karyozeulton, $210 f ., 212$, 255 ; T. lewisi, 197, 198, 203, 204, 205, $207 f$., $208 f ., 210,211$ f., 214 , 216, 217, $219 f ., 222$, $225 f_{\cdot}, 226 f_{.}, \quad 229$, 251 n., 252 ; $T$. mega, 212,255 ; $T$. nanum, 209 ; $T$. nelspruitense, $210 f ., 255$; T. noctuae, $198,202,205,208 f$., $213,218,219 f$., 223, $233,242,243,247,248$; $T$. paddae, $253 ; T$. polyflectri, 209 ; T. raiae, $204,209,216,224,228$, 231,255 f. ; T. remaki, $204,209,210 f_{\text {. }}, 216$; T. rotatorium, 208, 209, $210 f_{.}, 216,248$ n., $250 n$., 254 ; T. sanguinis avium, $195 f ., 254 ; T$. scyllii, 204, $255 f$; $T$. soleae, $210 f$., 217 ; $T$. theileri, $199 \mathrm{f}$., 209, 251 n., 253 ; $T$. transvatiense, 216,$253 ; T$. ngandense, $252 ; T$. undulans, $255 ; T$. varium, 227 ; T. ziemanni, 205, $208 f_{\text {. }}, 233,237 f_{\text {. }}, 238$, 253

Trypanosomaticlae, 248

Trypanosomes, 193, $213 f$. Trypanozoon, 248

Tsetse-fly, 196, $201 n$.

Tubulina, 63 ; T. stipitata, 56

Tubulinaceae, 63

Tulloch, 199, 200, 203, 230

Tuscarora nationalis, 122 $f_{.}, 150 f$.

Tuscaroridae, 150

Tuscurusa globosa, $150 \mathrm{f}$.

Ulothria, 156

Ulotrichaceae, 156

Umbilicosphaera, 176

Undulina, 250

Urceolus, 171

Uroglena, 158, 173, 176 ; $U$. ranarum, $166 f$. 194 $f ., 195,254$; $U$. volvox, $166 f$.

Urophagus, 169

Vacuolaria, 174

Vahlkampf, 70, 92

Valentin, 194

Vampyrella, 2, 3, $4 f$., 5, $7 f$., 15, 33

Vampyrellidium, 2, 3, 8

Veley, 71, 81, 92

Vernon, 98, 152

Verworn, 13, 95, 152

Voges, 272

Volvocina, 181

Volvox, 158, 181 ; I. aureus, $182 ; V$.globator, $166 f ., 181$; V. minor, $166 f . ; V$. tertius, 182

Wagnerella, 34

Wasielewsky, 212, 272

Watase, 189

Wenyon, 192

West, G. S., 13, 36, 192 
Wolfenden, 113, 152

Wondcock, 193 n., 268

Woronin, 3, 13, 58, 66, 173,192

Wright, S., 13, 256, 273

Xanthellae, 22

Xenophyophoridae, ?.86
Xiphicantha alata, 128, zooxanthellae, in Radio$142 f ., 143$

Zederbauer, 192

Zoochlorella actinosphaerii, 22

Zoospores, in Mycetozoa, 40; in Proteomyxa, 4

Zooteirea, 33 laria, 97

Zopf, 2, 5, 6 n., 13, 39, $60,61,67,280,281$, 283

Zuelzer, 71, 86, 87, 92

Zygacantha, 146 ; Z. septentrionalis, 146

Zygoselmis, 171 



\section{|||||||||||||||||||||||||||||||||||}


